\section{Epidemiology and Prevention of Cardiovascular Disease}

\author{
Effect of long-term salt substitutes on blood \\ pressure: a meta-analysis of randomized \\ control trails \\ Yaguang Peng, Xiaoxiao Wen, Ying Li, Wei Li, \\ Liancheng Zhao \\ State Key Laboratory of Cardiovascular Disease, Fuwai Hospital, \\ National Center for Cardiovascular Disease, Chinese Academy of \\ Medical Sciences and Peking Union Medical College
}

Objective The objective of this meta-analysis was to evaluate the long-term effects of salt substitutes on blood pressure (BP) reduction.

Methods Following PRISMA guidelines for RCT meta-analysis, studies were identified by systematic search on PubMed, Embase and Cochrane Library, Wan Fang database and CNKI (China National Knowledge Infrastructure) databases, through December 2013. Inclusion criteria were as follows: (1) RCT of salt substitute or low sodium salt as an independent intervention, (2) follow-up period $\geq 6$ months, (3) primary outcome BP, including both systolic blood pressure (SBP) and diastolic blood pressure (DBP). Collected data included the study/trial name, published year, study design, population, intervention duration, substitute salt ingredient, randomize approach, type of blinding, allocation concealment method, statistical analysis principle, mean differences (MD) between intervention group and control group before and after intervention in both SBP and DBP respectively, the standard errors (SE) and $95 \%$ confidence intervals $(95 \% \mathrm{CI})$ of the mean difference. The main outcome of the study was difference in BP reduction (mean $\pm \mathrm{SD} / \mathrm{mm} \mathrm{Hg}$ in SBP and DBP) between the intervention and control groups. A random-effect model, according to the heterogeneity $\left(\mathrm{I}^{2}\right)$, was performed to pool the BP reduction, both in SBP and DBP. Subgroup analysis in hypertensive and normotensive participants, and sample sizes ( $\geq 300$ vs $<300$ participants) were conducted as well. Publication bias was evaluated using the Egger's test and visual inspection of a funnel plot. A sensitivity analysis exploring the effect of a single study on the pooled results was conducted by omitting one study at a time. Metaanalysis was applied using meta procedure by STATA 11.0 (Stata Corp. Texas, USA).

Result A total of 645 articles were identified from Pubmed, Embase and Cochrane Library, and 136 articles from Wan Fang database, 20 articles from CNKI. One hundred and eight duplicates and 670 irrelevant articles, which only appeared search key words, were excluded. The full text of the remaining 23 articles was reviewed and an additional 18 articles were excluded because they did not meet the study design criteria. Therefore, 6 cohorts in 5 articles consisting of 1974 participants (1 trial enrolled two cohorts for independent intervention), with high quality (all over 4 scores), were qualified for this meta-analysis. On the basis of randomized effect models, the pooled results showed that salt substitute had significant effect on SBP [Mean difference (MD) $=-4.9$ $\mathrm{mm} \mathrm{Hg}$; 95\% CI: $-7.3--2.5 ; \mathrm{P}<0.001]$ and DBP $(\mathrm{MD}=-1.5 \mathrm{~mm}$ $\mathrm{Hg} ; 95 \% \mathrm{CI}:-2.7-0.3 ; \mathrm{P}=0.013)$. The heterogeneity was found for the outcomes of SBP $\left(\mathrm{I}^{2}=76.7 \%\right)$ and DBP $\left(\mathrm{I}^{2}=65.8 \%\right)$, respectively. Subgroup analysis according to predefined characteristics indicated a decrease in heterogeneity. In the hypertensive subgroup, the SBP and
DBP were significantly reduced $5.7(8.2-3.2) \mathrm{mm} \mathrm{Hg}$ and 2.4 (4.5-0.2) $\mathrm{mm} \mathrm{Hg}$, respectively (all $\mathrm{P}$ values $<0.05$ ). The potential of lowering $\mathrm{BP}$ was also found in the normotensive subgroup, however, the MD of SBP and DBP were -2.7 (-6.1- 0.8) $\mathrm{mm} \mathrm{Hg}$ and -1.3 (-4.5-1.9) mm $\mathrm{Hg}$ with no statistical significance. The BP reduction in studies with $\geq$ 300 participants sample sizes ranged from $3.0 \mathrm{~mm} \mathrm{Hg}$ in SBP and 0.7 $\mathrm{mm} \mathrm{Hg}$ in DBP $(\mathrm{P}<0.05)$. In $<300$ participants sample sizes subgroup, were $7.3 \mathrm{~mm} \mathrm{Hg}$ in $\mathrm{SBP}$ and $3.1 \mathrm{~mm} \mathrm{Hg}$ in $\mathrm{DBP}(\mathrm{P}<0.05)$. Sensitivity analysis indicated that the pooled effects of salt substitute on SBP and DBP were not change significantly and publication bias assessment showed no significant publication bias in this meta-analysis of SBP and DBP. There was no evidence of publication bias as indicated by funnel plots and Egger's tests ( $\mathrm{P}=0.17$ and 0.22 for SBP and DBP, respectively).

Conclusion This meta-analysis suggested that salt substitute strategy for lowering BP was effective and could lower SBP and DBP. It proved the long-term effect of salt substitute on anti-hypertension. Further long-term RCTs in normotensives to evaluate the effect of salt substitutes on BP are needed to confirm these findings.

\section{Health status, risk factors, and medical conditions among hypertension of different income adults in southwest China \\ Xiaohua Liang ${ }^{1}$, Tingyu $\mathrm{Li}^{1}$, Youxue Liu ${ }^{1}$, Jie Chen ${ }^{1}$, Lun Xiao ${ }^{2}$, Xuejun Tan ${ }^{2}$, Daxing $\mathrm{Li}^{2}$, Daili Lan ${ }^{2}$ 1. Ministry of Education Key Laboratory of Child Development and Disorders, Key Laboratory of Pediatrics in Chongqing, Chongqing International Science and Technology Cooperation Center for Child Development and Disorders, Chongqing, China \\ 2. Centers for Disease Control and Prevention of Jiulongpo District}

Objective To document the health care needs and health risks of different income hypertensions in the developing area of China. These data will help physicians and social medical insurance programs prepare for the possible expansions for low-income hypertensions.

Methods Stratified random cluster sampling was used to choose four communities, including two city and two countryside communities, and 239 hypertensions who had income information were included in the analysis. Data from the national basic public health services and telephone survey were used to analyze health conditions among 128 samples with income no more than 30000 yuan per-year compared with 111 samples with income more than 30000 yuan per year. Control of hypertension, hyperglycaemia and hyperlipemia based on examinations and laboratory tests, measures of self reported health status including medical conditions, income and risk factors such as measured obesity status were analyzed.

Result Compared with those high income adults, low income adults were less likely to have abdominal obesity, hyperlipemia and alcoholic and tea drinking and less likely to have the family history of hypertension, hypercholesterolemia, diabetes and heart disease. They also were less likely to have free medicare and social medical insurance $(18 \%$ vs $65 \%$ ). And $27 \%$ of low income hypertensions compared with $52 \%$ of those high income individuals treated disease at county hospital or grade 3 and first-class hospital $(\mathrm{P}<0.01)$. Besides, low income hypertensions were less likely to take two or more kinds of drugs combining (19\% vs $32 \%$ ), and less likely to have blood pressure controlled. For example, the systolic blood pressure and diastolic blood pressure were lower in low income individuals than that of high income adults (SBP: $138.23 \mathrm{~mm}$ $\mathrm{Hg}$ vs $133.97 \mathrm{~mm} \mathrm{Hg}, \mathrm{P}=0.03$; DBP: $87.03 \mathrm{~mm} \mathrm{Hg}$ vs $79.57 \mathrm{~mm} \mathrm{Hg}$, $\mathrm{P}<0.01$ ). After adjusted covariables of sex, region, education, treating agency, medical insurance and the kinds of drug taking, the effect still 
existed.

Conclusion Compared with adults with high-income, low-income adults are potentially just to have a lower level of medical insurance and medical care. A substantial proportion of low income adults with hypertension did not have good blood pressure control, although intensive medical care of these adults was conducted by general practitioner.

\section{Associations of non-high density lipoprotein cholesterol and traditional blood lipid profiles with hyperuricemia among middle-aged and elderly chinese population}

\section{Juan $\mathrm{Xu}^{1}$, Hao Peng ${ }^{1}$, Qinghua $\mathrm{Ma}^{2}$, Xiaohua Zhou ${ }^{2}$, Wenxin $\mathrm{Xu}^{2}$, Liang Huang ${ }^{2}$, Jiarong $\mathrm{Hu}^{2}$, Yonghong Zhang ${ }^{1}$ 1. Department of Epidemiology, School of Public Health, Medical College of Soochow University \\ 2. The Third People' s Hospital of Xiangcheng District}

Objective To compare the associations of non-high density lipoprotein cholesterol (non-HDL-C) and other traditional blood lipid profiles with hyperuricemia in a middle-aged and elderly Chinese population.

Methods Data was collected from 9580 participants undergoing routine physical examinations in Xiangcheng district of Suzhou. Serum uric acid (SUA), total cholesterol (TC), triglyceride (TG), low density lipoprotein cholesterol (LDL-C), high density lipoprotein cholesterol (HDL-C), and other traditional biomarkers were examined. Non-HDL-C was calculated by subtracting HDL-C from TC. The associations of hyperuricemia with blood lipid parameters were examined in men and women, respectively. The areas under Receiver Operating Characteristic (ROC) curves (AUCs) were compared to assess the discriminatory value of blood lipid parameters for predicting hyperuricemia.

Result All blood lipid parameters significantly correlated with SUA (all $\mathrm{P}$ values $<0.001$ ). The correlation coefficient between SUA and TG was the highest in both genders. The correlation coefficient of nonHDL-C was higher than HDL-C in males and was higher than TC and LDL-C but followed HDL-C in females. In male group, AUC of TG (0.659) was greater than that of non-HDL-C $(0.595)(\mathrm{P}<0.001)$. The AUC values of HDL-C, TC and LDL-C were lower; respectively 0.581 , 0.559 and 0.552 . In female group, AUC was highest for TG (0.678) followed by HDL-C (0.616), non-HDL-C (0.610), LDL-C (0.559) and TC (0.557) (all $\mathrm{P}<0.001)$.

Conclusion In both genders, serum TG has the strongest association with hyperuricemia among blood lipid parameters. Non-HDL-C is also a significant predictor of hyperuricemia.

\section{Research on cigarette smoking and risk factors of cardiovascular and cerebrovascular disease mortality in tianjin: a population based case control study \\ Dezheng Wang, Guohong Jiang, Zhongliang Xu, Hui Zhang, Guide Song, Ying Zhang, Chengfeng Shen, Wei Li Non-Communicable Disease Control and Prevention, Tianjin Centers for Disease Control and Prevention}

Objective The purpose of this study is to explore the distribution characteristics and cigarette smoking, as well as other risk factors of cardiovascular and cerebrovascular disease, and to provide basis for prevention and control of cardiovascular and cerebrovascular disease in Tianjin.

Methods A case-control study was conducted with surveillance data from the all-cause mortality surveillance system maintained by the Tianjin Centers for Disease Control and Prevention (CDC). Cases ( $\mathrm{n}=$ $20408)$ and controls $(n=6839)$ were men aged 18 to 69 years who had died between 2010 and 2012 from cardiovascular and cerebrovascular disease and non-smoking-related causes, respectively. Each record was coded using the International Classification of Diseases (ICD). The data was analyzed by both single factors and multiple factors unconditional logistic regression analysis using SPSS software version 11.5.

Result By single logistic analysis, risk factors of cardiovascular and cerebrovascular disease mortality were as follows: age (Wald $\chi^{2}$ $=3498.072, \mathrm{P}=0.000 ; \mathrm{OR}=1.084,95 \% \mathrm{CI}$ : 1.081-1.087); married patients (Wald $\chi^{2}=7.005, \mathrm{P}=0.008 ; \mathrm{OR}=1.054,95 \%$ CI: 1.014-1.096); lower education (Wald $\chi^{2}=5.528, \mathrm{P}=0.019$; $\mathrm{OR}=0.970,95 \% \mathrm{CI}$ : $0.945-0.995)$; in urban areas (Wald $\chi^{2}=437.640, \mathrm{P}=0.000 ; \mathrm{OR}=0.521$, 95\% CI: $0.490-0.554$ ); current or former smokers (Wald $\chi^{2}=152.754$, $\mathrm{P}=0.000 ; \mathrm{OR}=1.231,95 \% \mathrm{CI}: 1.191-1.273)$; much more cigarette consumption per day (Wald $\chi^{2}=218.467, \mathrm{P}=0.000 ; \mathrm{OR}=1.327,95 \%$ CI: $1.278-1.378$ ); long-term smokers (Wald $\chi^{2}=173.439, \mathrm{P}=0.000$; OR $=1.243,95 \%$ CI: $1.203-1.284)$. By multiple logistic analysis, risk factors of cardiovascular and cerebrovascular disease mortality were much more cigarette consumption per day (Wald $\chi^{2}=111.161, \mathrm{P}=0.000$; OR $=1.235,95 \%$ CI: $1.187-1.284$ ), in urban areas (Wald $\chi^{2}=270.868, \mathrm{P}=$ 0.000 ; OR $=0.546,95 \%$ CI: 0.508-0.586), lower education level (Wald $\chi^{2}=28.215, \mathrm{P}=0.000 ; \mathrm{OR}=0.915,95 \%$ CI: 0.886-0.946).

Conclusion Cardiovascular and cerebrovascular disease mortality was higher and strongly correlated with cigarette smoking. Our study has provided important information to develop smoking free law and establish heath education on risk factors of cardiovascular and cerebrovascular disease that can deliver effective measures and disseminate information for cardiovascular and cerebrovascular disease prevention, in order to decrease the burden of cardiovascular and cerebrovascular disease.

\section{The predictive value of waist-to-height ratio with ischemic stroke in a population- based prospective cohort study among inner mongolian men from China \\ Juan Xu, Yonghong Zhang \\ Department of Epidemiology, School of Public Health, Medical College \\ of Soochow University, Suzhou, China}

Objective The purpose of the current study is to evaluate the hypothesis that waist-to-height ratio (WHtR) is better obesity predictor of ischemic stroke than body mass index (BMI) and waist circumference (WC) in Inner Mongolian men.

Methods A population-based prospective cohort study was conducted from June 2003 to July 2012 in Inner Mongolia, an autonomous region in north China. A total of 1034 men aged 20 years and older free of cardiovascular disease at baseline were selected from two adjacent townships located in the counties of Kezuohou Banner and Naiman Banner. During a mean follow-up of 9.3 years, 47 ischemic strokes were recorded. The subjects were divided into four groups by WHtR $<0.40,0.40-0.50,0.51-0.60,>0.60$. The cumulative survival rates of ischemic stroke among the four groups were estimated with 
the Kaplan-Meier curves and compared by log-rank. Cox proportional hazards models and Receiver Operating Characteristic (ROC) curves were employed to evaluate the association between obesity indices (WHtR, BMI, WC) and ischemic stroke.

Result The cumulative incidence of ischemic stroke was $4.55 \%$, the incidence density was 507.61/100 00 person-years. After adjustment for age, smoking, alcohol consumption, family history of cardiovascular disease, fasting plasma glucose, lipids and blood pressure, the hazard ratio for ischemic stroke was $3.58(1.2-11.46)$ for the group of WHtR $>0.60$ compared with WHtR $(0.40-0.50)$. The Kaplan-Meier survival curves showed that the cumulative survival rate in group with WHtR $>$ 0.60 was lower than other three groups and was significantly difference compared with the group of WHtR 0.40 to 0.50 (log-rank test, $\mathrm{P}=0.025$ ). The AUC values for each index were as follows: 0.543 for $\mathrm{WC} ; 0.566$ for BMI; 0.586 for WHtR. WHtR was significantly better than WC at predicting ischemic stroke, with a significance level of $\mathrm{P}=0.005$. The AUC of WHtR was larger than BMI although they were not significantly different $(\mathrm{P}=0.797)$. No significant association between $\mathrm{BMI}, \mathrm{WC}$ and ischemic stroke was observed.

Conclusion Our study indicated that, WHtR, especially extreme WHtRn $(>0.60)$, was associated with ischemic stroke in Inner Mongolian men. It was a better adiposity index than BMI and WC for predicting the risk of ischemic stroke in this population. WHtR, as an appropriate index for abdominal obesity, may be a more useful clinical screening tool for ischemic stroke.

\section{Association between the Prol2Ala, C1431T and C681G polymorphisms in PPAR $y$ and lipid serum levels \\ Shujun $\mathrm{Gu}^{1,2}$, Zhengyuan Zhou ${ }^{1}$, Zhirong $\mathrm{Guo}^{2}$, Ming $\mathrm{Wu}^{3}$, Mingao $\mathrm{Xu}^{1}$, Ning Zhang ${ }^{1}$, Xiaoyan $\mathrm{Xu}^{1}$ \\ 1. Center for Disease Control of ChangShu, Suzhou, JiangSu, China \\ 2. Department of Epidemiology, School of Public Health, Soochow \\ university, Suzhou, Jiangsu, China Department of Epidemiology, School of Public Health, Soochow university, Jiangsu, China}

Objective To investigate the association of the Prol2Ala, C1431T and $\mathrm{C} 681 \mathrm{G}$ polymorphisms in PPAR $\gamma$ with lipid serum levels.

Methods 820 subjects (270 males, 550 females) were randomly selected from the PMMJS (Prevention of Multiple Metabolic Disorders and MS in Jiangsu) cohort populations and no individuals were related. The genotypes of C681G, Prol2Ala and C1431T polymorphism were identified by TaqMan PCR. The line regression model was used to examine the association between these polymorphisms and lipid metabolism. SNPstats was used to explore the haplotype association analyses.

Result After adjustment for age, gender, smoking, alcohol consumption and so on, in the both codominant and dominant models, the PA, AA, $(\mathrm{PA}+\mathrm{AA})$ gene carriers of Pro12Ala were associated with a significant increased levels of TG $(\mathrm{P}<0.001)$. In the both codominant and dominant models, the CT, TT, $(\mathrm{CT}+\mathrm{TT})$ gene carriers of $\mathrm{C} 1431 \mathrm{~T}$ were associated with a significant increased levels of TG, TC and nonHDL $(\mathrm{P}<0.001)$. In the codominant model, the GG gene carriers of C681G were associated with a significant decreased levels of TC and non-HDL $(\mathrm{P}<0.05)$. When the most common haplotype $\mathrm{P}-\mathrm{C}-\mathrm{C}$ was treated as the reference group, the A-C-C, P-T-T, A-T-C and A-C-T haplotypes were associated with a significantly increased levels of TG ( $\mathrm{P}<0.05)$, P-T-C, A-T-C haplotypes were associated with a significantly increased levels of TC ( $\mathrm{P}<0.05)$, P-T-C haplotype was associated with a significantly increased levels of non-HDL $(\mathrm{P}=0.049)$.

Conclusion PPAR $\gamma$ polymorphisms and haplotypes were associated with lipid metabolism.

\section{Utility of the atherosclerotic cardiovascular disease pooled cohort risk equations in a chinese inner mongolian population \\ Xiaoqing Bu, Fanlong Kong, Juan Xu, Hao Peng, \\ Hongmei Li, Yonghong Zhang \\ Department of Epidemiology, School of Public Health, Medical College \\ of Soochow University, Suzhou, Jiangsu Province, China}

Objective To assess the validation of the Pooled Cohort risk equations proposed by the 2013 American College of Cardiology/ American Heart Association (ACC/AHA) guidelines in an Inner Mongolian population from China.

Methods A prospective cohort study was conducted from June 2003 through July 2012 among 2589 participants without clinical cardiovascular diseases or statin using at baseline in Inner Mongolia, China. Calibration and discrimination of the Pooled Cohort prediction model were performed in all participants and, separately, in those for whom statin initiation should be based on estimated 10-year atherosclerotic cardiovascular disease (ASCVD) risk [ie., participants who are between 40 and 79 years of age without diabetes and with a lowdensity lipoprotein cholesterol (LDL-C) level between 70 and $189 \mathrm{mg}$ / dl].

Result Up to July 3120122583 participants were successfully followed up for an average of 9.2 years, and a total of 23292 personyears were observed. 200 ASCVD events (124 strokes and 76 coronary heart diseases) occurred during the follow-up period. The Pooled Cohort risk prediction model provided a good calibration with a moderate to good discrimination both in overall population [Hosmer-Lemeshow $\mathrm{C} 2=11.83, \mathrm{P}=0.16 ; \mathrm{C}$ statistic $=0.79(95 \% \mathrm{CI}: 0.76-0.82)]$ and the subpopulation [Hosmer-Lemeshow $\mathrm{C} 2=11.51, \mathrm{P}=0.17$; $\mathrm{C}$ statistic $=$ 0.71 (95\% CI: 0.66-0.76) ].

Conclusion In conclusion, aside from a small amount of underestimation, this risk prediction model formed reasonably well in the Inner Mongolian population, especially among those for whom statin initiation should be based on predicted ASCVD risk.

\section{Investigation on levels of leptin in Xinjiang uygur population and association of leptin with metabolic syndrome}

Qin Luo, Nanfang Li, Feiya Suo, Ling Zhou, Jing Hong, Hongmei Wang, Xiaoguang Yao, Yunwei Bi The Center of Hypertension of the People' s Hospital of Xinjiang Uygur Autonomous Region; Hypertension Institute of Xinjiang

Objective The study aims to investigate the levels of leptin in Xinjiang Uygur population and explore the association of leptin with metabolic syndrome (MS) in this population.

Methods A total of 781 individuals (249 men and 532 women) were selected from the cross-sectional study of MS from Hetian area in Xinjiang Uygur Autonomous Region, aged from 30 to 70 years, included 403 MS subjects and 378 non-MS controls. All subjects underwent a medical examination and fasting blood measurement, including blood pressure (BP), height, weight, waist circumference (WC), fasting serum leptin, fasting glucose (GLU), fasting insulin (FIN), HDL-cholesterol 
and triglycerides (TG) et al.

Result The median of leptin was $1.23 \mathrm{ng} / \mathrm{ml}$ in Xinjiang Uygurgeneral population. The median of leptin in MS was $1.72 \mathrm{ng} / \mathrm{ml}$, which was significantly higher than non-MS controls $(0.81 \mathrm{ng} / \mathrm{ml}, \mathrm{P}<0.01)$. Leptin levels were 3 times higher in women than in men $(\mathrm{P}<0.01)$. With the increasing levels of leptin, frequency of MS, levels of WC, body mass index (BMI), diastolic blood pressure (DBP) and TG were increased in both men and women. Systolic blood pressure (SBP) level also increased with the increasing of leptin level in women but not in men. Spearman correlation of levels of leptin with metabolic factors showed that BMI ( $\mathrm{r}=0.46$ in man, $\mathrm{r}=0.50$ in women $)$ and $\mathrm{WC}(\mathrm{r}=$ 0.42 in man, $r=0.46$ in women) were the strongest factors associated with leptin in men while in women. The following association factors were HOMA-IR, TG, DBP in men, and were DBP, SBP, TG, age, HOMA-IR in women, respectively. Leptin was a predictor of MS on ROC analysis, with area under the curve (AUC) $=0.68$ in men and $\mathrm{AUC}=0.66$ in women.

Conclusion Elevated levels of leptin were positively associated with MS and its important components and somewhat predicted MS in Xinjiang Uygur population. BMI and WC were the strongest factors associated with leptin in men while in women. But there were some differences between men and women, the correlation between leptin and insulin resistance was stronger in men than in women, and the correlation between leptin and blood pressure was stronger in women than in men, SBP was associated with leptin only in women.

\section{Association of genetic variants in NPR3 gene with salt sensitivity of blood pressure in chinese population: the GenSalt study \\ Shufeng Chen, Xiangfeng Lu, Jianfeng Huang, Jie Cao, Laiyuan Wang, Jianxin Li, Hongfan Li, Xueli Yang, Dongfeng Gu \\ Division of Population Genetics, State Key Laboratory of Cardiovascular Disease, Cardiovascular Institute and Fuwai Hospital, National Center for Cardiovascular Diseases, Chinese Academy of Medical Sciences and Peking Union Medical College}

Background Variation of blood pressure (BP) response to dietary salt intake, a phenomenon described as BP salt sensitivity, is associated with an increased risk of hypertension and cardiovascular disease. An individual's genetic profile might play an important role in their susceptibility to salt intake. Genetic variants in the natriuretic peptide (NP) pathway have been associated with hypertension. However, few study focused on the relationship of NP pathway gene with BP responses to the dietary salt intervention. We aimed to explore the association of NP pathway (NPPA, NPPB, NPPC, NPR1, 2, and 3) with sodium-sensitivity of BP in GenSalt study.

Methods GenSalt Study conducted a 7-day low-sodium intervention $(51.3 \mathrm{mmol} /$ day $)$ followed by a 7-day high-sodium intervention (307.8 mmol/day) in rural area of northern China. Among the 1906 eligible participants from 620 families, 1871 (98.2\%) and 1860 (97.6\%) completed the low-sodium and high-sodium interventions, respectively. BP measurements were obtained at baseline and the end of each intervention period using a random-zero sphygmomanometer. A total of 17 tag single-nucleotide polymorphisms were genotyped in our study. The most likely haplotypes within each block for individuals were inferred using the MERLIN program. The associations of SNPs/ haplotypes with salt sensitivity were tested under additive genetic model. Both single-marker and haplotype association analyses were conducted using linear mixed models. Age, gender, and body-mass index were adjusted in multivariable analyses. All statistic analyses were conducted using SAS 9.3.

Result On average, study participants were 38.7 years old and had a mean BMI of $23.3 \mathrm{~kg} / \mathrm{m}^{2}$, mean SBP of $116.9 \mathrm{~mm} \mathrm{Hg}$, and mean DBP of $73.7 \mathrm{~mm} \mathrm{Hg}$ at baseline. We have found that SBP response to highsodium intervention increased with the number of $G$ alleles of marker rs1147225 in natriuretic peptide receptor 3 (NPR3) gene $(\mathrm{P}=0.02)$. Mean SBP change for rs1147225 genotypes AA, GA and GG were $4.47 \mathrm{~mm} \mathrm{Hg}, 4.96 \mathrm{~mm} \mathrm{Hg}$ and $5.46 \mathrm{~mm} \mathrm{Hg}$, respectively. Two distinct linkage disequilibrium blocks were identified in the NPR3 gene. Linkage disequilibrium block 2 (rs888692-rs1147225) contained four common haplotypes including: A-A, A-G, G-A, and G-G. Similar to findings from single-marker analysis, $\mathrm{SBP}$ response to high-sodium intervention increased with the number of copies of the A-G haplotype $(\mathrm{P}=0.017)$. We did not observe any association between SNPs/haplotypes in these 6 genes and BP responses to the low-sodium intervention.

Conclusion These data suggest that genetic variants of the NPR3 gene play roles in salt sensitivity in Chinese population. Replication of these results in other populations is warranted.

\section{HMGB1 gene polymorphism is associated with essential hypertension in han chinese population \\ Yingshui Yao ${ }^{1}$, Daoxia Guo ${ }^{1}$, Song Yang ${ }^{3}$, Yuelong Jin ${ }^{1}$, Lianping $\mathrm{He}^{1}$, Jinfeng $\mathrm{Chen}^{2}$, Xianghai Zhao ${ }^{3}$, \\ Yanchun Chen ${ }^{3}$, Wei Zhou ${ }^{3}$, Chong Shen ${ }^{2}$ \\ 1. School of Public Health, Wannan Medical College, Wuhu, China \\ 2. Department of Epidemiology and Biostatistics, School of Public \\ Health, Nanjing Medical University \\ 3. Department of Cardiology, Affiliated Yixing Peoples Hospital of \\ Jiangsu University, Peoples Hospital of Yixing City}

Background High-mobility group box 1 protein (HMGB1) acts as a proinflammatory cytokine by activating pattern recognition receptors (PRRs), including Toll-like receptor 4 (TLR4) and the receptor of AGE (AGER) with oxidative injury. Animal study proved that HMGB1 contributed to the pathogenesis of experimental pulmonary hypertension via activation of TLR4. Previous Evidence suggested that with serum HMGB1 level increasing, the risk of CAD in non-diabetic and type 2 diabetic patients increased significantly. The aim of this study is to test whether HMGB1 harbor genetic susceptibility to essential hypertension (EH) in a Chinese population.

Methods Unpaired Student's t-test was used to test the differences of all quantitative variables presented as the means \pm SD between cases and controls. Qualitative variables, the allele and genotype frequency distributions between cases and controls were compared by the twosided Chi-square $\left(\chi^{2}\right)$ test. P value of $\leq 0.05$ was defined to be statistically significant. Among controls, genotype frequencies for each SNP were tested by Fisher's exact $\chi^{2}$ test using the program Hardy-Weinberg equilibrium (HWE). The statistical power was estimated by Program Quanto. The association between genotypes and EH was expressed by computing the odds ratios (ORs) and $95 \%$ confidence intervals $(95 \%$ CIs). Logistic regression model was used to evaluate the genetic effects of SNPs polymorphisms on HT. Additionally, general linear model (GLM) was applied to compare blood pressure levels between genotypes in cases and controls.

Result Single locus analysis showed that there was no statistical association of three tagSNPs with EH after adjustment for the covariates. 
Association analysis of haplotype constructed of rs2249825, rs1412125 and rs1045411 with EH showed that comparing with the reference Haplotype Hap1 (C-T-G), no significant haplotype was identified. Further stratification analysis found that rs2249825 was significantly associated with $\mathrm{EH}$ in $\geq 55$ years groups, ORs $(95 \% \mathrm{CI})$ of additive model and dominant model were $1.208(1.029-1.417)$ and $1.212(1.020$ 1.441), and $P$ values were 0.021 and 0.029 respectively. Quantitative trait analysis indicated that diastolic blood pressure (DBP) had a linear decrease with the variations of rs2249825 in both untreated EH group $(\mathrm{P}=0.002)$ and control group $(\mathrm{P}=0.034)$ respectively after adjusted for covariates. Also, there is significant difference of DBP between the genotypes of rs1045411 $(\mathrm{P}=0.015)$.

Conclusion Our finding suggests that rs2249825 of HMGB1 genetic polymorphisms are significantly associated with EH and DBP, and the genetic effect on $\mathrm{EH}$ is modulated by age.

\section{A novel association of adenosine deaminase with paroxysmal atrial fibrillation: a propensity score analysis from a case-control study \\ Liang Liu, Yongming He, Xin Zhao, Feng Xue, \\ Xiangjun Yang \\ The First Affiliated Hospital of Soochow University}

Objective To identify novel biochemical markers for incident paroxysmal atrial fibrillation (PAF).

Methods Men or women aged $\geq 18$ years that were hospitalized between 1st Jun. 2010 and 31st Dec. 2013 for PAF and for paroxysmal supraventricular tachycardia (non-PAF) were included. We used propensity score methods to mitigate the influence of the nonrandom selection of PAF and non-PAF patients. A total of 1802 eligible patients were identified. Of these, 895 patients had at least 1 exclusion criterion. A total of 124 patients per group were finally included for analysis. Conditional logistic regression analysis was performed on tricuspid pressure gradient, right atrial diameter, left atrial diameter, creatinine, adenosine deaminase, MV regurgitation and TV regurgitation that were significantly different between the both groups.

Result The PAF and non-PAF groups were well matched on demographic and clinical characteristics after propensity matching. Risk factors for PAF included tricuspid pressure gradient $(\mathrm{OR}=1.04, \mathrm{P}=0.004$, 95\% CI: $1.01-1.06)$, right atrial diameter $(\mathrm{OR}=1.08, \mathrm{P}=0.012,95 \% \mathrm{CI}$ : $1.02-1.14)$, left atrial diameter $(\mathrm{OR}=1.09, \mathrm{P}=0.001,95 \% \mathrm{CI}: 1.04-1.15)$, creatinine $(\mathrm{OR}=1.02, \mathrm{P}=0.042,95 \% \mathrm{CI}: 1.00-1.03)$, $\mathrm{MV}$ regurgitation $(\mathrm{OR}=3.64, \mathrm{P}=0.001,95 \% \mathrm{CI}: 1.68-7.90)$ and $\mathrm{TV}$ regurgitation $(\mathrm{OR}=$ $2.01, \mathrm{P}=0.014,95 \%$ CI: $1.15-3.50)$. Only the adenosine deaminase was a protective factor for $\mathrm{PAF}(\mathrm{OR}=0.92, \mathrm{P}=0.038,95 \% \mathrm{CI}$ : $0.85-1.00)$.

Conclusion The adenosine deaminase, reflecting patients' immunological function, seems to be associated with PAF. The current study provides a new insight into prevention and treatment of PAF.

\section{Antithrombotic therapy in chinese patients with atrial fibrillation: results from a multicenter, prospective registry \\ Han Zhang, Yanmin Yang, Jun Zhu, Xinghui Shao, Juan Wang, Li Tian, Bi Huang \\ Emergency and Intensive Care Center, Fuwai Hospital, Chinese \\ Academy of Medical Sciences and Peking Union Medical College}

Background Aim of this study was to describe the antithrombotic therapy in Chinese patients with atrial fibrillation (AF) and identify factors associated with warfarin use at baseline and 12-month follow-up.

Methods We conducted a multicenter, prospective registry to evaluate patterns of antithrombotic use in Chinese patients with $\mathrm{AF}$ at baseline and at follow-up. A multivariate regression model was developed to identify predictors of warfarin use, respectively.

Result Among 319 patients with valvular atrial fibrillation, 132 at baseline and 111 at follow-up were on warfarin, while 43 (32.6\%) and $64(57.7 \%)$ have international normalized ratio (INR) levels between 2.0 to 3.0, respectively. In patients with non-valvular atrial fibrillation, warfarin was most used in patients with the $\mathrm{CHADS}_{2}$ score $=1$, and proportion of patients met the targer INR levels increased as rising of the $\mathrm{CHADS}_{2}$ score. Use of aspirin also increased with risk score rising. Over half of low-risk patients received antithrombotic drugs $(60.2 \%$ and $55.3 \%$, respectively). History of stroke/TIA and prior diagnosis of AF were determinants of warfarin use at baseline, while there was a decreased likelihood of warfarin use with increasing age, increasing HR, and history of CAD. Same factors were identified at follow-up.

Conclusion In Chinese AF patients, antithrombotic therapy is frequently not used according to guidelines, but overuse in patients at low risk and underuse in those at high risk of stroke. History of stroke/ TIA, prior diagnosis of AF, age, heart rate, and history of CAD were associated with warfarin use.

\section{Obesity paradox in patients with atrial fibrillation and heart failure \\ Juan Wang, Yanmin Yang, Jun Zhu \\ Emergency and Intensive Care Center, State Key Laboratory of Cardiovascular Disease, Fuwai Hospital, National Center for Cardiovascular Disease, Chinese Academy of Medical Science and Peking Union Medical College, Beiijng, China.}

Background An obesity paradox, a "paradoxical" decrease in morbidity and mortality with increasing body mass index (BMI), has been shown in patients with cardiovascular diseases. However, whether this phenomenon exists in atrial fibrillation (AF) patients with concomitant heart failure (HF) is not known.

Methods This study consecutively enrolled AF patients presenting to an emergency department at 20 hospitals in China from Nov 2008 to Oct 2011. AF concomitant HF patients were categorized as underweight (BMI $<18.5 \mathrm{~kg} / \mathrm{m}^{2}$ ), normal (BMI 18.5 to $<24 \mathrm{~kg} / \mathrm{m}^{2}$ ), overweight (BMI 24 to $<28 \mathrm{~kg} / \mathrm{m}^{2}$ ), and obese (BMI $\geq 28$ all $\mathrm{kg} / \mathrm{m}^{2}$ ). Multivariate Cox proportional hazards regression was used on all the patients. End points of the analyses were all-cause mortality and cardiovascular mortality.

Result A total of 806 AF patients with concomitant HF were included in the analysis, mean BMI was $(23.3 \pm 4.1) \mathrm{kg} / \mathrm{m}^{2}$. A total of 153 deaths occurred over the 12-month follow-up, all-cause mortality was $19 \%$ in the whole patients, in underweight patients, normal weight, overweight, obese categories were $28.7 \%, 23.6 \%, 10.4 \%$, and $11.8 \%$ respectively $(\mathrm{P}<0.001)$. The cardiovascular mortality in whole patients was $14 \%$, in underweight, normal weight, overweight, obese categories were $21.8 \%, 17.2 \%, 7.8 \%$ and $8.8 \%$ respectively $(\mathrm{P}=0.001)$. On multivariate analysis, as a continuous variable, BMI was not a risk factors for all-cause mortality $(\mathrm{HR}=0.91,95 \% \mathrm{CI}: 0.87-0.95 ; \mathrm{P}<0.001)$, and for cardiovascular mortality ( $\mathrm{HR}=0.91,95 \% \mathrm{CI}$ : 0.86-0.96; $\mathrm{P}<$ $0.001)$; as a category variable, obesity $(\mathrm{HR}=0.50,95 \% \mathrm{CI}$ : $0.26-0.94$, $\mathrm{P}=0.032)$ and overweight $(\mathrm{HR}=0.40,95 \% \mathrm{CI}: 0.25-0.63, \mathrm{P}<0.001)$ were significantly associated with a lower risk of all-cause mortality, and overweight also with a lower cardiovascular death $(\mathrm{HR}=0.45,95 \% \mathrm{CI}$ 
$0.26-0.76, \mathrm{P}=0.003$ ) compared to normal weight patients.

Conclusion Our study showed an "obesity paradox" exists in AF patients with concomitant HF. Obesity was not a risk factor for 12-month mortality in $\mathrm{AF}$ and $\mathrm{HF}$ patients. Overweight $\mathrm{AF}$ patients have better survival and outcomes than normal weight (BMI 18.5-24 kg/m²) patients.

\section{The effect of exercise on endothelial function of patient with coronary heart disease}

Jia Lu, Yingjie Zhang

Department of Cardiology, the First Hospital Affliated to Liaoning Medical

University Jinzhou

Objective To observe the effect of exercise on endothelial function of patient with coronary heart disease.

Methods 100 patients with coronary heart disease were divided into two groups randomly (50 per group). The control group received medicine such as asprin, beta blockers, nitrates, calcium channel blocker and statin, The exercise group gives individualized exercise prescription, medicine were the same as the control group. The level of serum high sensitivity $\mathrm{C}$ reactive protein (hsCRP), nitric oxide (NO), endothelin (ET) were measured before and after 3 months' treatment. Flowmediated vasodilation of the brachial artery (FMD) was observed by using two-dimensional ultrasound before and after 3 months' treatment. Cardiovascular events were follow-up for 3 months.

Result The baseline informations of two groups have no statistical difference before treatmentment $(\mathrm{P}>0.05)$. The FMD of the two groups were improved after treatment (all $\mathrm{P}<0.01$ ), while the exercise group was obviously superior to the control group $[(10.08 \pm 1.96) \%$ vs $(11.51$ $\pm 1.84) \%, \mathrm{P}<0.01]$. The level of hsCRP and ET of the two groups were decreased after treatment (all $\mathrm{P}<0.01$ ). The exercise group was obviously superior to the control group [ hsCRP $(5.67 \pm 0.32) \mathrm{mg} / \mathrm{L}$ vs $(4.40 \pm 0.51)$ $\mathrm{mg} / \mathrm{L}, \mathrm{ET}(64.80 \pm 6.45) \mathrm{ng} / \mathrm{L}$ vs $(55.90 \pm 4.43) \mathrm{ng} / \mathrm{L}$, all $\mathrm{P}<0.01]$. The level of serum $\mathrm{NO}$ of the two groups increased after treatment (all $\mathrm{P}<0.01$ ), and more siginificantly increased in the exercise group $[(50.87 \pm 2.92) \mu \mathrm{mol} /$ $\mathrm{L}$ vs $(56.93 \pm 3.75) \mu \mathrm{mol} / \mathrm{L}, \mathrm{P}<0.01]$. There is not significant difference of cardiovascular events between two group $(\mathrm{P}>0.05)$.

Conclusion Exercise can improve vascular endothelial function of patients with coronary heart disease. It may be attributable to increase in $\mathrm{NO}$, and reduce in hsCRP and ET.

\section{Association of RANTES gene polymorphisms with takayasu arteritis in Chinese Han population \\ Yuehua $\mathrm{Li}^{1}$, Dan Wen ${ }^{2}$, Xiongjing Jiang ${ }^{1}$, Haiying $\mathrm{Wu}^{1}$, Huimin Zhang ${ }^{1}$, Xianliang Zhou \\ 1. Fuwai Hospital, National Center for Cardiovascular Disease, Chinese Academy of Medical Science and Peking Union Medical College \\ 2. Beijing Anzhen Hospital}

Objective Takayasu arteritis is a chronic vasculitis that affects primarily large elastic arteries such as the aorta, its main branches, and the pulmonary arteries. The mechanism of Takayasu arteritis is unclear. We try to explore the association of RANTES-403G/A and $-28 \mathrm{C} / \mathrm{G}$ gene polymorphysm with the risk of Takayasu arteritis in Han population.

Methods We used a case-control study to assess the relationship between RANTES-403G/A and $-28 \mathrm{C} / \mathrm{G}$ gene polymorphysm and the risk of Takayasu arteritis. TaqMan scope was used to exlpore the genotype.

Result For the RANTES-403G/A, the odds ratio (OR) and their corresponding 95\% confidence interval (CI) was 1.515 (1.065-2.156; $\mathrm{P}=0.021) ; 1.499$ (1.136-1.978; $\mathrm{P}=0.004) ; 2.305$ (1.192-4.455; $\mathrm{P}=$ 0.013 ) in dominant model, addictive model and recessive model when we did not adjust the relevant risk factors; the OR and $95 \%$ CI was 1.593 (1.101-2.305; P 0.014); 1.568 (1.174-2.096; P = 0.002); 2.474 (1.2534.884; $\mathrm{P}=0.009)$ in dominant model, addictive model and recessive model when we adjusted the relevant risk factors. For the RANTES$28 \mathrm{C} / \mathrm{G}$, the $\mathrm{OR}$ and $95 \% \mathrm{CI}$ was $1.776(1.171-2.693, \mathrm{P}=0.007) ; 1.742$ ( $1.254-2.420, \mathrm{P}=0.001) ; 2.510(1.053-5.984, \mathrm{P}=0.038)$ in dominant model, addictive model and recessive model when we did not adjust the relevant risk factors; the OR and 95\% CI was $1.929(1.249-2.978, \mathrm{P}=$ $0.003)$; 1.846 (1.309-2.604, $\mathrm{P}=0.003) ; 2.792$ (1.107-6.672, $\mathrm{P}=0.029)$ in dominant model, addictive model and recessive model when we adjusted the relevant risk factors.

Conclusion RANTES-403G/A and -28C/G gene polymorphism are risk factors to the risk of Takayasu arteritis.

\section{Practice patterns in the use of spironolactone during hospitalization for acute myocardial infarction in China: China PEACE-retrospective AMI study, 2001, 2006 and 2011 \\ Wenchi Guan ${ }^{1}$, Karthik Murugiah ${ }^{2}$, Jing Li ${ }^{1}$, Qing Wang ${ }^{1}$, Joseph Ross ${ }^{2,5}$, Nihar Desai ${ }^{2,5}$, Frederick Masoudi ${ }^{3}$, John Spertus ${ }^{4}, \mathrm{Xi} \mathrm{Li}^{1}$, Harlan Krumholz ${ }^{2,5}$, Lixin Jiang ${ }^{1}$ 1. National Clinical Research Center of Cardiovascular Diseases, State Key Laboratory of Cardiovascular Disease, Fuwai Hospital, National Center for Cardiovascular Diseases, Chinese Academy of Medical Sciences and Peking Union Medical College, Beijing, Peoples Republic of China \\ 2. Center for Outcomes Research and Evaluation, Yale-New Haven Hospital, New Haven, Connecticut, United States \\ 3. Division of Cardiology, University of Colorado Anschutz Medical Campus, Aurora, Colorado, United States \\ 4. St. Luke' s Mid America Heart Institute/University of Missouri Kansas City, Kansas City, Missouri, United States \\ 5. Yale University School of Medicine, New Haven, Connecticut, United States}

Objective To describe patterns of spironolactone therapy among patients with acute myocardial infarction (AMI) in China. We analyzed use in patients with either diabetes or clinical heart failure stratified into four groups: ideal patients [left ventricular ejection fraction (LVEF) $\leq 40 \%$ and without contraindications], patients with contraindications (e.g. hyperkalemia), patients with neither indications nor contraindications, and those for whom indications were unknown (LVEF unmeasured).

Methods We determined the proportions of patients in each subgroup treated with spironolactone during hospitalization in a nationally representative sample of AMI patients in 162 Chinese hospitals in 2001, 2006, and 2011. The significance of trends over time was tested using Cochran-Armitage test.

Result Among 6906 patients, 637 (9.2\%) patients were ideal candidates, 759 (11.0\%) patients had contraindications to spironolactone, 2677 (38.8\%) with a LVEF $>40 \%$, indicates no indications, 2833 (41.0\%) patients did not undergo LVEF assessment thus their indications for spironolactone were unclear. Spironolactone use increased in all four groups from 2001 to 2011, especially among ideal patients (ideal 
patients: $28.6 \%$ to $72.4 \%$, those with contraindications: $11.4 \%$ to $27.5 \%$, those with neither indications nor contraindications: $27.5 \%$ to $38.3 \%$, and patients with indications unknown: $27.3 \%$ to $35.1 \%$. All $\mathrm{P}<0.01$ for trend. Patients with heart failure at admission were more likely to be treated in all patients groups.

Conclusion The use of spironolactone for patients with AMI in China has increased substantially over time in candidates for treatment. However, increasing use in patients not likely to benefit suggests opportunities to improve patient selection for this therapy. Finally, wider use of LVEF testing would better characterize patients' candidacy for this potentially beneficial therapy.

\section{Egg consumption and risk of colorectal cancer: a meta-analysis \\ Yuehua Li, Hanjun Pei, Chenghui Zhou, Xianliang Zhou, Yongjian Wu, Lihuan Li \\ Fuwai Hospital, National Center for Cardiovascular Diseases, Chinese Academy of Medical Science and Peking Union Medical College}

Objective Egg consumption is very prevalent in our daily life. Egg serves as the major source dietary cholesterol. High egg consumption has been reported to increase the risk of cardiovascular diseases. But the relationship between egg consumption and risk of colorectal cancer is undetermind. So we tried to assess the dose-response relationship between egg intake and the risk of colorectal cancer.

Methods We systematically searched medline through Jan 2014 Random-effects model was used to pool the relative risks (RR) and their $95 \%$ confidence intervals (CI). Subgroup analyses was performed to explore the potential sources of heterogeneity. Weighted linear regression model was used to estimate the dose-response relationship.

Result We identified 3899 articles by database and manual searching. Fourteen studies involving 151388 subjects were included. The pooled RRs of the risk of colorectal cancer for the highest vs lowest egg intake was 1.44 (95\% CI: $1.26-1.65, \mathrm{P}=0.000$; i-squared $=44.5 \%$, $\mathrm{P}$ for heterogeneity $=0.008$ ). Subgroup analyses showed that studies without adjusted age have a higher risk of colorectal cancer than the studies with adjusted age $(\mathrm{RR}=2.32,95 \% \mathrm{CI}$ : $1.68-3.21$ vs $\mathrm{RR}=1.28$, $95 \%$ CI: $1.15-1.42, \mathrm{P}=0.000$ for subgroup difference). For each 4/week increment in egg intake, the RRs of the risk for colorectal cancer was 1.32 (95\% CI: 1.23-1.42).

Conclusion Our study suggests that there is a dose-responsed positive association between egg consumption and the risk of colorectal cancer.

\section{Factors influencing the spontaneous closure of ventricular septal defect in infants \\ Yang Xu, Sirui Yang, Min Liu \\ First Affliated Bethune Hospital, Jilin University. Center of Pediatrics, Institute of Pediatrics,}

Objective Predictors for spontaneous closure of ventricular septal defects (VSD) in infants are still unknown. The current study is designed to prospectively evaluate the potential value of maternal and infantile variables as predictors for the spontaneous VSD closure in infants.

Methods Consecutive infants, who were less than six-monthold when diagnosed with VSD, were followed-up for at least 5 years. Demographic, clinical and maternal factors were evaluated for the possible associations of incidence of spontaneous VSD closure.
Result Of the 425 eligible infants, 93 (21.88\%) had spontaneous VSD closure, $78.50 \%$ of which occurred when the patients were under 3 years of age. Results of logistic analyses suggested that diameter of the defect (dvsd), ratio between diameter of the defect and aortic root diameter (dvsd/dar), left atrium sizes, left ventricle sizes, main pulmonary forwardblood flow, infection scores, shunt ratio (qp/qs), VSD locations, and comorbidities including patent ductus arteriosus (PDA), and membranous septal aneurysm were independent predictors of spontaneous closure. The most appropriate cut off value for the prediction of spontaneous closure is evaluated ( $\mathrm{dvsd}<2.85 \mathrm{~mm}, \mathrm{dvsd} /$ dar $<0.23$, left atrium sizes $<19.5 \mathrm{~mm}$, left ventricle sizes $<32.5 \mathrm{~mm}$, main pulmonary forward blood flow $<1.01 \mathrm{~cm} / \mathrm{s}$, infection scores $<2.43$, and qp/qs $<1.53$ ). However, maternal factors (such as heavy drinking) during the first 3 months of pregnancy and VSD infants with down syndrome did not affect the spontaneous closure of infants with VSD.

Conclusion Dvsd, dvsd/dar, left atrium sizes, left ventricle sizes, main pulmonary forwardblood flow, infection scores, qp/qs, VSD location, comorbidities including PDA, or membranous septal aneurysm may be used as potential independent predictors of spontaneous VSD closure in infants. But the maternal factors and with other diseases do not affect the spontaneous closure of infants with VSD.

\section{Early clopidogrel therapy in chinese patients with acute myocardial infarction in 2006 and 2011: insights from the China PEACE- retrospective AMI study \\ Lihua Zhang ${ }^{1}$, Nihar Desai, ${ }^{2,3}$, Jing $\mathrm{Li}^{1}$, Shuang $\mathrm{Hu}^{1}$, Xi Li ${ }^{1}$, Frederick Masoudi ${ }^{4}$, John Spertus ${ }^{5}$, Sudhakar Nuti ${ }^{2}$, Sisi Wang ${ }^{2}$, Harlan Krumholz ${ }^{2,3,6,7}$, Lixin Jiang ${ }^{1}$ 1. National Clinical Research Center of Cardiovascular Diseases, State Key Laboratory of Cardiovascular Disease, Fuwai Hospital, National Center for Cardiovascular Diseases, Chinese Academy of Medical Sciences and Peking Union Medical College, Beijing, Peoples Republic of China. \\ 2. Center for Outcomes Research and Evaluation, Yale-New Haven Hospital, New Haven, Connecticut, United States. \\ 3. Section of Cardiovascular Medicine, Department of Internal Medicine, Yale University School of Medicine, New Haven, Connecticut, United States. \\ 4. Division of Cardiology, University of Colorado Anschutz Medical Campus, Aurora, Colorado, United States. \\ 5. Saint. Luke' s Mid America Heart Institute/University of Missouri- Kansas City, Kansas City, Missouri, United States. \\ 6. Robert Wood Johnson Clinical Scholars Program, Department of Internal Medicine, Yale University School of Medicine, New Haven, Connecticut, United States. \\ 7. Department of Health Policy and Management, Yale School of Public Health, New Haven, Connecticut, United States.}

Background Clopidogrel reduces the risk of adverse events when administered early to patients with acute myocardial infarction (AMI). However, there have been no studies to evaluate early clopidogrel therapy for patients with AMI in China.

Methods Data from the China Patient-Centered Evaluative Assessment of Cardiac Events Retrospective Study of Acute Myocardial Infarction were analyzed for 11944 eligible patients from 162 randomly selected Chinese hospitals in 2006 and 2011 to determine the use of early clopidogrel therapy, defined as use initiated within 24 hours of hospital admission. 
Result Among the 11944 eligible patients, the rate of early clopidogrel therapy increased from $41.5 \%$ in 2006 to $77.1 \%$ in 2011 (P $<0.001)$ in China. There was significant hospital-level variation in early clopidogrel use by region in both years, with rates ranging from $1.5 \%$ to $58.0 \%$ in $2006(\mathrm{P}<0.001)$ and $48.7 \%$ to $87.7 \%$ in $2011(\mathrm{P}<0.001)$. While early clopidogrel use was uniformly high in urban hospitals in 2011 (median $89.3 \%$; interquartile range $80.1 \%$ to $94.5 \%$ ), there was marked heterogeneity among rural hospitals (median $50.0 \%$; interquartile range $11.5 \%$ to $84.4 \%$ ). Patients with prior myocardial infarction or ischemic stroke and those admitted to rural hospitals were less likely to be treated.

Conclusion While the utilization of early clopidogrel therapy in patients with AMI has increased in China, there is persistent underuse, particularly in rural hospitals. Quality improvement initiatives to increase the early use of clopidogrel for patients with AMI are needed to improve clinical outcomes.

\section{Genetic variant in hepatocyte nuclear factor 1 alpha is associated with left main coronary artery disease \\ Hanning Liu ${ }^{1}$, Shengshou $\mathrm{Hu}^{1}$, Zhe Zheng ${ }^{1}$, Xiao Teng ${ }^{1}$, Haiyong $\mathrm{Gu}^{1}$, Rui Liu ${ }^{1}$ \\ 1. State Key Laboratory of Cardiovascular Disease, Fuwai Hospital, National Center for Cardiovascular Diseases, Chinese Academy of Medical Sciences and Peking Union Medical College, China. \\ 2. Department of Cardiothoracic Surgery, Affiliated Peoples Hospital of Jiangsu University, Zhenjiang, China.}

Objective Coronary artery disease (CAD) is the leading cause of mortality and morbidity in the world. Left main coronary artery disease (LMCAD) is a particularly severe phenotypic form of CAD and is heritable. We hypothesized that some inflammation- and hyperhomocysteinemia-related gene polymorphisms may contribute to LMCAD susceptibility in a Chinese population.

Methods We studied the association between polymorphisms in the genes hepatocyte nuclear factor 1 alpha (HNF1A; rs7310409, G/A), CRP (rs1800947 andrs3093059 T/C), methylenetetrahydrofolate reductase (rs1801133, C/T), and methylenetetrahydrofolate dehydrogenase (rs1076991, A/G) in 402 LMCAD and 804 more peripheral coronary artery disease patients in a Chinese population. Patients were consecutively recruited from Fuwai Hospital between December 2007 and December 2008. All patients were diagnosed using angiography. LMCAD was defined as a lesion compromising the lumen by $>50 \%$. Genotyping was performed using the matrix-assisted laser desorption/ ionization time-of-flight mass spectrometry method. A chi-squared test was used to evaluate the differences in demographic variables, clinical features, and genotypes of the five SNP variants. Odds ratios (ORs) and 95\% confidence intervals (CIs) was calculated to estimate the associations between the five SNPs and the risk of CAD phenotypes using logistic regression analyses.

Result LMCAD patients were significantly older than MPCAD patients $(62.24$ years vs 60.14 years, $\mathrm{P}<0.001)$. There were no significant differences between the two patient groups for other clinic risk factors. When the HNF1Ars 7310409 GG homozygote genotype was used as the reference group, both the individual, $\mathrm{GA}(\mathrm{OR}=1.46, \mathrm{P}$ $=0,009)$ and $\mathrm{AA}(\mathrm{OR}=1.45, \mathrm{P}=0.033)$, and combined GA/AA $(\mathrm{OR}$ $=1.45, \mathrm{P}=0.006$ ) genotypes were associated with an increased risk of LMCAD. This single nucleotide polymorphism (rs7310409) is strongly associated with plasma CRP levels.
Conclusion In conclusion, the present study provides strong evidence that the HNF1Ars7310409G/A functional polymorphism may contribute to the risk of LMCAD.

\section{Association between 24-hour urinary sodium excretion and metabolic syndrome in chinese adults: a cross-sectional study \\ Zeng $\mathrm{Ge}^{1}$, Xiaolei Guo ${ }^{2}$, Xiaorong Chen ${ }^{1}$, Junli Tang ${ }^{2}$, \\ Liuxia Yan ${ }^{1}$, Jiyu Zhang ${ }^{2}$, Jianwei Xu ${ }^{1}$, Hao Liang ${ }^{1}$, \\ Jixiang $\mathrm{Ma}$ \\ 1. Division of Cardiovascular Disease Control and Prevention, National \\ Center for Chronic and Noncommunicable Disease Control and \\ Prevention, China CDC \\ 2. Shandong Center for Disease Control and Prevention}

Objective To examine the association of 24-hour urinary sodium excretion with metabolic syndrome in Chinese adults.

Methods A population-based cross-sectional study (the Shandong and Ministry of Health Action on Salt and Hypertension) was conducted among 2112 participants aged 18-69 years in Shandong province in 2011. Data on 24-hour urinary sodium excretion and other variables were obtained and components of metabolic syndrome were examined. Participants were divided into three groups according to tertile of 24hour urinary sodium excretion. Logistic model was used to examine the association between 24-hour urinary sodium excretion and metabolic syndrome.

Result A total of 1977 participants were retained for data analysis. Of these participants, 474 (24\%) were with metabolic syndrome. The mean 24 hour urinary sodium excretion was $232.60 \mathrm{mmol}$. After adjustment for age, gender, education, residence, smoking, drinking and physical activity, compare participants with 24-hour urinary sodium excretion $<195.41 \mathrm{mmol}$, the RR $(95 \% \mathrm{CI})$ of MS for participants with 24-hour urinary sodium excretion 195.41-251.98 and $\geq 251.98$ was 1.30 (0.78-2.17) and $2.20(1.32-3.68)$, respectively. In addition, a significant dose response association between 24-hour urinary sodium excretion and metabolic syndrome was observed (P for linear trend $<0.001$ ). An increase of $100 \mathrm{mmol}$ in 24-hour urinary sodium excretion was associated with $86 \%$ higher risk of metabolic syndrome.

Conclusion Chinese adult with higher 24-hour urinary sodium excretion maybe at risk for MS. Therefore, the reduction in salt intake should be a potential component for preventing metabolic syndrome.

\section{Association between serum uric acid level and metabolic syndrome and its sex difference in a chinese community elderly population \\ Miao Liu ${ }^{1,2}$, Yao $\mathrm{He}^{1,2}$, Bin Jiang ${ }^{3}$, Lei Wu ${ }^{1,2}$, \\ Jianhua Wang ${ }^{1,2}$, Xiaoying $\mathrm{Li}^{4}$ \\ 1. Institute of Geriatrics, Chinese PLA General Hospital \\ 2. Beijing Key Laboratory of Aging and Geriatrics, Chinese PLA General Hospital \\ 3. Department of Chinese Traditional Medicine and Acupuncture, Chinese PLA General Hospital \\ 4. Department of Geriatric Cardiology, Chinese PLA General Hospital}

This study aimed to evaluate the association between serum uric acid (SUA) and metabolic syndrome (MetS) among Chinese community elderly and explore the sex difference. We conducted a populationbased cross-sectional study in a representative urban area of Beijing 
between 2009 and 2010. A two-stage stratified clustering sampling method was used and 2102 community elderly were included in the study. The prevalence of hyperuricemia and MetS was $16.7 \%$ and $59.1 \%$ respectively. There was a strong association between SUA level and all five components of MetS in women and only three components in men. Multiple logistic regression analysis showed the odds ratio (OR) of hyperuricemia for MetS was 1.67 (95\% CI: 1.11-2.50) in men and 2.73 (95\% CI: 1.81-4.11) in women. Even in the normal range, the ORs for MetS and its individual components increased gradually according to SUA levels. Compared with the lowest quartile of SUA, the adjusted ORs were 0.97 (95\% CI: 0.66-1.43), 1.32 (95\% CI: 0.76-2.30), 1.52 (95\% CI: 1.03-2.23) in men and 1.59 (95\% CI: 1.15-2.19), 1.85 (95\% CI: $1.34-2.55), 3.05$ (95\% CI: 1.86-5.02) in women for the second, third and top quartiles. A significantly liner-trend increasing in the component number of MetS across quartile of SUA was observed in both sexes (adjusted $\mathrm{P}$ for trend $<0.001$ ). This study suggests that higher SUA levels are positively associated with MetS and its components among Chinese community elderly, and it's stronger associated in women than men. Physicians should recognize MetS as a frequent comorbidity of hyperuricemia and take early action to prevent subsequent chronic disease burden.

\section{Early $\beta$-blocker therapy in patients with acute myocardial infarction in China, 2001-2011: The China PEACE-retrospective AMI study \\ Haibo Zhang ${ }^{1}$, Frederick A Masoudi ${ }^{2}$, Jing $\mathrm{Li}^{1}$, Qing Wang ${ }^{1}$, Xi Li ${ }^{1}$, John A Spertus', Joseph S Ross ${ }^{4}$, Nihar R Desai ${ }^{4}$, Harlan M Krumholz ${ }^{4}$, Lixin Jiang ${ }^{1}$ 1. National Clinical Research Center of Cardiovascular Diseases, State Key Laboratory of Cardiovascular Disease, Fuwai Hospital, National Center for Cardiovascular Diseases, Chinese Academy of Medical Sciences and Peking Union Medical College \\ 2. Division of Cardiology, University of Colorado Anschutz Medical Campus \\ 3. Saint Luke' s Mid America Heart Institute/University of Missouri 4. Center for Outcomes Research and Evaluation, Yale-New Haven Hospital}

Background Clinical practice guidelines recommend providing $\beta$-blocker therapy early in the course of acute myocardial infarction (AMI) for many patients who are not at high risk of shock. We sought to characterize patterns of beta blocker use during hospitalization for AMI over the past decade in China.

Methods We conducted medical record review of a nationally representative sample of patients with AMI and without absolute contraindications to $\beta$-blocker therapy during 2001, 2006, and 2011. We evaluated the use, type, and dose of $\beta$-blocker therapy within the first 24 hours of admission over time and identified predictors of not using this treatment.

Result 6426 patients with AMI and no contraindications to early $\beta$-blocker therapy were identified from 162 hospitals in China. Of these, 3419 patients were ideal candidates for therapy and 3007 had at least one risk factor for cardiogenic shock. Use in ideal patients was $54.9 \%, 68.2 \%$, and $61.8 \%$ in 2001, 2006 and 2011, respectively ( $\mathrm{P}=$ 0.36 for trend). Clinical predictors of non-treatment included older age, lower systolic blood pressure or heart rate, absence of chest discomfort and admission to a non-teaching hospital. Use in patients with risk factors for cardiogenic shock was $43.7 \%, 59.6 \%$, and $53.6 \%$ in the three years respectively $(\mathrm{P}=0.32$ for trend). Metoprolol was used most frequently (91.5\%). Dosages of Metoprolol were often below guideline recommendations $(73.6 \%$ received cumulative dosage of no more than $25 \mathrm{mg}$ in the first 24 hours of admission, only $4.0 \%$ received more than $50 \mathrm{mg}$ ), and did not change significantly over time.

Conclusion The use of early $\beta$-blocker therapy for patients with AMI in China is suboptimal with underuse of patients who could benefit and substantial use among those who might be harmed. The use of $\beta$ blockers, which has not improved over time, is an important target for efforts to improve quality of care for AMI.

\section{Prevalence and risk factor of hypertension among Shanghai children and adolescents, 2013 \\ Yi Zhang ${ }^{1}$, Weili Yan ${ }^{1}$, Yanqiang $\mathrm{Wu}^{2}$, Yang Yang ${ }^{2}$, \\ Shaoke Chen ${ }^{1}$, Yi Chen ${ }^{1}$, Fang Cao ${ }^{1}$, Qian Zhao ${ }^{1}$ \\ 1. Department of Clinical Epidemiology, Children's s Hospital of Fudan \\ University, Shanghai, China \\ 2. Shanghai Municipal Center for Students' Physical and Health, \\ Shanghai, China}

Objective To know the prevalence of hypertension and its relevant factors among children and adolescents in Shanghai.

Methods We conducted a school-based cross-sectional study in children and adolescents aged 6-19 years in ten districts of Shanghai. The anthropometrics including height, weight, waist circumference and blood pressure was measured. Family history of hypertension and some related factors were investigated with the uniform questionnaire completed by parents. BP values were typically based on repeated measurements over three visits. Those with $\mathrm{BP}>=95^{\text {th }}$ precentile, measured by automated Omron HEM-7012 monitor for first and second visit would enter the next visit. The diagnosis of hypertension was based on mercurial sphygmomanometer measurement at the third visit. Multivariate logistic stepwise regression analysis was performed to identify the relationship between parental family history and childhood hypertension with adjustment of possible confounders.

Result A total of 6692 consecutive subjects, aged 6 to 19 years, were enrolled in the screening. The prevalence of hypertension was $22.41 \%, 10.15 \%$ and $4.62 \%$ on the first, second and third time. Multivariate analysis indicated that father's level of education (OR = 0.79, 95\% CI: 0.65-0.98) had a positive effects on blood pressure, BMI $(\mathrm{OR}=1.25,95 \%$ CI: $1.18-1.32)$ and father's hypertension history (OR $=1.76,95 \%$ CI: $1.02-3.03$ ) was associated with a high likelihood of hypertension.

Conclusion It should be recommended that measuring BP on separate visits before characterizing a child as having hypertension. BMI and father's hypertension history are the leading cause of pediatric HTN.

\section{The gender difference in impact of family history of hypertension on children and adolescents in Shanghai \\ Yi Zhang ${ }^{1}$, Weili Yan ${ }^{1}$, Yang Yang ${ }^{2}$, Yanqiang Wu ${ }^{2}$, \\ Yi Chen ${ }^{1}$, Qian Zhao ${ }^{1}$, Shaoke Chen ${ }^{1}$, Fang Cao ${ }^{1}$, Kai Mu \\ 1. Department of Clinical Epidemiology, Children's S Hospital of Fudan \\ University, Shanghai, China \\ 2. Shanghai Municipal Center for Students' Physical and Health, Shanghai, China}

Objective This study aims to determine the gender difference 
in impact of positive family history on hypertension in children and adolescent in Shanghai.

Methods We conducted a school-based cross-sectional study in children and adolescents aged 6-19 years in ten districts of Shanghai. The anthropometrics including height, weight, waist circumference and blood pressure was measured. Family history of hypertension and some related factors were investigated with the uniform questionnaire completed by parents. Parents were considered to have hypertension if they have been diagnosed by doctors or told by health care providers that they have elevated BP and need medication. BP values were typically based on repeated measurements over three visits. Those with $\mathrm{BP} \geq 95^{\text {th }}$ precentile, measured by automated Omron HEM-7012 monitor for first and second visit would enter the next visit. The diagnosis of hypertension was based on mercurial sphygmomanometer measurement at the third visit. Multivariate logistic stepwise regression analysis was performed to identify the relationship between parental family history and childhood hypertension with adjustment of possible confounders.

Result A total of 6692 subjects were screened. The prevalence of hypertension was $22.41 \%, 10.15 \%$ and $4.62 \%$ at the first, second and third visit. Results indicated that father education level (OR $=0.69,95 \%$ CI: $0.60-0.81)$ was associated with higher blood pressure, BMI z-score $(\mathrm{OR}=1.65,95 \% \mathrm{CI}: 1.46-1.87)$ and hypertensive father $(\mathrm{OR}=1.57$, 95\% CI: 1.05-2.37) was associated with a higher risk of hypertension. After adjusting obesity, paternal (OR $=2.1,95 \%$ CI: $1.29-3.4)$, maternal $(\mathrm{OR}=2.7,95 \% \mathrm{CI}: 1.11-6.54)$ and both parental hypertension history $(\mathrm{OR}=1.45,95 \%$ CI: $0.17-12.1)$ increased the risk of hypertension in boys. While for girls, mother hypertension history $(\mathrm{OR}=1.73,95 \% \mathrm{CI}$ : 1.1-2.74) was the only significant risk factor.

Conclusion Impact of paternal and maternal hypertension history on childhood hypertension differs in genders. Mother hypertension history was the only significant impact on hypertension in girls.

\section{Study on the incidence of sudden cardiac death in rural area in Beijing \\ Linfeng Zhang ${ }^{1}$, Ying $\mathrm{Li}^{1}$, Jianmei $\mathrm{Gao}^{2}$, Caixia Liu ${ }^{2}$, \\ Haiyan Zhang ${ }^{2}$, Yan Dai ${ }^{1}$, Shu Zhang ${ }^{1}$, Wei Hua ${ }^{1}$ \\ 1. Fuwai Hospital of the Chinese Academy of Medical Sciences \\ and Peking Union Medical College, and the National Center for \\ Cardiovascular Disease \\ 2. Center for Disease Control and Prevention in the Huairou District of Beijing}

Objective To explore the incidence of sudden cardiac death (SCD) in the Beijing rural residents in Huairou district.

Methods A community based epidemiology surveillance was carried out in the residents in Huairou district in Beijing from October in 2008 to September in 2009. Data on the population size, age, sex and vital status of the subjects monitored, and the death cause and related information of the decedent were collected from the household administrative workers, the family members of the decedent, the hospital personnel and the witnesses.

Result 277851 subjects (140 052 men and 137799 women) were monitored between October 1, 2008 and September 30, 2009, a total of 1697 deaths (995 men and 702 women) occurred and 130 sudden cardiac deaths (92 men and 38 women) were determined. The incidence of SCD was 65.7 (95\% CI: 52.3-79.1) per 100000 per year for men and $27.6(95 \%$ CI: 18.8-36.3) per 100000 per year for women. SCD accounted for $9.2 \%$ of mortality in men and $5.4 \%$ of mortality in women. There were a total of $108 \mathrm{SCD}$ cases after the age of 55, which accounted for $83.1 \%$ of the
SCD cases occurred in the study population and the incidence of SCD increased significantly with age after the age of 55 .

Conclusion Sudden cardiac death is a great threat to the residents in Beijing Huairou district and most of the cases occurred after the age of 55. So it is important to take preventive measures in the population aged 55 years and over.

\section{The red blood cell distribution width and its relationship in elder patients with coronary artery disease \\ Kai Zhao, Yongjian Li, Sheng Gao \\ Department of Cardiology, Tianjin Nankai Hospital}

Objective Red blood cell distribution width (RDW), which is routinely reported in complete blood counts, is a measure of the variability in size of circulating erythrocytes. Previous studies suggest the higher RDW the greater the risk of mortality in patients with coronary artery disease (CAD). However, the relationship between RDW and coronary artery lesion severity in elder patients remains unclear. The aim of the present study is to analyze the relationship between RDW level and coronary artery lesion severity in elder patients, then evaluate whether RDW is an independent risk factor of CAD.

Methods According to the coronary angiography results, 376 elder patients were divided into four groups: Non-CAD group ( $\mathrm{n}=$ 106), single-branch group $(\mathrm{n}=87)$, double-branch group $(\mathrm{n}=91)$, and multiple-branch group $(\mathrm{n}=92)$. Analyze and compare the relevant clinical data, echocardiography results and CAD influencing factors among these groups. Multivariate logistic regression analysis was used to evaluate the independent risk factors of CAD.

Result With the increase in the number of coronary lesions, RDW level gradually increased. The level of RDW in multiple-branch group $(14.02 \% \pm 1.07 \%)$ was higher than those in double-branch group $(13.89 \%$ $\pm 0.97 \%)$ and single-branch group $(13.64 \% \pm 0.84 \%)$. The level of RDW in double-branch group was higher than those in single-branch group $(\mathrm{P}<0.05)$. Left atrial diameter $(\mathrm{LAD})$ and left ventricular end-diastolic diameter (LVED) in CAD group were significantly larger than those in Non-CAD group $(\mathrm{P}<0.05)$. Left ventricular posterior wall thickness (LVPWT) and ejection fraction (EF) in CAD group were smaller than those in Non-CAD group $(\mathrm{P}<0.05)$. Multivariate logistic regression analysis showed that the independent risk factors predicting CAD included low-density lipoprotein-cholesterol, fasting plasma glucose, C-reactive protein, age and RDW.

Conclusion The level of RDW is closely correlated with the severity of coronary artery stenosis in elder patients, RDW is an independent influencing factor for CAD.

\section{Effects of tea intake on blood pressure: a meta- analysis of 21 randomized controlled trials \\ Gang Liu, Xinxin Zheng, Jie Lu, Xiaohong Huang Department of Special Medical Treatment Center, Fuwai Hospital, National Center for Cardiovascular Diseases, Chinese Academy of Medical Sciences \& Peking Union Medical College}

Objective The effect of tea intake on blood pressure (BP) is controversial. We undertook a meta-analysis of randomized controlled trials to determine changes in systolic and diastolic BP due to the intake black and green tea.

Methods MEDLINE, EMBASE, and the Cochrane Controlled 
Trials Register were searched from 1966 until January 2014 for studies in parallel group or crossover design in which BP was assessed before and after receiving black or green tea for at least 1 week. The weighted mean difference was calculated for net changes in BP by using fixed-effects or random-effects models. Previously defined subgroup analyses were performed to explore the influence of study characteristics.

Result Twenty-one eligible randomized controlled trials with 1323 subjects were enrolled. After the tea intake, the pooled mean systolic and diastolic BP were $-1.8 \mathrm{~mm} \mathrm{Hg}(95 \% \mathrm{CI}$ : -2.4--1.1) and -1.4mm $\mathrm{Hg}$ (95\% CI: -2.2--0.6) lower, respectively, compared with the tea-free controls. Subgroup analyses showed that the BP-lowering effect was apparent in the subjects who consumed a tea over a median of 12 weeks (systolic/diastolic BP, -2.6/-2.1 mm Hg, both $\mathrm{P}<0.001$ ). Stratified by type of tea, green tea significantly reduced systolic and diastolic BP of -2.1 (95\% CI: $-2.9--1.2)$ and -1.7 (95\% CI: $-2.9--0.5) \mathrm{mm} \mathrm{Hg}$, and black tea significantly reduced systolic and diastolic BP of $-1.4(95 \%$ CI: $-2.4-0.4)$ and -1.1 (95\% CI: $-1.9-0.2) \mathrm{mm} \mathrm{Hg}$, respectively. The benefits of tea intake were not influenced by ethnicity, treatment dose of tea catechins, individual health status, or caffeine intake.

Conclusion The meta-analysis showed that long-term ( $\geq 12$ weeks) ingestion of a tea (green and black tea) resulted in a significant reduction in systolic and diastolic BP.

\section{Both Fasting and 2-hour post load glycemic progression predicts subsequent cardiovascular events in persons with impaired glucose tolerance: 23-year follow-up of the Da Qing diabetes prevention study \\ Xiaoxia Shen ${ }^{1}$, Yali An ${ }^{1}$, Ping zhang ${ }^{2}$, Jinping Wang ${ }^{3}$, Edward W Gregg ${ }^{2}$, Bo Zhang, Hui Li ${ }^{3}$, Qiuhong Gong ${ }^{1}$, Yanyan Chen ${ }^{1}$, Xiaoyan Xing, Michael Engelgau ${ }^{2}$ Yinghua $\mathrm{Hu}^{3}$, Peter $\mathrm{H}$ Bennett ${ }^{4}$, Guangwei $\mathrm{Li}^{1,5}$ \\ 1. Center of Endocrinology and Cardiovascular disease, Fuwai Hospital, Beijing, China \\ 2. Division of Diabetes Translation, CDC, Atlanta \\ 3. Department of Cardiology, Da Qing First Hospital, China \\ 4. Phoenix Epidemiology and Clinical Research Branch, NIDDK, \\ Phoenix, USA \\ 5. Department of Endocrinology, China-Japan Friendship Hospital, Bejing, China}

Background We sought to determine if both fasting (FPG) and 2-hour post-load (2hPG) glycemic progression in persons with impaired glucose tolerance (IGT) after initiation of a lifestyle intervention trial predicted cardiovascular events.

Methods In 1986, 576 adults aged 25 years and older with IGT in Da Qing China were randomly assigned by clinic to control or diet, exercise, and diet plus exercise intervention groups for a 6-year period. Subsequently participants received medical care in their local clinics. In 2009, we tracked participants to ascertain cardiovascular events and other outcomes.

Result Among the 542 subjects who completed the 6-year trial, 182 first non-fatal and fatal cardiovascular (CVD) events (myocardial infarction or stroke) occurred during the subsequent 17-years. Epidemiological analysis found that after controlling for age, sex, bodymass index, blood pressure and smoking at the baseline, one $\mathrm{mmol} / \mathrm{l}$ increase in FPG and 2hPG levels at the end of 6-year trial was associated with $8 \%(\mathrm{HR}=1.08 ; 95 \% \mathrm{CI}: 1.05-1.12)$ and $5 \%(\mathrm{HR}=1.05 ; 95 \% \mathrm{CI}$ : $1.02-1.09$ ) increase in having a first CVD event. In contrast, there were no significant associations between the baseline glucose levels and first CVD events (FPG: $\mathrm{HR}=1.19,95 \%$ CI: $0.98-1.45$; 2hPG: $\mathrm{HR}=1.06$, $95 \%$ CI: $0.90-1.25)$. Classifying participants by diabetes status according to either their 6-year FPG or $2 \mathrm{hPG}$ values, controlling for the same covariates, showed that those who had developed diabetes had a higher incidence of subsequent CVD events than those who had not (either FPG or $2 \mathrm{hPG}$ : HR $=1.45,95 \% \mathrm{CI}: 1.07-1.95)$.

Conclusion We concluded that both fasting and post-load glucose levels at the end of the 6-year trial predicted the development of first CVD events in adult Chinese with IGT and that a faster glycemic progression predicted a higher risk of subsequent CVD events.

\section{Framingham risk score for the prediction of coronary artery disease in patients with chronic rheumatic heart disease \\ Yaowang Lin, Xuebiao Wei, Anping Cai, Yingling Zhou, Danqing $\mathrm{Yu}$ \\ Guangdong Provincial People's Hospital, Guangzhou, China}

Objective Patients with chronic rheumatic heart disease (CRHD) have concomitant coronary artery disease (CAD), but the model to detect coexistent coronary artery stenosis prior to surgery has not been validated. Our study investigated whether the Framingham risk score (FRS) is a valid predictor of CAD in patients undergoing surgery for CRHD.

Methods A total of 989 rheumatic patients were enrolled between 2005 and 2010. They were subdivided into two groups according to coronary angiography $(\mathrm{CAG})$ results to identify potential factors in the development of CAD. Finally, all patients were assessed using the FRS to examine the association between the 10-year cardiovascular disease (CVD) risk and CAD.

Result There were statistically significant inter-group differences in terms of age, sex, smoking, hypertension, bypass surgery and cardiac function in the New York Heart Association (NYHA) classification status $(\mathrm{P}<0.05)$. We showed that the FRS had high accuracy in predicting $\mathrm{CAD}$ in female and male patients with CRHD. In the male group, the area under the curve (AUC) for predicting CAD was 0.904, with a specificity of $90.3 \%$ and sensitivity of $76.1 \%$. In the female group, the AUC for predicting CAD was 0.924 , with a specificity of $77.5 \%$ and sensitivity of $90.9 \%$, respectively. With a cut-off point of a 10 -year CVD risk of $12.5(\%)$ in the male group and a 10-year CVD risk of $2.5(\%)$ in the female group, the FRS identified 746 low-risk patients, including $11(4.3 \%)$ with CAD in the male group and $4(0.8 \%)$ with CAD in the female group. None of the patients needed an indication for Coronary Artery Bypass Grafting (CABG).

Conclusion The FRS model can accurately predict the prevalence of significant CAD and can reliably identify low-risk patients in whom routine pre-surgical angiography could be avoided.

\section{Thresholds of central systolic blood pressure in chinese middle-aged population}

Guang Hao, Zengwu Wang, Linfeng Zhang, Zuo Chen, Xin Wang, Min Guo, Ye Tian, Lan Shao, Manlu Zhu State Key Laboratory of Cardiovascular Disease, Fuwai Hospital, National Center for Cardiovascular Disease, Peking Union Medical College \& Chinese Academy of Medical Sciences

Objective Recently, an increasing body of evidence has emerged 
that central systolic blood pressure (CSBP) is more relevant to cardiovascular diseases than brachial BP. However, threshold values of CSBP have been less investigated. The aim of this study was to define the threshold values of the CSBP in Chinese population.

Methods 11623 participated were recruited in our survey, of them 10012 (86.14\%) participants were eligible for analysis. The mean age was $49.61 \pm 8.05$ years; $4699(46.93 \%)$ were men. To generate a healthy adult population sample, we excluded 4586 participants because of hypertension, diabetes, hyperlipidemia, or history of cardiovascular diseases, and obtained a healthy reference sample with 5426 participants. We defined abnormally high CSBP as exceeding the 90th percentile healthy referent sex-specific cut-point.

Result We establish a 90th percentile cutoff value for CSBP based on a healthy referent sample without major CV risk factors or disease, which was $125 \mathrm{~mm} \mathrm{Hg}$ both in men and women. A significant difference in CSBP according to diabetes, hypertension, history of CV disease, and hyperlipidemia $(\mathrm{P}<0.001)$ were found in our analysis. The proportion of patients with enlarged CSBP values was increased with age, for both genders $(\mathrm{P}<0.001)$.

Conclusion We established $125 \mathrm{~mm} \mathrm{Hg}$ in men and women as threshold value for CSBP in Chinese population. We will confirm these results with the prospective data of this study in the future.

\footnotetext{
PPAR $\delta$ polymorphisms as protection factors for dyslipidemia in a Chinese Han population

Zhengyuan Zhou ${ }^{1}$, Zhirong Guo ${ }^{2}$, Shujun $\mathrm{Gu}^{1,2}$,

Ming $\mathrm{Wu}^{3}$, Ning Zhang ${ }^{1}$

1. Center for Disease Control of ChangShu, Suzhou, JiangSu, China

2. Department of Epidemiology, School of Public Health, Soochow

University, Suzhou, Jiangsu, China

3. Department of Epidemiology, School of Public Health, Soochow

University, Suzhou, Jiangsu, China
}

Objective Cardiovascular disease (CVD) represents the leading cause of death in adults worldwide. Alterations in lipidand lipoprotein metabolisms have been investigated asimportant CVD risk factors. Many recent functional studies suggest that peroxisome proliferator activated receptor $\delta(\operatorname{PPAR} \delta$ ) is critically involved in the regulation of lipid, lipoprotein and glucose metabolism in multiple tissues including adipose tissue, skeletal muscle and the heart. This study aimed to investigate the association between PPAR $\delta$ polymorphisms and dyslipidemia.

Methods The studied population consisted of 820 subjects who were randomly selected from the Prevention of Multiple Metabolic Disorders and MS in Jiangsu province cohort populations. All the subjects were subjected to clinical interviews and blood collection for laboratory examinations and DNA extraction. The Taqman SNP genotyping assay was used for polymorphism genotyping. Individual polymorphism and haplotype data were available for analyses.

Result Single-locus analyses showed that rs2016520 was significantly associated with dyslipidemia, the OR (95\% CI) was 0.69 (0.52-0.92). However, rs9794 did not exhibit the significant association with dyslipidemia. After adjusted for age, sex, smoking, alcohol consumption and so on, rs2016520 was still significantly associated with dyslipidemia, the OR (95\% CI) was 0.68 ( $0.51-0.91)$. When the most common haplotype T-C was treated as the reference group, the C-C haplotype wassignificantly associated with decrease in the risk of dyslipidemia, the OR $(95 \%$ CI) were $0.72(0.55-0.93)$.

Conclusion PPAR $\delta$ polymorphisms and haplotypes may be the genetic protection factors for dyslipidemia.
Association of visceral and total body fat with hypertension and prehypertension in middle- aged chinese population
Zengwu Wang, Linfeng Zhang, Zuo Chen, Xin Wang, Manlu Zhu, Wen Wang, Weiwei Chen Department of Prevention and Community Health, National Center for Cardiovascular Disease; Fuwai Hospital, Peking Union Medical College \& Chinese Academy of Medical Sciences, Beijing, China

Objective The aim of this study was to investigate the relative effects of percent body fat (PBF) and visceral fat index (VFI) on the prevalence of hypertension (HTN) and prehypertension (PHT), and evaluate if excess visceral deposition of body fat increased the risk of HTN or PHT.

Methods A general population-based sample of men $(n=5297)$ and women $(n=6232)$, aged 35 to 64 years, was studied. Based on blood pressure (BP) levels, study participants were classified as normotensive, prehypertensive, and hypertensive. Multivariate linear regression models with systolic or diastolic BP as a dependent variable and multivariate ordinal logistic regression models with BP categories as a dependent variable were performed.

Result In the normotensive, prehypertensive, and hypertensive subjects, the mean (standard deviation) PBF was 27.83 (6.85), 28.91 (6.65), and 30.2 (6.73), respectively, while VFI was 6.97 (3.78), 8.89 (4.35), and 10.75 (5.05), respectively. After adjusting for age, smoking, alcohol consumption, diabetes, and family history, PBF and VFI (in separate models) showed a strong and positive association with HTN or PHT. However, this association did not exist when both PBF and VFI were adjusted for in the same model, but interestingly, VFI remained positively associated with HTN and PHT for both genders $(\mathrm{P}<0.0001)$. A similar positive association was reported with the PBF/VFI ratio.

Conclusion An excess visceral body fat is strongly associated with higher risk of HTN and PHT, measurements of VFI or VFI/PBF ratio, in addition to PBF, will provide a better understanding of adiposity-related risks for HTN and PHT.

\section{Association between the level of high-sensitivity C-reactive protein and cardiovascular risk factors in middle aged chinese: a cardiovascular epidemiological study in 2009-2010 \\ Zengwu Wang, Zuo Chen, Linfeng Zhang, Xin Wang, Manlu Zhu, Wen Wang, Weiwei Chen \\ Department of Prevention and Community Health, National Center for Cardiovascular Disease; Fuwai Hospital, Peking Union Medical College \& Chinese Academy of Medical Sciences, Beijing, China}

Objective To evaluate the association between the level of highsensitivity C-reactive protein (hs-CRP) and cardiovascular risk factors in middle aged Chinese.

Methods Hs-CRP levels were measured among 11623 subjects selected from 12 study populations across China in 2009-2010, demographics of them also were collected. We excluded the subjects with chronic inflammatory diseases; therefore, there were 8931 subjects in the present study. The correlations between the hs-CRP level and cardiovascular risk factors (age, smoking, hypertension, etc.) were investigated.

Result The mean hs-CRP $(\mathrm{mg} / \mathrm{L})$ level was $2.04 \pm 5.95$. Men 
had a higher hs-CRP level than women $(2.19 \pm 6.20 \mathrm{vs} 1.81 \pm 4.37$, $\mathrm{P}<0.0001)$. The levels of hs-CRP were significantly higher in the presence of cardiovascular disease risk factors [such as age, high density lipoprotein cholesterol (HDL-C), body mass index (BMI), systolic blood pressure (SBP), diastolic blood pressure (DBP), glucose, hypertension, diabetes, high cholesterol] than in the absentation of the risk factors, whereas, low density lipoprotein cholesterol (LDL-C) level and current smoking didn't affect the levels of hs-CRP. There was a positive correlation between the levels of hs-CRP and age, as well as LDL-C, BMI, SBP, DBP, triglyceride, total cholesterol (TC), glucose, hypertension, diabetes, LDL-C/ HDL-C, LDL-C/ TC, high cholesterol, with the exception of HDL-C, HDL-C/TC. In addition, the subjects with hypertension and diabetes at the same time had the highest hs-CRP levels.

Conclusion The relationship between hs-CRP levels and cardiovascular risks was significant, especially for the age, HDL-C, High Cholestrol, etc. Therefore, we may predict the risk of cardiovascular diseases (CVD) according to the different hs-CRP levels.

\section{Hypertension prevalence, awareness, treatment and control between han and ethnic minorities in China \\ Hongqiu $\mathrm{Gu}^{1}$, Koon Teo ${ }^{1}$, Wei Li ${ }^{1}$, Jingang Yang ${ }^{1}$, \\ Yang Wang ${ }^{1}$, Jian Bo ${ }^{1}$, Li Xi ${ }^{1}$, Salim Yusuf ${ }^{2}$ \\ 1. State Key Laboratory of Cardiovascular Disease, Fuwai Hospital, \\ National Center for Cardiovascular Diseases, Chinese Academy of Medical Sciences and Peking Union Medical College, Beijing, China 2. Population Health Research Institute, Hamilton Health Sciences and McMaster University, Hamilton, Canada}

Objective To compare hypertension prevalence, awareness, treatment and control between Han and Ethnic Minorities in China.

Methods A cross-sectional study description of the baseline data from Prospective Urban Rural Epidemiology (PURE) study focusing on ethnic minorities (Uygur, Mongolian, Hui and Dai ethnic minority) of China. Hypertension was defined as individuals with selfreported, treated hypertension or with an average of 2 blood pressure measurements of at least 140/90 mm Hg using an automated digital device. Awareness was based on self-reports, treatment was based on the regular use of blood pressure lowering medications, and control was defined as individuals with blood pressure lower than 140/90 mm Hg. Adjusted rate were estimated through logistic regression after age, sex, location and education were controlled.

Result Among the total of 6814 participants (Han: 1379, Ethnic minorities: 5435), 1249 (18.3\%) had hypertension based on self-reports. Hypertension prevalence, awareness, treatment and control was $41.2 \%$, $40.9 \%, 29.7 \%, 4.9 \%$, respectively. Of those 834 treated population, only 137 (16.4\%) were controlled. Results from adjusted rate showed that hypertension prevalence in ethnic minority was significantly higher than Han ethnic ( $42.6 \%$ vs $35.3 \%, \mathrm{P}<0.0001)$, while control $(4.5 \%$ vs $7.1 \%, \mathrm{P}=0.0198)$ and treated control rate $(15.1 \%$ vs $23.2 \%, \mathrm{P}=0.02)$ was lower. Uygur had a higher prevalence $(29.2 \%$ vs $20.7 \%, \mathrm{P}=0.0073)$ and lower control rate $(3.8 \%$ vs $12.8 \%, \mathrm{P}=0.0424)$ compared to Han locally. Mongolian had a lower control rate $(4.9 \%$ vs $14.5 \%, \mathrm{P}=0.0078)$ and treated control rate ( $14.9 \%$ vs $50.2 \%, \mathrm{P}=0.0021)$ compared to Han locally.

Conclusion Compared to Han, ethnic minority had higher hypertension prevalence, especially for Uygur, and lower control rate, especially for Mongolian.

\section{Hypertension prevalence, awareness, treatment and control in China: analysis of nationwide data \\ Dianjiang $\mathrm{Li}^{1}$, Jun $\mathrm{Lv}^{1}$, Fangchao $\mathrm{Liu}^{2}$, Pei Liu ${ }^{1}$, Mo Hao 1. Research Institute of Health Development Strategies, School of Public Health, Fudan University, Shanghai, China \\ 2. Department of Evidence Based Medicine, Fuwai Hospital, National Center for Cardiovascular Diseases, Peking Union Medical College and Chinese Academy of Medical Sciences, China}

Objective To estimate the overall prevalence, awareness, treatment and control of hypertension in China and in various regions in 2010.

Methods We conducted a systematic literature search for relevant studies in three electronic databases (CNKI, Wanfang and PubMed) from January 2003 to March 2014, as well as a manual search of bibliographies of retrieved articles. We identified studies that reported age- and genderspecific prevalence and/ or age-specific awareness, treatment and control of hypertension for at least three separate age-groups within the 20-79 year range in representative population samples from 31 provinces. All data were extracted independently by two investigators using a standardized protocol and data-collection form from the articles or the authors. Prevalence rates were directly age and sex standardized to the China 2010 Census population. The percentages (awareness, treatment, and control of hypertension) were age standardized with the national hypertensive estimates for 2010 used as the standard population.

Result A total of 65 studies were included, with 49 reporting prevalence data covering all 31 provinces and 29 reporting awareness, treatment, and control data from 24 provinces. Overall, $26.7 \%$ of Chinese adults aged $20-79$ years had hypertension ( $28.9 \%$ of men and $24.5 \%$ of women) in 2010. Of these, the awareness, treatment, and control of hypertension were $44.6 \%, 35.2 \%$, and $11.2 \%$, respectively. The prevalence of hypertension was higher in east $(32.2 \%)$ compared with all other regions (30.5\% in northeast, $30.0 \%$ in north, $25.4 \%$ in northwest, $21.9 \%$ in south central and $19.5 \%$ in southwest region), and men had higher prevalences than women in all the six regions. Hypertension awareness, treatment and control among hypertensive patients exhibited strong geographic variations, the control (awareness and treatment) rate was $14.0 \%(42.2 \%$ and $35.0 \%)$ in east, $13.1 \%(50.3 \%$ and $40.3 \%)$ in south central, $12.0 \%(47.6 \%$ and $38.9 \%)$ in north, $6.5 \%$ (49.5\% and $30.9 \%$ ) in northwest, $5.9 \%$ (46.9\% and $36.4 \%$ ) in northeast, and $4.8 \%$ (31.6\% and $22.4 \%$ ) in southwest region, respectively.

Conclusion This analysis demonstrates that hypertension is a major public health challege in China. Effective prevention strategies are urgently needed, especially in the regions with high hypertension prevalence and low control rate such as northeast China.

\section{Hypertension burden in 2010 and projections for 2030 in China \\ Dianjiang $\mathrm{Li}^{1}$, Fangchao Liu ${ }^{2}$, Pei Liu ${ }^{1}$, Mo Hao ${ }^{1}$, Jun Lv 1. Research Institute of Health Development Strategies, School of Public Health, Fudan University, Shanghai China \\ 2. Department of Evidence Based Medicine, Fuwai Hospital, National Center for Cardiovascular Diseases, Peking Union Medical College and Chinese Academy of Medical Sciences, Beijing, China}

Objective To estimate the number of people with hypertension for the years 2010 and 2030 in China.

Methods We searched for relevant studies in three electronic 
databases (CNKI, Wanfang and PubMed) from January 2003 to March 2014, supplemented by a manual search of bibliographies of retrieved articles. We identified studies that reported age- and gender-specific prevalence of hypertension for at least three separate age-groups within the 20-79 year range in representative population samples from 31 provinces. All data were extracted independently by two investigators using a standardized protocol and data-collection form from the articles or the authors. Sex- and age-specific prevalence of hypertension were applied to the 2010 population to estimate the number of individuals with hypertension in each province and the entire country. In addition, the prevalence were applied to China 2030 population projections estimated by the United Nations Population Division to forecast the number of individuals with hypertension in 2030.

Result A total of 49 studies were included, covering all 31 provinces. In 2010, the estimated total number of people with hypertension (20-79 years) was 265 million (145 million among men, and 120 million in women); this number was projected to increase by $32.8 \%$ to a total of 352 million in 2030 , at an annual growth of $1.4 \%$, which is more than three times the annual growth of the total national adults aged 20-79 years. Between 2010 and 2030, the number of adults with hypertension was predicted to increase by $27.6 \%$ (185 million) in men and $39.2 \%$ (167 million) in women because of projected changes in the age distribution of the population.

Conclusion In 2010, there were an estimated 265 million hypertensive patients (20-79 years) in China, making this country the largest people living with hypertension in the world. China will incur serious socioeconomic losses in the 21 st century if no effective strategies are conducted to limit the growing burden of hypertension.

\section{Inhaled budesonide for the prevention of acute mountain sickness in unacclimatization young men: a double-blind randomized controlled trial Xiangjun Li Cardiovascular Department of Xinqiao Hospital, Third Military Medical University}

Background Oral glucocorticoids can prevent Acute Mountain Sickness (AMS); however, these drugs are associated with multiple systemic side effects. The effects of inhaled Budesonide, an alternative AMS therapy, remains unknown.

Methods The 80 healthy young male plain residents (17-33 years old) were recruited. Potential participants were excluded if they had a high altitude ( $>2500 \mathrm{~m}$ ) exposure history in the last year or organic diseases such as congenital heart disease, arrhythmia, liver or kidney dysfunction, psychological or neurological disorder. The subjects were randomly assigned to receive inhalation of budesonide (BUD, 200 $\mu \mathrm{g}$, bid), procaterol tablet (PT, $25 \mu \mathrm{g}$, bid), inhalation of budesonide/ fomoterol (BUD/FM, $160 \mu \mathrm{g} / 4.5 \mu \mathrm{g}$, bid) or placebo (1 tablet, bid) (n $=20$ subjects, respectively). Subjects began treatment three days before an ascent to $3700 \mathrm{~m}$ from $500 \mathrm{~m}$ plain within $2.5 \mathrm{~h}$ by air. The treatment stopped after arrival. The Lake Louis AMS questionnaire, blood pressure (BP), heart rate (HR), and oxygen saturation $\left(\mathrm{SpO}_{2}\right)$ were scored at $20 \mathrm{~h}$, $72 \mathrm{~h}$, and $120 \mathrm{~h}$ following exposure to high altitude. Pulmonary function was measured after $20 \mathrm{~h}$ exposure.

Result Compared with placebo, BUD reduced the incidence of AMS $(70 \%$ vs $25 \%$ at $20 \mathrm{~h}$ after exposure, $\mathrm{P}<0.05 ; 10 \%$ vs $5 \%$ at 72 $\mathrm{h}, \mathrm{P}>0.05 ; 10 \%$ vs $5 \%$ at $120 \mathrm{~h}, \mathrm{P}>0.05$ ) without side effects, relative risk is 0.357 , and the attributable risk is $0.45 . \mathrm{SpO}_{2}$ was higher in $\mathrm{BUD}$, $\mathrm{BUD} / \mathrm{FM}$ and $\mathrm{PT}$ groups compared to placebo at $20 \mathrm{~h}(\mathrm{P}=0.0001)$. All subjects' $\mathrm{SpO}_{2}$ dropped following ascent $(98.1 \%$ to $88.12 \%, \mathrm{P}<0.01)$ and increased gradually but still lower at $120 \mathrm{~h}$ than that at plain $(92.04 \%$ vs $98.1 \%, \mathrm{P}<0.01)$. Pulmonary function was not different among the four groups at $20 \mathrm{~h}$. There was no HAPE or HACE reported.

Conclusion BUD can prevent AMS without side effects. The alleviation of AMS may be related to increased $\mathrm{SpO}_{2}$ rather than pulmonary function. The registration number of this clinical trial was ChiCTR-PRC-12002748.

\section{Association of plasma PCSK9 levels with white blood cell count and its subsets in patients with stable coronary artery disease \\ Sha Li, Yuanlin Guo, Ruixa Xu, Yan Zhang, Chenggang Zhu, Jing Sun, Ping Qing, Naqiong Wu, LiXin Jiang, Jianjun Li Division of Dyslipidemia, State Key Laboratory of Cardiovascular Disease, Fuwai Hospital, National Center for Cardiovascular Diseases, Chinese Academy of Medical Sciences, Peking Union Medical College, Beijing, China.}

Objective Recent studies have suggested that proprotein convertase subtilisin/kexin type 9 (PCSK9) is associated with atherosclerosis and plays a potential role in inflammation. However, the correlation between PCSK9 and white blood cell count (WBCC) has not yet been assessed. The objective of the present study was to examine the association of the WBCC and its subset counts with plasma PCSK9 levels in patients with stable coronary artery disease (CAD).

Methods In this cross-sectional study, a total of 251 consecutive, stable CAD patients who were not treated with lipid-lowering drugs were enrolled at our center between October 2012 and October 2013. The baseline clinical characteristics were collected, and the plasma PCSK9 levels were determined using ELISA. The associations of plasma PCSK9 levels with the WBCC and its subsets were investigated.

Result In the overall population, plasma PCSK9 levels were positively associated with the WBCC $(r=0.167, \mathrm{P}=0.008)$. Multivariable regression analysis revealed that the plasma PCSK9 levels were significantly and independently associated with the WBCC $(\beta=$ $0.217, \mathrm{P}<0.001$ ) and its subsets (neutrophil $\beta=0.152, \mathrm{P}<0.05$; lymphocyte $\beta$ $=0.241, \mathrm{P}<0.001)$. However, the relationships between PCSK9 and WBCC and its subsets remained significant in men (WBCC $\mathrm{r}=0.234, \mathrm{P}=0.001$; neutrophil $\mathrm{r}=0.181, \mathrm{P}=0.014$; lymphocyte $\mathrm{r}=0.226, \mathrm{P}=0.002$ ) but were not significant in women when the analysis was performed based on gender.

Conclusion These data demonstrate that the plasma PCSK9 levels are independently associated with the WBCC and its subsets, suggesting a potential interaction between PCSK9 and chronic inflammation in patients with CAD.

\footnotetext{
Plasma PCSK9 Levels are associated with the severity of coronary stenosis in patients with atherosclerosis

Sha Li, Yuanlin Guo, Ruixia Xu, Yan Zhang, Chenggang Zhu, Jing Sun, Ping Qing, Naqiong Wu, Jianjun $\mathrm{Li}$

Division of Dyslipidemia, State Key Laboratory of Cardiovascular Disease, Fuwai Hospital, National Center for Cardiovascular Diseases, Chinese Academy of Medical Sciences, Peking Union Medical College, Beijing, China.
}

Objective Proprotein convertase subtilisin/kexin type 9 (PCSK9) 
plays a central role in the regulation of low density lipoprotein cholesterol (LDLC) concentration. Recently, experimental studies have demonstrated the association between functional changes of PCSK9 and atherosclerosis. However, the possible correlation of PCSK9 and severity of atherosclerosis is little known. The aim of present study was to tests the hypothesis that plasma PCSK9 levels may be associated with severity of coronary stenosis.

Methods In this cross-sectional study, we consecutively enrolled 243 patients who had angina-like chest pain and/or positive exercise tests and underwent coronary angiography. Baseline clinical characteristics were collected and plasma PCSK9 levels were determined by ELISA.

They were classified into the three groups according to tertiles of standard Gensini score (GS, low group 0-24, $\mathrm{n}=135$; intermediate group 25-53, $\mathrm{n}=54$; high group $\geq 54, \mathrm{n}=54$ ). The relationship between PCSK9 levels and the severity of coronary stenosis were evaluated.

Result Patients in high score group had significant elevated PCSK9 concentrations compared with who in low score group (PCSK9 median 208.61, 217.43, $246.81 \mathrm{ng} / \mathrm{ml}$, respectively). A positive association between plasma PCSK9 and the Gensini score was found $(r=0.191, \mathrm{P}$ $=0.003$ ). In multivariate regression analysis, PCSK9 was demonstrated to be an independent factor of coronary stenosis after adjustment for classical risk factors $(\mathrm{r}=0.139, \mathrm{P}=0.034)$. In addition, patients with mutivessel stenosis tend to have higher PCSK9 levels according to nonparametric test $(\mathrm{P}=0.011)$.

Conclusion The data provides the first line of evidence that plasma PCSK9 levels were associated with severity of coronary stenosis in patients with atherosclerosis.

\section{A cross-sectional study of pulmonary arterial hypertension in congenital heart disease by right heart catheterization \\ Wen $\mathrm{Li}^{1}$, Yunjuan Sun ${ }^{2}$, Kunjing Pang ${ }^{1}$, Hao Wang ${ }^{1}$, Tao Yang ${ }^{1}$, Qing $\mathrm{Gu}^{1}$, Zhihui Zhao ${ }^{1}$, Changming Xiong ${ }^{1}$, Xinhai $\mathrm{Ni}^{1}$, Zhihong Liu ${ }^{1}$, Jianguo $\mathrm{He}^{1}$ \\ 1. State Key Laboratory of Cardiovascular Disease, Fuwai Hospital, National Center for Cardiovascular Diseases, Chinese Academy of Medical Sciences and Peking Union Medical College \\ 2. The First Hospital Affiliated to Suzhou University}

Objective To investigate the prevalence of pulmonary arterial hypertension (PAH) in congenital heart disease, and analyze its independent risk factors.

Methods Recruited patients with congenital heart disease, who were admitted to Fuwai Hospital for the first time, during the period from May 2007 to December 2008. Their baselinedata including age, gender, type of CHD, systemic artery systolic blood pressure and systemic artery diastolic blood pressure were collected, and right heart catheterizations (RHC) were performed by experienced cardiologists before surgical or interventional therapy. Haemodynamic parameters, like mean pulmonary arterial pressure (mPAP), were collected. Patients, whose mPAP $\geq 25 \mathrm{~mm} \mathrm{Hg}$ by RHC, were diagnosed as pulmonary arterial hypertension.

Result We totally enrolled 704 patients, including 319 men and 385 women, and their median age was 5-year-old (Q1: 1 year-Q3: 18 year). Among them, 185 patients had atrial septal defect, 452 patients had ventricular septal defect, 48 patients had patent ductus arteriosus and 19 patients had combinations of above lesions. Besides, 280 CHD patients had PAH, 424 CHD patients didn't have PAH and there was no significant difference between the prevalence of $\mathrm{PAH}$ in two genders $(\mathrm{P}=0.288)$. The prevalence of PAH in ASD, VSD, PDA and combinations of above lesions were $17.30 \%, 46.90 \%, 50 \%$ and $63.16 \%$ separately. In ASD and PDA, the risks of getting PAH in female patients were 4.14 times $(\mathrm{P}=0.007,95 \% \mathrm{CI}$ : $1.39-12.46)$ and 13.8 times $(\mathrm{P}=0.004,95 \% \mathrm{CI}$ : $1.58-120.37)$ separately. Logistic regression analysis found that age, systemic artery systolic blood pressure, ventricular septal defect, patent ductus arteriosus and combinations of lesions were independent risk factors of PAH in CHD. PAH risk would increase $1.7 \%$ with every 1 year increase of CHD patients` age. And VSD, PDA, combinations of above lesions would also increase the risk of PAH compared with ASD. But PAH risk would decrease $4.1 \%$ with every $1 \mathrm{~mm} \mathrm{Hg}$ increase of systemic artery systolic blood pressure (95\% CI: $0.944-0.975, \mathrm{P}<0.001)$.

Conclusion PAH is a common complication of CHD. Age, systemic artery systolic blood pressure and the type of lesion are independent risk factors of $\mathrm{PAH}$ in $\mathrm{CHD}$.

\section{Prevalence, awareness, treatment, and control of hypertension in high-, middle-, and low- income provinces of China \\ Pei Liu ${ }^{1}$, Jun Lv ${ }^{1}$, Fangchao Liu ${ }^{2}$, Dianjiang $\mathrm{Li}^{1}$, Mo Hao 1. Research Institute of Health Development Strategies, School of Public Health, Fudan University, Shanghai, China \\ 2. Department of Evidence Based Medicine, Fuwai Hospital, National Center for Cardiovascular Diseases, Peking Union Medical College and Chinese Academy of Medical Sciences, Beijing, China}

Objective To examine hypertension prevalence, awareness, treatment, and control in high-, middle-, and low-income provinces in China.

Methods We conducted a systematic search for relevant studies in three electronic databases (CNKI, Wanfang and PubMed) from January 2003 to March 2014, as well as a manual search of bibliographies of retrieved articles. We identified studies that reported age- and genderspecific prevalence and/or age-specific awareness, treatment and control of hypertension for at least three separate age-groups within the 20-79 year range in representative population samples from 31 provinces. The provinces in mainland China were divided into three categories according to their gross domestic product: high-income provinces (HIPs), middle-income provinces (MIPs), and low-income provinces (LIPs). Prevalence rates were directly age and sex standardized to the China 2010 Census population. The percentages (awareness, treatment, and control of hypertension) were age standardized with the national hypertensive estimates for 2010 used as the standard population, respectively.

Result A total of 65 studies were included, with 49 reporting prevalence and 29 reporting awareness, treatment, and control. In 2010, the estimated prevalence of hypertension was $21.2 \%, 27.7 \%$, and $29.4 \%$ in LIPs, MIPs, and HIPs, respectively. Among patients with hypertension, the percentages aware (44.5\% in HIPs, $46.3 \%$ in MIPs, and $37.3 \%$ in LIPs) and treated (35.9\% in HIPs, $38.1 \%$ in MIPs, and $27.6 \%$ in LIPs) were lower in LIPs than those in HIPs and MIPs. In addition, the control of hypertension was related with economic development, the control rate was $6.5 \%$ in LIPs, $10.2 \%$ in MIPs, and $13.0 \%$ in HIPs, respectively.

Conclusion This study indicates that hypertension has become an important public health burden not only in low-income, but also in middle- and high-income provinces. Effective government responses are needed to tackle hypertension in China, especially in socioeconomically disadvantaged subpopulations. 


\section{Effect of acute high altitude exposure on lung function and relationship between lung function and AMS}

Pan Song, Junqin Zhang, Ji Hang, Xubin Gao, Jie Yu, Lan Huang

Cardiovascular Department of Xinqiao Hospital, the Third Military

Medical University

Objective To investigate the effect of acute high altitude exposure on lung function and the relationship between lung function and AMS.

Methods We collected the lung function and Lewis Lake data of 73 subjects ( age 18 to 26, male ) at sea-level and junmachang (after five days Exposure to 3000 m, 3900 m).

Result Compared with sea-level, lung function decreased in FVC, MMF, $\mathrm{V}_{50}, \mathrm{~V}_{25}$ While $\mathrm{FEV}_{1}, \mathrm{PEF}, \mathrm{V}_{75}$ did not change; FVC, FEV ${ }_{1}$, $\mathrm{PEF}, \mathrm{MMF}$ was used to analyze the relationship between lung function and AMS, there is no differences in lung function between AMS group and Non-AMS group at sea-level, but lung function of AMS group is statistically significant lower than Non-AMS group in FVC, MMF at high altitude; there is differences between AMS group and Non-AMS group in the rate of change of FVC, MMF; logistic regression analysis showed that the rate of change of the FVC was independent risk factors, correlation analysis showed that the change of FVC and the change of oxygen saturation is relative.

Conclusion Lung function showed restriction decreased after acute high altitude exposure, the changes of lung function will increase the hypoxia and susceptible AMS.

\section{Alteration in right heart function upon high- altitude exposure and its roles in acute mountain sickness \\ Xi Liu \\ Cardiovascular Department of Xinqiao Hospital, Third Military Medical University}

Background People who rapidly ascend to the high altitude above $3000 \mathrm{~m}$ or a higher altitude than residence without adaptation will not acclimatize to high altitude in the combined effect of cold, physical overloading labour and hypobaric hypoxia that reduce tolerance hypoxia environment. Most of maladaptive symptoms will disappeared due to compensatory ability in the short term. However, there are still metabolic changes and aggravated and persist symptoms in many people. Generally, the incidence of acute mountain sickness (AMS) increased as the increasing of altitude. The blood oxygen content due to hypoxia will reduce the adaptive compensations for body initiation, for example, the increase of heart rate and acceleration of blood flow velocity, thereby establishing the adaptive mechanism to hypoxic conditions. In case of loss of compensation, the body will have corresponding symptoms, or show the concomitant symptoms after compensating to a certain degree, among which, the changes of cardio-pulmonary functions are most obvious. The symptoms related to cardiovascular system include palpitation, shortness of breath, edema, sleep disorder, etc. We aimed to observe the alterations of right heart function by different altitudes and time phase, explore its roles in AMS and try to provide new clues and direction in clinical diagnosis, treatment and prevention for AMS.

Methods The AMS was diagnosed by Lake Louise international diagnostic criteria including the following five symptoms: headache, dizziness, gastrointestinal symptoms, sleep difficulties and fatigue weakness.

Result Among 264 subjects who rapidly ascended to $3700 \mathrm{~m}$ high altitude, the incidence of AMS was $25.8 \%$ (68 out of 196). Compared with plain, basic follow-up observations of 264 subjects' radical plateau $24 \mathrm{~h}$ right heart function parameters variation is consistent with the findings in the first chapter. Enter the altitude of $3700 \mathrm{~m}$ plateau $24 \mathrm{~h}$ within the $\mathrm{E} / \mathrm{A}_{\text {ratio }}(\mathrm{P}<0.05)$ reduced significantly, while Tei index, $\mathrm{PVR}$, mPAP was increased significantly (all $P$ values were less than 0.05 ). Tei index was higher $(\mathrm{P}<0.05)$ in AMS group than that in Non-AMS group, $\mathrm{E} / \mathrm{A}_{\text {ratio }}$ was lower $(\mathrm{P}<0.05)$ in AMS group than that in Non-AMS group. However, there were no significant differences in PVR, mPAP between AMS and Non-AMS groups.

Conclusion Thehypobaric hypoxic environment of high altitude would lead to a series of changes in right ventricular functions mainly reflected in increase of systolic function, reduction of diastolic function, as well as increase of right ventricular afterload. Although there was no significant increase of right ventricular stroke work when exposing to the higher elevations after temporary adaption at $3700 \mathrm{~m}$, yet the diastolic function degraded gradually, indicating that the body would initially enter into the acclimatization stage with the extension of stay in highaltitude hypoxic environment. The occurrence and symptom severity of AMS were certainly associated with the related right ventricular function indexes of Tei index and $\mathrm{E} / \mathrm{A}_{\text {ratio, }}$, which were also available for prediction and screening susceptible populations.

\section{Liberal versus restricted fluid administration in heart failure patients: a meta-analysis of 6 randomized trials \\ Biao $\mathrm{Fu}$ \\ Cardiovascular Department of Xinqiao Hospital, Third Military Medical \\ University}

Objective International guidelines have recommended fluid restriction for patients with chronic heart failure (CHF). However, this recommendation lacks scientific evidence. This study sought to evaluate effect of fluid restriction on patients with heart failure in randomized controlled trials.

Methods Randomized controlled trials were identified in MEDLINE, EMBASE, and Cochran data base by using the searchkeyword of fluid and heart failure. The liberal fluid intake and restricted fluid therapy was compared in heart failure patients. The risk ratio (RR) and mean difference (MD) were calculated from abstracted data. The studies focused on decompensated heart failure were separated from compensated heart failure as a sensitivity analysis.

Result 6 randomized trials were included. Between liberal and restricted fluid groups, there was no difference in readmission $(\mathrm{RR}=1.32$; 95\% CI: $0.86-2.01 ; \mathrm{P}=0.2)$, mortality $(\mathrm{RR}=1.50 ; 95 \%$ CI: $0.87-2.57$; $\mathrm{P}=0.14$ ), perceived thirst ( $\mathrm{MD}=-0.7 ; 95 \% \mathrm{CI}:-2.58-1.17 ; \mathrm{P}=0.46$ ), duration of intravenous diuretics $(\mathrm{MD}=0.17 ; 95 \% \mathrm{CI}$ : $-1.26-1.6$; $\mathrm{P}=0.81$ ) and serum sodium ( $\mathrm{MD}=-1.61 ; 95 \% \mathrm{CI}$ : $-3.28-0.07 ; \mathrm{P}=$ 0.06 ). With significant heterogeneity in those trials, the pooled $\mathrm{MD}$ of creatinine and BNP in the liberal versus restricted fluid group was 0.20 (95\% CI: 0.15-0.25; P < 0.00001) and 172.59 (95\% CI: 67.38-277.8; $\mathrm{P}=0.001)$ respectively. All endpoints showed no difference between liberal and restricted fluid groups without heterogeneity after removing the study that recruited patients with severe impairment of left ventricular ejection fraction (LVEF).

Conclusion Compared with liberal fluid intake, restrictive fluid prescription did not show more benefit for patients with heart failure, 
especially for those without severe impairment of LVEF. However, the total sample size for each outcome measured was too small to detect true differences between groups.

\section{Myocardial performance index and ejection time in healthy adult males after rapid ascent to $3700 \mathrm{~m}$ \\ Mingyue Rao \\ Cardiovascular Department of Xingiao Hospital, Third Military Medical University}

Objective The purpose of this study was to examine the relationship between acute mountain sickness (AMS) and left ventricular function, and aimed to explore objective diagnostic parameters for AMS by traditional echocardiographic aspects.

Methods One hundred and thirty nine China healthy male servicemen participated in the field trials. Structured case report form (CRF) questionnaires were used to record demographic data, physiological data, previous high altitude exposure and symptoms related to AMS. Resting oxygen saturation $\left(\mathrm{SaO}_{2}\right)$, blood pressure (BP) and heart rate (HR) were measured in 139 all subjects at sea level, within 24 hours after arrival at $3700 \mathrm{~m}$. Left ventricular functions were also examined in these subjects by Doppler echocardiography. AMS was diagnosed with Lake Louise score (LLS). Comparisons of the relevant parameters were made between subjects with and without AMS.

Result Upon acute high altitude exposure, 75 of 139 (53.96\%) subjects met the criteria for AMS. Cardiac output (CO), ejection fraction (EF) and HR were significantly increased, but $\mathrm{SaO}_{2}, \mathrm{E} / \mathrm{A}_{\text {ratio }}$ obviously decreased in the two groups after acute high altitude exposure. The AMS group had remarkable shorter ejection time (ET), but higher left ventricular myocardial performance index (LV Tei index), HR and systolic blood pressure (SBP) than the non-AMS group. LV Tei index, HR, SBP showed significantly positive correlations with LLS, but ET negatively correlated with LLS. In addition, ET cutoff value of $0.274 \mathrm{~ms}$ for diagnosis of AMS was identified by receiver operating characteristic (ROC) curves analysis [area under the curve (AUC), 0.779; 73.97\% sensitivity; $76.74 \%$ specificity]. LV Tei index cutoff value of 0.463 for diagnosis of AMS was identified (AUC, 0.711; 67.12\% sensitivity; $69.77 \%$ specificity).

Conclusion Upon acute high altitude exposure, LV Tei index and ET are significantly correlated with LLS and have relative accurateness in diagnosis of AMS by ROC analysis. Our findings will provide novel parameters for objective diagnosis of AMS.

\section{Sleep quality changes in insomniacs and non- insomniacs after acute altitude exposure and its relationship with acute mountain sickness \\ Xugang Tang \\ Cardiovascular Department of Xinqiao Hospital, Third Military Medical University}

Objective To observe the changes in subjective sleep quality among insomniacs and non-insomniacs after acute ascending to $3700 \mathrm{~m}$, and the possible relationship with acute mountain sickness (AMS).

Methods 600 adult men were recruited. Sleeping quality was evaluated prior to ascent and in the first, third, seventh, fourteenth, and sixtieth days at $3700 \mathrm{~m}$, using the Athens Insomnia Scale (AIS) and Lake Louise scoring system. Arterial oxygen saturation $\left(\mathrm{SaO}_{2}\right)$ was measured.
Result Despite resolving insomnia in few people, the incidence of insomnia among insomniacs remained stable at $90 \%$ after rapid ascent to $3700 \mathrm{~m}$. However, among non-insomniacs, the incidence of insomnia sharply increased to $32.92 \%$ in the first day of altitude exposure, which progressively reduced to $4.26 \%$ by the sixtieth day of altitude stay. At $3700 \mathrm{~m}$, the incidence of AMS among insomniacs was $77.11 \%, 58.95 \%$, and $31.65 \%$ on the first, third, and seventh days, respectively, which was significantly higher than that among non-insomniacs $(56.53 \%, 26.67 \%$, and $12.22 \%$, respectively). Furthermore, the $\mathrm{SaO}_{2}$ were significantly lower amonginsomniacs than among non-insomniacs in the first days of altitude exposure. Multivariate regression revealed that elevated AIS scores are an independent risk factor for AMS (adjusted OR $=1.405$, 95\% CI: 1.346-1.467, P < 0.001) at high altitude. Additionally, high $\mathrm{SaO}_{2}$ and long duration of altitude exposure are protective factors against AMS.

Conclusion Our results suggest that the effect of high-altitude exposure on subjective sleep quality is more marked among noninsomniacs than among insomniacs, whereas AMS is especially common among the latter. Moreover, poor-quality sleep is a risk factor for AMS.

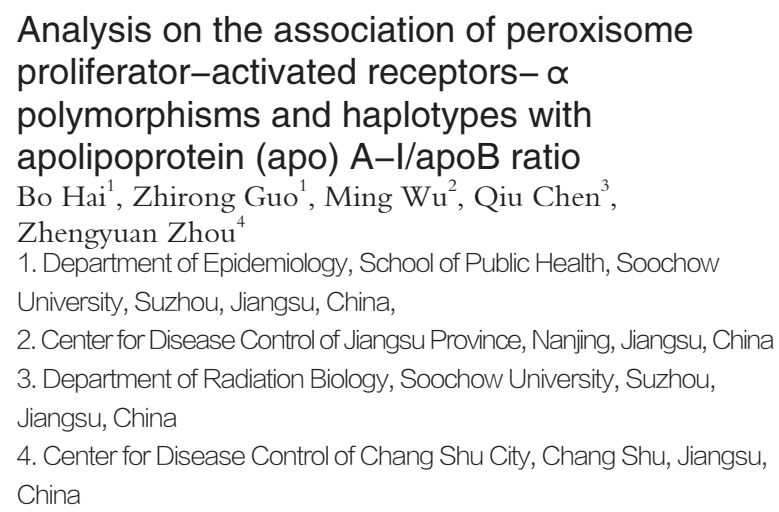

Objective The peroxisome proliferator-activated receptors- $\alpha$ (PPAR- $\alpha$ ) is one of the ligand-activated transcription factors in the nuclear hormone receptor superfamily that function as the master regulators of fatty acid oxidation and lipoprotein metabolism, energy balance, cell proliferation and differentiation and atherosclerosis. The study aimed to investigate the association between PPAR- $\alpha$ polymorphisms and haplotypes with apoA-I/apoB ratio level. About 630 subjects were genotyped for the three single-nucleotide polymorphisms used as genetic markers for the PPAR- $\alpha$ gene (rs1800206, rs135539 and rs4253778).

Methods 630 subjects (212 males, 418 females) were randomly selected from the Prevention of Metabolic syndrome (MS) study in Jiangsu province cohort populations. The genotypes of rs 1800206 , rs135539 and rs4253778 polymorphisms were identified by TaqMan PCR. A linear regression model was used to analyze the relations between gene polymorphism and apoA-I/ apoB ratio level. Mean difference (Difference) and 95\% confident interval (95\% CI) were calculated. Generalized multifactor dimensionality reduction (GMDR) was used to explore the gene-gene interaction. Then we chose the polymorphisms in significant GMDR model to make haplotype.

Result The three SNPs were within Hardy-Weinberg equilibrium $(P>0.05)$. Linkage analysis showed that there was no significant linkage disequilibrium between these polymorphism $\left(\mathrm{D}^{\prime}<0.75\right)$. After adjustment for age, sex, smoking, alcohol consumption, the $\mathrm{C}$ allele 
$(\mathrm{GC}+\mathrm{CC})$ of $\mathrm{rs} 4253778$ was significantly associated with the increase in the risk of apoA-I/ apoB ratio level [Difference ( $95 \% \mathrm{CI}): 0.76$ (0.04 1.48), $\mathrm{P}=0.039$ ]. The $\mathrm{V}$ allele ( $\mathrm{LV}+\mathrm{VV}$ ) of $\mathrm{rs} 1800206$ was significantly associated with the decrease in the risk of apoA-I/ apoB ratio level [Difference (95\% CI): -1.21 (-1.91 - - 0.51), P = 0.008]. However, rs135539 was not significantly associated with apoA-I/ apoB ratio level. Generalized multifactor dimensionality reduction analysis showed that there was a potential gene-gene interaction between rs1800206 and rs135539 $(\mathrm{P}=0.0010)$. The $\mathrm{A}-\mathrm{V}$ and the $\mathrm{C}-\mathrm{V}$ haplotype (established by rs135539 and rs1800206) were significantly associated with decrease in the risk of apoA-I/ apoB ratio level, the Difference $(95 \% \mathrm{CI})$ were -1.14 $(-1.96--0.32)$ and $-1.3(-2.25--0.36)$, respectively.

Conclusion This study suggested that the rs4253778 and rs1800206 polymorphisms were associated with apoA-I/ apoB ratio level and haplotype analysis showed for the first time that the $\mathrm{A}-\mathrm{V}$ and the $\mathrm{C}-\mathrm{V}$ haplotype (established by rs135539 and rs1800206) may be a genetic marker of apoA-I/ apoB ratio level in the Chinese Han population.

\section{Comparative study on the achievement in primary school children with congenital heart disease accepted cardiac surgical operation after or before primary school admission \\ He Li, Meiling Shi, Mulan Deng, Xiaxing Jiang, Jimei Chen, Yifeng Cai, Jian Zhuang \\ Guangdong Cardiovascular Institute, Guangdong General Hospital, \\ Guangdong Academy of Medical Sciences}

Objective With comparing the achievement in the primary school children suffering from congenital heart disease (CHD), who have accepted the cardiac surgical operation after or before the primary school admission, to analyze whether the cardiac surgical operation age will affect their school achievement.

Methods The $265 \mathrm{CHD}$ subjects (age $=10.51 \pm 2.40$, male 165 and female 100), been operated in the year 2006-2008, were investigated with self-designed questionnaire. The questionnaire was mailed and finished by the subjects or parents with the informed consent. The t-test, t'-test and Rank-sum test were applied for the significant difference test. The significant level was set at 0.05 .

Result The results showed: The operation age in the groups after and before the primary school admission (120 subjects in the after group, 145 subjects in the before group) was $9.38 \pm 1.72$ and $5.49 \pm 1.18$ years old, respectively, with significant difference $(\mathrm{t}=21.04, \mathrm{P}<0.0001)$. The primary school admission age in the groups after and before the primary school admission was $6.66 \pm 0.82$ and $6.96 \pm 0.88$ years old, respectively, with significant difference $(\mathrm{t}=2.82, \mathrm{P}=0.0051)$. The achievement in CHD's primary school children been affected in mild, moderate and severe degrees in the groups after and before the primary school admission were $40.00 \%, 15.83 \%, 5.83 \%$ and $15.17 \%, 10.34 \%, 5.52 \%$, respectively, with significant difference (Rank-sum test: $Z=4.2437, \mathrm{P}<$ 0.0001).

Conclusion Whether the cardiac surgical operation age might affect the CHD primary school children's achievement is still little known. Which is better to accept the operation after or before the primary school admission based on the achievement? Although the primary school children operated before the primary school admission will delay the primary school admission age for 3.6 months at an average, the achievement in those operated after the primary school admission will be affected severer than that of those operated before the primary school admission. It's possible those accepted the operation after primary school admission might need the rest time at home while they were at the primary school stage, which will affect their primary school achievement. Or those at some big-age stage might have a big effect on their psychology, or their psychological feelings are more sensitive than that of those small-age ones, which might decrease the achievement or performance. So it is suggested that CHD children should have a cardiac surgical operation before the primary school admission if it is possible based on the achievement in the CHD's primary school children, which should be pay more attention by those coming from the rural areas or faraway district.

\section{Interaction of peroxisome proliferator-activated receptors-delta gene haplotypes and current smoking status on abdominal obesity risk \\ Wei Fan ${ }^{1}$, Zhirong Guo ${ }^{1}$, Zhengyuan Zhou ${ }^{2}$, \\ Mengmeng $\mathrm{Liu}^{1}$, Bo Hai ${ }^{1}$, Huijian Xie ${ }^{1}$ \\ 1. Department of Epidemiology, School of Public Health, Medical College of Soochow University, Suzhou, China \\ 2. Center for Disease Control of Changshu City, Suzhou, China}

Objective In this study, We are now focus on the interaction of PPARD gene haplotypes and current smoking status on abdominal obesity and detect whether the interaction exsists.

Methods A total of 820 individuals were recruited in our current study. Two SNPs that covered rs2016520 and rs9794 were chosen for our research. And We mainly focused on the interaction of PPARD haplotype and the environmental risk factor, current smoking status and their main effects on abdominal obesity. Association between haplotypes and abdominal obesity were analysed by Haplo.score, and Haplo.glm in $\mathrm{R}$ package was applied to detect the interaction.

Result Two SNPs of PPARD gene satisfied Hardy Weinberg equilibrium. Four Haplotypes were constructed base on two SNPs, and the frequency of each haplotype was $0.2686,0.0351,0.1857$, 0.5107 , respectively. In the results of Haplo.score, Haplotype 1 (T-C) and Haplotype 4 (C-C) showed significant association with abdominal obesity (empirical P value $=0.007$ and 0.001 , respectively). Main effects of PPARD haplotype and smoking status were observed during the generalized linear model. While We didn't discover that any of PPARD haplotypes interacted with smoking on abdominal obesity (set Haplotype 4 as reference, all interaction terms didn't showed significant association with abdominal obesity, $\mathrm{P}$ value was $0.7074,0.6149,0.7627$, respectively.

Conclusion Haplotype of PPARD gene and current smoking status have significant main effects on abdominal obesity. But the interaction between PPARD gene haplotype presented here and smoking on abdominal obesity is absent.

\section{Additional use of a phosphodiesterase 5 inhibitor in patients with pulmonary hypertension secondary to chronic systolic heart failure Xiaojing Wu Cardiovascular Department of Xinqiao Hospital, Third Military Medica University}

Objective Increased indiscriminate use of pulmonary artery hypertension targeted drugs has been observed in patients with pulmonary hypertension $(\mathrm{PH})$ secondary to heart failure. We performed a meta-analysis to evaluate the chronic effects of using phosphodiesterase 
5 (PDE5) inhibitors to treat patients with $\mathrm{PH}$ secondary to chronic systolic heart failure.

Methods and Result PubMed, EMBASE, and the Cochrane Library were searched through October 2013 for randomized controlled trials (RCTs) assessing PDE5 inhibitor treatments in PH patients secondary to chronic heart failure. Six RCTs involving 206 chronic systolic heart failure patients with $\mathrm{PH}$ complications were included. Sildenafil was used in all trials. Sildenafil treatment resulted in fewer hospital admissions compared with the placebo treatment $(3.15 \%$ vs $12.20 \%$; RR $=0.29 ; 95 \%$ CI: $0.11-0.77)$. Various hemodynamic parameters were improved with additional sildenafil treatment, including reduced mean pulmonary artery pressure [weighted mean differences (WMD), $-5.71 \mathrm{~mm} \mathrm{Hg}, \mathrm{P}<0.05]$, and pulmonary vascular resistance (WMD, -81.5 dynes $\cdot \mathrm{cm}^{-5}, \mathrm{P}<0.00001$ ), increased left ventricular ejection fraction (WMD, $3.95 \%, \mathrm{P}<0.001$ ), and unchanged heart rate and blood pressure. The exercise capacity improved (Oxygen consumption at peak exercise, WMD, $3.20 \mathrm{ml} /(\mathrm{min} \cdot \mathrm{kg}), \mathrm{P}<0.00001$; Ventilation to $\mathrm{CO}_{2}$ production slope, WMD, $-5.89, \mathrm{P}<0.00001$ ), and the clinical symptoms were relieved based on the breathlessness (WMD, 7.72, $\mathrm{P}<0.00001$ ), fatigue (WMD, 2.28, $\mathrm{P}<0.05$ ), and emotional functioning (WMD, 5.92, $\mathrm{P}<0.00001)$ scores.

Conclusion Additional sildenafil treatment is a potential therapeutic method to improve pulmonary exercise capacity and quality of life by ameliorating $\mathrm{PH}$ in patients with chronic systolic heart failure.

\section{Effect evaluation on the model of third party service for standard management of hypertesion in occupational population Quanyong Xiang ${ }^{1}$, Qiuhua $\mathrm{Wu}^{2}$, Lin Zhang ${ }^{3}$, Yu Qin ${ }^{1}$, Zengwu Wang ${ }^{4}$, Xin Wang ${ }^{4}$, Weiwei Chen ${ }^{4}$, Yan Xu ${ }^{1}$ 1. Jiangsu Center for Disease Control and Prevention 2. Nanjing Yijia Health Management \\ 3. Nanjing Jiangbei Peoples Hospital \\ 4. National Center for Cardiovascular Disease China, Fuwai Hospital}

Objective To explore the model of third party service participate the standard management of hypertension in occupational population, and to evaluate the effect of this model, to provide the evidence for the making of health management strategy of the occupational population.

Methods 10 occupational areas (branch factory or base operations) from 2 large national-owned enterprises in Nanjing were selected by the method of stratified cluster random sampling. All employees in these 10 areas were investigated for basic health information and screened for hypertension. The contents of investigation included demographic status, disease history, and life behavior, and so on. The contents of body medical examination included body height, body weight, blood pressure, blood fat, and blood glucose, and so on. Many kinds of health education and health promotion were carried out in 8 occupational areas. 204 hypertension cases from these 8 areas were randomly recruited for standard management. Meanwhile, the other 2 occupational areas, and 80 hypertension cases which were randomly selected from these 2areas, were set up as control areas and control group. The effects were evaluated after intervention and standard management for 1 year.

Result One year later, except the control areas and control group, the rates of awareness of health knowledge, smoking in males, control for cooking oil, control for smoking in males, and suitable exercise were significant improvement. The rates of hypertension awareness, treatment, and blood pressure control were significant increased from $65.3 \%$, $63.4 \%$, and $31.2 \%$ to $100 \%, 74.5 \%, 62.8 \%$ after one year standard management. The average of systolic blood pressure (SBP) and diastolic blood pressure (DBP) were significant decreased for $10.3 \mathrm{~mm} \mathrm{Hg}$ and $5.4 \mathrm{~mm} \mathrm{Hg}$ respectively. The improvement of correlation risk factors and biochemical indicators of hypertension were significant after one year standard management, the differences were also significant when compared with the control group.

Conclusion The effects of the hypertension standard management in occupational population were significant when the third party service participated. So the third party professional service was the suitable and ideal model for occupational population health management.

\section{Association between the level of fasting blood glucose and carotid intima-media thickness in Han, Uygur and Kazak population from Xinjiang \\ Haixia Li, Xiaomei Li, Yining Yang, Yitong Ma, Fen Liu, Bangdang Chen \\ Department of Cardiology, First Affiliated Hospital of Xinjiang Medical University, China}

Objective To investigate the relationship between different level of fasting blood glucose and carotid intima-media thickness (IMT) in Han, Uygur and Kazak adult population from Xinjiang.

Methods The 13935 inhabitants among Han, Uygur and Kazak adult population in Xinjiang were investigated and the subjects were divided into three groups according to different fasting blood glucose levels: normal, impaired fasting glucose (IFG) and Diabetes mellitus (DM). IMT values of carotid artery were measured by B mode colorful Doppler Ultrasonography.

Result The IMT values in different nationality and gender increased with fasting blood glucose level increases. To male, the IMT were ( 0.77 $\pm 0.26) \mathrm{mm},(0.79 \pm 0.27) \mathrm{mm}$ and $(0.82 \pm 0.29) \mathrm{mm}$ in normal, IFG and DM croups respectively. To female, the IMT in normal, IFG and $\mathrm{DM}$ croups were $(0.70 \pm 0.25) \mathrm{mm},(0.74 \pm 0.26) \mathrm{mm}$ and $(0.75 \pm 0.29)$ $\mathrm{mm}$ respectively. The results showed statistically significant differences $(\mathrm{P}<0.05)$. Person correlation analysis showed that fasting blood glucose levels and carotid IMT values have a positive correlation. And Multivariate regression analysis showed that age, systolic blood pressure, triglyceride, fasting blood glucose were independent risk factors of IMT.

Conclusion To Han, Uygur and Kazak adult population in Xinjiang, the level of fasting blood glucose is associated with carotid intima-media thickness, it may be an independent influence factor of IMT.

\section{Different target organ venerability with several noninvasive arterial stiffness measurements \\ Junli Zuo, Guili Chang, Pingjin Gao, Dingliang Zhu, \\ Shaoli Chu \\ Department of Hypertension, Ruijin north Hospital, Shanghai Jiaotong University School of Medicine}

Objective To validate the predictor impact of organ venerability with the noninvasive arterial stiffness measurements.

Method We studied a cohort of 508 patients (Diabetes excluded) with essential hypertension $(\mathrm{EH})$. The mean age was $(63.1 \pm 9.3)$ years, 193 patients with pure EH. Target organ damages were defined on the basis of including chronic kidney disease (EH-CKD), brain stroke (EHST) and cardiovascular disease (EH-CHD).

Result Compared with EH, the EH-CKD, EH-ST and CHD 
showed higher age, higher level in UACR, lower eGFR and CG levels ( $\mathrm{P}$ $<0.05)$. The EH-CKD, EH-ST showed longer duration of hypertension $(\mathrm{P}<0.01)$. Carotid-femoral pulse wave velocity (c-f PWV), carotid artery plaque, brachial pulse pressure (BPP) and central pulse pressure (CPP) with target organ damage were significantly higher than those with the pure $\mathrm{EH}(\mathrm{P}<0.05)$, and central augmentation pressure (CAP) was also significantly higher in the EH-CKD group. After adjusting confounding factors, c-f PWV was higher in the presence of EH-CHD and EH-ST $(\mathrm{P}<0.05)$. When EH-CKD was stratified by gender, there was no significantly difference in both subgroups either. After adjusting for confounding factors, each SD $(3 \mathrm{~m} / \mathrm{s})$ increased in c-f PWV entailed a 1.51, 1.77 times higher risk of EH-ST and EH-CHD organ damage. Each SD (8 mm Hg) increased in CAP entailed a 2.38, 2.25 times higher risk of EH-CHD and EH-CKD. Furthermore, the risk of EH-CHD and EH-ST with crotid arerial plague was 2.29, 2.39 times higher those without plague.

Conclusion These data demonstrate that c-f PWV and carotid arteray plague can predict the EH-CHD, EH-ST but not for EH-CKD. While CAP may have good predictive value in EH-CKD and EH-CHD.

\section{Polymorphisms of the TGFBRAP1 gene in relation to blood pressure variability and plasma TGF- $\beta 1$ \\ Daoxia Guo ${ }^{1}$, Chong Shen, Yanchun Chen ${ }^{3}$, Song Yang ${ }^{3}$, Linhong Wang ${ }^{1}$ Yuelong Jin ${ }^{1}$, Lianping $\mathrm{He}^{1}$, Jinfeng Chen ${ }^{2}$, Xianghai Zhao ${ }^{3}$, Wei Zhou ${ }^{3}$, Hailong Zhao ${ }^{2}$, Yingshui Yao 1. School of Public Health, Wannan Medical College \\ 2. Department of Epidemiology and Biostatistics, School of Public Health, Nanjing Medical University \\ 3. Department of Cardiology, Afflilated Yixing Peoples Hospital of Jiangsu University, Peoples Hospital of Yixing City}

Objective TGF- $\beta$ receptor-associated protein 1 (TGFBRAP1) is a cytoplasmic protein shown to bind to TGFBR1 and helped Smad4 to participate in vascular development and remodeling mainly through TGF- $\beta$ /Smad Signaling by bringing Smad 4 into the vicinity of the activated receptor complex and facilitating its transfer to the receptoractivated Smad proteins as a chaperone for Smad4. The purpose of this study is to investigate whether the TGFBRAP1 gene contributes genetic susceptibility to blood pressure regulation, essential hypertension (EH), or plasma TGF- $\beta 1$ levels.

Methods A case-control study comprising 2012 hypertension cases and 2210 controls was used to evaluate the association of TGFBRAP1 gene with essential hypertension, blood pressure regulation and plasma TGF- $\beta 1$ levels. Logistic regression model was used to adjust confounding factor for $\mathrm{EH}$ and general linear model (GLM) was applied to compare blood pressure levels and plasma TGF- $\beta 1$ levels between genotypes in cases and controls. Furthermore, a validation research in a children population was used to replicate the association generated from adult population. 1071 children was selected from 2045 children aged 5-15 years to perform case-control study, which included 343 cases with elevated blood pressure which was defined according to high quartile of SBP and/or DBP over $75^{\text {th }}$ percentile within the same sex and age group, and 728 controls with normal or low blood pressure which was defined according to lower quartile of SBP under $25^{\text {th }}$ percentile and DBP under $\mathrm{P}_{75}$, or DBP under $\mathrm{P}_{25}$ and SBP under $\mathrm{P}_{75}$.

Result There was no statistical association with $\mathrm{EH}$ after adjusted for the covariates in this study population. However, quantitative trait analysis indicated that DBP had a linear decrease with the variations of rs2679860 ( $\mathrm{P}=0.005)$ after adjustment for confounding factor. And normally distributed square root of TGF- $\beta 1(\mathrm{pg} / \mathrm{ml})$ had a linear increased with the variations of rs2679860 $(\mathrm{P}=0.042)$ after adjusting covariates. Further quantitative trait analysis for the Z-scores of SBP and DBP in children indicated that AA genotype carriers had a lower Z-scores of DBP than the AG+GG genotype carriers $(P=0.040)$ in city children after adjusted for covariates. But the direction of this genetic effect was opposite of that in the adult population.

Conclusion Our finding suggests that TGFBRAP1 genetic polymorphisms are significantly associated with DBP variation and plasma levels of TGF- $\beta 1$. The variation of rs2679860 might influence the direct modulatory effect of TGF- $\beta 1$ on the blood pressure by regulating the plasma levels of TGF- $\beta 1$.

\section{Relationship between circadian blood pressure rhythm and renal function in obese elderly women \\ Jianchao $\mathrm{Xu}^{1,2}$, Zhendong Liu ${ }^{2}$, Fanghong $\mathrm{Lu}^{2}$ \\ 1. School of Medicine and Life Sciences, University of Jinan-Shandong \\ Academy Science \\ 2. handong Academy of Medical Science}

Objective To investigate the relationship of circadian blood pressure rhythm to renal function in obese elderly women.

Methods Data were extracted from 353 obese elderly women [age $(73.71 \pm 8.02)$ years, body mass index $\left.=(27.2 \pm 2.4) \mathrm{kg} / \mathrm{m}^{2}\right]$. Ambulatory blood pressure was collected in all participants. And divided into two groups by the nocturnal blood pressure drop rate (nBPDR): dipper group $(\mathrm{nBPDR} \geq 10 \%, \mathrm{n}=189)$, non-dipper group $(\mathrm{nBPDR}<10 \%, \mathrm{n}=$ 164). Renal function (RF) was assessed by creatinine clearance rate (Ccr) ( $\mathrm{ml} / \mathrm{min})$. Cer was calculated using the Cockroft and Gault formula: Ccr $=(140$-age $) \times$ weight $(\mathrm{kg}) /($ serum creatinine $\times 72[\times 0.85$ for women $])$. The lower limit of Ccr for normal RF was $70 \mathrm{ml} / \mathrm{min}$.

Result Ccr in dipper group $(77.8 \pm 34.2) \mathrm{ml} / \mathrm{min}$ was significantly higher than they in the non-dipper group $[(70.9 \pm 33.2) \mathrm{ml} / \mathrm{min} ; \mathrm{P}<0.05]$. And in non-dipper group the renal insufficiency rate $(64.6 \%, \mathrm{n}=106$ cases) was significantly higher than they in the dipper group $(49.7 \%, \mathrm{n}$ =94) $\left(\lambda^{2}=7.937, \mathrm{P}<0.01\right)$. Logistic regression analyses showed that age $(\mathrm{OR}=1.079,95 \% \mathrm{CI}: 1.034-1.127 ; \mathrm{P}<0.05)$, glucose $(\mathrm{OR}=1.682$, 95\% CI: $1.066-2.653$; $\mathrm{P}<0.05)$, BMI (OR $=1.500,95 \%$ CI: $1.051-$ $2.140 ; \mathrm{P}<0.05)$, and circadian blood pressure rhythm are important factors for renal function $(\mathrm{OR}=1.048,95 \% \mathrm{CI}: 1.014-1.083 ; \mathrm{P}<0.05)$.

Conclusion The disorder of circadian blood pressure rhythm may cause Ccr reduce in obese elderly women.

\section{The Incidence of congenital heart disease from the congenital heart disease network study in Guangdong province, China \\ Yanji Qu, Zhiqiang Nie, Xiaoqing Liu, Jimei Chen, Wei Pan, Jinzhuang Mai, Xiangmin Gao, Yong Wu, Yanqiu Ou, Jian Zhuang \\ Guangdong Cardiovascular Institute, Guangdong General Hospital,}

Objective To explore the incidence of congenital heart disease (CHD) based on the prevention and treatment network of CHD in Guangdong province.

Methods The prevention and treatment network of CHD in 
Guangdong province comprises 35 general hospitals and maternal and child care service centers from 18 cities distributed in all directions of Guangdong province. Fetus and infants less than 1 year old were monitored for CHD in all included clinical sites from 2004 to 2012. The overall incidence of CHD were calculated and stratified according to outcomes, gender, residence and age of mothers. Temporal trend and regional distribution of the incidence of CHD were explored. The frequency of different forms of CHD were identified according to the outcomes including total birth, live birth and still birth.

Result Totally, 638791 fetus and infants were monitored in our network from Jan 1, 2004 to Dec 31, 2012 and 6045 were diagnosed with CHD. The overall incidence of CHD was 9.46 per 1000 and it was significantly higher in still birth ( $34.03 \%$ vs $9.02 \%$ in live birth, $\mathrm{P}=0.00$ ), infants died in 7 days after birth $(40.03 \%)$, male ( $8.05 \%$ os $7.27 \%$ in female, $\mathrm{P}=0.00)$, urban residence $(9.37 \%$ o vs $8.79 \%$ in rural residence, $\mathrm{P}$ $=0.016$ ) and gravidas younger than 25 or older than 35 . The incidence of CHD showed an uprising tendency from 2004 to 2012 and the regional distribution changed during this period. $126(42.42 \%)$ in 297 cases diagnosed prenatally were therapeutic terminated and this proportion was higher than in other countries. VSD, PDA and ASD were the most common CHD forms on the whole.

Conclusion The CHD network study of Guangdong province is the first province-wide hospital-based study of CHD in China. The incidence of CHD in this study was higher than previous results from China. There was an uprising incidence rate from 2004 to 2012 which mainly attributed the improvement of diagnostic techniques. The prenatal diagnosis rate was low, while the therapeutic termination of the pregnancy rate was high. Frequencies of different lesion forms in Guangdong province were comparable with other studies.

\section{Clinical characteristics and prognosis of 60 patients with midventricular obstructive hypertrophic cardiomyopathy \\ Lirong Yan, Yinjian Yang, Xiying Guo, Zhimin Xu, Yishi Li, Chaomei Fan \\ Key Laboratory of Clinical Trial Research in Cardiovascular Drugs, Ministry of Health, State Key Laboratory of Cardiovascular Diseases, FuWai Hospital, National Center for Cardiovascular Diseases, Chinese Academy of Medical Sciences and Peking Union Medical College, Beijing, China}

Background Midventricular obstructive hypertrophic cardiomyopathy (MVOHCM) is a rare form of hypertrophic cardiomyopathy. Knowledge regarding the diagnosis, morbidity and cardiovascular mortality is limited. In this study, we aimed to describe the long-term outcomes of patients with MVOHCM followed in a tertiary referral center.

Methods A retrospective study of 60 patients with MVOHCM diagnosed at Fuwai Hospital was performed. Clinical features, mortality and cardiovascular morbidity were analyzed.

Result The 60 patients with MVOHCM represented $2.9 \%$ of all the hypertrophic cardiomyopathy cases $(\mathrm{n}=2068)$. At diagnosis, the mean age was $(40.2 \pm 15.0)$ years. During $(7.1 \pm 6.3)$ years of follow-up after diagnosis, the cardiovascular mortality was $15.0 \%$. The probability of survival at 10 years was $(77.0 \pm 8.0) \%$. The following two predictors of cardiovascular mortality were identified: severe ventricular septal hypertrophy $\geq 30 \mathrm{~mm}(\mathrm{HR}=3.19 ; \mathrm{P}=0.031)$ and unexplained syncope $(\mathrm{HR}=4.59 ; \mathrm{P}=0.002)$ at baseline. Thirty patients $(50.0 \%)$ had one or more morbid events, and the most frequent was non-sustained ventricular tachycardia (NSVT). Apical aneurysm formation was identified in 20\% of patients, and the patients with apical aneurysms were more inclined to experience NSVT than patients without apical aneurysm $(58.3 \%$ vs $16.7 \% ; \mathrm{P}=0.003)$. Peak pressure gradient $\geq 70 \mathrm{~mm} \mathrm{Hg}(\mathrm{HR}=3.00 ; \mathrm{P}=$ 0.01 ) at baseline was identified as the only predictor of apical aneurysm.

Conclusion In Chinese patients, MVOHCM is associated with an unfavorable prognosis of cardiovascular mortality. One-half of these patients experience major cardiovascular events, and 20\% develop an apical aneurysm, which significantly increases arrhythmia events. These data warrant measures to ensure the early recognition of MVOHCM followed by appropriate therapeutic interventions.

\section{Shift work and risk of metabolic syndrome: a meta-analysis of cohort studies \\ Chen Huang, Zunsong Hu, Jianfeng Huang, Dongfeng Gu State Key Laboratory of Cardiovascular Disease, Fuwai Hospital, Department of Evidence Based Medicine, National Center for Cardiovascular Diseases, Chinese Academy of Medical Sciences and Peking Union Medical College, Beijing, China}

Objective Accumulating epidemiological studies have suggested that shift work would increase risk of metabolic syndrome (MS), but the results are controversial. We performed a meta-analysis to summarize the evidence relating shift work and risk of MS.

Methods Articles were systematically searched in PubMed, EMbase and the Cochrane Library Database up to May 2014. And reference lists of the retrieved articles were also reviewed. Observational studies that reported the estimates with $95 \%$ confidence intervals (CIs) for MS in relation to shift work, using daytime work or general population as reference, were included. Random-effects models were used to pool the overall associations between shift work and MS. The Cocharne Q test and $\mathrm{I}^{2}$ statistics were used to test heterogeneity among studies.

Result 1258 individuals developed MS cases among 12039 participants from six studies were included in this meta-analysis. The pooled relative risk (RR) and 95\% CI of shift work for the development of MS is 1.73 (1.19-2.54). Stratified analyses indicated a significant association among men but not for women, with pooled RR $(95 \% \mathrm{CI})$ of 1.37 (1.02-1.84) and 1.64 (0.34-7.94), respectively. For studies carried out in Asia, the pooled RR (95\% CI) of MS was 1.52 (1.05-2.21). For studies with over $100 \mathrm{MS}$ cases, the pooled RR $(95 \% \mathrm{CI})$ was 1.49 $(1.15-1.92)$

Conclusion Our meta-analysis suggested that shift work may increase the risk of development of MS, especially among men and Asian populations. Education and potential work schedule adjustment for the workers, especially in high risk group of MS may have an important public health implication.

Shift work and risk of diabetes: a meta-analysis of prospective studies

Zunsong Hu, Chen Huang, Jianfeng Huang, Dongfeng Gu State Key Laboratory of Cardiovascular Disease, Fuwai Hospital, Department of Evidence Based Medicine, National Center for Cardiovascular Disease, Chinese Academy of Medical Sciences and Peking Union Medical College, Beijing, China.

Objective The aim of this meta-analysis was to synthesize the evidence on the potential relationship between shift work and diabetes.

Methods We searched PubMed, EMBASE, and the Cochrane 
Library up to May 2014 and references of relevant retrieved articles for studies comparing incidence of diabetes between workers in shift work and those with no-shift work. Prospective cohort studies were included if relative risk (RR) or hazard ratio and $95 \%$ confidence intervals (CIs) for diabetes according to shift work status was reported. The NewcastleOttawa Scale was used to assess the quality of included studies. The pooled estimates were calculated by random-effect models.

Result The search yielded 6 eligible cohort studies with 11 631 diabetes cases among 224645 participants. In the primary meta-analysis, the pooled RR (95\% CIs) of diabetes for individuals with shift work was 1.17 (1.07-1.27). Both male shift workers and female shift workers had increased risk of diabetes, with pooled RR of 1.32 (1.18-1.50) and 1.09 (1.07-1.27), respectively. Further subgroup analyses demonstrated that both factory shift workers and hospital nurses were at an increased risk of diabetes. And significant associations between shift work and diabetes were found in Asian, European and American populations. However, these findings were limited by unmeasured confounding.

Conclusion Our analysis showed shift work is associated with increased risk of diabetes, which may have implications for public policy and occupational medicine.

\section{Association study of nos3 gene polymorphisms and hypertension in han chinese population Linhong Wang ${ }^{1}$, Daoxia Guo ${ }^{1}$, Song Yang ${ }^{3}$, Yuelong Jin ${ }^{1}$, Lianping $\mathrm{He}^{1}$, Jinfeng Chen ${ }^{2}$, Xianghai Zhao ${ }^{3}$, \\ Yanchun Chen ${ }^{3}$, Wei Zhou ${ }^{3}$, Chong Shen ${ }^{2}$, Yingshui Yao ${ }^{1}$ \\ 1. School of Public Health, Wannan Medical College, wuhu, China \\ 2. Department of Epidemiology and Biostatistics, School of Public Health, Nanjing Medical University, China \\ 3. Department of Cardiology, Affliated Yixing Peoples Hospital of \\ Jiangsu University, Peoples Hospital of Yixing City, China}

Background Recent studies have reported that nitric oxide synthase (NOS) gene plays an important role in cardiovascular pathology whereas the association of endothelial nitric synthase (NOS3) gene and hypertension (HT) was controversial between African Americans and European whites. Here we aimed to further investigate the NOS3 genetic effect on the susceptibility of HT in Han Chinese population.

Methods The association of three clinically relevant polymorphisms s4496877, rs1808593 and rs3918186 to HT was tested in a case-control study consists of 2012 HT patients and 2210 controls. Logistic regression was applied to evaluate the association of genotype and haplotypes of the NOS3 gene and HT, and general linear model was performed to compare blood pressure traits between genotypes.

Result Association analysis showed that there wasn't significant association found between the three SNPs rs4496877, rs1808593 and rs3918186 and HT in the whole study population after adjusted for the covariates including age, sex, glucose, lipids, body mass index, smoking and drinking. Further stratification analysis indicated that dominant model of rs3918186 was significantly associated with HT in $\geq 55$ years population, the odds ratio (OR) and $95 \%$ confidence interval $(\mathrm{CI})$ were $1.245,1.010-1.534, \mathrm{P}=0.04$. The recessive models of $\mathrm{rs} 4496877$ and rs1808593 were significant associated with HT in male population ( $\mathrm{P}$ $=0.015)$ and $<55$ years population $(\mathrm{P}=0.025)$ respectively, $\mathrm{OR}(95 \%$ CI) were 3.254 (1.257-8.425) and 1.683 (1.066-2.657). Furthermore, quantitative trait analysis showed that there were significant differences of SBP and DBP among the genotypes (AA, AT and TT) of rs3918186 in non-treatment hypertension populations $(\mathrm{P}=0.016)$ and the genotypes
(GG, GT and TT) of rs4496877 ( $\mathrm{P}=0.025)$ in control populations respectively after adjusted for the covariates.

Conclusion The findings of this study indicated that NOS3 gene contributes to the genetic susceptibility of HT and blood pressure trait in Chinese population and age, sex might modify the genetic effects on HT. We recommend further exploration of the relationship between NOS3 gene and HT in Asian population.

\section{Association study of profilin-1 gene polymorphisms and essential hypertension in han chinese population \\ Daoxia Guo ${ }^{1}$, Yanchun Chen ${ }^{2}$, Yingshui Yao', \\ Xianghai Zhao ${ }^{2}$, Wei Zhou ${ }^{2}$, Yuelong Jin ${ }^{1}$, Lianping $\mathrm{He}^{1}$, \\ Jinfeng Chen ${ }^{3}$, Chong Shen ${ }^{3}$, Song Yang ${ }^{2}$ \\ 1. School of Public Health, Wannan Medical College, China \\ 2. Department of Cardiology, Affiliated Yixing Peoples Hospital of \\ Jiangsu University, Peoples Hospital of Yixing City, China \\ 3. Department of Epidemiology and Biostatistics, School of Public \\ Health, Nanjing Medical University, China}

Objective Profilins are cytoplasmic protein which was shown to bind to many ligands such as actin, the actin-related protein (Arp) 2, gephyrin, Vasodilator-stimulated phosphoprotein (VASP), etc. Profilin-1 is expressed in all tissues, in contrast, profilin-2 functions uniquely in brain because it is expressed at high levels mainly in the brain. As an actin regulatory protein, profilin-1 makes a role in endothelial cell shrinkage and increase vascular permeability. Many studies have reported that the expression of profilin-1 is increased in artery atheromatous plaque of the patients with coronary heart disease (CHD). Animal studies suggested that profilin-1 may contribute to vascular remodeling in hypertension through advanced glycation end products (AGEs) and related pathways. The purpose of this study is to investigate whether the profilin- 1 gene contributes genetic susceptibility to blood pressure regulation, essential hypertension (EH), plasma profilin-1 levels, plasma AGER levels, and whether the profilin-1 is related to AGER.

Methods A case-control study comprising 2012 hypertension cases and 2210 controls was used to evaluate the association of profilin-1 gene with $\mathrm{EH}$ and blood pressure regulation. Logistic regression model was used to adjust confounding factor for $\mathrm{EH}$ and general linear model (GLM) was applied to compare blood pressure levels, plasma profilin-1 levels and plasma AGER levels between genotypes in cases and controls. Correlation analysis was used to assess the correlation of profilin- 1 levels and AGER levels in plasma.

Result There was no statistical association with EH after adjusting covariates in this study population. Quantitative trait analysis indicated that both SBP and DBP had a tenuous decrease with the variations of rs238239 after adjusting covariates whereas neither the correlations reach statistical significance $(\mathrm{P}=0.299, \mathrm{P}=0.177)$. And there was no significant difference of both normally distributed square root of AGER ( $\mathrm{pg} / \mathrm{ml}$ ) or normally distributed Ln of profilin-1 between genotypes of the two SNPs. Correlation analysis of plasma profilin-1 and AGER didn't reveal the positive correlation which was reported in previous study $(\mathrm{P}=$ 0.578).

Conclusion The results of this study didn't support the positive associations of profilin-1 gene and the genetic susceptibility of EH, blood pressure trait and plasma profilin1 as well as plasma profilin-1 and AGER. Thus, the association evidence of profilin1 and hypertension from animal model study should be cautiously cited to research the molecular mechanism human's hypertension. On the other hand, further 
study could be warranted to explore the relationship between profilin-1 gene and other cardiovascular disease such as stroke and coronary heart.

\section{The Clinical Features of Patients with Stable Coronary Artery Disease and Atrial Fibrillation Kuo Zhang, Wenyao Wang, Xun Yuan, Yang Guo, Min Yang, Mu Mu, Yida Tang State Key Laboratory of Cardiovascular Disease, Fuwai Hospital, National Center for Cardiovascular Diseases, Chinese Academy of Medical Sciences and Peking Union Medical College, Beijing, China}

Objective Stable coronary artery disease (CAD) with presence of atrial fibrillation is not rare, which makes clinical management more complicated especially in coronary stent implantation condition. We conducted this retrospective study to assess the risk factors that render stable CAD patients susceptible to arterial fibrillation.

Methods We consecutively collected baseline characteristics and clinical features of stable CAD patients in Fuwai hospital from January 2012 to December 2012. Patients are divided into 2 groups according to atrial fibrillation status, i.e. atrial fibrillation group and control group. Continuous data were expressed as mean \pm standard deviation. Categorical data were expressed as number (percentage). Two-sample $t$ test and the Fisher exact test were used for comparison of continuous and categorical data.

Result 2538 stable CAD patients with complete information were analyzed in our study including 170 atrial fibrillation patients. The incidence of atrial fibrillation among stable CAD patients is $6.7 \%$. Patients with atrial fibrillation are older than those without $(\mathrm{P}<0.001)$. Compared with patients absent of atrial fibrillation, more female patients $(\mathrm{P}=0.036)$, more hypertension patients $(\mathrm{P}<0.001)$, larger left atrial diameter $(\mathrm{P}<$ $0.001)$, larger left ventricular end diastolic diameter $(\mathrm{P}<0.001)$, lower $\operatorname{LVEF}(\mathrm{P}=0.021)$, more frequent abnormal thyroid function $(\mathrm{P}=0.003)$ were observed among stable CAD patients with atrial fibrillation. In addition, patients in atrial fibrillation group tended to have higher levels of uric acid $(\mathrm{P}<0.001)$, NT-proBNP $(\mathrm{P}<0.001)$ and $\mathrm{CRP}(\mathrm{P}=0.04)$.

Conclusion Our findings suggest that female, old age, hypertension, left atrial and ventricular enlargement and abnormal thyroid function are associated with atrial fibrillation among stable CAD patients.

\section{MG53 gene polymorphisms are associated with systolic blood pressure in type 2 diabetes mellitus patients with hyperglycemia \\ Hailong Zhao ${ }^{1}$, Yanchun Chen ${ }^{2}$, Ming Wu ${ }^{3}$, Jie Yang ${ }^{3}$, Daoxia Guo ${ }^{4}$, Song Yang ${ }^{2}$, Xianghai Zhao ${ }^{2}$, Wei Zhou ${ }^{2}$, Yingshui $\mathrm{Yao}^{4}$, Chong Shen ${ }^{1}$ \\ 1. Department of Epidemiology and Biostatistics, School of Public \\ Health, Nanjing Medical University, China \\ 2. Department of Cardiology, Affiliated Yixing Peoples Hospital of \\ Jiangsu University, Peoples Hospital of Yixing City, China \\ 3. Division of Chronic Disease Control, Center for Disease Control of \\ Jiangsu Province, Nanjing, China \\ 4. School of Public Health, Wannan Medical College, China}

Background A recent report published in Nature identified a novel role of Mitsugumin 53 (MG53) as an E3 ligase that targets insulin receptor and insulin receptor substrate 1 for degradation, thus contributes to severe insulin resistance and metabolic syndrome. The aim of this study was to explore whether polymorphisms in MG53 gene influence blood pressure trait in different glucose level.

Methods We recruited 957 normal glucose tolerance (NGT) subjects, 522 impaired fasting glucose (IFG) subjects and 7762 diabetes mellitus (T2DM) patients from community based population. Among them 300 individuals had a history of taking anti-diabetic medicine in 2 weeks before measurement of blood pressure. Two tagging SNPs rs7186832 and rs12929077 were genotyped and general linear model was applied to compare the blood pressure among different genotypes.

Result In NGT and IFG subjects, neither of the variants of MG53 showed statistical association with systolic blood pressure (SBP) and diastolic blood pressure (DBP). Remarkably, linear decrease of SBP was also observed with the variants of $\operatorname{rs} 12929077(\mathrm{P}=0.024)$ in untreated T2DM patients excluding subjects with anti-hypertension medicine history, and SBP of AA, AG and GG genotypes were $134.24 \pm 21.23 \mathrm{~mm}$ $\mathrm{Hg}$, (130.46 \pm 16.39$) \mathrm{mm} \mathrm{Hg}$ and (123.67 \pm 19.67$) \mathrm{mm} \mathrm{Hg}$ respectively. SBP levels of TC and CC genotypes of rs7186832 (125.46 \pm 13.51$) \mathrm{mm}$ $\mathrm{Hg}$ was significantly higher than that of TT genotype (133.97 \pm 20.82$)$ $\mathrm{mm} \mathrm{Hg}, \mathrm{P}=0.017$. In patients with anti-hypertension medicine history, similar correlations were also found. There was no statistical difference of SBP and DBP between different genotypes of MG53 gene in treated T2DM patients without anti-hypertension medicine history.

Conclusion The results suggest that common variants of MG53 do not influence blood pressure of individual in normal glucose tolerance or impaired glucose tolerance yet not developed to diabetes. But in the status of hyperglycemia, the genetic variants of MG53 confer a significant decrease of SBP. These findings indicate that hyperglycemia modifies the genetic effect of MG53 on the traits of blood pressure and further replication and functional research should be warranted.

\section{The study of relationship between red cell distribution width and coronary bifurcation lesions \\ Pinxiao Liu, Peng Qu \\ The Second Affiliated Hospital of Dalian Medical University}

Objective To study the relationship between red cell distribution width -CV level and coronary bifurcation lesions.

Methods 1173 consecutive hospitalized patients with ACS were enrolled in this study from January 2008 to January 2012. The medical history of all the patients was recorded. The red cell distribution width coefficient of variation (RDW-CV) was a measurement derived from the red blood cell distribution curves generated on automated hematology analyzers and was an indicator of variation in RBC size within a blood sample. According to the rules of SYNTAX score, the bifurcation lesion score (Bif-score) was calculated in each patient depending on Medina bifurcation lesion classification, the severity of stenosis and the site of bifurcation lesions. Bifurcation lesion ratio (Bif-ratio), namely "Bif-ratio $=($ Bif-score/SYNTAX Score $) \times 100 \%$ ", was calculated for each patient with coronary bifurcation lesion. According cohort study, we studied the relationship between RDW-CV and coronary bifurcation lesions.

Result According to the presence or absence of coronary bifurcation lesions in patients were divided into two groups, the mean RDW-CV of two groups was $(12.33 \pm 0.92) \%$ and $(12.50 \pm 1.17) \%$. There has significantly statistical difference of RDW-CV found between two groups ( $\mathrm{P}<0.05)$. According to the SYNTAX scores system, patients were divided into three levels, the mean RDW-CV of three levels was $(12.35 \pm 1.07) \%,(12.62 \pm 1.18) \%,(12.96 \pm 1.27) \%$. There has significantly statistical difference of RDW-CV found between three levels. According to RDW-CV level, patients were divided into two 
levels: high-level and slight-level, the mean SYNTAX scores of two levels had significantly statistical difference. Two levels continued to compare with Bif-score, the mean Bif-score of two levels was $(8.48$ $\pm 7.78)$, (10.15 \pm 8.73$)$; the mean Bif-ratio of two levels was (43.46 \pm $26.86) \%,(46.76 \pm 28.52) \%$. There has significantly statistical difference of Bif-score and Bif-ratio found between two levels $(\mathrm{P}<0.05)$. There was a good correlation between RDW-CV and the SYNTAX score (regression coefficient $=0.126, \mathrm{P}<0.001$ ). There was a good correlation between RDW-CV and Bif-score $(\mathrm{R}=0.090, \mathrm{P}<0.05)$. There was a good correlation between RDW-CV and Bif-ratio $(\mathrm{R}=0.061, \mathrm{P}<0.05)$. The elevation of RDW-CV remains an independent factor that associated with Bif-score.

Conclusion Bif-score and Bif-ratio were good indicators to evaluate coronary bifurcation lesions. The elevation of RDW-CV was not only highly coronary artery lesion severity, but also was high incidence of coronary bifurcation lesions and highly coronary bifurcation lesions severity. RDW was one as the independent risk factor of bifurcation lesions.

\section{Trends in cardiac biomarker testing in China for patients with acute myocardial infarction, 2001 to 2011: China PEACE-retrospective AMI study} Lijuan Zhan ${ }^{1}$, Frederick A. Masoudi ${ }^{2}, \mathrm{Xi} \mathrm{Li}^{1}$, Shuang $\mathrm{Hu}^{1}$, Arjun K. Venkatesh ${ }^{3,4,5}$, John A. Spertu ${ }^{6}$, Zhenqiu Lin ${ }^{7}$, Nihar R. Desai ${ }^{4,7}$, Jing Li ${ }^{1}$, Harlan M. Krumholz ${ }^{3,4,7,8,9}$, Lixin Jiang ${ }^{1}$

1. National Clinical Research Center of Cardiovascular Diseases, State Key Laboratory of Cardiovascular Disease, Fuwai Hospital, National Center for Cardiovascular Diseases, Chinese Academy of Medical Sciences and Peking Union Medical College, Beijing, Peoples Republic of China 2. Division of Cardiology, University of Colorado Anschutz Medical Campus, Aurora, Colorado, United States

3. Robert Wood Johnson Clinical Scholars Program, Yale School of Medicine, New Haven, Connecticut, United State

4. Department of Internal Medicine, Yale School of Medicine, New

Haven, Connecticut, United State

5. Department of Emergency medicine, Yale School of Medicine, New

Haven, Connecticut, United State

6. Saint Luke's Mid America Heart Institute/University of MissouriKansas City, Kansas City, Missouri, United States

7. Center for Outcomes Research and Evaluation, Yale-New Haven

Hospital, New Haven, Connecticut, United States

8. Section of Cardiovascular Medicine, Yale School of Medicine, New Haven, Connecticut, United States

9. Department of Health Policy and Management, Yale School of Public Health, New Haven, Connecticut, United States

Objective Cardiac biomarker testing is standard in high-income countries, but little is known about the availability and use of cardiac biomarker testing in low- and middle-income countries (LMICs) such as China. The aim of this study is to describe trends in the availability of biomarker testing in Chinese hospitals and how practice complies with established standards for the diagnosis of acute myocardial infarction (AMI).

Methods Based on a nationally representative sample of 162 Chinese hospitals in 2001, 2006 and 2011, we describe the temporal trends and regional differences in the hospital capability and rates of use of cardiac biomarker testing, as well as the variation in use across hospitals with testing capability, for patients labeled with the diagnosis of
AMI.

Result The proportion of hospitals with biomarker testing capability was $57.4 \%$ in 2001 [ $25.0 \%$ troponin and $32.4 \%$ creatine kinase MB fraction (CK-MB) only] and 96.3\% (81.4\% troponin and 14.9\% CKMB only) in 2011. The proportion of hospitals with troponin testing capability in 2011 was significantly higher in urban compared with rural hospitals $(96.8 \%$ vs $71.4 \%, \mathrm{P}<0.001)$. In 2011 , only $55.9 \%$ of hospitals with troponin testing capability (71 out of 127 hospitals) used the assay for more than $80 \%$ of their patients with AMI. Among hospitals with either biomarker testing capability, there was marked variation in use in both rural (from $7.1 \%$ to $100.0 \%$ of patients) and urban hospitals (from $57.9 \%$ to $100.0 \%$ of patients). In $2011,36.1 \%$ of the patients with AMI did not have troponin tested and $4.9 \%$ did not have either biomarker measured.

Conclusion The recommended biomarker tests for AMI diagnosis are not universally available or consistently applied in China. 


\section{Basic Cardiovascular Medicine}

\author{
Hypoxia preconditioned mesenchymal stem \\ cells prevent cardiac fibroblast activation and \\ collagen production via leptin \\ Panpan Chen ${ }^{1,2}$, Xinyang $\mathrm{Hu}^{1,2}$, Jianan Wang ${ }^{1,2}$ \\ 1. Department of Cardiology, Second Affliated Hospital, Zhejiang \\ University School of Medicine \\ 2. Key lab of cardiovascular disease, Second Affliated Hospital Zhejiang \\ University School of Medicine
}

Background Activation of cardiac fibroblasts into myofibroblasts constitutes a key step in cardiac remodeling after myocardial infarction (MI), due to interstitial fibrosis. Mesenchymal stem cells (MSCs), especially hypoxia preconditioned MSCs (H-MSCs), have been shown to be able to improve post-MI remodeling. Leptin has been shown to promote cardiac fibrosis. The expression of leptin is significantly increased in MSCs after hypoxia preconditioning. However, it is still unknown whether and how leptin participates in the therapeutic effects by MSCs, especially the fibrosis process. The objective of this study was to identify the role of leptin from MSCs in cardiac fibroblasts activation.

Methods The activation of cardiac fibroblasts was induced by hypoxia $\left(0.5 \% \mathrm{O}_{2}\right)$. The effects of MSCs on the fibroblast activation were analyzed by co-culturing MSCs with CFs, and detecting the expression change of $\alpha$-SMA and SM22 $\alpha$, as well as collagen I $\alpha$ I in CFs with western blot, immunofluorescence and Sirius red staining. In vivo MSCs fibrotic effects on left ventricular remodeling were investigated using an acute MI model that was induced by permanent ligation of the left anterior descending coronary artery.

Result Co-cultured MSCs decreased fibroblast activation, hypoxia preconditioned MSCs showed stronger effects on preventing fibroblast activation than normoxia treated MSCs. However, leptin deficit MSCs from $\mathrm{Ob} / \mathrm{Ob}$ mice did not exhibit such effect. The in vivo study also showed that H-MSCs significantly inhibited cardiac fibrosis after MI, along with the decrease of TGF- $\beta /$ Smad 2 and MRTF-A in CFs, which again were absent when leptin in MSCs was deficient.

Conclusion Our data demonstrate that activation of cardiac fibroblast can be inhibited by MSCs, hypoxia preconditioned MSCs enhanced the inhibition effect. Leptin in MSCs played a key role in inhibiting cardiac fibrosis, possibly through blocking both TGF- $\beta / \mathrm{Smad} 2$ and MRTF-A signal pathways.

\footnotetext{
Mast cell plays pivotal role in nicotine-induced atherosclerotic plaque progress and instability

Chen Wang ${ }^{1,2}$, Han Chen ${ }^{1,2}$, Yinchuang $\mathrm{Xu}^{1,2}$,

Xinyang $\mathrm{Hu}^{1,2}$, Jianan Wang ${ }^{1,2}$

1. Department of Cardiology, Second Affliated Hospital, Zhejiang

University School of Medicine

2. Key lab of cardiovascular disease, Second Affliated Hospital,

Zhejiang University School of Medicine
}

Objective Nicotine has been identified topromote atherosclerosis.
But the mechanism of nicotine induced atherogenesishas not been well elucidated. Mast cell plays an important role in high-fatdiet induced atherogenesis. This study focuses on the role of mast cell innicotine induced atherogenesis and plaque instability.

Methods Peritoneal administration of $100 \mathrm{mM}$ disodium cromoglicate (DSCG) was introduced to inhibit mast cell degranulation. 45 ApoE deficient mice weredivided into 3 groups: high-fat diet, highfat diet+nicotine and high-fat diet+nicotine+DSCG ( $\mathrm{n}=15$ each). After 12 weeks of treatments atherosclerotic lesion size of theaortas were quantified. Toluidine blue and tryptase staining identified mastcell count and activation at the lesion. Immuno-staining of CD68 and CD45 were used to evaluate the inflammatory filtration. SMA Ki-67 and sirus red staining were used to study smooth muscle cell proliferation and collagen content in thelesion. In vitro, bone marrow-derived mast cells (BMMCs) were harvested anddivided into 5 groups, which are PBS treatment group as a negative control, compound 48/80 treatment group as a positive control, $100 \mu \mathrm{g} / \mathrm{ml}$ nicotinetreatment group, 100 $\mu \mathrm{g} / \mathrm{ml}$ nicotine treatment with $100 \mathrm{mM}$ DSCG pretreatmentgroup, and nicotine $100 \mu \mathrm{g} / \mathrm{ml}$ with $10 \mu \mathrm{g} / \mathrm{ml}$ mecamylamine pretreatment group. At $0.5 \mathrm{hr}, 1 \mathrm{hr} 2 \mathrm{hrs}$, supernatants were harvested to analyze the mast celldegranulation level. Futhermore conditioned medium were also used to induce themacrophage migration and foam cell formation.

Result Nicotine increases plaque size andmacrophage infiltration decreases smooth muscle collagen content along with theincreases in mast cells count and activation ratio at the lesion which could beinhibited by DSCG. Nicotine induced mast cell degranulation at 2 hours comparing to PBS ( $43.60 \%$ vs $2.3 \%$ ) which could be inhibited by mast cell stablizer DSCG (23.7\%) and nAChR blocker mecamylamine (20.35\%). Macrophage migration ability in the compound $48 / 80$ and nicotine conditional medium group were significantly higher comparing to PBS DSCG and mecamylamine group. Foam cell formation ratioin the compound $48 / 80$ and nicotine conditional group were significantly higher comparing to PBS DSCG and mecamylamine group.

Conclusion Nicotine might induce mast cell degranulation through $\mathrm{nAChR}$ and thenactivate mast cell to release a range of proinflammatory mediators to increasethe migration ability of macrophages as well as the foam cell formation anddestabilize theatherosclerostic plaque. Administration of mast cell stabilizerrevealed the potential of applying mast cell stabilizer in preventing nicotineinduced atherogenesis.

\section{MiR-211 mediated hypoxia induces bone marrow mesenchymal stem cells migration through STAT5A \\ Xinyang $\mathrm{Hu}^{1,2}$, Panpan Chen ${ }^{1,2}$, Jianan Wang ${ }^{1,2}$ \\ 1. Department of Cardiology, Second Affliated Hospital, Zhejiang University School of Medicine \\ 2. Key lab of cardiovascular disease, Second Affliated Hospital, \\ Zhejiang University School of Medicine}

Background Efficacy of intravenous administration of mesenchymal stem cells (MSCs) for myocardial infarction (MI) is limited by low cell migration to the damaged myocardium. Our previous study demonstrated that migration ability of MSCs enhanced by hypoxia preconditioning (HPC). miRNA microarray displayed that miR-211 exhibited the most significant change between HPC and normoxia cultured MSCs. The aim of this study is to identify whether miR-211 regulates MSCs migration and the potential mechanism.

Methods In vitro, transwell assay were used to assess the migration ability of MSC moduated by miR-211 using miR-211 overexpressing 
and knockdown lentivirus. The target gene of miR-211 predicted by Targetscan, which was verified by PCR, western blot and luciferase assay. We knocked down the target gene and assessed the migration capability again. Chromatin immunoprecitation (ChiP) were used to explore the transcription factors that regulate the expression of miR-211. To evaluate the effect of miR-211 on MSCs migration in vivo, miR-211mimic and miR-211-shRNA MSCs from male mice were intravenously delivered $24 \mathrm{~h}$ after MI, the engraft cells were detected by RT-PCR of SRY gene.

Result Quatitative RT-PCR showed that miR-211 expression of MSCs upregulated by HPC. MiR-211 mimic improved MSCs migration by $31.03 \%(\mathrm{P}<0.05)$, however, knockdown miR-211 using shRNA attenuated MSCs migration ability significantly. Signal transducer and activator of transcription 5A (STAT5A) was predicted as one of target genes of miR-211, and was confirmed by PCR and Western blot, it showed miR-211 overexpression dramatically decreased STAT5A expression, while miR-211 knockdown upregulated STAT5A. The luciferase assay showed the similar results. Functional experiment showed that STAT5A knockdown reversed the inhibition of MSCs migration induced by miR-211-shRNA. Interestingly, ChiP assay showed that STAT5A can combine to the promoter of miR-211, which lead to the regulation of miR-211 transcription. In vivo data showed that MiR-211 overexpression enhanced MSCs homing to ischemic myocardium, the long-term experiment illustrated miR211 overexpression MSCs improved cardiac function $28 \mathrm{~d}$ post-MI. However, miR-211 knockdown decreased MSCs homing and hampered cardiac function recovery.

Conclusion These results indicate that miR-211 plays a important role in regulating MSCs migration through targeting STAT5A, meanwhile STAT5A regulates miR-211 transcription.

\section{Transplantation of SIRT1 engineered aged MSCs improves cardiac function in a rat myocardial infarction model \\ Huiqiang Chen ${ }^{1,2}$, Xianbao Liu ${ }^{1,2}$, Jianan Wang ${ }^{1,2}$ \\ 1. Division of Cardiology, Key Division of Ministry of Health, Second \\ Affiliated Hospital, Zhejiang University School of Medicine, Hangzhou, China \\ 2. Provincial Key Laboratory of Cardiovascular Research, Second Affiliated Hospital, Zhejiang University School of Medicine, Hangzhou, China}

Background Previous studies demonstrated that biological aging exerted a negative effect on the therapeutic effects of mesenchymal stem cells (MSCs) -based therapy. Here, using a rat myocardial infarction (MI) model, we tested the hypothesis that SIRT1 may ameliorate the phenotype and improve the function of aged MSCs and hence enhance the efficacy of aged MSCs-based therapy.

Methods Sixty female rats experienced left anterior descending coronary artery ligation and were randomly assigned to receiving intramyocardial injection of cell culture medium (DMEM group), SIRT1 overexpression vector treated-aged MSCs (SIRT1-aged MSC group) obtained from aged male SD rats or empty vector treated-aged MSCs (vector-aged MSC group). Another twenty sham operated rats underwent open-chest surgery without coronary ligation and any other intervention served as controls.

Result SIRT1-aged MSCs group exhibited enhanced blood vessel density in the border zone of MI hearts, which was associated with reduced cardiac remodeling, leading to an improved cardiac performance. Consistent with the in vivo data, our in vitro experiments also demonstrated that SIRT1 overexpression ameliorated aged MSCs senescent phenotype and recapitulated the pro-angiogenesis property of MSCs and conferred the anti-stress response capabilities, as indicated by increases in pro-angiogenic factors, angiopoietin 1 (Ang1) and basic fibroblast growth factor (bFGF), expressions and a decrease in antiangiogenic factor thrombospondin-1 (TBS1) at mRNA levels, and increases in Bcl-2/Bax ratio at protein level.

Conclusions Up-regulating SIRT1 expression could enhance the efficacy of aged MSCs-based therapy for MI which is related to the amelioration of senescent phenotype and hence improved biological function of aged MSCs.

\section{Cerebral protection against ischemia/ reperfusion injury by ischemic preconditioning during moderate hypothermia low flow procedures \\ Zhifei Ma, Wen Chen, Changchun Cao, Xin Chen \\ Nanjing First Hospital, Nanjing Medical University, China}

Objective To determine the effects of ischemic preconditioning (IP) on cerebral protection against apoptosis induced by brain ischemia/ reperfusion $(\mathrm{I} / \mathrm{R})$ in a rat model of moderate hypothermic low flow (MHLF).

Methods Adult male Sprague-Dawley rats were randomly and equally divided into surgery group, sham-surgery and IP-treated surgery group. Surgery group underwent gradual body temperature reduction and bilateral common carotid artery occlusion (CCA) for 120 $\min$ at $25 \pm 0.5^{\circ} \mathrm{C}$, followed by artery reopening and rewarming. The occlusion was excluded in sham-surgery group rats. IP-treated surgery group was pre-treated with four cycles of 2 min occlusion of bilateral CCAs and 5 min reperfusion, before $120 \mathrm{~min}$ of brain ischemia. The regional cerebral blood flow (rCBF) was determined by laser Doppler flowmetry. We investigated brain cell apoptosis levels using the TUNEL assay and assessed mitochondrial apoptosis pathway components by immunohistochemistry, real time PCR and western blot at various time points following reperfusion.

Result rCBF was decreased to around 15\% during bilateral CCA occlusion. IP-treated group displayed decreased numbers of apoptotic cerebral cells and apoptotic protein expression levels. Western analysis revealed IP lead to more efficient apoptotic signaling pathway inhibition.

Conclusion IP suppressed the mitochondrial apoptosis signaling pathway and alleviated cerebral ischemia/reperfusion injury after MHLF in rats. These findings highlight the potential of IP as a neuroprotective therapy for surgery involving MHLF procedure.

\footnotetext{
Overexpression of cardiac ankyrin repeat protein induces dilated cardiomyopathy

Na Zhang ${ }^{1,2}$, Xiaojie Xie ${ }^{1,2}$, Jianan Wang ${ }^{1,2}$

1. Department of Cardiology, Second Affliliated Hospital, Zhejiang

University School of Medicine, Hangzhou, China

2. Key lab of cardiovascular disease, Second Affiliated Hospital,

Zhejiang University School of Medicine, Hangzhou, China
}

Background Cardiac ankyrin repeat protein (CARP) is a nuclear transcription co-factor regulating atrial natriuretic factor (ANF), ventricular myosin light chain 2 (MLC-2v), cardiac troponin C (cTnC) gene expression, and a component of a titin-associated stretch-sensing 
complex in the muscle sarcomere. Moreover, CARP was found to be strongly upregulate in the hearts of hypertrophic animal models and heart failure patients with dilated cardiomyopathy (DCM), ischemic heart disease or arrhythmogenic right ventricular cardiomyopathy. Recent clinical research has indicated that CARP mutation maybe involve in DCM and hypertrophic cardiomyopathy. Despite this substantial experiment and clinical evidence demonstrating the role of CARP in cardiomyocyte and cardiovascular diseases, the question whether it play its demonstrated roles in vivo model and the results of its overexpression remain unsolved.

Methods and Result To investigate the role of CARP in the heart, we generated cardiac-specific CARP transgenic mice used $\alpha$-MHC-CARP construct. Wild type littermates were used as controls. Echocardiography analysis indicated that CARP overexpression induced left ventricular chamber dilation (LVIDd, $3.61 \pm 0.14$ vs $2.43 \pm 0.20 \mathrm{~mm}, \mathrm{P}<0.001)$ and thinned ventricular wall, systolic function was also impaired compared to wile type littermates [ejection fraction, $(50.73 \pm 4.30) \%$ vs $(73.78 \pm 5.03) \%$, $\mathrm{P}<0.01$; fraction shorting, $(26.26 \pm 4.27) \%$ vs $(43.01 \pm 3.14) \%, \mathrm{P}$ $<0.01]$. Histological analysis also showed ventricular dilation and increased cardiomyocyte cross-sectional area $[(432.57 \pm 23.30)$ $\mu \mathrm{m}^{2}$ vs $\left.(305.87 \pm 10.56) \mu \mathrm{m}^{2}, \mathrm{P}<0.01\right]$ without obvious collagen alterations. Further, analysis of ultarstructure in cardiomyocyte by electron microscopy indicated sarcomere disarray and swallowed mitochondria in CARP transgenic mice. In mRNA and protein levels measured by quantitative real time polymerase chain reaction and western blot in CARP transgenic heart, ANF, MLC-2v and cTnC expression was observed significantly down-regulation.

Conclusion Over-expression of CARP in the heart would induce dilated cardiomyopathy with chamber dilation and systolic dysfunction, associated with transcriptional inhibition of cardiac gene expressions and disrupt the normal structure of sarcomere.

\section{A potentiated anti-atherosclerosis in complete bone marrow cell transplantation of $\mathrm{ApoE}^{-/-}$ mice than C57/BL6 mice through inactivation of C-C chemokine receptor 5 \\ Quanwei Tao ${ }^{1,2}$, Xiangyang Xia ${ }^{1,2}$, Na Zhang ${ }^{1,2}$, Qunchao $\mathrm{Ma}^{1,2}$, Hong $\mathrm{Yu}^{1,2}$ \\ 1. Division of Cardiology, Key Division of Ministry of Health, Second Affiliated Hospital, Zhejiang University School of Medicine, Hangzhou, China \\ 2. Provincial Key Laboratory of Cardiovascular Research, Second Affiliated Hospital, Zhejiang University School of Medicine, Hangzhou, China}

Bone marrow cell transplantation to ameliorate atherosclerosis has been studied recently while the relating molecular mechanism is still ambiguous. We want to demonstrate the different profiles between ApoE/- mice and C57/BL6 mice to investigate the diversity of bone marrow cells in atherosclerosis. Isolated bone marrow cells from GFP-labeled ApoE-/- mice and GFP-labeled C57/BL6 mice were quantified to $1 \times 10^{6}$ cells into $100 \mu \mathrm{l}$ volume and then transplanted into 6-week old ApoE/- mice via tail vein injection every other week with high fat diet at the same time ( $\mathrm{n}=12$ in each group). Same volume of PBS was injected as the negative control group $(n=9)$. After the cell injection, 12 weeks later, mice were sacrificed and aortas were extracted to calculate the plaques proportion. The plaques in ApoE-/- mice bone marrow cell group was significantly lower than C57/BL6 group and PBS group (42.2 $\mathrm{mm}^{2} \pm$ $\left.0.4 \mathrm{~mm}^{2}, \mathrm{P}<0.05\right)$. Blood physiobiochemical index of atherosclerosis depicted that a significant decrease of cholesterol $[(52.3 \pm 0.3 \mathrm{mmol} / \mathrm{L})$, $\mathrm{P}<0.01]$ and triglycerides $[(43.7 \pm 0.6) \mathrm{mmol} / \mathrm{L}, \mathrm{P}<0.05]$ in circulating blood of ApoE-/- mice bone marrow cells group than others. Elisa detection of inflammatory factor demonstrated IL-10 was up regulated in ApoE -/- mice bone marrow cells group. Immunofluorescence of GFP in plaques showed more bone marrow cells engraftment in ApoE -/- cell group compared to $\mathrm{C} 57$ cell group $(145 \% \pm 1 \% \mathrm{P}<0.05)$ which indicate a stronger cell recruitment and engraftment of ApoE-/- mice than normal cells. We further screened all inflammatory-related genes by RT-PCR then picked the highest expression genes of Jak2 and AKT and a lacked expression of CC chemokine receptor 5 (CCR5) in ApoE-/mice cell group. Using adenovirus into Apoe-/- mice cells to up-regulate the CCR5, the plaque areas were increased into a same level of control group $\left(61.4 \pm 1.7 \mathrm{~mm}^{2}\right)$. Thus we concluded that Apoe knockout induced bone marrow cells to shut down the expression of CCR5 and to provide a more protective role in cell transplantation of atherosclerosis treatment than C57/BL6 mice bone marrow cells.

\section{PKC epsilon translocation is essential for mesenchymal stem cell paracrine factors mediated cardioprotective effect against ischemia and reperfusion injury \\ Yu Zhou ${ }^{1,2}$, Zhi Jiang ${ }^{1,2}$, Xinyang $\mathrm{Hu}^{1,2}$, Wei Zhu ${ }^{1,2}$, Yingchao Wang ${ }^{1,2}$, Lengmei Wang ${ }^{1,2}$, Jianan Wang ${ }^{1,2}$ 1. Division of Cardiology, Key Division of Ministry of Health, Second Affliated Hospital, Zhejiang University School of Medicine, Hangzhou, China \\ 2. Provincial Key Laboratory of Cardiovascular Research, Second Affliated Hospital, Zhejiang University School of Medicine, Hangzhou, China}

Objective Mesenchymal stem cells (MSCs), considered as a competent candidate for cell-based therapy, protect myocardium after acute myocardial infarction. More and more evidences reported that MSC cardio-protection was relied on its paracrine effect. However, whether MSC paracrine factors can protect myocardium against ischemia and reperfusion (IR) injury, and its mechanism is unknown.

Methods and Result MSC paracrine factors were abstract from condition medium of MSCs. Mice underwent tail vein injection with MSC paracrine factors, saline or inhibitors, followed by reperfusion after 30-minute occlusion. MSC paracrine factors significantly reduced myocardial IR injury, enhanced cardiac function recovery and reduced cardiomyocytes apoptosis. PKC epsilon activation and translocation into mitochondrial was detected in $\mathrm{I} / \mathrm{R}$ injured heart. Inhibition of PKC epsilon activity attenuated protective effect of MSC paracrine factors. In vitro, MSC paracrine factors reduced ROS production, apoptosis and lipid oxidative stress in reoxygenation-injured cardiomyocytes via PKC epsilon pathway. In PKC epsilon dominant negative mice, cardioprotective effect of MSC paracrine factor was limited compared with WT mice. Furthermore, we observed that eNOS/NO pathway was activated with MSC paracrine factor treatment, and inhibitor of eNOS attenuated PKC epsilon nitrification and translocation into mitochondria, which indicated that eNOS/NO pathway is essential in PKC epsilon activation and translocation.

Conclusion MSC paracrine factors can protect myocardium against IR injury by reducing oxidative stress releasing and anti-apoptosis effect. In addition, PKC epsilon activation and translocation plays an essential role in cardioprotection of MSC paracrine factors. 


\section{MicroRNA-223 governs profibrillatory L-type calcium channel subunits protein changes in atrial fibrillation \\ Fengping He, Xin Xu, Shuguo Yuan, Liangqiu Tang, Fenglian Liu \\ Department Institute of Cardiovascular Diseases, The Yuebei \\ People' s Hospital, Medical College, Shantou University, China}

Background Small noncoding microRNAs regulate gene and protein expression in cardiac development and disease and have been implicated in the rheumatic heart disease and in the regulation of extracellular matrix proteins. However, their role in L-type $\mathrm{Ca}^{2+}$ channel protein and atrial fibrillation (AF) was not well understood. The present study was designed to decipher molecular mechanisms underlying atrial electrical remodeling and $\mathrm{AF}$.

Methods The miRNA transcriptome was analyzed by microarray and verified by real-time reverse-transcription (RT) polymerase chain reaction (PCR) with left atrial samples from dogs of AF established by right atrial tachypacing for 8 weeks and from human atrial samples from $\mathrm{AF}$ patients with mitral stenosis (MS) of rheumatic heart disease. The expression of L-type $\mathrm{Ca}^{2+}$ channel protein were measured using westernblot analysis.

Result The miRNA-223 was found to be upregulated by $>2$ fold, expression of protein of cardiac L-type $\mathrm{Ca}^{2+}$ channel $\alpha 1$ cand $\beta 1$ subunits were upregulated, respectively. The close relationship between the levels of miRNA-223 and L-type $\mathrm{Ca}^{2+}$ channel protein. Adenovirus- mediated expression of miRNA-223 enchanced AF vulnerability. Effects of miRNA-223 overexpression on AF and associated electrical remodeling. In contrast, miRNA-223 knockdown, inhibition, or downregulation of intracellular $\mathrm{Ca}^{2+}$ overload and overexpression of L-type $\mathrm{Ca}^{2+}$ channel protein and suppressed AF in dogs.

Conclusion The level of miRNA-223 was significantly upregulated in dogs and patients with AF, contributing to upregulated of L-type $\mathrm{Ca}^{2+}$ channel protein involved in this process of atrial electrical remodelling in patients with rheumatic heart disease and $\mathrm{AF}$, which suggesting potention novel marker reflective of the process of atrial electrical remodeling, could be used in predictive AF of rheumatic heart disease.

\section{The altered microRNA expression profiling of lung tissue with or without ulinastatin pulmonary perfusion in a deep hypothermic low-flow cardiopulmonary bypass induced lung injury model \\ Wenlei Li, Shoujun Li, Hao Zhang, Fuxia Yan, Jinping Liu, Sen Zhang, Qiuming Chen \\ Center of Pediatric Cardiac Surgery, Fuwai Hospital and Cardiovascular Institute, Chinese Academy of Medical Sciences, Peking Union Medical College, Beijing, China}

Background The lung injury is severe in infants who received deep hypothermia with low-flow (DHLF) cardiopulmonary bypass (CPB) during cardiac surgery. Pulmonary perfusion with ulinastatin during CPB ameliorated some extent of lung injury. The aim of this study was to investigate the changed miRNAs and their potential target genes and relevant function in neonatal piglet lungs in response to DHLF-CPB and lung protection with pulmonary perfusion with ulinastatin.

Methods Piglets $(\mathrm{n}=15)$ aged $18.5 \pm 1.6$ days, were randomly divided into sham-operated (Group S, $\mathrm{n}=5$ ), DHLF-CPB group (Group
$\mathrm{C}, \mathrm{n}=5$ ) and pulmonary perfusion with ulinastatin group (Group $\mathrm{U}, \mathrm{n}$ $=5$ ). The group $\mathrm{S}$ only received median sternotomy. In Group $\mathrm{C}$ and Group U, the DHLF-CPB was obtained by cooled to $25^{\circ} \mathrm{C}$ with lowflow during in the 2-hour aortic cross-clamping. In Group U, pulmonary artery was perfused with protective solution after cardiac arrest. At 2 hours after weaning from $\mathrm{CPB}$, the lung tissue was harvested. We applied miRNA microarray and qRT-PCR analysis to determine miRNA expression. Meanwhile, predicted mRNA targets were obtained from miRanda. A Gene Ontology analysis and KEGG analysis performed by DAVID to obtain the functional annotation and pathway mapping of target genes. qRT-PCR and ELISA are used for further validation.

Result Using miRNA microarray we found the expression of 16 miRNAs in Group C, 8 miRNAs in Group U were significantly changed comparing to Group S. The qRT-PCR analysis verified up-regulation of miR-21 and down-regulation of miR-127, miR-145 and miR-204 in Group C. The same trend was also found in Group U except miR-204. But there are up-regulation of miR-127, miR-204 and down-regulation of miR-21 in Group U when compared to Group C. The results of DAVID analysis suggested the differentially expressed miRNAs related to series of important biology pathway related to CPB-induced lung injury, including $\mathrm{T}$ Cell receptor pathway, PI3K-Akt-NF- $\mathrm{KB}$ pathway and RAS pathway. Further validation of putatively target genes ACE, PTGS2 and PIK3CG revealed its upregulation in Group C. We found no difference in PIK3CG between Group $\mathrm{U}$ and Group S, when there is an up-regulation of ACE and PTGS2 in group U. And the extent of upregulation of the 3 target genes in Group C was higher than Group U.

Conclusion The changes of putative anti-inflammatory miRNA: miR-127, miR-145, miR-204 and putative pro-inflammatory miRNA: miR-21 indicated miRNA involved in the process of lung injury with close correlation to inflammatory response and oxidative stress. While the degree of miRNA change was lower in Group U suggested that ulinastatin alleviated DHLF-CPB induced lung injury by regulating relevant miRNA.

\section{Endurance training forestalling aging-related myocardial contractile dysfunction and ischemic intolerance: AMPK vs Akt signaling divergence \\ Chen $\mathrm{Li}^{1}$, Tiannan Jiang ${ }^{2}$, Yue Yin ${ }^{1}, \mathrm{Lu} \mathrm{Yu}{ }^{3}$, Heng Ma ${ }^{1}$ \\ 1. Department of Physiology, Fourth Military Medical University, Xi' an, China \\ 2. Department of Cardiology, Tangdu Hospital, Fourth Military Medical \\ University, Xi' an, China \\ 3. Department of Pathology, Xijing Hospital, Fourth Military Medical \\ University, Xi' an, China}

Heart senescence is associated with both myocardial functional deterioration and ischemic intolerance. Recent evidence has described potential role of AMPK and autophagy in exercise induced cardioprotection. The present study was designed to explore whether endurance training protect aged heart against contractile dysfunction and ischemic intolerance via AMPK regulated autophagy. 10-week free-loading swimming exercise $(1 \mathrm{~h} / \mathrm{d}, 5$ days/week $)$ as endurance training model were introduced in young $(4 \mathrm{mo})$ and aged $(22 \mathrm{mo})$ male C57BL/6 mice. In young mice, endurance training elicited a marked left ventricular hypertrophy with increased AMPK and Akt phosphorylation (all $\mathrm{P}<0.05$ ). In aged mice, however, the extent of endurance training induced no obvious left ventricular hypertrophy, accompanied with partially reversed AMPK phosphorylation but not Akt. Moreover, a reduced myocardial glycogen content and phosphorylated mTOR was 
also detected in aged mice after endurance training. In accordance with inhibited mTOR signaling, aged heart showed an impairment in autophagy capacity as evidenced by decreased autophagosomes markers (beclin-1, Atg5, LC3-II-to-LC3-I ratio), myocardial LAMP1, and increased lipofuscin, which could be reversed by endurance training $(\mathrm{P}<0.05)$. Both endurance training and mTOR inhibition by Rapamycin significantly restored myocardial autophagy capacity, leading to increased contractility in aged cardiomyocyte with increases of PS, $\pm \mathrm{dL} / \mathrm{dt}$ and TR90 $(\mathrm{P}<0.05)$, and protect aged heart against I/R injury, as evidenced by reduced infarct size, $\mathrm{CK}$ and caspase-3 activities (all $\mathrm{P}<0.05$ ). But endurance training induced cardioprotective effect and upregulation of cardiac autophagy were blunted by AMPK inhibition in aged mice. Neither Akt level nor p-Akt/Akt ratio affected by endurance training in both aged WT and KD mice. In conclusion, the present study demonstrated that endurance training mainly lead to AMPK but not Akt activation in aged heart. Upregulated autophagy induced by endurance training enhanced intrinsic aged myocardial resistance to ischemic injury, partly mediated by AMPK dependent mTOR pathway.

\section{Role of GRK4 in the regulation of arterial at1 receptor in hypertension \\ Ken Chen ${ }^{1,2}$, Chunjiang $\mathrm{Fu}^{1,2}$, Caiyu Chen ${ }^{1,2}, \mathrm{Li} \mathrm{Liu}^{1,2}$, Hongmei Ren ${ }^{1,2}$, Yu Han ${ }^{1,2}$, Jian Yang ${ }^{1,2}$, Duofen $\mathrm{He}^{1,2}$, Chunyu Zeng ${ }^{1,2}$ \\ 1. Department of Cardiology, Daping Hospital, Third Military Medical University \\ 2. Chongqing Institute of Cardiology, Chongaing}

Objective G protein-coupled receptor kinase 4 (GRK4) gene variants, via impairment of renal dopamine receptor and enhancement of renin-angiotensin system functions, cause sodium retention and increase blood pressure. Whether or not GRK4 and the angiotensin type 1 receptor (AT1R) interact in the aorta is not known.

Methods GRK4 expression in vascular smooth muscle cells (VSMCs) of the aorta was analyzed by confocal microscopy of doublestained, RT-PCR and immunoblotting. AT1R protein expression and function in GRK4 variant $142 \mathrm{~V}$ transfected A10 cells and WT cells was quantified by immunoblotting and AT1R-mediated intracellular calcium concentration. AT1R phosphorylation level was determined by immunoprecipitation. The interaction between GRK4 and AT1R was determined by immunoprecipitation and confocal microscopy of doublestained. NF-kB activity was analyzed by electrophoretic mobility shift assay (EMSA). Angiotensin II-mediated vasoconstriction of the aorta from $142 \mathrm{~V}$-transgenic mice and WT mcie was analyzed by tension measurement of the artery rings.

Result We report that GRK4 is expressed in vascular smooth muscle cells (VSMCs) of the aorta. Heterologous expression of the GRK4g variant $142 \mathrm{~V}$ in A10 cells increased AT1R protein expression and AT1R-mediated increase in intracellular calcium concentration. The increase in AT1R expression was related to an increase in AT1R mRNA expression via the NF-kB pathway. As compared with control, cells expressing GRK4g 142V had greater NF-kB activity with more NF-kB bound to the AT1R promoter. The increased AT1R expression in cells expressing GRK4g 142V was also associated with decreased AT1R degradation, which may be ascribed to lower AT1R phosphorylation. There was a direct interaction between GRK4g wild-type (WT) and AT1R that was decreased by GRK4g $142 \mathrm{~V}$. The regulation of AT1R expression by GRK $4 \mathrm{~g} 142 \mathrm{~V}$ in
A10 cells was confirmed in GRK4g 142V transgenic mice; AT1R expression was higher in the aorta of GRK4g $142 \mathrm{~V}$ transgenic mice than control GRK4g wild-type (WT) mice. Angiotensin II-mediated vasoconstriction of the aorta was also higher in GRK4g $142 \mathrm{~V}$ than WT transgenic mice.

Conclusion This study provides a mechanism by which GRK4, via regulation of arterial AT1R expression and function, participates in the pathogenesis of conduit vessel abnormalities in hypertension.

\section{Impact of currently prescribed lipid-lowering drugs on plasma PCSK9 concentration: single or in combination study \\ Yan Zhang, Jun Liu, Sha Li, Ruixia Xu, Jing Sun, Jianjun Li Division of Dyslipidemia, State Key Laboratory of Cardiovascular Disease, Fu Wai Hospital, National Center for Cardiovascular Diseases, Chinese Academy of Medical Sciences, Peking Union Medical College, China.}

Background An emerging data suggested a significant impact of statins on PCSK9 concentration, while the rapid and long term effects of other lipid-lowering drugs such as ezetimibe and Xuezhikang alone or in combination on PCSK9 and lipid profile have not been assessed.

Methods Sixty-three rats were randomly divided into six groups and orally administrated with placebo $(\mathrm{n}=13)$, ezetimibe $10 \mathrm{mg} / \mathrm{kg}$ daily, Xuezhikang $1200 \mathrm{mg} / \mathrm{kg}$ daily, ezetimibe10 mg/kg plus Xuezhikang $1200 \mathrm{mg} / \mathrm{kg}$ daily, pitavastatin $10 \mathrm{mg} / \mathrm{kg}$ daily orpitavastatin $10 \mathrm{mg} / \mathrm{kg}$ plus ezetimibe $10 \mathrm{mg} / \mathrm{kg}$ daily for 3 days ( $\mathrm{n}=10$ for eachgroup). The patients $(\mathrm{n}=16)$ without previous lipid-lowering drug treatment for dyslipidemia received Xuezhikang $1200 \mathrm{mg}$ daily for 8 weeks. Blood samples were obtained atday 3 for rats, and at baseline and week 8 for patients. Plasma PCSK9 levels and lipid profile were measured.

Result In rats, ezetimibe, Xuezhikang and pitavastatin alone and Xuezhikang plus ezetimibeas well as pitavastatin plus ezetimibe increased PCSK 9 levels by $124 \%, 56 \%, 111 \%, 63 \%$ and $204 \%$ respectively ( $\mathrm{P}<0.05$ vs control). However, Xuezhikang plus ezetimibe did not enhance greater PCSK9 levels compared to monotherapy. Ezetimibe and pitavastatin in combination induced higher PCSK9 levels than pitavastatin monotherapy or co-therapy with ezetimibe plus Xuezhikang. There was no significant difference between any groups with regard to lipid profile levels at day $3(\mathrm{P}>0.05)$. At week 8, PCSK9 levels increased by $34 \%$ and total cholesterol and low-density lipoprotein cholesterol decreased by $22 \%$ and $28 \%$ in patients ( $\mathrm{P}<0.05$ vs baseline).

Conclusion Lipid-lowering drugs have significant impact on PCSK9 in a rapid and long-term manner in rats and humans. Elevated PCSK9 concentration was found prior to the alterations of lipid profile in rats. Combination of Xuezhikang and ezetimibe significantly attenuated increase in PCSK9 compared to ezetimibe plus pitavastatin, suggesting that the former combination may be better than the latter inclinical application.

\footnotetext{
Role of GRK4 $y$ variant $142 \mathrm{~V}$ in the regulation of renal ETB receptor in hypertension

Meixiang $\mathrm{Li}^{1,2}$, Caiyu Chen ${ }^{1,2}$, Shuo Zheng ${ }^{1,2}$,

Chunyu Zeng Zeng ${ }^{1,2}$

1. Department of Cardiology, Daping Hospital, Third Military Medical

University

2. Chongqing Institute of Cardiology
}

Background The endothelin receptor B (ETBR) regulates blood 
pressure and water and electrolyte balance by engendering natriuresis. In hypertensive states, the ETBR mediated diuresis and natriuresis is impaired. However, the underlying mechanisms are not clear. G proteincoupled receptor kinase 4 (GRK4), whose gene locus 4p16.3 is linked to essential hypertension, caused sodium retention and increased blood pressure via impairment of renal dopamine receptor and enhancement of renin-angiotensin system functions. Due to the higher activity of GRK4 in kidney from spontaneously hypertensive rats (SHRs) and hypertensive patients, we hypothesized that GRK4 might be the cause of ETBR impairment in hypertension.

Methods Experiments were carried out in male anaesthetic spontaneously hypertensive rats (SHR) and in normotensive WistarKyoto (WKY) rats. The ETB receptor agonist, BQ-3020 (0.1, 0.5, 1.0 $\mu \mathrm{g} / \mathrm{kg} / \mathrm{min}$ ) were infused via supra-renal artery at a rate of $0.04 \mathrm{ml} / \mathrm{min}$ for 40 minutes. The same experiments were conducted in GRK4 A142V and GRK4 Wild Type transgenic mice. The ETBR function was also checked in the wild-type and A142V transfected renal proximal tubule (RPT) cells from mice.

Result We found that diuresis and natriuresis of ETBR agonist, BQ3020, in Wistar-Kyoto (WKY) rats, which was impaired in SHRs. The GRK4 expression was higher in renal cortex from SHRs as compared with WKY rats. In GRK4 A142V transgenic mice, it resulted that ETBR-mediated diuresis and natriuresis was impaired compared with Wild type. In wild-type transfected cells, activation of ETBR inhibited $\mathrm{Na}^{+}-\mathrm{K}^{+}$-ATPase activity; while in A142V transfected cells, the inhibitory effect was lost. There are co-localization and coimmunoprecipitation between ETBR and GRK4 inRPT cells. The linkage of ETBR/GRK4 was higher in wilde-type cells than in A142V cells. Similar phenomenon was found in the kidney from WKY and SHRs, SHRs had higher ETBR/GRK4 linkage, accompanied with higher ETBR phosphorylation, which might account for the impaired ETBR function in hypertension.

Conclusion This study provides a mechanism by which GRK4, via regulation of renal ETBR function, participates in the pathogenesis of hypertension.

\section{Telmisartan effects on left ventricular remodeling in spontaneously hypertensive rats Huan Lin, Xiuying Li, Jiyan Leng The First Hospital of Jilin University}

Objective To explore the influence of telmisartan in left ventricular remodeling of spontaneously hypertensive rats (SHR).

Methods 16 only 12 weeks of male SHR, were randomly divided into telmisartan group and SHR blank control group, each group of eight; Setting up age-matched Wistar rats (WKY) 8 only to normal control group. Treatment group was given telmisartan $10 \mathrm{mg}$ lavage, $\mathrm{kg}$ ${ }^{1} \cdot \mathrm{d}^{-1}$, put to death animals after feeding $8 \mathrm{w}$. Measuring left ventricular and myocardial thickness, weight calculation of left ventricular weight and body weight (LVW/BW). By Van Gieson staining to observe left ventricular myocardial collagen fibers, the left ventricular myocardial collagen volume fraction (CVF) and perivascular collagen area (PVCA) were analyzed. Electron microscopy (sem) and HE staining to observe left ventricular myocardial ultrastructure.

Result Compared with normal control group WKY rats, SHR blank tail artery systolic blood pressure (SBP), LVW/BW, left ventricular wall thickness, CVF, PVCA were significantly increased $(\mathrm{P}<0.01)$. Compared with SHR blank control group, telmisartan group can effectively reduce SHR SBP, improve left ventricular hypertrophy $(\mathrm{P}<$
$0.01)$, reduce myocardial interstitial and myocardial collagen around the small artery $(\mathrm{P}<0.01)$. Histopathology and electron microscopy showed that telmisartan treatment can significantly improve SHR left ventricular remodeling.

Conclusion Telmisartan can effectively reduce the SHR blood pressure, improve left ventricular remodeling.

\section{Up-regulation of caveolin-1 suppresses vascular smooth muscle cells proliferation and neointimal formation in balloon injured rat carotid artery \\ Wei Lu \\ Cardiovascular Department of Xinqiao Hospital, Third Military Medica University}

Background Caveolin-1 (cav-1) is the major coat protein responsible for caveolae assembly, regulating signaling via proteinprotein interactions with resident caveolar proteins, but its biological mechanism in vascular smooth muscle cells (VSMCs) is still unclear. The aim of this study was to evaluate the role of cav-1 on VSMCs proliferation and the neointimal formation in balloon injured rat carotid artery.

Methods Left common carotid arteries from Sprague-Dawley rats were injured by a balloon catheter, and the injured arteries were incubated with $30 \mu \mathrm{L}$ solution of Ad-cav-1 adenoviral vectors, Ad-GFP adenoviral vectors or PBS for $30 \mathrm{~min}$. The rats were euthanized for morphometric and immunohistochemical analysis, real-time PCR and western blot analysis at 2 weeks after balloon injury and gene transfer. The cultured rat VSMCs transduced with Ad-cav-1 or Ad-GFP adenoviral vectors were used for cell proliferation assay, real-time PCR and western blot analysis. The vascular or intracellular ROS level was also detected.

Result Adenoviral vectors encoding cav-1 cDNA could increase cav-1 expression both in mRNA and protein levels in balloon injured artery walls and cultured rat VSMCs. Upregulation of cav-1 significantly suppressed VSMCs proliferation and intimal formation. Over-expression of cav-1 could reduce vascular or intracellular ROS level and decrease the phosphorylation of the ERK1/2 in balloon injured artery walls and cultured rat VSMCs.

Conclusion Our study suggests that overexpression of cav1 significantly suppresses VSMCs proliferation and progression of neointimal formation after vascular injury.

\section{Phosphatase and tensin homologue on chromosome 10 regulates angiotensin II- induced proliferation and collagen metabolism in neonatal rat cardiac fibroblasts through the Akt/P27 signaling pathway \\ Ning Lie \\ Cardiovascular Department of Xinqiao Hospital, Third Military Medical \\ University}

Cardiac fibroblasts (CFs) play a key role in cardiac fibrosis by regulating the balance between extracellular matrix production and breakdown. Although phosphatase and tensin homologue (PTEN) on Chromosome 10 has been found to play an important role in cardiovascular disease, it is not clear whether PTEN is involved in functional regulation of CFs. In the present study, PTEN was 
overexpressed in neonatal rat CFs by gene transfer of recombinant adenovirus. Then, we investigated whether PTEN played an important role in Angiotensin (Ang) II-induced CF proliferation, apoptosis, the cell cycle, and collagen metabolism as well as the possible molecular mechanisms. Compared with the untreated and green fluorescent protein-expressing adenovirus (Ad-GFP) groups, PTEN adenovirus (AdPTEN) infection increased PTEN protein and mRNA expression in CFs. Ad-PTEN increased the proportion of CFs in G1/S phase events with no change in cell apoptosis. In addition, Ad-PTEN decreased mRNA expression and the protein synthesis rate of collagen type I and III, and antagonized Ang II-induced collagen synthesis. Overexpression of PTEN also decreased Ang II-induced matrix metalloproteinase-2 (MMP-2) and tissue inhibitor of metalloproteinase-1 (TIMP-1) production as well as gelatinase activity. Moreover, Ad-PTEN decreased Akt expression and increased P27 expression with or without Ang II stimulation. These results suggested that PTEN could regulate Ang II-induced collagen metabolism in neonatal rat $\mathrm{CFs}$ through the Akt/P27 signaling pathway, thus providing a new possible target for the treatment of cardiac fibrosis.

\section{Combination of hyaluronic acid and bone marrow-derived mesenchymal stem cells promotes myocardial repair after infarction Qingqing Shang, Kai Li, Jian Meng, Xuewen Liu, Jun Li, Jianye Zhou, Shengshou Hu State Key laboratory of Cardiovascular Disease, Fu wai Hospital, National Center for Cardiovascular Diseases, Peking Union Medical College and Chinese Academy of Medical Sciences, Beijing, 100037, China}

Objective The poor retention and survival of donor cells implanted into the myocardium limit the efficacy of cell therapy for myocardial infarction. Embedding cells in natural or synthetic biomaterials is a strategy to address this issue. Therefore, in the present study, we planned to evaluate: 1. HA hydrogel enhancing retention and survival rate of the implanted cells; 2. BMSCs encapsulated in HA hydrogel systematically improving cardiac function after myocardial infarction; 3. the mechanism of BMSCs and HA hydrogel in promoting damaged heart repair.

Methods A biodegradable and in situ polymerizable hydrogel based on hyaluronic acid (HA) was used as the carrier for bone marrow-derived mesenchymal stem cells (BMSC) obtained from male rats. Infarcted hearts of female rats (LAD ligation) were randomized to injection of PBS, HA, BMSCs, or HA+BMSC complex respectively. The survival rate and apoptosis of implanted cells and the expression of MMP2, VEGF, Tbeta4 and c-Kit were examined at post-injection day 1, week 1 and week 2 by RT-PCR, TUNEL staining and Western blot, respectively. At post-injection week 4, changes of cardiac microstructure and function were evaluated by histological examination and echocardiography.

Result HA hydrogel significantly enhanced the cell retention and survival rate at post injection day 1 and week 2 . The expressions of VEGF and MMP2 in the infarct zone treated with BMSCs alone were significantly up-regulated at week 1 . At week $2, \mathrm{HA}+\mathrm{BMSC}$ group has a higher expression of VEGF and MMP2 than HA group; the levels of Tbeta4 and c-Kit in hearts injected with HA+BMSC complex or either component alone were increased. Histological analysis revealed that thicker infarct wall in HA group, smaller cardiomyocyte size and higher vessel density in BMSC group, the largest benefits in combined group as indicated by reversing cardiac remodeling, angiogenesis, and more viable myocardium tissue in the infarct zone. The relative changes of EF and FS further reflected that the HA+BMSCs combined treatment yielded the best recoveries of cardiac function.
Conclusion HA hydrogel can act as BMSCs delivery vehicles and add to the beneficial effects of implanted BMSCs in repairing damaged heart via enhancing cell retention and survival, and prolonging paracrine effects of engrafted cells. In addition, HA hydrogel alone supplies injured heart with mechanical support and endogenous stem cells recruitment.

\section{Tissue distribution of exogenous estrogens and $\beta-(3,4-$ dihydroxyphenyl) $-\alpha$-hydroxypropionate in ovariectomized rat speciality: internal medicine \\ Qianrong Zhang ${ }^{1}$, Xiaopu Zheng ${ }^{1}$, Hongling San $^{2}$, \\ Xiaohui Zheng ${ }^{3}$ \\ 1. Department of Cardiology, First Hospital of Xi' an Jiaotong University, Key Laboratory of environment and Genes Related to Diseases, \\ Ministry of Education, China) \\ 2. Department of Medicine, Monash University Central Clinical School, \\ Commercial Road, Prahran, Melbourne, VIC 3181, Australia \\ 3. Institute of Analytical Science/Shaanxi Provincial Key Laboratory of \\ Electroanalytical Chemistry, Northwest University}

Objective To observe the Tissue distribution of exogenous estrogens and b- (3, 4-dihydroxyphenyl) -a-hydroxypropionate in ovariectomized rat.

Methods The conditions of post-menopause were established by an artificial bilateral ovariectomy (de-ovaries) in female SpragueDawley rats, with the de-ovary for 1 week and 16 weeks to mimick early and late menopause, respectively. Normal control rats accepted a sham surgical operation supplementation of exogenous estrogen and progesterone (HRT) and DBZ. De-ovaries groups (OVX) were injected estradiol (E2, $0.07 \mathrm{mg} / \mathrm{kg}$ ), plus progestrone $(0.2 \mathrm{mg} / \mathrm{kg})$ or DBZ (20 $\mathrm{mg} / \mathrm{kg}$ ) once per day at the beginning (OVX 1-week group) and 16th week (OVX 16-week group) of the de-ovary, and the normal control group (NC) accepted an injection of vehicle. The tension of aortic rings was examined by determining the relaxation response to acetylcholine $(\mathrm{ACH})$, an endothelium-dependent vasodilator. The aortics were isolated, sectioned, HE stained, and observed under light and electronic microscopes.

Result Tissue distribution levels of estrogens increase more in a supplement of HRT at early than late phase of menopause. At the same time, the number of ERa, but not ERb, in myocardium can also increase following the HRT. DBZ improves the tissue distribution of estrogens, possibly through an influence of endogenous estrogen and estrogen receptor production, thus leading to some effects similar to the early HRT. It is still unknown why the DBZ group has a relatively much high distribution of estrogen in uterus, which needs to have a further research. Both DBZ and EHRT groups present some protective effects on vascular endothelial function, with that of DBZ better than EHRT.

\section{New-onset heart failure after short-term permanent right ventricular apical pacing in patients with third degree atrioventricular block and normal left ventricular function \\ Nana Liu \\ Fuwai Hospital and Cardiovascular Institute, Peking Union Medical College, Chinese Academy of Medical Sciences}

Objective Emerging data have suggested that long-term right 
ventricular (RV) apical pacing results in progressive left ventricular (LV) dysfunction and contributes to the development of heart failure (HF), commonly the duration takes more than 3 years. However new-onset $\mathrm{HF}$ were found to occur shortly after permanent pacing in a very small number of the patients with third degree atrioventricular (AV) block. This study aimed to investigate the prevalence and genetic mutations for the development of new-onset HF within 3 years' RV apical pacing in patients with third degree $\mathrm{AV}$ block who require permanent pacing.

Methods We studied the clinical outcomes after short-term RV apical pacing for third degree AV block in 877 patients without a prior history of HF and carried out genetic testing (Target Region Sequencing) in the ones developed HF.

Result After a median follow-up of 35.4 months, 31 patients (3.5\%) developed new-onset $\mathrm{HF}$ after RV apical pacing. In those patients who developed HF, 19 (2.2\%) suffered myocardial infarction and 4 (0.5\%) developed atrial fibrillation before the implantation of cardiac pacemaker, the last 8 individuals showed none cardiovascular risk factors. Four of the eight patients underwent genetic testing and showed some LMNA mutations which can result in atrioventricular block and heart failure.

Conclusion After a follow-up of 35.4 months, permanent RV apical pacing was associated with $\mathrm{HF}$ in $3.5 \%$ of patients. Myocardial gene mutation may play an important role in the development of atrioventricular block in the ones without any risk factors. More importantly, patients with genetic backgrounds are apt to develop heart failure after the implantation of pacemakers.

\section{Cortistatin inhibited calcification of rat vascular smooth muscle cells induced by $\beta$-glycerophosphate through GHSR1a \\ Yue Liu ${ }^{1}, \mathrm{Yu} \mathrm{Fu}{ }^{1}$, Xiaohui Zhang, ${ }^{1}$, Fang Lin ${ }^{1}$, \\ Yongfen $\mathrm{Qi}^{2}$, Xinhua Yin \\ 1. Department of Cardiology, the First Affiliated Hospital of Harbin \\ Medical University, Harbin, China \\ 2. Department of Physiology and Pathophysiology, Peking University Health Science Center, Beijing, China}

Objective Vascular calcification is an active cell-mediated mineralization process, involving vascular smooth muscle cells (VSMCs), mast cells, peritubular cells and macrophages. Accumulative evidences have indicated that VSMCs plays a pivotal role in the development of vascular calcification. Therefore, we investigated effect of cortistatin (CST) on $\beta$-glycerophosphate-Induced calcification of rat VSMCs in vitro, and further its probable mechanism.

Methods VSMCs were incubated with $\beta$-glycerophosphate ( $\beta$-GP) for in vitro calcification model. Calcium content, alkaline phosphatase (ALP) activity and calcified lesion were measured by o-cresolphthalein complexone colorimetric method, colorimetric method using a commercial kit and von Kossa staining, respectively, in cultured rat VSMCs.

Result ALP activity and calcium content of $\beta$-GP-Induced calcified VSMCs were significantly increased in Ca group as compared with control group $(\mathrm{P}<0.05)$. There was the formation of a large number of multicellular nodules in Ca group compared with control group by the use of light microscope. Moreover, result analysis of von Kossa staining for calcification confirmed that those were many dark/brown positive particles. $10^{-9}-10^{-7} \mathrm{~mol} / \mathrm{L}$ CST could reverse the augmentation of ALP activity and calcium deposition of VSMCs induced by $\beta$-GP in $\mathrm{Ca}+\mathrm{CST}$ group than $\mathrm{Ca}$ group alone $(\mathrm{P}<0.05)$. And then, $1 \times 10^{-8} \mathrm{~mol} / \mathrm{L} \mathrm{CST}$ also obviously decreased the formation of multicellular nodule and von Kossa-staining-positive granules of rat VSMCs in $\mathrm{Ca}+\mathrm{CST}$ group compared with Ca group alone. However, there was no significant difference on ALP activity and calcium content between group of different doses of CST $(\mathrm{P}<0.05)$. In order to validate whether growth hormone secretagogue receptor 1a (GHSR1a) participated in anticalcification of CST on $\beta$-GP-induced calcification of rat VSMCs in vitro, ghrelin, a GHSR1a agonist, and D-GHRP-6, a GHSR1a antagonist were applied in cell experiment. The results showed that both $1 \times 10^{-7}$ $\mathrm{mol} / \mathrm{L}$ ghrelin and $1 \times 10^{-8} \mathrm{~mol} / \mathrm{L} \mathrm{CST}$ could exert similar inhibition of ALP activity and calcium deposition of rat calcified VSMCs in $\mathrm{Ca}+$ ghrelin group or $\mathrm{Ca}+\mathrm{CST}$ group than $\mathrm{Ca}$ group alone $(\mathrm{P}<0.05)$. However, $1 \times 10^{-7} \mathrm{~mol} / \mathrm{L}$ D-GHRP-6 incubation could reverse effect of CST on rat VSMC calcification $(\mathrm{P}<0.05) .1 \times 10^{-7} \mathrm{~mol} / \mathrm{L}$ D-GHRP-6 incubation did not affect ALP activity and calcium deposition of VSMCs in $\mathrm{Ca}+\mathrm{D}-\mathrm{GHRP}-6$ group compared with Ca group alone $(\mathrm{P}>0.05)$.

Conclusion Cortistatin could reduce ALP activation, calcium deposition and calcified cytological damage of cultured rat VSMCs induced by $\beta$-GP in a dose-independent way, where the inhibitory effect of CST was mediated by GHSRla, thus providing an experimental foundation for the application of cortistatin in the prevention and treatment of vascular calcification.

\section{Inhibition of focal adhesion kinase (FAK) suppresses hypoxia-induced differentiation and collagen synthesis in neonatal mice cardiac fibroblasts \\ Guangpu Fan, Hui Zhao, Wei Wang \\ State Key Laboratory of Cardiovascular Disease, Fuwai Hospital, \\ National Center for Cardiovascular Disease, Chinese Academy of \\ Medical Sciences and Peking Union Medical College, China}

Objective To explore the effect of focal adhesion kinase (FAK) on mediating hypoxia-induced differentiation and collagen synthesis in neonatal mice cardiac fibroblasts.

Methods Primary cultures of mouse cardiac fibroblasts (CFs) were isolated from 1-day old newborn C57BL/6 mice according to the protocol of our lab, and CFs at culture passage 2 (P2) were used for further experiments. CFs at $80 \%$ confluence were induced to transform into myofibroblasts (myoCFs) in DMEM containing 1\% FBS in a sealed hypoxic GENbox jar fitted with a catalyst for different times $(6 \mathrm{~h}, 12 \mathrm{~h}$, $24 \mathrm{~h}$ ). To assess the effect of FAK, CFs were treated with or without PP2 (10 $\mu \mathrm{M}$ as our previous study) at the same time of hypoxia. Quantitative real-time PCR (qRT-PCR) was used to detect the expression of FAK, alpha-smooth muscle actin (alpha-SMA) and collagenI-alpha1 after treatment. And the myoCFs differentiation related proteins, transforming growth factor-beta (TGF- $\beta$ ) and $\alpha$-SMA, as well as phosphorylation of FAK were evaluated by Western-blot.

Result Hypoxia did not produce much morphological changes in CFs, as the CFs and myoCFs could not be distinguished by the phenotype of the cells. Analysis of gene expression by qRT-PCR of the cells indicated that hypoxia activates alpha-SMA and collagenIalpha1 mRNAs, along with FAK mRNA, indicating the differentiation of CFs to myoCFs and increase of collagen synthesis, but inhibition of FAK attenuated the myoCFs transition in response to reduced oxygen. Assessment of TGF- $\beta$, alpha-SMA and phosphorylation of FAK protein levels demonstrated that their release by CFs was upregulated in hypoxic conditions but attenuated by PP2 treatment.

Conclusion This study demonstrates that FAK would be a therapeutic target on hypoxia-induced fibrosis, and therefore illuminates 
the promising role of inhibition of FAK on the healing of heart remodeling after hypoxia injury.

\section{Umbilical cord blood mesenchymal stem cell conditioned medium inhibits hypoxia induced apoptosis in $\mathrm{H} 9 \mathrm{c} 2$ cardiomyoblasts by activation of Akt}

Guangpu Fan, Xuhe Gong, Guogan Wang, Wei Wang State Key Laboratory of Cardiovascular Disease, Fuwai Hospital, National Center for Cardiovascular Disease, Chinese Academy of Medical Sciences and Peking Union Medical College, China

Objective To study the beneficial role of human umbilical cord blood derived mesenchymal stem cell-conditioned medium (MSC-CM) in hypoxia-induced apoptosis in $\mathrm{h} 9 \mathrm{c} 2$ cardiomyoblasts, in which the serine/threonine kinases (Akt) pathway would be involved.

Methods Serum-free DMEM medium was replaced when the MSCs were grown to $80 \%$ confluence, then the MSC-CM was collected and sterilized after 24 hours culture. H9c2 cardiomyoblasts were divided into the following groups: control group, hypoxia group, MSC-CM intervention group (CM group), MSC-CM + Akt phosphorylation inhibitor (LY294002) group (LY group). Cell apoptosis of the cardiomyoblasts was tested with chromatin dye Hoechst 33342, and FITC-conjugated Annexin V apoptosis detection kit by flow cytometer after a Hypoxia/Serum deprivation treatment for $24 \mathrm{~h}$. The apoptosis related proteins were evaluated by Western-blot.

Result On Ho33342 apoptosis staining, the cardiomyoblasts morphology displayed a lower proportion of apoptosis in CM group than those in hypoxia group, while apoptosis was increased in LY group; Flow cytometric analysis revealed the apoptosis ratio in CM group is lower than the hypoxia group $[(2.84 \pm 1.20) \%$ vs $(10.15 \pm 1.61) \%, \mathrm{P}<$ $0.05]$, while the LY group was significantly higher $[(14.32 \pm 2.07) \%$ vs $(2.84 \pm 1.20) \%, \mathrm{P}<0.01]$. Active caspase-3 expression was increased in hypoxia group than that in control group $(\mathrm{P}<0.05)$, but decreased in $\mathrm{CM}$ group $(\mathrm{P}<0.01)$.

Conclusion The umbilical cord blood mesenchymal stem cell conditioned medium can inhibit hypoxia-induced H9c2 cardiomyoblasts apoptosis, and in which the activation of Akt phosphorylation is involved to achieve the protective effect.

\section{Trimetazidine protects mesenchymal stem cells against hypoxia and serum deprivation induced apoptosis by activation of Akt \\ Xuhe Gong, Guangpu Fan, Wei Wang, Guogan Wang Emergency and Critical Care Center of Cardiovascular Department, State Key Laboratory of Cardiovascular Disease, Fuwai Hospital, National Center for Cardiovascular Diseases, Chinese Academy of Medical Sciences and Peking Union Medical College, Beijing, Peoples Republic of China.}

Objective Mesenchymal stem cells (MSCs) transplantation has been shown to be a safe effective strategy for treating ischemic heart disease. However, the efficacy is limited by poor viability of MSCs transplanted into the infarcted heart, only few cells play a role through cellular differentiation and paracrine. Therefore, promoting the survival of implanted MSCs is important. Recent findings have implicated trimetazidine (TMZ) enhance the survival of stem cells under various conditions. However, Stem cells in these studies are animal derived, to our knowledge, little information is available about the human MSCs in protection against Hypoxia/Serum deprivation (H/SD) induced apoptosis. Herein, we proposed that TMZ administration may improve human umbilical cord MSCs' viability through micro-environmental amelioration, especially through phosphatidylinositol 3-kinase (PI3K) / Akt pathway.

Methods Human umbilical cords MSCs were prepared from the Wharton's jelly and grown in DMEM/F12 with 10\% FBS. MSCs were identified by cell morphology and cell surface antigen CD34, CD44, CD45 and CD105 expression. Cells were used at the third passage for all experiments. For TMZ protection experiments, MSCs were pretreated with $10 \mu \mathrm{M} \mathrm{TMZ}$ for $12 \mathrm{~h}$ of $\mathrm{H} / \mathrm{SD}$ with or without the PI3K inhibitor LY294002 $(25 \mu \mathrm{M})$. The morphological changes were assessed using the chromatin dye Hoechst 33342. Apoptosis was evaluated by FITCconjugated Annexin V apoptosis detection kit. The apoptosis related proteins (Bcl-2 and phosphorylation of Akt) expression were performed by Western-blot.

Result The number of adherent cells increased with time and became spindle shaped within 7 days. After 10-15 days, a number of classical MSCs colonies appeared. Flow cytometric analysis revealed positive staining with CD44 (99.62\%), CD105 (99.15\%) and negativity for CD34 (0.01\%), CD45 (0\%). H/SD triggered apoptotic MSCs death by nuclear shrinkage, chromatin condensation, and decrease in cell size. TMZ had significant survival effects on apoptotic and necrosis as compared with the H/SD group $(\mathrm{P}<0.01)$. After hypoxia for $12 \mathrm{~h}$, the proportion of early stage apoptotic cells decreased significantly from $6.5 \%$ to $1.0 \%$, and the late stage apoptotic cells decreased from $54.8 \%$ to $36.7 \%$ in the group of TMZ+MSCs as compared with that in the group of MSCs. The expression of anti-apoptosis protein Bcl-2 and p-Akt was increased significantly in TMZ+MSCs group $(\mathrm{P}<0.05)$.

Conclusion Trimetazidine protects human umbilical cord MSCs from Hypoxia/SD induced apoptosis via PI3K/Aktpathways, suggesting that it may provide a useful therapeutic adjunct for transplanting MSCs into damaged heart after myocardial infarction.

\section{Hydrogen sulphide protects HUVECs against high-glucose induced senescence \\ Zhiming Song ${ }^{1}$, Yong Liu ${ }^{1}$, Shujie Yu ${ }^{1}$, Shuiying Huang ${ }^{1}$, Lin Chen ${ }^{1}$, Xiaoxian Qian ${ }^{1,2}$ \\ 1. Department of Cardiology, The Third Afflilated Hospital, Sun Yat-sen \\ University, Guangzhou, China \\ 2. Institute for Integrated Traditional Chinese and Western Medicine, Sun Yat-sen University, China}

Objective Hydrogen sulphide $\left(\mathrm{H}_{2} \mathrm{~S}\right)$ is the 3rd gaseous mediator in mammals and has exhibited numerous functions in cardiovascular system. Endothelial senescence contributes much to endothelial dysfunction. So this study is designed to investigate the effect of exogenous $\mathrm{H}_{2} \mathrm{~S}$ on HUVEC senescence induced by high glucose.

Methods High glucose (33 mM) was applied to induce premature senescence in HUVECs and senescence was confirmed by $\beta$-galactosidase (SA- $\beta$ gal) staining. MTT assay was used to assess cell viability. PAI-1, SOD1 and NF-kB p65 was detected by western blot. MDA level was measured using a commercial kit.

Result High glucose induced a senescence-like phenotype in HUVECs as shown by slower proliferation, more SA- $\beta$ gal positive cells and increased protein expression of PAI-1. In senescent cells, the SOD1 expression was reduced dramatically, but NF- $\mathrm{KB}$ p65 activity and MDA 
production was increased significantly. However, sodium hydrosulphide (NaHS, $\mathrm{H}_{2} \mathrm{~S}$ donor, $100 \mu \mathrm{mol} / \mathrm{L}$ and $200 \mu \mathrm{mol} / \mathrm{L}$ ) was able to promote cell proliferation, decrease the number of SA- $\beta$ gal positive cells and reduce PAI-1 expression. In the meantime, NaHS increased SOD1 expression, inhibited the activity of NF- $\mathrm{kB}$ p 65 and decreased MDA production.

Coclusion Exogenous hydrogen sulphide prevents HUVECs against high glucose-induced senescence by modulating oxidative stress and NF- $\mathrm{kB}$ p65 activity. Our results may shed light on the accelerated endothelial dysfunction in diabetic patients whose $\mathrm{H}_{2} \mathrm{~S}$ level are decreased.

\section{Next generation sequencing as a fast molecular diagnosis for Marfan syndrome in a Chinese family with mutation in FBN1 \\ Yan Xiao ${ }^{1}$, Chaoxia $\mathrm{Lu}^{2}$, Yaxin Liu ${ }^{1}$, Kunqi Yang ${ }^{1}$, \\ Fang Liu ${ }^{2}$, Jifang Xiao ${ }^{2}$, Tao Tian ${ }^{1}$, Xue Zhang ${ }^{2}$, \\ Xianliang Zhou \\ 1. Hypertension Center, State Key Laboratory of Cardiovascular \\ Disease, Fuwai Hospital, National Center for Cardiovascular Disease, \\ Chinese Academy of Medical Sciences, Peking Union Medical College, China. \\ 2. McKusick-Zhang Center for Genetic Medicine, State Key Laboratory of Medical Molecular Biology, Institute of Basic Medical Sciences, \\ Chinese Academy of Medical Sciences and Peking Union Medical \\ College, China.}

Objective Marfan syndrome (MFS) is a common autosomal dominant inherited disorder with high risk of aortic aneurysm and dissection. The diagnosis of MFS relies on clinical manifestations and genetic detection of mutation in FBN1 or TGFBR2. Although more than a thousand mutations have been identified in FBN1, next generation sequencing (NGS) has not applied as a diagnostic method for familial MFS patients yet. This study aimed to identify the diseasing-causing mutation of a Chinese family with MFS by NGS and to establish genotype-phenotype correlations of MFS.

Methods Thorough examinations were performed to screen patients of MFS in a four-generation Chinese family by physical examination, slit lamp examination, echocardiography, $\mathrm{X}$ ray and magnetic resonance with Ghent Criteria. Genomic DNA extracted from venous blood of the proband was tested by NGS sequencing using a custom designed panel on IonTorrent PGM. Variants were screened by bioinformatic analysis and the pathogenic mutation was confirmed by Sanger sequencing. Correlations between mutations in exon 46 of FBN1 and clinical features of published literatures were analyzed by statistical analysis.

Result Total mutation analysis (library preparation, NGS sequencing, bioinformatic data analysis and Sanger sequencing) was completed in 3 days. The reported mutation c.5684G $>$ A (p.C1895Y) in exon 46 of FBN1 was identified as the diseasing-causing mutation of the family. Thorough examinations indicated this mutation may be associated with the development of aortic dissecting aneurysm, sudden death, mild skeletal features and dural ectasia but no ectopia lentis. Genotype-phenotype analyses indicated a higher probability of ascending aortic aneurysm were found in patients with cysteine mutations than a mutations without cysteine involved ( $91 \%$ vs $47 \%$, $\mathrm{P}$ $<0.05$ ). No significant difference in the proportions of ectopia lens was found between patients with cysteine mutations and patients without cysteine involved ( $45 \%$ vs $53 \%, \mathrm{P}>0.05)$. Significant reduction in aortic root dimension ( $62 \mathrm{~mm}$ vs $60 \mathrm{~mm}$ ) was detected after 3 months' losartan treatment in the proband, while no progressive dilation in other affected family members.

Conclusion Next generation sequencing will be established as a robust diagnostic method of familial patients with Marfan syndrome, which may be beneficial to strengthen clinical management and to predict the prognosis of the patients. The cysteine mutations occurring in exon 46 of FBN1 appeared to be an indicator of severe aortic aneurysm, and may not always develop ectopia lens. Losartan treatment could slowdown the progression of aortic dilation.

\section{CARD3 mediates cardiac remodeling after myocardial infarction through p38-dependent signaling pathway \\ Liangpeng Li, Xin Chen \\ Department of Thoracic and Cardiovascular Surgery, Nanjing Hospital \\ Affiliated to Nanjing Medical University, China}

Background Caspase activation and recruitment domain 3 (CARD3) is a critical regulator of the Toll-like receptor-mediated signaling pathway. Previous research on the function of CARD3 has focused on its role in the immune response and inflammatory diseases. However, the functional significance of CARD3 in cardiac remodeling following myocardial infarction (MI) remains unclear. This study was aimed to identify the effect of CARD3 in post-infarction cardiac remodeling and its underlying mechanisms.

Methods Gene-manipulated CARD3-knockout (KO) mice, transgenic mice that overexpress $\mathrm{CARD} 3$ in the heart and their respective wild-type (WT) and non-transgenic controls were subjected to MI by left anterior descending artery ligation.

Result Our preliminary studies demonstrated CARD3 to be strongly induced in the infarcted murine myocardium and in left ventricular (LV) tissue samples obtained from patients who had died after an acute MI. In vivo cardiac structure and function was relatively improved in CARD3KO mice at 28 days after MI when compared with MI-treated wild-type littermates. Deficiency of CARD3 reduced apoptosis, limited infarct size, prevented left ventricle dilation, and reduced mortality in CARD3-KO mice. Furthermore, cardiomyocyte cross-sectional areas and myocardial collagen deposition was significantly attenuated in CARD3-KO mice, which were accompanied by down-regulation of hypertrophic genes and profibrotic genes. However, CARD3 overexpression displayed the opposite phenotype after MI. Similar results were observed in ex vivo cultured neonatal rat cardiomyocytes exposed to hypoxia. Further analysis of signaling events suggests that CARD3 mediates cardiac remodeling after MI via the positive regulation of the p38 signaling pathways.

Conclusion Taken together, our findings suggest that CARD3 serves as a positive regulator of cardiac remodeling after $\mathrm{MI}$ and that this function largely occurs via the regulation of $\mathrm{p} 38$ signaling.

\section{Mechanism of resisting delayed cardiac xenograft rejection by inhibiting the NF-kappa B signaling pathway \\ Wenxue Ye, Xiaomei Ten, Zhe Yu, Zhenya Shen Department of Cardiovascular Surgery of the First affiliated Hospital, Institute for Cardiovascular Science of Soochow University, Suzhou, Jiangsu}

Objective Heart transplantation has become an effective approach 
for the treatment of end-stage heart failure. To alleviate the clinical shortages of donors for allotransplantation, increasing number of studies have reported the broad use of xenotransplantation. However, the effects of inhibiting the nuclear factor-kappa B (NF- $\mathrm{kB})$ signaling pathway on delayed xenograft rejection (DXR) and the myocardial apoptosis mechanisms involved still need to be further testified.

Methods The donor and recipient animals were randomly divided into the control group, the small interfering RNA (siRNA) negative control (siRNA-NC) group, and the siRNA group. Hematoxylin and eosin (HE) staining and transmission electron microscopy (TEM) were used to observe the myocardial histopathological and ultrastructural changes separately. Terminal deoxynucleotidyl transferase (TdT) -mediated deoxyuridine triphosphate (dUTP) nick end-labeling (TUNEL) assay was used to detect the myocardial apoptosis indices. The mitochondrial membrane potential $(\Delta \Psi \mathrm{m})$ was determined by specific probe. Enzyme-linked immunosorbent assay (ELISAs) was used to measure caspase-3 and inducible nitric oxide synthase (iNOS) activities. A colorimetric assay was used to measure NO content, and the expressions of apoptosis-related proteins were examined.

Result The inhibition of NF- $\mathrm{KB}$ p65 significantly improved the histopathological and ultrastructural changes in the myocardial tissue after transplantation, prolonging the survival times. This inhibition also significantly reduced the apoptosis indices of the myocardial tissues, inhibited the reduction in the mitochondrial membrane potentials, and decreased the caspase- 3 activity. Furthermore, the inhibition of NF- $\mathrm{KB}$ p65 not only significantly inhibited the iNOS activity, reducing the NO content, but also significantly reduced Fas and Fas-L expression.

Conclusion Inhibiting or blocking the activation of NF- $\mathrm{kB}$ p65 pathway can effectively delay the occurrence of DXR. The underlying mechanisms may relate to the reduced occurrence of myocardial apoptosis in the DXR process.

\section{PEDF: a novel protective factor for ischemic hearts \\ Hao Zhang ${ }^{1,3}$, Zheng Wang ${ }^{3}$, Shoujie Feng ${ }^{3}$, Guangda Yuan ${ }^{3}$, Wei Yan ${ }^{3}$, Lei Xu ${ }^{3}$, Hexian Shi ${ }^{3}$, Lili Chen ${ }^{3}$, Zhongming Zhang, ${ }^{1,3}$, Hongyan Dong ${ }^{2}$ 1. Department of Thoracic and Cardiovascular Surgery, the First Afflilated Hospital of Nanjing Medical University \\ 2. Department of Biology, Xuzhou Medical College \\ 3. Institute of Cardiovascular Disease, Affliliated Hospital of Xuzhou Medical College}

Objective Pigment epithelium-derived factor (PEDF) is a pleiotropic gene with anti-inflammatory, antioxidant and anti-angiogenic properties. However, the role of PEDF in the heart is unclear. The present study evaluates the effect of PEDF on functional recovery in the ischemic heart.

Methods Adult Sprague-Dawley rat models of acute myocardial infarction (AMI) were surgically established. PEDF-small interfering RNA (siRNA) -lentivirus (PEDF-RNAi-LV) or PEDF-LV was delivered into the ischemic myocardium to reduce or overexpress PEDF, respectively. Expression levels of PEDF, VEGF, VEGFR2 and HIF-1a in the ischemic myocardium were determined by immunoblotting at 4 weeks after AMI. Vessel density, permeability and functionality were analyzed by histological observation and immunofluorescence assay. Additionally, myocardial inflammation, cardiomyocyte apoptosis and animal cardiac function were also evaluated.

Result The results indicated that the expression levels of PEDF and VEGF were opposite each other in the ischemic myocardium. In the siPEDF group, an increase in cardiomyocyte apoptosis and myocardial inflammation were observed. In contrast, PEDF overexpression significantly reduced the cardiomyocyte apoptosis and inflammation reaction in the ischemic myocardium. Compared with the siPEDF and the ischemic control groups, echocardiography examination showed a significant enhancement of cardiac function in the PEDF group, although the number of local endothelial cells sprouting and angiogenesis were increased in the siPEDF group.

Conclusion PEDF is an indispensable environmental factor in stabilizing the microenvironment of the normal heart. PEDF overexpression exerts an important biological effect on improving cardiac function by cardiomyocyte protection in the ischemic heart. Thus, PEDF is a novel protective factor for ischemic hearts.

\section{Effects of renal sympathetic denervation on the atrial electrophysiology in dogs with pacing- induced heart failure \\ Xiaozhan Wang, Qingyan Zhao, Hongping Deng, \\ Xule Wang, Zongwen Guo, Zixuan Dai, Jinping Xiao, \\ Peixing Wan, Congxin Huang \\ Renmin Hospital of Wuhan University}

Objective Heart failure (HF) and atrial fibrillation (AF) are associated with sympathetic activation. Renal sympathetic denervation (RSD) can suppress AF vulnerability. This article wanted to reasearch the impact of RSD on atrial electrophysiology in experimental HF.

Methods Twenty-two beagles were randomized into control, HF and HF+RSD groups. Control dogs were implanted cardiac pacemakers without pacing. Dogs in the HF group underwent right ventricular pacing for 3 weeks at $240 \mathrm{bpm}$ to induce HF. The dogs in the HF+RSD group received RSD and underwent the same HF-inducing procedure.

Result The P-wave dispersion was higher in HF dogs than in control and HF+RSD dogs $(19 \pm 3.1 \mathrm{~ms}$ vs $13 \pm 2.3 \mathrm{~ms}$ and $15 \pm 2.9 \mathrm{~ms}$, $\mathrm{P}=0.04)$. Conduction time $(\mathrm{CT})$ within the inter-atrium was significant longer in HF dogs than that in control and HF+RSD dogs [CTRA-LA: (39 $\pm 4) \mathrm{ms}$ vs $(31 \pm 3) \mathrm{ms}, 33 \pm 4 \mathrm{~ms} ; \mathrm{P}=0.03$ ]. Window of vulnerability (WOV) of AF was widened in HF dogs than in HF+RSD dogs (RA: 37 $\pm 5 \mathrm{~ms}$ vs $14 \pm 3 \mathrm{~ms} ; \mathrm{P}<0.01$ ), while $\mathrm{AF}$ could not be induced ( $\mathrm{WOV}=0$ ) in control dogs during S1S2 stimulation. The voltage in the threshold for $\mathrm{AF}$ inducilibity was lower during ganglionated plexi stimulation in $\mathrm{HF}$ dogs than in control and HF+RSD dogs[ $[(1.8 \pm 0.6) \mathrm{V}$ vs $(2.5 \pm 0.6) \mathrm{V}, 2.4$ $\pm 0.4 \mathrm{~V} ; \mathrm{P}=0.04]$

Conclusion RSD could reverse the atrial electrical remodeling and decrease $\mathrm{AF}$ inducibility in dogs with pacing-induced HF.

\section{Tongxinluo reduces infarct size in reperfused diabetic heart by maintaining endothelial cell- cell junctions integrity via PI3K/Akt pathway \\ Kang Qi, Yuejin Yang, Xiangdong Li, Chen Jin, Hehe Cui, Leipei Jiang, $\mathrm{Na} \mathrm{Li}$ \\ State Key Laboratory of Cardiovascular Disease, Fuwai Hospital, National Center for Cardiovascular Disease, Chinese Academy of Medical Sciences and Peking Union Medical College}

Objective Ischemia/reperfusion disrupts structure and function of microvascular barrier, resulting uncontrollable inflammation and ischemia/reperfusion injury (IRI). High glucose worsens microvascular 
barrier function and may aggravate myocardial IRI. As a cardioprotective drug, Tongxinluo (TXL) may stablize endothelial junctions, thereby limiting myocardial edema, netrophil transendothelial migration, inflammation and consequent necrosis. In order to confirm the effect of different types of endothelial cell-cell junctions in the TXL-mediated cardiac protection, studies in vitro and in vivo were conducted.

Methods HCMECs were cultured for $48 \mathrm{~h}$ respectively followed by glucose-oxygen-serum deprivation (GOSD) for $2 \mathrm{~h}$ and reoxygenation for $2 \mathrm{~h}$. PI3K/Akt inhibitor wortmannin $(100 \mathrm{nM})$, insulin $(10 \mathrm{nM})$ and TXL $(800 \mu \mathrm{g} / \mathrm{ml})$ were supplemented. Endothelial monolayer permeability was assessed. Endothelial junctional proteins including VE-cadherin, p120-catenin, ZO-2, occludin, JAM-A, integrin-alpha5, integrin-beta1, connexin-43 and connexin-37 expressions were detected by western blot. ZDF rats with T2DM underwent 45 min ligation and 3 $\mathrm{h}$ reperfusion of LAD. Insulin $(5 \mathrm{U} / 100 \mathrm{~g})$ and TXL $(800 \mu \mathrm{g} / \mathrm{ml})$ were administered before ischemia. Cardiac microvascular permeability and infarct size were determined by FITC-dextran and TTC staining.

Result The fluorescence intensity in the TXL group (1917 \pm 124$)$ was dramatically decreased when compared with control group (3548 \pm 181). Membrane/total expression of VE-cadherin, p120-catenin, ZO-2, occludin, JAM-A, integrin-alpha5, integrin-beta1 but not connexin-43 or connexin-37 was significantly increased by TXL or insulin both in vitro and in vivo. Infarct size in control group $(53.5 \pm 3.3) \%$ was significantly reduced when compared with TXL group $(42.5 \pm 4.2) \%$. These protective effects of TXL were inhibited by wortmannin.

Conclusion TXL protects reperfused diabetic heart through attenuating paracellular hyperpermeability in CMECs. This protection may be related to TXL-mediated enhancement of adhesion junction, tight junction and focal adhesion, but not connexins.

\section{Angptl4 functions as a protective factor in reperfused diabetic heart by reducing endothelial paracellular permeability \\ Kang Qi, Yuejin Yang, Na Li, Xiangdong Li, Hehe Cui, Leipei Jiang, Qing Li \\ State Key Laboratory of Cardiovascular Disease, Fuwai Hospital, National Center for Cardiovascular Disease, Chinese Academy of Medical Sciences and Peking Union Medical College}

Objective Structural and functional disruption of microvascular barrier caused by ischemia/reperfusion results in uncontrolled inflammation and ischemia/reperfusion injury (IRI). Hyperglycemia may aggravate myocardial IRI since it worsens the barrier function. Angiopoietin-like 4 (Angpt14) has the potential to improve the endothelial barrier function through its alteration of the endothelial F-actin, and it may be involved in reperfused diabetic heart protection. In order to confirm the effect and mechanism Angpt14-mediated cardiac protection, studies in vitro and in vivo were conducted.

Methods HCMECs were cultured in normal $(5.5 \mathrm{mM})$ and high glucose $(18 \mathrm{mM})$ for $48 \mathrm{~h}$ respectively followed by glucoseoxygen-serum deprivation (GOSD) for $2 \mathrm{~h}$ and reoxygenation for 2 h. RhAngptl4 (1 $\mu \mathrm{g} / \mathrm{ml})$, PI3K/Akt inhibitor wortmannin (100 nM) were supplemented. Endothelial monolayer permeability was assessed. Internalization of adhesion junction proteins (VE-cadherin, p120catenin), tight junction proteins (ZO-2, occludin, JAM-A) and focal adhesion proteins (integrin-alpha5, integrin-beta1) and connexin proteins (Connexin-43, Connexin-37) were detected by western blot. ZDF rats with Type 2 diabetes mellitus (T2DM) underwent 45 min ligation and 3 $\mathrm{h}$ reperfusion of LAD. Microvascular permeability and infarct size were determined by FITC-dextran and TTC staining.

Result Compared with the control group, rhAngptl4 dramatically decreased the fluorescence intensity (1485 \pm 106 vs $3284 \pm 158)$ and the internalization of adhesion junction proteins (VE-cadherin, p120catenin), tight junction proteins (ZO-2, occludin, JAM-A) and focal adhesion proteins (integrin-alpha5, integrin-beta1) but not connexin proteins (Connexin-43, Connexin-37). RhAngptl4 significantly reduced size of myocardial permeability and infarct size $[(38 \pm 3) \%$ vs $(58 \pm 5) \%$ ] of ZDF rats with T2DM in AMI/reperfusion model compared with control group. These protective effects of rhAngptl4 were inhibited by wortmannin.

Conclusion Accordingly, rhAngptl4 protects reperfused diabetic heart through attenuating endothelial paracellular hyperpermeability.

\section{Hyperglycemia and oxidative stress stimulate abnormal proliferation of atrial fibroblast through NADPH oxidase-JNK 1/p38-MMP9 signal pathway \\ Tong Liu, Qitong Zhang, Xinghua Wang, Xue Liang, Guangping Li \\ Tianjin Key Laboratory of lonic-Molecular Function of Cardiovascular \\ Disease, Department of Cardiology, Tianjin Institute of Cardiology, Second Hospital of Tianjin Medical University}

Introduction Atrial fibrosis is one of the fundamental pathophysiological mechanisms in the development of atrial fibrillation, and the abnormal proliferation and polarization of atrial fibroblast stimulated by kinds of pathological factors participate in this process. Several epidemiological studies have indicated that diabetes mellitus is an independent risk factor of the onset and perpetuation of atrial fibrillation. Previous studies found that oxidative stress is a significant trigger of the abnormal proliferation and polarization of atrial fibroblast by up-regulation of the activity of nicotinamide adenine dinucleotide phosphate-oxidase (NADPH oxidase) and mitogen-activated protein kinases (MAPK) signaling transduction. However, the variation of atrial fibroblast in hyperglycemic and oxidative stress state needs more exploration.

Methods Atrial fibroblast isolated from adult rabbits' left atrium is cultured and stimulated respectively by high levels of glucose, hydrogenperoxide $\left(\mathrm{H}_{2} \mathrm{O}_{2}\right)$ and their combination and the NADPH oxidase inhibitor apocynin. We test the variation of proliferation activity of the fibroblast by MTS colorimetricmethod and the alteration of protein expressions of NADPH oxidase, MAPK signal pathway and matrix metalloproteinases (MMP2 and MMP9).

Result High levels of glucose and $\mathrm{H}_{2} \mathrm{O}_{2}$ promote the proliferation of atrial fibroblast and $\mathrm{H}_{2} \mathrm{O}_{2}$ have the similar ability of acceleration compared with hyperglycemia. However, the combined stimulus of hyperglycemia and $\mathrm{H}_{2} \mathrm{O}_{2}$ reveals even more remarkable enhancement of proliferation of fibroblast $(\mathrm{P}<0.01)$. And all the promotion by these agents can be depressed by apocynin $(\mathrm{P}<0.01)$. The expression of Rac1, the regulatory subunit of NADPH oxidase is up-regulated by activation of high concentration of glucose, $\mathrm{H}_{2} \mathrm{O}_{2}$ and the combined stimulus $(\mathrm{P}<0.01)$. And this provocation can be reduced by apocynin. However, the protein expression of NADPH oxidase's subunit, p22phox and gp91phox have no significant difference among all the groups. Regarding the MAPK pathway, the phosphorylation level of JNK 1 and p38 are up-regulated and apocynin can reduce the protein expression levels of their phosphorylation $(\mathrm{P}<0.01)$. The downstream fibrosisrelated protein MMP9 is over-expressed by up-mentioned stimulation 
and can be reduced by apocynin $(\mathrm{P}<0.05)$.

Conclusion High concentration of glucose and $\mathrm{H}_{2} \mathrm{O}_{2}$ promote the proliferation of atrial fibroblast and the combined stimulus has more remarkable effects. Similarly to the mechanism of $\mathrm{H}_{2} \mathrm{O}_{2}$, glucose enhance the expression of fibrosis-related protein MMP 2 and MMP 9 through up-regulating the function of NADPH oxidase and the expression and phosphorylation level of JNK 1 and p38. Apocynin, a NADPH oxidase inhibitor, can reduce the up-regulation of NADPH oxidase-JNK 1/p38MMP 9 signal pathway and suppress the abnormal proliferation of atrial fibroblast.

\section{Gene knock-in mouse models for heart development and regeneration studies \\ Jianyun Yan ${ }^{1,2}$, Chenleng Cai ${ }^{2}$ \\ 1. Department of Histology and Embryology, Southern Medical \\ University \\ 2. Department of Developmental and Regenerative Biology, Mount Sinal School of Medicine}

Objective Tnnt2, encoding thin-filament sarcomeric protein cardiac troponin $\mathrm{T}$, plays critical roles during mouse heart development. This study aims to develop a conditional and inducible gene knockout approach for heart development and regeneration studies, and establish a mouse model for understanding the origins of regenerated cardiomyocytes.

Methods We generated a new Tnnt2: MCM (Tnnt2 $\left.{ }^{\mathrm{MCM} /}\right)$ knockin mouse line that allows cardiomyocyte-specific gene disruption and lineage tracing after tamoxifen treatment. Rosa26 reporter mouse lines were used to examine the efficiency and specificity of Tnnt2 inducible Cre recombinase expression. In addition, we also generated a new Tnnt2: nlacZ-H2BGFP (Tnnt2 $2^{\text {nlacZ-H2BGFP// }}$ ) knock-in mouse line to uncover the source of regenerated cardiomyocytes during injury. $\mathrm{X}$-gal and immunostaining were used to determine Tnnt2 expression in cardiomyocytes.

Result We demonstrated that Tnnt2 Cre recombinase was specifically and robustly expressed in the cardiomyocytes of embryonic and adult hearts following tamoxifen induction. Additionally, we also found that Tnnt2: nlacZ was highly expressed in the cardiomyocytes. Tnnt2: H2BGFP mouse line was obtained by crossing ProtamineCre recombinase transgenes with Tnnt $2^{\text {nlacz-H2BGFP/t }}$ knock-in mice. Nuclear GFP expression mirrored that of the endogenous Tnnt2 gene in myocardial cells of Tnnt2: H2BGFP mice.

Conclusion These results show that the novel mouse line Tnnt $2^{\mathrm{MCM} /+}$ can be used for analysis of spatiotemporal gene function and cardiomyocyte lineage tracing during heart development and regeneration. Tnnt $2^{\text {nlacZ-H2BGFP/+ }}$ mouse line can be used as a useful model to identify the source of regenerated cardiomyocytes for cardiac regeneration and heart diseases.

\section{Expression of ZO-1 in iPS cells mediated by lentiviral vector \\ Tao Sheng ${ }^{1}$, Jianping Zeng ${ }^{2}$, He Huang ${ }^{2}$, Hui $\mathrm{Li}^{2}$, \\ Zhishan $\operatorname{Sun}^{2}$ \\ 1. The Second Xiangya Hospital of Central South University \\ 2. Xiangtan Central Hospital}

Objective The fundamental researches of cardiovascular disease verified that intercalated disc-related gene $\mathrm{ZO}-1$ varis in different myocardial pathologic conditions, and it interacts with $\mathrm{Cx} 43$. This research is about to construct recombinant lentivirus over-expressing vector of porcine ZO-1 gene, to infect iPS cells, to obtain iPS cells carrying this target gene.

Methods The ZO-1gene sequence publicated on Genbank was analyzed, and designed a couple of primer containing both BamHI and SalI. The porcine ZO-1 gene was amplified by PCR, and cloned into lentiviral expression vector, to construct the recombinant Lenti-EF1 $\alpha$ EGFP-TRE-ZO1 vector, the vector was confirmed by DNA sequencing. The production of lentivirus were obtained by using pVSVG, delta 8.91 and fugene to contransfect $293 \mathrm{~T}$ cells. The titer of lentivirus was testd with fluorescent expression, to infect iPS cells.

Result The recombinant lentivirus plasmid carrying ZO-1 was correctly constructed, it was identified correct by double enzyme digestion and DNA sequencing. The lentiviral packaging was succeed, and the titer of lentivirus was $1.42 \times 10^{8} \mathrm{IU} / \mathrm{ml}$.

Conclusion Lenti-EF1 $\alpha$-EGFP-TRE-ZO1 lentiviral vector has been successfully constructed. The titer of lentivirus was $1.42 \times 10^{8} \mathrm{IU} /$ $\mathrm{ml}$, it was used to infect iPS cells, which will be useful for further study in vivo, to understand its function and its unique effects under different pathological status of heart, and open new doors for the treatment of cardiovascular diseases.

\section{Study on atrial fibrillation related miRNA and mRNA profiling and the establishment of miRNA-mRNA regulatory network}

Jie Gao, Jian Zhou, Pixiong Su

Beijing Chao Yang Hospital, Capital Medical University

Objective The study is screening for the AF-related miRNAs and their target gene by microarray. Then microRNA-mRNA regulatory net was established based on prediction of miRNAs target gene and negative correlation between the miRNA and mRNA. The results will provide bases for the pathogenesis, diagnosis and treatment of the disease.

Methods miRNA arrays (miRCURY ${ }^{\mathrm{TM}}$ LNA expression array) were used to evaluate the microRNA expression signature of the left atrial appendages of AF patients with healthy heart donors in sinus rhythm. At the same time, the expression profiling of mRNA were analysed by oligonucleotide microarray. GOmir was used to predict the potential miRNA targets and to analyze their functions. Pathway analysis was applied to predict the potential pathway that the differential expressed genes are involved in. Furthermore, correlations between differentially expressed miRNA and mRNA were analyzed, and miRNA-mRNA regulatory network was established. qRT-PCR analysis were used to validated the expression of key miRNAs and gene in the network. Bioinformatics analyses were performed to understanding the global regulating roles of miRNAs-mRNA network.

Result 26 miRNAs were identified to differentially expressed in AF patients compared with healthy controls, including 16 up-regulated and 10 down-regulated, respectively. microRNA regulatory network was constructed, showing the key regulatory microRNA were hsa-miR-144, hsa-miR-3613-3p, hsa-miR-1284, hsa-miR-185, hsa-miR-101 and hsamiR-1827. GOmir analysis showed they are involved in transcription, development, signal transduction, protein phosphorylation, cell adhesion and ion transport, et al. $610 \mathrm{mRNAs}$ (fold change $>2, \mathrm{P}<0.05$ ) were shown to be significantly changed in AF patients compared with healthy controls. The signal pathway effected by up-regulated genes were immune response etc. While the signal pathway effected by downregulated genes were proliferation (TGF- $\beta$ signal pathway, MAPK signal 
pathway), ion transport. The results were in accordance with the miRNA. The target prediction and negative correlations analyses with the mRNA array have identified 20 dysregulated miRNAs and their 107 target genes and corresponding miRNA-mRNA network were constructed. The miRNAs including miR-144, miR-1284, miR-1827, miR-1, miR-36133p, miR-101 and target mRNAs containing PTEN, TAOK1, RUNX1 and TPM 3 were found to be in the central part of the network and their expression were further verified by qRT-PCR analysis. Bioinformatics analysis reflects the basis of electrophysiology, atrial fibroblast, left atrium dilation and fetal gene expression of AF remodeling.

Conclusion This study identified 26 miRNAs and 610 genes which may play important roles in the occurrence and progression of $\mathrm{AF}$, demonstrated the global expression difference of miRNA-mRNA between permanent AF and sinus rhythm. qRT-PCR analysis verified the differential expression of some key miRNA and target genes. AFrelated miRNA-mRNA regulatory network were established and key miRNA-gene pairs were identified. These results provide a basis for understanding the mechanism and searching for targets in diagnosis and treatment of permanent AF.

\section{EETs mediated anti-oxidative stress effect on prevention against aortic adventitial remodeling Junxiong Chen, Daowen Wang Department of Internal Medicine and Gene Therapy Center, Tongji Hospital, Tongji Medical College, Huazhong University of Science and Technology, Wuhan, China}

Objective Vascular adventitial remodeling is an important pathologic process in multiple vascular fatal diseases. Reactive oxygen species (ROS) are an important triggering factor involved in adventitial remodeling. EETs (epoxyeicosatrienoic acids) level was increased by CYP2J2 overexpression or soluble epoxide hydrolase inhibitors (sEHIs) administration. And EETs exert diverse protective effects on cardiovascular system. However, we don't know much about the effect of EETs on prevention against adventitial remodeling. Herein, we investigated whether CYP2J2-EETs-sEH pathway prevented adventitial remodeling via reducing oxidative stress.

Methods Aortic adventitial remodeling in C57BL/6J mice was induced by Angiotensin II (Ang II, $1 \mathrm{mg} / \mathrm{kg} / \mathrm{d}$ ) infusion. Recombinant adeno-associated virus (rAAV) -mediated CYP2J2 gene delivery and TUPS (one of the sEHIs with better oral bioavailability) were performed both in vivo and in vitro. Sections of mice aortas were used for H\&E, sirus red, immunohistochemical and DHE staining. Primary aortic adventitial fibroblasts (AoAFs) were cultured from rats' aortas. CCK8 and Brdu-ELISA, transwell chambers and scratch test, western blotting, enzyme activity kits and ROS detection were used to evaluate remodeling effects and oxidative stress.

Result Ang II significantly induced aortic adventitial remodeling, including adventitial hyperplasia, collagen accumulation, transformation (indicated by alpha-SMA) and inward migration of adventitial fibroblasts (indicated by fibroblasts marker ER-TR7). Importantly, CYP2J2 and TUPS markedly reduced the adventitial remodeling. In primary AoAFs, CYP2J2, TUPS and 11, 12-EET significantly prevented remodeling (indicated by proliferation, migration, and expression of type I collagen and alpha-SMA) and ROS increasing induced by Ang II. In addition, increasing of $\mathrm{H}_{2} \mathrm{O}_{2}$ level and phosphorylation of p38MAPK and JNK1/2 were markedly decreased by 11,12 -EET. We then excluded the diverse inductive effects of Ang II by using $\mathrm{H}_{2} \mathrm{O}_{2}$. Results showed that 11, 12-EET markedly prevented AoAFs remodeling induced by $\mathrm{H}_{2} \mathrm{O}_{2}$.
Furthermore, 11, 12-EET reduced the increased expression of gp91phox and reversed the inhibition of Ang II on expression and activities of $\mathrm{Cu}$ / $\mathrm{Zn-SOD}$ and catalase. However, both 11, 12-EET and Ang II had no effect on Mn-SOD. Interestingly, GW9662 (inhibitor of PPAR-gamma) significantly blockaded the beneficial effect of 11, 12-EET on ROS inhibition.

Conclusion These results suggest that CYP2J2 and TUPS attenuates remodeling and oxidative stress both in vivo and in primary AoAFs. Taken together, the anti-remodeling effect of CYP2J2 and TUPS might be partially due to their direct effects on AoAFs. Furthermore, EET-mediated anti-oxidative stress effect was involved in these favorable profiles. Exploitation of this beneficial activity may shed light on designing novel therapeutic approaches for remodeling in multiple kinds of vascular diseases.

\section{Exosome-carried miRNA-185-5P regulates the transcription of metalloprotease ADAMTS13 \\ Yuanyuan Fu, Hang Yang, Qinqin Xiang, Zhou Zhou State Key Laboratory of Cardiovascular Disease, Center of Molecular Diagnostics, Fuwai Hospital, National Center for Cardiovascular Diseases, Chinese Academy of Medical Sciences and Peking Union Medical College,}

Objective Coronary artery disease (CAD) is one of the leading causes for mortality. Previous studies shown that metalloprotease ADAMTS13 in plasma specifically cleaves von Willebrand Factor (VWF) to regulate thrombi formation and its reduction is closely associated with the initiation and progress of CAD. Hepatic stellate cell (HSC) is the major source for ADAMTS13 production in plasma. Recent studies have shown that miRNAs actively regulated the transcription of ADAMTS family proteins. Exosome, a small vesicle known to mediate intercellular communication by delivering nucleic acids and proteins, draws attention because of its ability in packaging miRNAs and protecting them from degradation. To further understand the molecular mechanisms of ADAMTS13 production and provide insights for CAD progression, we focused on the regulation of ADAMTS13 transcription by miRNAs in both liver fibrotic mouse model and primarily cultured HSCs, especially those shuttled through exosomes.

Methods CCL4 was delivered intraperitoneally into male Swiss Webster mice (6-8 weeks) three times per week for 5 weeks to induce liver fibrosis. Olive oil was used as vehicle control. Primary HSCs were isolated by in situ enzymatic dissociation and density gradient centrifugation from livers of CCL4-/oil-injected or normal male Swiss Webster mice, and processed for real-time polymerase chain reaction (RT-PCR). Sirius red staining and immunofluorescent (IF) staining of ADAMTS13 was performed on paraffin-embedded liver sections to detect collagen deposition and ADAMTS13 protein levels. Candidate miRNAs regulating ADAMTS13 transcription were predicted based on microRNA. org database. In-situ hybridization (ISH) on liver sections was done to detect the reciprocal correlation between candidate miRNAs and ADAMTS13. The efficiency of respective miRNA in binding ADAMTS13 3'-UTR was examined by luciferase assay. The profiling of miRNAs changes in fibrotic or control mice serum was performed to verify the changes.

Result We showed that the down-regulation of ADAMTS13 in fibrotic livers, or in activated primary mouse HSC was associated with a reciprocal up-regulation of miRNA-185-5P (miR-185-5P). miRNA185-5P was predicted to bind ADAMTS13 3'-UTR to regulate its transcription. Both ISH and RT-PCR using liver tissue showed declined 
ADAMTS13 mRNA and elevated miR-185-5P levels, indicating a reciprocal correlation between them. By co-transfecting either FireCtx containing vehicle vector, wild-type ADAMTS13 3'-UTR, or 3'UTR with a mutation in the miR-185-5P binding site along with premir-185-5P into HSC, the luciferase assay showed miR-185-5Pdependent regulation of a wild-type ADAMTS13 3'-UTR, but not of a mutant ADAMTS13 3'-UTR lacking the miR-185-5P binding site. Additionally, miR-185-5P was present in both mouse serum and HSC exosomes, which were bi-membrane vesicles, $50-150 \mathrm{~nm}$ in diameter, negatively charged $(-19 \mathrm{mV})$, and positive for CD9 and flotilin. miRNA profiling of serum exosomes from fibrotic mice showed significantly increased miR-185-5P. Accordingly, ADAMTS13 mRNA of serum exosomes significantly decreased in fibrotic mice.

Conclusion Exosomal transfer of miR-185-5P may be a paradigm for the regulation of ADAMTS13 transcription.

\section{The regulation role of P2X7R to NLRP3 inflammasome in atherosclerosis}

Kuang Peng, Hongyan Wang, Xiaoqing Wang, Lili Wang, Dan He, Peng Qu Department of Cardiology, Second Affiliated Hospital of Dalian Medical University.

Objective Observation of the roles of P2X7R in atherosclerosis. Investigate the mechanism of NLRP3 inflammasome activation in atherosclerosis.

Methods The expression levels of P2X7R and NLRP3 in monocytes were observed by Real-time PCR and western blot. The plasma and medium levels of oxLDL and IL- $1 \mathrm{~b}$ were analyzed by ELISA. The expressions of P2X7R and NLRP3 in the atherosclerosis plaque of coronary artery were observed by immunohistochemistry or immunofluorescence. The specificity siRNA was used to inhibit NLRP3 and P2X7R. The co-immunoprecipitation was used to assay the interaction between PKR phosphorylation and NLRP3.

Result The expressions of P2X7R and NLRP3 in CAD were higher than in Non-CAD patients. The expression of P2X7R in the CD68 positive area in artery has high intensity. And expression of NLRP3 is also very high in these locations. Increased amount of proIL$1 \mathrm{~b}$ and mature IL-1b in THP- 1 were produced by oxLDL. The mRNA and protein expression of P2X7R and NLRP3 in THP-1 were also elevated. After NLRP3 or P2X7R was knocked down by siRNA, the expression of proIL-1b was keeping the high level, but the level of IL$1 \mathrm{~b}$ was significantly lower than the negative control. OxLDL could improve PKR phosphorylation, up-regulate NLRP3 expression, and promote caspase-1 production. When P2X7R was suppressed, the effect was attenuated. Co-immunoprecipitation showed PKR phosphorylation by oxLDL was related with NLRP3. When P2X7R was inhibited by siRNA, PKR phosphorylation by oxLDL decreased significantly, and less NLRP3 was interacting with PKR phosphorylation. The activated caspase-1 was also reduced. With 12 weeks high cholesterol diet, atherosclerotic plaque was found in apoE-/-mice aortic sinus. In the plaque, P2X7R had intensive expression, especially in foam cells genesis. Otherwise, compared to apoE-/- and apoE-/-NC, the atherosclerosis in apoE-/-P2X7R-/- was attenuated. The IL-1b level in plasma of apoE-/P2X7R-/- mice was much lower than control and negative control.

Conclusion P2X7R and NLRP3 expressions in macrophages may participate in human atherosclerosis onset and development. By oxLDL, P2X7R regulates the assembly and activation of NLRP3 inflammasome by controlling the level of PKR phosphorylation, but P2X7R could not regulate the expression of NLRP3 mRNA and protein directly. P2X7R plays important roles in the apoE-/- mice atherosclerosis development.

\section{Attenuation of CLOCK-BMAL1 transcriptionally decreases the expression of beta1-AR to anticipate the occurrence of ventricular arrhythmia after chronic heart failure \\ Jiamin Yuan, Zhiyong Qian, Peibing Ge, Yao Wang, \\ Yanhong Chen, Xiaofeng Hou, Jiangang Zou \\ Department of Cardiology, the First Affiliated Hospital of Nanjing Medical \\ University, Nanjing, Jiangsu, China}

Circadian rhythms influence the incidence of sudden cardiac death in chronic heart failure (CHF) but the underlying mechanisms are not well defined. We sought to investigate the role of the beta-adrenergic receptor (beta-AR) in cardiac circadian disorders and ventricular arrhythmia (VA) after CHF.

CHF was created by transverse aorta constriction. Circadian variations of the myocardial expressions of $\beta 1-\mathrm{AR}, \beta 2-\mathrm{AR}$ and circadian genes CLOCK and BMAL1 were examined by real time reverse transcription polymerase chain reaction, Western blot and immunohistochemistry. Chromatin immunoprecipitation assay (ChIP) and luciferase (LUC) were applied to determine whether CLOCKBMAL1 regulate $\beta 1-A R$ expression. Electrocardiograms were recorded in vivo and/or ex vivo. Ventricular tachyarrhythmias were induced by isoprenaline and programmed electrical stimulation (PES).

Normal guinea pigs showed circadian oscillations in both the myocardial expression of beta1-AR and CLOCK-BMAL1, but not for beta2-AR. However, these circadian rhythms were significantly blunted or even abolished in guinea pigs with CHF. And the expression of $\beta 1$ AR and CLOCK-BMAL1 were attenuated in guinea pigs with CHF. ChIP-qPCR analysis and luciferase revealed that BMAL1 can bind to enhancer of $\beta 1-A R$ to regulate arrhythmia severity after CHF.

Circadian rhythms of myocardial $\beta 1$-AR and CLOCK-BMAL1 activities are disturbed after CHF. Attenuation of CLOCK-BMAL1 transcriptionally decreases the expression of $\beta 1$-AR to anticipate the occurrence of ventricular arrhythmia after CHF.

\section{Ox-LDL modulates vascular calcification via TLR4-NK- $k$ B signaling}

Yan Song ${ }^{1}$, Jianyun Yan ${ }^{2}$, Menglin Hou ${ }^{1}$, Qin Zhou ${ }^{3}$,

Lihe $\mathrm{Lu}^{1}$

1. Department of Pathophysiology, Zhongshan Medical School, Sun

Yat-Sen University, China

2. Department of Histology and Embryology, Southern Medical

University, China

3. Department of Anesthesiology, The First Afflilated Hospital, Sun YatSen University, China

Vascular calcification is a major feature of advanced atherosclerosis and highly associated with cardiovascular diseases. Oxidized low density lipoprotein (Ox-LDL) has been recognized as a critical risk factor for atherosclerosis and calcification of vascular smooth muscle cells (VSMCs). Previous studies demonstrated that toll-like receptor 4 (TLR4) is highly expressed in atherosclerotic lesions and participates in the progression of atherosclerosis. However, the role of TLR4 in vascular calcification is unknown. In this study, we investigated whether TLR4 modulates vascular calcification induced by Ox-LDL. TLR4 
expression was up-regulated in cultured human VSMCs treated by Ox-LDL. Knockdown of TLR4 by small interfering RNA (siRNA) significantly reduced Ox-LDL-induced calcification, detected by alizarin red staining and calcium content assay. TLR4 siRNA also decreased the mRNA expression of bone-related proteins including Msx2, osterix and BMP2, but increased the expression of VSMC contractile proteins including SMA and SM22 $\alpha$ in VSMCs. In addition, Ox-LDL stimulated the phosphorylation of the inhibitors of nuclear factor kappa B (IкB), and induced nuclear translocation of NK-кB p65. These effects of OxLDL on VSMCs were reversed by TLR4 siRNA. Furthermore, NK$\kappa \mathrm{B}$ inhibitor, pyrrolidine dithiocarbamate (PDTC), attenuated OxLDL-induced VSMC calcification, which was rescued by C2-ceramide treatment. In conclusion, these findings suggest that TLR4 regulates VSMC calcification induced by Ox-LDL through activation of nuclear factor kappa B (NK- $\mathrm{B})$, highlighting TLR4 as a novel potential therapeutic target for vascular calcification.

\section{Decreased MiR-451 in hypertrophic cardiomyopathy regulates autophagy by targeting TSC1 \\ Jizheng Wang ${ }^{1}$, Ming Su ${ }^{1}$, Yubao Zou ${ }^{1}$, Xiaojian Wang ${ }^{1}$, Yilu Wang ${ }^{2}$, Hongli Cui ${ }^{3}$, Peng Zhao ${ }^{3}$, Shuiyu Wang ${ }^{1}$, Rutai Hui ${ }^{1}$, Lei Song ${ }^{1}$ \\ 1. Fuwai Hospital, National Center for Cardiovascular Disease, Chinese Academy of Medical Sciences and Peking Union Medical College, \\ Beijing, China \\ 2. China Meitan General Hospital, Beijing, China \\ 3. Department of Pathology, Affiliated Hospital of Qingdao University Medical College, Qingdao, China}

Objective The molecular mechanisms that drive the development of cardiac hypertrophy in hypertrophic cardiomyopathy (HCM) remain elusive. Accumulated evidence suggests that microRNAs are essential regulators of cardiac remodeling. We hypothesized that microRNAs could play a role in the process of HCM. In the present study, we identified the differentially expressed microRNAs in HCM and reveal the role of miR-451 in regulating cardiac hypertrophy.

Methods and Result To uncover which microRNAs were changed in their expression, microRNA microarrays were performed on heart tissue from HCM patients $(\mathrm{n}=7)$ and from healthy donors (n $=5$ ). Among the 13 microRNAs that were differentially expressed in HCM, miR-451 was the most down-regulated. Ectopic overexpression of miR-451 in neonatal rat cardiomyocytes (NRCM) decreased the cell size, whereas knockdown of endogenous miR-451 increased the cell surface area. Luciferase reporter assay analyses demonstrated that tuberous sclerosis complex 1 (TSC1) was a direct target of miR-451. Overexpression of miR-451 in both HeLa cells and NRCM suppressed the expression of TSC1. Furthermore, TSC1 was significantly upregulated in HCM myocardia, which correlated with the decreased levels of miR-451. As TSC1 is a known positive regulator of autophagy, we examined the role of miR-451 in the regulation of autophagy. Overexpression of miR-451 in vitro inhibited the formation of the autophagosome. Conversely, miR-451 knockdown accelerated autophagosome formation. Consistently, an increased number of autophagosomes was observed in HCM myocardia, accompanied by up-regulated autophagy markers, and the lipidated form of LC3 and Beclin-1.

Conclusion Our findings indicate that miR-451 regulates cardiac hypertrophy and cardiac autophagy by targeting TSC1. The down- regulation of miR-451 may contribute to the development of HCM and may be a potential therapeutic target for this disease.

\section{Genetic polymorphisms in ZFHX3 are associated with atrial fibrillation in a chinese han population \\ Yaowu Liu, Xinguang Chen, Zhen Fang, Liyan Zhao, Minglong Chen, Bing Yang, Fengxiang Zhang \\ Department of Cardiology, the First Affliliated Hospital of Nanjing Medical University, Nanjing, Jiangsu, China}

Background The gene zinc finger homeobox 3 (ZFHX3) encodes a transcription factor with cardiac expression and its genetic variants are associated with atrial fibrillation (AF). We aimed to explore the associations between single nucleotide polymorphisms (SNPs) of ZFHX3 and the risk of AF in a Chinese Han population.

Methods We genotyped eight SNPs, including seven potentially functional SNPs and one previously reported SNP by using the middlethroughput iPLEX Sequenom MassARRAY platform. Odds ratios (ORs) and $95 \%$ confidence intervals (CIs) were calculated in logistic regression models.

Result We enrolled a total of 1593 Chinese Han origin individuals in the study, including 597 AF patients and 996 non-AF controls. Logistic regression analyses revealed that potentially functional SNPs rs6499600 and rs 16971436 were associated with a decreased risk of AF (adjusted $\mathrm{OR}=0.73,95 \% \mathrm{CI}$ : $0.63-0.86, \mathrm{P}=1.07 \times 10^{-4}$; adjusted $\mathrm{OR}=$ $0.74,95 \%$ CI: $0.56-0.98, P=0.039$, respectively). In addition, rs 2106261 showed a robust association with an increased risk of $\mathrm{AF}$ (adjusted OR $=1.71,95 \%$ CI: $\left.1.46-2.00, \mathrm{P}=1.85 \times 10^{-11}\right)$. After multiple comparisons, rs 16971436 conferred a borderline significant association with the risk of AF. Stratification analysis indicated that the risks of AF were statistically different among subgroups of age for rs2106261, and the effect for rs 16971436 was more evident in subgroups of patients with coronary artery disease.

Conclusion In summary, our study investigated the role of genetic variants of ZFHX3 in AF and two SNPs (rs2106261, rs6499600) showed significant associations while rs 16971436 conferred a borderline significant association with AF risk in Chinese Han populations. However, further large and functional studies are warranted to confirm our findings.

\section{The effect of non-invasive ventilation on erythrocyte deformation in patients with hypoxemia after acute myocardial infarction Xifu Wang, Ming Ye, Huimin Zhang, Yanfang Li, Shaoping Nie Emergency Department, Beijing Anzhen Hospital, Capital Medical University}

Objective Noninvasive mechanical ventilation treatment is an effective method in curing acute heart failure and chronic obstructive pulmonary disease with hypercapnia and hypoxemia through reducing pulmonary interstitial and alveolar exudates, increasing the area of the respiratory membrane, lowering respiratory membrane thickness, lowering the intrapleural pressure, reducing the left ventricular transmural pressure and the cardiac afterload. The aim of this study is to observe the effect of non-invasive ventilation (NIV) treatment on the erythrocyte deformation index and plasma lipid peroxidation in Patients 
with Hypoxemia after acute myocardial infarction.

Methods Fifty patients with acute myocardial infarction were recruited after percutaneous coronary intervention (PCI). The patients still showed hypoxemia even when the high-flow oxygen inhalation was given. Non-invasive ventilation (NIV) was given to the pateints, and after 1 and 2 hours, the blood gas, erythrocyte deformability, and plasma malondialdehyde were measured.

Result We found that, at 1 hour after NIV, the blood $\mathrm{PaO}_{2}$ resumed to the normal level and the erythrocyte deformation index (DI) showed an increase. At 2 hours after NIV, DI significantly increased as compared with that of pre-NIV $(\mathrm{P}<0.05)$ while the blood MDA level decreased remarkably $(\mathrm{P}<0.05)$.

Conclusion The results suggested that NIV treatment could improve oxygen content in patients of hypoxemia with acute myocardial infarction after PCI, decrease plasma lipid peroxidation injury, and increase RBC deformation index, which would be beneficial to functional recovery of the ischemic cardiomyocytes, especiall acute myocardial infarction.

\section{Effect of rosuvastatin on blood pressure, mesenteric arteries structure and vasodilatation function in spontaneously hypertensive rats \\ Zhoufei Fang, Liangdi Xie \\ First Affliliated Hospital of Fujian Medical University}

Objective To study the effect of Rosuvastatin (Rsv) on blood pressure, vascular structure and vasodilatation function of $3^{\text {rd }}$ grade branch mesenteric in spontaneously hypertensive rats (SHRs).

Methods Thirty-two male SHRs at 12 wks old were randomly divided into 2 groups: rosuvastatin [SHR-R, $\mathrm{n}=16,10 \mathrm{mg} /(\mathrm{kg} \cdot \mathrm{d})$ and untreated controls (SHR, $\mathrm{n}=16$ ). Age- and weight-matched WKY rats served as controls (WKY, $\mathrm{n}=16$ ). The control rats were administrated equivalent distilled water. Systolic blood pressure (SBP) was determined by tail-cuff method before treatment, 4 and 8 wks after treatment. The wall-to-lumen area ratios (W/L), the ratios of wall thickness (WT) to lumen radius (LR) of $3^{\text {rd }}$ grade branch mesenteric arteries were assessed morphometrically. Endothelium-dependent relaxation (EDdR), endothelium-independent relaxation (EDiR) were measured by PowerLab biological signal analytical system.

Result SBP in SHRs was remarkably higher than that in WKY from 12 to 20 wks of age $(P<0.01)$. SBP in Rsv-treated rats was significantly lower than that in untreated rats after $4,8 \mathrm{wks}$ of treatment (SBP/mm Hg, 4 wks: SHR-R $180.01 \pm 14.56$ vs SHR $200.27 \pm 13.94$; 8 wks: SHR-R $180.13 \pm 13.46$ vs SHR $189.88 \pm 10.06$, both $\mathrm{P}<0.01$ ). $\mathrm{W} / \mathrm{L}$ and WT/LR in SHR-R showed a descending tendency while exited no statistical difference after 4 wks treatment $(\mathrm{P}>0.05)$. Compared with untreated SHR, W/L (SHR-R $0.51 \pm 0.21$ vs SHR $1.82 \pm 0.96, \mathrm{P}<0.01$ ) and WT/LR (SHR-R $0.23 \pm 0.04$ vs SHR $0.53 \pm 0.29, \mathrm{P}<0.01)$ of $3^{\text {rd }}$ grade branch mesenteric arteries in Rsv-treated rats were markedly lower than that of untreated SHR after 8wks treatment. WT/LR in Rsv-treated rats was similar to the level as that of WKY $(\mathrm{P}>0.05)$. EDdR and EDiR of $3^{\text {rd }}$ grade branch mesenteric arteries were reduced in untreated SHR. After 4 wks treatment, EDdR of $3^{\text {rd }}$ grade branch mesenteric arteries was increased (Emax/\%: SHR $29.10 \pm 7.35$, SHR-R $47.41 \pm 10.74$, WKY $83.85 \pm 5.17$; pD2: SHR $5.96 \pm 0.58$, SHR-R $6.39 \pm 0.90$, WKY 8.34 \pm 0.21 , both $\mathrm{P}<0.01)$. EDiR was also enhanced (Emax $/ \%$ : SHR 46.13 \pm 11.45 , SHR-R $75.23 \pm 20.10$, WKY $96.28 \pm 2.68$; pD2: SHR $5.56 \pm$ 0.23 , SHR-R $6.72 \pm 0.44$, WKY $7.84 \pm 0.13$, both $\mathrm{P}<0.01$ ). After 8 wks treatment, its continuing enhancement was not statistically significant for
EDdR and EDiR.

Conclusion The treatment of Rosuvastatin may lower blood pressure, attenuate remodeling of $3^{\text {rd }}$ grade branch mesenteric arteries, and ameliorate vasodilatation function in SHRs. The increase of vasodilatation function was prior to improvement of vascular structure.

\section{Effect of xiongdan on blood pressure, mesenteric vascular structure and function in spontaneously hypertensive rats \\ Zhoufei Fang, Liangdi Xie \\ First Affiliated Hospital of Fujian Medical University}

Objective To observe the effect of Xiongdan on blood pressure, vascular structure and dilatation function of 3rd grade branch mesenteric arteries in spontaneously hypertensive rats (SHRs).

Methods Sixteen male SHRs at 12 wks old were randomly divided into 2 groups: Xiongdan (SHR-X, $\mathrm{n}=8$, a Traditional Chinese Medicine compound, $800 \mathrm{mg} /(\mathrm{kg} \cdot \mathrm{d})$ and untreated controls (SHR, n $=8$ ). Age-and weight-matched WKY rats served as controls (WKY, $\mathrm{n}$ $=8$ ). Systolic blood pressure (SBP) was measured by tail-cuff method before treatment, 4 and 8 wks after treatment. The wall-to-lumen area ratios (W/L), the ratios of wall thickness (WT) to lumen radius (LR) of $3^{\text {rd }}$ grade branch mesenteric arteries were assessed morphometrically. Endothelium-dependent relaxation (EDdR), endothelium-independent relaxation (EDiR) were measured by PowerLab biological signal analytical system.

Result SBP in SHRs was higher than that in WKY from 12 to 20 wks of age. SBP in $X$ treated rats was significantly lower than that in untreated rats (SBP/mm Hg, 4wks: SHR-X $176.45 \pm 11.44$ vs SHR $200.27 \pm 13.94$; 8wks: SHR-X $169.43 \pm 11.97$ vs SHR $189.88 \pm 10.06$, both $\mathrm{P}<0.01)$. W/L and WT/LR of $3^{\text {rd }}$ grade branch mesenteric arteries in X treated rats were markedly lower than that of untreated SHR (W/L: SHR-X $0.53 \pm 0.09$ vs SHR $1.82 \pm 0.96$; WT/LR: SHR-X $0.24 \pm 0.08$ vs SHR $0.53 \pm 0.29$, both $\mathrm{P}<0.01)$, similar to the level that of WKY (P $>$ 0.05). Compared with SHR, EDdR (Emax/\%: SHR-X 59.29 \pm 15.15 vs SHR: $20.69 \pm 6.31, \mathrm{pD} 2$ : SHR-X $8.24 \pm 0.13$ vs SHR $5.82 \pm 0.23$; both $\mathrm{P}<0.01$ ) and EDiR (Emax/\%: SHR-X $96.37 \pm 1.87$ vs SHR $29.04 \pm$ $4.56, \mathrm{pD} 2$ : SHR-X $7.79 \pm 0.15$ vs SHR $5.31 \pm 0.14$; both $\mathrm{P}<0.01)$ of $3^{\text {rd }}$ grade branch mesenteric arteries were increased in $\mathrm{X}$ treated after $8 \mathrm{wks}$ treatment.

Conclusion The treatment of Xiongdan may lower blood pressure, ameliorate the vascular structure and dilatation function of 3rd grade branch mesenteric arteries in SHRs.

\section{PEDF and PEDF-derived peptide 44mer protect cardiomyocytes against hypoxia-induced apoptosis and necroptosis via anti-oxidative effect \\ Xiao Gao ${ }^{1}$, Hao Zhang ${ }^{1}$, Wei Zhuang ${ }^{2}$, Guangda Yuan ${ }^{1}$, Teng Sun ${ }^{1}$, Xia Jiang ${ }^{1}$, Honghua Yuan ${ }^{2}$, Zhongxin Zhou ${ }^{1}$, Zhongming Zhang ${ }^{1}$, Hongyan Dong ${ }^{2}$ \\ 1. Department of Thoracic \& Cardiovascular Surgery, Affiliated Hospital of Xuzhou Medical College \\ 2. Center of Neurobiological Research, Xuzhou Medical College}

Pigment epithelium-derived factor (PEDF) possess many biological activities such as anti angiogenesis, vascular permeability resistance, anti-inflammatory, anti-oxidation, anti-tumor, cytoprotection and 
neuronal protection. Segmental functional peptides of PEDF play part of the biological function similar to that of the holoprotein. Among these functional peptides, two different epitopes 34mer (Asp44-Asn77) and 44 mer (Val78-Thr121) draw most attention. 34mer can induce apoptosis in endothelial cells and inhibit angiogenesis, while 44mer showed neurotrophic and cytoprotective effect. But it's not known whether PEDF and its functional peptides could protectagainst hypoxia-induced cell death and the mechanisms are still unclear. We used cultured H9c2 cells and primary cardiomyocytes to show that apoptosis and necroptosis were significantly increased after hypoxia. Both PEDF and its fuctional peptides $44 \mathrm{mer}$ reduced apoptosis and necroptosis rates and inhibited the expression of cleaved caspase 3 and receptor-interacting protein 3 (RIP3). Furthermore, PEDF and 44mer could up-regulate super oxide dismutase (SOD), catalase (CAT) and glutathione peroxidase (GPx) levels, promote clearing of reactive oxygen species (ROS) and malondialdehyde (MDA). While, 34mer, another functional peptides had no effect on cell apoptosis and necroptosis. Hereby this is the first evidence that PEDF and its functional peptide 44mer protect cultured H9c2 cells and primary cardiomyocytes against apoptosis and necroptosis under hypoxic condition via the anti-oxidative mechanism.

\section{Gene expression signature of increased heart rate in shensongyangxin-treated bradycardia rabbits \\ Zhouying Liu, Roumu Hu, Jian Huang, Jielin Pu State Key Laboratory of Cardiovascular Disease, Physiology and Pathophysiology Laboratory, Fuwai Hospital, National Center for Cardiovascular Diseases, Chinese Academy of Medical Sciences and Peking Union Medical College}

Objective Effective drug aimed at heart rate increase for a long time is in demand. The basis for the effectiveness of traditional Chinese medicine ShenSongYangXin (SSYX) treatment on bradycardia is incompletely understood. The present study investigated the genomic profile of SSYX on mRNA expression.

Methods Seventeen adult Oryctolagus cuniculus were used (5 insham, 6 incontrol and SSYX group), with mean body weight $2.5 \pm 0.5$ $\mathrm{kg}$. Sterilized cotton bud with formaldehyde (37\%, SCRC) was fixed near the entrance of the superior vena cava until heart beat decreased 25\%-35\%. Slow heart rate was confirmed after electrocardiogram monitoring for continuous two weeks. Rabbits were supplied orally with either $220 \mathrm{mg} \cdot \mathrm{kg}^{-1} \cdot \mathrm{d}^{-1}$ dry powder (dissolve in purified water) of SSYX for SSYX group or purified water for control group. Electrocardiogram monitoring was recorded for 4 weeks. In sham group, formaldehyde was replaced by purified water; other operations were the same as the other group. Total RNA was isolated using mirVana ${ }^{\mathrm{TM}}$ mRNA isolation kit (Ambion-1561) according to manufacturer's instructions. RNA was quantified by the NanoDrop ND-2000 (Thermo Scientific) and the RNA integrity was assessed using Agilent Bioanalyzer 2100 (Agilent Technologies). To minimize variations attributable to individual rabbit, each experiment was performed with RNA pooled from 3 atria. Total RNA were transcribed to double strand cDNA, then synthesized into cRNA and labeled with Cy3. Labeled cRNAs were hybridized onto Agilent Rabbit Gene Expression Chip (4*44K, Design ID: 020908) according to manufacturer's protocol and scanned by Agilent Scanner G2505C (Agilent Technologies). Data normalization was performed using Genespring. The differentially expressed genes were selected based on fold change greater than 2.0. Gene ontoloogy database and KEGG were applied to determine functions of these differentially expressed
mRNAs. SYBR Green quantitative real-time reverse transcription-PCR (RT-PCR) was performed on the genes BDNF, FN1, TBX20, KCNJ11, ERBB2, GUCY1B3, PRKG1 and 18S rRNA (as an internal control) to confirm the results of chip. Primers were designed with LightCycler Probe Design software 2.0 (Roche Applied Bioscience). The cDNA was synthesized at $37^{\circ} \mathrm{C}$ for $15 \mathrm{~min}$ in a $10 \mu \mathrm{l}$ reaction. All PCR reactions were carried out in triplicate. The expression of each gene was normalized as $\Delta \mathrm{C}_{\mathrm{t}}$ using $18 \mathrm{~S}$ rRNA as the control.

Result There is no difference in basic heart rate of three groups $(\mathrm{P}>0.05)$. Chemical injury of sinoatrial node decreased the mean RR interval by $37 \%$ (from $277 \pm 20$ in sham group to $379 \pm 23$ in control group, $\mathrm{P}<0.05)$ after six weeks. This effect was reversed by four-week SSYX treatment $(220 \mathrm{mg} /(\mathrm{kg} \cdot \mathrm{d})$, from $379 \pm 23$ in control group to 233 \pm 7 in SSYX group, $\mathrm{P}<0.05$ ). SSYX had no significant effect on atrial, atrioventricular, ventricular conduction and ventricular repolarization parameters, since the P, PR, QRS and corrected QT interval were not modified. 1049 down-regulated (S-C-Down) genes and 891 up-regulated (S-C-Up) genes were detected in sham vs control. Similarly, there are 799 down-regulated (C-T-Down) and 890 up-regulated (C-T-Up) genes in control vs SSYX-treated rabbits. Then we picked out the congenerous genes between C-T-Down vs S-C-Up and C-T-Up vs S-C-Down. Finally, 535 and 311 genes were identified, respectively. According to our results, reversed heart rate is partially played by these reversely expressed genes. Significant overexpressed gene items are involved in muscle contraction, cellular calcium ion homeostasis, cardiac muscle fiber development etc. In contrast, genes in mitotic cell cycle, cell division, DNA replication, mitotic prometaphase were down-regulated in SSYX group. RTPCR verified the deregulated genes and demonstrated that the trend of mRNA expression changes validated by RT-PCR was in agreement with expression chip.

Conclusion The present study shows that SSYX is effective in treating bradycardia. Long term SSYX treatment reversed the expression of genes related to muscle contraction, calcium ion homeostasis, cell proliferation etc. in pathological condition. Various ion channel and genes in diverse pathways were directly or indirectly affected by SSYX treatment. In conclusion, these datas provide future avenues for investigation of the underlying mechanisms of long term SSYX treatment.

\section{Up-regulated lipocalin-2 in pulmonary hypertension involving in pulmonary artery SMC resistance to apoptosis}

Guoliang Wang, Shenghua Liu, Wang Li, Liukun Meng, Hongliang Zhang, Yingjie Wei

State Key Laboratory of Cardiovascular Disease, Fuwai Hospital, National Centerfor Cardiovascular Diseases, Chinese Academy of Medical Sciences and Peking Union Medical College

Background and Objective A key feature of pulmonary arterial hypertension (PAH) is the remodeling of small pulmonary arteries, which is due to abnormal pulmonary artery smooth muscle cell (PASMC) proliferation and resistance to apoptosis. While the cellular mechanisms of how the normal PASMC switched to resistant to apoptosis remains unknown. Lipocalin 2 (Lcn2), also known as neutrophil gelatinaseassociated lipocalin (NGAL), is reported recently play roles in cell survival in a wide array of benign and malignant conditions. In this study, we examined the levels of Lcn2 in a rat PH model induced with monocrotaline and in congenital heart disease associated $\mathrm{PH}$. We also investigated whether Lcn2 regulate human PASMC apoptosis and the 
mechanism involved.

Methods Rat model of PAH was induced by monocrotaline (60 $\mathrm{mg} / \mathrm{kg}$, subcutaneous injection). Plasma Lcn2 level was measured by ELISA. Cell apoptosis was induced by $\mathrm{H}_{2} \mathrm{O}_{2}$ and measured by TUNEL assay and flow cytometry, and cleaved caspase- 3 and caspase- 3 activity. Real-time RT-PCR and Western-Blotting was used to measure protein expression levels. Cellular ROS was measured use Reactive Oxygen Species Assay Kit through flow cytometry.

Result Both western blot and RT-PCR revealed that the expression level of Lcn2 was increased significantly after MCT injection. Immunostaining for Lcn2 also showed an up-regulation in lungs of rat after 3 weeks of MCT injection. The mean plasma Lcn2 level of congenital heart disease patients associated $\mathrm{PH}$ was $(46.5 \pm 7.18) \mathrm{ng} /$ $\mathrm{ml}$, significantly higher than patients but no PH $(32.6 \pm 6.37) \mathrm{ng} / \mathrm{ml}$. Len2 significantly inhibited HPASMC apoptosis induced by $\mathrm{H}_{2} \mathrm{O}_{2}$. This was shown by detection of DNA fragmentation, membrane phosphatidylserine exposure, cleaved caspase-3 and caspase-3 activity. We also observed that $\mathrm{Lcn} 2$ caused down-regulation of the proapoptotic protein Bax. Furthermore, Lcn2 lowers the cellular ROS and upregulates the expression of superoxide dismutases (SOD1 and SOD2) compared with control.

Conlusion Len2 significantly inhibited HPASMC apoptosis via a mechanism involving decreased intracellular ROS and elevated SODs. Up-regulation of Lcn2 in a rat PH model and CHD-PH patients may be involved in the pathological process of $\mathrm{PH}$.

\section{The expression of IL33/ST2 in congenital pulmonary hypertension \\ Guoliang Wang, Shenghua Liu, Li Wang, Liukun Meng, Hongliang Zhang, Yingjie Wei \\ State Key Laboratory of Cardiovascular Disease, Fuwai Hospital, National Center for Cardiovascular Diseases, Chinese Academy of Medical Sciences and Peking Union Medical College}

Objective Inflammatory processes are prominent in various types of human and experimental pulmonary hypertension $(\mathrm{PH})$ and are increasingly recognized as major pathogenic components of pulmonary vascular remodeling. Macrophages, $\mathrm{T}$ and B lymphocytes, and dendritic cells (DC) are present in the vascular lesions of PH. IL33 is an important chemoattractant for macrophages and DC. ST2L is the membrane receptor of IL33 and SST2 is the soluble receptor of IL33. We investigated the serum level of IL33/ST2 in patients of congenital pulmonary hypertension and lung tissue of PAH rats.

Methods Rat model of PAH was induced by monocrotaline (60 $\mathrm{mg} / \mathrm{kg}$, subcutaneous injection). The expression level of IL33 and its receptors (ST2L and sST2) were measured by real-time RT-PCR. Serum SST2 level was measured by protein affinity microarray. Circulating SST2 levels of patients with congenital heart disease associated with (or without) $\mathrm{PH}$ was compared.

Result IL33 expression level in lung tissue of rat was decreased rapidly after monocrotaline injection and maintained with low level. The lowest level was reached at week 2 , about $40 \%$ of the control. However, the expression level of sST2, soluble receptor of IL33, increased significantly after monocrotaline injection, with a top level of 16 times of control at week 2. The expression level of ST2L, membrane receptor of IL33, decreased slightly after monocrotaline injection and has no significant compared with the control. The mean serum sST2 level of congenital heart disease patients with severe PH was $293 \pm 26 \mathrm{pg} / \mathrm{ml}$, significantly higher than the patients without PAH (about 4 times).
Conclusion Our preliminary results showed serum IL33 level was decreased significantly and level of its soluble receptor, sST2, was increased significantly in rat PAH model and congenital pulmonary hypertension patients. This study emerged the probability of serum IL33/ ST2 being new biomarkers of PAH.

\section{The novel DSG2 mutations and the functional changes of desmoglein2 caused by DSG2 mutations in ARVC patients \\ Li Wang, Guoliang Wang, Shenghua Liu, Hongliang Zhang, Yingjie Wei \\ State Key Laboratory of Cardiovascular Disease, Fuwai Hospital, \\ National Center for Cardiovascular Diseases, Chinese Academy of \\ Medical Sciences and Peking Union Medical College}

Objective Arrhythmogenic right ventricular dysplasia/ cardiomyopathy $(\mathrm{ARVD} / \mathrm{C})$ is an inherited myocardial disease associated with ventricular arrhythmias that may lead to sudden death, especially in young people and athletes. We explored the effects of mutations of DSG2 in pathogenesis of ARVC according to protein location and expression in myocardial tissue for the sake of clarifying the molecular genetics of ARVC.

Methods DNA was extracted from myocardial tissue of ARVC patients who underwent heart transplantation and sequenced by highthroughput sequencing, then verified by Sanger sequencing. Diseased tissue samples were studied for desmosomal protein DSG2 distribution and expression using immunohistochemistry and western blot.

Result We identified 2 mutations in DSG2 in 3 of 26 probands (11\%), one mutation is F531C, another is L797E. The results of immunohistochemistry showed these two mutations did not affect the location of DSG2 in intercalated disc; and the results of western blot exhibited protein level were significant decreased in myocardial tissue carrying F531C mutation, in contrast, the protein level of DSG2 in L797E carrier was similar compared to normal control.

Conclusion DSG2 F531C and L797E were both disease-causing mutations in ARVC, F531C may affect protein expression level in myocardial tissue and could not influence the location of DSG2 in intercalated disc, however, L797E had no effects on protein distribution and expression in myocardial tissue, concluding the protein DSG2 with L797E incorporated into desmosome. Our results strengthen the view that DSG2 is an important pathogenic gene in ARVC and ARVC is a disease of desmosome.

\section{The intervention target of atherosclerotic vulnerable plaque based on the literature \\ Yanyang $\mathrm{Li}^{1}$, Aiqin Zhong ${ }^{1}$, Yaping Zhu ${ }^{2}$, Junping Zhang ${ }^{2}$ \\ 1. Tianjin University of Chinese Medicine \\ 2. The First Hospital Affliated to Tianjin University of Chinese Medicine}

Objective To Comb atherosclerosis research status of vulnerable plaque and to investigate how to stable the targets of atherosclerotic vulnerable plaque.

Methods To establish a stable systematic analysis of vulnerable plaque literature repository, the literature review are used to this study which is based on atherosclerotic vulnerable plaque literature.

Result Through carding is known that biological characteristics of unstable plaque are: infiltration of inflammatory cytokines; larger lipid core formation and degradation of the fibrous cap and so on. Looking 
from a different angle and effective treatment for plaque stabilization can eliminate the factors affecting the stability of the plaque, thereby reducing the incidence of the risk event. By reviewing the literature, the stability of vulnerable plaques targets include: anti-inflammatory treatment, such as intervention MCP-1, PPAR $\gamma$ and inflammatory factors such as TLR; lowering therapy, the medicine which has clinical evidences such as statins; led down the extracellular matrix thin fibrous cap of the vulnerable plaque degradation of extracellular matrix synthesis or regulated, such as the use of MMP inhibitors; change plaque localized shear; inhibiting angiogenesis within the plaque.

Conclusion The stability of atherosclerotic plaques is more important than arterial stenosis. After unstable arterial plaque ruptured, the loss often led to acute coronary syndrome. With the in-depth study of the mechanism of occurrence of vulnerable plaque, more and more methods of stabilizing vulnerable plaques should be used in different ways. However, based on the concept of translational medicine, practical applications in clinical methods and drugs need to promote basic research results in current which can be translated into further quickly and effectively to reduce the incidence of cardiovascular events.

\section{Increased frequency of follicular helper T Cells in mice viral myocarditis is relevant with anti- ANT antoantibody}

Fan Yang, Wenhong Mo, Baoping Tan, Xiaomou Wei, Hong Wang

Department of Cardiology, the Fourth Affiliated Hospital of Guangxi Medical University

Background Recently, a new subset of $\mathrm{CD} 4^{+} \mathrm{T}$ helper cell termed Follicular helper T cells (Tfh), which play a pivotal role in B cell activation and differentiation in lymphoid structures, has been reported to participate in some certain autoimmune diseases. But whether Tfh cells are involved in the pathogenesis of VMC remains unclear.

Methods Male BALB/c mice were intraperitoneally (i.p) infected with CVB3 for establishing VMC models. Control mice were treated with phosphate-buffered saline i.p. On $0,1,2,3,4,6$ weeks post injection, frequencies of splenic Tfh cells were determined by flow cytometric analysis, productions of IL-21 and anti-adenine nucleotide translocator (ANT) autoantibody were detected by enzyme-linked immunosorbent assay. To further investigate the effects of IL-21, VMC mice were treated with anti-IL-21 neutralizing antibody. Heart pathology was examined histologically, and the frequencies of Tfh cells and the expressions of anti-ANT autoantibody were investigated after anti-IL-21 intervention. Spearman analysis was used to evaluate the relationship between the frequencies of Tfh cells and IL-21 levels with anti-ANT autoantibody.

Result The percentage of Tfh cells significantly increased in VMC mice from $1 \mathrm{~W}$ to $6 \mathrm{~W}$, the serum levels of IL-21 and ANT autoantibody were also significantly increased in VMC mice. Neutralization of IL21 with anti-IL-21 can ameliorate the myocardium inflammation and decrease Tfh cells and ANT autoantibody after IL-21 antibody intervention compared with those of the control ones $(\mathrm{P}<0.05)$. Both of the frequencies of Tfh cells and IL-21 levels were positively correlated with anti-ANT antibody levels $(\mathrm{R}=0.758, \mathrm{P}<0.05$ and $\mathrm{R}=0.88, \mathrm{P}<0.01$, respectively).

Conclusion Those results suggest that Tfh cells and IL-21 might involve in the pathogenesis of VMC and play an important role in anti-
ANT autoantibody production. Targeting the Tfh cell and IL-21 may be a new therapeutic target for the treatment of CVB3-induced VMC.

\section{Phosphoinositide 3-kinase $y$ pathway mediates the pro-inflammatory effects of high-density lipoprotein in patients with coronary heart disease \\ Hui Zhao, Hong Liu, Lin Chai, Lirong Yan, Yuexin Jiang, Bing Duan, Xiaoyuan Guan, Lu Hua, Lei Tian, Youhong Jia, Yishi Li \\ Key Laboratory of Clinical Trial Research in Cardiovascular Drugs, \\ Ministry of Health, Cardiovascular Institute and Fu Wai Hospital, CAMS and PUMC Beijing, China}

Objective Endothelial injury and subsequent inflammation are considered as a key role in coronary artery disease (CAD). High-density lipoprotein from healthy subjects $\left(\mathrm{HDL}_{\text {healthy }}\right)$ are proposed to exert endothelial protection through anti-inflammation which may represent its important anti-atherosclerotic property. Recent studies demonstrated that the function of HDL may transform to pro-atherogenic when CAD occured. So we aimed to investigate the effects of HDL from CAD patients $\left(\mathrm{HDL}_{\mathrm{CAD}}\right)$ on the stimulation of pro-inflammatory cytokines and pathways related.

Methods 15 CAD patients diagnosed by coronary angiography and 10 matched healthy volunteers were enrolled. HDL was isolated from fresh fasting venous plasma by density gradient ultracentrifugation. The purity of HDL sample was tested by SDS-PAGE, and the protein content was measured by BCA method. Cultured human umbilical vein endothelial cells (HUVECs) were treated with equal amount of $\mathrm{HDL}_{\mathrm{CAD}}$ and $\mathrm{HDL}_{\text {healthy, }}$ respectively. The concentraion of IL-6 in the supernatant was measured by ELISA method after 24 hours' incubation. To investigate the possible pathway involved, specific PI3K $\gamma$ inhibitor AS605240 was used to pretreat the HUVECs, and the IL- 6 concentration was examined in the same way. Differences between groups were compared with SPSS17.0.

Result Compared with $\mathrm{HDL}_{\text {healthy, }} \mathrm{HDL}_{\mathrm{CAD}}$ promoted more IL-6 secretion of HUVECs[(4.68 \pm 0.26$) \mathrm{ng} / \mathrm{ml}$ vs $(0.18 \pm 0.02) \mathrm{ng} / \mathrm{ml}, \mathrm{P}<$ $0.05]$. AS- 605240 significantly decreased the production of IL-6 induced by $\mathrm{HDL}_{\mathrm{CAD}}[(0.13 \pm 0.03) \mathrm{ng} / \mathrm{ml}$ vs $\left.(4.68 \pm 0.26) \mathrm{ng} / \mathrm{ml}, \mathrm{P}<0.05)\right]$.

Conclusion Present study confirmed that the beneficial function of $\mathrm{HDL}_{\mathrm{CAD}}$ shifted towards pro-inflammatory which was associated with PI $3 \mathrm{~K} \gamma$ pathway. This would provide novel insights into the mechanism study and clinical application in atherosclerosis.

\section{SIRT1 inactivation by carbonyl stress impairs the ischemic tolerance and responsiveness to ischemic preconditioning in aged hearts \\ Yishi Wang ${ }^{1}$, Zhifa Wang ${ }^{1}$, Yue Yin ${ }^{1}$, Chunhu $\mathrm{Gu}^{2}$, Heng $\mathrm{Ma}$ \\ 1. Department of Physiology, Fourth Military Medical University, Xi' an, China \\ 2. Department of Cardiovascular Surgery, Xijing Hospital, Fourth Military Medical University, Xi' an, China}

Sirtuin 1 (SIRT1) is essential for conferring cardioprotection in response to ischemic preconditioning (IPC) stimulus. However, the cardioprotective effect of IPC is impaired in aged hearts. Our previously study have demonstrated that impaired cardiac SIRT1 activity by 
carbonyl stress contributed to aging-related ischemic intolerance. We also have demonstrated that aldehyde dehydrogenase 2 (ALDH2) was a cardioprotective factor to ameliorate the carbonyl stress induced by cardiac toxicity of carbonyl stress and reduce ischemic damage. We hypothesized that SIRT1 inactivation by carbonyl stress be responsible for the loss of IPC cardioprotection in aged hearts. Thus, young (4-6 mo) and aged (22-24 mo) mice were subjected to in vivo regional myocardial ischemia for $30 \mathrm{~min}$ followed by reperfusion for $4 \mathrm{hrs}$ with or without a prior IPC stimulus. Our results show that aged heart exhibited increased endogenous carbonyl stress due to impaired ALDH2 activity concomitant with blunted SIRT1 activity $(\mathrm{P}<0.05)$, which leading to worse $\mathrm{I} / \mathrm{R}$ injury reflecting the increased infarct size and $\mathrm{CK}$ activity levels. IPC significantly increased SIRT1 activity and reduced the $\mathrm{I} / \mathrm{R}$ injury in the young hearts. In contrast, IPC failed to activate SIRT1 and resistant to protection with IPC stimulus in aged hearts. ALDH2 activation by Alda-1 led to a significant reduction of SIRT1 carbonylation in aged hearts and then rescued the protective effect of IPC in aged hearts $(\mathrm{P}<0.05)$. However, SIRT1 inhibitor blocked the protective effect of Alda-1 on IPC treated aged hearts. These results provide a new insight that impaired cardiac SIRT1 activity by carbonyl stress contributes to the age-induced loss of protection by IPC. Activation of ALDH2 can prevent SIRT1 activation and restore the IPC cardioprotection in aged heart.

\section{SIRT3 mediated cardioprotection prevents ischemia reperfusion arrhythmias \\ Tiannan Jiang ${ }^{1}$, Chen $\mathrm{Li}^{2}, \mathrm{Lu} \mathrm{Yu}{ }^{3}$, Yue Yin ${ }^{2}$, Qiangsun Zheng ${ }^{1}$, Heng Ma ${ }^{2}$ \\ 1. Department of Cardiology, Tangdu Hospital, Fourth Military Medical \\ University, Xi' an, China \\ 2. Department of Physiology, Fourth Military Medical University, Xi' an, China \\ 3. Department of Pathology, Xijing Hospital, Fourth Military Medical University, Xi' an, China}

Ischemia reperfusion induces a high percentage of lethal arrhythmias. Sirtuin 3 (SIRT3), a key nutrient sensing regulator by governing mitochondrial homeostasis, was also reported to protect heart from reactive oxygen species (ROS) assaults, but whether it is involved in ischemia reperfusion arrhythmias (IRA) and the mechanism underlying remains unknown. The present study was designed to investigate the role of cardiac mitochondrial SIRT3 in ischemia reperfusion arrhythmias. Thus, Sirt3 knockout (SIRT3 KO) mice and littermate wild-type (WT) mice were assigned into sham group, ischemia reperfusion (I/R) group and $\mathrm{I} / \mathrm{R}$ with $\mathrm{NAD}$ treated group (7 days, $1 \mathrm{mg}$ / $\mathrm{kg} /$ day) (NAD $+\mathrm{I} / \mathrm{R})$. ECG was examined during $\mathrm{I} / \mathrm{R}$. At the end of reperfusion period, the arrhythmia score, reactive oxygen species (ROS) production, cardiac SIRT3 and MnSOD levels were measured and analyzed. The results demonstrated that the cardiac SIRT3, MnSOD and Catalase expression were decreased in SIRT3 KO mice compared with the WT mice. Arrhythmia was detected in SIRT3 KO mice under sham. $\mathrm{I} / \mathrm{R}$ triggered serious arrhythmia in WT mice and aggravated arrhythmia in SIRT3 KO mice $(\mathrm{P}<0.05)$. SIRT3 KO mice showed increased ROS production after I/R compared with $\mathrm{WTI} / \mathrm{R}$ mice $(\mathrm{P}<0.05)$. NAD treatment significantly increased cardiac SIRT3 and MnSOD activity, inhibited ROS production, and consequently suppressed I/R-induced arrhythmia in WT mice. However, NAD induced cardioprotctive effects were blunted in SIRT3 KO mice. These findings indicated that impairment of SIRT3 expression with subsequent ROS production constitutes an important mechanism by which played an important role during the I/R induce arrhythmia. Therefore, protection of SIRT3 activity could be potential approaches to prevent I/R-induced arrhythmia.

\section{eNOS modified endothelial progenitor cells inhibit efficiently neointima formation and enhancement of vascular function \\ Bin Cui \\ Cardiovascular Department of Xinqiao Hospital, Third Military Medical University}

Background Loss of endothelial NO production after arterial injury may contribute to restenosis, characterized by neointima formation and elastic recoil. Previous studies have established that bone marrow-derived endothelial progenitor cells (EPCs) played an important role in vascular repair. In this study, we investigated that hypothesis that overexpression of vasculoprotective gene endothelial nitric oxide synthase (eNOS) in EPCs may restore NO production and inhibit neointimal hyperplasia.

Methods EPCs obtained from rat bone marrow were isolated using a Ficoll density gradient centrifugation, and expanded in endothelial basal medium. The endothelial characteristics of EPCs were identified by immunologic cell chemical staining a fluorescent labeling. EPCs were transduced with pseudotyped retroviral vectors expressing human eNOS (eNOS-EPCs) or green fluorescent protein (GFP-EPCs). eNOS or GFP modified EPCs were injected directly by intravenous tail vein after arterial injury and again 24 hours later. Two weeks after transplantation, eNOS proteins in the rat vessels were assayed by western blot. The morphology of arterial intima and media was studied by optical microscopy and image analysis system.

Result The adherent cells were considered EPCs which had spindle shape and form blood-island like structures during development. The adherent cells had many endothelial characteristics. Transduction efficiency of EPCs ex vivo was above $90 \%$. eNOS gene transfer augmented EPCs proliferative activity. eNOS proteins were detected in the rat vessels Transfused EPCs may home to the injury site and enhanced reendothelialization associated with decreased neointima formation. The antiproliferative effect of EPCs is further enhanced by overexpression of eNOS. Furthermore, eNOS overexpressed EPCs could increase significantly endothelium-dependent vasodilation function (EDVR).

Conclusion In vitro, eNOS gene transfer enhanced EPCs proliferative activity. In vivo, eNOS overexpressed EPCs could accelerated reendothelialization and inhibit neointimal hyperplasia. The results show that gene modified EPCs facilitate the strategy of cell transplantation for vascular dysfunction and prevention of restenosis after angioplasty.

\section{An autopsied case of a neonate with the origin anomaly of total coronary arteries from the pulmonary artery \\ Zhenglian Chen, Chunyu Shen, Xinshan Chen Department of Forensic Pathology, Tongji Medcial College, Huazhong University of Science and Technology}

Coronary Artery anomaly is not a common disease. A neonate with the origin of total coronary arteries from the pulmonary artery (TCAPA) is extremely rare and fetal, which has not been yet reported in China. Here an autopsied case of TCAPA is reported to provide reference for the 
medical colleagues. A female infant died 9 hours after born to 29-yearold healthy mother at 39 weeks of gestation. The fetal heart rate was 144 bmp before cesarean delivery. After born the infant's Apger scores was $3,0,3$ at 1, 5, 10 min respectively, along with pallor, marked cyanosis of fingernails and toenails, decreased heart rate $(60 / \mathrm{min})$ and bradypnea. Heart rate and breath became normal after oxygen and other therapies. Then she was transferred to another hospital for definite diagnose and further therapies. Symptoms persisted after continued therapy, and the laboratory examination manifested acidosis. Forensic autopsy was performed after 2 days. She was 19.69 inches tall and weighted 7.72 pounds. Both left and right coronary arteries originated from pulmonary artery, and no other malformations and lethal changes were found. The cause of death was identified as TCAPA. As there are nonspecific clinical presentations of TCAPA, it is easy to ignore by some doctors. Clinically coronary angiography is the golden standard of diagnosis of the disease. Once TCAPA is diagnosed, surgery should be done immediately in order to be available for supplying enough blood for heart.

\section{c-Met overexpression promotes reendothelialization and inhibits neointimal formation after balloon injury \\ Mingbao Song \\ Cardiovascular Department of Xinqiao Hospital, Third Military Medical University}

Objective To explore the effect of c-met overexpression in EPCs on reendothelialization after balloon injury.

Methods EPCs derived from mouse bone marrow were isolated and cultured. 3- (4, 5-dimethylthiazol-2-yl) -2, 5-diphenyltetrazolium bromide assays were used to evaluate EPC proliferation. Adenoviral vector expressing c-Met was generated using the AdEasy system. To evaluate the role of HGF/Met in vascular repair in vivo, we used ballooninjured rat carotid artery model. Evans Blue dye was administered to evaluate reendothelialization after 10 days injury, and the neointimal formation was assessed at 21 days following vascular injury.

Result The effect of HGF on EPC proliferation was examined $48 \mathrm{~h}$ after exposure to different quantities of HGF (range 2-20 ng/ml). The proliferation effect was strongly dose-dependent and significantly increased in c-met-EPCs group compared with EPCs group. After transfusion of c-met-EPCs or EPCs to balloon-injured rat via vessel, Evans Blue dye was administered to evaluate reendothelialization after balloon injury. Reendothelialized area was significantly larger in c-metEPCs group than in EPCs group $(64.25 \% \pm 8.90 \%$ vs $43.21 \% \pm 7.24 \%$, $\mathrm{n}=5, \mathrm{P}<0.01)$. A marked decrease in the neointimal area and I/M ratio were found in c-met-EPCs group compared with EPCs group at day 21 $(0.29 \pm 0.06$ vs $0.63 \pm 0.13, \mathrm{n}=5, \mathrm{P}<0.01)$

Conclusion c-Met overexpression improves EPCs proliferation, promotes reendothelialization and inhibits neointimal formation after balloon injury.

\section{Beneficial effects of a polysaccharide from Salvia miltiorrhiza on myocardial ischemia- reperfusion injury in rats. \\ Mingbao Song \\ Cardiovascular Department of Xinqiao Hospital, Third Military Medical University}

Objective To explore the effects of a polysaccharide from Salvia miltiorrhiza on myocardial ischemia-reperfusion injury in rats.

Methods In the present study, one water-soluble polysaccharide (SMP1) was isolated from the roots of Salvia miltiorrhiza. The cardioprotective potential of SMP1 was studied in the ischemia-reperfusion (I/R) model of rats in vivo.

Result Results showed that $30 \mathrm{~min}$ of left anterior descending coronary artery occlusion (LAD) followed by $4 \mathrm{~h}$ of reperfusion markedly decreased myocardial superoxide dismutase (SOD), $\mathrm{Na}^{+}-$ $\mathrm{K}^{+}$-ATPase and $\mathrm{Ca}^{2+}-\mathrm{Mg}^{2+}$-ATPase activities and increased myocardial malondialdehyde (MDA) level and serum activities of creatine kinase (CK) and lactate dehydrogenase (LDH) in I/R rats. An increase in infarct size and high apoptosis index of cardiac cell were also observed in IR rats. Administration of SMP1 400 and $800 \mathrm{mg} / \mathrm{kg}$ significantly reversed these biochemical parameters in the $\mathrm{I} / \mathrm{R}$ rats to the normal levels in sham control rats. The infarct sizes and the percent of TUNEL-positive cells were found significantly decreased in SMP1-treated groups compared to the I/R rats.

Conclusion Taken together, the present study clearly suggests SMP1 has a protective effect against myocardial I/R injury in rats by ameliorating oxidative stress and inhibiting myocardial apoptosis.

\section{Atorvastain attenuates TNF- $\alpha$-induced Increase of glucose oxidation through PGC- $1 \propto$ up-regulation in cardiomyocytes \\ Jiang Wang \\ Cardiovascular Department of Xinqiao Hospital, Third Military Medical University}

Backgroud Recent studies have shown that atorvastain has anti-inflammatory effect and could prevent cardiac hypertrophy. The development of cardiac hypertrophy and dysfunction is associated with an increase in cardiac glucose utilization.

Methods In this study, we investigated the effect of atorvastatin on glucose oxidation in tumour necrosis factor- $\alpha$ (TNF- $\alpha)$-stimulated cardiomyocytes (H9c2 cells) and the potential role of peroxisome proliferation-activated receptor co-activator $-1 \alpha(\mathrm{PGC}-1 \alpha)$ in this effect.

Result Exposure of $\mathrm{H} 9 \mathrm{c} 2$ cells to TNF- $\alpha$ inhibited the expressions of PGC-1 $\alpha$, pyruvate dehydrogenase kinase 4 (PDK4), carnitine palmityl transferase 1 (CPT1) and induced a significant increase in glucose oxidation rate. However, these effects of TNF- $\alpha$ were significantly reversed by atorvastatin. Selective silence of PGC-1 $\alpha$ in H9c2 cells resulted in the down-regulation of PDK4 and CPT1 and further increased the TNF- $\alpha$-induced glucose oxidation. Interestingly, the effect of atorvastatin on PGC-1 $\alpha$ was almost abolished by mevalonate and partially by farnesol, but not by geranylgeraniol.

Conclusion In conclusion, atorvastatin inhibits TNF- $\alpha$-induced glucose oxidation through PGC-1 $\alpha$ up-regulation in cardiomyocytes, which might be associated with the regulation of isoprenoid metabolites.

\section{Baseline serum uric acid level as a predictor of cardiovascular disease related mortality and all-cause mortality: A meta-analysis of prospective studies. \\ Gang Zhao \\ Cardiovascular Department of Xinqiao Hospital, Third Military Medica University}

Objective Serum uric acid (SUA) levels have been used to predict 
cardiovascular and all-cause mortality event, but the data have yielded conflicting results. We investigated whether SUA was an independent predictor for cardiovascular or all-cause mortality with prospective studies by meta-analysis.

Methods Pubmed and Embase were searched without language restrictions for publications available till April 2013. Only prospective studies on cardiovascular or all-cause mortality related to SUA levels were included. Pooled adjust relative risk (RR) and corresponding 95\% confidence intervals (CI) were calculated separately for the highest vs lowest category or the lowest vs middle category,

Result For the highest SUA, eleven studies with 172123 participants were identified and analyzed. Elevated SUA increased risk of all-cause mortality $(\mathrm{RR}=1.24 ; 95 \% \mathrm{CI}$ : $1.09-1.42)$ and cardiovascular mortality ( $R R=1.37$; $95 \%$ CI: $1.19-1.57)$. Subgroup analyses showed that elevated SUA significantly increased the risk of all-cause mortality among men (RR $=1.23$; 95\% CI: $1.08-1.42)$, but not in women $(\mathrm{RR}=$ $1.05 ; 95 \%$ CI: $0.79-1.39)$. Risk of cardiovascular mortality appeared to be more pronounced among women $(\mathrm{RR}=1.35$; $95 \% \mathrm{CI}$ : $1.06-1.72)$. The association between extremely low SUA and mortality was reported in three studies; we did not perform a pooled analysis because of high degree of heterogeneity in these studies.

Conclusion Baseline SUA level is an independent predictor for future cardiovascular mortality. Elevated SUA appears to significantly increase the risk of all-cause mortality in men, but not in women. Whether low SUA levels are predictors of mortality is still inconclusive.

\section{The interaction of transient receptor potential melastatin 7 with macrophages promotes vascular adventitial remodeling in transverse aortic constriction rats \\ Shujie Guo \\ Shanghai institute of hypertension, Ruijin Hospital, Shanghai Jiao Tong University School of Medicine, Shanghai, China}

Transient receptor potential melastatin 7 (TRPM7), a novel channel kinase, has been recently identified in the vasculature. However, its regulation and function in vascular diseases remain poorly understood. To address this lack of knowledge, we sought to examine whether TRPM7 can mediate the vascular remodeling process induced by pressure overload in the rightcommon carotid artery proximal to the band (RCCA-B) in male Sprague-Dawley rats with transverse aortic constriction (TAC). The contribution of TRPM7 to amplified vascular remodeling after TAC was tested using morphometric and western blot analyses. Pressure overload-induced vascular wall thickening, especially in the adventitia, was readily detected in RCCA-B. The TRPM7 level was increased with a simultaneous accumulation of macrophages in the adventitia of RCCA-B, whereas theanti-inflammatory molecule annexin-1, a TRPM7 downstream target, was decreased. After the addition of the TRPM7 inhibitor2-aminoethoxydiphenyl borate (2APB), significant reductions in macrophage accumulation as well as the expression of monocyte chemotactic protein-1, SM-22-a and collagen I were observed, whereas annexin-1 was rescued. Finally, in culturedvascular adventitial fibroblasts treated with macrophageconditioned medium, there were marked increases in the expression of TRPM7 and SM-22-a with a concurrent reduction in annexin-1 expression; these effects were largely prevented by treatment with 2-APB and specific anti-TRPM7 small interfering RNA. Our findings provide the first demonstration of the potential regulatory roles of TRPM7 in the vascular inflammation, pressure overload-mediated vascular adventitial collagen accumulationand cell phenotypic transformation in TAC rats. The targeting of TRPM7 has potential therapeutic importance for vascular diseases.

\section{The protective effects of a non-depolarizing solution on the endothelial function in vitro preservation \\ Mengmeng Chen, Feilong Hei \\ Department of Extracorporeal Circulation, State Key Laboratory of Cardiovascular Disease, Fuwai Hospital, National Center for Cardiovascular Diseases, Chinese Academy of Medical Sciences and Peking Union Medical College, China}

Objective The study was designed to investigate the protective effects of the non-depolarizing solution (NDP) on EDHF-mediated relaxation of porcine coronary arteries in comparision with KrebsHelenseit (KH) solution, St.Thomas (ST) solution, Histidine-TryptophanKetoglutarate (HTK) solution after vascular rings were incubated with these solutions at $37^{\circ} \mathrm{C}$ for 1 hour or at $4^{\circ} \mathrm{C}$ for 10 hour.

Methods Due to different preservation solutions used in different groups, the rings were divided into four groups: control (CON) group, St.Thomas (ST) group, Histidine-Tryptophan-Ketoglutarate (HTK) group and NDP group. Rings from porcine coronary arteries were studied in the organ chamber. After equilibration, the rings were incubated with Krebs-Helenseit solution in CON group, St.Thomas solution in ST group, Histidine-tryptophan-ketoglutarate solution in HTK group, non-depolarizing solution in NDP group at $37^{\circ} \mathrm{C}$ for 1 hour or at $4^{\circ} \mathrm{C}$ for 10 hour. Then Precontraction tone induced by prostaglandin F2 $\alpha$ U46619 and endotheliumdependent relaxation tone induced by bradykinin was measured in the presence of indomethacin (Indo) and N-nitro-L-arginine (L-NNA).

Result After incubation with different solutions at $37^{\circ} \mathrm{C}$ for 1 hour, the EDHF-mediated relaxation induced by bradykinin was much higher in the NDP group $(74.79 \% \pm 2.26 \%)$ than in the HTK group $(51.32 \% \pm$ $7.75 \%), \mathrm{CON}$ group $(24.03 \% \pm 2.83 \%)$ and ST group $(16.20 \% \pm 2.26 \%)$; after incubation with different solutions at $4^{\circ} \mathrm{C}$ for 10 hour, the EDHFmediated relaxation induced by bradykinin was much higher in the NDP group $(70.35 \% \pm 5.58 \%)$ than in the HTK group $(61.53 \% \pm 5.04 \%)$, CON group $(28.11 \% \pm 3.77 \%)$ and ST group $(20.16 \% \pm 3.02 \%)$, but statistical analysis showed no significant difference between NDP group and HTK group.

Conclusion NDP solution provides superior endothelium protective effect in comparision with ST and HTK solution at $37^{\circ} \mathrm{C}$ for 1 hour.NDP solution provides superior endothelium protective effect in comparision with ST and HTK solution at $4^{\circ} \mathrm{C}$ for 10 hour, but statistical analysis showed no significant difference between NDP group and HTK group.

\section{The role of potassium channels in protective effects of NDP solution on EDHF-mediated relaxation \\ Mengmeng Chen, Feilong Hei Department of Extracorporeal Circulation, State Key Laboratory of Cardiovascular Disease, Fuwai Hospital, National Center for Cardiovascular Diseases, Chinese Academy of Medical Sciences and Peking Union Medical College, China}

Objective Our previous study has found that NDP solution provides superior protective effect on EDHF-mediated relaxation. Based on that, this study was aimed to explore the mechanism of potassium channels 
in protective effects of NDP solution on EDHF-mediated vasodilation. The potassium channels involved in the study mainly includes calciumactivated potassium $\left(\mathrm{K}_{\mathrm{Ca}}\right)$ channels, inward rectifier potassium $\left(\mathrm{K}_{\mathrm{ir}}\right)$ channels, ATP-sensitive potassium channels $\left(\mathrm{K}_{\mathrm{ATP}}\right)$, voltage-dependent potassium $(\mathrm{Kv})$ channels.

Methods Rings from porcine coronary arteries were studied in the organ chamber, after equilibration for 1 hour, the control group rings were incubated with Indo and L-NNA, the other groups were incubated with Indo, L-NNA and different potassium channel blockers. Since $\mathrm{K}_{\mathrm{Ca}}$ can be divided into three types: Large-Conductance $\mathrm{K}_{\mathrm{Ca}}\left(\mathrm{BK}_{\mathrm{Ca}}\right)$, Intermediate-Conductance $\mathrm{K}_{\mathrm{Ca}}\left(\mathrm{IK}_{\mathrm{Ca}}\right)$ and Small-Conductance $\mathrm{K}_{\mathrm{Ca}}\left(\mathrm{SK}_{\mathrm{Ca}}\right)$, these blockers are iberiotoxin (a specific blocker of $\mathrm{BK}_{\mathrm{Ca}}$ channels), charybdotoxin (a blocker of $\mathrm{BK}_{\mathrm{Ca}}$ channels and $\mathrm{IK}_{\mathrm{Ca}}$ channels), apamin (a specific blocker of $\mathrm{SK}_{\mathrm{Ca}}$ channels), barium chloride $\left(\mathrm{BaCl}_{2}\right.$, a blocker of $\mathrm{K}_{\mathrm{ir}}$ channels), Glibenclamide (a blocker of $\mathrm{K}_{\mathrm{ATP}}$ channels), 4-Aminopyridine (4-AP, a specific blocker Kv channels). Then Precontraction tone induced by prostaglandin F2 $\alpha$ U46619 and endotheliumdependent relaxation tone induced by bradykinin were measured in the presence of Indo and L-NNA with/without different calcium-activated potassium channel blockers.

Result Compared with the control group $(65.71 \% \pm 5.60 \%)$ EDHF-mediated endothelium-dependent relaxation induced by bradykinin was significantly decreased in Charybdotoxin group (23.57\% $\pm 3.46 \%$ ), Charybdotoxin+Apamin group $(8.01 \% \pm 2.32 \%)$, while the relaxation of Apamin group $(59.52 \% \pm 4.85 \%)$ was not statistically significant in comparision with the control group $(65.71 \% \pm 5.60 \%)$; Compared with the control group $(71.88 \% \pm 6.23 \%)$, EDHF-mediated endothelium-dependent relaxation induced by bradykinin was significantly decreased in the Iberiotoxin group $(25.67 \% \pm 1.86 \%)$; Compared with the control group $(63.43 \% \pm 7.71 \%)$, EDHFmediated endothelium-dependent relaxation induced by bradykinin was significantly decreased in the $\mathrm{BaCl}_{2}$ group $(9.34 \% \pm 1.37 \%)$; Compared with the control group $(51.41 \% \pm 5.49 \%)$, EDHFmediated endothelium-dependent relaxation induced by bradykinin was partly inhibited in the Glibenclamide group (37.05\% $\pm 5.32 \%$ ); EDHFmediated endothelium-dependent relaxation induced by bradykin was $(63.43 \pm 7.71) \%$ and $(54.31 \pm 3.35) \%$ respectively in the control group and Glibenclamide group, but statistical analysis showed no significant difference between NDP group and HTK group.

Conclusion $\mathrm{BK}_{\mathrm{Ca}}$ channels, $\mathrm{SK}_{\mathrm{Ca}}$ channels, $\mathrm{K}_{\mathrm{ir}}$ channels and $\mathrm{K}_{\mathrm{ATP}}$ channels play a role in the protective effects of NDP solution on EDHFmediated vasodilation. Among these channels, $\mathrm{BK}_{\mathrm{Ca}}$ and $\mathrm{SK}_{\mathrm{Ca}}$ channels act in a coordinated manner. However, Kv channels do not play a role.

\section{Long-term exercise beginning at prehypertensive stage delays the development of hypertension in SHRs and underlying mechanisms \\ Haifeng Zhang ${ }^{1}$, Wenjuan Xing ${ }^{2}$, Feng $\mathrm{Gao}^{2,3}$ 1. Experiment Teaching Center, the Fourth Military Medical University 2. Department of Physiology, the Fourth Military Medical University \\ 3. Department of Cardiology, Xijing Hospital, the Fourth Military Medical University}

Objective Exercise training, a recommended nonpharmacological strategy for hypertensive patients, exerts beneficial effect on blood pressure modulation, but the underlying mechanisms remain elusive. Vascular insulin resistance is an early pathologic alteration in hypertensive vascular injury and plays a critical role in the development of hypertension. This study aimed to investigate whether long-term physical exercise starting at prehypertensive period prevents the development of hypertension via improving vascular insulin sensitivity.

Methods Young (4-week-old) prehypertensive spontaneously hypertensive rats (SHRs) and their normotensive Wistar-Kyoto controls (WKY) were subjected to a 10-week free-of-loading swim training session (60 min/day, 5 days/week). Insulin-induced mesenteric arteriolar vasorelaxation were determined.

Result SHRs showed higher systolic blood pressure (SBP) and decreased insulin sensitivity of the whole body and resistance vessels compared with those of WKY rats. SBP in the exercised SHRs was significantly lower than that in sedentary ones $(180.2 \pm 5.1$ vs $205.7 \pm$ $3.7 \mathrm{~mm} \mathrm{Hg}, \mathrm{n}=6, \mathrm{P}<0.05)$. Vascular insulin sensitivity in mesenteric arteries was improved after exercise training as evidenced by increased vasodilation response to insulin $(29.0 \% \pm 2.5 \%$ vs $18.4 \% \pm 5.9 \%$ to 1 $\mu \mathrm{mol} / \mathrm{L}$ insulin, $\mathrm{n}=6, \mathrm{P}<0.05$ ). In addition, exercise downregulated vascular GRK2 expression and activity, which further increased insulinstimulated vascular Akt/eNOS activation in exercised SHRs. More importantly, suppression of GRK2 with siRNA mimicked the effect of exercise-enhanced vascular insulin sensitivity, while upregulation of GRK2 by Chariot-mediated delivery reversed the exercise-induced vascular insulin sensitization.

Conclusion Long-term regular physical exercise beginning at prehypertensive stage improves vascular insulin sensitivity via downregulating GRK2 and consequently delays the development of hypertension.

\section{$\beta_{2}$ adrenergic receptor activation governs cardiac repolarization and arrhythmogenesis in a guinea pig model of heart failure \\ Yao Wang, Jiamin Yuan, Zhiyong Qian, Xiaofeng Hou, Jiangang Zou \\ Department of Cardiology, First Affiliated Hospital of Nanjing Medical University}

Background $\beta_{2}$ adrenergic receptor $\left(\beta_{2}-\mathrm{AR}\right)$ activation increases risk of sudden cardiac death (SCD) in heart failure (HF). Non-selective $\beta$-AR blocker has greater beneficial effect on survival than selective $\beta_{1}$ AR blocker in patients with chronic HF, indicating that $\beta_{2}$-AR or $\beta_{3}$ AR activation contributes to SCD in HF. Considering that $\beta_{3}$-AR is primarily expressed in the adipose and minimally in the heart, this study aims at investigating the role of $\beta_{2}$-AR activation on repolarization and ventricular arrhythmia (VA) in a guinea pig model of HF.

Methods HF was induced by descending aortic banding in guinea pigs and effective refractoriness period (ERP) in the left ventricle was recorded using programmed electrical stimulation (PES) based on Lagendorff perfusion. After treatment with selective $\beta_{2}$-AR agonist salbutamol (10 mmol/L) and antagonist ICI118551 (1 mmol/L), the corrected QT (QTc) and incidence of VA were recorded. In addition, the rapid component of delayed rectifier potassium current (Ikr) and action potential duration (APD) in myocytes from HF guinea pigs were examined by the whole cell patch clamp technique. Rp-cAMP (100 $\mathrm{mmol} / \mathrm{L}), \mathrm{KT} 5720$ (2.5 mmol/L), PTX (400 ng/L) and amrinone (30 $\mathrm{mmol} / \mathrm{L}$ ) were used to pretreat the myocytes, and then the changes of Ikr and APD in cells perfused with salbutamol were detected.

Result HF prolonged ERP and APD, and reduced Ikr in myocytes from HF guinea pigs. Salbutamol significantly prolonged QTc and increased the incidence of VA, which were prevented by ICI118551 in failing hearts. Rp-cAMP and KT5720 attenuated Ikr inhibition and 
APD prolongation induced by salbutamol in HF myocytes, but PTX and amrinone enhanced the effects.

Conclusion The results indicate that $\beta_{2}-\mathrm{AR}$ activation increases the incidence of VA in HF. Gs/cAMP/PKA and Gi/PDE pathways have opposite effects on $\beta_{2}$-AR activation-induced VA.

\section{Angiotensin II stimulates matrix metallopeptidase 2 upregulation in human aortic smooth muscle cells of thoracic aortic aneurysms by activating of ERK1/2, P38, JNK signaling \\ Chunmao Wang ${ }^{1,2}$, Penghong Liu ${ }^{1,2}$, Wenzhi liu, ${ }^{1,2}$, Qian chang $^{1,2}$ \\ 1. State Key Laboratory of Cardiovascular Disease, Fuwai Hospital, National Center for Cardiovascular Diseases, Chinese Academy of Medical Sciences and Peking Union Medical College \\ 2. Aorta Surgery Center}

Objective Several studies results demonstrated tissue samples from ascending aortic aneurysms had increased activity and expression of Matrix metalloproteinase-2 (MMP-2). However, the molecular and cellular mechanisms regulating MMP-2 expression in thoracic aortic aneurysms (TAAs) remain uncertain. Angiotensin II infusion promotes ascending aortic aneurysms in ApoE-/- mice. It provides direct evidence for angiotensin II induced TAAs formation. It is not yet known whether angiotensin II contributes to MMP-2 production and aneurysm progression in human TAAs. Therefore, in the present study, we demonstrated whether angiotensin II could induce MMP-2 upregulation in human TAA.

Methods We measured the plasma concentration of Ang II of 40 TAA patients ( 21 males and 19 females, $54.8 \pm 11.0$ years) by radioimmunoassay and MMP2 expression by western blot and qPCR of 20 TAA patients (14 males and 6 females, $55.2 \pm 9.8$ years). The plasma and aortic wall of control group (24 men and 16 women, $57.2 \pm 8.3$ years) were taken from coronary heart disease without ascending TAAs. Human aortic smooth muscle cells (HASMCs) were cultured by explant outgrowth from ascending aortic aneurysms walls obtained from TAA patients during surgery repair. We examined the effect of Ang II upon MMP2 production at different concentrations $(0 \mu \mathrm{M}, 0.01 \mu \mathrm{M}, 0.1 \mu \mathrm{M}$, $1 \mu \mathrm{M}, 10 \mu \mathrm{M})$ and time points $(0 \mathrm{~h}, 12 \mathrm{~h}, 18 \mathrm{~h}, 24 \mathrm{~h}, 48 \mathrm{~h})$ of Ang II by western blot. HASMCs were treated with $1 \mu \mathrm{M}$ Ang II for $0,2,5,10,30$, $60 \mathrm{~min}$ to measure MAPKs pathway activation. HASMCs were treated with $1 \mu \mathrm{M}$ Ang II for $48 \mathrm{~h}$ with or without pretreatment $30 \mathrm{~min}$ by 20 $\mu \mathrm{M}$ p38MAPK inhibitor SB203580, $10 \mu \mathrm{M}$ ERK1/2 inhibitor PD98059, and $10 \mu \mathrm{M}$ JNK inhibitor SP600125 respectively. Western blot was used for detecting MMP2 protein expression and the phosphorylation of ERK1/2, P38MAPK and JNK proteins.

Result Ang II plasma levels in ascending thoracic aortic aneurysm patients $(88.74 \pm 14.73 \mathrm{pg} / \mathrm{ml})$ were one times higher than that of CHD patients without TAAs $(41.48 \pm 2.89 \mathrm{pg} / \mathrm{ml})(\mathrm{P}<0.05)$. Compared with healthy population of which the value is $40.2 \pm 12.0 \mathrm{pg} / \mathrm{ml}$ when they lied down and eat normal diet, there is significantly high $(\mathrm{P}<0.05)$. Both the mRNA and protein expression level of MMP2 in ascending aortic aneurysm walls were 1.5 times and 3 times higher than that of MMP2 in aorta walls of CHD patients respectively $(\mathrm{P}<0.05)$. Western blotting results showed that Ang II increased MMP-2 expression in a dose-and time-dependant manner (Ang II 0.01, $0.1 \mu \mathrm{M}, \mathrm{P}<0.05$ vs $0 \mu \mathrm{M}$; Ang II $1,10 \mu \mathrm{M}, \mathrm{P}<0.01$ vs $0 \mu \mathrm{M}$ ) (Ang II $18 \mathrm{~h} \mathrm{P}<0.05$ vs 0 h; Ang II 24, 48 $\mathrm{h} \mathrm{P}<0.01$ vs 0 h). Ang II-induced MMP2 expression in HASMCs was significantly attenuated by MAPK inhibitors SB203580, PD98059, and
SP600125 respectively $(\mathrm{P}<0.05)$.

Conclusion These results indicate in human aortic smooth muscle cells from thoracic aortic aneurysms angiotensin II can induce MMP2 upregulation via JNK, ERK1/2 and p38 MAPK signaling pathways.

\section{A pro-atherogenic HDL profile in coronary heart disease patients: an iTRAQ labelling-based proteomic approach \\ Lirong Yan, Hong Liu, Hui Zhao, Xiaoxing Zhang, Lu Hua, Ping $\mathrm{Xu}$, Yishi Li \\ Key Laboratory of Clinical Trial Research in Cardiovascular Drugs, Ministry of Health, State Key Laboratory of Cardiovascular Diseases; Fuwai Hospital, National Center for Cardiovascular Diseases, Peking Union Medical College and Chinese Academy of Medical Sciences, Beijing, China}

Objective This study aims to compare the protein composition of high-density lipoprotein (HDL) particles in coronary heart disease (CHD) patients and controls by proteomic methods.

Background HDL has been reported to exert pro-atherogenic properties in $\mathrm{CHD}$ patients. Accumulating evidence indicates that HDL composition, rather than the HDL-C level, determines its functions. The changes in HDL composition involved in the conversion of anti-atherogenic to pro-atherogenic properties in CHD patients are currently unknown.

Methods and Result iTRAQ combined with nanoLC-MS/MS was performed to obtain a differential expression profile of the HDL pooled samples of the male age-matched CHD patients and controls (n =10/group). Of the 196 proteins identified in the examined HDL, 12 were differentially expressed between the CHD patients and the controls, including five up-regulated proteins and seven down-regulated proteins. Using GO analysis, we determined that the up-regulated proteins were mostly involved in inflammatory reactions, displaying a potential proatherogenic profile. In contrast, the down-regulated proteins were mostly involved in lipid metabolism processes, displaying anti-atherogenic properties.To confirm the proteomic results, serum amyloid A (SAA) and apoC-I were selected and quantified by ELISA, in the same population as the proteomic analysis, as well as another independent population $(\mathrm{n}=$ 120/group). Consistent with the proteomic results, the amount of SAA was significantly increased, and apoC-I was significantly decreased in the HDL particles of CHD patients compared with those of controls $(\mathrm{P}<0.05)$.

Conclusion Our study shows that the HDL proteome changes to a pro-atherogenic profile in $\mathrm{CHD}$ patients, which might compromise the protective effects of HDL. Proteomic analysis of HDL composition may provide more relevant information regarding their functional properties than steady-state HDL-C levels.

\footnotetext{
Comparison of overexpression of matrix metallopeptidase 2 in human ascending thoracic aortic aneurysms associated with bicuspid aortic valves and tricuspid aortic valves

Chunmao Wang ${ }^{1,2}$, Qian Chang,

1. State Key Laboratory of Cardiovascular Disease, Fuwai Hospital, National Center for Cardiovascular Diseases, Chinese Academy of Medical Sciences and Peking Union Medical College 2. Aorta Surgery Center
}

Objective Recently, several study results suggest that abnormal 
matrix metalloproteinase activity may be associated with the formation of thoracic aortic aneurysms. Bicuspid aortic valves are associated with an intrinsic aortic pathology that predisposes to formation of proximal thoracic aneurysms while tricuspid aortic valves are not. The objective of this study was to compare the activities of matrix metalloproteinase-2 (MMP-2) in thoracic aneurysms of patients with bicuspid and tricuspid aortic valves.

Methods Ascending thoracic aortic aneurysm and valve samples taken from patients with tricuspid aortic valve $(n=20,51.5 \pm 7.6$ years $)$ and patients with bicuspid aortic valve $(n=20,47.4 \pm 6.3$ years) when Bentall surgery were examined for matrix metalloproteinase 2 by Realtime quantitative PCR (qPCR), western blot and immunohistochemistry (IHC). All aneurysmal walls were stained with haematoxylin-eosin, Weigert-van Gieson for microscopic examinations.

Result By histological examination all aortic aneurysm walls showed some degree of cystic medial degeneration with fragmentation of elastic fibers. The presence of cystic medial necrosis was variable. No specimen showed histological evidence of atherosclerosis. No important differences were seen between the groups with regard to age, gender, smoking, aneurysm size, hypertension, any vascular dysfunction history, coronary artery disease, use of anti-hypertension drugs such as angiotensin-converting enzyme inhibitors, beta-blockers and calcium channel blockers. However, a trend was noted toward younger age in the bicuspid group. Matrix metalloproteinase-2 expression was greater in valves and aortic aneurysm tissues from ascending thoracic aortic aneurysm associated with bicuspid valves when compared with those from tricuspid valves, irrespective of the level of mRNA and protein. Western blot results demonstrated the matrix metalloproteinase-2 expression was significantly greater by increase of $80 \%$ in the walls of aneurysms associated with bicuspid aortic valves compared with tricuspid aortic valves $(P=0.01)$. Western blot results showed the matrix metalloproteinase- 2 expression in valves specimens of bicuspid aortic valves patients was greater by increase of $38.8 \%$ as compared with tricuspid aortic valves patients $(\mathrm{P}=0.03)$.

Conclusion The up-regulation of matrix metalloproteinase- 2 in the walls of aneurysms associated with bicuspid aortic valves may partly elucidate the predilection to aneurysm formation in these patients.

\section{The effect of valsartan on tumor necrosis factor $-\alpha($ TNF $-\alpha)$ in rat hearts during myocardial ischemia reperfusion \\ Yingie Zhang, Renguang Liu Cardiology Department of the First Affliliated Hospital of Liaoning Medical University, Jinzhou, China}

Objective To explore the effect of valsartan on tumor necrosis factor-alpha (TNF- $\alpha$ ) in buffer-perfused isolated rat hearts during myocardial ischemia reperfusion and cardiac myocytes exposed to anoxia-reoxygenation.

Methods Using the modified Langendorff model, 24 isolated rat hearts randomly divided into 3 equal groups: control group, ischemia/ reperfusion (I/R) group and valsartan group.TNF-aprotein expression was examined by immunohistochemistry stain and Western blot. mRNA expression was examined by Northern blot. Single myocytes isolated from neonatal rat hearts were subjected to the conditions mimicking ischemia and reperfusion. Ischemic condition was produced by superfusing myocytes with hypoxic substrate-free solutions containing elevated concentrations of $\mathrm{K}^{+}, \mathrm{H}^{+}$, and lactate. The myocytes were devided into 5 groups. Control group, hypoxia/reoxygenation (H/R) group, valsartan group, angiotensinII group and angiotensinII+valsartan group. The extent of cellular damage was accessed by the amount of released lactate dehydrogenase (LDH). The levels of TNF in the coronary effluent and supernatants of incubated cardiac myocytes were determined by ELISA.

Result After ischemia reperfusion, TNF- $\alpha$ expression in the myocardial cytoplasm. Compared to I/R group, The TNF- $\alpha$ mRNA and protein expression declined $(1.28 \pm 0.13$ vs $0.8 \pm 0.04,0.9 \pm 0.08$ vs 0.47 \pm 0.02 , respectively, all $\mathrm{P}<0.01$ ) in valsartan-treated hearts, the effluent TNF levels were declined in valsartan-treated group $(99.8 \pm 18.0 \mathrm{ng} / \mathrm{L}$ vs $59.6 \pm 38.3 \mathrm{ng} / \mathrm{L}, \mathrm{P}<0.01)$. Concentrations of TNF- $\alpha$ in supernatants of incubated cardiac myocytes in $\mathrm{H} / \mathrm{R}$ group and treated with $1 \times 10^{-6} \mathrm{~mol} /$ liter of Ang-II were $55.3 \pm 8.8 \mathrm{ng} / \mathrm{L}$ and $58.7 \pm 15.6 \mathrm{ng} / \mathrm{L}$, respectively $(\mathrm{P}$ $>0.05$ ). When pretreated with $1 \times 10^{-6} \mathrm{~mol} /$ liter of valsartan, TNF- $\alpha$ was declined. A parallel change of $\mathrm{LDH}$ release in supernatants of incubated cardiac myocytes was observed.

Conclusion I/R increased TNF- $\alpha$ levels, the same as angiotensin II. Valsartan protect myocardium from ischemia-reperfusion injury by reducing cardiac tumor necrosis factor-alpha content.

\section{Molecular genetics of liddles syndrome \\ Kunqi Yang ${ }^{1}$, Yan Xiao ${ }^{1}$, Tao Tian ${ }^{1}$, Linggen $\mathrm{Gao}^{2}$, Xianliang Zhou ${ }^{1}$ \\ 1. Hypertension Center, Department of Cardiology, Fuwai Hospital, \\ National Center for Cardiovascular Disease, Chinese Academy of \\ Medical Sciences and Peking Union Medical College \\ 2. Department of Geriatric Cardiology, General Hospital of the Chinese \\ Peoples Liberation Army}

Objective Liddle's syndrome, an autosomal dominant form of monogenic hypertension, is characterized by salt-sensitive hypertension with early penetrance, hypokalemia, metabolic alkalosis, suppression of plasma rennin activity and aldosterone secretion, and a clear-cut response to epithelial sodium channel $(\mathrm{ENaC})$ blockers but not spironolactone therapy. Our understanding of ENaCs and $\mathrm{Na}^{+}$transport defects has expanded greatly over the past two decades and provides detailed insight into the molecular basis of Liddle's syndrome. The purpose of this review is to offer an overview of recent advances in the molecular genetics of Liddle's syndrome.

Methods Studies were analyzed which included features of EnaCs, pathogenetic genes related to Liddle's syndrome, molecular mechanisms and genetic testing.

Result $\mathrm{ENaC}$ is considered as the rate-limiting step for sodium absorption in the distal nephron, and it is composed of alfa, beta and gamma subunits that share similar structures characterized by two transmembrane domains, a large extracellular loop, a relatively short cytoplasmic $\mathrm{C}$ - and $\mathrm{N}$-terminal domain. More than 20 mutations in SCNN1B and SCNN1G which encode the beta and gamma subunits of $\mathrm{ENaC}$, respectively, have been identified as the cause of Liddle's syndrome. All mutations reported in Liddle's syndrome delete or alter a conserved proline-rich amino acid sequence in the cytosolic C-terminus, PPPXY, referred to as the PY motif, which prevent binding between the $\mathrm{ENaC}$ and Nedd4-2, a specific ubiquitin ligase. As a result, internalization/ degradation of ENaCs is inhibited, leading to constitutive activation of the $\mathrm{ENaC}$ with increased $\mathrm{Na}^{+}$reabsorption, plasma volume expansion and hypertension. Genetic testing, based on PCR and direct sequencing analysis of the pathogenetic genes, has made it possible to make accurate diagnoses and develop tailored therapies for mutation carriers.

Conclusion Despite decades of clinical and molecular investigation 
of Liddle's syndrome, it is still considered as a rare-form disorder by most physicians. And many Liddle's patients with misdiagnosis suffer from severe cardiovascular and cerebrovascular complications at their early age. A genetic analysis of the amiloride-sensitive $\mathrm{ENaC}$ is recommended when assessing patients with low-renin, salt-sensitive hypertension not responsive to spironolactone treatment.

\section{The relationship between circulating estradiol levels and cardiac structure and function in patients with hypertrophic cardiomyopathy Baojing Sun, Shi Chen, Jiansong Yuan, Shubin Qiao, Fujian Duan, Rong Liu, Jingang Cui, Fenghuan Hu, Weixian Yang, Youzhou Chen, Yuejin Yang \\ Department of Cardiology, Cardiovascular Institute and Fuwai Hospital, National Center for Cardiovascular Diseases, Peking Union Medical College and Chinese Academy of Medical Sciences, Beijing, China}

Objective The goal of this study was to test the hypothesis that estrogen has preventive effects on diastolic function in hypertrophic cardiomyopathy (HCM).

Methods Fifty-six male patients with HCM were divided into quartiles based on plasma $17 \beta$-estradiol concentrations. Satistical analysis of the clinical characteristics, echocardiographic measures of cardiac structure and function, and myocardial fibrosis biomarkers were carried out.

Result The 17ß-estradiol levels in these patients were not correlated with Doppler diastolic parameters such as peak early and late diastolic transmitral filling flow velocities ratio (E/A ratio), deceleration time (DT), isovolumic relaxation time (IVRT) or the transmitral early left vetricular (LV) filling velocity to the early diastolic mitral annulus velocity (E/Earatio), hewever, the 17/-estradiol was negatively associated with left atrial volume (LAV) and left atrial volume index (LAVI). Multiple regression analysis was performed using the LAVI as a dependent variable and the factors that may influence left atrial pressure as independent variables. The result of this analysis showed that the correlation between 17ß-estradiol and LAVI remained (beta $=-0.422, \mathrm{P}=0.008$ ) after adjustments were made for possible confounding variables.

Conclusion Plasma 17 $\beta$-estradiol levels were negatively associated with LAV, LAVI and PICP levels, which indicated that $17 \beta$-estradiol can hinder the aggravation of diastolic dysfunction in HCM patients partially by inhibiting synthesis of collagen.

\footnotetext{
Candesartan inhibitors angiotensin ii-induced matrix metalloproteinase 2 activation in human aortic smooth muscle cells via angiotensin II type 1 Receptor

Chunmao Wang ${ }^{1,2}$, Chuan Tian ${ }^{1,3,3}$, Penghong Liu ${ }^{1,2}$, Wenzhi Liu ${ }^{1,2}$, Qian Chang ${ }^{1,2}$

1. State Key Laboratory of Cardiovascular Disease, Fuwai Hospital, National Center for Cardiovascular Diseases, Chinese Academy of Medical Sciences and Peking Union Medical College

2. Aorta Surgery Center, Fuwai Hospital, National Center for Cardiovascular Diseases, Chinese Academy of Medical Sciences and Peking Union Medical College

3. Adult Surgery Center, Fuwai Hospital, National Center for Cardiovascular Diseases, Chinese Academy of Medical Sciences and Peking Union Medical College
}

Objective Several research results showed that angiotensin II (Ang
II) can induced the production of matrix metalloproteinase 2 (MMP2) in human aortic smooth muscle cells, but the relative contribution of angiotensin II type 1 (AT1) and type 2 (AT2) receptors remains unknown. In this study, we examined which receptor was involved in Ang II-induced MMP-2 expression.

Methods Human primary aortic smooth muscle cells were purchased from American Type Culture Collection (ATCC). Passages 2-8 of the human aortas were used for the experiments. We examined the effect of Ang II upon MMP2 production at different concentrations $(0 \mu \mathrm{M}, 0.01 \mu \mathrm{M}, 0.1 \mu \mathrm{M}, 1 \mu \mathrm{M}, 10 \mu \mathrm{M})$ and time points $(0 \mathrm{~h}, 12 \mathrm{~h}, 18$ h, 24 h, 48 h) of AngII by western blot. Human aortic smooth muscle cells (HASMCs) were treated with $1 \mu \mathrm{M}$ AngII for $48 \mathrm{~h}$ with or without pretreatment $60 \mathrm{~min}$ by $1 \mu \mathrm{M}$ angiotensin II type 1 receptor (AT1R) inhibitor Candesartan and by $10 \mu \mathrm{M}$ angiotensin II type 2 receptor (AT2R) inhibitor PD123319 respectively. Quantitative reverse transcriptase-PCR (qPCR) and western blot were used for detecting MMP2 mRNA and protein expression. The phosphorylation of $\mathrm{p} 38 \mathrm{MAPK}, \mathrm{ERK} 1 / 2$ and JNK proteins was detected by western blot.

Result Western blotting and qPCR results showed that Ang II increased MMP-2 expression in a dose-and time-dependant manner. The AT1 receptor blocker Candesartan significantly blocked Ang II-induced expression of MMP-2 as compared with only Ang II group $(\mathrm{P}<0.05)$, whereas the AT2 receptor antagonist PD123319 did not block these responses. Candesartan also blocked the Ang II-induced phosphorylation of ERK1/2 and JNK. ERK1/2 inhibitor PD98059 and JNK inhibitor SP600125 suppressed AngII-induced expression of MMP-2. Both Candesartan and PD123319 had no effect on the up-regulation of MMP2 in the absence of Ang II stimulation.

Conclusion These results suggest that candesartan inhibitors angiotensin II-induced matrix metalloproteinase 2 activation in human aortic smooth muscle cells via angiotensin II type 1 receptor.

\section{Transgenic overexpression of peroxiredoxin II results in the decreased cardiac contractile function in vivo \\ Xiaojing Shi ${ }^{1}$, Wenjuan Zhou ${ }^{1}$, Huimin Wang ${ }^{1}$, Xuepeng Geng ${ }^{1}$, Kai Tang ${ }^{1}$, Shuang Zhang ${ }^{1}$, \\ Hongmin Liu ${ }^{1}$, Evangelia G. Kranias ${ }^{2}$, Wen Zhao \\ 1. Zhengzhou University School of Pharmaceutical Sciences \\ 2. University of Cincinnati College of Medicine, Department of Pharmacology \& Cell Biophysics}

Objective Peroxiredoxin II (Prx II) is a member of the antioxidant peroxiredoxin superfamily, which reduces hydrogen peroxide and hydroperoxides to $\mathrm{H}_{2} \mathrm{O}$ and ethanol. Previously, Prx II was found to be increased in two hyperdynamic genetic-engineered hearts, with either phospholamban deficiency or protein phosphatase inhibitor overexpression. However, the role of this protein in cardiac contractile performance remains unclear. The objective of this study was to gain insight in the role of Prx II in cardiac contractility and its underlying mechanisms in vivo.

Methods A transgenic vector, containing cardiac specific promoter $\alpha-\mathrm{MHC}$, Prx II cDNA and HGH PolyA was constructed and verified by DNA sequence analysis. Then, the vector was microinjected into zygotes of mice. The genomic DNA was extracted from the descendant bred mice. Finally, Prx II positive transgenic mice were screened using PCR method.

Result We have successfully obtained six lines of Prx II transgenic mice. Quantitative immunoblotting indicated that Prx II expression levels were increased to 1.9-2.5 fold in Prx II transgenic mouse hearts, 
compared to wild-types. Ex vivo cardiac function was then assessed by Langendorff perfusion. We found that the increased expression of Prx II was associated with depressed cardiac contractility in the organ levels: the rates of contraction (24\%) and relaxation (23\%), and left ventricular end systolic pressure (36\%) were significantly decreased in transgenic hearts, compared with wild-types. Interestingly, Prx II overexpresssion was accompanied by decreased protein levels of PLN-Ser16, whereas the protein levels of PLN, SERCA2 and heart/body ratio remained unaltered.

Conclusion These results suggest that Prx II is involved in the regulation of myocardial contractile function, which may be associated with the decreases in phosphorylation of PLN-Ser16.

\section{Chemerin induces insulin resistance in rat cardiomyocytes in part through the ERK1/2 signaling pathway \\ Yongjun Li, Ruining Zhang, Sunyun Liu, Bingyan Guo, Liang Chang \\ The Second Hospital of Hebei Medical University}

Objective Chemerin is a novel adipokine that is now known to be synthesized by adipocytes and is closely associated with cardiovascular diseases and glucose homeostasis. This study aimed to investigate the effects of chemerin on insulin resistance in rat cardiomyocytes.

Methods Chemerin expression was measured by real-time PCR and western blot in neonatal rat cardiomyocytes and cardiac fibroblasts. The cardiomyocytes were treated with high concentrations of glucose and TNF- $\alpha$, chemerin expression was measured by real-time PCR. Then, the cardiomyocytes were treated with chemerin and insulin. Glucose uptake was evaluated using a fluorescence microplate reader. Western blot analysis was used to evaluate the phosphorylation of Akt, insulin receptor substrate (IRS) -1, p38 mitogen-activated protein kinase (MAPK), extracellular signal-regulated kinase (ERK) 1/2, AMPactivated potein kinase (AMPK) $\alpha$, as well as chemerin protein levels.

Result Chemerin was found to be expressed in rat cardiomyocytes and cardiac fibroblasts, and chemerin expression in cardiomyocytes was increased by administration of high concentration of glucose and TNF- $\alpha$. Pretreatment with chemerin caused decreases in phospho-Akt, glucose uptake and phosphoAMPK in insulin-stimulated cardiomyocytes. Furthermore, chemerin activated the phosphorylation of p38 MAPK and ERK1/2 ininsulinstimulated cardiomyocytes. Inhibition of ERK partially prevented chemerin-induced insulin resistance.

Conclusion Chemerin is a novel adipokine that induces insulin resistance in rat cardiomyocytes, in part, through the ERK1/2 pathway.

\section{To dynamically observe a severe familial hypercholesterolemia child with seven-year follow up in China: a call for action \\ Long Jiang ${ }^{1}$, Feng Gao ${ }^{1}$, Liyuan Sun ${ }^{1}$, Xiaodong Pan ${ }^{1}$, Jie Lin ${ }^{1}$, Qiang Yong ${ }^{2}$, Qian Wang ${ }^{2}$, Ya Yang ${ }^{2}$, \\ Shiwei Yang ${ }^{1}$, Luya Wang ${ }^{1}$ \\ 1. Beijing An Zhen Hospital, Capital Medical University, The Key Laboratory of Remodeling-related Cardiovascular Diseases, Ministry of Education, Institute of Heart, Lung and Blood Vessel Diseases, China 2. Department of Imaging, Beijing An Zhen Hospital, Capital Medical University, China}

Background Familial hypercholesterolemia $(\mathrm{FH})$ is a severe autosomal codominant disorder that is characterized by an elevated concentration of low-density lipoprotein cholesterol (LDL-C) and a high prevalence of premature coronary heart disease. We reported clinical cardiovascular data from one case of homozygous FH in China after a seven-year study.

Methods We obtained $50 \mathrm{FH}$ patients with homozygous phenotypes who were admitted to Anzhen Hospital between 2005 and 2007 and selected one patient who was diagnosed with severe hyperlipidemia with early symptoms of cardiovascular disease. After diagnosis of $\mathrm{FH}$, genetic testing included gene capture chip sequencing and LDLR full length cDNA sequencing were performed and cholesterol-lowering drugs were administered. Follow-up clinical data were collected over seven years.

Result Genetic testing confirmed the diagnosis of compound heterozygous FH. The patient had mutations in exon 2 Q12X, exon 6 N296T, and exon 6 892delA of the LDL-R gene. Although the patient's TC and LDL-C concentrations were reduced by $28 \%$ and $6 \%$, respectively, with a combination of cholesterol-lowering drugs, both levels remained higher than their target values. Clinical imaging data collected over seven years showed that the left chamber of the patient's heart was persistently dilated with severe mitral insufficiency, myocardial ischemia due to multiple coronary artery stenoses, and multivessel plaque formation.

Conclusion In China, there are approximately 2.6 million potential FH patients. However, FH patients are underdiagnosed and undertreated in China because both doctors and patients lack knowledge of FH. The Chinese FH population will have poor outcomes without treatment, potentially increasing costs for the country. Therefore, we hope our report will encourage the government to devote more attention to this disease.

\section{Attenuation of LSD1 inhibitor on ISO-induced cardiac contractile response \\ Ke Yang, Xiaojing Shi, Wen Zhao \\ Zhengzhou University School of Pharmaceutical Sciences}

Objective It has been reported that lysine-specific histone demethylase 1 (LSD1), one of the epigenetic regulatory proteins, played an important role in the development of heart. LSD1 gene knockout in mouse can lead to deficiency of left ventriular septal. However, it remains unclear whether LSD1 affects myocardial contractile function. Previously, we designed, synthesized and screened a series of new LSD1 inhibitors, which are low toxicity and side effects through highthroughput screening model. In the current study, the Langendorff heart perfusion model was used to investigate the effects of these compounds on cardiac function and the involved mechanism.

Methods 25 ICR mice were randomly divided into five groups, which were subjecting to continuous infusions of increasing concentrations of isoproterenol $(0 \mu \mathrm{m}, 0.1 \mu \mathrm{m}, 0.25 \mu \mathrm{m}, 0.5 \mu \mathrm{m}, 1 \mu \mathrm{m})$. A small molecule compound ( $8 \mathrm{k}$ ), which showed significant inhibitory activity of LSD1 in vitro, was selected. According to the pre-experiment, the concentration of the drug was calculated to be $6 \mu \mathrm{M}$. We observed the effect of the drug on isolated heart in mice which was pretreated by isoproterenol as above, including the contractile parameyers, such as LVSP, $+\mathrm{dp} / \mathrm{dt}$, -dp/dt, before and aftertaking drug. Each heart was then collected and homogenized to determine cardiac contractile protein expression by Western Blotting.

Result Compared to the control group, the $\beta$ receptor agonist (ISO) is associated with increases in the force of contraction, which was in a dose-dependent manner and accompanied with rapid heart 
rate. However, the LSD1 inhibitor $8 \mathrm{~K}$ significantly attenuated the excitatory effects of ISO on the heart. Comparing with the high-dose ISO group, the expression of sarcoplasmic reticulum calcium pump SERCA2a in ISO+8K group did not change. However, we found that the expression level of serine 16 phosphorylation of PLN was significantly decreased.

Conclusion LSD1 inhibitors depressed the positive inotropic effect of ISO on the myocardium. The mechanism may be related to changes in the phosphorylation level of PLN. This finding shows that LSD1 may be involved in the regulation of cardiac contractility.

\section{The effect of PEAR1 genetic variants on platelet reactivity \\ Yi Yao', Xiaofang Tang ${ }^{1}$, Jing Wang ${ }^{1}$, Jiahui Zhang', Yuanliang $\mathrm{Ma}^{1}{ }^{1}$, Jingjing $\mathrm{Xu}^{1}{ }^{1}, \mathrm{Ru} \mathrm{Liu}^{1}$, Xianmin Meng ${ }^{2}$, Jinqing Yuan \\ 1. Department of Cardiology, Fuwai Hospital and Cardiovascular Institute, National Center for Cardiovascular Diseases, Chinese Academy of Medical Sciences and Peking Union Medical College 2. State Key Laboratory of Cardiovascular Disease, Fuwai Hospital and Cardiovascular Institute, National Center for Cardiovascular Diseases, Chinese Academy of Medical Sciences and Peking Union Medical College}

Objective Insufficient inhibition of platelet aggregation is associated with higher risk of high platelet reactivity and adverse clinical events. Platelet endothelial aggregation receptor-1 (PEAR1) is a newly reported platelet transmembrane protein which can be activated by agonist stimulation or platelet-platelet contact. Some researches indicate that PEAR1 may play an important role on platelet aggregation. The aim of this study is to investigate the effect of PEAR1 genetic variants on platelet reactivity in chinese coronary heart disease patients after percutaneous coronary intervention, which has not yet been reported.

Methods 1493 patients with coronary heart disease received percutaneous coronary intervention in Fuwai hospital were enrolled in the study. Adenosine diphosphate (ADP) -induced platelet reactivity was measured by thromboelastography, data were recorded as the percentage inhibition of platelet aggregation (IPA). For patients, IPA less than 30\% (means high platelet reactivity) were included in the experimental group; IPA greater than $70 \%$ (means normal platelet reactivity) were included in the control group. 16 candidate single nucleotide polymorphisms (SNPs) of PEAR1 searched in HAPMAP or ENSEMBLE were detected by the method of improved multiple ligase detection reaction.

Result Baseline characteristics were similar between the experimental group $(n=203)$ and the control group $(n=205)$, except for gender composition and average LDL level $(\mathrm{P}<0.05)$. Among 16 candidate SNPs, 4 SNPs showed a significantly different between the two groups, they were C-allele at rs11264580 ( $\mathrm{P}=0.033)$, G-allele at rs2644592 $(\mathrm{P}=0.048)$, T-allele at rs3737224 $(\mathrm{P}=0.033)$ and T-allele at rs41273215 ( $\mathrm{P}=0.025)$; all of them displayed a higher carrying frequency in the experimental group compared to the control group. After adjust for potential confounders, besides rs2644592, the other three allels were still significantly related to high platelet reactivity ( $\mathrm{P}$ $<0.05)$.

Conclusion The genetic variants of PEAR1 are strongly associated with ADP-induced platelet aggregation. SNPs (rs11264580, rs3737224, rs41273215) in our study have been proved related to high platelet reactivity.

\section{Transgenic overexpression of Prx II protects hearts from ischemia-reperfusion injury in vivo \\ Wen Zhao, Huimin Wang, Xiaojing Shi, Wenjuan Zhou, Ke Yang, Xuepeng Geng, Shuang Zhang, Jingjing Wang, Hongmin Liu \\ Zhengzhou University School of Pharmaceutical Sciences}

Objective Peroxiredoxin II (Prx II), a member of the antioxidant enzyme family, is abundant in myocardial cytosol. In the previous study, we found that the expression levels of Prx II were decreased in mouse hearts upon ischemia-reperfusion. Adenovirus-mediated Prx II overexpression protects cardiomyocytes from oxidative stress-induced injury. However, the protective effects of this protein in the whole animal and organ levels remain to be investigated.

Methods and Result In this study, a transgenic mouse with cardiac specific Prx II overexpression was selected. Using langendorff model, myocardial ischemia-reperfusion was induced as follows: after 20-30 minutes of equilibration time, the hearts were subjected to 40 minute ischemia followed by 60 minute reperfusion with Krebs-Henseleit buffer. During this period, systolic function parameters of isolated hearts were monitored to evaluate the injury extent of ischemia-reperfusion. Under basal condition, the systolic functional parameters were decreased in Prx II-overexpressing hearts, compared with wild-types. After I/R, the recovery of the rates of contraction and relaxation $\left( \pm \mathrm{dp} / \mathrm{dt}_{\max }\right)$ were significantly improved in Prx II-overexpressing hearts, compared to WTs (10\% and $20 \%$ ). $1 \%$ of 2, 3, 5-triphenyltetrazolium chloride (TTC) stains showed smaller infarct size in Prx II transgenic (Tg-Prx II) hearts than those in wild-types (WT). The enhanced systolic functionwas associated with specific increases in phosphorylation of phospholamban, relieving its inhibition of the apparent Ca affinity of SERCA2a. We also found that after I/R, some relative proteins in the downstream of mitochondrial apoptosis pathway, including Bax, were decreased, the activities of caspases 3, 9 and 12 were attenuated; Bcl-2 expression was increased in the Prx II-overexpressing hearts, compared with WTs, using quantitative immunoblotting.

Conclusion These findings suggest that Prx II-overexpression protects the hearts from ischemia-reperfusion injury, through mitochondria-mediated anti-apoptotic pathway.

\section{Impaired hippocampal synaptic plasticity in angiotensin II-dependent hypertensive mice Hailong Dai ${ }^{1,2,3}$, Xuefeng Guang ${ }^{1}$, Ming Zhang ${ }^{2}$, Zhicheng Xiao, ${ }^{2,3}$, Weiyuan $\mathrm{Hu}^{2}, \mathrm{Xin} \mathrm{Li}^{2}$ \\ 1. Yanan Affiliated Hospital of Kunming Medical University \\ 2. Kunming Medical University \\ 3. Monash University}

Objective Elevated blood pressure is associated with increased risk for cognitive impairment. However, its pathogenesis has not been sufficiently clarified. To investigate the underlying synaptic mechanism in a mouse model of chronic angiotensin II-dependent hypertension.

Methods We used subcutaneous mini-pumps containing a concentration of angiotensin II (1900 $\mathrm{ng} / \mathrm{kg} / \mathrm{min})$ that induces malignant hypertension or a saline solution for 28 days. Blood pressure was carefully monitored by a non-invasive tail-cuff method every week throughout the experiment. Long-term potentiation (LTP) was measured by vitro electrophysiology on hippocampal slices at the Schaffer-CA1 pathway, the density and length of the dendritic spines on the CA1 
pyramidal cells were examined by confocal microscopy after Golgi staining.

Result Compared to the saline-treated control group, the treatment of Ang II significantly raised the systolic blood pressure in adult male mice from the 1st week (control, $104.1 \pm 2.09 \mathrm{~mm} \mathrm{Hg}$; Ang II, 154.14 $\pm 7.71 \mathrm{~mm} \mathrm{Hg}$ ), the 2nd week (control, $104.1 \pm 1.99 \mathrm{~mm} \mathrm{Hg}$; Ang II, $159.1 \pm 7.15 \mathrm{~mm} \mathrm{Hg}$ ), the 3rd week (control, $103.8 \pm 1.99 \mathrm{~mm} \mathrm{Hg}$; Ang II, $155.43 \pm 8.96 \mathrm{~mm} \mathrm{Hg}$ ) to the 4th week (control, $105.3 \pm 2.61 \mathrm{~mm}$ Hg; Ang II, $155.14 \pm 6.74 \mathrm{~mm} \mathrm{Hg}$ ) (all P < 0.05). Hippocampal CA1 LTP was significantly impaired in Ang II-dependent hypertensive mice $(102.65 \pm 3.14$ vs $153.41 \pm 5.08, \mathrm{P}<0.01)$. The density $(/ 30 \mu \mathrm{m})$ and length $(\mu \mathrm{m})$ of the dendritic spines on the CA1 pyramidal cells were reduced in Ang II-dependent hypertensive mice (15.7 \pm 2.5 vs $27.3 \pm 2.1$, $\mathrm{P}<0.01 ; 0.64 \pm 0.25$ vs $1.01 \pm 0.23, \mathrm{P}<0.001)$.

Conclusion Hippocampal synaptic plasticity was impaired in angiotensin II-dependent hypertensive mice, supporting the idea that hypertension is a risk factor for cognitive decline.

\section{Regulatory role of microRNA-216a in the proliferation and adhesion of senescent endothelial cells}

Xuenan Mi, Jingyao Fan, Shujun Yang, Yu Chen, Rutai Hui, Weili Zhang

State Key Laboratory of Cardiovascular Disease, Fuwai Hospital, National Center for Cardiovascular Diseases, Chinese Academy of Medical Sciences and Peking Union Medical College, China

Objective Aging is an independent risk factor for cardiovascular diseases. Cellular senescence of endothelial cells has been proposed to be involved in endothelial dysfunction and atherogenesis. However, the regulations of endothelial cells senescence are yet to be clarified. It is reported that microRNA-216a (miR-216a) was upregulated in senescent endothelial cells. But the role of miR-216a in senescent endothelial cells and mechanism is still unclear. The present study was designed to study the role of miR-216a in the abilities of proliferation and adhesion of senescent endothelial cells.

Methods Primary human umbilical vein endothelial cells (HUVECs) were cultured and population-doubling levels (PDLs) were calculated during passages. The senescent status of HUVECs was verified by in situ staining for senescence associated $\beta$-galactosidase (SA- $\beta$-gal), cell cycle related proteins detection and telomere length detection. To compare the expression of miR-216a in PDL8 and PDL44 HUVECs, miR-216a was detected by real-time PCR. MiR-216a mimics and inhibitors were separately transfected into PDL44 HUVECs, and then the abilities of cell proliferation and adhesion, were assayed by MTS test and cell adhesion test, respectively.

Result Compared with PDL8 HUVECs, the percentage of SA$\beta$-gal positive cells was increased in PDL44 HUVECs by 4.7 -folds ( $P$ $<0.001)$. The expression of p53 and p21 was unregulated in PDL44 HUVECs by $69 \%(\mathrm{P}=0.003)$ and $61 \%(\mathrm{P}=0.01)$, respectively. And the telomerelength was shorter in PDL44 HUVECs by $42 \%$ $(\mathrm{P}=0.01)$. The expression of miR-216a was increased in PDL44 HUVECs by $59 \%(P=0.035)$ than PDL 8 HUVECs. In vitro analysis of PDL44 HUVECs, overexpression of miR-216a inhibited endothelial proliferation by $6 \%(\mathrm{P}=0.024)$, while miR-216a inhibitors can increase endothelial proliferation by $12 \%(\mathrm{P}=0.027)$. In the cell adhesion assay, overexpression of miR-216a increased the adhesion capabilities of THP- 1 cells to HUVECs by $53 \%(\mathrm{P}=0.003)$, in contrast, miR-216a inhibitors inhibited the adhesion capabilities by $29 \%(\mathrm{P}=0.014)$.

Conclusion Inhibition of miR-216a can enhance proliferation and attenuate adhesion of senescent endothelial cells, which it may serve as a target in regulating endothelial senescent associated cardiovascular diseases.

\section{Cardiosignal-TF is a cardiovascular transcription regulation knowledge base \\ Yisong Zhen \\ State Key Laboratory of Cardiovascular Disease, Fuwai Hospital \& \\ Cardiovascular Institute, Chinese Academy of Medical Sciences, Peking \\ Union Medical College}

Objective Information about cardiovascular gene transcription is fragmented and far behind the current requirement at the systems biology level to crack developmental biology problems. To facilitate deep learning of deluge of genomic data, we have constructed CardioSignalTF database, which is committed to collect cardiovascular information regarding transcriptional factors, their corresponding binding sites and position weight matrixes.

Methods We used natural language processing method (NLP), Naive Bayes algorithm to classify literature to sift all Weinsteinlike papers deposited in PubMed. GNAT was then used to pick out corresponding gene names. Local Perl scripts were used to integrate them into our own platform by using MariaDB management system. R scripts were implemented to analyze and build graph results.

Result Cardiovascular transcription factors from fly, ciona, zebrafish, chicken, mouse and human were collected based upon human version curated by the Wingender group. Cardiovascular mouse homologs defined by NCBI pipeline were verified by four independent sources with their Entrez GeneID as the key and were deposited in the database. Position weight matrixs from Jaspar, hPDI and Uniprobes databases were deposited in the database and can be retrieved by their original database primary ID. Enhancer regions from various sources of ChIP-seq data were integrated into the database.

Conclusion The database can be used as a portal to construct the transcriptional networks in cardiac development.

\section{Integrating genetic and gene expression evidence into genome-wide association analysis identified novel genes for lipid traits Xingbo Mo ${ }^{1,2}$, Huan Zhang ${ }^{2}$, Shufeng Lei ${ }^{1,2}$, Yonghong Zhang, \\ 1. Center for Genetic Epidemiology and Genomics, School of Public Health, Medical College of Soochow University, Jiangsu, China 2. Department of Epidemiology, School of Public Health, Medical College of Soochow University, Jiangsu, China}

Background Genetic factors contribute to the variation of lipid traits. The aim of this study was to use these methods to identify more susceptibility genes for lipid levels.

Methods Based on the public available SNP-based P value, we performed an initial gene-based GWAS in about 100000 individuals. Furthermore, we performed differential expression analysis of GEO data and protein-protein interaction analysis to find supplementary evidence to support the significance of the identified genes. Gene set enrichment analysis was used to detect lipids related pathways.

Result About 21883 genes on the human genome were 
analyzed for lipid levels in gene-base GWAS. A total of 419 genes were found to be statistically significant $\left(\mathrm{P}<2.3 \times 10^{-6}\right)$ after Bonferroni correction. Differential expression analysis of four studies further supported the significant association of 51 genes with total cholesterol, 37 genes with triglycerides, 41 genes with high-density lipoprotein cholesterol and 40 genes with low-density lipoprotein cholesterol. More convincingly, 21 of these genes were supported by at least two expression studies. Several of these novel genes, such as AKT2, INSIG2, HSPA1A, RORA and AGPAT1, play important roles in lipid metabolism. Fourteen biological pathways (e.g. lipid and fatty acid transport, apolipoprotein, transport, LXR/RXR activation and FXR/RXR activation) were found to be significantly enriched for lipid traits associations.

Conclusion The present study highlighted the high power of genebased and set-based analysis methods in finding novel lipids associated genes and pathways. Our findings provide more insights into the genetic basis of lipid metabolism.

\section{Down-regulation of the small-conductance calcium-activated potassium channels in diabetic mouse atria promotes arrhythmia induction \\ $\mathrm{Fu} \mathrm{Yi}{ }^{1}$, Tong $\mathrm{Lu}^{2}$, Xiaoli Wang ${ }^{2}$, Wenkuang Shen ${ }^{3}$, Hon-Chi Lee ${ }^{2}$ \\ 1. Department of Cardiovascular disease, Xijing Hospital, Fourth Military Medical University \\ 2. Department of Intemal Medicine, Division of Cardiovascular Diseases, Mayo Clinic, Rochester, Minnesota \\ 3. Department of Internal Medicine, Division of Cardiovascular Diseases, Mayo Clinic, Phoenix, Arizona}

The small-conductance calcium-activated potassium (SK) channels are abundantly expressed in atrial myocytes and are associated with the pathogenesis of atrial fibrillation (AF). Diabetes mellitus (DM) is an independent risk factor of $\mathrm{AF}$, however underlying ionic mechanism remains unclear. We hypothesized that the SK channel dysfunctions participated in atrial arrhythmogenesis in DM. Using cellular biology and electrophysioguical techniques in the study, we found that resting potentials of atrial strips were similar between control and streptozotocin-induced diabetic mice, but APD90s and APD50s were significantly longer in diabetic mice. Spontaneous premature beats, early after-depolarizations (EADs) and EADsinduced activities were frequently observed in atrial preparations of diabetic mice, but absent in those of controls. Exposure to apamin significantly prolonged APD90 in controls and no effect was found in diabetic mice. Patch clamp study confirmed that SK currents from freshly isolated atrial myocytes were markedly reduced in diabetic mice. Moreover, protein expressions of SK2 and SK3 were down-regulated by $85 \%$ and $92 \%$ respectively without change of SK1 expression in diabetic mouse atriums and in HL-1 cells with high-glucose culture, compared to those of control mice and normal-glucose cultured HL-1 cells. There was significant increase in production of reactive oxygen species in diabetic atriums and in high-glucose cultured HL-1 cells. Notably, downregulation of SK2 and SK3 expression was mimicked by a 24-h incubation of $\mathrm{H}_{2} \mathrm{O}_{2}$ in HL-1 cells with normal-glucose culture. Hence, our results provide a new insight into mechanisms of cardiac electrical remodeling in diabetic atria and may help us better understand atrial arrhythmogenesis in DM.

\section{Dipeptidyl peptidase (DPP) -4 inhibitor attenuates apoptosis in isoproterenol-induced myocardial infarcted rats by inhibiting oxidative stress \\ Yongjun Li, Bingyan Guo, Suyun Liu, Hui Zhang, \\ Rong Yang, Xin Jin \\ The Second Hospital of Hebei Medical University}

Background Cardiac apoptosis and oxidative stress play important roles in the pathology of myocardial infarction. The protective effects of dipeptidyl peptidase (Dpp) -4 inhibitor (vildagliptin) on cardiac apoptosis were evaluated in isoproterenol induced myocardial infarcted rats.

Methods Male wistar rats are treated intravenously with vildagliptin $(2 \mathrm{mg} / \mathrm{kg} /$ day $)$ daily for a period of 21 days. After 21 days of pretreatment, isoproterenol $(100 \mathrm{mg} / \mathrm{kg})$ was injected subcutaneously to rats at an interval of $24 \mathrm{~h}$ for 2 days (on 22th and 23th day) to induce myocardial infarction. Cardiac diagnostic markers, heart lipid peroxidation, antioxidant system, histopathological changes of the heart and apoptosis were evaluated in isoproterenol induced myocardial infarcted rats.

Result Isoproterenol induced myocardial infarcted rats showed a significant increase in the levels of serum cardiac diagnostic markers, heart lipid peroxidation products and a significant decrease in the activities/levels of heart antioxidants, compared with normal rats. Additionally, histopathological findings of myocardial infarcted rats revealed marked necrosis. Real time polymerase chain reaction study revealed an increase in the myocardial expression of bax, caspase- 8 and fas genes and a decrease in the myocardial expression of bcl-2 and bcl$\mathrm{xl}$ genes. Vildagliptin $(2 \mathrm{mg} / \mathrm{kg} /$ day) pretreatment decreased the levels of serum cardiac marker enzymes, reduced heart lipid peroxidation and minimized the alterations the activities/levels of heart antioxidants of isoproterenol-induced myocardial infarcted rats. Histopathological study evidenced that the pretreatment with vildagliptin inhibited myocardial damage. Vildagliptin pretreatment also showed protective effects on apoptosis. The in vitro study also revealed the free radical scavenging and anti-apoptosis activity of vildagliptin.

Conclusion Thus, vildagliptin protected the myocardial infarcted rat's heart against apoptosis by inhibiting oxidative stress.

\section{SFRP5 expression in rat cardiomyocytes is up-regulated in Ang II-induced cardiomyocyte hypertrophy \\ Yongjun Li, Xin Jin, Bingyan Guo, Suyun Liu, Liang Chang The Second Hospital of Hebei Medical University}

Objective Secreted frizzled-related protein 5 (SFRP5), as a novel adipokine, is now known to be synthesized by differentiated adipocytes and skeletal muscle cells with insulin-sensitising and anti-inflammatory properties similar to adiponectin. This study aimed to examine the patterns of SFRP5 expression in normal and hypertrophic cardiomyocyte and to explore the underlying mechanisms that could be involved.

Methods SFRP5 expression was measured by real-time PCR and western blot in normal neonatal rat cardiomyocytes. Then the cardiomyocytes were treated with various concentrations of Ang II, SFRP5 expression was measured by real-time PCR and western blot. Then, the cardiomyocytes were treated with Telmisartan and PD123319 which were used to block Ang II type-1 and type-1 receptors 
respectively. Y-27632, a selective inhibitor of ROCK was used to inhibit Rho/ROCK signalling pathway, real-time PCR and western blot were used to measure SFRP5 expression.

Result SFRP5 was found to be expressed in rat cardiomyocytes and SFRP5 expression in cardiomyocytes was significantly increased by Ang II at concentrations from $10^{-6}$ to $10^{-8} \mathrm{M}$. It was dose and time dependent. Angiotensin type-1 receptor activation is required for Ang II-stimulated effects on SFRP5. A selective inhibitor of ROCK blocked Ang II-mediated upregulation of SFRP5.

Conclusion SFRP5 is a novel adipokine that can be expressed by rat cardiomyocytes. The increased SFRP5 expression during Ang II induced cardiomyocyte hypertrophy was mainly through the AT1R-Rho/ ROCK signal pathway.

\section{CYP2J2-derived EETs attenuate angiotensin II-induced adventitial remodeling \\ Chi Zhou, Xizhen Xu, Daowen Wang \\ Cardiovascular Division, Department of Internal Medicine, Tongji \\ Hospital, Tongji Medical College, Huazhong University of Science and \\ Technology}

Objective Cytochrome P450 (CYP) epoxygenases metabolize arachidonic acids (AA) to form epoxyeicosatrienoic acids (EETs), which exert beneficial roles in the therapy of cardiovascular diseases, including hypertension, atherosclerosis, abdominal aortic aneurysm and aortic dissection. But little is known about its role in adventitial remodeling.

Methods In this study, we utilized primary adventitial fibroblasts (AFs) cell culture and Angiotensin (Ang) II osmotic pump infusion animal model to investigate the effect of CYP2J2 and its metabolites EETs on Ang II-induced adventitial remodeling.

Result We first identified that CYP/sEH system did exist in adventitia, and was activated in adventitial remodeling. Ang II stimulation promoted $\mathrm{sEH}$ expression via AT1 receptor. In cultured primary adventitial fibroblasts, exogenous EETs remarkably inhibited Ang II-induced AFs activation, including differentiation, proliferation, migration and collagen synthesis, while these protective effects could be reversed by PPAR gamma antagonist GW9662 addition or SOCS3 siRNA transfection. Two mechanisms of inflammatory pathways were found to be involved. Firstly, we confirmed that EETs activated PPAR gamma, and markedly suppressed Ang II-induced IкB alpha phosphorylation, NF- $\mathrm{KB}$ nuclear translocation and inflammatory cytokines secretion in AFs. Secondly, EETs reduced Ang II-induced JAK2, STAT1, STAT3 phosphorylation and p-STAT1, p-STAT3 nuclear translocation, which were mediated by SOCS3 induction but independent on PPAR gamma. rAAV-CYP2J2 injection led to abundant expression of CYP2J2 in aortic tissue and elevated serum circulating EETs level. Our results indicated that CYP2J2 overexpression alleviated Ang II-induced hypertension. Moreover, CYP2J2 gene delivery reduced vessel wall thicken, adventitial fibroblasts differentiation and collagen deposition in aorta induced by Ang II infusion, which is mediated by NF- $\mathrm{KB}$ and JAK/STAT-SOCS3 pathways. Interestingly, Hydralazine therapeutic group exerted no obviously function, which suggested that the protective effect of CYP2J2 was independent on BP lowering. It affected AFs directly.

Conclusion Taken together, we conclude that CYP2J2 and its metabolite EETs attenuate Ang II-induced adventitial remodeling via PPAR gamma dependent NF- $\mathrm{KB}$ and PPAR gamma independent JAK/ STAT-SOCS3 pathways. These results suggest that increasing EETs by CYP2J2 gene delivery could be considered as a novel approach to mitigate adventitial remodeling.

\section{Polymorphisms in the MMP-2 gene and chronic heart failure in henan han population \\ Liwei Guo ${ }^{1}$, Guifang Wang ${ }^{2}$, Xining Zhang ${ }^{2}$ \\ 1. State Key Laboratory of Cardiovascular Disease, Fuwai Hospital, National Center for Cardiovascular Disease, Peking Union Medical \\ College \& Chinese Academy of Medical Sciences \\ 2. Department of Cardiology, the First People's Hospital of Xinxiang, Henan}

Objective Chronic heart failure (CHF) is a clinical syndrome that represents the end-stage phenotype of a number of different cardiovascular diseases. Matrix metalloproteinase-2 (MMP-2), a collagenase encoded by the MMP-2 gene, degrades the type IV collagen and is responsible for inflammatory responses. It was reported that the serum levels of MMPs increase during CHF and the MMP-2 are related to a poor prognosis. Single nucleotide polymorphisms (SNP) of MMP2 genewas implicated in human CHF, in this study we investigated the association of single nucleotide polymorphisms (SNP) of MMP-2 gene with CHF in Henan Han population.

Methods We conducted a case-control study and a total of 253 $\mathrm{CHF}$ cases and 348 unrelated control subjects were recruited from north Henan province. Three selected SNPs, rs243866, rs17859821 and rs243864 of the MMP-2 gene were genotyped using Polymerase chain reaction-restriction fragment length Polymorphism (PCR-RFLP), A mixed linear model was used to estimate the association between each allelic associations of SNPs and SNP haplotypes with CHF.

Result The frequency of rs243866 A allele was much lower in CHF patients compared with controls ( $\mathrm{P}$ values $=0.043)$. The frequency of rs243864 T allele was just the reverse $(\mathrm{P}$ values $=0.002)$, the frequency of TT genotype was also increased significantly compared with controls ( $\mathrm{P}$ values $=0.027$ ). We also identified one risk haplotype (GGG; P values $=0.007)$, There was no significant difference in genotype and allelic frequency of rs17859821 between healthy subjects and patients.

Conclusion The rs 243866 A allele might confer lower risk of CHF, While the rs243864 T allele and Haplotype GGG increased the risk of CHF, rs17859821 have no association with chronic heart failure in Henan Han population.

\section{Prokaryotic expression, purification and activity evaluation of an antioxidant protein: peroxiredoxin II \\ Wen Zhao, Wenjuan Zhou, Xiaojing Shi, Yichao Zheng, Huimin Wang, Xuepeng Geng, Shuang Zhang, \\ Jingjing Wang, Hongmin Liu \\ Pharmacy College of Zhengzhou University}

Ojective As a member of antioxidant protein superfamily, peroxiredoxins (Prx) are widely expressed in the body. The mammalian Prxs include Prx I to VI. Peroxiredoxin II, mainly located in the cytoplasm, is highly conserved in its three cysteines, which have reductive activity in its structure. When Prx II participates in the anti-oxidative reaction, the thiol group of a peptide chain will react with another thiol group of the peptide to form an intermolecular disulfide that can provide hydrogen for redox. Then, depending on the system of Thioredoxin (Trx) and Thioredoxin reductase (TrxR), the $\mathrm{H}^{+}$of NADPH will be transferred to 
Prx to restore its reduced state. It has been reported that the expression of peroxiredoxin significantly increases in a variety of human maligant tissues and many other diseases (such as myocardial ischemia disease, diabetes, neurodegeneration). Prxs can be regarded as a therapeutic target for these diseases. This study was designed to generate Prx II prokaryotic expression vector and set up an activity evaluation system of PrxII in vitro.

Methods The expression vector PET28bPrx II, was constructed and transformed into the E. coli strain BL21 (DE3) to induce the expression of Prx II. The expressed proteins were extracted with phosphate buffer. The Prx II proteins were then purified through the nickel column. Then, according to the principle that the system of Trx and TrxR can transfer the $\mathrm{H}^{+}$of NADPH to Prx II, we can determine the antioxidant activity of Prx II by measuring the change of the absorbance of NADPH at $340 \mathrm{~nm}$.

Result By expressing and purifying the proteins of Prx II, we have successfully constructed Prx II activity evaluation system in vitro. Combined with the small purified molecule compounds that our research group has synthesized and isolated from natural products, the screening and evaluation of Prx II inhibitors in vitro is in progress.

Conclusion Prx II is a promising protein in the regulation of heart function. We will apply the selected small molecule inhibitors to further explore the mechanism of how Prx II impacts on the cardiac function and protects myocardial injury.

\section{Regulation of cardiomyocyte differentiation and proliferation by cnot3 \\ Junwei Liu, Li Wang, Jiawei Shi, Tucheng Sun, \\ Nianguo Dong \\ Cardiovascular Surgery, Union Hospital, Huazhong University of \\ Science and Technology}

Stem cell therapy has been considered as a promising strategy to repair damaged myocardium caused by infarction and ischemic heart diseases. Despite breakthrough in deriving cardiomyocytes from human pluripotent stem cells, the survival and maturation of those cells after transplantation into infarcted myocardium are still major obstacles hindering their clinical application. Thus, it is critically important to find novel molecular targets participating in cardiac development to improve the therapeutic effects of stem cell transplantation. Cnot3 has been identified as a factor required for normal heart function in an RNAi screen in Drosophila. Herein, we sought to determine its role in cardiac development and evaluate its potential in improving outcomes of cardiac stem cell therapy. We show that Cnot 3 is highly expressed at the cardiac progenitor stage, both during human embryonic stem cell (hESC) cardiac differentiation and mouse heart development. Cnot3 silencing inhibits cardiomyocytes differentiation and represses their proliferation, while Cnot3 overexpression has the opposite effects. Importantly, the pro-proliferative effect of Cnot3 is also evident in mature cardiomyocytes. In vitro, Cnot 3 expression promotes proliferation in hESC-derived mature cardiomyocytes. In vivo, Cnot3 heterozygous deletion inhibits proliferation of cardiomyocytes in adult mice. Furthermore, Cnot3 expression in human heart decreases during the natural course of aging, correlating with the loss of proliferation of cardiac cells. Taken together, our data suggest that Cnot3 plays a key role in regulating cardiomyocyte proliferation both during development and in the adult stage. We are further testing the potential therapeutic benefits of Cnot3 overexpression in the treatment of myocardial infarction in coronary artery disease.

\section{Prenatal lipopolysaccharide exposure results in dysfunction of renal dopamine D1 receptor in offspring rats}

\author{
Xinquan Wang ${ }^{1,2}$, Haoluo Luo ${ }^{1,2}$, Jialiang Wang ${ }^{1,2}$, \\ Caiyu Chen ${ }^{1,2}$, Yue Cai ${ }^{1,2}$, Shuo Zhen ${ }^{1,2}$, \\ Zhou Zhou ${ }^{1,2}$, Chunyu Zeng ${ }^{1,2}$ \\ 1.Department of Cardiology, Daping Hospital, The Third Military \\ MedicalUniversity \\ 2.Department of Cardiology, Daping Hospital, The Third Military \\ MedicalUniversity
}

Objective Adverse environmental exposure in utero predisposes to adult disease, including hypertension. Exposure to lipopolysaccharide (LPS) results in increased blood pressure in offspring, but the exact mechanisms are not clear. Our previous study shows dysfunction of renal D1 receptor (D1R) is ascribed to the pathogenesis of hypertension, which is associated with reactiveoxidative stress (ROS). In this study, we test whether dysfunction of renal D1R is involved in fetal programmed hypertension, and whether oxidative stresscontribute to this process.

Methods Pregnant Sprague-Dawley (SD) rats were intraperitoneally injected with LPS $(0.79 \mathrm{mg} / \mathrm{kg})$ or saline $(0.5 \mathrm{ml})$ at gestation day 8, 10 and 12. After birth, the blood pressure is measured, and treated with or without antioxidant tempol in tapwater for 3 weeks at postnatal 12 week.

Result As compared with control rats, the LPS-treated offspring rats showed higher blood pressure, decreased renal sodium excretion with increased plasma ROS activity. After treatment with tempol for 3 week, the increased blood pressure, decreased sodium excretion were reversed to normal levels in LPS rats. Further study found LPS rats had lower renal D1R expression, higher D1R phosphorylation, and D1Rmediated natriuresis and diuresis were lost. As an important kinase of D1R phosphorylation, G coupled receptor protein kinase 4 (GRK4) expression was increased in LPS rats. Tempol treatment reversed the decreased D1R expression, increased D1R phosphorylation and GRK4 expression. Moreover, the impaired D1R-mediated natriuresis and diuresis were restored to the control levels inLPS rats after tempol treatment.

Conclusion Prenatal LPS exposure, via impairment of ROS on renal D1R function, leads to hypertension in offspring. Of renal D1R function by alleviation of ROS might be a target for therapy of fetal programming hypertension.

\footnotetext{
Decreased soluble vascular endothelial growth factor receptor 2 expression in hypertensive diabetic patients

Shujie $\mathrm{Yu}^{1}$, Lixia Jian ${ }^{1}$, Zhiming Song ${ }^{1}$, Zhen $\mathrm{Wu}^{1}$,

Xiaoxian Qian ${ }^{1,2}$, Lin Chen ${ }^{1}$

1. Department of Cardiology, the Third Affiliated Hospital, Sun Yat-sen

University

2. Institute for Integrated Traditional Chinese and Western Medicine, Sun Yat-sen University
}

Objective To evaluate the expression of soluble vascular endothelial growth factor receptor 2 (sVEGFR2) in plasma of the hypertensive diabetic patients.

Methods In this cross-sectional study, fifty-seven cases were enrolled, which were divided into hypertensive diabetic group $(\mathrm{n}=31)$ 
and control group $(n=26)$. Blood pressure (BP) was obtained from each participant with mercury sphygmomanometer. The expressions of sVEGFR2 and superoxide dismutase (SOD) were measured by enzymelinked immunosorbent assay (ELISA). Serum lipid profile, glucose and glycosylated hemoglobin A1C $\left(\mathrm{GHbA}_{\mathrm{lc}}\right)$ levels were detected.

Result The levels of total cholesterol (TC), triglyceride (TG), low density lipoprotein cholesterol (LDL-C), body mass index (BMI), waist circumference (WI) were significantly higher in the hypertensive diabetic group than those in control group $(\mathrm{P}<0.05)$. The mean plasma levels of sVEGFR2 and SOD in hypertensive diabetic group were significantly decreased compared to that in the normal group $(\mathrm{P}<0.05)$. Moreover, there was a significantly positive correlation between SVEGFR2 and $\mathrm{SOD}$ in plasma in the whole study population $(\mathrm{r}=0.289, \mathrm{P}=0.029)$.

Conclusion Soluble VEGFR2 expression is decreased in hypertensive diabetic patients compared to that in the normal group. This study indicates that decreased SOD expression may contribute to the reduction of sVEGFR2 in hypertensive diabetic patients. As is wellknown, angiogenesis in these patients is impaired. So sVEGFR2 in plasma may be used as a biomarker to assess angiogenesis impairment in patients with hypertension and diabetes mellitus.

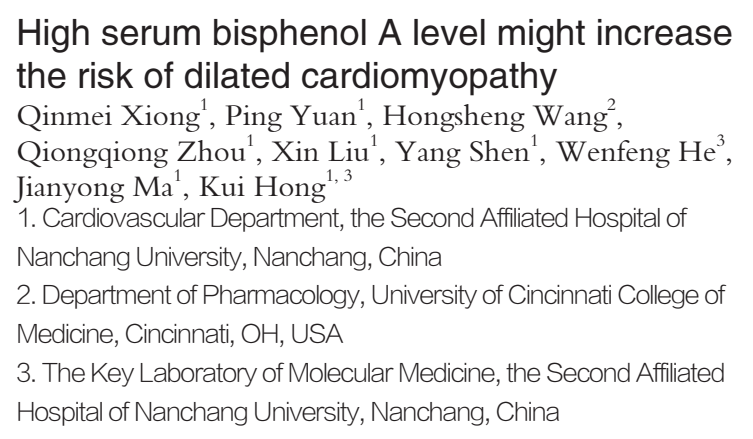

Objective Bisphenol A (BPA) is a widespread industrial chemical used in the synthesis of polycarbonate plastics and epoxy resins. Recent epidemiological investigations have disclosed the associations between BPA exposure and some heart diseases. Evidence from experimental animal studies suggests that BPA can act as an estrogenizing endocrine disruptor and impact cardiac calcium homeostasis. The present study aimed to determine serum BPA concentrations in patients with dilated cardiomyopathy (DCM) as well as the association between BPA and hormonal parameters compared to a control group.

Methods 88 DCM patients and 88 age- and gender-matched healthy controls were included from the second affiliated hospital of Nanchang University. Serum BPA levels and some hormonal parameters, including total testosterone (T), sex hormone-binding globulin (SHBG), estradiol (E2), Estrogen Receptor Alpha (ER $\alpha$ ), and Estrogen Receptor Beta (ER $\beta)$, were measured using corresponding ELISA Kits accordingly. Data analysis was performed using the statistical package SPSS version 18.0 (SPSS, Inc.).

Result BPA levels were significantly higher in the total DCM group compared with the controls $(6.9 \pm 2.7 \mathrm{ng} / \mathrm{ml}$ vs $3.8 \pm 1.9 \mathrm{ng} /$ $\mathrm{ml}, \mathrm{P}<0.001)$. Significant difference was also observed in SHBG and free androgen index (FAI) between DCM patients and controls, $76.9 \pm 30.9 \mathrm{nM} / \mathrm{L}$ vs $41.0 \pm 15.6 \mathrm{nM} / \mathrm{L}$ and $2.9 \pm 3.5$ vs $5.3 \pm 2.6, \mathrm{P}<$ 0.001 , respectively. There were similar tendency in the male and female subgroup. Mean T level was lower in DCM group than in control group, $540.8 \pm 186.0 \mathrm{pg} / \mathrm{ml}$ vs $656.3 \pm 112.9 \mathrm{pg} / \mathrm{ml}, \mathrm{P}<0.001$. Linear regression analysis has shown that increasing serum BPA levels were statistically significantly associated with increased SHBG levels. However, no statistic difference was noted for E2, ER $\alpha$ and ER $\beta$.

Conclusion Our findings firstly demonstrated that BPA exposure (evident in serum BPA levels) increased in DCM patients compared with healthy controls, while FAI and T levels decreased. Among all hormonal parameters, only SHBG presented a positive association with BPA. 


\section{Clinical Cardiology}

\author{
Effects of lipophilic statins for heart failure: a \\ meta-analysis of 13 randomized controlled \\ trials \\ Gang Liu, Xinxin Zheng, Xiaohong Huang \\ Department of Special Medical Treatment Center, State Key Laboratory \\ of Cardiovascular Disease, Fuwai Hospital, National Center for \\ Cardiovascular Diseases, Chinese Academy of Medical Sciences and \\ Peking Union Medical College
}

Background and Objective The effects of lipophilic statins in heart failure (HF) were controversial. The goal of the present study was to systematically review all randomized controlled trials evaluating the effects of lipophilic statins in patients with HF.

Methods We performed a comprehensive literature search to identify eligible trials that prospectively randomized patients with HF to lipophilic statins or control. Primary end points were all-cause mortality, cardiovascular mortality, hospitalization for worsening HF, left ventricular ejection fraction (LVEF), and low-density lipoprotein cholesterol. Risk ratios (RRs) and Weighted mean differences (WMDs) were calculated using fixed-effects models or randomeffects models.

Result A total of 13 randomized trials with 1532 subjects were included in this analysis. Ten trials randomized patients to atorvastatin, two to simvastatin, and one to pitavastatin. Overall, lipophilic statins significantly decreased all-cause mortality $(\mathrm{RR}=0.53, \mathrm{P}<0.001)$, cardiovascular mortality $(\mathrm{RR}=0.66, \mathrm{P}=0.04)$, and hospitalization for worsening $\mathrm{HF}(\mathrm{RR}=0.60, \mathrm{P}<0.001)$. Subgroup analyses showed that the effects of lipophilic statins in HF were not modified by age, baseline LVEF, and cause of HF. In addition, patients randomized to lipophilic statins had a significant increase in LVEF $(\mathrm{WMD}=3.91 \%, \mathrm{P}<0.001)$ and decrease in low-density lipoprotein cholesterol $(\mathrm{WMD}=0.90 \mathrm{mmol} /$ $\mathrm{L}, \mathrm{P}<0.001)$.

Conclusion It appears that further studies are needed to determine if lipophilic statins are beneficial for HF patients.

\section{Clinical characters and risk factors of left ventricular thrombus after acute myocardial infarction: a matched case-control study \\ Yuexin Jiang ${ }^{1}$, Linde Jing ${ }^{2}$, Youhong Jia ${ }^{1}$, Yishi $\mathrm{Li}^{1}$ \\ 1. Key Laboratory of Clinical Trial Research in Cardiovascular Drugs, Ministry of Health, State Key Laboratory of Cardiovascular Diseases, Fuwai Hospital, Chinese Academy of Medical Sciences and Peking Union Medical College \\ 2. QingHai Provincial People's Hospital}

Background and Ojective Left ventricular thrombus (LVT) is reported to be a common complication in acute myocardial infarction (AMI) patients. Its clinical importance lies in the risk of system embolism because LVT presents a source of emboli. The aim of this retrospective study was to evaluate the clinical characters and the risk factors of LVT after AMI.

Methods Ninety-six cases were identified from Fuwai Hospital's electronic medical records system from 2003 to 2012. Controls were 141 sex and age matched AMI patients without LVT during the same period. Conditional logistic regression (fitted by Cox model) was performed to identify the independent risk factors of LVT after AMI.

Result In a comparison with matched control cases, univariate analysis indicates that Killip classification on admission, anterior myocardial infarction (especially extensive anterior myocardial infarction), lower left ventricular ejection fraction (LVEF), severe regional wall motion abnormalities (RWMA), pericardial effusion, and left ventricular aneurysm are all related to LVT after AMI. While the independent risk factors after analyzed by conditional logistic regression are infarction locations, which are LVEF $(\mathrm{OR}=0.862$, 95\% CI: 0.782-0.951), severe RWMA (OR $=9.652,95 \%$ CI: 1.000 93.136), extensive anterior myocardial infarction (OR $=6.859,95 \%$ CI: $1.480-31.778)$ and left ventricular aneurysm $(\mathrm{OR}=5.008,95 \%$ CI: 1.007-24.897).

Conclusion This study suggests that lower LVEF, severe RWMA, extensive anterior myocardial infarction, as well as left ventricular aneurysm are independent risk factors of LVT after AMI.

\section{Comparition between statin-induced myopathy: clinical analysis of 160 cases of statin-induced myopathy \\ Yuexin Jiang ${ }^{1}$, Ying Lou ${ }^{1}$, Yuqing Liu ${ }^{1}$, Liming Zhang ${ }^{2}$, Yingqun Zhou ${ }^{2}$, Yishi $\mathrm{Li}^{1}$ \\ 1. Key Laboratory of Clinical Trial Research in Cardiovascular Drugs, Ministry of Health, Fuwai Hospital, Chinese Academy of Medical \\ Sciences and Peking Union Medical College \\ 2. Beijing Center for ADR Monitoring}

Objective To analyze the clinical outcomes of statin-induced myopathy, and to provide reference for safe drug use in clinical practice.

Methods The reports of statin-induced myopathy were reported as adverse drug reaction (ADR) to the Beijing Center for ADR Monitoring during January 2007 to December 2012. The above reports are defined as the general level of myopathy group and rhabdomyolysis group, according to the severity of ADR. The clinical outcomes medication history, sequelae were discussed. Differences of these issues were also analyzed.

Result The average age of the 160 statin-induced myopathy cases were $(64.22 \pm 13.55)$ yrs, $51.2 \%$ of which were male $(n=82)$. The ADR occurred from the first medication time to 4 years after medication. Observed clinical features were myalgia, Myositis, Symptomless creatine kinase (CK) elevation or rhabdomyolysis. 54 rhabdomyolysis cases $(33.8 \%)$ were identified from the 160 cases. Compared with the general level of myopathy group (160 cases), the average age were $(68.54 \pm 15.41)$ yrs and $(62.02 \pm 12.41)$ yrs, respectively $(\mathrm{P}=0.004)$, while the percentage of male were $46.3 \%$ and $53.8 \%(\mathrm{P}=\mathrm{NS}) .44 .4 \%$ of rhabdomyolysis were in a high dose statin treatment, while the occupation of that in normal myopathy was $16.5 \%\left(\chi^{2}=16.45, \mathrm{P}<0.001\right)$. Moreover, the ratio of simvastatin taken in the two group were $70.4 \%$ and $32.1 \%$, respectively $\left(\chi^{2}=21.20, \mathrm{P}<0.001\right)$.

Conclusion Rhabdomyolysis may more likely occur when patients are older, taking high dose statin or simvastatin. Clinicians should inform patients who intent to take statins that potential ADR may occur during their medication and that they need to seek for immediate medical help when those symptoms appear. 


\section{Effect of continuous positive airway pressure on nocturia in obstructive sleep apnea patients with coronary heart disease \\ Zhiwei Huang, Zhihong Liu, Zhihui Zhao, Qin Luo, Qing Zhao State Key Laboratory of Cardiovascular Disease, Fuwai Hospital, National Center for Cardiovascular Diseases, Chinese Academy of Medical Sciences and Peking Union Medical College}

Objective To investigate the effect of continuous positive airway pressure (CPAP) on nocturia in obstructive sleep apnea (OSA) patients with coronary heart disease (CHD).

Methods This prospective clinical study recruited CHD patients referred to the Fuwai Sleep Centre with diagnosed moderate to severe OSA. Patients were randomized to controls or CPAP group. Nocturia was assessed at four time points: baseline, 1 month, 3 months and 6 months.

Result 95 patients completed the study. CPAP group and controls had similar characteristics at baseline. The mean age was $(60 \pm 5)$ yrs, body mass index was $(29 \pm 3) \mathrm{kg} / \mathrm{m}^{2}$, and apnea-hypopnea index (AHI) was $(29.5 \pm 11.0)$ events/h. The mean time of CPAP therapy was $(4.9 \pm$ 1.3) $\mathrm{h} /$ night. The number of nocturia was similar between CPAP group and controls [CPAP, $(2.7 \pm 1.2)$ time/night vs $(2.8 \pm 1.3)$ times/night; P $=0.852]$ before treatment. The mean number of awakenings to void had a significant difference between groups [CPAP, $(0.8 \pm 0.5)$ time/night vs $(2.7 \pm 1.5)$ times/night; $\mathrm{P}<0.001)$ after treatment. Correlations were found in the number of nocturia before treatment with the $\mathrm{AHI}(\mathrm{r}=0.36$, $\mathrm{P}=0.02)$.

Conclusion CPAP treatment significantly reduced the frequency of OSA-related nocturia in CHD patients and improved life of quality.

\section{Comparison of transplantation of bone marrow- derived stem cells, adipose-derived stem cells and endometrium-derived stem cells in the infarcted heart \\ Xinyang Hu, Kan Wang, Jianan Wang \\ 1. Department of Cardiology, Second Affliated Hospital, Zhejiang \\ University School of Medicine; \\ 2. Key lab of cardiovascular disease, Second Affliated Hospital, Zhejiang University School of Medicine}

Background A variety of adult stem cells have been transplanted into the infarcted heart to cure myocardial infarction (MI), however, comparative studies are lacking to show more suitable source of cells for transplantation. Mesenchymal stem cells hold promise for myocardial re generation therapy. Derivation of these cells from the endometrium tissue might be easier compared to bone marrow and adipose tissue. However, the in vivo fate of endometrium stem cells (EnSCs) in the infarcted heart has never been compared directly to mesenchymal cells derived from bone marrow (BMMSCs) and adipose tissue (AdMSCs).

Methods EnSCs, AdMSCs and BMMSCs were isolated from healthy donors were characterized using flow cytometry for surface markers identification and microscopy for cell morphology. They were characterized with $\beta$-actin promoter driving firefly luciferase and green fluorescent protein (Fluc-GFP) double fusion reporter gene, and were characterized using flow cytometry, bioluminescence imaging (BLI) and luminometry. Cell proliferation was tested by CCK-8 kit, colony forming unit (CFU) was stained by crystal violet staining and apoptosis ratio were detected by TUNEL assay. Rat ( $\mathrm{n}=8 /$ group) underwent myocardial infarction followed by intramyocardial injection of $5 \times 10^{5}$ EnSCs, AdMSCs and BMMSCs, or saline (negative control). Cell survival was measured using BLI for 6 weeks and cardiac function was monitored by echocardiography and hemodynamics analysis. Ventricular morphology was assessed using histology.

Result EnSCs, AdMSCs and BMMSCs were CD29+, CD90+, CD105+, shared similar morphology, but EnSCs had best proliferation, colony-forming and anti-apoptosis activity of 3 types of MSCs. Cells expressed Fluc reporter genes in a number-dependent fashion, as confirmed by luminometry. After cardiac transplantation, transplantation of EnSCs was better capable of preserving ventricular function and dimensions than others, as confirmed by echo test, PV-loops and histology.

Conclusion This is the first study comparing the in vitro and in vivo behavior of 3 types of MSCs in the infarcted heart. AdMSCs an d BMMSCs do not tolerate well in the cardiac environment, resulting in more cell death andworse cardiac function than EnSCs groups.

\section{Pretreated MSCs with a growth hormone releasing hormone agonist enhanced their therapeutic properties of angiogenesis for hindlimb ischemia in mice \\ Xiangyang Xia, Qunchao Ma, Quanwei Tao, Hong Yu \\ 1. Division of Cardiology, Key Division of Ministry of Health, Second \\ Affliated Hospital, Zhejiang University School of Medicine \\ 2. Provincial Key Laboratory of Cardiovascular Research, Second \\ Affliated Hospital, Zhejiang University School of Medicine}

Objective Growth hormone releasing hormone (GHRH) is an endocrine hormone produced by pituitary gland and stored and released to the circulatory system. GHRH receptor has been confirmed to exist on cardiomyocytes and mesenchymal stem cells except on pituitary cells. Cell therapy with MSC has been widely tested in clinic practice while still draws a relative low efficiency mainly by the low survival rate. A GHRH analogue JI-34 is better at the stability and stimulating efficiency through by less degradation compared with physiological GHRH. Therefore, to testify a newly use of GHRH agonist JI-34 whether it can enhance the survival of MSC will be attractive.

Methods The survival, viability, migration angiogenesis ability and anti-apoptosis rate of MSC were compared between two groups of mice MSC pretreated with JI-34 or not. CCK-8, transwell assay, Western blot, quantitive RT-PCR, western blot, tube formation assay and TUNEL staining kit were used in this paper. In vivo study we revealed the angiogenesis ability of pretreated MSC by cell injection into ischemic hindlimb of 6 weeks old C57/BL6 mice.

Result After the super agonist of GHRH pretreatment for 24hours, MSCs' viability had increased by $(23 \pm 0.5) \%$ compared with controls in normoxic condition and increased $(26 \pm 1) \%$ in hypoxia conditions $\left(0.5 \% \mathrm{O}_{2}\right)$. Cell apoptosis rate decreased to $(31.0$ $\pm 1.2) \%$ in JI-34 group compared to hypoxia and serum deprivation group $(61 \pm 2.3) \%$. MSC migration capacity can also be raised (27 $\pm 2) \%, P<0.05$. Similarly, the supernatants of MSC pretreated with JI-34 co-cultured with Human Umbilical Vein Endothelial Cells (HUVEC) depicted a higher possibility of tube formation with longer tube length $(754 \pm 37) \mathrm{mm}$ than control ones $(580 \pm 40) \mathrm{mm}(\mathrm{P}<$ 0.05 ). RT-PCR and Western Blot showed that a highly augment of pro-angiogenic factors of VEGF-A and SDF-1 with 8 fold and 7.5 fold increase relating to control respectively. Western blot showed 
that STAT3 and other signaling pathway molecules acted as the switch could be activated after JI-34 treatment and these results could not be observed by adding specific inhibitor of STAT3.Ultrasound Doppler displayed that mice injected with treated GFP labeled-MSC gained a better recovery of blood perfusion in day 3 after femoral artery ligation surgery and sustained to day 14 than MSC and PBS group $(n=12)$. Immunofluorescence of CD31, a-SMA, TUNEL costaining with GFP and immunohistochemistry also supported the results that treated with JI-34, MSC increased vascularation and angiogenesis of mice hindlimb ischemia. Using male MSC injected into female mice to detect Sry gene expression we demonstrated a better survival of injected cells after JI-34 pretreatment in day 3 and day 7 .

Conclusion The agonist of GHRH can activate the receptor of MSC to enhance its viability and paracrine effect through STAT3 signaling pathway to promote the angiogenesis of ischemic hindlimb of mice.

\section{The prevalence and prognosis of extreme left ventricular hypertrophic cardiomyopathy in China \\ Xiying Guo ${ }^{1}$, Shihua Zhao ${ }^{2}$, Fujian Duan ${ }^{3}$, Zhimin Wang ${ }^{3}$, Yinjian Yang ${ }^{1}$, Shuoyan $\mathrm{An}^{1}$, Yishi $\mathrm{Li}^{1}$, Chaomei Fan ${ }^{1}$ State Key Laboratory of Cardiovascular Disease, Fuwai Hospital, National Center for Cardiovascular Disease Chinese Academy of \\ Medical Sciences and Peking Union Medical College \\ 1.Key Laboratory of Clinical Trial Research in Cardiovascular Drugs, \\ 2. Department of Radiology \\ 3. Department of Ultrasound}

Objective Extreme left ventricular hypertrophy (LVH) is a relatively rare form in patients with hypertrophic cardiomyopathy (HCM) and has been associated with a poor prognosis and an obvious risk factor of sudden cardiac death in previous study, however relevant data have not been revealed in China.This study was design to investigate the prevalence, clinical characteristics, hemodynamic properties, and longterm outcomes in Chinese HCM patients with extreme LVH.

Methods A total of 194 consecutive HCM patients with extreme LVH (maximal left ventricular wall thickness $\geq 30 \mathrm{~mm}$ at end-diastolic period in 2-dimensional echocardiography) in Fuwai hospital from 2002-2013 were enrolled in this study. All patients underwent history taking, physical examination, electrocardiography, echocardiography and Holter analysis.Magnetic resonance imaging (MRI) was used for better evaluation of ventricular morphology and function. Survival data were collected at subsequent clinic visits or by communication with patients. Clinical features, cardiovascular mortality and morbidity were analyzed. The survival estimates were calculated using the Kaplan-Meier method and log-rank test. Risk factors for cardiovascular death and events were determined by multivariate Cox proportional hazards regression models. For all texts, $\mathrm{P}$ value $<0.05$ was considered a significant difference.

Result The 194 patients with extreme LVH represented $8.0 \%$ of all $2413 \mathrm{HCM}$ patients. The average age of diagnosis was $(38.5 \pm 15) \mathrm{yrs}$ and with $75.8 \%$ males involved. 184 patients had a mean follow-up of $(7.8 \pm 4.4)$ yrs. 18 patients $(10 \%)$ experienced cardiovascular mortality including 9 patients with SCD, 3 patients with heart failure-related death and 6 patients with appropriate ICD therapies for ventricular fibrillation. $48.9 \%$ patients had 1 or more cardiovascular events of which syncope $(19 \%)$ was the most common. The multivariate cox regression analysis indicated 3 independent clinical predictors of cardiovascular death including age $\leq 18$ years old with a hazard ratio of 8.93 (95\% CI: 1.945 12.730; $\mathrm{P}=0.0008), \mathrm{LV}$ end diastolic dimension $\geq 45 \mathrm{~mm}$ with a hazard ratio of 1.16 (95\% CI: $1.044-1.283 ; \mathrm{P}=0.0056)$ and New York Heart Association (NYHA) class $\geq$ III with a hazard ratio of $2.56(95 \% \mathrm{CI}$ : 1.104-5.957; $\mathrm{P}=0.0285)$. Pre-syncope syndrome was an independent predictors of cardiovascular morbidity with a hazard ratio of $1.56(95 \%$ CI: $1.037-2.341 ; \mathrm{P}=0.0329$ ).

Conclusion The prevalence of extreme LVH in patients with HCM is relatively low, however $10 \%$ and $48.9 \%$ patients may develop cardiovascular death or events respectively in China. Age $\leq 18$ yrs, LV end diastolic dimension exceeding $45 \mathrm{~mm}$ and NYHA class $\geq \mathrm{III}$ are the predictors for cardiovascular death in HCM patients with extreme LVH. Timely recognition of such patients for early consideration of appropriate management strategies is important.

\section{Variations in the management and outcome of patients with acute myocardial infarction in China \\ Yuejin Yang, Jingang Yang, Xiaojin Gao, Haiyan Xu, Yuan Wu, Lei Song, Wei Li, Yang Wang, Xinran Tang, Li Chen, Yi Sun, Rui Fu, Yunqing Ye, Hui Sun, \\ Xinxin Yan, Qiuting Dong, Chen Jin \\ State Key Laboratory of Cardiovascular Disease, Fuwai Hospital, National Center for Cardiovascular Diseases, Chinese Academy of Medical Sciences and Peking UnionMedical College}

Objective Detailed data on patients admitted for acute myocardial infarction (AMI) in China are lacking. We sought to describe the variation in the management and outcome of patients withAMI among different parts of China and between tertiary and secondaryhospitals using data from China Acute Myocardial Infarction (CAMI) Registry.

Methods Among 30 provinces, municipalities or autonomous districts in China, at least one tertiary and secondaryhospital was selected. Between January 2013 and March 2014, we consecutively registered all patients with AMI within 7 days after the onset of AMI in 108 participating medical institutions. A standardized case report form was used to register all the patients. Data on baseline characteristics, type of AMI, management, and complications were recorded using a dedicated electronic form. In every province or municipality, we have selected one provincial-level academic hospital, one to three district-level hospitals, one to three county-level hospitals. These three levels represent typical Chinese governmental and administrative models in China.

Result Overall, 16023 patients [mean age $(62 \pm 15)$ yrs; $26 \%$ women] were included in the study. 11769 (73.5\%) patients had STsegment elevation myocardial infarction (STEMI). Primary percutaneous coronary interventions (PCI) were performed in 4987 (42.9\%) of STEMI patients, and $364(8.8 \%)$ of non-ST elevation myocardial infarction (NSTEMI) patients. Among patients with STEMI, less patients in county-level hospitals $(43.0 \%)$ had reperfusion therapy, compared to district-level hospitals (49.7\%) and provincial-level hospitals (62.4\%); The highest use of primary PCI was found provincial-level hospitals $(59.4 \%)$ and the lowest in county-level hospitals (15.2\%). Compared with secondary hospitals, tertiary hospitals performed more primary PCI $(49.0 \%$ vs $20.1 \%, \mathrm{P}<0.001)$ and less fibrinolysis $(7.1 \%$ vs $25.0 \%$, $\mathrm{P}<0.001)$. At discharge, there was no difference regarding aspirin, thienopyridines and statin prescription between tertiary and secondary hospitals and among county-level, district-level and provincial-level hospitals. The inhospital mortality rate in secondary hospitals was 
doubled (6.6\%) compared to the tertiary hospitals (3.5\%), and was lower in provincial-level hospitals $(2.3 \%)$ than that in district-level hosptals (4.3\%) and county-level hospitals (8.4\%).

Conclusion: The variation in the management and outcome in patients with AMI by region and by type of hospitals reported in this study in Chinamerit further investigation to reduce the observed disparities

\section{The effects of anticoagulant therapy on coagulant state and platelet function following transcatheter closure of atrial septal defect \\ Guocai Chen \\ Cardiovascular Department of Xinqiao Hospital, Third Military Medical University}

Background Several studies have demonstrated coagulant system was activated after transcatheter closure of ASD, but changes of platelet function still remain controversial. Currently, it is not clear which anticoagulant regiment is more effective to prevent thrombosis and embolic events after device implantion. This study was to compare the effects of three anticoagulant regiments on coagulant state and platelet function following transcatheter closure of atrial septal defect (ASD).

Methods A total 138 patients who underwent transcatheter closure of ASD were randomized into three groups to receive different anticoagulant therapy: unfractionated heparin (UFH) for $24 \mathrm{~h}$, low molecular weight heparin (LMWH) for $24 \mathrm{~h}$, and LMWH for $72 \mathrm{~h}$ (pLMWH). Aspirin was given to all patients for 6 months after intervention. The laboratory measurements included betathromboglobulin $(\beta-\mathrm{TG})$, platelet factor $4(\mathrm{PF} 4)$ and prothrombin fragment $1+2(F 1+2)$ which were done before intervention as baseline, immediately after, and day 1, 2, 3, 7, 30 and 90 after intervention.

ResultIn 3 groups, $\beta$-TG, PF4 and F1+2 elevated immediately after implantation procedure. $\beta$-TG and PF4 declined slightly on day 1 and 2 , and rose to a highest level on day 3 , then fell down to baseline on day 7 . The F1+2 gradually returned to baseline on day 90 . However, the F1+2 in pLMWH group was markedly lower than that in UFH and LMWH groups on day 3. No thrombo-embolic events were noted during followup.

Conclusion Transcatheter closure of ASD was associated with significant activation of both platelets and coagulation. These findings support an antithrombotic regiment after procedure including anticoagulant and antiplatelet agents.The F1+2 level fell down earlier in pLMWH group. However, there were no differences of clinical outcomes among three groups on day 90 after intervention. Therefore, a larger size and longer follow-up study is needed to further clarify this issue.

\section{Coronary risk factors profiling in chinese patients with acute myocardial infarction Xiaojin Gao, Yuejin Yang, Jingang Yang, Haiyan Xu, Yuan Wu, Wei Li, Yang Wang, xinran Tang, Yi Sun, Lei Song, Rui Fu, Yunqing Ye, Hui Sun, Xinxin Yan, Qiuting Dong, Peiyuan He, Chen Jin State Key Laboratory of Cardiovascular Disease, Fuwai Hospital, National Center for Cardiovascular Diseases, Chinese Academy of Medical Sciences and Peking Union Medical College}

Background and Objective Recently, there has been an increase in the prevalence of coronary risk factors, which are associated with an increased incidence of acute myocardial infarction (AMI) in China. However, few large-scale studies provided detailed information of risk factors in Chinese patients with AMI. This study aimed to determine the frequency of risk factors in Chinese patients presenting with AMI.

Methods Among 31 provinces, municipalities or autonomous districts in China, at least one tertiary and secondary hospital was selected.From January 2013 to November 2013, 10474 AMI patients (7784 males, 2690 females) within 7 days who were admitted to 93 hospitals and with complete data for statistical analyses were divided into younger ( $\leq 55 \mathrm{yrs}$; mean age $47.6 \mathrm{yrs} ; \mathrm{n}=2970 ; 28.4 \%$ ) and older $(>55$ yrs; mean age 67.7 yrs; $n=7504 ; 71.6 \%$ ) patients. A standardized case report form was used to register all the patients. Data were centralized at the National Center of Cardiovascular Disease.

Result ST-elevation myocardial infarction (STEMI) was diagnosed in $7442(71.1 \%)$ patients, and non-STEMI in 3032 $(28.9 \%)$. Smoking was documented in 5737 (54.8\%) patients, hypertension in $5327(50.9 \%)$, diabetes in 2031 (19.4\%) and a family history of premature CAD was recorded in $383(3.7 \%)$ patients. $3837(36.6 \%)$ patients were overweight $(24 \mathrm{~kg} /$ $\left.\mathrm{m}^{2} \leq \mathrm{BMI}<28 \mathrm{~kg} / \mathrm{m}^{2}\right)$ while $1010(9.6 \%)$ were obese (BMI $\geq$ $\left.28 \mathrm{~kg} / \mathrm{m}^{2}\right) .2238(21.4 \%)$ patients were active physically. The risk factors that were more prevalent in the younger patients than in the older patients included: male, smoking, hyperlipemia and family history of premature CAD. $85.3 \%(n=8933)$ of all patients had at least one of cardiovascular risk factors (hypertension, smoking, diabetes, overweight/obesity and hyperlipemia) and only $14.7 \%$ (n $=1544$ ) of all had $\geq 3$ risk factors. The rate of prescriptions of antihypertensive in hypertensive patients was $44.7 \%$ and the rate of antihyperlipidemia drugs in patients with diabetes was $2.7 \%$.

Conclusion In this study, 8933 out of 10474 patients with AMI have at least one of conventional cardiovascular risk factors. Smoking, hypertension and overweight/obesity are leading risk factors.

\section{Clinical manifestations and long-term outcome for patients with takayasu's arteritis in China Lirui Yang ${ }^{1}$, Huimin Zhang ${ }^{1}$, Xiongjing Jiang ${ }^{1}$, \\ Yubao Zou ${ }^{1}$, Fang Qin ${ }^{1}$, Yaxin Liu ${ }^{1}$, Haiying Wu ${ }^{1}$, Lei Song ${ }^{1}$, Lianjun $\mathrm{Xu}^{2}$, Ting Guan ${ }^{3}$, Xianliang Zhou ${ }^{1}$, Jin Bian ${ }^{1}$, \\ Rutai Hui ${ }^{1}$, Deyu Zheng \\ 1. Hypertension Division, State Key Laboratory of Cardiovascular \\ Disease, Fuwai Hospital, National Center for Cardiovascular Diseases, Chinese Academy of Medical Sciences and Peking Union Medical College \\ 2. Cardiology, State Key Laboratory of Cardiovascular Disease, Fuwai Hospital, National Center for Cardiovascular Diseases, Chinese Academy of Medical Sciences and Peking Union Medical College 3. Intensive Care Unit, State Key Laboratory of Cardiovascular Disease, Fuwai Hospital, National Center for Cardiovascular Diseases, Chinese Academy of Medical Sciences and Peking Union Medical College}

Objective To describe a large cohort of patients with Takayasu's arteritis in China.

Methods We retrospectively analyzed 566 patients hospitalized in Fuwai Hospital between 2002 and 2013. Clinical characteristics, laboratory findings, angiographic features, treatment and long-term outcomes were collected.

Result The female to male ratio was 3.8: 1, and the mean age of onset was $(28.9 \pm 12.0)$ yrs. The most common inflammatory symptom, initial symptom and coexisting disease was fever (52, 
9.2\%), dizziness $(214,37.8 \%)$ and hypertension (392, 69.3\%), respectively. A total of $129(22.8 \%)$ of patients were diagnosed to be in the active phase. Secondary hypertension was detected in 330 $(58.3 \%)$ patients and partly associated with lesions of renal artery $(212,37.5 \%)$ or constriction of aorta $(118,20.8 \%)$. Pulmonary artery, coronary artery and aortic regurgitation was found in $83(14.7 \%)$, $66(11.7 \%)$ and $168(34.1 \%)$ patients, respectively. Elevation of erythrocyte sedimentation rate was observed in $131(23.1 \%)$ patients. Treatment included drugs, interventional therapy, autologous blood vessel transplant, artificial blood vessel transplant and aortic valve replacement. During a mean follow-up of $(5.0 \pm 0.2)$ yrs, 32 patients had died, which included 1 patient who had a sudden death during coronary angiography. Secondary hypertension, major complication and disease of progressive course were significant markers of prognosis.

Conclusion Hypertension, instead of fever, is the leading cause of patients with Takayasu's arteritis to see a doctor in China. Secondary hypertension, major complication and disease of progressive course are statistically significant predictors of survival. Due to cardiovascular events led by the disease, early diagnosis and treatment is urgent.

\section{Study on the influence of recipient gender on mid-term survival rate of heart transplant patients \\ Liying Shi, Jie Huang, Zhongkai Liao State Key Laboratory of Cardiovascular Disease, Fuwai Hospital, National Center for Cardiovascular Diseases, Chinese Academy of Medical Sciences and Peking Union Medical College}

Objective It was reported that female heart transplant (HT) recipients had a poor survival than male. In this single-center retrospective study, the effect of recipient gender on HT mid-term survival is evaluated.

Methods Study the clinical data and follow-up information of 398 HT patients in Fuwai hospital from June 2004 to December 2013. The patients were divided into two groups by gender. SPSS 17.0 was used for statistical analysis, which included the basic preoperative features and risk factors affecting HT survival reported by International Society for Heart and Lung Transplantation. Use multivariate COX regression analysis to analyze the risk factors. The mid-term survival rates of the two groups were analyzed by Kaplan-Meier method and compared by log-rank test.

Result Among the 398 patients, females accounted for $17.6 \%$ ( $72.5 \%$ with prior pregnancy). Compared results of male and female groups showed significant differences $(\mathrm{P}<0.05)$ in the recipient age $[(45.3 \pm 13.1)$ yrs vs $(41.3 \pm 15.3)$ yrs $)$, body mass index [(22.31 \pm 3.99$)$ $\mathrm{kg} / \mathrm{m}^{2}$ vs $\left.\left.(19.65 \pm 3.11) \mathrm{kg} / \mathrm{m}^{2}\right)\right]$, history of diabetes $(17.1 \%$ vs $5.7 \%)$, cigarette history $(60.4 \%$ vs $2.9 \%)$, preoperative mean pulmonary artery pressure $[(33.96 \pm 10.85) \mathrm{mm} \mathrm{Hg}$ vs $(29.76 \pm 11.70) \mathrm{mm} \mathrm{Hg})]$, total bilirubin level $[(31.46 \pm 19.38) \mu \mathrm{mol} / \mathrm{L}$ vs $(27.22 \pm 15.44) \mu \mathrm{mol} / \mathrm{L})$, NTproBNP levels [(2749.66 \pm 1493.10$) \mathrm{pg} / \mathrm{ml}$ vs $(3237.61 \pm 1789.37) \mathrm{pg} /$ $\mathrm{ml}$ ] and primary cardiac etiology: cardiomyopathy $(73.5 \%$ vs $90.0 \%)$. No significant difference $(\mathrm{P}>0.05)$ was found in preoperative creatinine clearance rate and PRA positive rate. The median follow-up is 1294.5 days. Multivariate COX regression analysis showed that preoperative total bilirubin was the factor associated with HT survival $(\mathrm{RR}=1.028$, 95\% CI: $1.010-1.046 ; \mathrm{P}=0.002$ ). There was no statistical difference between male and female survival rates: 1 year: $94.7 \%$ vs $97.1 \% ; 3$ year: $91.9 \%$ vs $93.2 \%$; 5 year: $88.6 \%$ vs $90.1 \%$.

Conclusion The present data demonstrate that though female patients have the advantage of lower preoperative total bilirubin level, gender of HT recipients doesn't have a significant effect on mediumterm survival rates.

\section{The clinical application of TEG in monitoring clotting in patients with coronary heart disease \\ Ying Guo, Youhong Jia \\ Key Laboratory of Clinical Trial Research in Cardiovascular Drugs, State \\ Key Laboratory of Cardiovascular Disease, Fuwai Hospital, National \\ Center for Cardiovascular Diseases, Chinese Academy of Medical \\ Sciences and Peking Union Medical College}

Objective In order to improve clinical practice and awareness, we analyze clinical application of thromboelastography (TEG) in monitoring clotting in patients with coronary heart disease.

Method 168 patients were randomly selected to investigate the application value of TEG in monitoring clotting in patients with coronary heart disease. Patients were divided into acute coronary syndrome (ACS) group (52 cases) and chronic coronary artery disease (CAD) group (116 cases). 91 patients in these 168 patients had undergone percutaneous coronary intervention (PCI). According to adenosine diphosphate (ADP) induced inhibition rates, $91 \mathrm{PCI}$ patients were divided into $\mathrm{ADP}<50 \%$ for non-effect group (group A) (41 cases), $50 \% \leq \mathrm{ADP} \leq 75 \%$ for effect group (group B) (22 cases), $>75 \%$ for better inhibitor group (group C) (28 cases). General condition and major adverse cardiac events (MACE) were recorded after 6 months.

Result The use of stentings, aspirin and clopidogrel are more common in ACS group compared with CAD group. Larger ADP values $[(63.98 \pm 28.34) \%$ vs $(50.57 \pm 26.77) \%]$ are found in ACS group than CAD group, differences are statistically significant $(\mathrm{P}<0.01)$. ACS group have $8 \mathrm{MACE}(15.38 \%)$ and $\mathrm{CAD}$ group have $16 \mathrm{MACE}$ (13.79\%). PCI patients have 15 MACE after 6 months, of which group A have 8 MACE (19.51\%), group B have 6 MACE (27.27\%), and group $\mathrm{C}$ have $1 \mathrm{MACE}(3.57 \%)$. MACE rates in the three groups are group $\mathrm{B}$ $>$ group $\mathrm{A}>$ group $\mathrm{C}$, differences are statistically significant $(\mathrm{P}<0.05)$.

Conclusion Patients with ACS have higher ADP inhibition rates after antiplatelet therapy. Patients with coronary heart disease need to be given adequate antiplatelet therapy. ADP inhibition rates measured by TEG can be helpful for antiplatelet therapy and postoperative prognosis in PCI patients.

\section{Early development of xanthoma and coronary disease in a young female with homozygous familial hypercholesterolemia \\ Xin Zhao, Liping Bu, Shengmei Qin, Dehong Kong, \\ Bing Fan, Junbo Ge \\ Shanghai Institute of Cardiovascular Diseases, Department of \\ Cardiology, Zhongshan Hospital, Fudan University}

Familial hypercholesterolemia has extremely high level of lowdensity lipoprotein cholesterol (LDL-C), high risk of premature aortic valvular stenosis and coronary atherosclerosis, as well as remarkable xanthoma.

A 17-year-old woman was admitted to emergency with repeated chest pain or discomfort for 4 years, worsen and became cough for 1 week. Hypercholesterolemia was found at her 2 years old but without management of dyslipidemias. Around 2005, the patient arosexanthoma. In 2009, she developed chest tightness and syncope 
after activities. Although echocardiograph indicated aortic stenosis and mitral regurgitation, the patient didn't receive treatment. In August, 2013, a coronary angiogram showed: irregularity but no stenosis in LM; diffuse stenosis in proximal and middle segment of LAD, as well as in proximal and middle segment of LCX; coarctation of ascending aorta, and stenosis at the RCA opening and proximal segment; stenosis at both carotid arteries; massive diastolic regurgitation of contrast medium to apex region of left ventricle. Under 4-month-medication of rosuvastatin, ezetimibe and clopidogrel, her symptoms still fluctuated. One week ago, she became cough, fever and inability to lie flat. She was hypersensitive to aspirin. Her family history cannot be traced since the girl was adopted and no one knew about her biological parents.

Skin and tendon xanthomas which are striking in hip, elbows, wrists, knees and ankles. Mild edema in lower limbs. A 3/6 systolic ejection murmur at the apex region and aortic region.

Lipid profile (Before therapy. August, 2013): LDL-C: $19.44 \mathrm{mmol} /$ L; TG: $1.60 \mathrm{mmol} / \mathrm{L}$; TC: $21.48 \mathrm{mmol} / \mathrm{L}$; HDL-C: $0.88 \mathrm{mmol} / \mathrm{L}$. Lipid profile (After therapy of rosuvastatin and ezetimibe. January, 2014.): LDL-C: $11.67 \mathrm{mmol} / \mathrm{L}$; TG: $1.93 \mathrm{mmol} / \mathrm{L}$; TC: $12.90 \mathrm{mmol} / \mathrm{L}$; HDL-C: $0.35 \mathrm{mmol} / \mathrm{L}$.

A homozygous c.541T $>$ C mutation of exon 3, LDLR gene, which was proven by method of Sanger sequence. It is a novel LDLR gene mutation based on LOVD system.

\section{Hypothyroidism predicts the mortality of idiopathic dilated cardiomyopathy \\ Wenyao Wang, Yida Tang, Mu Mu, Xuan Zhang, \\ Min Yang, Xun Yuan, Kuo Zhang, Yuejin Yang \\ State Key Laboratory of Cardiovascular Disease, Fuwai Hospital, \\ National Center for Cardiovascular Diseases, Chinese Academy of \\ Medical Sciences and Peking Union Medical College}

Objective Previous studies claiming the relationship between thyroid dysfunction and poor prognosis of heart failure (HF) had a major limitation that they included patients with different etiology. With full information of thyroid function profile from four hundred and fifty eight consecutive patients with idiopathic dilated cardiomyopathy, we tested the hypothesis that thyroid status can independently influence the mortality in patients with HF.

Methods and result The original cohort consisted of 572 consecutive patients with idiopathic dilated cardiomyopathy (IDCM), and 458 patients remained at the end of follow-up. All the patients took thyroid function test and other regular examinations in hospital. The risk of mortality was evaluated based on $\mathrm{FT}_{3}, \mathrm{TSH}$, and the whole thyroid function profile, respectively.The most frequent thyroid dysfunction was subclinical hypothyroidism $(n=41)$, followed by subclinical hyperthyroidism $(\mathrm{n}=35)$, euthyroid sick syndrome $(\mathrm{n}=$ 17), and hypothyroidism $(n=12)$. Logistic analysis showed log-TSH and $\mathrm{FT}_{3}$ as independent predictors of exacerbated cardiac function (NYHA III-IV vs NYHA I-II). During the follow-up (17 \pm 8 ) months, there were 111 cumulative deaths. Hypothyroidism was the strongest predictor of mortality ( $\mathrm{HR}=4.189,95 \%$ CI: $2.118-8.283)$, followed by low- $\mathrm{T}_{3}$ syndrome $(\mathrm{HR}=3.147,95 \% \mathrm{CI}: 1.558-6.355)$ and subclinical hypothyroidism ( $\mathrm{HR}=2.869,95 \% \mathrm{CI}: 1.817-4.532)$. Subclinical hyperthyroidism showed no significant impact.

Conclusion We found clear association between an increased risk of death in HF caused by IDCM and thyroid dysfunction. These results suggest that monitoring thyroid function in HF patients is necessary and further study about treatment is warranted.

\section{The effect of Shexiangbaoxinwan on ventricle remodeling, brain natriuretic peptide and C-response protein in patients with acute coronary syndrome \\ Jinliang Chen ${ }^{1,2}$, Jing Zhang ${ }^{1,2}$, Baomin Jia ${ }^{1}$, Xingang Mo ${ }^{1}$ 1. Department of Cardiology, No. 281 Hospital of Beijing Military Region \\ 2. Department of Cardiology, The People`s Hospital of Qinhuangdao City}

Objective To study the effect of shexiangbaoxinwan on ventricle demodeling and change brain natriuretic peptide (BNP) and c-response protein (CRP) in patients with acute coronary syndrome.

Methods 126 patients with acute coronary syndrome were divided randomly into two groups, one was control group treated with regular treatment, another was treatment group treated with shexiangbaoxinwan on the basis of above regular treatment. All the patients were followed up for 6 months and observed the effect of the ventricle demodeling, change of BNP, CRP and Blood lipid

Result After shexiangbaoxinwan treatment, left ventricular ejection fraction (LVEF) and high density lipoprotein cholesterol is obviously improved than control group $(\mathrm{P}<0.05)$, left ventricular end systolic diameter (LVESD) and left ventricular end diastolic diameter (LVEDD) was reductive than control group $(\mathrm{P}<0.05)$. BNP, CRP, total cholesterol and triglyceride levels both decreased significantly $(\mathrm{P}<0.01)$, in treatment group, BNP, CRP, total cholesterol and triglyceried levels more decreased significantly $(\mathrm{P}<0.05)$.

Conclusion Shexiangbaoxinwan can be the effective drug used in clinic for treating acute coronary syndrome.

\section{Diffuse aneurysmal and obstructive coronary artery disease, and concomitant atrial septal defect and anomalous origin of the right coronary artery \\ Meng Zhang, Yubao Zou, Haiyan Qian, Huimin Zhang, Xiongjing Jiang \\ Department of Cardiology, State Key Laboratory of Cardiovascular Disease, Fuwai Hospital, National Center for Cardiovascular Diseases, Chinese Academy of Medical Sciences and Peking Union Medical College}

Background Coronary artery aneurysms are uncommon but potentially hazardous entities found on a small percentage of coronary angiograms. The best management strategy remains to be determined; the coexistence of obstructive coronary artery disease is the main determinant of prognosis. Thrombosis within the aneurysm can lead to distal embolization and myocardial infarction.

Case Presentation We report the case of a middle-aged woman with mixed aneurysmal and obstructive coronary artery disease who has a history of congenital heart disease (atrial septal defect, an abnormal origin of coronary artery from the pulmonary artery), hypertension, and obesity, presenting as an unstable angina pectoris. The unusual severity and complexity of the angiographic presentation of this case, with involvement of all major coronary arteries, raises important issues in terms of the patient's treatment options. A conservative approach was unanimously decided upon, including maximization of antithrombotic therapy (antiplatelet therapy \& vitamin $\mathrm{K}$ inhibitor) and anti-ischemic therapy (Statin, ACE inhibitor, $\beta$-blocker, Calcium Channel blocker, and Nitrates.

Conculusion The severity of coexisting obstructive coronary artery 
disease is the main factor in deciding whether to proceed with surgery in patients with coronary aneurysms. However, surgical management of our patient was ruled out by the poor distal vessel runoff and a percutaneous approach was also considered impracticable. Patients not managed surgically must nevertheless be monitored very closely and treated with antiplatelet and anticoagulation therapy to prevent thrombus formation within the aneurysm.

\section{Pregnancy outcomes of patients with hypertrophic cardiomyopathy \\ Yinjian Yang, Xiying Guo, Yishi Li, Jinqing Yuan, Chaomei Fan \\ Key Laboratory of Clinical Trial Research in Cardiovascular Drugs, State Key Laboratory of Cardiovascular Disease, Fuwai Hospital, National Center for Cardiovascular Diseases, Chinese Academy of Medical Sciences and Peking Union Medical College}

Objective To analyze the pregnancy outcomes of patients with hypertrophic cardiomyopathy in China.

Method Search Wanfangdata.com.cn with the terms of "hypertrophic cardiomyopathy" and "pregnancy", with language and publish time unlimited.

Result Seven articles published between the year of 2001 and 2013 and 49 patients were included. Forty-five $(91.8 \%)$ patients gave birth of live infants. Forty-four (89.8\%) patients underwent cesarean section; one $(2.0 \%)$ patient underwent vaginal delivery. Premature and full-term delivery occurred in $10(20.4 \%)$ and $35(71.4 \%)$ patients, respectively. One $(2.0 \%)$ patient died of acute heart failure after premature delivery. Pregnancy was terminated with induced transvaginal (1 patient, $2.0 \%$ ) and cesarean (1 patient, $2.0 \%$ ) abortion in the first and second trimester, respectively. Cesarean section was taken to remove dead fetus in one patient $(2.0 \%)$ in the third trimester.

Conclusion Most of the patients with hypertrophic cardiomyopathy can give birth of live infants. It seems that a $2.0 \%$ perinatal mortality is not very high; however, considering perinatal mortality is generally less than one ten thousandth, patients with hypertrophic cardiomyopathy are at a very high risk of perinatal mortality. Worsening heart failure during pregnancy makes premature delivery happen more easily. Cesarean section is the main delivery mode, which can save the life of both mothers and infants. We should pay intense attention to these patients to avoid perinatal tragedy.

\section{Prevalence and predictors of left ventricular reverse remodeling in idiopathic dilated cardiomyopathy receiving tailored medication Changhong Zou ${ }^{1}$, Xuefei Wu ${ }^{2}$, Yan Huang ${ }^{1}$, Qiong Zhou ${ }^{1}$, Bingqi Wei ${ }^{1}$, Rong Lv ${ }^{1}$, Yuhui Zhang ${ }^{1}$, Jian Zhang ${ }^{1}$ \\ 1. State Key Laboratory of Cardiovascular Disease, FuWai Hospital, National Center for Cardiovascular Diseases, Chinese Academy of Medical Sciences and Peking Union Medical College 2. Beijing Water Conservancy Hospital}

Objective The present study was designed to determine the prevalence and predictors of left ventricular reverse remodeling (LVRR) in idiopathic dilated cardiomyopathy (IDCM) on current tailored medical therapy.

Methods A total of 185 consecutive hospitalized patients with IDCM and left ventricular ejection fraction (LVEF) $\leq 40 \%$ and left ventricular end-diastolic diameter (LVEDD) $>55 \mathrm{~mm}$ by echocardiography at presentation was followed for at least 12 months to identify those with LVRR, which was defined as an absolute increase in $\mathrm{LVEF} \geq 10 \%$ to a follow-up LVEF $\geq 50 \%$, and a relative decrease in LVEDD $\geq 10 \%$ to a follow-up LVEDD $\leq 55 / 50 \mathrm{~mm}$ for male/female. An array of baseline variables regarded as potentially relevant to LVRR was evaluated to identify predictors of LVRR using multivariate logistic regression analysis.

Result After a mean follow-up with repeat echocardiogram of $(29 \pm 13)$ months, 41 patients $(22.2 \%)$ achieved LVRR, showing a significant increase in LVEF from $(32.5 \pm 5.9) \%$ at baseline to $(55.8$ $\pm 4.4) \%$ at initial test revealing LVRR and significant decrease in LVEDD from $(63.0 \pm 4.1) \mathrm{mm}$ to $(51.3 \pm 3.2) \mathrm{mm}$. In contrast, patients without LVRR showed a mild increase in LVEF of $(5.8 \pm 10.0) \%$ and decrease in LVEDD of $(1.0 \pm 6.6) \mathrm{mm}$ at last follow-up. Multivariate Logistic analysis showed that shorter symptom duration of heart failure ( $\leq 6$ months) $(\mathrm{OR}=0.240, \mathrm{P}=0.002)$, Higher systolic blood pressure on admission (per $10 \mathrm{~mm} \mathrm{Hg}$ increase, $\mathrm{OR}=1.333, \mathrm{P}<0.001$ ) and lower tertile of LVEDD/LVEF ratio (i.e. $\leq 2.0,2.0-3.0$, and $\geq 3.0$ ) at presentation $(\mathrm{OR}=0.242, \mathrm{P}<0.001)$ were independent predictors of LVRR. Overall, this baseline model showed an excellent discriminatory capacity as determined by the area under the curve of 0.796 (95\% CI: $0.724-0.867, \mathrm{P}<0.001$ ) on receiver operating characteristic analysis.

Conclusion On current tailored medical therapy, some patients with IDCM may show a significant increase in LVEF and decrease in LVEDD, namely occurrence of LVRR, which was associated with some baseline clinical and echocardiographic parameters.

\section{A large-scale prospective cohort study on the current status of therapeutic modalities for acute myocardial infarction in China: initial results of the China acute myocardial infarction (CAMI) registry. \\ Jingang Yang, Yuejin Yang, Xiaojin Gao, Haiyan Xu, Yuan Wu, Lei Song, Wei Li, Yang Wang, Xinran Tang, Li Chen, Yi Sun, Rui Fu, Yunqing Ye, Hui Sun, \\ Xinxin Yan, Qiuting Dong, Chen Jin \\ State Key Laboratory of Cardiovascular Disease, Fuwai Hospital, National Center for Cardiovascular Diseases, Chinese Academy of Medical Sciences and Peking UnionMedical College}

Objective Little is known about the management of acute myocardial infarction (AMI) in the real world in China. The purpose of this study was to build a comprehensive database on Chinese patients with AMI to elucidate their characteristics.

Methods Among 30 provinces, municipalities or autonomous districts in China, at least one tertiary and secondary hospital was selected. Between January 2013 and March 2014, we consecutively registered all patients with AMI within 7 days after the onset of AMlin 108 participating medical institutions. A standardized case report form was used to register all the patients.

Result A total of 16023 patients consecutive patients was registered (11 855 men, 74\%) with a age of (62 \pm 15$)$ yrs. Only 1770 (11.1\%) patients used an ambulance as transportation to the hospital. Among 11769 patients (73.5\%) with ST elevation myocardial infarction (STEMI), 24.0\% were admitted within the first 3 h, $40.5 \%$ within $6 \mathrm{~h}$, $66.7 \%$ within 12 h, $77.7 \%$ within $24 \mathrm{~h}$ of onset of symptoms. Primary percutaneous coronary intervention (PCI) and thrombolysis were 
conducted for 4957 (42.6\%) and 1195 patients (10.3\%), respectively. Among 4229 patients $(26.5 \%)$ with NSTEMI, primary PCI were performedin 364 patients $(8.8 \%)$. During initial hospitalization, the overall inhospital mortality rate was $4.6 \%$ and $3.4 \%$ for STEMI and NSTEMI, respectively.

Conclusion Findings from the China Acute Myocardial Infarction Registry provide an overview of the treatment that patients actually receive and the outcome, providing the opportunity toassess daily practice in a large population of patients with AMI in China.

\section{Characteristics of coronary atherosclerotic heart disease in ankylosing spondylitis \\ Yuzi Zhou, Li Wang, Humin Pang, Lirong Yan, Lu Hua Key laboratory of Clinical Trail Research in cardiovascular Drugs, Ministry of Health, State key laboratory of Cardiovascular Disease, Fuwai Hospital, National center for Cardiovascular Disease, Chinese Academy of Medical Science and Peking Union Medical College}

Objective Evaluate the differences between coronary atherosclerotic heart disease (CAD) in patients with ankylosing spondylitis (AS) and without AS in demographic characteristics, traditional risk factors, severity of coronary artery and percentage of myocardial infarction (MI).

Methods This monocentric retrospective study included 28 consecutive patients (1 woman, 27 women) with AS in Fuwai hospital between January 2010 and January 2012. The control group included 28 subjects (1 woman, 27 women) who were free of AS. All of them had been confirmed as coronary disease by coronary angiography. The characteristics of both groups were compared including demographics, traditional cardiovascular risks and laboratory findings. The baseline condition, GESININ score and the rate of MI were recorded and compared between 2 groups by student's $t$ test (for continuous variables ) or the ${ }^{2}$ test (for dichotomous variables).

Result There were no significant differences about demographics, traditional cardiovascular between ASgroup and control group except hyperlipidaemia (HLP) (50\% vs $78.57 \% ; \mathrm{P}=0.026)$. The result of laboratory demonstrated that the levels of blood lipid, creatinine and uric acid between the two groups had no difference.However, inflammatory marker such as erythrocyte sedimentation rate (ESR) $(17.14 \pm 17.04$ vs $6.45 \pm 4.72 ; \mathrm{P}=0.002$ ) showed high levels in AS patients. Besides, GENSINI scores of the two groups were $61.95 \pm 41.90$ and $38.04 \pm$ $28.97(\mathrm{P}=0.019)$. Butpercentage of MI $(34 \%$ vs $6 \%, \mathrm{P}<0.001)$ were't different between each other.

Conclusion CAD patients in AS didn't show higher rate of traditional cardiovascular risk factors. AS patients showed more severity of coronary artery but not the percentage of myocardial infarction (MI)

\section{Efficacy, modifiable factors to efficacy, safety of B-type natriutetic peptide-guided heart failure therapy: a meta-analysis \\ Yuehua Li, Hanjun Pei, Xianliang Zhou, Yongjian Wu State key laboratory of Cardiovascular Disease, Fuwai Hospital, National center for Cardiovascular Disease, Chinese Academy of Medical Science and Peking Union Medical College}

Background B-type natriuretic peptide (NP), brain natriuretic peptide (BNP) and N-terminal pro-B-type natriuretic peptide (NTproBNP), are regarded as the most powerful and gold biomarkers of impaired left ventricular function. Results fromrandomized-controlled trials about efficacy, modifiable factors to efficacy, safety of B-type natriuretic peptides (NPs) -guided therapy in heart failure (HF) patients are inconsistent. We performed a meta-analysis to clarify these issues.

Methods The efficacy outcomes was all-cause mortality, cardiovascular mortality, HF-related hospitalization, all-cause hospitalization and net changes of ejection fraction (EF). The safety outcome was adverse events. Fixed or random effects-model was used to calculate the effect size.

Result Compared with usual care, NP-guided therapy was assciated with reduced all-cause mortality $(\mathrm{RR}=0.79,95 \% \mathrm{CI}: 0.67-0.92 ; \mathrm{P}=$ $0.004)$, decreased HF-related hospitalization ( $R R=0.67,95 \%$ CI: $0.46-$ $0.97 ; \mathrm{P}=0.03$ ), and improved ejection fraction (EF) (weighted mean differences $=3.12,95 \%$ CI: $2.24-4.01 ; \mathrm{P}=0.000$ ), without increasing adverse events $(\mathrm{RR}=1.15,95 \% \mathrm{CI}$ : 0.99-1.342; $\mathrm{P}=0.69)$. Subgroup analyses showed that young patients $(<70 \mathrm{yrs}$ ) have lower HF-related hospitalization $(\mathrm{RR}=0.44,95 \% \mathrm{CI}: 0.31-0.63$ vs $\mathrm{RR}=0.89,95 \%$ CI: $0.74-1.07, \mathrm{P}=0.0006$ ) than the older ones ( $\geq 70 \mathrm{yrs}$ ). Sensitivity analyses showed that NP-guided therapy was associated with reduced mortality (RR $=0.77,95 \%$ CI: $0.60-0.996 ; \mathrm{P}=0.046)$, and HF-related hospitalization $(\mathrm{RR}=0.57,95 \% \mathrm{CI}: 0.34-0.98 ; \mathrm{P}=0.000)$ in patients with reduecd $\mathrm{EF}$ (HFrEF).

Conclusion NP-guided therapy decreases all-cause mortality, especially in patients with HFrEF, reduces HF-related hospitalization limited to young patients, and improves left ventricular function, without increasing adverse events.

\section{Case report: A 58-year-old man with flushing and hypotension \\ Wei Chen ${ }^{1}$, Haiyan $\mathrm{He}^{2}$, Xiaoxiao Shi ${ }^{2}$, Quan Fang ${ }^{1}$, Wenling $\mathrm{Zhu}^{1}$ \\ 1. Department of Cardiology, Peking Union MedicalCollege \\ Hospital, Chinese Academy of Medical Sciences and Peking Union \\ MedicalCollege \\ 2. Department of Internal Medicine, Peking UnionMedical College \\ Hospital, Chinese Academy of Medical Sciences and Peking \\ UnionMedical College}

Systemic mastocytosis is a rare disorder characterized by abnormalproliferation and infiltration of mast cells in one or more organ systems suchas the skin, bone marrow, digestive tract, liver and spleen. Urticaria pigmentosa is a typical but infrequent manifestation of systemic mastocytosis. Other clinical presentations of the disease are nonspecific, varying frompruritus, hypotension to multiple organ dysfunctions. In patients who lack skinlesions, diagnosis of systemic mastocytosis is often difficult.

We reported a 58-year-old male, who presented with episodic flushing and syncope. Vasovagal syncope and pheochromocytoma had been suspected before admission. He was noted to have markedly increased neutrophil count and reduced potassium concentrations soon after onset of each episode, both of which returned spontaneously tonormal ranges within 12 hours. Serologic test, pathomorphology and immunohistochemistry features were observed.

Elevation of serum tryptase concentrations were detected (81.6 ng/ $\mathrm{ml})$ and remained high $(35.5 \mathrm{ng} / \mathrm{ml}) 24$ hours after episode. Multifocal bone marrow aspiration revealeddense infiltrates of mast cells ( $>$ 15 mast cells), with a positivity forToluidine blue and CD 117 stain. Systemic mastocytosis without skin lesions could be misdiagnosed.To those with episodic flushing and hypotension, comprehensive differential 
diagnoses are required to exclude allergies, endocrinologic diseases, or other internal disorders.

Serum tryptase serum test and multifocal bone marrow aspiration and biopsy are recommended to establish the diagnosis of systemic mastocytosis.

\section{Incidence of acute mountain sickness in young adults at 3200 meters-comparison of the lake louise scoring and chinese scoring systems}

Guozhu Chen

Cardiovascular Department of Xinqiao Hospital, Third Military Medical

University

Objective The purpose of this study was to compare two scoring systems used for diagnosis of acute mountain sickness (AMS): Lake Louise Scoring (AMS-LLS) and Chinese Scoring System (AMS-CSS).

Methods 339 healthy young adult volunteers, resided at sea level [Age: $(24.59 \pm 3.27)$ yrs; Height: $(173.93 \pm 5.18) \mathrm{cm}$; Weight: $(68.21 \pm$ $7.79) \mathrm{kg}$ ], ascended to $3200 \mathrm{~m}$ by train and bus, a total journey time of 48 $\mathrm{h}$, all the persons were ascend as same way, and were divided into three groups. Group $1(\mathrm{n}=88)$, group $2(\mathrm{n}=91)$ and group $3(\mathrm{n}=160)$ were assessed after one, two and three nights, respectively, at altitude.

Result The overall incidence of AMS was 17.11\% $(\mathrm{n}=58)$ and $29.79 \%(n=101)$ according to AMS-LLS and AMS-CSS, respectively. Two participants $(0.59 \%)$ experienced high altitude pulmonary edema. Both scoring systems showed the highest incidence of AMS after the second night at high altitude. There was a good correlation between AMS-CSS and AMS-LLS scores (Pearson $=0.820, \mathrm{P}<0.001$ ). AMSCSS identified all AMS subjects diagnosed by AMS-LLS, plus an additional 43 missed by AMS-LLS. The dominant symptoms were reduced exercise tolerance $(61.7 \%)$, fatigue $(49.05 \%)$, dizziness (28.9\%), chest distress $(28.3 \%)$ and headache (27.4\%). Compared with AMS-LLS, the sensitivity, specificity, and positive and negative predictive values of AMS-CSS were $100 \%, 84.7 \%, 57.43 \%$ and $100 \%$, respectively. There was no relationship between oxygen saturation $\left(\mathrm{SpO}_{2}\right)$ levels and AMS scores at $3200 \mathrm{~m}$.

Conclusion AMS-CSS is similar, but a little different details, with AMS-LLS. AMS positive diagnosis outnumbers the LLS standard, but there might be afalse positive. Headache was not the dominant symptom at $3200 \mathrm{~m}$ high altitude in this study, and $\mathrm{SpO}_{2}$ levels did not correlate with AMS scores. 


\section{Interventional cardiology}

\author{
In-hospital death causes of acute myocardial \\ infarction after primary $\mathrm{PCl}$ : retrospective \\ analysis from Beijing PCI registry 2010-2013 \\ Lei Song ${ }^{1,10}$, Yuejin Yang ${ }^{1,10}$, Shuzheng $\mathrm{Lv}^{2,10}$, \\ Lefeng Wang ${ }^{3,10}$, Wei Gao, ${ }^{40}$, Jincheng Guo, ${ }^{5}$, \\ Hengjian $\mathrm{Hao}^{6,10}$, Quan Fang ${ }^{7,10}$, Mingying Wu ${ }^{8}$, \\ Hongwei $\mathrm{Li}^{9}$ \\ 1. State Key Laboratory of Cardiovascular Disease, Fuwai Hospital, \\ National Center for Cardiovascular Diseases \\ 2. Beijing Anzhen Hospital \\ 3. Beijing Chaoyang Hospital \\ 4. Peking University Third Hospital \\ 5. Beijing Luhe Hospital \\ 6. Beijing Xuanwu Hospital \\ 7. Peking Union Medical College Hospital \\ 8. Beijing Tongren Hospital \\ 9. Beijing Friendship Hospital \\ 10. Beijing Cardiovascular Intervention Quality Control and Improvement \\ Center
}

Background Intense investigation continued on the quality control of primary percutaneous coronary intervention (PCI) because of its high mortality. However, little study focused on the death cause analysis in a large sample.

Methods Data of in-hospital death after Primary PCI were obtained from Beijing PCI Registry (NCT01417325). Each case was reviewed quarterly in the Beijing Cardiovascular Intervention Quality Control and Improvement Center.

Result The study included 19905 emergency PCI patients from 52 hospitals in Beijing from 2010-2013. In-hospital mortality was 2.30\% (458/19 905). 56 cases were excluded according to exclusion criteria. Of the remaining 402 cases, $91.8 \%$ were STEMI, with an average age of (70.4 \pm 11.2$)$ yrs. $81.3 \%$ of death occurred during the first week after primary PCI. 58.5\% infarction involved in anterior wall. Average attack to presenting time were 7 hour 52 minutes. Of all causes of death, the disease itself (69.2\%) were the first, including cardiogenic shock (36.3\%), mechanical complications (28.1\%), and others (4.7\%); Followed by the procedure factors $(20.1 \%)$, including early stent thrombosis $(9.0 \%)$, no reflow $(7.5 \%)$, acute thrombosis or embolism $(1.5 \%)$, perforation or dissection $(1.0 \%)$, retroperitoneal hematoma $(0.7 \%)$ and side branch closure $(0.5 \%)$; The third was the comorbidity factors $(7.2 \%)$, including septic shock (2.7\%), cerebral infarction (2.5\%), pulmonary embolism $(1.2 \%)$ and multiple organ failure $(0.7 \%)$; Drug factors (3.5\%), including intracranial hemorrhage $(2.0 \%)$, massive gastrointestinal hemorrhage $(1.2 \%)$ and anaphylactic shock due to contrast medium $(0.2 \%)$, should also not be ignored. $47.3 \%$ of the dead cases had some sort of medical defects, including indication (1.0\%), rescue measures (14.9\%), strategy selection $(26.6 \%)$ or procedure issue $(4.7 \%)$. No significant difference of the mortality from 2010 to $2013(2.23 \%, 2.55 \%, 1.94 \%$ and $2.48 \%$ respectively).

Conclusion Mortality of acute myocardial infarction remains high, which have a variety of causes. The three leading causes of death were cardiogenic shock, mechanical complications and early stent thrombosis. Current study may contribute to facilitate individualized preventive approach and develop a successful quality improvement solution.

\section{Is percutaneous closure superior to surgical repair in terms of heart blocks after closureof perimembranous ventricular septal defect? evidence from a meta-analysis \\ Yuan Bai, Yongwen Qin \\ Changhai Hospital, Second Military Medical University}

Objective Traditional surgical repair, as a standard treatment for perimembranous ventricular septal defect (VSD), has yielded great results in the past decades. Percutaneous device closure for VSD is also associated with excellent rates of closure and low morbidity. However, Compared with surgical repair in which atrio-ventricular block usually appears early after the operation, atrio-ventricular block can occur immediately or late after transcatheteroccluder closure. The aim of this study was to compare the incidence of atrio-ventricular block after VSD closure of percutaneous procedure and surgical repair, and to find whether percutaneous procedure yields better outcomes than surgical repair.

Methods We searched PubMed, the Cochrane Library, the Web of Science and Chinese Biomedical Literature databases, Chinese National Knowledge Infrastructure, the Chinese Science and Technology Journal Full-Text database, and Wangfang database. The latest data was accessed in March 2014. Review Manager 5.1 software was used for comprehensive quantification data analysis.

Result Sixteen studies were included in the Meta-analysis, composed of 4669 participants and reporting heart block complications. The Meta-analysis indicated no statistically significant difference in right bundle branch block and third degree atrio-ventricular block between the two approaches, except for left bundle branch block $(\mathrm{OR}=3.19 ; 95 \%$ CI: $1.42-7.15 ; \mathrm{P}=0.005 ; \mathrm{I}^{2}=33 \%$ ).

Conclusion Current evidence suggests that percutaneous approach may be a good alternative to the surgery technique for closure of perimembranous ventricular septal defect in terms of heart blocks after procedure.

\section{XTRM-FIT coronary stent system in combination with tirofiban in the treatment of elderly acute Coronary Syndrome \\ Peiliang Liu, Xuan Li, Lei Miao \\ Center emergency cardiovascular disease, JinQiu Hospital of Liaoning Province}

Objective This research of XTRM-FIT Coronary Stent System in combination with Tirofiban in the treatment of 35 cases of elderly Acute Coronary Syndrome (ACS) had a satisfactory result.

Methods Choose 60 ACS patients under PCI, according to random number table the test wasrandomly divided into two groups: Group A (XTRM - FIT + Tirofiban) had 30 cases, male 16 cases, female 14 cases; Group B (XTRM-FIT) had 30 cases, male 15 cases, female 15 cases. Choose the same stent brand for each patient. therewere no statistically significant in difference compared $(\mathrm{P}>0.05)$. According to the angiography results of coronary artery stenosis degree, the degree of stenosis $\geq 75 \%$ was defined as PCI treatment lower limit. The size of stent was choseby clinical doctor. After coronary artery guiding wire 
through Occlusion lesions, then give injection load for Tirofiban $10 \mu \mathrm{g} /$ $\mathrm{kg}$, the intravenous injection is completed with in 3 minutes. For the 70 -year-old or older patients or stroke patients with in 2 years, the dosing is reduced $20 \%$, then the micro-injection pump continuous to inject 36 $\mathrm{h}$ with $0.15 \mu \mathrm{g} /(\mathrm{kg} \cdot \mathrm{min})$. In the process of PCI, intravenous injection heparin $50 \mathrm{U} / \mathrm{kg}$, an extra $1000 \mathrm{U}$ with each more than $1 \mathrm{~h}$. The presence of stenocardia, any abnormal changes of electrocardiogram, myocardial enzyme, platelet count, hemoglobin, hematokrit (HCT), etc. outpatient follow-up or telephone follow-up for 30 days about the incidence of stenocardia, the incidence of subacute thrombosis, the rate of MACE, rehospitalization rate.

Result In the two groups, there were no statistically significant ( $\mathrm{P}$ $>0.05$ ) for the number of target vessel, stenosis degree, the number of stent implantation, the Peak pressure of stent release, operating time, PCI success rate, etc. The rate of acute in-stent thrombosis occurred for the two groups were $0 \%$ and $3.33 \%$ respectively $(\mathrm{P}<0.05)$. Bleedingcomplications: between the two groups $(\mathrm{P}>0.05)$. The platelet count, hemoglobin, Hematokrit (HCT) change between the two groups $(\mathrm{P}<0.05)$. 30-day follow-up results after PCI: the rate of target vascular reconstruction, subacute thrombosis, MACE rate and rehospitalization between the two groups $(\mathrm{P}>0.05)$.

Conclusion XTRM-FIT coronary stent systemis the latest generation coronary artery stent which is manufactured by Blue Medical Devices B.V (the Netherlands). It adopts unique "Dylyn" biological coating technology, reducesthe intravascular endothelial hyperplasia and inflammatory reaction. both XTRM-FIT Coronary Stent System in combination with Tirofiban and onlystent implantation all have good security, but have significant differences for effectiveness. For the combination therapy, the incidence of stenocardia, MACE rate, the incidence of acute and subacute thrombosis and hospitalization rates were significantly lower.

\section{The association between post- $\mathrm{PCl}$ bleeding and 1 year adverse clinical outcomes in elderly patients \\ Peiyuan He, Yuejin Yang, Shubin Qiao, Bo Xu, Yongjian Wu, Jinqing Yuan, Jue Chen, Haibo Liu, Runlin Gao \\ Department of Cardiology, State Key Laboratory of Cardiovascular Diseases, Fuwai Hospital, National Center for Cardiovascular Diseases, Chinese Academy of Medical Sciences and Peking Union Medical College}

Objective Post-PCI bleeding was associated with short or longterm adverse cardiovascular events, and elderly patients are of higher risk of post-PCI bleeding. So we tried to evaluate the association between post-PCI bleeding and 1 year adverse cardiovascular events in elderly patients.

Methods 1105 elderly patients undergoing PCI were included. 153 patients had post-procedure bleeding. Bleeding Academic Research Consortium (BARC) definition was used to adjudicate and classify bleeding events. The primary endpoint was defined as 1 year adverse cardiovascular events which was a composite of death and myocardial infarction. Cox proportional hazards model was performed to evaluate the independent effect of bleeding on the composite endpoint.

Result Bleeding occurred in $13.8 \%$ of all elderly patients undergoing PCI. BARC 2 grade bleeding accounted the most (68.6\%). The rate of $\mathrm{BARC} \geq 2$ grade bleeding was $11.8 \%$; BARC $\geq 3$ grade bleeding was $2.3 \%$, and the access site-related bleeding accounted for $62.7 \%$ of all bleeding. The rate of 1 year death and myocardial infarction was higher in patients with bleeding $(9.2 \%$ vs $4.2 \% ; \mathrm{P}=0.008)$. The 1 year cardiac death was also higher in patients with bleeding (3.9\% vs $0.8 \% ; \mathrm{P}=0.007)$, but non-cardiac death was similar between the two groups. BARC $\geq 2$ grade bleeding was an independent predictor of 1 year death and myocardial infarction $(\mathrm{OR}=2.147$; 95\% CI: $1.133-4.069)$.

Conclusion Post-PCI bleeding was associated with 1 year adverse outcomes for elderly patients undergoing PCI. By reducing bleeding events, elderly patients' prognosis can be improved.

\section{Effect of gender difference on the 1 year follow- up outcomes for patients undergoing primary $\mathrm{PCl}$ \\ Peiyuan $\mathrm{He}^{1}$, Yuejin Yang ${ }^{1}$, Shubin Qiao ${ }^{1}, \mathrm{Bo} \mathrm{Xu}^{1}$, Yongjian $\mathrm{Wu}^{1}$, Jinqing Yuan ${ }^{1}$, Jue Chen ${ }^{1}$, Haibo Liu ${ }^{1}$, Xinran Tang ${ }^{2}$, Yang Wang ${ }^{2}$, Wei $\mathrm{Li}^{2}$, Runlin Gao ${ }^{1}$ State Key Laboratory of Cardiovascular Diseases, Fuwai Hospital, National Center for Cardiovascular Diseases, Chinese Academy of Medical Sciences and Peking Union Medical College \\ 1. Department of Cardiology 2. Medical Research \& Biometrics Center}

Objective Previous studies reported that post-PCI outcomes for women are worse compared with men in some selected patients. So we tried to evaluate the effect of gender difference on the in-hospital and 1 year follow-up outcomes for STEMI patients undergoing primary PCI in the era of drug-eluting stents.

Methods 687 STEMI patients who have undergone primary PCI were consecutively recruited. The baseline features, in-hospital outcomes and 1 year clinical prognosis were compared between men and women. Logistic regression analysis was performed to adjust for the baseline disparities.

Result Compared with men, women were older, and accompanied with a higher rate of multivessel disease. The adjusted rates of in-hospital and 1 year major adverse cardiovascular events (MACE) were similar between men and women[0.731 (0.250-2.136), $\mathrm{P}=0.566$, and 1.508 (0.660-3.443), $\mathrm{P}=0.330]$. The adjusted rates of bleeding academic research consortium (BARC) $\geq 2$ bleeding $[0.551$ (0.33-0.918), $\mathrm{P}=$ $0.022]$ was higher in women. Access site complications occurred more in women than in men $(9.6 \%$ vs $4.9 \%, \mathrm{P}=0.048)$, and the recurrent angina was also more frequent in women than in man at 1 year follow-up $(10.4 \%$ vs $3.9 \%, \mathrm{P}=0.003$ ).

Conclusion There is no gender difference for the in-hospital or 1 year term outcomes in STEMI patients undergoing primary PCI. Women have a higher risk of bleeding and access site complications. To avoid bleeding is the major goal in elevating the PCI quality in women.

\section{Association between peak neutrophil count, clopidogrel loading dose, and left ventricular systolic function in patients with primary percutaneous coronary intervention}

Xinyu Wang, Haiyi Yu, Zhaoping Li, Liuning Li, Youyi Zhang, Wei Gao

Department of Cardiology, Institute of Vascular Medicine, Peking University Third Hospital; Laboratory of Cardiovascular Molecular Biology and Regulatory Peptides, Ministry of Health and Key Laboratory of Molecular Science, Ministry of Education, and Beijing Key Laboratory of Cardiovascular Receptors Research.

Objective Inflammation plays an important role in plaque 
development and left ventricular remodeling during acute myocardial infarction (AMI). Clopidogrel may exhibit some anti-inflammatory properties and high loading dose of clopidogrel results in improved clinical outcomes in patients with AMI. The aim of this study was to establish the association of loading dose of clopidogrel and left ventricular systolic function in patients with STEMI.

Methods 357 patients received successful primary percutaneous coronary intervention from January 2008 to March 2011 in the Peking University Third Hospital were included in this study.Different loading dose of clopidogrel (300 mg, $450 \mathrm{mg}$ or $600 \mathrm{mg}$ ) was given at the discretion of the clinician.

Result Neutrophil reached their peak values on the first day after AMI. The higher levels of peak neutrophil and lower left ventricular ejection fraction (LVEF) were found in patients of low clopidogrel loading dose group (300 $\mathrm{mg}$ or $450 \mathrm{mg}$ ). After adjusting for the related confounders, a logistic regression model showed low clopidogrel loading dose remained an independent predictor of low $\mathrm{LVEF}(\mathrm{LVEF} \leq 50 \%$, OR $=1.959,95 \%$ CI: $1.058-3.626 ; \mathrm{P}=0.032$ ).

Conclusion Low clopidogrel loading dose was associated with higher peak neutrophil count and poor left ventricular systolic function, suggesting an important role of clopidogrel loading dose in the improvement of left ventricular function and high loading dose may exhibit better anti-inflammatory properties.

\section{An Angiographic tool for risk prediction of side branch occlusion in coronary bifurcation intervention: the RESOLVE score system \\ Kefei Dou, Dong Zhang, Bo Xu, Dong Yin Department of Cardiology, State Key Laboratory of Cardiovascular Diseases, Fuwai Hospital, National Center for Cardiovascular Diseases, Chinese Academy of Medical Sciences and Peking Union Medical College}

Background The risk of side branch (SB) occlusion is the most important consideration affecting the selection of an optimal intervention strategy in patients undergoing coronary bifurcation intervention. We aim to establish a scoring system to evaluate the risk of SB occlusion.

Methods 1545 consecutive patients undergoing percutaneous coronary intervention for bifurcation lesions (1601 lesions treated with a single stent technique or main vessel (MV) stenting first strategywere studied. 1200 lesions were used for the construction of the risk model and score system, 401 lesions were used for validating the model. A multivariable risk score was constructed with incremental weights attributed to each component variable according to their estimated coefficients. SB occlusion after MV stenting was defined as any decrease in TIMI flow grade or absence of flow in SB after MV stenting.

Result SB occlusion occurred in 118 (7.37\%) of 1601 bifurcation lesions. In multivariable analyses, 6 variables were independently associated with the risk of SB occlusion (model C-statistic $=0.80$, 95\% CI: $0.75-0.85$ with good calibration). Lesions divided into groups according to the quartile of RESOLVE score: quartile I was determined as low risk group (score: $0-2$ ); quartiles II and III were combined into an intermediate risk group (score: 3-9); and quartile IV described high risk group (score: $\geq 10$ ). For the 401 lesions included in the validation cohort, the RESOLVE score had a C-statistic $=0.77$ (95\% CI: 0.69-0.86), with good calibration. SB occlusion rates in the validation cohort increased significantly across different risk groups from $0.0 \%$ in low risk group to $3.8 \%$ in intermediate risk group, to $19.8 \%$ in high risk group $(\mathrm{P}<0.001)$.

Conclusion The RESOLVE score, a novel angiographic risk stratification tool, can help identify patients at risk for SB occlusion during bifurcation intervention.

\section{The comparison of clinical outcomes between incomplete revascularization and complete revascularization in the coronary artery disease \\ Jiehui Li, Kun Hua, Xiubin Yang Department of Cardiology, State Key Laboratory of Cardiovascular \\ Diseases, Fuwai Hospital, National Center for Cardiovascular Diseases, Chinese Academy of Medical Sciences and Peking Union Medical College}

Objective To compare the clinical outcomes of incomplete revascularization (IR) for coronary disease and complete revascularization $(\mathrm{CR})$.

Background There has been a considerable debate for a long time about whether IR for coronary disease could achieve the effect of CR. Some clinicians hold on some don't.

Methods Articles were searched from MEDLINE and major international cardiology conferences. Outcomes of "All-Cause Death (ACD) " and "Cardiac Death (CD) " from individual studies were pooled to calculate relative risks (RRs) and Weighted Mean Difference (WMD) with $95 \%$ confidence intervals (CI). Baseline factors as age, diabetes mellitus (DM), hypertension (HTN) and SYNTAX Score were also recorded and analyzed.

Result 26 articles were enrolled in our study eventually. The IR group had a more serious results of RR with $95 \%$ CI on ACD 1.43 (1.28-1.59), no matter in subgroup PCI 1.53 (1.31-1.79) or CAB 2.12 (1.718-2.667), and CD 2.12 (1.718-2.667). Besides, baseline factors presented a similar situation: WMD with $95 \%$ CI of age and SYNTAX Score were $0.959(0.291-1.628)$ and 6.530 (3.572-9.488); RR with $95 \%$ CI of DM and HTN were 1.226 (1.120-1.342) and 1.088 (1.039-1.139).

Conclusion IR has led to a worse outcome of ACD and CD, but patients on IR also have a worse preoperative status than CR. We need to do more prospective studies to control the imbalance of baseline. Only two groups of patients standing on the same starting line, results deduced from which could give us a convincing answer.

\section{IVUS guided true lumen seek and tracking technique for complex chronic total occlusion lesions, experience from fuwai hospital \\ Lei Song, Yuejin Yang, Liang Xu, Bo Xu, \\ Shubin Qiao, Yongjian Wu, Hongbing Yan, Kefei Dou Department of Cardiology, State Key Laboratory of Cardiovascular Diseases, Fuwai Hospital, National Center for Cardiovascular Diseases, Chinese Academy of Medical Sciences and Peking Union Medical College}

Background The major obstacle of a complex chronic total occlusion (CTO) is passing the guidewire through the true lumen, the guidance of intravascular ultrasound (IVUS) could be helpful in the procedure, even in the era that the retrograde approach has increased steadily. We reported the strategy and results of IVUS guided true lumen seeking and tracking technique for percutaneous coronary intervention (PCI) of complex CTO lesions, focusing on the safety and efficacy of IVUS guided true lumen seeking and tracking techniques.

Methods In this retrospective study, we included CTO cases from January 2013 to April 2014 in our center and one interventional doctor 
practiced all procedures. The definition of complex CTO is a lesion meet at least one of the following criteria: length $>20 \mathrm{~mm}$, blunt stump, branching off at the site of occlusion, severe calcification, or previous failed history. A group composed by three experts reviewed each case and confirmed the complexity of the lesion.

Result Total 198 CTO PCI performed, 30 simple CTO (all succeed) were excluded. 168 complex (J-Score $\geq 1,84.8 \%$ ) procedure, 161 lesions $/ 158$ patients were included. $77.4 \%$ of the procedures were trans-radial approach. 63 (40.1\%) were angiogram only and 105 (59.9\%) under the guidance of IVUS. IVUS guided lesions were more complex, documented by higher J-Score $(2.37 \pm 0.80$ vs $1.97 \pm 0.72, \mathrm{P}=0.001)$, higher percentage of lesions that length $>20 \mathrm{~mm}(91.4 \%$ vs $77.8 \%, \mathrm{P}=$ $0.013)$ and blunt stump $(69.5 \%$ vs $54.0 \%, \mathrm{P}=0.042)$. Success rate for complex CTO was $69.6 \%$ (61.9\% no IVUS vs $74.2 \%$ IVUS guided, $\mathrm{P}=$ 0.064). IVUS guided PCI had longer procedure duration $[(131.7 \pm 58.0)$ $\min$ vs $(66.2 \pm 34.6) \mathrm{min}, \mathrm{P}=0.000]$, length of stents $[(67.9 \pm 26.1) \mathrm{mm}$ vs $(52.9 \pm 23.2) \mathrm{mm}, \mathrm{P}=0.007]$, and more balloons ( $1.95 \pm 0.83$ vs 2.41 $\pm 1.39, \mathrm{P}=0.027$ ), compared with angiogram only PCI. The overall complication were infrequent, however, the IVUS guided procedure had fewer coronary perforation $(4.8 \%$ vs $14.3 \%, \mathrm{P}=0.031)$ and more intramural hematoma or dissection $(13.3 \%$ vs $3.2 \%, \mathrm{P}=0.030)$. The history of previous $\mathrm{CABG}(\mathrm{RR}=0.133,95 \% \mathrm{CI}$ : $0.032-0.559, \mathrm{P}=0.006)$ and in-stent occlusion ( $\mathrm{RR}=0.277,95 \% \mathrm{CI}$ : $0.083-0.921, \mathrm{P}=0.036$ ) was the independent predictive factors of failure.

Conclusion These results indicate that the IVUS guided true lumen seeking and tracking techniques are safe and useful for complex CTO lesions.

\section{Use of novel protective ballooning technique with provisional stenting for treatment of non-left main coronary bifurcation lesions: A feasibility pilot study \\ Haibo Liu, Bo Xu, Shubin Qiao, Man Wang, Liang Xu, Fenghuan Hu, Kefei Dou, Yuejin Yang, Runlin Gao Department of Cardiology, State Key Laboratory of Cardiovascular Diseases, Fuwai Hospital, National Center for Cardiovascular Diseases, Chinese Academy of Medical Sciences and Peking Union Medical College}

Background and Objective Percutaneous coronary intervention (PCI) of bifurcation lesions is associated with a higher risk of adverse events. Recent studies support the use of provisional side branch (SB) stenting, but the risk of SB closure after main vessel (MV) stenting remains an important concern. We sought to establish and demonstrate the feasibility and preliminary efficacy of a novel protective ballooning technique (PBT) for SB protection and treatment during MV stenting.

Methods The rationale of $\mathrm{PBT}$ for SB protection is to preposition a small balloon $(2.0 / 20 \mathrm{~mm})$ in the SB before MV stenting while the proximal makers of the balloon and the MV stent are being aligned. During deployment of the MV stent (14-16 atm), the uninflated jailed balloon under the stent struts serves to reduce both carina and plaque shifts due to its spatial occupation in the SB ostium. There after the jailed balloon is inflated at 8-14 atm to dilate the ostium. After removing the jailed balloon MV stent's balloon is inflated again at 14-16 atm to correct stent deformation or malapposition. If SB flow is preserved after MV stenting, the jailed wire will be removed from SB; otherwise it could be used as a marker to facilitate rewiring SB, and further kissing balloon inflation or provisional SB stenting will be performed to restore SB flow. Final intravascular ultrasound (IVUS) examinations were selectively performed in some patients to check the MV stent. Procedural and immediate clinical outcomes were recorded and reviewed.

Result This novel technique was successfully adopted in 92 patients with 99 bifurcation lesions. The majority of patients had Median class 1, 1, 1 bifurcation lesions (81\%). Final TIMI 3 flow was achieved in $100 \%$ of MV and $98 \%$ of SB. IVUS revealed optimal deployment of MV stent after final inflation in all checked cases $(n=13)$. Only one patient (1\%) had lesions that required rewiring and provisional stenting of the SB. TIMI 2 flow occurred in one patient (1\%). SB loss occurred in one patient (1\%), who suffered a periprocedural myocardial infarction (MI). No jailed balloon or wire was entrapped during any PCI.

Conclusion Provisional stenting of complex coronary bifurcation lesions using a PBT is associated with a high procedural success rate, improved SB patency, and a low rate of immediate cardiac events in this feasibility study; however, randomized trial is needed to further confirm its clinical benefits.

\section{Three-year clinical follow-up of the firehawk abluminal groove-filled biodegradable polymer sirolimus-eluting stent in the treatment of single de novo native coronary lesions: the TARGET | trial \\ Bo Xu', Yelin Zhao ${ }^{1}$, Yuejin Yang ${ }^{1}$, Martin Leon ${ }^{2}$, \\ Changdong Guan ${ }^{1}$ \\ 1. Department of Cardiology, State Key Laboratory of Cardiovascular \\ Diseases, Fuwai Hospital, National Center for Cardiovascular Diseases, \\ Chinese Academy of Medical Sciences and Peking Union Medical \\ College \\ 2. Cardiovascular Research Foundation, New York, United States}

Objective We sought to investigate the long-term outcomes of an abluminal groove-filled biodegradable polymer sirolimus-eluting stent FIREHAWK (MicroPort Medical, Shanghai, China) compared to an everolimus-eluting stent (EES) XIENCE V in the randomized TARGET I trial. Previously reported results demonstrated FIREHAWK stent was non-inferior to XIENCE V EES for the primary endpoint of 9-month instent late lumen loss (LLL) $[(0.13 \pm 0.24) \mathrm{mm}$ vs $(0.13 \pm 0.18) \mathrm{mm}, \mathrm{P}=$ 0.94; difference and $95 \%$ confidence interval $0.00(-0.04-0.04) \mathrm{mm}$; $\mathrm{P}$ for non-inferiority $<0.0001$ ], and had a comparable clinical outcome at 2 years. This paper reports the 3 -year clinical outcomes of TARGET I trial.

Methods A total of 458 patients with single de novo native coronary lesions $\leq 24 \mathrm{~mm}$ in length and a coronary artery $\geq 2.25$ to $\leq 4.0$ $\mathrm{mm}$ in diameter were enrolled in the TARGET I study, a prospective, randomized, non-inferiority trial. The primary endpoint was in-stent LLL) at 9-month follow-up. The secondary endpoint, target lesion failure (TLF), was defined as the composite of cardiac death, target vessel myocardial infarction (TV-MI), and ischemia-driven target lesion revascularization (iTLR). Clinical follow-up was scheduled at 1-, 6- and 12-month, and annually up to 5 years for all enrolled patients. All adverse clinical events were adjudicated by an independent committee.

Result The 3-year clinical follow-up was completed in 449 of 458 patients $(98 \%)$, the groups had similar baseline characteristics $(\mathrm{P}=$ 0.34). Treatment with FIREHAWK stent compared with XIENCE V EES still showed no significant difference rates of all adverse events. There were no statistically significant differences in TLF between study groups through 3-year follow-up. At 3 years, the observed rate of TLF was $4.1 \%$ for FIREHAWK and $3.5 \%$ for XIENCE V $(P=0.75)$. The rate of TV-MI, cardiac death and iTLR events in FIREHAWK compared with XIENCE V group was $1.4 \%$ vs $2.2 \%(\mathrm{P}=0.72), 0.9 \%$ vs $0.4 \%$ 
$(\mathrm{P}=0.62)$ and $1.8 \%$ vs $1.3 \%(\mathrm{P}=0.75)$. There was a trend of lower revascularization rate in FIREHAWK group compared with XIENCE $\mathrm{V}$ group $(4.5 \%$ vs $7.5 \%, \mathrm{P}=0.19)$. Over all those 3 years there was no definite/probable stent thrombosis occurred in FIREHAWK group, while 1 occurred in the XIENCE V group ( $0 \%$ vs $1.4 \%, \mathrm{P}=1.00)$.

Conclusion In the multicenter randomized TARGET I trial, the 3-year follow-up results confirmed that the novel FIREHAWK stent had a durable safety and efficacy profile, which was comparable to the XIENCE V EES for the treatment of single de novo native coronary lesions.

\section{Safety and efficacy of a novel abluminal groove-filled biodegradable polymer sirolimus- eluting stent for the treatment of de novo coronary lesions: 2-year results from the prospectively patient-level pooled analysis of the TARGET I and TARGET || trials \\ Yelin Zhao ${ }^{1}$, Bo Xu ${ }^{1}$, Yuejin Yang ${ }^{1}$, Yong $\mathrm{Huo}^{2}$, Alexandra Lansky ${ }^{3}$, Martin Leon ${ }^{4}$, Changdong Guan ${ }^{1}$ 1. Department of Cardiology, State Key Laboratory of Cardiovascular Diseases, Fuwai Hospital, National Center for Cardiovascular Diseases, Chinese Academy of Medical Sciences and Peking Union Medical

Background and Objective Early reports revealed that a novel abluminal groove-filled biodegradable polymer sirolimus-eluting stent (named target eluting technology) FIREHAWK (MicroPort Medical, Shanghai, China) was safe and effective in 1-year follow-up in a large cohort of patients from the TARGET clinical program. We aimed to investigate the long-term clinical outcomes of this novel stent.

Methods An objective performance criterion (OPC) study was required by the China Food and Drug Administration for new drugeluting stent. The primary endpoint, target lesion failure (TLF), was defined as the composite of cardiac death, target vessel myocardial infarction (TV-MI), or ischemia-driven target lesion revascularization (iTLR) at 12 months. A patient-level pooled data derived from TARGET I RCT $(\mathrm{n}=227)$, TARGET I long stent cohort $(\mathrm{n}=50)$, and TARGET II registry $(\mathrm{n}=730)$ was prospectively collected and analyzed in 1007 patients with de novo native coronary lesions. All patients were exclusively treated with FIREHAWK stent and the follow-up visits are at 1-, 6-, 12-month, and annually up to 5 years.

Result 2-year clinical follow-up information was available on 992 (98.5\%) patients implanted with FIREHAWK stents. Between 1 and 2 years, $62 \%$ of patients discontinued DAPT and there were only 7 newly occurred TLF events (39, 3.9\% at 1year vs. $46,4.6 \%$ at 2 year), including 3 cardiac death ( $5,0.5 \%$ at 1 year vs $8,0.8 \%$ at 2 year $), 1$ TV-MI $(28,2.8 \%$ at 1 year vs $29,2.9 \%$ at 2 year) and 3 iTLR $(9,0.9 \%$ at 1 year vs $12,1.2 \%$ at 2 year). The patient-oriented composite endpoint (PoCE) rate at 2 year was $7.8 \%$ (77), 21 patients more than 1 year outcomes. Mainly because 12 revascularization patients, however only 3 myocardial infarction was observed. Importantly, no definite/probable stent thrombosis (ST) was observed in this cohort through 1 year. Lesion length $\geq 30 \mathrm{~mm}$ was an independent predictor of TLF at 2 years ( $\mathrm{HR}=2.44,95 \% \mathrm{CI}$ : $1.32-4.53$, $\mathrm{P}<0.01)$.

Conclusion The sustained 2-year clinical data indicates that FIREHAWK stent could enable safety and efficacy by a novel target eluting technology in mild to moderate risk patients. However, large scale randomized trials (TARGET EU and III) are needed to further confirm its clinical benefits in treating most complex patients/lesions.

\section{Two-year clinical outcome after successful implantation of drug-eluting and bare metal stents in large ( $\geqslant 3.5 \mathrm{~mm}$ ) coronary arteries \\ Dong Yin, Kefei Dou, Yuejin Yang, Bo Xu \\ Department of Cardiology, State Key Laboratory of Cardiovascular \\ Diseases, Fuwai Hospital, National Center for Cardiovascular Diseases, Chinese Academy of Medical Sciences and Peking Union Medical College}

Objective Randomized trials have demonstrated that drug-eluting stents (DES) reduce the risk of target vessel revascularization (TVR) compared to bare-metal stents (BMS). This benefit is less pronounced as artery diameter increases. Whether DES is superior to BMS for larger coronary arteries in the setting of routine clinical practice is unknown. This study sought to evaluate the safety and effectiveness of DES compared to BMS for patients with large coronary vessels.

Methods From April 2004 to October 2006, 2408 consecutive patients undergoing de novo lesion PCI with reference vessel diameter greater than or equal to $3.5 \mathrm{~mm}$ at Fu Wai Hospitalwere prospectively enrolled into this study. All enrolled patients were divided into DES group $(\mathrm{n}=1621)$ and BMS group $(\mathrm{n}=787)$. We obtained 24-month clinical outcome including death, myocardial infarction (MI), thrombosis, target lesion revascularization (TLR), target vessel revascularization (TVR), and major adverse cardiac events (the composite of death, MI, and TVR). We used Cox's proportional-hazards models to assess relative risks of all the outcome measures after propensity match.

Result After propensity match, 515 DES-treated patients were matched to 515 BMS-treated patients. The patients treated with BMS were associated with higher risk of TLR ( $\mathrm{HR}=2.24,95 \%$ CI: $1.41-3.57$, $\mathrm{P}=0.0007)$, TVR (HR $=1.80,95 \% \mathrm{CI}: 1.21-2.68, \mathrm{P}=0.004)$ and MACE $(\mathrm{HR}=1.475,95 \% \mathrm{CI}: 1.05-2.07, \mathrm{P}=0.024)$. All ARC definition stent thrombosis at 24-month were comparable in the two groups.

Conclusion In patients requiring stenting of large coronary arteries, use of DES was associated with significant reductions in the risks of TLR, TVR and MACE at long-term follow-up.

\section{Lower mortality of paclitaxel-coated balloon compared with paclitaxel-eluting stent for the treatment of DES In-stent restenosis: two-year follow-up of the PEPCAD China ISR trial \\ Yelin Zhao ${ }^{1}$, Bo Xu ${ }^{1}$, Junbo $\mathrm{Ge}^{2}$, Yuejin Yang ${ }^{1}$, Changdong Guan ${ }^{1}$ \\ 1. Department of Cardiology, State Key Laboratory of Cardiovascular Diseases, Fuwai Hospital, National Center for Cardiovascular Diseases, Chinese Academy of Medical Sciences and Peking Union Medical College \\ 2. Zhongshan hospital Fudan University}

Background and Objective Previous studies demonstrated that angioplasty with a paclitaxel-coated balloon (PCB) was non-inferior to paclitaxel-eluting stent (PES) implantation when used to treat drugeluting stent in-stent restenosis (DES-ISR). There is a paucity of data regarding the long-term safety and efficacy of using PCB in treating DES-ISR. We sought to present for the first time the 2-year clinical 
outcomes from PEPCAD China ISR trial.

Methods PEPCAD China ISR was a 220 patient randomized (1: 1), single blind prospective multicenter trial conducted in China. Patients with DES-ISR received either PCB (SeQuent ${ }^{\circledR}$ Please, B.BraunMelsungen AG, Germany) or PES (Taxus ${ }^{\circledR}$ Liberté, Boston Scientific, Natick, MA, USA) treatment. The primary endpoint was in-segment late lumen loss (LLL) at 9 months. Secondary endpoints included 9-month\% diameter stenosis (DS), binary restenosis rate, and 1and 2-year target lesion failure (TLF) defined as the composite of cardiac death, target vessel myocardial infarction or ischemia-driven target lesion revascularization. In addition, definite/probable stent thrombosis (ST) rates were documented.

Result The primary endpoint of in-segment LLL at 9 months did not differ between PCB and PES group $[(0.46 \pm 0.51) \mathrm{mm}$ vs $(0.55 \pm$ $0.61) \mathrm{mm}, \mathrm{P}=0.32$ ]. At 9 months, $\% \mathrm{DS}$ and binary restenosis were not significantly different between PCB and PES group neither (29.0 \pm 21.3 vs $30.8 \pm 25.3 ; 18.6 \%$ vs $23.8 \% ; \mathrm{P}>0.05$ respectively). There were no adverse ischemic events occurred between 1 to 2 years in PCB group except 1 non-target vessel revascularization, no additional definite or probable stent thrombosis or myocardial infarction were reported in both arms through 1 year. Two-year rate of TLF was similar between two groups $(16.8 \%$ vs $18.6 \%, \mathrm{P}=0.73)$, comparing with oneyear outcomes only 2 cardiac death (one of them also had ischemiadriven TLR) occurred in the PES group. The patient-oriented composite which including all-cause death, any myocardial infarction, any revascularization, showed non-significantly difference between PCB and PES, despite fewer all-cause death in patients with PCB ( $0 \%$ vs $4.9 \%, \mathrm{P}$ $=0.03$ ).

Conclusion The prolonged 2-year clinical follow-up showed that there was no newly occurred death, MI, TVR, or ST in PCB group between 1 and 2 years, and statistically, a lower all-cause mortality of PCB compared to PES for the treatment of DES-ISR.

\section{Association of dual antiplatelet therapy cessation with ischemic and bleeding events following cobalt-chromium everolimus-eluting stent implantation in chinese population: first two-year reports of the prospective multicenter SEEDS study \\ Yelin Zhao, Bo Xu, Yuejin Yang, Changdong Guan Department of Cardiology, State Key Laboratory of Cardiovascular Diseases, Fuwai Hospital, National Center for Cardiovascular Diseases, Chinese Academy of Medical Sciences and Peking Union Medical College}

Background and Objective Appropriate duration of dual antiplatelet therapy (DAPT) after drug-eluting stent implantation is still controversial. We sought to assess the associations between DAPT cessation, ischemic events and bleeding risks following PCI in patients with complex lesions treated with the cobalt-chromium $(\mathrm{CoCr})$ everolimus-eluting stent (EES).

Methods 1900 patients with small vessel (reference diameter $<2.75$ $\mathrm{mm}$ ), long lesion (Length $>25 \mathrm{~mm}$ ), or multivessel ( $>2$ Target vessels) disease who underwent PCI with EES (Xience V, Abbott Vascular, Santa Clara, CA, USA) in the prospective SEEDS study were categorized into 4 groups according to DAPT duration (1-6 months, 6-12 months, 12-24 months after PCI and no DAPT cessation). All events were classified into "on DAPT" or "off DAPT" group according to the DAPT status at the time of event occurred. Clinical follow-up data at 2 years were analyzed for all. The major outcomes were ischemia-driven target vessel failure (ID-TVF), a composite of cardiac death, target vessel myocardial infarction, or ischemia-driven target vessel revascularization, definite/ probable stent thrombosis (ST), and BARC bleeding $2-5$ at 2 years.

Result DAPT cessation after 12 months following the index PCI decreases the risks of ID-TVF, but not ST compared to no DAPT cessation group. As showed in the results, ID-TVF rate in the DAPT cessation at 12-24 months group compared with no DAPT cessation group was $4.63 \%$ vs $9.21 \%(\mathrm{P}=0.001)$, while the $\mathrm{ST}$ rate in those two group was $0.77 \%$ vs $0.6 \%(\mathrm{P}=0.75)$. Among the 13 definite/probable ST events, 12 (92.9\%) of them occurred on DAPT and 1 occurred off DAPT. On the other hand, use of DAPT was a strongly independent predictor of BARC bleeding ( $\mathrm{HR}=9.82,95 \% \mathrm{CI}$ : 4.64-20.80, $\mathrm{P}<$ $0.0001)$ at 2 years. Overall, adverse events occurred on DAPT was increased overtime.

Conclusion The present study demonstrated that DAPT cessation was not associated with an increase in adverse ischemic events and might suggest that shorter DAPT duration is reasonable to decrease bleeding complications in Chinese patients receiving a CoCr EES.

\section{Double-kissing culotte stenting technique in coronary bifurcation disease: angiographic follow-up and 12-month clinical outcomes \\ Tao $\mathrm{Hu}$ \\ Department of Cardiology, Xijing Hospital, Fourth Military Medical \\ University}

Objective The culotte technique is a widely used method. However, this technique has its own limitations. We therefore developed a double kissing (DK) balloon method to optimize the final result of culotte stenting. To study this technique, and to compare it to the classical crush stenting technique, we performed a prospective, randomized trial aimed at determining the differences in the rates of final kissing balloon inflation (FKBI) and long-term clinical outcomes from January 2006 to June 2013 at our institution.

Methods We prospectively enrolled patients undergoing PCI of a true coronary bifurcation lesion with a Medina classification of $(1,1$, 1) with drug-eluting stents (Firebird) in our institution. Patients were randomly selected to undergo treatment using either the classical Culotte stenting or the DK Culotte stenting technique with DES. Angiographic follow-up was scheduled between 6 and 9months. Clinical follow-up was available up to 12 months. All patients underwent monthly followup by telephone or clinic review.

Result A total of 124 bifurcation lesions (58 patients with 63 lesions in the classical Culotte stenting group, and 59 patientswith 61 lesions in the DK Culotte stenting group were enrolled in this study. Procedural angiographic success was achieved in all lesions. FKBI were performed in $89 \%$ of the classical Culotte group and in $100 \%$ of the DK Culotte group $(\mathrm{P}<0.001)$, and there was a lower rate of unsatisfactory final kissing balloon inflations ( 16.51 vs $3.3 \%, \mathrm{P}<0.01)$ in the DK Culotte stenting group; The overall restenosis rate was $12.6 \%$ the classical Culotte group, however, the restenosis rate for the DK Culotte group was only $8.7 \%(\mathrm{P}<0.001)$; The cumulative MACE were higher in the classic Culotte stent group than those in the DK Culotte group (12.18\% vs $4.71 \%, \mathrm{P}=0.001)$; Predictors of angiographic restenosis were older age, increasing bifurcation angle, more severe distal main branch stenosis, and smaller side branch reference diameter, kissing balloon post-dilatation tended to have a protective effect.

Conclusion The DK Culotte stenting technique is associated with 
high procedural success by optimizing the FKBI rates and by allowing a complete stent expansion at the side branch ostium and a relatively low risk of angiographic restenosis. The improved clinical outcomes were sustained even at a 12 month follow-up period.

\section{Duration of dual antiplatelet therapy and outcomes after left main percutaneous coronary intervention \\ $\mathrm{Tao} \mathrm{Hu}$ \\ Department of Cardiology, Xijing Hospital, Fourth Military Medical University}

Objective Despite treatment recommendations for at least 12 months of dual antiplatelet therapy (DAPT) following drug-eluting stent revascularization, the optimal duration of DAPT after left main percutaneous coronary intervention remains controversial. We sought to evaluate differences in late safety outcomes relative to DAPT duration in patients treated with drug eluting stents in left main coronary artery.

Methods 216 patients undergoing successful primary percutaneous left main coronary intervention of dual antiplatelet therapy with Aspirin $(100 \mathrm{mg} / \mathrm{d})$ and Clopidogrel $(75 \mathrm{mg} / \mathrm{d})$ were eligible for enrolment in this randomised, double-blind, placebo-controlled trial from September 2008 to October 2013 at our institution. Patients were analyzed according to continuation or discontinuation of DAPT at a 12-month landmark, excluding patients with events prior to the landmark, and outcomes were followed up to 36- months after stenting. Among patients who were event-free at 12-months, clopidogrel was discontinued in 88 patients and was continued for longer than 36-months in 94 patients. The main outcome for our current analysis was Target Vessel Failure (TVF), defined as target vessel-related cardiac death or myocardial infarction and target vesselrevascularization. Secondary outcomes included stent thrombosis (ST).adjusted hazard ratios (HR) for TVF were calculated with Cox regression.

Result Through 3 years, risk-adjusted ischemic event rates did not significantly differ between groups: 12 versus $\geq 36$ months: death ( $2.6 \%$ vs $2.3 \%$ ), myocardial infarction (MI, $0.2 \%$ vs $1.0 \%$ ), and definite/ probable stent thrombosis (ST, $0.2 \%$ vs $0 \%$ ). Composite events also did not statistically vary between DAPT durations. In multivariable analysis, 12-month versus longer DAPT duration was not associated with increased likelihood of thrombotic events at 3-year follow-up. Even after addition of identified independent predictors for TVF, adjusted TVF hazards were comparable. Major bleeding was negligible across groups.

Conclusion 12-month dual antiplatelet therapy seems to be feasible after left main percutaneous coronary intervention in Chinese patients.

\section{The cardiac resynchronization therapy can decrease microvolt $T$ wave alternans \\ Zhen Chen, Xiaofeng Hou, Yuanyuan Tang, \\ Feng Zhang, Jiangang Zou \\ Department of Cardiology, First Afflilated Hospital of Nanjing Medical \\ University}

Background and Objective Cardiac resynchronization therapy (CRT) has been shown to reverse the ventricular remodeling and reduce the ventricular arrhythmias in heart failure patients. But it is not clear whether CRT can affect microvolt $\mathrm{T}$ wave alternans (MTWA). We sought to study the effect of CRT on MTWA.

Methods The new CRT patients were included. The echocardiographic parameters included left ventricular end systolic volume (LVESV) and ejection fraction (EF). MTWA was tested immediately after CRT and repeated at more than 6-month follow-up period at 110 biventricular pacing rate using modified moving average analysis method. All patients were divided into CRT responders group and non-responders group according to whether LVESV decreased $\geq 15 \%$ after CRT. The MTWA changes were compared between responders and nonresponders.

Result 29 patients (20 males) were included, average age of ( $8.5 \pm$ 11.4) y/o. Among 29 patients, 16 were CRT responders. There were the same LVEF[(31.4 \pm 7.2$) \%$ vs $(30.3 \pm 4.8) \%, \mathrm{P}=0.66]$, same LVESV $[(211.30 \pm 86.91) \mathrm{ml}$ vs $(197.49 \pm 91.41) \mathrm{ml}, \mathrm{P}=0.68]$ and same MTWA $[(14.38 \pm 11.59) \mu \mathrm{V}$ vs $(14.15 \pm 9.59) \mu \mathrm{V}, \mathrm{P}=0.96]$ at baseline between responders and nonresponders. After the mean follow-up period of (8.1 $\pm 3.9)$ month, responders had significant increase of LVEF [(43.32 \pm $9.77) \%$ vs $(32.3 \pm 6.07) \%, \mathrm{P}=0.002]$, decrease of LVESV [(130.90 $\pm 77.67) \mathrm{ml}$ vs $(202.62 \pm 84.30) \mathrm{ml}, \mathrm{P}=0.02]$ and decrease of MTWA amplitude $[(6.37 \pm 5.19) \mu \mathrm{V}$ vs $(14.00 \pm 12.46) \mathrm{Mv}, \mathrm{P}=0.03]$ than nonresponders. Meanwhile, the changes of LVESV $[(-80.40 \pm 35.64) \mathrm{ml}$ vs $(5.13 \pm 20.89) \mathrm{ml}, \mathrm{P}=0.000]$ and $\mathrm{MTWA}[(-8.00 \pm 9.86) \mu \mathrm{V}$ vs $(-0.15$ $\pm 13.23) \mu \mathrm{V}, \mathrm{P}=0.08]$ of the two groups are also significantly different. A significant positive correlation was found between the change of MTWA and the change of LVESV $(r=0.4878, \mathrm{P}=0.007)$.

Conclusion MTWA amplitude changed with heart function after CRT. When heart function improved, MTWA decreased.

\section{RESOLUTE China randomized controlled trial: two-year results of resolute zotarolimus eluting stent versustaxusliberte paclitaxel eluting stent Bo Xu \\ Department of Cardiology, State Key Laboratory of Cardiovascular Diseases, Fuwai Hospital, National Center for Cardiovascular Diseases, Chinese Academy of Medical Sciences and Peking Union Medical College}

Objective We sought to compare the 2-year clinical outcomes with theResolute ${ }^{\mathbb{B}}$ zotarolimus-eluting stent (R-ZES) vsthe Taxus ${ }^{\mathrm{TM}}$ Liberte $^{\mathrm{TM}}$ paclitaxel-eluting stent (PES) in a Chinese population.

Methods The RESOLUTE China multicenter trial randomized patients to R-ZES or PES. Inclusion criteria included de novo coronary artery lesions in native coronary arteries with a reference vessel diameter of $2.25 \mathrm{~mm}$ to $4.0 \mathrm{~mm}$. Clinical outcomes included target lesion failure (TLF), a composite of death from cardiac causes (CD), target vessel myocardial infarction (TV-MI), and clinically-driven target lesion revascularization (TLR); target vessel failure (TVF), a composite of target vessel revascularization (TVR), CD, and TV-MI; and Major Adverse Cardiac Events (MACE), a composite of death, MI, emergent coronary bypass surgery, or TLR. Between 26 September 2011 and 30 November 2011, 400 patients (198 R-ZES vs 202 PES) were enrolled at 16 study sites in China. Baseline characteristics were similar in patients treated with R-ZES vs PES: mean age was $(59.7 \pm 9.9)$ vs $(59.6 \pm 10.6)$ years $(\mathrm{P}=0.986)$, and men comprised $77.8 \%$ vs $80.7 \%(\mathrm{P}=0.538)$ of the study group, respectively; however, acute MI within 72 hours at baseline was $8.9 \%$ with R-ZES vs $3.2 \%$ with PES $(\mathrm{P}=0.029)$. The primary endpoint, 9-month late lumen loss, was significant lower for R-ZES vsPES $[(0.16 \pm 0.38) \mathrm{mm}$ vs $(0.33 \pm 0.52) \mathrm{mm}, \mathrm{P}<0.001)$, and 1 -year clinically-driven TLR was significantly lower for R-ZES vs PES (1.5\% vs $7.0 \%, \mathrm{P}=0.011$ ).

Result Follow-up at 2 years is available for 194 R-ZES patients 
and 194 PES patients. R-ZES as compared with PES continued to have a significantly lower rate of clinically-driven TLR $(2.6 \%$ vs $7.7 \%, \mathrm{P}=$ $0.036)$, clinically-driven TVR (3.1\% vs $9.7 \%, \mathrm{P}=0.012)$, TVF $(7.2 \%$ vs $14.3 \%, \mathrm{P}=0.033)$ and $\mathrm{MACE}(6.7 \%$ vs $13.3 \%, \mathrm{P}=0.042)$, and a numerically lower TLF $(6.7 \%$ vs $12.2 \%, P=0.083)$, respectively. There was no significant difference between R-ZES and PES in the incidence of cardiac death ( $1.0 \%$ vs $1.0 \%)$, TV-MI $(4.1 \%$ vs $3.6 \%, \mathrm{P}=0.799)$, or ARC definite/probable ST (1.0\% vs $1.0 \%)$, respectively.

Conclusion Use of R-ZES as compared with PES in a Chinese population was associated with a significantly lower rate of TLR, TVR, TVF, and MACE that was sustained to two years post enrollment, and TLF continued to trend lower with R-ZES as compared to PES. These results confirm the excellent and sustained clinical safety and efficacy of R-ZES also in a Chinese population.

\section{The platelet receptor $\mathrm{P}_{2 Y_{12}}$ gene polymorphisms could predict bleeding risk in clopidogrel-treated chinese patients after percutaneous coronary intervention \\ Jiahui Zhang, Jinqing Yuan, Xianmin Meng, Xiaofang Tang, Jing Wang, Yi Yao, Yuanliang Ma, Jue Chen, \\ Yuan Wu, Zhan Gao, Runlin Gao \\ Department of Cardiology, State Key Laboratory of Cardiovascular \\ Diseases, Fuwai Hospital, National Center for Cardiovascular Diseases, Chinese Academy of Medical Sciences and Peking Union Medical \\ College}

Objective Polymorphisms in the platelet receptor $\mathrm{P}_{2} \mathrm{Y}_{12}$ gene have been suggested to influence the pharmacodynamics of Clopidogrel and prognosis of patients treated with Clopidogrel. The effect of $\mathrm{P} 2 \mathrm{Y}_{12}$ polymorphisms on bleeding events of Clopidogrel treatment have not yet been reported in Chinese patients after percutaneous coronary intervention (PCI). This study sought to investigate the impact of $\mathrm{P}_{2} \mathrm{Y}_{12}$ polymorphisms on the risk of bleeding in Clopidogrel-treated Chinese patients after PCI.

Methods Between January 2011 and June 2012, 504 consecutive patients with acute coronary syndromes (ACS) who received coronary angiography or an uneventful PCI and were exposed to Clopidogrel treatment for 12 months, were enrolled in the single-center registry. 18 tag single nucleotide polymorphisms (SNPs) selected from $\mathrm{P}_{2} \mathrm{Y}_{12}$ gene and the gain of function CYP2C19*17 allele were detected by the ligase detection reaction. The primary clinical safety end point was the incidence of major bleeding defined according to the Bleeding Academic Research Consortium (BARC) criteria, including type 3 and 5 in the analysis. The primary clinical efficacy end point was a composite of cardiovascular death, non-fatal myocardial infarction, unplanned target vessel revascularization, and stent thrombosis. The follow-up period was 12 months.

Result Major bleeding events occurred in 47 patients (9.3\%) according to BARC criteria, including 12 patients or $2.4 \%$ with BARC type $3 \mathrm{~b}$ bleeding, 35 patients or $6.9 \%$ with BARC type 3a bleeding. Binary logistic regression analysis identified two tag SNPs rs6785930 $(\mathrm{OR}=2.305,95 \%$ CI: $1.180-4.503, \mathrm{P}=0.015), \mathrm{rs} 6809699(\mathrm{OR}=$ $3.227,95 \% \mathrm{CI}: 1.587-6.558, \mathrm{P}=0.001)$ ) as significant independent predictors of BARC type $\geq 3$ bleeding in the context of gain of function CYP2C19*17 allele. A total of 34 ischemic events occurred. However, no significant influence of tag SNPs of $\mathrm{P}_{2} \mathrm{Y}_{12}$ on the occurrence of ischemic events was found.

Conclusion In our study population, the $\mathrm{P}_{2} \mathrm{Y}_{12}$ genetic locus harbors SNPs that could have influence on bleeding events after PCI.

\section{Video-assisted thoracoscopic pericardial fenestration for diagnosis and management of pericardial effusions \\ Zili Meng ${ }^{1}$, Hongwei $\mathrm{Li}^{2}$, Shucai $\mathrm{Wu}^{1}$, Hui $\mathrm{Li}^{1}$, Yonghui Liu ${ }^{1}$ 1. Hebei Chest Hospital \\ 2. The Second Affliated Hospital of Zhengzhou University}

Objective Pericardial effusion is a clinical condition requiring multidisciplinary management. In the study we evaluate the efficacy of video-assisted thoracoscopic pericardial fenestration (VATSPF) approach.

Methods Since May 2004 to January 2014, 23 patients (14 men, 9 women; mean age 49 yrs) with echocardiographically diagnosed pericardial effusion underwent video-assisted thoracoscopy at our hospital.Pericardial fenestrations were created under general anesthesia and single-lung ventilation using 2 to 3 trocars. A left thoracic approach was used in 16 patients and a right thoracic approach in 7.

Result The mean duration of the surgery was $(32.1 \pm 12) \mathrm{min}$. Microbiology and virology cultures of the pericardial fluid were negative. The definitive diagnoses were tuberculosis in $30.43 \%$, malignant processes in $26.09 \%$ of cases, including bronchogenic carcinoma and breast cancer. Histologic findings were compatible with tuberculosis in 6 cases and infiltrating breast carcinoma, and infiltrating nonsmall cell carcinoma in 1 case each. In the remaining patients, the histologic diagnosis was chronic or subacute nonspecific pericarditis. There was no surgical mortality and complications of the thoracoscopy technique.

Conclusion Video-assisted thoracoscopic pericardial fenestration is an effective and safe technique for the diagnosis and treatment of chronic pericardial effusions, and which also makes possible the study of pleural cavity, the obtaining of parietal pleura samples and, if necessary, the performance of talcpleurodesis.

\section{Safety and efficacy of polymer free vs based drug-eluting stents \\ Xiaohui Zhao \\ Cardiovascular Department of Xinqiao Hospital, Third Military Medical \\ University}

Background Polymer free drug-eluting stents (PF-DES) have recently been developed to improve the delayed healing and hypersensitive reaction in the vessel.However, Uncertainty exists regarding the relative performance of PF-DES versus polymer basedDES (PB-DES) in percutaneous coronary intervention. The aim of this study was to perform a Meta-analysis of randomized controlled trial (RCT) comparing the safety and efficacy profile of PF-DES vs PB-DES.

Methods and Result PubMed, Embaseand Cochrane Central Register of Controlled Trials (CENTRAL) were searched for RCTs that compared PF-DES and PB-DES, enrolling at least 100 patients.Efficacy endpoints were target-lesion revascularization (TLR) and in-stent late loss (ISLL). Safety endpoints werecardiac-death, death, myocardial infarction (MI), composite of definite and probable stent thrombosis (ST). The meta-analysis included 9 RCTs $(n=6364)$ with a median follow-up of 26 months clinical outcomes and 7 RCTs $(n=5027)$ with interest angiography results. The results showed that there was no differencebetween PF-DES and PB-DESwith regard to cardiac death, 
death, ST, MI, ISLL or TLR.

Conclusion PF-DES is comparable as PB-DES with regard tocardiac death, death, ST, MI, ISLL and TLR. Further large RCTs with longer follow-up are warranted to better define the relative merits of these drug-eluting stents.

\section{Cardiovascular changes during pericardial window by videothoracoscope for pericardial effusions \\ Zili Meng ${ }^{1}$, Hongwei $\mathrm{Li}^{2}$, yonghui Yang ${ }^{1}$, Qiang $\mathrm{Li}^{1}$ \\ 1. Hebei Chest Hospital \\ 2. The Second Affliated Hospital of Zhengzhou University}

Objective Pericardial windows by videothoracoscopic (VATS) is effective for diagnosis and management of pericardial effusions, however, cardiovascular changes during pericardial window for pericardial effusions by videothoracoscope are not well understood, and most studies are of systemic effects and not of right ventricular performance. In the study, we evaluate the cardiovascular changes during pericardial window by videothoracoscope.

Methods Since May 2004 to July 2013, 17 patients (11 men, 6 women) with echocardiographically diagnosed pericardial effusion underwent video-assisted thoracoscopy with a low-pressure pneumothorax $(6 \mathrm{~mm} \mathrm{Hg})$ at our hospital. Pericardial windows were created under general anesthesia and single-lung ventilation using 2 to 3 trocars. Cardiac output was measured by thermodilution with the patient anaesthetized in the supine position before the procedure; in the right lateral position after a low-pressure pneumothorax had been established; and after drainage of the pericardial effusion.

Result The mean duration of the surgery was $(35.2 \pm 10) \mathrm{min}$. Before the procedure, cardiac output was low and central venous pressure and pulmonary artery occlusion pressure were increased. Systemic vascular resistance and arterial blood pressure were within normal limits. After the pericardial windows cardiac index increased and systemic and pulmonary vascular resistances were reduced.There was no surgical mortality and complications of the thoracoscopy technique.

Conclusion Pericardial effusion reduces right ventricular distensibility, right and left systolic ventricular function, and cardiac output. Pericardial windows of pericardial effusions allows cardiac distensibility to increase and cardiac performance changes to increase ejection.

\section{Safety and efficacy of degradable vs permanent polymer drug-eluting stents \\ Xiaohui Zhao \\ Cardiovascular Department of Xinqiao Hospital, Third Military Medical \\ University}

Background Degradable polymer drug-eluting stent (DP-DES) represents a promising strategy to improve the delayed healing and hypersensitive reaction in the vessel. However, the efficacy and safety of DP-DES vs permanent polymer drug-eluting stents (PP-DES) are less well defined. The aim of this meta-analysis was to compare the total, short ( $<30 \mathrm{~d})$, mid (30 d-1 y) and long ( $>1 \mathrm{y})$ term outcomes of DPDES vsPP-DES.

Methods and Result PubMed, Embase, and Cochrane Central Register of Controlled Trials (CENTRAL) were searched for randomized clinical trials to compare any of approved DP-and PP-DES.
Efficacy endpoints were target-lesion revascularization (TLR) and instent late loss (ISLL). Safety endpoints were death, myocardial infarction (MI), composite of definite and probable stent thrombosis (ST). The meta-analysis included 19 RCTs $(n=18395)$ with interest results. As compared with DES, there was a significantly reduced very late ST (OR $=0.42,95 \%$ CI: $0.24-0.77, \mathrm{P}=0.852)$ and ISLL $(\mathrm{OR}=-0.07,95 \% \mathrm{CI}$ : $-0.12-0.02, \mathrm{P}=0.000)$ in DP-DES patients. However, there were no difference for other safety and efficiency outcomes between DP-DES and PP-DES, except that the stratified analysis showed a significant decreased TLR with DP-DES as compared to paclitaxel-eluting stent (OR $=0.41,95 \%$ CI: $0.20-0.81, \mathrm{P}=0.457$ ).

Conclusion DP-DES is more effective in reducing very late ST and ISLL, as well as comparable to PP-DES with regard to death, TLR and MI. Further large RCTs with long-term follow-up are warranted to better define the relative merits of DP-DES.

\section{Application value of balloon angioplasty in patients of rheumatic heart disease combined with left atrioventricular valve stenosis and right atrioventricular valve regurgitation \\ Zhangqiang Chen \\ Jiangxi province people’s hospital}

Objective To investigation apply value of percutaneous balloon mitral valvuloplasty (PBMV) in patients of rheumatic heart disease combined with left atrioventricular valve stenosis and right atrioventricular valve regurgitation.

Methods 1700 patients selected in our hospital from January 2000 to June 2012 due to rheumatic heart disease with left atrioventricular valve stenosis and undergoing PBMV, of which 220 patients with tricuspid valve regurgitation, average age $(45 \pm 12)$ yrs, male 90 cases, female 130 cases, mitral valve area [average of $(0.9 \pm 0.3) \mathrm{cm}^{2}$ ], tricuspid regurgitation area [average $(11.2 \pm 6.5) \mathrm{cm}^{2}$ ]. Incremental approach to expanding with contrast agent. the end of expansion: left atrial pressure decreased more than $50 \%$, or the average left atrial pressure below $15 \mathrm{~mm} \mathrm{Hg}$, the rumbling murmur in mitral valve area disappeared or significantly reduced. Diuretics were combined with use and maintain more than $2000 \mathrm{ml}$ urine volume a day for patients with severe tricuspid regurgitation. within the first 2-3 d after operation, we reviewed left atrioventricular valve area (MVA), right atrioventricular valve regurgitation area (TRA), left atrial diameter (LAD), right atrial diameter (RAD), pulmonary artery pressure (PAP) and left ventricular ejection fraction (LVEF) by transthoracic echocardiography. After PBMV, regular telephone or in outpatient were followed up for 6 months to 9 years, mean $(75 \pm 32)$ months and at the end of follow up these indicators (ie. MVA, TRA, LAD, RAD, PAP and LVEF) were reviewed by color Doppler echocardiography, the incidence of cardiovascular events was observed such as thromboembolism, heart failure, arrhythmia and death, etc.

Result The mitral valve areas after percutaneous balloon mitral valvuloplasty increased significantly than the preoperative $(\mathrm{P}<0.01)$; the postoperative tricuspid regurgitation areas significantly reduced than the preoperative $(\mathrm{P}<0.01)$; the postoperative left atrial diameter significantly reduced than the preoperative $(\mathrm{P}<0.01)$, and the postoperative right atrial diameter significantly reduced than the preoperative $(\mathrm{P}<0.01)$; the postoperative average of left atrium pressure significantly reduced compared with the preoperative $(\mathrm{P}<0.01)$, the postoperative right atrial pressure decreased significantly compared with preoperative $(\mathrm{P}<0.01)$; The pulmonary arterial pressure decreased significantly after PBMV (P 
$<0.01)$. Rumbling -like diastolic murmur from mitral valve weakened from severe to mild degree, Systolic murmur-like hair from mitral valve from the operative $0-1 / 6$ degree up to $1-2 / 6$ degree, The symptom of chest tightness, shortness of breath and other symptoms were relieved, cardiac function significantly improved. All cases followed up for 6 months to 9 years [average $(75 \pm 32)$ months], 2 patients with severe regurgitation died (one case of massive cerebral infarction, and 1 case of heart failure after 6 years and 8 years respectively), two cases lost access. At the end of follow-up, MVA has been reduced compared with the postoperative $(\mathrm{P}<0.05)$; LAD, RAD slightly increased compared with the postoperative $(\mathrm{P}<0.05)$, but did not reach the preoperative level. TRA slightly increased compared with the post-operation, but the difference was not statistically significant $(\mathrm{P}>0.05)$; The PAP and LVEF appeared no statistical difference compared with the postoperative, the remaining patients without serious complications.

Conclusion Percutaneous balloon dilatation for treatment of rheumatic heart disease combined with left atrioventricular valve stenosis and mild to moderate right atrioventricular valve regurgitation.in patients could alleviate the symptoms and reduce size of tricuspid regurgitation, It'sefficacy sure; For patients with severe tricuspid valve regurgitation, It can also improve the quality of life, recent and mid-term efficacy is certain. the long-term efficacy remains to be observed. 


\section{Cardiac Surgery in the Adult}

\section{Effects of dexmedetomidine on perioperative sedation in coronary artery bypass grafting surgery patients: A meta-analysis}

Fangfang Cao, Haitao Zhang, Xue Feng

State Key Laboratory of Cardiovascular Disease, Fuwai Hospital,

National Center for Cardiovascular Diseases, Chinese Academy of

Medical Sciences and Peking Union Medical College

Objective To assess the effect of dexmedetomidineon perioperative sedation in coronary artery bypass grafting surgery patients.

Method Electronic databases including Cochrane library, Pubmed, EMBase, Highwire, MEDLINE, CBM, CNKI and CSJD were searched. A meta-analysis of all randomized controlled trials (RCTs) comparing dexmedetomidine with control in perioperative coronary artery bypass grafting surgery was performed.Study selection and meta-analysis were conducted which according to the Cochrane Handbook for systematic reviews. Data were extracted from these trials by 3 reviewers independently and analyzed by RevMan 5.0 software.

Result A total of 10 RCTs including 562 patients were assessed in this study. Compared with control group, dexmedetomidinegroup decreased ventilation time significantly $(\mathrm{RR}=-2.26,95 \% \mathrm{CI}:-4.44$ $-0.07 ; \mathrm{P}=0.04)$, ventricular tachyarrhythmias $(\mathrm{RR}=0.21,95 \% \mathrm{CI}$ : $0.09-0.53 ; \mathrm{P}=0.0009)$, and incidence of delirium $(\mathrm{RR}=0.53,95 \%$ CI: $0.29-0.98 ; \mathrm{P}=0.04)$. No difference was existed in intensive care unit time $(\mathrm{RR}=-0.27,95 \% \mathrm{CI}:-0.56-0.02 ; \mathrm{P}=0.06)$, mortality $(\mathrm{RR}=$ $0.77,95 \%$ CI: $0.20-3.05 ; \mathrm{P}=0.71)$, bradycardia $(\mathrm{RR}=1.46,95 \% \mathrm{CI}$ : $0.88-2.44 ; \mathrm{P}=0.14)$, hypotension $(\mathrm{RR}=1.55,95 \% \mathrm{CI}: 0.98-2.46, \mathrm{P}=$ $0.06)$, application of vasoactive agents $(\mathrm{RR}=0.99,95 \% \mathrm{CI}$ : $0.56-1.76$; $\mathrm{P}$ $=0.98)$, atrial-fibrillation $(\mathrm{RR}=0.82,95 \% \mathrm{CI}: 0.58-1.17 ; \mathrm{P}=0.27)$.

Conclusion Dexmedetomidine applied in perioperative coronary artery bypass grafting surgery patients could decreased ventilation time, incidence of ventricular tachyarrhythmias and delirium and no increase in complications, thereby improved the prognosis of patients.

\section{The meta-analysis of the comparison of conventional coronary artery bypass grafting and hybrid coronary revascularization \\ Jiehui Li, Kun Hua, Xiubin Yang \\ State Key Laboratory of Cardiovascular Disease, Fuwai Hospital, National Center for Cardiovascular Diseases, Chinese Academy of Medical Sciences and Peking Union Medical College}

Objective To compare the prognosis of hybrid coronary revascularization (HCR) and conventional coronary artery bypass grafting (CABG).

Methods We performed the electronic search of the PubMed. We defined the "hybrid coronary revascularization" as the operation based on combining the excellent long term patency of LIMA-LAD graft by surgical procedure and minimal invasiveness of interventional procedures to achieve complete revascularization. The primary end point of this meta-analysis was the mortality of all cause death (ACD) in the follow-up after two ways of revascularization (HCR, CABG), the rate of "repeat revascularization" and "major adverse cardiac events" (MACE) or "major adverse cardiac and cerebral events (MACCE) ". The secondary end point was the number of bridge vessels used in operation.

Result We found 965 articles from the internet, of which, we considered and retrieved 6 citations with 5572 patients for possible inclusion in the meta-analysis. The mean age of participants in individual trials varied from 60 to 65 years, and the length of follow-up ranged from 1 to 60 months. It had been found that the HCR therapy has no significant benefit of the prognosis of ACD compared with CABG $(0.58 \%$ vs $1.68 \%$; $\mathrm{RR}=0.434,95 \% \mathrm{CI}: 0.144-1.31 ; \mathrm{P}=0.14)$. The comparison of MI also conducted to the same result, which could not find the significant difference between $\mathrm{HCR}$ and CABG $(0.27 \% \mathrm{vs} 0.67 \%$; RR $=$ $0.39,95 \%$ CI: $0.04-3.89 ; \mathrm{P}=0.43$ ). We finally found that patients treated with HCR definitely had a higher probability than conventional coronary artery bypass grafting of repeat revascularization $(5.23 \%$ vs $0.77 \%$; $\mathrm{RR}=2.52,95 \%$ CI: $1.47-4.32 ; \mathrm{P}<0.001)$. And we found that patients treated with HCR therapy had a better result of MACE, compared with CABG (3.52\% vs 3.67\%; RR $=0.32,95 \%$ CI: $0.17-0.61$; $\mathrm{P}<0.001)$. There was no doubt that patients treated with HCR had a lower number bridge vessels used in operation than CABG (1.08 vs 2.98; RR $=0.711$, $95 \%$ CI: $0.663-0.762, \mathrm{P}<0.001)$. However, though operations of HCR may use fewer saphenous veins, they also used stents of bare metal and drug eluting, which consequently led to a longer length of stay in operation room and higher cost because of the hybrid medical process and supplies.

Conclusion It could be concluded that HCR therapy has a significant benefit of MACE and usage of bridge vessels, compared with conventional CABG.

\section{The comparison of short and long term prognosis about the aortic dissection between open surgery and endografting or hybrid procedure \\ Jiehui Li, Meng Peng, Xiubin Yang Cadiac Surgery, State Key Laboratory of Cardiovascular Disease, Fuwai Hospital, National Center for Cardiovascular Diseases, Chinese Academy of Medical Sciences and Peking Union Medical College}

Objective To compare the short and long term prognosis about the aortic dissection between open surgery and endografting or hybrid procedure.

Methods We searched Medline and Embase for published studies reporting on the comparison between open surgery and endografting or hybrid procedure. We defined the "hybrid procedure" as the endovascular procedures were performed either concomitantly or in a delayed fashion, less than 30 days after the open surgery. The primary end point of this meta-analysis was the mortality of all cause death (ACD) in hospital or in 30 days discharged, and the long term mortality. The secondary end point was the morbidity of neurologic complications in hospital.

Result We had found 1230 articles on the internet, and enrolled 14 citations with 2141 patients for the Meta-analysis at last. There could be found that the endografting or hybrid procedure has the significant benefit about the prognosis of ACD, compared with the conventional open surgery in hospital and 30 days discharged (13.95\% vs $21.25 \%$; RR $=0.61,95 \%$ CI: $0.49-0.77 ; \mathrm{P}<0.001)$, however, we could not conduct 
the same result in the long term mortality $(22.44 \%$ vs $27.47 \%, \mathrm{RR}=0.75$, $95 \%$ CI: $0.54-1.03 ; \mathrm{P}=0.08$ ). We could find that there is a significant difference about the morbidity of neurologic complications in hospital, which shows a lower occurrence of the endografting or hybrid procedure (10.09\% vs $11.12 \%, \mathrm{RR}=0.73,95 \%$ CI: $0.54-0.98 ; \mathrm{P}=0.04)$.

Conclusion The endografting or hybrid procedure could conduct to a better result in hospital, compared with conventional open surgery. And the long term prognosis shows the same trend; however, we need more clinical trial to confirm it.

\section{Preliminary explore of acute kidney injury associated with deep hypothermic circulatory arrest \\ Yingjie Du ${ }^{1}$, Guyan Wang ${ }^{1}$, Lijing Yang ${ }^{1}$, Sheng Shi ${ }^{1}$, \\ Jun $\mathrm{Li}^{1}$, Bingyang $\mathrm{Ji}^{2}$, Zhe Zheng \\ State Key Laboratory of Cardiovascular Disease, Fuwai Hospital, \\ National Center for Cardio-vascular Diseases, Chinese Academy of \\ Medical Sciences and Peking Union Medical College \\ 1. Department of Anesthesiology \\ 2. Department of Cardiopulmonary Bypass \\ 3. Department of Cardiac Surgery}

Objective To confirm whether deep hypothermic circulatory arrest can lead to acute kidney injury. To investigate the relationship between endoplasmic reticulum stress and acute kidney injury associated with deep hypothermic circulatory arrest. To investigate the relationship between inflammatory response and acute kidney injury associated with deep hypothermic circulatory arrest. Preliminary explore of the influence of different hypothermic circulatory arrest temperature on postoperative renal function.

Methods Twenty eight male Sprague-Dawley rats were divided into five groups randomly: group A (deep hypothermic circulatory arrest: $15^{\circ} \mathrm{C}-20^{\circ} \mathrm{C}, \mathrm{n}=6$ ), group $\mathrm{B}$ (moderate hypothermic circulatory arrest: $20^{\circ} \mathrm{C}-25^{\circ} \mathrm{C}, \mathrm{n}=6$ ), group $\mathrm{C}$ (mild hypothermic circulatory arrest: $25^{\circ} \mathrm{C}-30^{\circ} \mathrm{C}, \mathrm{n}=6$ ), group $\mathrm{D}$ (cardiopulmonary bypass, $\mathrm{n}=6$ ) and group $\mathrm{E}(\mathrm{sham}, \mathrm{n}=4)$. In the whole process of the experiment, hemodynamicparameterswas real-time monitored. After removing from cardiopulmonary bypass, the rats received mechanical ventilation for 1 hour, thenwere executed, the kidney and blood were harvested. The serum creatinine values (classic indicators for renal function) were compared bewteen the five groups. We observed the pathological morphological changes of different groups for renal tubular injury using light microscope, and renal tissue ultrastructure changes (including mitochondria, endoplasmic reticulum and nucleus) using scanning electron microscope. TUNEL method was used to detect apoptosis index of the kidney. ELISA method was used to detect the release of proinflammatory factors, IL-1, IL-6 and TNF-alafa.We used Western Blot method to detect the expression of protein, including endoplasmic reticulum stress related protein CHOP, GRP-78, Caspase-12, NF-kappa $\mathrm{B}$ and the apoptosis related proteins Bax, Bcl-2, caspase-3. The mRNA level of caspase-3, CHOP and GRP-78 were detected by RealTime PCR.

Result The serum creatinine levels of three hypothermic circulatory arrest group A, B, C, were significantly higher than cardiopulmonary bypass group $\mathrm{D}$ and the sham group $\mathrm{E}(\mathrm{P}<0.05)$, but no difference were detected between the three groups. The release of proinflammatory factors, including IL-1, IL- 6 and TNF- $\alpha$, in the three hypothermic circulatory arrest groups was significantly higher than that in sham group $\mathrm{E}(\mathrm{P}<0.05)$. Renal tubular injury of three hypothermic circulatory arrest groups were worse than cardiopulmonary bypass group $\mathrm{D}$ and sham group E. The higher temperature was, the more severe renal tubular injury was.Ultrastructures were observed using scanning electron microscopy for mitochondria and endoplasmic reticulum, and the result was consistent with the renal tubular injury. In terms of apoptosis, renal apoptosis cells were rare in all groups. CHOP protein and mRNA were significantly higher in three hypothermic circulatory arrest groups than cardiopulmonary bypass group $\mathrm{D}$ and the sham group $\mathrm{E}(\mathrm{P}<0.05)$. There was no significant difference in other protein and mRNA.

Conclusion This study confirmed that deep hypothermic circulatory arrest can lead to acute kidney injury. It was based on multi-level and multi-angle evidence, including the classic renal function indicator serum creatinine, pathological morphological change (light microscopy, scanning electron microscope), the release of proinflammation factors, and the expression of protein $\mathrm{CHOP}$ and its mRNA.

Taking the ultrastructure change for endoplasmic reticulum and the expression of maker for endoplasmic reticulum (CHOP and its mRNA) into consideration, we speculate that acute kidney injury associated with deep hypothermic circulatory arrest may be induced by endoplasmic reticulum stress.

Considering the expression of CHOP and its mRNA, proinflammatory factors, and conclusions of recent studies, we speculate that CHOP may lead to acute kidney injury associated with deep hypothermic circulatory arrest through regulating inflammatory reaction.

Although we did not find the differences in acute kidney injury induced by different hypothermic circulatory arrest temperatures in terms of serum creatinine, proinflammatory factors and CHOP, but the pathological morphology indicate that the higher temperature are, the more severe renal tubular injury is. The clinical practice is complex and changeful, we should carry out more rigorous animal experiments and large-scale prospective clinical trials, to further study the influence of different hypothermic circulatory arrest temperature on postoperative renal function.

\section{Asymptomatic pulmonary embolism following off-pump coronary artery bypass: outcomes of a prospective observational study \\ Jian Wang, Hengchao Wu, Bin Lv, Hansong Sun State Key Laboratory of Cardiovascular Disease, Fuwai Hospital, National Center for Cardiovascular Diseases, Chinese Academy of Medical Sciences and Peking Union Medical College}

Background Despite pulmonary embolism (PE) is the third cause of death due to cardiovascular diseases, the reported incidence of $\mathrm{PE}$ following coronary artery bypass graft (CABG) ranged widely. Furthermore, the accurate incidence and predisposing factors of $\mathrm{PE}$ in patients receiving off-pump coronary artery bypass (OPCAB) within the early postoperative period before discharge is still uncertain.

Methods and Result An analysis of prospectively collected data was conducted of 582 consecutive patients receiving isolated OPCAB during October 2009 to September 2012. All the patients had undergone coronary CT angiography (CCTA) to screen $\mathrm{PE}$ on mean postoperative day 5. CCTA detected silent PE in 11 patients. Byunivariate analysis, the proportion of preoperative left ventricular segmental wall motion abnormality (LVSWMA) in patients with PE was higher than in cases without $\mathrm{PE}(72.7 \%$ vs $36.4 \%)$ which bordered on significance $(\mathrm{P}=0.058)$. Preoperative Length of hospital stay was of borderline significance. 1: 5 paired logistic regression analysis showed that preoperative LVSWMA (P $=0.034)$ was an independent predictor for PE. Anticoagulant treatment lasted 3 months for $9 \mathrm{PE}$ patients.Redone CCTA showed PE disappeared. 
All 11 patients survived in the fellow-up.

Conclusion This study showed a 1.9\% incidence of asymptomatic PE duringthe first week after OPCAB. Postoperative CCTA should beadvocated to detect silent PE, especially in patients showing LVSWMA on preoperative echocardiogram. Warfarin is effective in the treatment of $\mathrm{PE}$, and anticoagulant therapy may be taken into account in $\mathrm{OPCAB}$ patients for the prophylaxis of $\mathrm{PE}$.

\section{Ischemia related revascularization could lead to a better prognosis for patients of coronary artery disease, compared with angiography related \\ Jiehui Li, Xiaoli Zhang, Xiubin Yang Cadiac Surgery, State Key Laboratory of Cardiovascular Disease, Fuwai Hospital, National Center for Cardio-vascular Diseases, Chinese Academy of Medical Sciences and Peking Union Medical College}

Background and Objective It is rarely found that the single photon emission computed tomography (SPECT) has been used as a guideline to estimate the prognosis of patients with coronary artery disease (CAD) who treated with incomplete revascularization (IR) or complete revascularization (CR). Our aim was to determine whether the SPECT could be used as the guidance in the treatment of CAD

Methods The study enrolled the suspected cases of CAD in the out-patient department with a positive result of SPECT, who accepted the coronary angiography (CA) test and the therapy in our hospital afterwards from 2006 to 2008 . We divided patients into four groups: group A treated by percutaneous coronary intervention (PCI), group B treated by coronary artery bypass grafting $(\mathrm{CABG})$, group $\mathrm{C}$ treated by medicine, group $\mathrm{D}$ without $\mathrm{CAD}$ treatment. We defined $\mathrm{CR}$ as if every lesion had been treated by PCI or CABG, otherwise as IR. If the left main artery (LM), left anterior descending (LAD), left circumflexus (LCx), or right coronary artery (RA) had a stenosis greater than $50 \%$, or the main branch had a stenosis greater than $75 \%$, we treated them as the lesions by CA. If any stressed difference score (SDS) of three main arteries, of which 17 segments divided to three main vascular areas, was greater than 1 , we treated them as lesions by SPECT. We divided patients by two ways because of two definitions of IR/CR, signed as group 1/I (CR), 2/II (IR), 3/III (group C), 4/IV (group D) of CA (SPECT).

Result 326 patients had finished follow-up with a mean age of $(64.2 \pm 9.85)$ yrs and $66.56 \%(n=217)$ were males. 182 patients were treated by PCI, 35 by CABG, 69 by medicine, and 38 without CAD treatment. 107 patients finished CR and 110 IR estimated by CA, compared 120 and 97 by SPECT. We had found all-cause death (ACD) $(\mathrm{n}=34)$, cardiac death $(\mathrm{CD})(\mathrm{n}=18)$, myocardial infarction (MI) $(\mathrm{n}=$ 39), repeat revascularization $(\mathrm{RR})(\mathrm{n}=30)$ and main adverse cardiac events (MACE) $(n=63)$. The cumulative ACD-free survival of patients Grouped by therapeutics during the follow-up period was $85.14 \%$ in group A, $83.17 \%$ in $\mathrm{B}, 68.01 \%$ in $\mathrm{C}, 85.13 \%$ in $\mathrm{D}(\log \operatorname{rank} \mathrm{P}<0.001)$. The cumulative survival of CD-free MI-free, RR-free, and MACEfree had the same results. And all these trends could also been found in groups divided by CA and SPECT. All of 217 patients treated by PCI or CABG were picked out for a further division by two ways of IR/ CR. Group $\alpha$ : angiography related CR plus ischemia related CR (ACR $+\mathrm{ICR}$ ); Group $\beta$ : angiography related IR plus ischemia related CR (AIR $+\mathrm{ICR}$ ); Group $\gamma$ : angiography related CR plus ischemia related IR (ACR $+\mathrm{IR}$ ); Group $\delta$ : angiography related IR plus ischemia related IR (AIR + IIR).

The cumulative ACD-free survival during the follow-up period was
$90.8 \%$ in Group $\alpha, 80.17 \%$ in Group $\beta, 74.48 \%$ in Group $\gamma, 67.59 \%$ in Group $\delta$ (Log rank $\mathrm{P}=0.017$ ), and the cumulative RR-free survival had the same result. However, the cumulative survival of CD-free (Log rank $\mathrm{P}=0.083)$, and MI-free (Log rank $\mathrm{P}=0.227)$, MACE-free (Log rank $\mathrm{P}$ $=0.078$ ) could not conduct to the significant results. Multivariate Cox analysis showed that the SDS, LVEF, and IR by SPECT were risk factors of CAD patients.

Conclusion Patients treated with CR could conduct to a better prognosis in future. And ischemia related revascularization may reflect a better prognosis compared with angiography related. Maybe we could use it to guide the therapy of coronary artery disease.

\section{The comparative application study of continuous blood purification and peritoneal dialysis for severe acute renal failure after cardiac surgery \\ Maozhou Tian ${ }^{1}$, Weijian Huang ${ }^{1}$, Pengfei Sun ${ }^{1}$, \\ Yongfeng Lai ${ }^{1}$, Lei Zheng ${ }^{1}$, Chuanlong Zhao ${ }^{1}$, Wenyi Liu ${ }^{1}$, Qinming Fan ${ }^{1}$, Zhenyu Wei ${ }^{1}$, Hourong Sun ${ }^{2}$ \\ 1. Shandong Yantaiyuhuangding Hospital \\ 2. Qilu Hospital of Shandong University}

Objective Acute renal failure (ARF) is a common and serious complication after cardiac surgery using cardiopulmonary bypass (CPB). In recent years, with CPB-related technology advanced, surgical handle and perioperative management improved, the incidence of ARF has been reduced, but if patients in the event of the ARF, the mortality rate is still high. Therefore, how to properly handle ARF after cardiopulmonary bypass still has a great significance.In this paper, the clinical use of the CBP and PD during ARF in cardiac surgery and the optimal time of clinical application of CBP treatment were investigated. We further analyzed the effects of CBP and PD treatment and specific mechanism involved.

Methods 36 patients with ARF after heart surgery from August 2005 to May 2010 in Qilu Hospital of Shandong University receiving continuous blood purification, while 21 patients with ARF receiving PD. All patients have no abnormal renal function before surgery. By percutaneous femoral vein indwelling double lumen catheter vascular access, All patients were divided into survival group $(n=24)$ and death group $(n=12)$ in CBP according to the prognosis, while $13 \& 8$ in PD ones. Clinical data were retrospectively analyzed, including ARFoccurence time after cardiac surgery, the number of days, the initiation of dialysis time, total duration times of hemodialysis, intensive care unit stay times and the APACHE III scores were observed in all patients before and after $12 \mathrm{~h}, 24 \mathrm{~h}, 48 \mathrm{~h}$ and $72 \mathrm{~h}$ treatment. Changes of inflammatory factorswere also observed during hemodialysis.

Result A total of 13 patients after CBP treatment discharged, with utter recovery of renal function and normal urine output. 8 cases still died after CBP treatment during hospitalization (12 cases in survival group and 8 cases in death group at $48 \mathrm{~h} ; 11$ cases in survival group and 6 cases in death group at $72 \mathrm{~h}$ ), of which 5 cases died of multiple organ failure, 2 cases died of heart failure and 1 case died of infection. Before using CBP, compared with the survival group, the indicators including $\mathrm{CO}$, LDH $(\mathrm{P}<0.05), \mathrm{CI}, \mathrm{BUN}, \mathrm{Cr}$, AST and CPK $(\mathrm{P}<$ 0.01 ) has statistically significance in death group. LVEF, CO and CI form two groups of patients with ARF at $48 \mathrm{~h}$ and $72 \mathrm{~h}$ after CBP were significantly improved. $72 \mathrm{~h}$ later, LVEF values in survival group significantly increased $(\mathrm{P}<0.01)$ and it was also in death group $(\mathrm{P}<0.05)$. Indicators of $\mathrm{CO}, \mathrm{CI}$ in two groups at $72 \mathrm{~h}$ after hemodialysis were both 
significantly increased $(\mathrm{P}<0.01)$, BUN and $\mathrm{Cr}$ were gradually decreased with the using of hemodialysis, compared to preoperative value, the measured value at $24 \mathrm{~h}, 48 \mathrm{~h}$, and $72 \mathrm{~h}$ were significantly decreased $(\mathrm{P}<$ 0.01). Before treatment of hemodialysis, difference of indicator Lac (P $<0.05$ ) has statistically significance in the death group vssurvival group. $72 \mathrm{~h}$ after hemodialysis, the MAP, HR was significantly improved in two groups $(\mathrm{P}<0.05)$, the level of Lac was remarkably reduced compared with the preoperation $(\mathrm{P}<0.01)$, respiratory function was also improved significantly, $\mathrm{PaO}_{2} / \mathrm{Fio}_{2}$ was significantly increased $(\mathrm{P}<0.05)$ at $24 \mathrm{~h}$ after hemodialysis, and continue to increase at $48 \mathrm{~h}, 72 \mathrm{~h}$ after hemodialysis $(\mathrm{P}$ $<0.01)$. Hemodialysis had no significant effect $(\mathrm{P}>0.05)$ on WBC and PLT. PLT in death group at $72 \mathrm{~h}$ treatment was decreased significantly compared with that of before hemodialysis $(\mathrm{P}<0.05)$ and it was also decreased significantly compared with that of in survival group at $12 \mathrm{~h}$ after treatment $(\mathrm{P}<0.05)$.

Conclusion $\mathrm{CBP}$ is an effective treatment for ARF following cardiac surgery. The earlier diagnosis of ARF after cardiac surgery and faster treatment measures to be implemented will greatly reducing the incidence of other complication, such as multiple organ dysfunction syndrome and mortality in patients.

\section{1-year outcomes comparison among medical therapy, percutaneous coronary intervention and coronary artery bypass graft in patients with three-vessel or left main coronary artery disease: results of a prospective registry in single institution \\ Chenfei Rao, Shengshou Hu, Zhe Zheng, Kun Hua, Xin Yuan, Heng Zhang, Huawei Gao State Key Laboratory of Cardiovascular Disease, Fuwai Hospital, National Center for Cardiovascular Diseases, Chinese Academy of Medical Sciences and Peking Union Medical College}

Objective The aim of this study is to compare medical therapy, coronary artery bypass grafting $(\mathrm{CABG})$ and percutaneous coronary intervention $(\mathrm{PCI})$ for complex coronary artery disease $(\mathrm{CAD})$ in realworld practice.

Methods Between July 2011 and September 2012, patients diagnosed as three-vessel or left-main CAD were consecutively enrolled. Medical records were reviewed to abstract detailed in-hospital information. The Corelab calculated SYNTAX Scores according to coronary angiograms. Follow-up was conducted at 12 months after the index hospitalization. The end point is major adverse cerebral and cardic events (MACCE) including death, myocardial infarction, stroke and additional revascularizatin. We use baseline variables and SYNTAX Scores for propensity socres to estimate the probability, that patients would be selected for revascularization and CABG according to different level comparison. Inverse probability weighting was used as the primary tool to adjust for differences between treatment groups.

Result 1707 patients were consecutively enrolled. 15\%, 44\% and $41 \%$ underwent medical therapy, PCI and CABG respectively. At 1 year, after adjustment using inverse probability weighting adjusted model, medical therapy is associated with higher rates of all-cause death (medical therapy: $4.5 \%$; revascularization: $1.6 \% ; \mathrm{P}<0.001)$ and MACCE $(12.4 \%$ vs $6.9 \%, \mathrm{P}<0.001)$. Compared with $\mathrm{CABG}$, the rates of all-cause death (PCI: $2.8 \%$, CABG: 0.7\%, $\mathrm{P}<0.001$ ), additional revascularization (PCI: 7.6\%, CABG: $1.1 \%, \mathrm{P}<0.001$ ), and MACCE (PCI: $11 \%$, CABG: $2.6 \%$, $\mathrm{P}<0.001)$ in the $\mathrm{PCI}$ group are significantly higher.

Conclusion For patients with 3-vessel disease or left main CAD,
$15 \%$ were treated with medical therapy, which was associated with higher rates of all-cause death and myocardial infarction.Compared with PCI, CABG had significantly lower rates of all-cause death and additional revascularization.

\section{Impact of $\mathrm{AHA} / \mathrm{ACC}$ coronary artery bypass grafting and percutaneous coronary intervention guidelines on real-world treatment strategies for complex coronary artery heart disease.}

Chenfei Rao, Shengshou Hu, Zhe Zheng, Xin Yuan, Huawei Gao, Kun Hua, Heng Zhang State Key Laboratory of Cardiovascular Disease, Fuwai Hospital, National Center for Cardiovascular Diseases, Chinese Academy of Medical Sciences and Peking Union Medical College

Objective 2011 ACCF/AHA coronary artery bypass grafting $(\mathrm{CABG})$ guideline and percutaneous coronary intervention (PCI) guideline were published on December 2011. The aim of this study is to explore the impact of guidelines' publication on real-world treatment for three-vessel or left-main coronary artery disease (CAD).

Methods Between July 2011 and September 2012, patients diagnosed as three-vessel or left-main CAD at Fuwai hospital were consecutively enrolled. Medical records were reviewed to abstract detailed in-hospital information. The Corelab calculated SYNTAX Scores according to coronary angiograms. Follow-up interview was conducted at 12 months after the index hospitalization. The end point was major adverse cerebral and cardic events (MACCE) including death, myocardial infarction, stroke and additional revascularizatin. Patients were then divided into pre-guideline group and post-guideline group according to the conary angiography date. Binary viariables logistic model and Cox model were uesd for baseline and 1-year outcomes comparison respectively.

Result 1746 patients were consecutively enrolled. 37.9\% (662) were in pre-guideline group, $62.1 \%(1084)$ in post-guideline group. There were no significant differences in demographic factors, cardiac surgery risks and SYNTAX Scores between pre- and post-guideline groups. After the publication of $2011 \mathrm{ACCF} / \mathrm{AHA}$ guidelines, doctors recommende more patients to choose CABG (pre: $49.0 \%$, post: $55.6 \%$, $\mathrm{P}<0.05$ ), the surgeons' consultation rate increased (pre: $41.9 \%$, post: $49.9 \%, \mathrm{P}<0.05$ ), the rate of ad hoc PCI ( $\mathrm{PCI}$ immediately after coronary angiography) decreased (pre: $47.5 \%$, post: $40.9 \%, \mathrm{P}<0.05$ ). After adjustment for coronary anatomy complexity and cardiac surgery risks using Cox model, pre-guideline group was associated with significantly higher rates of MACCE $(\mathrm{HR}=1.62,95 \% \mathrm{CI}: 1.15-2.27)$.

Conclusion The publication of 2011 ACCF/AHA CABG and PCI guidelines significantly changed real-world treatment for complex $\mathrm{CAD}$ in Fuwai hospital, and further improved outcomes.

Prevalence, predictors and 1-year outcomes of ad hoc pci for complex coronary artery disease: a patient-centered observational study Chenfei Rao, Shengshou Hu, Zhe Zheng, Xin Yuan, Huawei Gao, Kun Hua, Heng Zhang State Key Laboratory of Cardiovascular Disease, Fuwai Hospital, National Center for Cardiovascular Diseases, Chinese Academy of Medical Sciences and Peking Union Medical College

Objective 2011 ACCF/AHA coronary artery bypass grafting 
(CABG) guideline and percutaneous coronary intervention (PCI) guideline recommended the forbidden use of ad hoc PCI (PCI immediately after coronary angiography) for complex coronary artery disease $(\mathrm{CAD})$. The aim of this study is to explore the prevalence, risk factors and outcomes of ad hoc PCI for three-vessel or left-main CAD.

Methods Between July 2011 and September 2012, patients diagnosed as three-vessel or left-main CAD at Fuwai hospital were consecutively enrolled. Medical records were reviewed to abstract detailed in-hospital information. The Corelab calculated SYNTAX Scores according to coronary angiograms. Follow-up interview was conducted at 12 months after the index hospitalization. The endpointweremajor adverse cerebral and cardic events (MACCE) including death, myocardial infarction, stroke and additional revascularizatin. Patients were then divided into ad hoc PCI group and staged treatment group. Binary viariables logistic model and Cox model were uesd for baseline and 1-year outcomes comparison respectively.

Result 1746 patients were consecutively enrolled. 36.7\% (640) undertook ad hocPCI, 63.3\% (1106) received staged treatment (290 choosed medical therapy, 113 undertook non-ad hoc PCI, 688 received $\mathrm{CABG})$. Compared with staged treatment, the patients in ad hoc PCI group were younger [ad hoc PCI: $(59.3 \pm 10.6)$ yrs, staged treatment group: $(62.2 \pm 9.5)$ yrs, $\mathrm{P}=0.002)$, had lower SYNTAX Scores (ad hoc PCI: $21.1 \pm 8.0$, staged treatment group: $26.6 \pm 9.7, \mathrm{P}<0.001]$ and SinoSCOREs (ad hoc PCI: $0.5 \pm 2.9$, staged treatment group: $1.7 \pm$ 3.6, $\mathrm{P}<0.001$ ). After adjustment for coronary anatomy complexity and cardiac surgery risks using Cox model, ad hoc PCI was associated with significantly higher rates of MACCE $(\mathrm{HR}=0.644,95 \% \mathrm{CI}$ : $0.446-0.930$; $\mathrm{P}=0.019)$ and additional revascularization $(\mathrm{HR}=0.485,95 \% \mathrm{CI}: 0.306$ 0.769; $\mathrm{P}=0.002$ ).

Conclusion For patients with 3-vessel disease or left main CAD, $36.7 \%$ undertook ad hoc PCI, which was associated with higher rates of MACCE and additional revascularization.

\section{Impact of cardiac surgery on plasma levels of tenascin-C in patients with hypertrophic obstructive cardiomyopathy \\ Zhiyan Jiang, Fei Xu, Yun Liu, Yunhu Song, Xin Wang Department of Cardiovascular Surgery, State Key Laboratory of Cardiovascular Disease, Fuwai Hospital, National Center for Cardio- vascular Diseases, Chinese Academy of Medical Sciences and Peking Union Medical College}

Background and Objective Tenascin-C (TN-C) is one of the most important molecules in tissue remodeling, and was found playing a critical role in ventricular remodeling and myocardial fibrosis. In previous studies, TN-C was proved to be related to the prognosis of hypertrophic cardiomyopathy, patients with higherplasma levels of TN-C were more likely to suffer from heart failure. However, the relationship between plasma level of TN-C and the prognosis of patients with hypertrophic obstructive cardiomyopathy received surgical therapy remains unknown. The purpose of this study is to examine prospectively the effect of cardiac surgery on plasma concentration of TN-C in patients with hypertrophic obstructive cardiomyopathy.

Methods From July 2013 to March 2014, 35 patients with hypertrophic obstructive cardiomyopathy who needed cardiac surgery were involved in this study. Ventricular septal myectomy with modified Morrow procedure was chosen as the standard surgical procedure, and all operations were conducted in FuWai Hospital. TN-C plasma concentration was measured by enzyme-linked sandwich immunoassay in all patients on the day before, seven days after and three months after surgical therapy. To evaluate the result of the therapy, echocardiography was performed before and after surgery.TN-C levels were compared to perioperative data.

Result Before the operation, the mean systolic gradient between the left ventricle and the aorta from echocardiography was $(84 \pm 30) \mathrm{mm}$ $\mathrm{Hg}$ (ranging from 70 to $135 \mathrm{~mm} \mathrm{Hg}$ ). There was moderate or severe systolic anterior motion (SAM) in 31 cases and mitral regurgitation in 24 cases. Almost all patients were discharged without complications except one suffered from third degree A-V block and had pacemaker implantation. After the operation, the mean systolic gradient between the left ventricle and the aorta decreased to $(15 \pm 9) \mathrm{mm} \mathrm{Hg}(\mathrm{t}=12.36, \mathrm{P}<$ $0.05)$, and the thickness of ventricular septum decreased from $(25 \pm 4)$ $\mathrm{mm}$ to $(10 \pm 3) \mathrm{mm}(\mathrm{t}=12.65, \mathrm{P}<0.05)$. Mitral regurgitation and SAM were significantly improved or disappeared $(\mathrm{P}<0.05)$. TN-C decreased significantly $(\mathrm{P}<0.05)$ after cardiac surgery from mean $9.39 \mu \mathrm{g} / \mathrm{ml}$ to mean $5.91 \mu \mathrm{g} / \mathrm{ml}$ and remained significantly declined during the first three months $(\mathrm{P}<0.05)$. Postoperative TN-C plasma concentration was correlated to TN-C level before surgery $(\mathrm{r}=0.58, \mathrm{P}<0.05)$, and to the reduction of the mean systolic gradient between the left ventricle and the aorta $(\mathrm{r}=0.52, \mathrm{P}<0.05)$.

Conclusion In patients with hypertrophic cardiomyopathy, plasma TN-C decreased after cardiac surgery. The correlation to the reduction of the mean systolic gradient between the left ventricle and the aorta might be explained by the improvement of hemodynamic after cardiac surgery. The relationship between the plasma level of TN-C and the prognosis of patients with hypertrophic obstructive cardiomyopathy received surgery needs to be further studied in the future.

\section{Surgical treatment for pediatric hypertrophic cardiomyopathy \\ Jun Yan, Ronglong Guo, Yajuan Zhang, Qiang Wang, Guohua Luo \\ State Key Laboratory of Cardiovascular Disease, Fuwai Hospital, National Center for Cardiovascular Diseases, Chinese Academy of Medical Sciences and Peking Union Medical College}

Objective Children under 6 years of age with hypertrophic cardiomyopathy (HCM) are patients at high risk of death. For a long time, operation is considered to be the forbidden area of treatment due to its high risk, this topic of research aims at evaluating how surgical treatment influences HCM children's natural outcome, and its security, reliability and superiority are also under analysis.

Methods Between September 2013 and May 2014, the ward 2 of the pediatric cardiac surgery center of our hospital totally received 6 cases of HCM patients under the age of 6, among which 4 cases are male, and 2 cases are female. Their age ranges from 4 years and 5 months to 6 years old, all of them are confirmed the diagnosis of HCM according to the clinical manifestation, chest X-ray and echocardiography. Preoperative left ventricular outflow tract pressure gradient is between $70.6 \mathrm{~mm} \mathrm{Hg}$ and $148.8 \mathrm{~mm} \mathrm{Hg}$, and their left ventricular outflow tract obstruction is relieved by surgical treatment.

Result There is no death for the whole group, Left ventricular outflow obstructions are terminated satisfactorily. Left ventricular outflow tract pressure gradients drop to $13-25 \mathrm{~mm} \mathrm{Hg}$ after operation, SAM phenomenons disappear, and $\mathrm{LVEF} \% \geq 60 \%$, there is no postoperative conduction block and all of them were discharged successfully. There is no death, no residual obstruction after follow-up of 3 to 8 months. And at 
the same time their clinical symptoms improved significantly.

Conclusion For children under 6 years of age with hypertrophic cardiomyopathy (HCM), surgical operation should not be treated as the forbidden treatment. Operation can thoroughly solve the left ventricular outflow tract and right ventricular outflow tract obstruction, the success rate of which is high, and the incidence of postoperative complications and residual obstruction is very low. During follow-up period, although we found that patient's clinical symptoms improved significantly, but the results of long-term followup still remain to be further evaluated.

\section{Exploratory clinical research of the treatment with bosentan for congenital heart disease combined critical pulmonary hypertension with operation indication \\ Jun Yan, Ronglong Guo, Yajuan Zhang, Qiang Wang, Guohua Luo \\ State Key Laboratory of Cardiovascular Disease, Fuwai Hospital, National Center for Cardiovascular Diseases, Chinese Academy of Medical Sciences and Peking Union Medical College}

Objective Treating with bosentan exploratory for congenital heart disease combined with critical pulmonary hypertension and operation indication, groping for preoperative treatment course makes it possible for patients with high operation risks to get safer operations.

Methods Between September 2012 to May 2014, 14 cases of children with congenital heart disease combined with clinical pulmonary hypertension received cardiac catheterization, among which 11 cases are male, 3 cases are female, age ranges from 10 months to $7 \mathrm{yrs}$, and the average age is $4.2 \mathrm{yrs}$, all of them received the review of the cardiac catheterization after 3 to 6 months of bosentan treatment.

Result Matching test analysis shows that before and after bosentan treatment, the average of pulmonary vascular resistance is 14.33 wood and 11.42 wood respectively, there is statistical difference between them $(\mathrm{P}<0.05)$, but the average of femoral arterial blood oxygen saturation is $97.33 \%$ and $97.08 \%$ respectively, there is no statistical difference $(\mathrm{P}>0.05)$, also the average of mean pulmonary artery pressure is $78.29 \mathrm{~mm} \mathrm{Hg}$ and $73.57 \mathrm{~mm} \mathrm{Hg}$, there is no statistical difference too $(\mathrm{P}>0.05)$. among 14 cases received the treatment of bosentan, 8 cases of children completed operations successfully, including 7 cases of ventricular septal defect, 1 case of patent ductus arteriosus. 3 cases of children with ventricular septal defect need to reserve about $3 \mathrm{~mm}$ one-way live disc during operation due to their high preoperative pulmonary resistance (17.81 wood, 13.46 wood, 12.37 wood), the first two cases of which even need to perform a second operation to place the pericardial drainage tube for the review of echocardiography showed the existent of hydropericardium, and their drainage time were 44 days and 10 days respectively. 1 month after operation, the review of echocardiography for all of these 3 cases suggested that there is small amounts of left to right shunt. There is no surgical death, also we have not observed any death after follow-up of 4 to 10 months, but we can observe that their clinical symptoms improved significantly.

Conclusion Bosentan can effectively reduce the pulmonary vascular resistance, which creates surgery opportunity for congenital heart disease children with critical pulmonary hypertension. For patients which high preoperative pulmonary resistance, we need to reserve a one-way live disc during operation for decompression, and their probability of postoperative hydropericardium is rather high, therefore, for these patients, their pericardial drainage time should be extended appropriately.

\section{Genetic variant in glycoprotein receptor I associated with major adverse cardiac and cerebrovascular events after coronary artery bypass graft surgery \\ Hanning Liu ${ }^{1}$, Shengshou $\mathrm{Hu}^{1}$, Zhe Zheng ${ }^{1}$, Haiyong $\mathrm{Gu}^{2}$, Xiao Teng ${ }^{1}$ \\ 1. State Key Laboratory of Cardiovascular Disease, Fuwai Hospital, National Center for Cardiovascular Diseases, Chinese Academy of Medical Sciences and Peking Union Medical College \\ 2. Department of Cardiothoracic Surgery, Affiliated Peoples Hospital of Jiangsu University}

Background and Objective A high occurrence of major adverse cardiac and cerebrovascular events (MACCE) after coronary artery bypass graft $(\mathrm{CABG})$ surgery still exists. It has been suggested that variations in genes related to platelet function are independently associated with MACCE after CABG.

Methods Two separate cohorts of patients undergoing CABG surgery were examined, and MACCE after the index CABG was recorded. In a discovery cohort of 1576 patients, 44 single-nucleotide polymorphisms (SNPs) related to platelet function were tested. Cox proportional hazard models were used to identify clinical and genomic multivariate predictors of MACCE. The positive SNPs were verified in a replication cohort with 646 patients.

Result In discovery cohort, the average age of MACCE cases was significantly higher than that of patients without MACCE $(\mathrm{P}=$ 0.010 ) and $\beta$-blocker users were significantly lower in MACCE cases as compared with patients without MACCE $(\mathrm{P}=0.0003)$. And for other clinical factors, there was no difference. Three SNPs (PTGS2 rs5277 C/G, $\mathrm{HR}=1.554, \mathrm{P}=0.039 ; \mathrm{F} 13 \mathrm{~A} 1 \mathrm{rs} 5982 \mathrm{C} / \mathrm{T}, \mathrm{HR}=1.402, \mathrm{P}=0.017$; $\mathrm{GP}$ Ia rs $1126643 \mathrm{C} / \mathrm{T}, \mathrm{HR}=1.429, \mathrm{P}=0.014$ ) were independently associated with significantly increased risk of MACCE. After adjusted by multiple clinical risk factors, they still showed positive to increase the risk of MACCE. In the replication cohort of 646 patients from a multi-center study, we verified that GPIa rs $1126643 \mathrm{C} / \mathrm{T} \mathrm{SNP}(\mathrm{HR}=1.733, \mathrm{P}=0.020)$ was associated with a significantly increase of MACCE.

Conclusion In 2 independent cardiac surgery cohorts, we firstly reported GP Ia rs $1126643 \mathrm{C} / \mathrm{T}$ SNP was associated with increased risk of MACCE after CABG.

\section{Analysis of risk factors for mid-term survival of 435 heart transplant patients in Fuwai Hospital \\ Shengshou Hu, Jie Huang, Yunhu Song, Wei Wang, Zhe Zheng, Zhongkai Liao \\ Heart transplant center, State Key Laboratory of Cardiovascular Disease, Fuwai Hospital, National Center for Cardiovascular Diseases, Chinese Academy of Medical Sciences and Peking Union Medical College}

Objective This single-center retrospective study aims to analyze the preoperative risk factors associated with survival and put forward clinical strategy for treatment to improve the of heart transplant (HT) patients' survival.

Methods and Result In the past decade, the center has completed 435 cases of HT, among which male accounted for $82.8 \%$. The mean 
age of the total is $(44.6 \pm 13.6)$ years old. Follow-up to May 2014 and the median follow-up is 1220 days (428-2053). The survival rate of HT recipients was at one year, 94.9\%; 3-year, 91.6\%; 5-year, 86.5\%; 7-year, $82.6 \%$; 9-year, $75.1 \%$. Univariate COX regression analysis showed that preoperative serum creatinine level $(\mathrm{RR}=1.009,95 \mathrm{CI}$ : $1.005-1.013$; $\mathrm{P}$ $<0.001)$, blood urea nitrogen level $(\mathrm{RR}=1.013,95 \mathrm{CI}: 1.048-1.161 ; \mathrm{P}$ $<0.001)$, serum uric acid levels ( $\mathrm{RR}=1.002,95 \mathrm{CI}$ : 1.000-1.004; $\mathrm{P}=$ 0.012), total bilirubin ( $\mathrm{RR}=1.018,95 \mathrm{CI}: 1.005-1.031 ; \mathrm{P}=0.008)$, highsensitivity C-reactive protein levels $(\mathrm{RR}=1.040,95 \mathrm{CI}$ : $1.001-1.081 ; \mathrm{P}=$ $0.045)$ and the primary cardiac etiology $(R R=0.425,95 \mathrm{CI}: 0.232-0.779$; $\mathrm{P}=0.006$ ) were the factors affecting HT survival. Multivariate COX regression analysis showed that preoperative serum creatinine levels (RR $=1.01$, 95CI: $1.005-1.014 ; \mathrm{P}<0.001)$, serum uric acid levels $(\mathrm{RR}=1.002$, 95CI: $1.000-1.004 ; \mathrm{P}=0.048)$ and primary cardiac etiology $(\mathrm{RR}=0$. 367, 95CI: $0.185-0.726 ; \mathrm{P}=0.004$ ) remained the preoperative factors affecting the mid-term survival of HT patients.

Conclusion Through a rigorous screening for indications of HT recipients, the current national HT survival was satisfactory. Preoperative serum creatinine and uric acid levels are risk factors affecting the midterm survival of HT patients; Cardiomyopathy as one of the primary cardiac etiology is a protective factor for survival. The results are consistent that reported by the International Society for heart and lung transplant.

\section{Comparative study on the early outcomes following three different approaches of CABG in moderate to high risk patients evaluated by sinoSCORE \\ Junlong Hu, Junjie Sun, Zhaoyun Cheng, Ziniu Zhao, Xiaoqiang Quan \\ Peoples Hospital of Zhengzhou University; Henan Provincial People`s Hospital; Henan Cardiovascular Hospital}

Objective To compare the early outcomes following three different approaches of CABG in moderate to high-risk patients by SinoSCORE, as well as to discuss the surgical procedures' selection strategies for those patients.

Methods Retrospectively analysis the 430 patients who were enrolled to the Chinese Cardiac Surgery Registry system and received selective isolated CABG by the same surgical team in our center from April 2013 to March 2014. A coronary operation risk evaluation with the criteria of SinoSCORE was undertaken on every patient, among whom 223 cases were defined as moderate to high-risk patients with a riskscore higher than They were selected and divided into 3 groups by their surgical procedure approaches, ie. the conventional on-pump CABG group (CCABG, $n=44$ ), the On-pump beating heart $\mathrm{CABG}$ group (OnPump BH, $\mathrm{n}=24)$ and the off-pump $\mathrm{CABG}$ group $(\mathrm{OPCAB}, \mathrm{n}=155)$. The perioperative clinical data of 3 groups were collected and early clinical outcomes compared.

Result The On-Pump BH group's mean risk score (6.63 \pm 4.19$)$ was higher than the other 2 groups $(5.34 \pm 3.28$ and $5.70 \pm 2.75)$, without statistical difference among them. Mean age of the OPCAB group was significantly greater than CCABG group [(66.45 \pm 8.03$)$ yrs vs $(61.67 \pm 9.38)$ yrs, $\mathrm{P}<0.01]$, whereas the On-Pump BH group's age has no statistical difference with the other 2 groups. The last preoperative echocardiography revealed that the On-Pump BH groupmean EF $[(45.70 \pm 8.14) \%$ ] was statistically higher than the other 2 groups $[(54.59 \pm 9.08) \%$ and $(53.09 \pm 7.86) \%]$. No significant difference was seen on 3 groupother preoperative clinical data. The mean number of grafts in the On-PumpBH group (3.79 \pm 0.78$)$ was significantly higher than the CCABG group $(3.22 \pm 0.82)$ and the OPCAB group $(3.30 \pm$ 0.85 ). mean duration of operation, ventilator-support time, ICU stay and post-operative hospital stay of the OPCAB group were significantly lower than the CCABG group, whereas the On-Pump BH group were all ranked at the moderate place among the 3 groups and has no statistical difference with the others. No significant difference of the oxygenation index was seen among the 3 groups pre-operavely and shortly after anesthesia induction, however the CCABG group's began to decrease more sharply than the other 2 groups at the several following time points. The CCABG group's CTnI was significantly higher than the 2 othersgroup at the 1st hour after arriving ICU and the 2nd POD. The 3 groups' pre-operative serum creatine was statistically alike, but a significant difference appeared at the 1st POD between the CCABG and the OPCAB group, however each group's risenvalue wasstatistically different with the others. As the perioperative blood products consumption, the OPCAB group's red blood cell and plasma dosage were both statistically lower than the CCABG group. No significant differences were seen between the CCABG group and the On-Pump BH group. The OPCAB group's volume of chest drainage was statistically lower than the On-Pump BH group, no significant differences were seen between other groups. The OPCAB groupmortality rate was lower than the On-Pump BH group, whereas no statistical differences were found between the other groups. The CCABG group has the highest compositive complication rate, which was statistically higher than OPCAB group, the On-Pump BH group ranked the moderate place without statistical differences with the other groups.

Conclusion OPCAB and On-Pump BH CABG can benefit more from suffering less perioperativepulmonary injury, myocardialinjury, renal injuryand related complications than $\mathrm{CCABG}$ in moderate to high risk patients. On-Pump BH CABG has a higher revascularization index than $\mathrm{OPCAB}$, and has more advantages than $\mathrm{CCABG}$, which deserves more attempts in high risk patients with left ventricle dysfunction.

\section{Pulmonary surfactant changes in neonates with and without ALI/ARDS underwent cardiopulmonary bypass \\ Rongyuan Zhang, Xu Wang, Shoujun Li, Jun Yan, Juxian Yang, Shengli Li, MinZeng, Leilei Duan State Key Laboratory of Cardiovascular Disease, Fuwai Hospital, National Center for Cardiovascular Diseases, Chinese Academy of Medical Sciences and Peking Union Medical College}

Background and Objective Infants younger than 1 month of age with congenital heart disease are at increased risk for respiratory tract infections, while postoperatively they are at risk for acute lung injury (ALI) and acute respiratory distress syndrome (ARDS) precipitated by cardiopulmonary bypass (CPB). Pulmonary surfactant dysfunction and inactivation are key contributors to the pathophysiology. This study aimed to explore the changes of surfactant in neonates undergoing cardiovascular surgery with $\mathrm{CPB}$, and to characterize pulmonary surfactant properties in these infants with ALI/ARDS.

Methods Thirty pediatric patients (age $<31$ days, RACHS- 1 degree 3-5) with congenital heart defects undergoing cardiovascular surgery with $\mathrm{CPB}$ were collected for plasma surfactant level detected. Plasma levels of surfactant proteins A, B, C and D (SP-A, SP-B, SP-C and SP-D) were measured after $\mathrm{CPB}$ as the baseline, as well as 2, 12, 24, 72 and $120 \mathrm{hrs}$ after admission to the pediatric intensive care unit. Twenty patients (age < 31days, RACHS-1 degree 3-5) suffered from 
ALI/ARDS after cardiovascular surgery were choose for pulmonary surfactant properties detected, as twenty patients without ALI/ARDS were choose as the control group. Total protein (TP), total phospholipids (TPO) and saturated phosphatidylcholine (SatPC) concentrations were assessed in native tracheal aspirates; plasma level of SP-A was assessed in ALI/ARDS group and the control.

Result After cardiac operations with cardiopulmonary bypass surgery, SP-A was increased on 2, 12, 24, 72 and $120 \mathrm{~h}[(142.16 \pm 39.15)$ $\mathrm{ng} / \mathrm{ml},(159.91 \pm 25.86) \mathrm{ng} / \mathrm{ml},(160.39 \pm 23.42) \mathrm{ng} / \mathrm{ml},(175.71 \pm 27.77)$ $\mathrm{ng} / \mathrm{ml},(177.98 \pm 31.19) \mathrm{ng} / \mathrm{ml}, \mathrm{P}<0.01$ Compared with baseline), SP-B $[(69.78 \pm 16.49) \mathrm{ng} / \mathrm{ml},(67.35 \pm 12.82) \mathrm{ng} / \mathrm{ml},(67.65 \pm 11.75) \mathrm{ng} /$ $\mathrm{ml},(64.48 \pm 14.45) \mathrm{ng} / \mathrm{ml},(71.22 \pm 15.39) \mathrm{ng} / \mathrm{ml}, \mathrm{P}>0.05$ compared with baseline], SP-C [(8.68 \pm 1.54$) \mathrm{ng} / \mathrm{ml},(8.57 \pm 1.53) \mathrm{ng} / \mathrm{ml},(7.91 \pm$ 1.39) $\mathrm{ng} / \mathrm{ml},(8.46 \pm 1.25) \mathrm{ng} / \mathrm{ml},(8.22 \pm 1.40) \mathrm{ng} / \mathrm{ml}, \mathrm{ng} / \mathrm{ml}, \mathrm{P}>0.05$ compared with baseline) $]$ and SP-D [(440.76 \pm 98.85$) \mathrm{ng} / \mathrm{ml},(425.05 \pm$ $82.33) \mathrm{ng} / \mathrm{ml},(431.06 \pm 73.61) \mathrm{ng} / \mathrm{ml},(388.31 \pm 84.85) \mathrm{ng} / \mathrm{ml}, \mathrm{P}>0.01$ compared with baseline] were unchanged. The SatPC/TPL and SatPC/ TP were both decreased in ALI/ARDS group compared with the control group (SatPC/TPL P $<0.05$, SatPC/TP P $<0.01$ ). Plasma SP-A level was higher $(\mathrm{P}<0.01)$ in ALI/ARDS group $[(148.18 \pm 19.18) \mathrm{g} / \mathrm{ml}]$ than the control $[(93.06 \pm 12.39) \mathrm{ng} / \mathrm{ml}]$. We found three children with congenital heart disease and ARDS who had significant high level of SP-A ( > 200 $\mathrm{ng} / \mathrm{ml}$ ) had a life-threatening hypoxemia.

Conclusion CPB in infants younger than 1 month of age induce profound changes in the surfactant system. Both phospholipids and protein components of the surfactant system biophysical function disturbed in infants with ALI/ARDS undergoing cardiovascular surgery with CPB. SP-A provides a better indicator of postoperative patients with ALL/ARDS.

\section{The radial artery for coronary revascularization: experience and clinical results spanning 12 years}

Zhaoyun Cheng, Ziniu Zhao, Xiaoqiang Quan, Zhenwei Ge, Liang Zhao, Junjie Sun, Junlong Hu, Chuanyu Gao Henan Provincial People's Hospital

Henan Cardiovascular Hospital

Background and Objective The radial artery (RA) is becoming a popular conduit for coronary artery bypass grafting (CABG), yet data reporting the long-term results are rare. We reported our clinical, angiographic and intravascular ultrasound findings on 93 patients who had the RA used as part of the conduit for the CABG procedures during a 12-year period from June 2001 to June 2013.

Methods A total of 118 radial artery conduits were harvested in 87 males and 6 females, age from 28 to 66 (mean 49.9) years. An "intraoperative Allen's Test" was developed to safeguard blood supply to the arm and hand. A "Double-clip \& scissors-cut" technique was carried out to minimize the thermal injury to the radial artery from the diathermy. The left radial artery was used in 67 patients, the right in one, and bilateral radial arteries in 25 patients. One hundred and twenty-two out of 272 distal anastomoses (44.9\%) were constructed with radial arteries, with an average of 2.9 grafts per patient (range 2-6).

Result Follow-up angiography and intravascular ultrasound study at 3-139 postoperative months (mean 59 months) revealed a $93.1 \%$ radial artery patency. String sign occurred in one patient in whom the radial artery was directed to a big right coronary artery with a stenosis of around $50 \%$. The patency for the internal mammary artery was $96.4 \%$.

Conclusion The radial artery is an excellent conduit that broadens the options for total arterial coronary artery bypass grafting surgery. Good graft patency could be achieved through careful harvesting techniques and choice of proper target coronary vessels.

\section{Predictors of early prognosis in patients with ischemic mitral regurgitation undergoing mitral annuloplasty combined with revascularization \\ Shen Liu, Yunhu Song, Feng Lv, Shuiyun Wang, \\ Chao Dong, Yan Yang, Ruofan Liu, Minghui Tong, \\ Jianping $\mathrm{Xu}$ \\ Adult Cardiac Surgery Center, State Key Laboratory of Cardiovascular \\ Disease, Fuwai Hospital, National Center for Cardiovascular Diseases, Chinese Academy of Medical Sciences and Peking Union Medical College}

Objective The aim of this study was to identify the predictors of early prognoses and survivals in patients with ischemic mitral regurgitation (IMR) who had undergone mitral annuloplasty (MA) combined with revascularization.

Methods From January 2011 to December 2012, 71 patients with IMR who had undergone MA combined with coronary artery bypass graft $(\mathrm{CABG})$ in our institution were enrolled. Mitral valve annuloplasty rings were used in all patients. Patients with flexible rings $(\mathrm{n}=38)$ and rigid rings $(\mathrm{n}=33)$ were analyzed. Patients' mean age was $58.9 \pm 7.8$ years, New York Heart Association (NYHA) class III or IV was $43.7 \%$, preoperative left ventricular end-diastolic diameter (LVEDD) was (58.7 $\pm 6.9) \mathrm{mm}$ and ejection fraction (EF) was $(50.9 \pm 11.2) \%$. The study endpoints were postoperative death, reoperation, recurrence of moderate or severe mitral regurgitation and heart failure.

Result Follow-up data were obtained in all patients. Mean follow-up was $(20.3 \pm 8.5)$ months. Preoperative characteristics were comparable between the two groups. Only mitral annular dilatation (AD) was significantly different between the groups $(57.9 \%$ vs $30.3 \%, \mathrm{P}=0.02)$. $\mathrm{AD}(\mathrm{P}<0.05)$, NYHA class III or IV ( $\mathrm{P}<0.05)$, ventricular aneurysm (VA) ( $\mathrm{P}$ $<0.05)$ were powerful risk factors of endpoint events on univariate analysis. After adjustments, Cox proportional-hazards regression analysis showed that $\mathrm{AD}(\mathrm{P}<0.01)$, atrial fibrillation ( $\mathrm{P}<0.01)$, aortic cross clamp time $(\mathrm{P}<0.01)$ were the risk factors of early prognoses. Moreover, patients with rigid rings showed significantly higher rate of endpoint events than those with flexible rings $(\mathrm{HR}=5.49,95 \% \mathrm{CI}: 1.04-28.81, \mathrm{P}<0.05)$.

Conclusion In patients with IMR, annular dilatation, atrial fibrillation, and long aortic cross clamp time were independent risk factors of early prognoses after MA combined with CABG. Patients with rigid rings showed higher rate of endpoint events than those with flexible rings.

\section{Association between mitral annular dilatation and early prognosis of concomitant mitral valve repair with revascularization in ischemic mitral regurgitation \\ Shen Liu, Yunhu Song, Feng Lv, Shuiyun Wang, Chao Dong, Yan Yang, Ruofan Liu, Minghui Tong, Jianping $\mathrm{Xu}$ Adult Cardiac Surgery Center, Fuwai Hospital, National Center for Cardiovascular Disease, Chinese Academy of Medical Sciences and Peking Union Medical College}

Objective To identify the association between mitral annular 
dilatation and early prognosis in patients with ischemic mitral regurgitation (IMR) who had undergone mitral valve repair (MVR) combined with revascularization.

Methods From January 2011 to December 2012, 77 patients with IMR who had undergone MVR combined with coronary artery bypass graft $(\mathrm{CABG})$ in our institution were enrolled. Annular dilatation (AD) was defined as the mitral annulus dimension exceeded $35 \mathrm{~mm}$ when measured by transthoracic or transesophageal echocardiography. The patients with $(n=36)$ or without $(n=41)$ AD were analyzed. Patients' mean age was $(58.8 \pm 7.7)$ yrs and New York Heart Association class III or IV was $45.5 \%$. Preoperative left ventricular end-diastolic diameter (LVEDD) was $(58.7 \pm 7.3) \mathrm{mm}$ and ejection fraction (EF) was (50.7 $\pm 10.4) \%$. The study endpoints were postoperative death, reoperation, recurrence of moderate or severe mitral regurgitation and heart failure.

Result Follow-up data were obtained in all patients. Mean followup was $(19.7 \pm 8.8)$ months. Preoperative characteristics were comparable between the 2 groups. Only EF was significantly lower in the $\mathrm{AD}$ group $[(46.5 \pm 10.3) \%$ vs $(54.4 \pm 11.2) \%, \mathrm{P}=0.002]$. $\mathrm{AD}$ was a powerful predictor of endpoint events on univariate analysis $(\mathrm{OR}=4.6,95 \% \mathrm{CI}: 1.3-16.0 ; \mathrm{P}=0.011)$. Patients without $\mathrm{AD}$ were showed significantly higher rate of freedom from events than the AD group (Log Rank test, $\mathrm{P}=0.008)$. Cox proportional-hazards regression analysis showed that $\mathrm{AD}(\mathrm{HR}=4.10,95 \% \mathrm{CI}$ : $1.19-14.10$; $\mathrm{P}=0.025)$, atrial fibrillation $(\mathrm{P}=0.033)$ and ventricular aneurysm ( $\mathrm{P}$ $<0.05$ ) were the risk factors of early prognosis of patients who had undergone MVR combined with CABG.

Conclusion In patients with IMR undergoing MVR and CABG, mitral annular dilatation, atrial fibrillation and ventricular aneurysm were the independent risk factors which associated with early prognosis. Therefore, patients with AD should be discreetly considered for MVR during $\mathrm{CABG}$.

\section{Cost-effectiveness analysis for surgical, interventional, or medical treatments for patients with triple-vessel or left main coronary artery disease: 1 -year follow-up results from single institution \\ Kun Hua, Zhe Zheng, Xin Yuan, Chenfei Rao, Wei Li, Shengshou $\mathrm{Hu}$ \\ State Key Laboratory of Cardiovascular Disease, Fuwai Hospital, National Center for Cardiovascular Diseases, Chinese Academy of Medical Sciences and Peking Union Medical College}

Background The China Patient-Centered Evaluative Assessment of Cardiac Events Prospective Study of 3-Vessel Disease (China PEACE-3VD) included patients with stable triple-vessel or left main coronary artery disease. Patients underwent coronary artery bypass graft surgery $(\mathrm{CABG})$, percutaneous coronary intervention $(\mathrm{PCI})$, or medical treatment (MT) alone. This investigation compares the economic outcome at 1-year follow-up of the 3 treatment strategies.

Methods and Result Between July 2011 and September 2012, 971 patients were included in the study. 133 patients received MT, in revascularization treatment (RT) group 419 patients underwent $\mathrm{PCI}$ and another 419 underwent CABG. Costs were assessed from the perspective of the China healthcare and medical insurance system. Health state utilities were assessed using the EuroQOL 5 dimension 3 level (EQ-5D) questionnaire. We used inverse-weighted estimates of the probability of treatment to adjust for treatment selection bias. After 1 year, average total costs were $24684 \mathrm{RMB}$ for MT versus $87379 \mathrm{RMB}$ for RT patients, a difference of 62695 RMB (95\% CI: 56172-69218 RMB), whereas average total costs were 90789 RMB for PCI vs 85823 RMB for CABG patients, a difference of $4966 \mathrm{RMB}$ (95\% CI: 18.4-9912.9 RMB). The 1-year event-free costs were 27208 RMB for MT; 90460 RMB for RT; 102355 RMB for PCI; and 89492 RMB for CABG. The comparison of the event-free costs showed that there was a significant difference favoring MT versus $\mathrm{RT}(\mathrm{P}<0.0001)$ and $\mathrm{CABG}$ versus $\mathrm{PCI}(\mathrm{P}<0.0001)$. In addition, respectively, MT presented 0.79 quality-adjusted lifeyear (QALY); PCI presented 0.80 QALY; and CABG also demonstrated 0.80 QALY.

Conclusion In the short-term economic analysis, for the prevention of a composite primary end point, MT was more cost-effective than RT, and $\mathrm{CABG}$ was more cost-effective than PCI. However, the long-term economic analysis is warranted.

\section{Effect of preoperatively continued aspirin use on early and mid-term outcomes in off-pump coronary artery bypass grafting: a propensity score-matched study of 1418 patients \\ Fucheng Xiao, Hengchao Wu, Hansong Sun, Shiwei Pan, Jianping $\mathrm{Xu}$, Yunhu Song \\ Department of Cardiovascular Surgery, State Key Laboratory of \\ Cardiovascular Disease, FuWai Hospital and Cardiovascular Institute, National Center for Cardiovascular Diseases, Chinese Academy of Medical Sciencesand Peking Union Medical College}

Objective To date, studies regarding the effect of preoperatively continued aspirin administration have yielded inconsistent results and have been conducted mainly in conventional on-pump coronary artery bypass grafting $(\mathrm{CABG})$. We aimed to assess the effect of preoperatively continued aspirin use on early and mid-term outcomes in patients receiving off-pump CABG.

Methods From October 2009 to September 2013, 5787 patients with only aspirin use for anti-platelet therapy underwent elective isolated off-pump CABG at the Fuwai Hospital. Of those, 720 patients continued aspirin use preoperatively. Propensity score constructed from core patient characteristics was used to match preoperative aspirin user with preoperative nonaspirin users to obtain risk-adjusted outcome comparisons between the two groups (709 patients for each group). Early outcomes were in-hospital death, stroke, postoperative blood loss, reoperation for bleeding and blood product transfusion. Major adverse cardiac events (death, myocardial infarction or repeat revascularization), angina recurrence, and readmission for cardiac reasons were considered as mid-term endpoints.

Result There were no significant differences between the aspirin and nonaspirin use group in baseline characteristics. The average postoperative blood loss $(895.8 \mathrm{ml}$ vs $879.8 \mathrm{ml}, \mathrm{P}=0.60)$, blood transfusion requirements $(25.1 \%$ vs $24.4 \%, \mathrm{P}=0.76)$ and composite outcome of in-hospital death, stroke and reoperation for bleeding $(2.8 \%$ vs $1.6 \%, \mathrm{P}=0.10$ ) were similar in aspirin and nonaspirin use group. At a mean follow-up of $(18.8 \pm 12.4)$ months, no significant difference was observed among the aspirin and nonaspirin use group in major adverse cardiac events free survival estimates $(95.7 \%$ vs $91.5 \%, \mathrm{P}=0.23)$ and freedom from readmission for cardiac reasons ( $88.5 \%$ vs $85.3 \%, \mathrm{P}=0.77$ ) whereas the angina recurrence free survival rates was $83.7 \%$ and $73.9 \%$ in the aspirin use group and nonaspirin use group respectively $(\mathrm{P}=0.02)$, with hazard ratio for preoperative aspirin estimated at $0.71(95 \% \mathrm{CI}$ : 0.49-1.04, $\mathrm{P}=0.08$ ).

Conclusion Preoperatively continued aspirin use was not associated 
with increased risk of postoperative blood loss, blood transfusion requirements and composite outcome of in-hospital death, stroke and reoperation for bleeding in off-pump CABG. Preoperative aspirin use tended to decrease the hazard of mid-term angina recurrence.

\section{Real-world comparing outcomes of revascularization strategies for multivessel disease in patients with diabetes \\ Heng Zhang ${ }^{1}$, Xin Yuan ${ }^{1}$, Bo Xu ${ }^{2}$, Yang Wang ${ }^{3}$, \\ Shengshou $\mathrm{Hu}^{1}$, Zhe Zheng ${ }^{1}$ \\ State Key Laboratory of Cardiovascular Disease, Fuwai Hospital, \\ National Center for Cardiovascular Diseases, Chinese Academy of \\ Medical Sciences and Peking Union Medical College \\ 1. Department of Cardiovascular Surgery \\ 2. Department of Cardiology \\ 3. Statistic Center for Medical Research,}

Background Randomized trials comparing revascularization strategies for patients with diabetes report that coronary-artery bypass grafting $(\mathrm{CABG})$ has a better outcome than percutaneous coronary intervention (PCI). While evidence from a more generalizable population of patients is still lacking. This study investigated clinical outcomes in a large cohort of patients with DM and multivessel coronary artery disease (CAD) after CABG or PCI in contemporary practice.

Methods All 4148 consecutive patients who underwent isolated CABG $(n=1853)$ or PCI $(n=2295)$ at our institution for the years 2004 through 2008 were included. Both in-hospital and 4-year major adverse cardiovascular events (MACE) including death, myocardial infarction (MI), and repeat revascularization were compared with the use of the inverse probability weighted method.

Result After adjustment for differences in baseline risk factors, the in hospital risk of cardiac death $(\mathrm{OR}=3.94,95 \% \mathrm{CI}$ : $1.36-11.40$; $\mathrm{P}=0.011)$, MI $(\mathrm{OR}=4.32 ; 95 \% \mathrm{CI}: 1.67-11.19 ; \mathrm{P}=0.003)$ and repeat revascularization $(\mathrm{OR}=15.91 ; 95 \% \mathrm{CI}$ : 4.18-60.54; $\mathrm{P}=0.003)$ was higher for patients undergoing PCI than CABG. The adjusted hazard ratios for 4-year cardiac death $(3.89,95 \% \mathrm{CI}$ : $1.75-8.63$; $\mathrm{P}=0.001)$ and MACE (7.79, 95\% CI: 5.88-10.80; $\mathrm{P}<0.001)$ were significantly higher after PCI than CABG. For patients insulin treated diabetes, similar results were obtained when mortality and MI were compared between PCI and CABG. While revascularization with PCI resulted in higher MACE for both patients with three-vessel disease ( $\mathrm{HR}=8.12,95 \% \mathrm{CI}$ : 5.81-11.37) and patients with left main disease ( $\mathrm{HR}=12.40,95 \% \mathrm{CI}$ : 6.04-25.43).

Conclusion In contemporary practice, for patients with diabetes and two or more diseased coronary arteries, there is a significant advantage of CABG over PCI in overall survival, and reduction of myocardial infarction and repeat revascularization.

\section{Long-term incidence of stroke and transient ischemic attack after coronary artery bypass grafting}

Heng Zhang, Xin Yuan, Huawei Gao, Chenfei Rao, Wei Wang, Shengshou Hu, Zhe Zheng Department of Cardiovascular Surgery, State Key Laboratory of Cardiovascular Disease, Fuwai Hospital, National Center for Cardiovascular Diseases, Chinese Academy of Medical Sciences and Peking Union Medical College

Objective To examine the epidemiology, risk factors and healthcare burden of new-onset stroke and transient ischemic attack (TIA) during the long term following coronary artery bypass grafting (CABG).

Methods All 9172 consecutive patients who underwent isolated CABG and discharged alive between January 1999 and December 2008 were included and analyzed for long-term new-onset stroke and TIA and repeat hospitalization after the procedure. Patients were defined with stroke or TIA, according to the definitions of the 2013 American Heart Association criteria.

Result After an average of 3.7 years of follow-up, the prevalence of new-onset ischemic stroke, hemorrhagic stroke, undetermined stroke and TIA was $5.6 \%, 0.3 \%, 0.5 \%$ and $2.3 \%$, respectively. The prevalence of stroke or TIA was $2.2 \%, 4.9 \%, 9.7 \%, 14.4 \%$ and $18.1 \%$ at 1 year, 3 years, 5 years, 8 years and 10 years after the CABG. The relative risks of myocardial infarction was 4.33 (95\% CI: 2.68-6.99) for patients developed new-onset stroke or TIA. Among patients who developed stroke and TIA, 78.8\% of them experienced at least once repeat hospitalization. Following adjustment for patient and surgical characteristics, preoperative atrial fibrillation $(\mathrm{HR}=1.48,95 \% \mathrm{CI}$ : 1.01-2.16), insufficient physical activity ( $\mathrm{HR}=1.72,95 \%$ CI: $1.41-2.11)$ and underuse of $\beta$-blockers (HR $=1.20,95 \%$ CI: $1.04-1.39$ ) were significantly associated with increased risk of new-onset stroke or TIA during the follow-up.

Conclusion This is by far the first and largest study evaluating the prevalence and risk burden in a Chinese cohort. Morbidity attributed to the postoperative stroke and TIA was high after CABG. Reasonable prevention strategies should be designed to attenuate the healthcare burden of new-onset stroke and TIA following the primary procedure.

\section{Perioperative period of efficacy in high risk patients undergoing off-pump versus on- pump coronary artery bypass grafting: a Meta- analysis \\ Shixiong Wang, Debin Liu, Wei Wang, Li Pan, \\ Weifan Wang, Bingren Gao \\ Department of Cardiac Surgery, the Second Hospital Lanzhou \\ University}

Objective To assess differences in perioperative efficacy and safety of high-risk patients undergoing OPCABG versus CCABG.

Methods Relevant clinical trials, published in English and Chinese before March 2014, were searched in PubMed, EMbase, China National Knowledge Infrastructure (CNKI), China Biomedicine Literature Database (CBM), VIP Wan fang database and CochraneLibrary (Issue 3 2014). Manual searches of bibliographies were also performed. Two reviewers independently selected eligibletrials, performed quality assessment, abstracted relevant data, and cross-checked each other finally. The meta-analysis was performed with software RevMan 5.1.

Result A total of 16 studies, including 2 randomized controlled trials and 14 retrospective studies were identified. A total of 6441 highrisk patients, involving 2948 in the OPCABG group and 3493 in the CCABG group were included. The results of meta-analyses showed: The difference of stroke, the IABP using, recurrent myocardial infarction, respiratory dysfunction and mortality incidence in the perioperative period, the ventilation time, the time in ICU, hospitalization time, postoperative drainage and blood transfusion were significant differences between OPCABG and CCABG patients were significant, and the OR/ MD (95\% CI) value was $0.53(0.38-0.74), 0.57(0.41-0.79), 0.62$ (0.41$0.86), 0.54(0.40-0.71), 0.62(0.48-0.80),-6.65(-9.24--4.12),-12.66$ (-18.03- -7.92), -1.88 (-3.62 - -0.14), -338.28 (-552.08 - -124.48), $-254.86(-265.40--244.31)$ respectively.While there was no statistical 
difference in atrial fibrillation, acute renal injury, wound infection and reoperation rate.

Conclusion Compared with CCABG in the perioperative period, using OPCABG for high-risk patients is safe and effective and with less trauma, lower operative mortality, rapider postoperative recovery, fewer complications advantages, but because of the number of included studies limited and most is the Non-RCT, OPCABG can not replace CCABG, However, the results and long-term efficacy still need to be confirmed by higher-quality, more multi-center, large-sample and randomized doubleblind controlled trials in the future.

\section{Sequential vein bypass grafting is not associated with an increase of either in- hospital or mid-term adverse events in off- pump coronary artery bypass grafting \\ Fucheng Xiao, Jian Wang, Hengchao Wu, Hansong Sun Department of Cardiovascular Surgery, State Key Laboratory of Cardiovascular Disease, FuWai Hospital and Cardiovascular Institute, National Center for Cardiovascular Diseases, Chinese Academy of Medical Sciencesand Peking Union Medical College}

Objective The impact of sequential vein bypass grafting on clinical outcomes remains controversial and is less known in off-pump coronary artery bypass grafting (CABG). We aimed to evaluate the effects of sequential vein bypass grafting on clinical outcomes in off-pump CABG.

Methods From October 2009 to September 2013 at the Fuwai Hospital, 127 patients with at least one sequential venous graft were matched with 127 patients of individual venous grafts only, using propensity score matching method. In-hospital measurement was composite outcome of in-hospital death, myocardial infarction, stroke, requirement for intra-aortic ballon pump (IABP) assistance and prolonged ventilation. Major adverse cardiac events (MACEs: death, myocardial infarction or repeat revascularization) and angina recurrence were considered as mid-term endpoints.

Result There were no differences between individual and sequential venous grafts groups with regards to composite outcome of in-hospital mortality, myocardial infarction, stroke, IABP assistance and prolonged ventilation ( $11.0 \%$ vs $14.2 \%, \mathrm{P}=0.45)$. Individual in-hospital measurement also did not differ significantly between the two groups. At about 4 years follow-up, the MACEs free survival estimates $(92.5 \%$ vs $97.3 \%, \mathrm{P}=0.36)$ and survival rate free of angina recurrence $(80.9 \%$ vs $85.5 \%, \mathrm{P}=0.48$ ) were similar among individual and sequential venous grafts groups. In the Cox regression analysis, sequential vein bypass grafting was not identified as an independent predictor of both MACEs and angina recurrence.

Conclusion Compared to individual vein bypass grafting, sequential vein bypass grafting was not associated with an increase of either in-hospital or mid-term adverse events in patients undergoing offpump CABG.

\section{Dilatation of the remaining ascending aorta root after aortic valve replacement for bicuspid versus tricuspid aortic valve: a single center experience}

Jing Zhang, Guangpu Fan, Hui Zhao, Xu Wang, Zhiwei Wang, Peide Zhang, Wei Wang

State Key Laboratory of Cardiovascular Disease, Fuwai Hospital, National Center for Cardiovascular Disease, Chinese Academy of

\section{Medical Sciences and Peking Union Medical College}

Objective The natural history and management of ascending aorta root (AAr) dilatation in patients with bicuspid aortic valve (BAV) after aortic valve replacement (AVR) remains controversial. Our aim is to identify dilatation of the remaining aorta root after AVR in patients with BAV compared with patients with tricuspid aortic valve (TAV)

Method We identified total 817 patients with bicuspid and tricuspid aortic valve ( $\mathrm{n}=264 ; \mathrm{n}=553$; and $72.7 \%$ vs $71.8 \%$ males; respectively) undergoing isolated aortic valve replacement between January 2002 and December 2008 at our center. Follow-up $(5.26 \pm 2.6)$ yrs was by review of clinical records, phone call questionnaire and out-patient examination. AAr dilatation was defined as aortic root diameters $\geq 45 \mathrm{~mm}$ or expanding at $1.5 \mathrm{~mm} / \mathrm{year}$, adverse aortic events were defined as the need for proximal aortic surgery or the occurrence of aortic dissection/rupture or sudden death during follow-up.

Result Median ages of patients with BAV or TAV were (48.5 $\pm 13)$ yrs and $(51.6 \pm 14)$ yrs, respectively $(\mathrm{P}<0.01)$. Preoperative diameter of AA in the BAV group with no aneurysm $(34.9 \pm 5.5) \mathrm{cm}$ was significantly higher than in the TAV group $(33.5 \pm 5.1) \mathrm{cm}(\mathrm{P}<0.001)$. In either BAV or TAV groups who underwent AVR, the dimension of AAr was significant expanded post-operation $[(37.3 \pm 6.3) \mathrm{cm}$ vs $(35.3$ $\pm 6.1) \mathrm{cm}$ ] with paired-t test $(\mathrm{P}<0.001)$, and majority of dilatation happened in the BAV group (21.5\% vs $14.1 \% ; \mathrm{P}<0.05)$. However, the Cox regression showed $\mathrm{BAV}$ or TAV did not make contribution to the enlargement of the $\mathrm{AAr}(\mathrm{P}=0.27)$, moreover, the aortic dilatation did not predict adverse aortic events and mortality $(\mathrm{P}=0.89,0.71$, respectively).

Conclusion Significant dilatation of the AAr was observed after AVR, which showed a more pronounced tendency in patients of BAV than in patients with TAV after operation in our study. However, the dilatation in both groups did not predict adverse aortic events and mortality, so the necessity of contemporary aortoplasty or AAr replacement at non-aneurysmal AVR was considered to be inadequate. 


\section{Pediatric Cardiology}

\author{
The comparative study for metoprolol sustained \\ release tablets and propafenone treating \\ children with frequent premature ventricular \\ contractions \\ Yan Zhou, Lizhi Han, Jinhua Piao \\ The pediatric cardiovascular department of the first hospital of JiLin \\ University
}

Objective Propafenone most commonly used in treatment for children with frequent premature ventricular contractions (PVC). The number of PVC is not reduced but increased significantly after using the propafenone in some patients. Metoprolol is a selective $\beta 1$ receptor blocker. $\beta$ receptor blockers are currently the only recognized drugs which can reduce the long-term mortality of arrhythmia. The purpose of this research is to compare the effect and prognosis of metoprolol sustained release tablets and propafenone for treating children's PVC.

Methods We used a prospective study method. The research objects were 71 cases of children aged 1-12 yrs, all the things have been finished according to the orders in the follow-up period, which contained 37 males and 34 females, the average age was 5.7 yrs. All the children were diagnosed with frequent PVC through Holter and normal ECG, and they had no organic heart diseases, such as myocarditis, cardiomyopathy, congenital heart diseases, cardiac insufficiency. All the children had obvious clinical symptoms, for example, chest tightness, short of breath, fatigue, Sweating. All the PVC are belong to Lown's class 3 or above, and need treatment by anti-arrhythmic drugs. The children were randomly divided into two groups, the treatment group of metoprolol sustained release tablets have 36 children (The starting dose was $0.5 \mathrm{mg}$ / $\mathrm{kg}$, one time per day, take orally, gradually increase the amount), the treatment group of propafenone have 35 children $(5 \mathrm{mg} / \mathrm{kg}$, three times per day, take orally). We reviewed the Holter and monitored the number of premature ventricular contractions at one week, two weeks, one month, two months, six months, one year after the oral medication, and we reviewed the liver function at the same time.

Result The treatment group of metoprolol sustained release tablets: After giving the starting dose one week, 12 patients $\mathrm{PVC}$ reduced significantly, but which of the other 19 patients had no significant reduction, after gradually increasing the dose of metoprolol, the number of PVC reduced gradually. And 2 patients' PVC had not reduced, 3 patients had an increased number of PVC. We changed the anti-arrhythmic drugs for the 3 patients, 1 patient was treated by radiofrequency ablation. During follow-up period, liver function of 3 patients were abnormal.

The treatment group of propafenone: PVC of 21 patients significantly reduced in two weeks, but the number of PVC increased again in the 9 patients of the 21 patients in the next follow-up period. 6 patients had no significant improvement in one month. PVC of 8 patients increased significantly in one week after using this drug, so we stopped using propafenone and began to use other anti-arrhythmic drugs. 3 patients of them were treated by radiofrequency ablation. During followup period, liver function of 6 patients were abnormal.

Conclusion Compared with propafenone, metoprolol sustained release tablets have better therapeutic effects, higher effective rates, and fewer side effects for treating children with PVC. At last, metoprolol sustained release tablets have a long half-life, the patients only need to take it once a day, the compliance is good for children.

\section{The systemic-to-pulmonary artery shunt in patients with pulmonary atresia, ventricular septal defect and hypoplastic pulmonary arteries \\ Yonghui Zhang, Shoujun Li, Kai Ma, Qiuming Chen, Wenlei Li \\ State Key Laboratoryof Cardiovascular Disease, FuwailHospital, National Center forCardiovascular Diseases, ChineseAcademy of Medical Sciencesand Peking Union MedicalCollege}

Objective To compare the differrences of the efficacy and safety in the common systemic-to-pulmonary artery shunts to rehabilitate the hypoplastic pulmonary arteries in patients with pulmonary atresia and ventricular septal defect.

Methods From December 2009 to August 2012, 47 consecutive patients [mean age $(1.78 \pm 1.82)$ yrs, range $0.2-8.1 \mathrm{yrs}$ ] diagnosed with pulmonary atresia, ventricular septal defect and pulmonary artery hypoplasia [Nakata index $(86.62 \pm 45.02) \mathrm{mm}^{2} / \mathrm{m}^{2}$; McGoon ratio 1.03 $\pm 0.40]$ were included in this retrospective study. 28 (59.6\%) patients underwent the procedure of modified blalock-taussing shunt (BT shunt) and the left 19 paitents receiewed the central shunt, mainly based on the surgeon habits and preoperative pulmonary vascular development. The nakata index in the BT group was significantly smaller than that in the central pulmonary shunt group $(\mathrm{P}<0.01)$. Some patients received transcatheter occlusion of major aortopulmonary collaterals and/or pulmonary angioplasty. Mean follow-up was $(2.36 \pm 1.07)$ yrs.

Result After the systemic-to-pulmonary artery shunts, pulmonary vascular of two groups had significant growth (Nakata index: $207.94 \pm$ 101.47, McGoon ratio: $1.55 \pm 0.44$ ), but there was no difference between the two groups $(\mathrm{P}>0.05)$. The central shunt group had more prolonged mechanical ventilation time and ICU monitoring time than the BT shunt group ( $\mathrm{P}<0.01) .15$ (31.9\%) patients underwent the anatomical repair after palliative surgery in an average of $1.1 \mathrm{yrs}(0.6-1.6 \mathrm{yrs})$. They were all in the BT shunt group. There were no early dearth and late dearth. All survivors who underwent anatomical repair were in New York Heart Association class I-II.

Conclusion Although the systemic-to-pulmonary artery shunts can effectively promote the hypoplastic pulmonary arteries growth in the PAVSD patients, but ultimately only $1 / 3$ of the patients can get biventricular repiar. The central shunt group had worse early and late results than the BT group, maybe for poorer preoperative pulmonary vascular development.

\section{Comparison of the cardioprotection between blood cardioplegia and STH cardioplegia in infant cardiac surgery: a Meta-analysis}

Shixiong Wang, Debin Liu, Wei Wang, Li Pan, Bingren Gao, Weifan Wang

Department of Cardiac Surgery of the Second Hospital Lanzhou

University

Objective To compare the cardioprotection effect between blood and STH cardioplegia during cardiacsurgery in infant patients, and 
provide a theoretical basis for optimal myocardial protection strategies.

Methods A Meta-analysis of randomized controlled trials (RCTs) studies about comparing blood and STH cardioplegia in infant patients undergoing cardiac surgery were performed. Cochrane library (Issue 4, 2014), MEDLINE, EMBase, PubMed, Springer Link, Science Direct, $\mathrm{CBM}, \mathrm{CNKI}$ and Wan fang were searched from increation to April 2014. Studies were assessed according to the Cochrane Handbook for systematic reviews. Data were extracted from these trials and analyzed by RevMan 5.1 software.

Result Fourteen trials were included, Meta-analysis indicated that, There were significant differences between blood cardioplegia and STH cardioplegia in the incidence of postoperative arrhythmias $(\mathrm{OR}=0.24$, 95\% CI: 0.08-0.76; $\mathrm{P}=0.02)$, days in hospital $(\mathrm{OR}=-1.09,95 \% \mathrm{CI}$ : $-2.03-0.15 ; \mathrm{P}=0.02)$, resuscitation time $(\mathrm{OR}=-73.20,95 \% \mathrm{CI}:-111.65$ - -34.75; $\mathrm{P}=0.0002)$, but there were no significant differences in the incidence of postoperative auto-beating $(\mathrm{OR}=2.55,95 \% \mathrm{CI}$ : 0.57-11.40; $\mathrm{P}=0.22)$, the incidence of postoperative low cardiac output $(\mathrm{OR}=0.24$, $95 \% \mathrm{CI}: 0.01-7.60 ; \mathrm{P}=0.41)$ and the hours of ventilation $(\mathrm{OR}=-0.975$, 95\% CI: $-24.00-4.51 ; \mathrm{P}=0.18)$.

Conclusion The current evidence shows that The protective effects of blood cardioplegia on immature myocardium in infants cardiac surgery are obviously better than STH cardioplegia. However, given the low methodological quality and the limited sample size of most included studies, this conclusion still needs to be further proved by conducting more strictly-designed, high-quality and large-scale RCTs. The long-term effectiveness of those two treatment modalities also needs to be observed in a longer follow-up duration.

\section{The palliated right ventricle to pulmonary artery connection in Patients with Pulmonary atresia, ventricular septal defect and hypoplastic pulmonary arteries \\ Shoujun Li, Yonghui Zhang, Kai Ma, Qiuming Chen, Wenlei Li \\ State Key Laboratoryof Cardiovascular Disease, FuwaiHospital, National Center forCardiovascular Diseases, ChineseAcademy of Medical Sciencesand Peking Union MedicalCollege}

Objective To determine the effect and safety of the right ventricle to pulmonary artery connection to rehabilitate the hypoplastic pulmonary arteries in patients with pulmonary atresia and ventricular septal defect.

Methods From December 2009 to August 2012, 45 consecutive patients [mean age $(1.61 \pm 1.63)$ yrs, range $0.2-7.2 \mathrm{yrs})$ diagnosed with pulmonary atresia, ventricular septal defect and pulmonary artery hypoplasia [Nakata index: $(88.25 \pm 29.27) \mathrm{mm}^{2} / \mathrm{m}^{2}$; McGoon ratio: 0.94 $\pm 0.14]$ were included in this retrospective study. All patients underwent the procedure of right ventricle to pulmonary artery connection, during which most of them received transcatheter occlusion of major aortopulmonary collaterals and/or pulmonary angioplasty. Mean followup was $(2.06 \pm 0.83)$ yrs.

Result There were no early deaths, but one patient died of myocarditis. Significant pulmonary artery growth was obtained [Nakata index: $(215.1 \pm 107.79) \mathrm{mm}^{2} / \mathrm{m}^{2}, \mathrm{P}<0.001$; McGoon ratio: $1.6 \pm 0.5$, $\mathrm{P}<0.001]$ in all of the 47 patients. Restenosis occurs in two patients' pulmonary arterial brach and one parient's outflow tract of right ventricle. All of the restenosis were relieved though percutneoustrnsluminlblloon dilttion. 28 patients $(62.2 \%)$ whose pulmonary growth was considered adequate obtained a complete repair. There were 1 early dearth and no late dearth. All survivors who underwent anatomical repair were in New
York Heart Association class I-II. The patients with right ventricle/left ventricle pressure ratio more than 0.6 after the anatomical repair had prolonged mechanical ventilation time and ICU monitoring time.

Conclusion Connection of the right ventricle to the pulmonary artery is safe and effective to promote the growth of the native pulmonary arteries in patients with pulmonary atresia, ventricular septal defect and hypoplastic native pulmonary arteries who ultimately have anatomical repair. The right ventricle/left ventricle pressure ratio is the important impact of early results

\section{Surgical treatment of coronary artery fistula: single-center experience spanning 12 years \\ Fang Li, Dianyuan Li, Jiawei Qiu, Jun Yan, \\ Qiang Wang, Shoujun Li \\ State Key Laboratoryof Cardiovascular Disease, Fuwail Hospital, \\ National Center forCardiovascular Diseases, ChineseAcademy of \\ Medical Sciencesand Peking Union MedicalCollege}

Objective Coronary artery fistula (CAF) is an uncommon form of congenital heart disease. It is often diagnosed incidentally during angiograms. We have reported on clinical characteristics, diagnosis, and surgical treatment of CAF in Fuwai Hospital spanning 12 years.

Methods Retrospective review of Fuwai Hospital database identified 223 patients with CAF between October 1996 and December 2013. We have noted 223 patients diagnosed as coronary artery fistula. Age weight and drainage site were reviewed. Mean follow-up was (25.63 \pm 19.53 ) months.

Result A total of 223 patients with coronary artery fistula at a single institution were included in our analysis. Patients complaints were directly associated with the presence of the fistula. Chest pain was the admission symptom in all of the patients with isolated coronary artery fistula. The median age and weight was $25.2 \mathrm{yrs}$ and $46.3 \mathrm{~kg}$. The drainage site is the pulmonary artery in $21.9 \%$ (49/223) of cases, the right ventricle in $34.5 \%$ (77/223), the right atrium in $17.9 \%$ (40/223), the left ventricle in $19.2 \%$ (43/223), the coronary sinus in $2.2 \%(5 / 223)$, and the left atrium in $4.0 \%(9 / 223)$. Eleven patients $(4.9 \%)$ died during followup.

Conclusion In contrast to our report, right ventricle was found to be the most frequent region of the fistula drainage. Clinical presentations were variable depending on type, size of fistula, and the presence of other cardiac conditions. Management of CAF is still controversial and treatment of adult asymptomatic patients with nonsignificant shunting is still a matter of debate.

\section{Study the relationship of left atrial volume index and vasovagal syncope \\ Xia Yu ${ }^{1}$, Bin Wang ${ }^{2}$, Jinghui Sun ${ }^{1}$ \\ 1. the First Hospital of Jilin University \\ 2. Jilin Tumor Hospital}

Objective Through detecting the left atrial volume index (LAVI) and cardiothoracic ratio, to explore the relationship between the volum and function of the heart and the vasovagol syncope (VVS).

Methods The 68 cases in observation group were hospitalization in the First Hospital of Jilin University and diagnosised VVS, from January 1, 2012 to December 31; The 60 cases in control group were selected in children and adolescents healthy physical examination. All patients were given the heart color Doppler ultrasound, head up tilt test 
(HUT), body height, weight, chest X-Ray and account the LAVI and cardiothoracic ratio. Measuring the LAVI by method of biplane arealength, LAVI $\left(\mathrm{ml} / \mathrm{m}^{2}\right)=$ left atrial volume $(\mathrm{ml}) /$ body surface area $\left(\mathrm{m}^{2}\right)$. Left atrial volume $=(\pi / 6) \cdot(\mathrm{LAd}) \cdot(\mathrm{Lx}) \cdot(\mathrm{Sx})$. Left atrial diameter $(\mathrm{LAd})$ was Mtypeultrasound measured in parasternal end-systole from the aorta posterior wall to left atrium behind the distance $(\mathrm{cm})$. Lx was left atrial area long axis recorded by cardiac apex four chamber trace chart $(\mathrm{cm})$. Sx was a short axis. The top of the right diaphragm method to calculate the cardiothoracic ratio. The data of experimental results was analysed using the statistical software SPSS 17.0. Measurement data was used $\mathrm{t}$ test. And numeration data was used $\chi^{2}$ test, $\mathrm{P}<0.05$ was statistically significant.

Result There were 6 cases VVS vascular inhibition (VVS-VI) patients, 24 cases VVS cardiac inhibition (VVS-CI) and 38 cases VVS mixed inhibition (VVS-MI) in the observation group. The average age were $(12.19 \pm 2.01) \mathrm{yrs}$ and $(12.15 \pm 2) \mathrm{yrs}$ in the observation group and the control group respectively $(\mathrm{t}=0.10, \mathrm{P}>0.05)$. The two groups had no statistically significant difference in age. The observation group had 23 boys and 45 girls, and the control group had 31 boys and 29 girls $\left(\chi^{2}\right.$ $=4.16, \mathrm{P}<0.05$ ). There was statistical significance in two groups, The observation group had more girls, The LAVI values were $(21.06 \pm 2.59)$ $\mathrm{ml} / \mathrm{m}^{2}$ and $(23.45 \pm 3) \mathrm{ml} / \mathrm{m}^{2}$, in observation group and the control group respectively $(\mathrm{t}=4.29, \mathrm{P}<0.05)$, there was statistical significance in two groups, the observation group LAVI was lower than control group. The LAVI values were $(21.06 \pm 2.59) \mathrm{ml} / \mathrm{m}^{2}$ and $(23.45 \pm 3.01) \mathrm{ml} / \mathrm{m}^{2}$ in VVS-VI and control group respectively, $(\mathrm{t}=3.36, \mathrm{P}<0.05)$, there was statistical significance in two groups.VVS-VI LAVI value was lower than the control group; LAVI values were $(21.41 \pm 2.98) \mathrm{ml} / \mathrm{m}^{2}$ and $(23.45 \pm 3.01) \mathrm{ml} / \mathrm{m}^{2}$, in VVS-MI and control group respectively $(\mathrm{t}=$ $3.27, \mathrm{P}<0.05)$, there was statistical significance in two groups. VVS-MI LAVI is lower than control group; LAVI values were $(21.23 \pm 3.22) \mathrm{ml} /$ $\mathrm{m}^{2}$ and $(23.45 \pm 3.01) \mathrm{ml} / \mathrm{m}^{2}$ in VVS-CI and control group respectively $\left(\mathrm{t}^{\prime}=1.61, \mathrm{P}>0.05\right)$, there were no statistical significance in two groups. The cardiothoracic ratio were $0.43 \pm 0.07$ and $0.46 \pm 0.06$ in two groups respectively $(\mathrm{t}=3.05, \mathrm{P}<0.05)$ with statistical significance, the observation group is lower than that of the control group.

Conclusion VVS is related to the size and function of left heart. The smaller LAVI and cardiothoracic ratio children and teenagers is the more susceptible to VVS.

\section{Comparison of fenestrated and nonfenestrated undergoing modified fontan operation at the early period of postoperation \\ Fan Fan \\ State Key Laboratoryof Cardiovascular Disease, FuwaiHospital, National Center forCardiovascular Diseases, ChineseAcademy of Medical Sciencesand Peking Union MedicalCollege}

Objective The purpose of this study is to compare the early clinical outcome of postoperation between Fenestrated (F, 49 patients) and Nonfenestrated (NF, 41 patients) modified Fontan patients.

Methods 90 Patiens from November 2008 to June 2013 having a modified Fontan operation were retrospectively selected for this study. We compared the perioperative files between the F group and NF group include age, anatomy, chest tube volume and duration, central venous pressure, ICU and hospital stay.

Result Cohorts were similar in anotomy and age. The duration [F $(11.1 \pm 9.2) \mathrm{d}, \mathrm{NF}(15.0 \pm 10.7) \mathrm{d}, \mathrm{P}=0.160]$ and volume[F $(1480.7 \pm$ 1729.5) $\mathrm{ml}, \mathrm{NF}(1858.1 \pm 2201.1) \mathrm{ml}, \mathrm{P}=0.494]$ of chest tube between cohorts were not significantly different. The ICU stay (F 3.1 \pm 1.6, NF 3.9 $\pm 4.4, \mathrm{P}=0.894)$ and postoperative $\mathrm{CVP}[\mathrm{F}(11.4 \pm 4.1) \mathrm{mm} \mathrm{Hg}, \mathrm{NF}(12.1$ $\pm 3.8) \mathrm{mm} \mathrm{Hg}, \mathrm{P}=0.894]$ were also similar between the two cohorts. But the outcomes of $\mathrm{F}$ group were a little better than the NF group. The cost and complications were similar between the two cohorts. However, the hospital stay $(\mathrm{P}=0.025)$ of $\mathrm{F}$ group was obvious longer than the NF group.

Conclusion In the aspect of duration and volume of chest tube the F group was a little better than the NF group. On the other hand, the hospital stay of $\mathrm{F}$ group was longer than NF group. Fenestration was helpless for the Fontan operation at the early peroid of post operation. It is unnecessary to routinely fenestrate anFontan. 


\section{Cardiac Electrophysiology}

\author{
Increased mortality in patients with secondary \\ diagnosis of atrial fibrillation: report from \\ chinese AF registry \\ Xinghui Shao, Yanmin Yang, Jun Zhu, Han Zhang, \\ Yao Liu, Xin Gao, Litian Yu, Lisheng Liu \\ Emergency and Intensive Care Center, State Key Laboratory \\ of Cardiovascular Disease, Fuwai Hospital, National Center for \\ Cardiovascular Diseases, Chinese Academy of Medical Sciences and \\ Peking Union Medical College
}

Background Atrial fibrillation (AF) is associated with a substantial risk of death. The relationship between mortality and the primary diagnosis in AF patients is poorly recognized. The purpose of the study is to compare the differences on mortality in patients with a primary or secondary diagnosis of $\mathrm{AF}$ and to identify risk factors amenable to treatment.

Methods A total of 2015 patients with confirmed AF from the Chinese AF registry were divided into two groups according to their primary diagnosis. For admitted patients, a follow-up was completed to obtain the outcomes during 1 year.

Result AF was the primary diagnosis in $40.9 \%(n=825)$ of them. Compared with primary AF diagnosis group, the secondary AF diagnosis group was older $(69.8$ yrs vs 66.6 yrs, $\mathrm{P}<0.001)$ with more comorbidities (eg. heart failure, stroke and diabetes) and more probably be prescribed to ACE inhibitor, diuretics and digoxin (all $\mathrm{P}<0.01$ ). At 1 year, the unadjusted mortality was $7.8 \%$ (64 deaths) in the primary AF diagnosis group and $18.2 \%$ (213 deaths) in the secondary AF diagnosis group. In Cox regression analysis with adjustment for confounding factors, patients with secondary AF diagnosis were associated with an increased mortality (relative risk 1.723; 95\% CI: 1.283-2.315, P < 0.001). On multivariate analysis, age $\geq 75 \mathrm{yrs}$, left ventricular systolic dysfunction, chronic obstructive pulmonary disease and diabetes were independently predictors of mortality in patients with primary AF diagnosis, while for the secondary AF diagnosis group, the risk factors were age $\geq 75$ yrs, heart failure and previous history of stroke.

Conclusion Patients presenting to ED with secondary diagnosis of $\mathrm{AF}$ was suffering from higher mortality risks compared with primary $\mathrm{AF}$ diagnosis patients. Physicians should distinguish these two groups and pay attention to their risk factors on treatment.

\section{Comparison of the clinical features and outcomes in two age-groups elderly patients with atrial fibrillation}

Xinghui Shao, Yanmin Yan, Jun Zhu, Han Zhang, Yao Liu, Xin Gao, Litian Yu, Lisheng Liu

Emergency and Intensive Care Center, State Key Laboratory of Cardiovascular Disease, Fuwai Hospital, National Center for Cardiovascular Diseases, Chinese Academy of Medical Sciences and Peking Union Medical College

Background Atrial fibrillation (AF) disproportionately affects older adults. However, the directly comparison of the clinical features, medical therapy and outcomes in AF patients between age 65-74 yrs and age $\geq 75 \mathrm{yrs}$ is rare. The objective of the present study is to evaluate the differences in clinical characteristics and prognosis in these two agegroups geriatric patients with $\mathrm{AF}$.

Methods 1336 individuals aged $\geq 65$ yrs old from Chinese AF registry were assessed in the present study. 570 were in the 65-74 yrs group, and 766 were in the $\geq 75$ yrs group. Multivariable Cox hazards regression was performed to analyse the major adverse cardiac events (MACE) between groups.

Results In our population, the older group were more likely to have coronary artery disease, hypertension, previous stroke, cognitive disorder or chronic obstructive pulmonary disease (COPD) and had 1.2 higher mean $\mathrm{CHADS}_{2}$ score but with a less probably being prescribed with drugs. Compared with 65-74 yrs individuals, the older group had higher risks of death (HR 2.881;95\% CI: 1.981-4.189; $\mathrm{P}<0.001$ ) or MACE (HR $=2.202$; 95\% CI: 1.646-2.945; P < 0.001) during 1 year follow-up. In multivariable Cox analyses, secondary AF diagnosis, a history of COPD, and left ventricular systolic dysfunction were independent predictors of MACE in the older group.

Conclusion Patients aged $\geq 75$ yrs had a worse prognosis than 65-74 yrs group and were associated with higher risks of both death or MACE. A better understanding of the age-specific risk factors may help inform strategies aimed at improving outcomes in elderly.

\section{Clinical characteristics and impact of diabetes mellitus on outcomes in patients with nonvalvular atrial fibrillation \\ Bi Huang, Yanmin Yang, Jun Zhu, Yan Liang, Han Zhang, Li Tian, Xinghui Shao, Juan Wang, Huaibin Wan Emergency and Critical Care Center, State Key Laboratory of Cardiovascular Disease, Fuwai Hospital, National Center for Cardiovascular Diseases, Chinese Academy of Medical Sciences and Peking Union Medical College}

Objective Studies have shown diabetes mellitus (DM) is a risk factor for cardiovascular disease including atrial fibrillation (AF), but the clinical characteristics and prognostic impact of DM in patients with nonvalvular AF haven't been well understood in China. The aim of present study was to analyse the clinical profiles, management, and the impact of DM on 1-year outcomes in patients with nonvalvular AF.

Methods Included were 1644 consecutive patients with nonvalvular AF. Endpoints included all-cause mortality, cardiovascular mortality, stroke, major bleeding and combined endpoint events (CEE) during 1-year follow up.

Result The prevalence of DM was $16.8 \%$ in nonvalvular AF patients. Compared with non-diabetic AF patients, diabetic AF patients were older and tended to coexist other cardiovascular diseases. Most of patients with DM (93.5\%) were eligible for anticoagulation by $\mathrm{CHADS}_{2}$ score. However, only $13.0 \%$ of patients received anticoagulation. During 1-year follow up, the all-cause mortality and CEE rate in DM group were significantly higher than that of non-DM group, while the incidence of stroke was comparable. After multivariate adjustments, DM was still an independent risk factor for 1-year all-cause mortality $(\mathrm{HR}=1.558,95 \%$ CI: $1.126-2.156, \mathrm{P}=0.007)$, cardiovascular mortality $(\mathrm{HR}=1.615,95 \%$ CI: $1.052-2.479, \mathrm{P}=0.028)$, and CEE $(\mathrm{HR}=1.523,95 \% \mathrm{CI}: 1.098$ $2.112, \mathrm{P}=0.012)$, but not for stroke $(\mathrm{HR}=1.119,95 \% \mathrm{CI}$ : $0.724-1.728$, $\mathrm{P}=0.614)$.

Conclusion DM is a common morbidity coexisting with 
nonvalvular $\mathrm{AF}$ and is associated with increased risk of 1-year all-cause mortality, cardiovascular mortality, and CEE. However, no increased risk of stroke was found during 1-year follow-up in patients with $\mathrm{AF}$ and DM.

\section{Effects of pre-excitation syndrome on terminal QRS vector observed in spatial vector \\ Yang Chen, Renguang Liu \\ The first affiliated hospital of Liaoning medical colleage}

Objective Pre-excitation syndrome could affect the terminal QRS vector. However, there is little information about the change of terminal QRS vector. In this study we made a comparison between three-dimensional vectorcardiogram (VCG) before and after ablation to observe the change of terminal QRS vector. Furthermore, the relationship between the change of terminal QRS vector and accessory pathway (AP) as well as the initial QRS vector was analyzed.

Methods The 30 patients who were proved to have a single accessory pathway (AP) by ablation were included. All the patients were divided into 7 groups based on the AP location ( 8 were left anterior $\mathrm{AP}, 5$ were left lateral AP; 3 were left posterior AP; 2 were right lateral AP; 4 were right posterior AP; 2 were right midseptal AP; 6 were right posteroseptal $\mathrm{AP}$ ). In order to observe the change of terminal and initial QRS vector, we made a comparison between VCG before and after ablation. The relationship between the change of terminal QRS vector and AP location as well as the change of initial QRS vector was analyzed.

Result All 30 patients had a change in terminal QRS vector (elevation and/or azimuth) in comparison to postablation VCG. The change of terminal QRS vector was related to the initial QRS vector and AP location. The agreement between the change of terminal QRS vector and the initial QRS vector was $91.65 \%(\mathrm{P}<0.01)$. The consistency rate between the change of terminal QRS vector and initial $\mathrm{QRS}$ vector was $0.846(\mathrm{P}<0.01)$.

Conclusion Both initial and terminal QRS vector are affected by the antegrade conduction of AP in pre-excitation syndrome. The change of terminal QRS vector is related to the AP location and the initial QRS vector. The effect of pre-excitation syndrome on QRS initial and terminal vector is showed more intuitive and easy in spatial vector loop in comparison to that in ECG, which is helpful for the diagnosis of preexcitation without evident delta wave and the localization of AP.

\section{Impact of life-threatening arrhythmias on the short-term outcomes in patients with ST segment elevation myocardial infarction (STEMI) undergoing reperfusion treatment \\ Bi Huang, Yanmin Yang, Jun Zhu, Yan Liang, \\ Huiqiong Tan, Litian Yu, Xin Gao, Jiandong Li \\ Emergency and Critical Care Center, State Key Laboratory of \\ Cardiovascular Disease, Fuwai Hospital, National Center for \\ Cardiovascular Diseases, Chinese Academy of Medical Sciences and \\ Peking Union Medical College}

Objective The aim of the present study was to evaluate the impact of life-threatening arrhythmias on the short-term outcomes in patients with ST-elevation myocardial infarction (STEMI) undergoing reperfusion treatment.

Methods Data of 4652 consecutive STEMI patients undergoing reperfusion treatment were retrospectively analyzed. Life-threatening arrhythmias included sustained ventricular tachycardia (VT) and ventricular fibrillation (VF), high grade atrioventricular block (AVB), and asystole or pulseless electrical activity (PEA). The major endpoints were 7 -and 30-day all cause mortality.

Result Of 4652 patients, 3792 patients received thrombolysis, 731 received PCI, and 129 received both. During hospital course, 138 (3.0\%) experienced high grade AVB, 199 (4.3\%) experienced sustained VT/VF, and 85 (1.8\%) experienced asystole or PEA. Patients with life-threatening arrhythmias were more likely to receive thrombolysis therapy while a relatively higher percentage of patients without lifethreatening arrhythmia received PCI therapy compared with those with life-threatening arrhythmias (all $\mathrm{P}=0.001$ ). The 7-day all cause mortality was $70.6 \%$ in patients with asystole or PEA, followed by sustained VT/ VF (37.2\%) and high grade AVB (14.5\%), lowest in patients without lifethreatening arrhythmia $(3.3 \%)(\mathrm{P}<0.001)$. The values within 30 days were $76.5 \%, 45.2 \%, 20.3 \%$, and $4.9 \%$, respectively $(\mathrm{P}<0.001)$. After adjusting for age, sex, weight, medical histories, onset-to-admission interval, admission vital signs, location of myocardial infarction, and treatment during hospitalization (reperfusion strategies and other medications), high grade AVB was associated with 3.4-fold increase in 30-day all cause mortality $(\mathrm{HR}=3.477,95 \% \mathrm{CI}: 2.156-5.607, \mathrm{P}<0.001)$, sustained VT/VF predicted nearly 10 -fold increased risk of 30-day all cause mortality $(\mathrm{HR}=9.951,95 \% \mathrm{CI}: 7.354-13.464, \mathrm{P}<0.001)$, and asystole or PEA was associated with 16-fold increase in 30-day all cause mortality $(\mathrm{HR}=16.512,95 \% \mathrm{CI}: 11.826-23.054, \mathrm{P}<0.001)$.

Conclusion Compared with those without life-threatening arrhythmias, patients experienced asystole or PEA had remarkably increased risk of short-term mortality, followed by those experienced sustained VT/VF and high grade AVB in addition to traditional risk factors. More attention should be paid to those experienced lifethreatening arrhythmias in clinical practice.

\section{Clinical characteristics and impact of hypertension on the 1-year outcomes in patients with atrial fibrillation and valvular diseases}

Bi Huang, Yanmin Yang, Jun Zhu, Yan Liang, Han Zhang, Li Tian, Xinghui Shao, Juan Wang, Huaibin Wan Emergency and Critical Care Center, State Key Laboratory of Cardiovascular Disease, Fuwai Hospital, National Center for Cardiovascular Diseases, Chinese Academy of Medical Sciences and Peking Union Medical College

Objective To study the clinical characteristics and impact of hypertension on the 1-year outcomes in patients with atrial fibrillation (AF) and valvular diseases.

Methods From November 2008 to October 2011, 2016 patients with AF in 20 hospitals around China were enrolled, of which 331 had valvular diseases. These patients with valvular diseases were divided into two groups according to whether they had concomitant hypertension. The major endpoints were 1-year all cause mortality and stroke.

Result Of 331 AF patients with valvular diseases, 90 patients (27.2\%) had concomitant hypertension. Compared with those without hypertension, AF patients with hypertension were more likely to be older, heavier, and had histories of stroke and diabetes mellitus (all $\mathrm{P}<0.05$ ). During 1 year follow- up period, patients with valvualr diseases were more likely to use beta-blocker, calcium channel blockers, angiotensin converting enzyme inhibitors (or angiotensin receptor antagonist), and 
statins (all $\mathrm{P}<0.05$ ). As far as antithrombolic therapy concerned, $\mathrm{AF}$ patients with valvular diseases and hypertension were more likely to receive aspirin ( $55.6 \% \mathrm{vs} 40.7 \%, \mathrm{P}=0.018)$, however, the percentage of patients that received anticoagulation with warfarin was low and with no significant difference between with and without hypertension (36.7\% vs $39.0 \%, \mathrm{P}=0.800$ ). The 1-year all cause mortality was $16.6 \%$ in patients without hypertension and $8.9 \%$ in those with hypertension $(\mathrm{P}=0.082)$. The incidence of stroke in patients with and without hypertension were $5.6 \%$ and $5.4 \%$, respectively $(\mathrm{P}=1.000)$. After adjusting for sex, age, medical histories, admission vital signs, medications during follow-up by multivariate Cox regression analysis, the presence of hypertension was a protector for 1 -year all cause mortality $(\mathrm{HR}=0.394,95 \% \mathrm{CI}$ : $0.179-0.867, \mathrm{P}=0.021)$, however, was not independently associated with increased risk of stroke ( $\mathrm{HR}=1.053,95 \% \mathrm{CI}$ : 0.204-5.438, $\mathrm{P}=$ 0.951).

Conclusion Our results suggested that hypertension appeared to have a protective role in the 1-year outcome in $\mathrm{AF}$ patients with valvular diseases, however, a long-term prognostic value of hypertension was needed to further study.

\section{Association of reperfusion strategies with sustained ventricular tachycardia/ventricular fibrillation and in patients with ST elevation myocardial infarction \\ Bi Huang, Yanmin Yang, Jun Zhu, Yan Liang, Huiqiong Tan, Litian Yu, Xin Gao, Jiandong Li Emergency and Critical Care Center, State Key Laboratory of Cardiovascular Disease, Fuwai Hospital, National Center for Cardiovascular Diseases, Chinese Academy of Medical Sciences and Peking Union Medical College}

Objective The aim of the present study was to evaluate the association of reperfusion strategies with sustained ventricular tachycardia (VT) /ventricular fibrillation (VF) in patients with STelevation myocardial infarction (STEMI).

Methods Data of 4652 consecutive STEMI patients undergoing reperfusion treatment were retrospectively analyzed. Sustained VT/VF was defined as those occurred during or after reperfusion treatment. The major endpoint was incidence of sustained VT/VF within 30 days after hospitalization.

Result Of 4652 patients, 3792 patients received thrombolysis, 731 received percutaneous coronary intervention (PCI), and 129 received both. Patients that received PCI treatment were more likely to be older, male gender, heavier, and have histories of diabetes mellitus, hypertension, and stroke (all $\mathrm{P}<0.05$ ). After hospitalization, patients received PCI treatment were more likely to use beta-blocker, Clopidogrel, Angiotensin converting enzyme inhibitors, and Statins, but less use diuretics (all $\mathrm{P}<0.05$ ). A total of 168 patients experienced sustained VT/VF during or after reperfusion treatment. The median timing of sustained VT/VF was 11.8 hours (interquartile range 5.0-38.1 hours) after symptom onset. The incidence of sustain VT/VF was 3.9\%, $2.2 \%$, and $3.9 \%$ in patients undergoing thrombolysis, $\mathrm{PCI}$, and both, respectively $(P=0.080)$. Survival curves reported that there were no significant difference among groups $(\mathrm{P}=0.081)$. After adjusting for age, sex, weight, medical histories, onset-to-admission interval, admission vital signs, location of myocardial infarction, and treatment during hospitalization, PCI was not associated with increased risk of incidence of sustained VT/VF (HR $=0.906,95 \%$ CI: $0.485-1.693, \mathrm{P}=0.757)$ compared with thrombolysis.
Conclusion Our results suggested that different reperfusion strategies appeared to have no influence on the incidence of sustained VT/VF in patients with STEMI.

\section{Effect of renal sympathetic denervation on the progression of paroxysmal atrial fibrillation in canines with long-term intermittent atrial pacing Xule Wang, Congxin Huang, Qingyan Zhao, He Huang, Yanhong Tang, zixuan Dai, Xiaozhan Wang, Zongwen Guo Department of Cardiology, Renmin Hospital of Wuhan University; Cardiovascular Research Institute of Wuhan University}

Objective The aim of the study was to explore the effect of renal sympathetic denervation (RSD) on the progression of paroxysmal AF in canines with long-term intermittent atrial pacing.

Methods Nineteen beagles were randomly divided into shamoperated group (6 dogs), control group (6 dogs), and RSD group (7 dogs). Sham-operated group were implanted with pacemakers without pacing; control group were implanted with pacemakers with longterm intermittent atrial pacing; and RSD group underwent catheterbased RSD bilaterally and were simultaneously implanted with pacemakers. Atrial pacing was maintained for 8 hours a day and a total of 12 weeks in control group and RSD group. Electrophysiological test and hemodynamic parameters were measured in all the animals at the baseline and endpoint of the protocol. The plasma levels of angiotensin II and aldosterone were examined by ELISA.

Result Compared with control group, RSD group had fewer incidences of $\mathrm{AF}$ and a shorter duration of $\mathrm{AF}(\mathrm{P}<0.05)$ after longterm intermittent atrial pacing. In addition to increased AERP and AF cycle length, AERP dispersion and P-wave duration and dispersion were significantly decreased in the RSD group compared with the control group $(\mathrm{P}<0.05)$. Compared with the control group, RSD prevented the increase of right atrial pressure, mean pulmonary artery pressure and pulmonary capillary wedge pressure induced by long-term intermittent atrial pacing $(\mathrm{P}<0.05)$. The plasma levels of angiotensin II and aldosterone increased significantly in control group, but the increasing trend was inhibited by RSD $(\mathrm{P}<0.05)$.

Conclusion Renal denervation inhibits the progression of paroxysmal AF, which might be related to the suppression of atrial electrophysiology and hemodynamic changes induced by long-term intermittent atrial pacing.

\section{Utility of modified moving average analysis of microvolt $t-$ wave alternans to predict ventricular arrhythmias in patients with arrhythmogenic right ventricular cardiomyopathy \\ Sheliang Xue, Xiaofeng Hou, Quanpeng Wang, Yao Wang, Haiping Ying, Jiangang Zou \\ Department of cardiology, The First Affiliated Hospital of Nanjing Medical University}

Background and Objective Arrhythmogenic right ventricular cardiomyopathy (ARVC) is an inherited cardiomyopathy characterized by right ventricular dysfunction and ventricular arrhythmias. Studies have shown that ARVC is present in up to $20 \%$ of individuals who experience sudden cardiac death (SCD) and is even more common among athletes who die suddenly. Improved prognostic indices are needed to identify risk for sudden cardiac arrest from ventricular 
arrhythmias in ARVC patients. Our study explored the characteristics of microvolt T-wave alternans (MTWA) in patients with arrhythmogenic right ventricular cardiomyopathy (ARVC) and its predictive value of ventricular arrhythmia events.

Methods MTWA was assessed with modified moving average (MMA) analysis method by treadmill exercise testing in 35 ARVC patients and 60 healthy volunteers. Alternation voltage of $T$ wave in the precordial leads $\mathrm{V}_{1}-\mathrm{V}_{6}$ was measured and the greatest alternation voltage of each individual in all precordial leads was defined as MaxAlt. All ARVC patients were followed up. 20 of these 35 patients underwent a second TWA examination during the follow-up. The clinical ventricular arrhythmia events included sudden cardiac death, sustained symptomatic ventricular tachycardia/fibrillation or appropriate ICD discharge.

Result The 35 ARVC patients (28 males) had an average age of (38. $6 \pm 11.0)$ yrs. Despite the different morphologic changes of the right ventricular, the patients have preserved left ventricular function with an average LVEF of $(65.0 \pm 5.5) \%$, There were significant differences between ARVC patients and healthy people in alternation voltage of lead $\mathrm{V}_{1}-\mathrm{V}_{6}$ and MaxAlt. The 75th percentile value of MaxValt in healthy people is $9.75 \mu \mathrm{V}$. Twenty-nine ARVC patients $(82.9 \%)$ had positive test result when MaxAlt $\geq 9.75 \mu \mathrm{V}$ was defined as MTWA positive. During $(69.9 \pm 4.9)$ months' follow-up, $15(42.9 \%)$ patients had clinical events. Among these 15 patients, 14 were MTWA positive and only one negative. The negative predictive value (NPV) and positive predictive value (PPV) of the MTWA test for the prediction of clinical events were $83.3 \%$ and $48.3 \%$ respectively. Neither the difference of clinical events rate between MTWA-negative and positive subjects nor the difference of alternation voltage value between patients with and without clinical events had been found. Kaplan-Meier survival curve showed that MTWA positive had no relation with clinical events in ARVC patients. 20 of these 35 patients were called for second TWA examination. All patients with negative MTWA value (4 patients) remained negative, and two patients with positive MTWA value turned into negative. No clinical ventricular arrhythmia events had been found in all these six negativevalue patients.

Conclusion ARVC patients have much higher level of T wave alternation voltage than healthy controls; MTWA test had good and stable negative predictive value of ventricular arrhythmia but was not able to predict the clinical events of this group of ARVC patients with preserved LV function.

\section{Prevalence and determinants of elevated high- sensitivity cardiac troponin I in emergency patients with atrial fibrillation \\ Han Zhang, Yanmin Yang, Jun Zhu \\ Emergency and Intensive Care Center, State Key Laboratory of Cardiovascular Disease, Fuwai Hospital, National Center for Cardiovascular Diseases, Chinese Academy of Medical Sciences and Peking Union Medical College}

Objective This study was designed to evaluate the prevalence and determinants of increased high-sensitivity cardiac troponin I (hs-cTnI) as a marker of cardiac injury in emergency patients with atrial fibrillation (AF).

Methods A total of consecutive patients with AF [52.9\% males; mean age $(63.3 \pm 14.3)$ yrs] between May 2013 and November 2013 were evaluated by measuring the level of serum hs-cTnI along with other clinical assessments.

Result There were $110(17.0 \%)$ patients with a minimum serum
hs-cTnT level of $0.02 \mathrm{ng} / \mathrm{ml}$. The median hs-cTnI level was $0.006 \mathrm{ng} /$ $\mathrm{ml}(0.003-0.014 \mathrm{ng} / \mathrm{ml})$. There were significant differences in age, sex, heart rate (HR), type of AF, reason for emergency department visit, concomitant valvular heart disease, hypertension, hyperlipidemia, creatinine, creatinine clearance rate (Ccr), left ventricular end-diastolic diameter (LVEDD) and left ventricular ejection fraction (LVEF) in echocardiography between the patients with hs-cTnI $<0.02 \mathrm{ng} / \mathrm{ml}$ and those with hs-cTnI $\geq 0.02 \mathrm{ng} / \mathrm{ml}$. However, after multivariate analysis, $\mathrm{HR}(\mathrm{OR}=1.014 ; 95 \% \mathrm{CI}: 1.003-1.025 ; \mathrm{P}=0.010), \mathrm{LVEF}(\mathrm{OR}=0.967$; 95\% CI: $0.946-0.989 ; \mathrm{P}=0.003)$, and GFR $(\mathrm{P}=0.002)$ remained as the independent determinants of elevated hs-cTnI in AF.

Conclusion The results demonstrated that hs-cTnI was elevated in a significant number of our AF patients. HR, Ccr, and LVEF can predict the elevation of hs-cTnI in AF patients.

\section{Reversible Tachycardia Mediated Cardiomyopathy after Radiofrequency Ablation of Idiopathic Left Ventricular Tachycardia \\ Jia He, Pihua Fang, Zheng Liu, Xiongbiao Chen, Jun Liu, Min Tang, Yuhe Jia, Shu Zhang Center for Arrhythmia Diagnosis and Treatment, State Key Laboratory of Cardiovascular Disease, Fuwai Hospital, National Center for Cardiovascular Diseases, Chinese Academy of Medical Sciences and Peking Union Medical College}

Objective Tachycardia mediated cardiomyopathy (TMC) is a reversible form of dilated cardiomyopathy characterized by new onset ventricular systolic and diastolic dysfunction simultaneous with prolonged tachycardia.Idiopathic left ventricular tachycardia (ILVT) related TMC and dynamic recovery of the ILVT related TMC after radiofrequency ablation has been rarely reported yet.

Methods and result A 20-year-old girl was admitted to our hospital with a chief complain of 12-day of palpitation and progressive shortness of breath. The symptoms of heart failure were consistent with New York Heart Association (NYHA) grade III. She had fever and cold symptoms 2-day before the onset of palpitation and heart failure symptoms but recovered soon, without other significant medical history. Physical examination demonstrates a heart rate of 170 beat per minute (bpm), a blood pressure of 70/40 $\mathrm{mm} \mathrm{Hg}$ and bibasilar rales. ECG revealed a relatively narrow QRS tachycardia of $170 \mathrm{bpm}$ with right bundle branch block (RBBB) morphology and a superior left axis configuration. At the emergency department, empirical administration of intravenous verapamil was injected and sinus rhythm was restored within 3 minutes with inverted $\mathrm{T}$ wave in lead II, III, aVF and $\mathrm{V}_{3-6}$. A chest $\mathrm{X}$-ray revealed bilateral plural effusion and increased cardiothoracic ratio. Transthoracic echocardiography performed demonstrated marginal left ventricular dysfunction [left ventricular ejection fraction (LVEF) of 50\%], with normal LV end-diastolic diameter (LVEDD) of $38 \mathrm{~mm}$ and cardiac effusion with fluid sonolucent area of $6 \mathrm{~mm}$. Electrophysiological study performed in the following day after administration induced narrow QRS tachycardia which was not replicate the clinical morphology and indicated atrio-ventricular tachycardia via left atrio-ventricular bypass, while the tachycardia of clinical morphology was not induced. The atrio-ventricular bypass was successfully ablated and the presystolic purkinje potentials were targeted, resulting in new Q wave in inferior leads of ECG which mimic left posterior fascicular block after ablation. Chest X-ray performed in the $2^{\text {nd }}$ day, the $3^{\text {rd }}$ day and the $40^{\text {th }}$ day revealed decreased and demission of plural effusion and decrease cardiothoracic ratio. Transthoracic echocardiography performed in the $3^{\text {rd }}$ 
day demonstrated normalization of left ventricular dysfunction (LVEF of $76.6 \%$ ) and cardiac effusion with fluid sonolucent area of $6 \mathrm{~mm}$, while in the $40^{\text {th }}$ day the cardiac effusion was diminished. ECG in the $40^{\text {th }}$ day revealed $\mathrm{T}$ wave restored upright. The patient remained free from arrhythmia for 2 months, without anti-arrhythmic medication.

Conclusion IVLT induced TMC is completely reversible demonstrated by recovery of clinical symptoms, ECG, chest X-ray and transthoracic echocardiography. Radiofrequency ablation is an effect approach for IVLT induced TMC.

\section{Baseline characteristics and 1-year follow-up of chinese atrial fibrillation patients according to age: A registry study \\ Tao Chen, Yanmin Yang, Hui qiong Tan, Yan Liang, Jun Zhu \\ Emergency and Critical Care Centre, State Key Laboratory of \\ Cardiovascular Disease, Fuwai Hospital, National Center for \\ Cardiovascular Diseases, Chinese Academy of Medical Sciences and \\ Peking Union Medical College}

Background and Objective The prevalence of atrial fibrillation (AF) increases with age, and may lead to complications and reduced quality of life. The aim of this study was to determine the characteristics, management and prognosis of Chinese AF patients and whether there were differences according to age.

Methods This registry-based study enrolled ambulatory, outpatient clinic or hospitalized patients with AF in four sites in China. Based on the Birmingham 2009 schema, patients without and with valve lesion were stratified into 3 groups according to age.

Result Between September 2008 and April 2011, 2016 patients were enrolled, including 1606 patients without valve lesion and 410 patients with valve lesion. Compared with the other two groups, patients $>74$ were more likely to have morbidity and a $\mathrm{CHADS}_{2}$ score $>1$, and less likely to receive oral anticoagulants and rhythm-control drugs. At the 1-year follow-up, patients $>74$ years were more likely to have died or suffered a cerebrovascular event or systemic embolism. Age as a continuous variable ( $\mathrm{SHR}=0.98,95 \% \mathrm{CI}$ : $0.96-1.01, \mathrm{P}=0.29$ ) was not associated with risk of a cerebrovascular event or systemic embolism at 1 -year but age $\geq 75$ yrs (SHR $=1.73,95 \%$ CI: $1.05-2.87, \mathrm{P}=0.03)$ was an independent risk factor for the outcome at 1-year when all $\mathrm{AF}$ patients were included.

Conclusion Elderly AF patients are inadequately studied and treated compared with younger patients. Education on evidence-based management and the design of randomized controlled trials specifically targeting the elderly, especially the Chinese elderly, should improve the management and prognosis of this frail segment of the $\mathrm{AF}$ population.

\section{Management of recurred atrial appendage tachycardia: repetitive catheter ablation or surgical atrial appendectomy? \\ Zheng Liu, Yuhe Jia, Pihua Fang, Zhe Zheng \\ State Key Laboratory of Cardiovascular Disease, Fuwai Hospital, National Center for Cardiovascular Diseases, Chinese Academy of Medical Sciences and Peking Union Medical College}

Objective Atrial tachycardias originated from atrial appendage (AATs) are rare entities in clinical practice. Pharmaceutics therapy is generally ineffective, and patients underwent radiofrequency catheter ablation (RFCA) is easy to recur. Patients with recurred AAT after initial failed RFCA can resorted to video-assisted thoracoscopic (VAT) atrial appendectomy or redo RFCA. The outcome of the different management of the relapsed AAT after initial radiofrequency catheter ablation (RFCA) is unknown. We tried to evaluate the mid/long- term outcomeof different management for patients with relapsed AAT after initial RFCA.

Methods From Feburary 2011 to July 2013, 19 patients diagnosed with AAT experiencing recurrence after initial radiofrequency catheter ablation procedures (RFCA) were studied. Five of them resorted to video-assisted thoracoscopic (VAT) atrial appendectomy (atrial appendectomy cohort) and 14 received re-do RFCA (RFCA cohort). We investigated the mid/long-term outcomes of the two cohorts.

Result After a mean follow up of $(353 \pm 217)$ days, no patients experienced recurrence in atrial appendectomy cohort, while 8 out of 14 patients $(57.1 \%)$ in the RFCA cohort experienced recurrence. Log-Rank analysis observed significant differences between two cohorts $(\mathrm{P}=0.049)$. No major complications were reported in either cohort. Larger earliest $10 \mathrm{~ms}$ activation area in the endocardial atrial appendage, as well as more antiarrhythmic drug resistance indicate recurrence for patients who underwent redo-RFCA.

Conclusion VAT atrial appendectomy may be a reasonable option for patients with relapsed AATs after the initial RFCA

\section{Algorithm for catheter ablation of orthodromic reciprocating tachycardia using left accessory pathways: based on bipolar polarity reversal mapping technique. \\ Zheng Liu, Yuhe Jia, Pihua Fang \\ The Department of Cardiac Arrhythmia, State Key Laboratory of Cardiovascular Disease, Fuwai Hospital, National Center for \\ Cardiovascular Diseases, Chinese Academy of Medical Sciences and Peking Union Medical College}

Background The bipolar polarity reversal mapping technique during consecutive mapping the mitral annulus was reported to be precise when ablating the orthodromic reciprocating tachycardia (ORT) using left accessory pathways (LAPs).

Methods 122 patients with LAPs who received successful ablation guided by conventional electrogram criteria were collected and the intracardiac tracing were analyzed retrospectively. Locations of the "bipolar reversal pairs" were used to compare with the successful fluoroscopic ablation sites, which was classified as "CS", "CS+", and "CS++". Then an algorithm based on indices of the bipolar polarity reversal mapping technique was developed and validated in the other prospective cohort with 74patients.

Result In the retrospective cohort, the successful ablation site was highly coincided with the locations of the "bipolar reversal pairs". The sensitivity, specificity and PPV were $95.4 \%, 88.3 \%$, and $91.6 \%$, respectively. Indices required for the "bipolar polarity reversal mapping technique" for the localization of LAPs were generated. Amplitude ratio (AR: Ratio of atrial electrogram amplitude during ORT/NSR) of the "proximal reversal bipole" with cut-off value of 0.201 can differentiate "CS" from "CS+" $(\mathrm{AUC}=0.891)$ and "reversal pair interval" (interval of Atrial electrogram between proximal and distal reversal bipole during ORT) with cut-off value of $-2.5 \mathrm{~ms}$ can differentiate "CS+" from " $\mathrm{CS}++$ " (AUC $=0.882$ ). In the prospective cohort, the algorithm was validated with an accuracy of $88.5 \%$, significantly reducing the procedure time and ablation attempts required to achieve an AP block[(34.99 \pm 8.79$)$ min vs (55.35 \pm 20.27$) \mathrm{min}, \mathrm{P}<0.001$; (1.15 \pm 0.358$)$ attempts/AP vs $(2.56 \pm$ 
2.098) attempts/AP, P $<0.001$, respectively) ]

Conclusion A new algorithm based on the bipolar polarity reversal mapping technique can be used to predict the exact ablating site of LAPs.

\section{Second generation of cryoballoon can improve efficiency of cryoablation for atrial fibrillation \\ Jun Liu, jinHong, Gerds-Li \\ State Key Laboratory of Cardiovascular Disease, Fuwai \\ Hospital, National Center for Cardiovascular Diseases, \\ Chinese Academy of Medical Sciences and Peking Union \\ Medical College}

Background To investigate whether a new second generation of cryoballoon can improve the efficiency and safety of cryoablation for atrial fibrillation (AF) as compared with a first generation of cryoballoon during ablation procedure and followup outcome.

Methods The data of AF patients who were consecutively treated by a single $28 \mathrm{~mm}$ cryoballoon were analyzed. The patients were divided to two groups according to the different cryoballoon used during procedure, the G1 group by the first generation of cryoballoon (ArcticFront) and the $\mathrm{G} 2$ group by the second generation of cryoballoon (ArcticFront Advance) respectively. The failure-treatment of cryoablation was defined as any detected episode of AF, atrial flutter, atrial tachycardia which lasted for 30 seconds after 3 months during followup. Left atrium diameter (LAD) was measured by the transthoracic echocardiography before cryoablation.

Result A total of 125 patients (G1/G2 group: 57/68) were enrolled. The mean total time of whole procedure, cryomapping and cryoablation in the G2 group were shorter than that in the G1 group $(\mathrm{P}<0.05)$. No complication happened in the G1 group. the rate of complication was higher to $8.8 \%$ in the G2 group. During mean $(12 \pm 4)$ months follow-up, the success rate of cryoablation was $76.0 \%(95 / 125)$, which was higher in the G2 group than that in the G1 group [89.7\% (61/68) vs 59.7\% (34/57), $\mathrm{P}<0.001]$. The failuretreatment patients had a larger LAD $[(48 \pm 8) \mathrm{mm}$ vs $(44 \pm 6) \mathrm{mm}, \mathrm{P}$ $=0.002]$ than the success-treatment patients. The type of cryoballoon $[\mathrm{RR}=5.75,95 \% \mathrm{CI}: 2.16-15.27, \mathrm{P}<0.0001]$ and the $\mathrm{LAD}[\mathrm{RR}=$ $0.90,95 \%$ CI: $0.83-0.97, \mathrm{P}=0.0043$ ] could individually related to difference of success rate after multivariate analysis.

Conclusion The new generation of cryoballoon ablation for AF is associated with higher PVI success rate and better outcome of cryoablation for AF patients. However, the higher rate of complication is occurred during the early stage of application with the $\mathrm{G} 2$ cryoballoon.

\section{Frequent spectrum characteristics of bipolar electrogram at the ablation site in patients with ventricular arrhythmia near the left fibrous trigone and the implication for outcome after catheter ablation. \\ Zheng Liu, Pihua Fang \\ Center of Arrhythmia, State Key Laboratory of Cardiovascular Disease, \\ Fuwai Hospital, National Center for Cardiovascular Diseases, Chinese \\ Academy of Medical Sciences and Peking Union Medical College}

Objective Ventricular arrhythmia (VAs) can originate from aortic sinus cusp (ASC), subvalvular left ventricular outflow tract (SVLOT), anterior mitral annulus (AMA) and LV summit. These regions are proximal to the left fibrous trigone of the heart (LFT). Electrogram recorded at the earliest activation site (EAS) can present as low frequent slurring morphology or high frequent distinct morphology. The detailed frequency spectrum characteristics of the bipolar electrogram in VAs from the Para-LFT region are not well investigated.

Methods 59 Consecutive patients underwent catheter ablation of VAs originated from the para-LFT regions were studied. Catheter ablation of the VAs was targeted at EAS. Patients were divided into two groups $(\mathrm{A}=$ successfully ablated and $\mathrm{B}=$ recurrence $)$ according to the clinical outcome during 1-year-follow up. Pace mapping at the targeted ablation site was performed before and after RF application. The bipolar electrogram of the EAS both before and after the RFCA were collected and underwent off-line fast Fourier transformation (FFT). Bipolar electrogram with Harmonics Frequency larger than $70 \mathrm{~Hz}$ in the spectrum were defined as high frequency electrogram (HFE).

Result 42 patients were successfully ablated without recurrence. In the group A, The HFE was documented in the distal bipole in 40 patients before ablation, and $35(87.5 \%)$ were eliminated after the effective ablation shot and in $5(12.5 \%)$ patients, the harmonic amplitude were reduced after an effective shot. While in the recurrence group $(\mathrm{n}=$ 17), the HFE were absent in the 10 patients before ablation shot. In the 6 patients, in whom the HFE were documented, HFE persist without diminish of the harmonic amplitude after the ablation shot. Eliminating the HFE by the RF shot was significantly different between two groups $(87.5 \%$ vs $5.9 \%, \mathrm{P}<0.001)$.

Conclusion Elimination of the HFE at the EAS was an adjunct approach and highly specific indicator.

\section{The survival analysis of relationship between ventricular arrhythmia and ischemic cardiomyopathy \\ Tianjie Feng, Keping Chen, Wei Hua, Shu Zhang Department of Cardiology, State Key Laboratory of Cardiovascular Disease, Fuwai Hospital, National Center for Cardiovascular Diseases, Chinese Academy of Medical Sciences and Peking Union Medical College}

Objective The effects of ventricular arrhythmia occurrence on ischemic cardiomyopathy patients in survival analysis of long term follow-up.

Methods The patients criteria included left ventricular eject fraction less than $35 \%$ confirmed by coronary angiogram and undergoing Holter recording for inspecting of ventricular arrhythmia occurrence. The patients were divided into ventricular arrhythmia group and no ventricular arrhythmia group. The data of demography, cardiovascular risk factors, blood biochemistry, echocardiographic measurements (in hospital), and the pharmacological treatment were collected. The following-up methods used in this study included: clinic consultation, telephone consultation and locating identity card. The items of followingup include recurrence of end-poings. The primary end-point means all cause deaths, the secondary end point include all cause death, sudden cardiac death, readmission of heart failure, ventricular tachycardia and/ or ventricular fibrillation events, heart transplantation. We compare the different characteristics between two groups and hope to identify the risk factors of primary and secondary end-point events.

Result Total 209 patients were recruited, male was dominant (86\%), mean age was $(59 \pm 8.4)$ yrs. $155(74 \%)$ patients had the history of hypertension. $53(25 \%)$ patients had the history of diabetes. $131(63 \%)$ patients had the history of anterior myocardial infraction. 25 (12\%) patients had the history of complete bundle branch. 184 (89\%) patients 
had narrow QRS width ( $<120 \mathrm{~ms}$ ) through ECG. 19 (9\%) patients were proved QRS width between $120 \mathrm{~ms}$ and $150 \mathrm{~ms}$ through ECG. $6(3 \%)$ patients were proved QRS width $>150 \mathrm{~ms}$ through ECG. 167 $(80 \%)$ patients were diagnosed the heart failure and received the regular treatment first time when they were in our hospital.During the follow-up time, 22 patients (10.5\%) reached study primary end-points, including 17 patients death from heart disease, 13 patients death from sudden cardiac death. 47 patients (23\%) reached the secondary end-points. The survival rates of 1 year, 2 year, and above 2 years were $99 \%, 96 \%$, and $89 \%$, respectively. The occurrence of secondary end-point events in ventricular arrhythmia group was higher than no ventricular arrhythmia group. The sudden death was account for most cause of death, the rate in ventricular arrhythmia group and no ventricular arrhythmia group is $60 \%$ and $58 \%$, respectively. Cox regression showed that the risk factors of secondary end-point included: anterior myocardial infarction, left ventricular eject fraction is less than $30 \%$ in echocardiography, the formation of ventricular aneurysm, the width of QRS $>150 \mathrm{~ms}$, and existing of ventricular arrhythmia.

Conclusion The sudden death was the main reason of all cause of death. The patients of ventricular arrhythmia group were more likely to reach the secondary end-point than no ventricular arrhythmia group. The risk factor of all cause death included male, complete left bundle branch block, anterior myocardial infraction, and ventricular arrhythmia occurrence. The use of $\beta$-blocker was the protective factor of all cause death. The risk factors of all cause death, readmission from heart failure, ventricular tachycardia and/or ventricular fibrillator occurrence, heart transplantation were anterior myocardial infarction, left ventricular eject fraction is less than $35 \%$ in echocardiography, the formation of ventricular aneurysm, the width of QRS $>150 \mathrm{~ms}$.

\section{Effects of different right ventricular pacing sites on QRS duration in patients receiving permanent dual chamber pacemakers \\ Xiaoqing Ren \\ Center for Arrhythmia Diagnosis and Treatment, State Key Laboratory of Cardiovascular Disease, Fuwai Hospital, National Center for Cardiovascular Diseases, Chinese Academy of Medical Sciences and \\ Peking Union Medical College}

Objective To investigate the influence of different right ventricular (RV) pacing sites on QRS width, and to provide a potential reference site for permanent right ventricular pacing in patients implanted with permanent pacemakers. Pacing at the site with the shortest QRS duration may reduce the deleterious effects of RV pacing on LV function, and therefore may have beneficial effects on patient's outcome.

Methods All consecutive patients who were planned to have permanent pacemaker implantation for a Class I or II a indication at our department from October 2010 to July 2012 were screened for participation in this prospective, single center, non-randomized study. The baseline surface ECG was analyzed for QRS width and morphology, respectively. During the implantation procedure patients were transiently paced at different RV locations [right ventricular apex, right ventricular inflow tract (RVIT), midseptum, high septum and right ventricular outflow tract (RVOT) ] before the lead was placed at its final position. During pacing at the different positions the surface 12 leads ECG was recorded. Based on the surface ECG QRS duration and morphology of the different right ventricular pacing sites were analyzed and compared with baseline and to each other.
Result A total of 216 patients [39\% female, mean age $(69 \pm 13) \mathrm{yrs}$, higher degree AV block 30.5\%] was enrolled in the study. Paced QRS duration differed significantly between all right ventricular pacing sites compared with the baseline ECG [baseline: $(106 \pm 21) \mathrm{ms}$; mean paced: $(158 \pm 16) \mathrm{ms} ; \mathrm{P}<0.001]$. RVA pacing showed the widest QRS $(168 \pm 16) \mathrm{ms}$. QRS duration with RVIT pacing was (166 \pm $15) \mathrm{ms}$, and with RVOT pacing $(165 \pm 15) \mathrm{ms}$, respectively. QRS duration was not significantly different between these 3 positions. Mid-septal pacing showed the narrowest QRS (139 \pm 19$) \mathrm{ms}$ compared to all other pacing sites $(\mathrm{P}<0.001)$. Pacing at the highseptum showed a broader QRS $(153 \pm 14) \mathrm{ms}$ than pacing at the mid-septum. Compared to other right ventricular pacing sites, QRS morphology at the mid-septum was close to normal, and electrical axis was unchanged as compared to baseline.

Conclusion Pacing at different right ventricular sites showed significant widening of the QRS compared to baseline. The shortest QRS duration was seen with mid-septal pacing. Therefore, mid-septal pacing may have less deleterious effects on LV function compared to other RV pacing locations and may be the optimal right ventricular pacing site in permanent pacemaker recipients.

\section{Low circulating big ET-1 predicts full response to 12 months of cardiac resynchronization therapy \\ Xinwei Yang, Wei Hua, Jing Wang, Zhimin Liu, Keping Chen, Shu Zhang \\ State Key Laboratory of Cardiovascular Disease, Arrhythmia Center, Fuwai Hospital, National Center for Cardiovascular Diseases, Chinese Academy of Medical Sciences and Peking Union Medical College, Beijing, 100037 China.}

Background Plasma big endothelin-1 (big ET-1) involved into the pathogenesis of adverse remodeling, associated with the severity of heart failure, and predicted poor prognosis in severe cases. We hypothesized that there might be a negative correlation between circulating big ET-1 and super-response to CRT.

Methods Data of 84 consecutive patients who underwent CRT implantation during January 2011 and December 2012 inour hospital were reviewed. Super-response was defined as absolute $\mathrm{LVEF} \geq 50 \%$ at 1 year follow-up.

Result Ten patients were excluded for device replacement, persistent atrial fibrillation, and/or renal dysfunction. Seventy-four patients [52 male, mean age $(61 \pm 12)$ years] entered into final data analysis. At one year follow-up, 15 patients $(20.3 \%)$ were identified as super-responders (SRs). SRs had less male gender (33\% vs $80 \%$, $\mathrm{P}=0.001)$, more LBBB $(87 \%$ vs $58 \%, \mathrm{P}=0.041)$, lower plasma creatinine $[(0.88 \pm 0.14) \mathrm{mg} / \mathrm{dl}$ vs $(1.04 \pm 0.25) \mathrm{mg} / \mathrm{dl}, \mathrm{P}=0.003]$ and lower big ET-1 $[(0.4 \pm 0.2) \mathrm{pmol} / \mathrm{L}$ vs $(1.0 \pm 0.9) \mathrm{pmol} / \mathrm{L}, \mathrm{P}=$ $0.034]$. Big ET-1 correlated with NYHA class $(\mathrm{r}=0.301, \mathrm{P}=0.009)$, NT-proBNP $(r=0.290, P=0.014), \operatorname{LAD}(r=0.270, P=0.020)$ and LVEDD $(r=0.329, P=0.004)$ positively, associated with superresponse $(r=-0.387, P=0.001)$ negatively. The best cutoff point for big ET-1 to predict hyper-response to CRT was $0.5 \mathrm{pmol} / \mathrm{L}$ (AUC: $0.778,95 \%$ CI: $0.657-0.899, \mathrm{P}=0.001$ ) with a sensitivity of $71 \%$ and specificity of $67 \%$. In multivariate analysis, big ET-1 $(\leq 0.5$ $\mathrm{pmol} / \mathrm{L}$ ) was an independent super-response predictor to CRT (OR $=8.7,95 \%$ CI: $1.9-38.8, \mathrm{P}=0.005)$, besides female gender $(\mathrm{OR}=$ 7.5, 95\% CI: $1.8-32.1, \mathrm{P}=0.007)$ and LBBB $(\mathrm{OR}=6.6,95 \% \mathrm{CI}$ : $1.1-40.5, \mathrm{P}=0.041)$ 
Conclusion Low circulating big ET-1 $(\leq 0.5 \mathrm{pmol} / \mathrm{L})$ could predict super-responders independently. Plasma big ET-1 is a promising humeral factor in defining disease severity and refining candidates.

\section{How bifurcation angle impact the fate of side branch after main vessel stenting: results from a large center \\ Dong Zhang, Kefei Dou, Bo Xu, Dong Yin \\ Department of Cardiology, Cardiovascular Institute and Fuwai Hospital, \\ National Center for Cardiovascular Disease, Chinese Academy of \\ Medical Sciences and Peking Union Medical College, Beijing 10037, \\ China}

Background Bifurcation angle (BA) was thought to impact the risk of side branch $(\mathrm{SB})$ occlusion in coronary bifurcation patients undergoing percutaneous coronary intervention (PCI). We aim to investigate the effect of BA on SB occlusion after main vessel (MV) stenting.

Methods 1171 consecutive patients undergoing PCI for bifurcation lesions (1200 lesions treated with a single stent technique or MV stenting first strategy) were studied. Lesions were divided into low angle and high angle groups using the median $\mathrm{BA}\left(52^{\circ}\right)$ : 600 lesions in low angle group $\left(\leq 52^{\circ}\right)$ and 600 lesions in high angle group $\left(>52^{\circ}\right)$. Clinical characteristics, coronary angiography findings, PCI procedural factors and quantitative coronary angiographic analysis data were collected. Multivariate logistic regression analysis was performed to identify independent predictors of SB occlusion. SB occlusion after MV stenting was defined as any decrease in TIMI flow grade or absence of flow in SB after MV stenting.

Result SB occlusion occurred in 88 (7.33\%) of 1200 bifurcation lesions treated with one stent technique or MV stenting first strategy. Rate of SB occlusion was significantly higher in high angle group $(67,10.1 \%)$ than low angle group $(21,3.9 \%)(\mathrm{P}<0.001)$. Rate of SB occlusion increased significantly across different quartiles of BA: from $3.63 \%$ in the first quartile of BA, to $4.71 \%$ in quartile II, to $8.14 \%$ in quartile III to $12.97 \%$ in quartile IV. Multivariable analysis showed that high angle was an independent predictor of SB occlusion $(\mathrm{OR}=1.026$, 95\% CI: 1.014-1.037). other angiographic and procedural factors were also significant and independent predictors of SB branch occlusion.

Conclusion High BA was significantly associated with SB occlusion after MV stenting. Plaque distribution at the same side of SB, MV TIMI flow grade before stenting, pre-procedural diameter stenosis of bifurcation core, diameter ratio between $\mathrm{MV} / \mathrm{SB}$ and diameter stenosis of SB before MV stenting were also independent predictors of SB occlusion.

\section{Comparative study of guiding radiofrequency catheter ablation to treat atrial flutter with three- dimensional mapping system and conventional methods \\ Yan He, Haide Liu, Zhengnan Zhang, Guoqiang Zhong Department of Geriatric Cardiology, First Affiliated Hospital of Guangxi Medical University, nanning 530021, Guangxi Zhuang Autonomous Region, China}

Objective To explore the value of guiding radiofrequency catheter ablation to treat senile atrial flutter by three-dimensional mapping system.

Methods Forty-two atrial flutter patients who agreed to undergo radiofrequency catheter ablation operation were selected by 12-lead electrocardiogram from June 2007 to August 2013.The conventional group which ablation under the conventional X-ray guidance had 18 cases. The median age $(66.5 \pm 7.8)$ yrs, including fifteen males and three females. The CARTO group which ablation by CARTO system had 14 cases. The median age was $(67.3 \pm 8.2)$ yrs, including ten males and four females. The Ensite Velocity group had 10 cases. The median age was $(68.2 \pm 9.7)$ yrs, including eight males and two females. Comparing the instant successful rate, fluoroscopic time and recurrence rate after four months of the three groups of operation. Comprehensive evaluation the good and bad of the three groups of operation to guide radiofrequency ablation of atrial flutter.

Result There were 37 cases with typical atrial flutter and 5 cases with atypical atrial flutter in 42 cases of patients with atrial flutter. After radiofrequency catheter ablation, 16 cases had instantaneous successful ablation among the conventional group. One case with unsuccessful ablation was the atrial flutter after congenital heart surgery and underwent second ablation with a successful result by CARTO system after 4 months. Another case with unsuccessful ablation was the atrial flutter dependented by tricuspid isthmus and terminated by intravenous ibutilide. The average fluoroscopic time was $(49 \pm 16) \mathrm{min}$. In CARTO group, all 14 cases had instantaneous successful ablation, and the average fluoroscopic time was (36 \pm 8 ) min. In Ensite Velocity group, all 10 cases had instantaneous successful ablation, and the average fluoroscopic time was $(33 \pm 7) \mathrm{min}$. The CARTO group and the Ensite Velocity group had no recurrence.

Conclusion Applied CARTO system or Ensite Velocity system to locate atrial flutter is more accurate and reliable than conventional method, especially apparent on the decrease of fluoroscopic time, and they are more efficient on the decrease of discharge times. They improve the success rate and they are safe and effective.

\section{The impact of pulmonary antrum radial-linear ablation vs pulmonary vein isolation on long- term outcome for paroxysmal atrial fibrillation} Xue Zhao ${ }^{1}$, Jiayou zhang ${ }^{1}$, Jianqiang $\mathrm{Hu}^{1}$, dening Liao ${ }^{1}$, Yinxiang Zhu ${ }^{1}$, Yanping Gui ${ }^{1}$, Wenliang $\mathrm{Lu}^{2}$, Yan Huang ${ }^{2}$, Xingui Guo ${ }^{2}$, Yawei Xu ${ }^{3}$, Yanzhou Zhang ${ }^{4}$, Hui Gong ${ }^{5}$, Zhenguo Liu ${ }^{6}$

1. Division of Cardiac Electrophysiology, Translational Medicine Center, Changzheng Hospital, Second Military Medical University, Shanghai, China

2. Department of Cardiology, Huadong Hospital, Fudan University, Shanghai, China

3. Department of Cardiology, Shanghai Tenth People's s Hospital,

Tongji University, Shanghai, China

4. Department of Cardiology, Renii Hospital, Shanghai Jiao Tong

University, Shanghai, China

5. Department of Cardiology, Jingshan Hospital, Fudan University, Shanghai, China

6. Davis Heart \& Lung Research Institute and Division of Cardiovascular Medicine, Ohio State University Medical Center, Columbus, Ohio, USA

Background The aim of this study was to investigate whether the pulmonary antrum radial-linear (PAR) ablation in a single ablation procedure yields higher success rates than pulmonary vein isolation (PVI) in patients with paroxysmal atrial fibrillation (PAF).

Methods The enrollment occurred between March 7, 2011, and August, 2011, with the last follow-up in May, 2014. One-hundred 
thirty four enrolled patients with documented PAF were blinded and randomized to PAR ablation group or PVI group. 24-hour Holter monitoring was conducted at months 6, 12 and 24, and 72-hour Holter at the final visit for all patients. Recurrent AF was defined as an occurrence of AF (including AFL and atrial tachycardia (AT) ) with duration of at least 30 seconds documented by an ECG or device recording system after the 3-month blanking period until the final visit.

Result At 38 (40-36) months of follow-up after single procedure, 43 of $66(65 \%)$ patients in PAR ablation group and 28 of 67 patients (42\%) in PVI group had no recurrence of AF off antiarrhythmic drug (AAD) $(\mathrm{P}=0.007)$; and 47 of 66 patients $(71 \%)$ in PAR ablation group and 32 of 67 patients (48\%) in PV isolation group had no recurrence of $\mathrm{AF}$ with $\mathrm{AAD}(\mathrm{P}=0.006)$. At 38 months, the burden of atrial fibrillation was significantly lower in PAR ablation group than in PVI group. Seven patients became long-standing atrial fibrillation in PVI group and none in PAR ablation group. No major adverse event related to the procedures occurred in both groups.

Conclusion This study suggests that PAR ablation is a simple, safe, and effective strategy for treatment of PAF.

\section{Pulmonary vein isolation with cryoballoon technique for the treatment of atrial fibrillation: early experience by a single operator in a chinese electrophysiology centre}

Gongbu Zhou, Jian Ma, Xiaogang Guo, Xu Liu, Shu Zhang State Key Laboratory of Cardiovascular Disease, Arrhythmia Center,

Fuwai Hospital, National Center for Cardiovascular Diseases, Chinese Academy of Medical Sciences and Peking Union Medical College, Beijing, 100037 China.

Objective To evaluate the recently approved cryoballon ablation approach for treating atrial fibrillation (AF) with respect to efficacy, safety and procedural properties.

Methods A total of 13 patients with drug refractory symptomatic AF underwent cryoballon ablation between December 2013 and April 2014 were included in this study. The pulmonary vein isolation (PVI) was performed with cryoballon catheter (Arctic Front ${ }^{\mathbb{B}}$, Medtronic Inc, diameter $28 \mathrm{~mm}$ ). During the cryoablation procedure, indices regarding occlusion level, number of freezing cycles and lowest cryoballon temperature were recorded. The phrenic nerve was continuously paced (1200 ms) from the superior caval vein and the motion of diaphragm was closely monitored during cryoablation of the right pulmonary veins (PV). In addition, real-time PV potential was recorded during cryoablation when available using an octapolar circumferential mapping catheter (Achieve ${ }^{\circledR}$, Medtronic Inc.). After the cryoablation procedure, the Achieve catheter was introduced to assess the electrical isolation of each PV. The conventional post-ablation follow-up protocol was applied to the patient cohort.

Results Of the 13 consecutive patients, the average age at inclusion was $(56.7 \pm 8.9)$ yrs (44-71 yrs) and the median duration of AF was 36 months (6-120 months). 2 patients (15.4\%) had persistent AF for cryoablation. The diameter of LA decided by transthoracic echocardiogram was $(38.5 \pm 4.4) \mathrm{mm}$. Overall, 51 of 53 (96.2\%) PVs were successfully isolated with $(4 \pm 0.5)$ freezing cycles for each PV. Real-time recording of PV potential were attempted in 5 patients and real-time visualization of PVI could be observed in 13/20 PVs (65\%). No cases of persistent phrenic nerve palsy, symptomatic PV stenosis, thromboembolism, cardiac tamponade, atrial-esophageal fistula and vascular access complications were observed. Average procedural time was $134.2 \pm 15.8$ minutes with fluoroscopy time of $(37.5 \pm 9.2) \mathrm{min}$. During a median follow-up period of 2.1 months (6.4-1.1 months), 10 patients $(76.9 \%)$ remained free of $\mathrm{AF}$ episodes without a blanking period.

Conclusion PVI with cryoballoon technique appeared to be effective and safe for the treatment of AF according to the early experience. Further investigation with larger patient population is warranted.

\section{Trends of LVEF in patients with long-term treatment of cardiac resynchronization therapy \\ Xinwei Yang, Wei Hua, Jing Wang, Zhimin Liu, \\ Keping Chen, Shu Zhang \\ State Key Laboratory of Cardiovascular Disease, Arrhythmia Center, \\ Fuwai Hospital, National Center for Cardiovascular Diseases, Chinese \\ Academy of Medical Sciences and Peking Union Medical College, \\ Beijing, 100037 China.}

Background The effectiveness of cardiac resynchronization therapy (CRT) has been established largely. However, little is known about the trends of LVEF after long-term treatment of CRT.

Methods Data of patients who underwent CRT implantation and survived more than 2 years in our hospital were reviewed. Those with regular follow-up at 3, 6, 12, and 24 months post-CRT were extracted. Response was defined as improvement in LVEF by $\geq 10 \%$ from baseline. Super-response was defined as absolute LVEF $\geq 50 \%$ at followups. Trends of LVEF were depicted in different response groups.

Result Overall of 62 patients [ 36 male, mean age, ( $60 \pm 10$ years) ] were involved into this study. All subjects completed 24 months follow-ups and 24 patients had visits at 36 months, another 11 patients had visits at 48 months. There were 11 non-responders (NRs), 27 responders (Rs), and 24 super-responders (SRs) identified as assessed by the maximal improvement in LVEF during follow-ups. Changes in LVEF were insignificant in average level in NRs. Large variation in LVEF was observed in responders. Most of responders presented with deterioration in LVEF after early amelioration. Only SRs had progressive improvements in LVEF during long-term follow-ups.

Conclusion Responders to CRT had inconsistent trends in LVEF during long-term monitoring. Super-responders had progressive improvements in LVEF post-CRT. 


\section{Hypertension}

\section{The relationship between resting heart rate and left ventricular hypertrophy in prehypertension \\ Yuqing Huang, Yingqing Feng, Yingling Zhou, Jiyan Chen Departmentof Cardiology, Guangdong Provincial Cardiovascular Institute, Guangdong General Hospital, Guangdong Academy of Medical Sciences}

Objective To investigate the relationship between resting heart rate (RHR) and left ventricular hypertrophy (LVH) and analysis the predictive value of LVH in prehypertension.

Methods All participants were from Guangdong General Hospital Cardiovascular internal medicine outpatient diagnosed recently and (or) did not taken medicine and all of them did not merge with other cardiovascular diseases. A total of 394 participatants were divided into three groups depending on the levels of resting heart rate: group A, RHR $<70$ bmp, group B, 70 bmp $\leq$ RHR $<80$ bmp, group C, RHR $\geq$ $80 \mathrm{bmp}$. After comparing the difference of basic information (such as age, sex, etc), the factors influence LVH were assessed by multi-factor Logistic regression model, using Characteristic curve (ROC) analysis the predictive value of RHR to LVH.

Result A total of 394 participatants in this study aged 45-60 yrs [male 145, female 249, age (52.0 \pm 4.4$)$ yrs], with $183(46.4 \%)$ subjects in group A, 136 (34.5\%) in group B, 75 (19.0\%) in group C. Among groups systolic blood pressure (SBP), diastolic blood pressure (DBP), interventricular septum (IVS), left ventricular posterior wall thickness (LVPW), left ventricular mass index (LVMI) and the incidence of LVH differed significantly (all $\mathrm{P}<0.05$ ), as well as the incidence of $\mathrm{LVH}$ among the three groups increased significantly $[2.7 \%, 16.9 \%, 48 \%, \mathrm{P}<$ 0.05]. Multiariable logistic regression analysis showed age $(\mathrm{OR}=1.983$, $\mathrm{P}=0.039)$, RHR $(\mathrm{OR}=1.254, \mathrm{P}<0.01)$ were risks factor for $\mathrm{LVH}$. The ROC curve of the relationship between RHR and LVH demonstrared that the largest area under the curve (AUC) was $0.860(\mathrm{P}<0.05)$, indicading RHR was valuable to predict $\mathrm{LVH}$.

Conclusion In prehypertension, age and RHR are risk factors for left ventricular hypertrophy, different levels of RHR have different influence on left ventricular hypertrophy and RHR may have certain predictive value of left ventricular hypertrophy.

\section{Evaluating the effect on different intervention models of rural urbanization community hypertension \\ Yuqing Huang, Yingqing Feng, Jiyan Chen, Yingling Zhou Departmentof Cardiology, Guangdong Provincial Cardiovascular Institute, Guangdong General Hospital, Guangdong Academy of Medical Sciences}

Objective As we all known, different intervention models have different impacts on hypertension. So our term plan to evaluate the effect on taking different intervention models of management for rural urbanization community hypertensions and try to explor a basis intervention model that is suitable for management for the area of rural urbanization community.

Methods All participants were from Liao Bu, Dongguan rural urbanization community and diagnosed by hypertension in 2010 , all patient divided into two groups: general management group and strengthen management group; Oral health education, including the definition, harmness, risk factors for hypertension and the importance of non-drug therapy of hypertension; following up patients regualr and taking management with chronic disease cards; each quarter in administrative zones taking hypertension knowledge lectures; each quarter follow-up blood pressure at a time. Strengthen management group: based on general management group mode, but add the following contents: more following-up, more oral health eduction and told all participants hypertension need treatment for lifelong and nondrug treatment measures detailed; according to Chinese hypertension prevention and treatment guide (2009 edition), the management of all participants based on the risk stratification and divided into low, moderate, high risk of three layers. All patients followed-up 2 years, and comparing with between two groups: including the effect of treatment, awareness rate, control rate, treatment rate.

Result Strengthen group blood pressure decreased $(6.41 \pm 20.24)$ / (3.27 \pm 12.28$) \mathrm{mm} \mathrm{Hg}$ and general group decrease $(1.61 \pm 23.27) /(0.74$ $\pm 14.21) \mathrm{mm} \mathrm{Hg}$, there was significant difference between before and after the intensive group intervention $(\mathrm{P}<0.001)$. The awareness rate, control rate and treatment rate increased from $25.78 \%, 26.88 \%, 34.83 \%$ to $45.80 \%, 38.7 \%, 42.61 \%$ between two groups, respectively (all $\mathrm{P}<0.001$ ).

Conclusion The awareness rate, control rate and treatment rate of hypertension can be improved significantly through strengthening management and eduction model in rural urbanization community.

\section{Effects of aldosterone antagonists on blood pressure in resistant hypertension: a meta- analysis \\ Gang Liu, Xinxin Zheng, Jie Lu, Xiaohong Huang Department of Special Medical Treatment Center, State Key Laboratory of Cardiovascular Disease, Fuwai Hospital, National Center for Cardiovascular Diseases, Chinese Academy of Medical Sciences and Peking Union Medical College}

Objective There is currently limited data on which drug should be used to improve blood pressure (BP) control in patients with resistant hypertension (RH). We performed a systematic review and metaanalysis of published studies evaluating the anti-hypertensive benefit of aldosterone antagonists (AA) as an add-on therapy in patients with RH.

Methods A systematic literature search for eligible studies was conducted until March 2014, using literature databases and hand search. Studies were stratified according to controlled vs. uncontrolled design and analyzed using random-effects meta-analysis models.

Result We identified 13 eligible studies involving a total of 2640 patients, consisting of 3 randomized controlled trials, and 10 observational studies without a control group. In controlled studies, there was a reduction in mean systolic and diastolic BP of $-16.5 \mathrm{~mm} \mathrm{Hg}(95 \%$ CI: $-30.0--3.0)$ and $-4.1 \mathrm{~mm} \mathrm{Hg}(95 \% \mathrm{CI}:-7.8--0.32)$, respectively, compared to control. In uncontrolled studies, there was a reduction in mean systolic and diastolic BP of -19.7 (95\% CI: $-23.2--16.2)$ and -9.1 (95\% CI: $-10.3--7.8) \mathrm{mm} \mathrm{Hg}$, respectively, compared to pre-AA therapy. Subgroup analysis showed that the systolic BP change was more pronounced in patients with baseline systolic $\mathrm{BP}>150 \mathrm{~mm} \mathrm{Hg}$ (WMD: $-23.1 \mathrm{~mm} \mathrm{Hg}$ ) than patents with $\leq 150 \mathrm{~mm} \mathrm{Hg}$ (WMD: -15.4 
$\mathrm{mm} \mathrm{Hg}$ ) (between groups $\mathrm{P}<0.05$ ), suggesting that the baseline systolic $\mathrm{BP}$ was a predictor of the BP response to AA treatment. Furthermore, AA demonstrated a mild increase in serum potassium and creatinine (both $\mathrm{P}$ $<0.0001)$.

Conclusion AA therapy was effective for lowering systolic and diastolic BP in patients with $\mathrm{RH}$.

\section{Association of serum sulfatide with target- organ damage in arterial hypertension \\ Jian Gu, Gang Li \\ Hebei General Hospital}

Objective Hypertension is a very common disorder with a substantial impact on public health because of its target-organ damage (TOD). Studies have shown that serum sulfatide play a possible role in the progress of hypertension. The study is going to investigate the association between serum sulfatide and TOD in patients with arterial hypertension.

Method Serum sulfatide was analyzed in 77 untreated patients with mild to moderate essential hypertension and in 52 healthy controls. Staging of TOD obtained according to World Health Organization guidelines by clinical evaluation, and laboratory tests including measurements of creatinine clearance, proteinuria, ophthalmoscopy, electrocardiography, echocardiography, and ultrasound examination of major arteries; levels of lipids, fibrinogen.

Result Blood pressure, duration of hypertension, and levels of total cholesterol, low-density lipoprotein cholesterol, serum sulfatide, and fibrinogen were significantly related to the presence and severity of TOD in univariate analysis. Stepwise multivariate analysis showed serum sulfatide levels $(\mathrm{P}<0.001)$ to be the best discriminator of the presence of TOD, followed by systolic blood pressure $(\mathrm{P}<0.001)$, duration of hypertension $(\mathrm{P}<0.05)$, and low-density lipoprotein cholesterol $(\mathrm{P}<$ $0.05)$. The serum sulfatide levels were related to TOD independent of the level of blood pressure.

Conclusion Serum sulfatide is sensitive indicators of the severity of TOD in patients with arterial hypertension, and its evaluation might permit identification of hypertensive subjects liable to the development of organ damage.

\section{The predictive value between the blood uric acid level and the carotid atherosclerosis of middle-aged patients with essential hypertension \\ Yuqing Huang, Xuebiao Wei, Jiaxin Zhan, Lei Jiang, Jiyan Chen, Yingling Zhou, Yingqing Feng \\ Department of Cardiology, Guangdong Provincial Cardiovascular \\ Institute, Guangdong General Hospital, Guangdong Academy of Medical Sciences}

Objective To investigate the relationship between the blood uric acid (UA) level and the carotid atherosclerosis (ACS) of middle-aged patients with essential hypertension and analyze the relationship between UA predictive value of ACS.

Methods From Fuwai Hospital led industry special funds for scientific research projects: risk factors for cardiovascular disease monitoring and standardization management has completed the household registration management of hypertension patients were randomly selected in the library completed echocardiography and carotid ultrasound examination, 339 cases of patients with Middle-aged essential hypertension $(\mathrm{EH})$ based on whether carotid intimal thickening and whether there are divided into carotid atherosclerotic plaque formation and normal carotid artery in the two groups, the analysis of blood uric acid levels in all groups and the relationship between carotid artery atherosclerosis; At the same time, according to the blood uric acid levels were divided into normal uric acid and hyperuricemia in the two groups, the analysis of the incidence of carotid atherosclerosis in each group, and establish the ROC curve, analyzing their predictive value of carotid artery atherosclerosis occurs.

Result 339 patients included in this study, among which 155 were male, 184 were female, mean age $(62.0 \pm 10.4)$ yrs, with the increase of UA, also increase the incidence of ACS, UA normal incidence of ACS group was $20.3 \%$, the HUA group was $66.7 \%$, the difference statistically significantly $(\mathrm{P}<0.05)$; For UA test variables, the ACS group as state variables, state variables is 1 , the ROC curve, in which male area under curve $(\mathrm{AUC})=0.798(\mathrm{P}<0.05)$, has good predictive value, 95\% CI: $0.723-0.723,70.80 \%$, sensitivity $89.90 \%$, was statistically significantly; Female area under curve $($ AUC $)=0.830(\mathrm{P}<0.05)$, also has good predictive value, $95 \% \mathrm{Cl}: 0.769-0.769,75.60 \%, 78.90 \%$ sensitivity, was statistically significantly $(\mathrm{P}<0.05)$.

Conclusion The middle-aged patients with essential hypertension (EH) the blood uric acid levels and carotid atherosclerosis in related, and female blood uric acid level have more higher value for the forecast of carotid artery atherosclerosis occurs than male.

\section{The relationship between the 24-hour ambulatory pulse pressure and left ventricular mass index in elderly patients with essential hypertension \\ Hongjian Li, Suxia Han \\ Department of Cardiology, the Fifth Affiliated Hospital of Xinjiang Medical University}

Objective To investigate the relationship of 24-hour ambulatory pulse pressure $(24 \mathrm{hPP})$ with left ventricular mass index (LVMI) in the elderly essential hypertension patients.

Methods The data of 110 elderly patients with essential hypertension from January 2012 to December 2012 were collected in the study. All patients received 24-hour ambulatory blood pressure monitoring and echoeardiographic examination. $24 \mathrm{hPP}$ and LVMI were calculated according to the results of 24-hour ambulatory blood pressure monitoring and echocardiographic measurements. The patients were divided into group A ( $24 \mathrm{hPP} \geq 60 \mathrm{~mm} \mathrm{Hg}, \mathrm{n}=70$ ) and group B ( $24 \mathrm{hPP}$ $<60 \mathrm{~mm} \mathrm{Hg}, \mathrm{n}=40$ ).

Result The 24-hour systolic blood pressure and $24 \mathrm{hPP}$ with patients in group $\mathrm{B}$ were significantly higher than those in group A (P $<0.001)$. Compared with group A patients, the interventricular septam thickness, left ventricular posterior wall thickness, left vetricular mass and left vetricular mass index were significantly higher in group $\mathrm{B}(\mathrm{P}<$ $0.05)$. Pearson correlation analysis showed that $24 \mathrm{hPP}$ had a positive correlation with LVMI in the elderly essential hypertension patients $(\mathrm{r}=$ $0.33, \mathrm{P}<0.001)$. Multiple stepwise regression analysis showed that 24 hPP was the main factors that increased LVMI in the elderly essential hypertension patients $(\beta=0.34, \mathrm{P}<0.001)$.

Conclusion The $24 \mathrm{hPP}$ is positive correlate with LVMI in the elderly essential hypertension patients. High $24 \mathrm{hPP}$ is an important risk factor for left ventricular structural damage in the elderly essential hypertension patients. 


\section{Genetic variations of STEAP2 Gene polymorphism and related inflammatory factors in obstructive sleep apnea syndrome \\ Ting Yin, Nanfang Li, Ruimei Han, Zhitao Yan, Wanyong Ma, Xiaoguang Yao, Yingchun Wang, Tao Li, Le Sun, Feiya Zu, Jiangqiong Kong, Xiangyang Zhang Hypertension Center of The Peoples Hospital Of Xinjiang Uygur \\ Autonomous Region, Hypertension Institute of Xinjiang}

Background and Objective Increasingly studies reported an independent association of obstructive sleep apnea syndrome (OSAS) with different components of metabolic syndrome (Mets), as well as considered a "manifestation of MetS" and an increasing risk of cardiovascular disease. Six-transmembrane protein of prostate 2 (STEAP 2) was an important factor that links inflamation and Mets, IR and obesity, represents a strong biological and positional candidate of susceptible factor for OSAS. The association between human STEAP 2 gene and OSAS is unclear. The aim of this study was designed to investigate whether STEAP 2 gene conferred susceptibility and related inflammatory factors to OSAS independently.

Methods The representative variations of STEAP 2 were selected based on the function (missense mutation) and linkage disequilibrxium $\left(\mathrm{r}^{2}>0.8\right)$. Taq-Man polymerase chain reaction method was used genotyping. Blood samples were collected from all subjects and genotyping was performed on DNA extracted from blood cells.

Result Four common SNPs of STEAP 2 gene (rs8122, rs1981529, rs34741656, and rs7810472) were detected in patients with OSAS ( $\mathrm{n}=$ $614)$ and controls $(n=399)$. SNPrs8122 and rs1981529 satisfied HEW $(\mathrm{P}>0.05)$. rs8122 was significantly associated with OSAS phenotype (additive $\mathrm{P}=0.022$, dominant $\mathrm{P}=0.010 ; \mathrm{OR}=1.366,95 \% \mathrm{CI}$ : 1.034 $1.805)$ after adjusting for male $(\mathrm{OR}=1.999,95 \% \mathrm{CI}: 1.478-2.688)$, $\mathrm{BMI} \geq 28 \mathrm{~kg} / \mathrm{cm}^{2}(\mathrm{OR}=3.686,95 \%$ CI: $2.725-4.985$; additive $\mathrm{P}=0.015$ and dominant $\mathrm{P}=0.037$, respectively). Level of AHI in carriers of rs8122 AA genetype is lower than in GG genetype, as well as ratios of severe OSAS.

Conclusion Our study demonstrated an association of common variation rs8122 in STEAP 2 gene with OSAS independently in Chinese general population.

\section{rs3740835 variant in KCNJ5 gene related to the ct scan-based adrenal hyperplasia in patients with primary aldsteronism \\ Xiaoguang Yao, Nanfang Li, Delian Zhang, Guijuan Chang, Keming Zhou, Qin Luo, Junli Hu \\ Hypertension Center of The Peoples Hospital Of Xinjiang Uygur \\ Autonomous Region, Hypertension Institute of Xinjiang}

Objective As a novel candidate gene contributing to primary aldosteronism (PA), KCNJ5 gene is believed to play roles in adrenal hyperplasia. The present study aims to explore the association of variants in KCNJ 5 gene with CT scan-based adrenal hyperplasia in patients with PA from Xinjiang.

Methods Followed the guideline of PA in 2008, a protocol for PA screening and diagnosis was performed in Center of hypertension in Xinjiang from 2008 to 2010. Finally, 457 cases of subjects with confirmed PA were enrolled and were divided into adrenal hyperplasia group and control group according to the findings of thin-section CT scan. The blood samples were collected for DNA extraction and genotype identification.

Result The average level of plasma potassium in patients with adrenal hyperplasia was $(3.63 \pm 0.42) \mathrm{mmol} / \mathrm{L}$, which was significantly lower than that in controls $(3.76 \pm 0.37) \mathrm{mmol} / \mathrm{L}$ with $\mathrm{P}=0.001$. But the baseline plasma aldostrone level $(22.78 \pm 26.52) \mathrm{ng} / \mathrm{dl}$, urine potassium excretion $(46.02 \pm 22.47) \mathrm{mmol} / \mathrm{L}$ in the adrenal hyperplasia group was higher than those in controls, which was $(18.13 \pm 7.60) \mathrm{ng} / \mathrm{dl}(\mathrm{P}=0.032)$ and $(42.37 \pm 15.66) \mathrm{mmol} / \mathrm{L}(\mathrm{P}=0.080)$. The age, mean blood pressure, body mass index, plasma sodium, urine sodium excretion as well as plasma rennin activity were matched. $41.3 \%$ subjects were diagnosed as obstructive sleep apnea syndrome, $75.7 \%$ patients had hypertension history and $33.0 \%$ patients had hypokalemia history. Further, more patients with adrenal hyperplasia had hypokalemia history than that without adrenal hyperplasia $(39.2 \%$ vs $20.7 \%, \mathrm{P}<0.001)$. The frequency of CC genotype of rs3740835 in KCNJ 5 gene was higher in control group rather than that in adrenal hyperplasia group $(67.7 \%$ vs $58.2 \%, \mathrm{P}$ $=0.047$ ), though the significance only reached the boardline. Logistic regression model was constructed to explore the potential factors that related to CT scan-based adrenal hyperplasia in whole study population. The results showed that the rs3740835 in KCNJ5 gene [0.642 (0.424 $0.971), \mathrm{P}=0.036]$ as well as plasma potassium[0.432 (0.261-0.715), $\mathrm{P}$ $=0.01]$ played protective roles in adrenal hyperplasia, adjusted for body mass index, gender, age.

Conclusion The rs3740835 variants in KCNJ5 gene as well as plasma potassium levels were contributing factors to adrenal hyperplasia in patients with PA from Xinjiang.

\section{Investigation of the influence of antidepression therapy on blood pressure and quality of life in elderly patients both with hypertension and depression \\ Lina Ma, Yun Li \\ Xuanwu Hospital of Capital Medical University}

Objective Essential hypertension (EH) and depression are common chronic diseases in the elderly in China, but the effect of antidepression medication is obscure. So our study is to explore the influence of antidepression therapy on blood pressure and quality of life in elderly patients both with hypertension and depression.

Methods A total of 67 elderly patients with hypertension and depression in the department of internal medicine of Xuanwu hospital during August 2008 and March 2011 were randomly divided into two groups based on the antihypertensive therapy: control group (amlodipine $5 \mathrm{mg} / \mathrm{d}$ ) and therapy group (amlodipine $5 \mathrm{mg} / \mathrm{d}$ and cipramil $20 \mathrm{mg} / \mathrm{d}$ ) for three months. The pretherapy and post-treatment changes in the two groups were compared between $24 \mathrm{hSBP}, 24 \mathrm{hDBP}$, dSBP, dDBP, nSBP, $\mathrm{nDBP}$ and scores of the short form health survey.

Result After treatment, $24 \mathrm{hSBP}[(128.74 \pm 3.79) \mathrm{mm} \mathrm{Hg}$ vs (139.42 $\pm 10.19) \mathrm{mm} \mathrm{Hg})], 24 \mathrm{hDBP}[(72.10 \pm 7.65) \mathrm{mm} \mathrm{Hg}$ vs $(78.64 \pm 8.03)$ $\mathrm{mm} \mathrm{Hg}$ ], dSBP $[(130.81 \pm 4.50) \mathrm{mm} \mathrm{Hg}$ vs $(143.39 \pm 12.32) \mathrm{mm} \mathrm{Hg})$, $\mathrm{dDBP}[(76.03 \pm 9.46) \mathrm{mm} \mathrm{Hg}$ vs $(81.53 \pm 9.20) \mathrm{mm} \mathrm{Hg}], \mathrm{nSBP}[(126.94$ $\pm 3.69) \mathrm{mm} \mathrm{Hg}$ vs $(136.53 \pm 9.03) \mathrm{mm} \mathrm{Hg}], \mathrm{nDBP}[(68.52 \pm 6.89) \mathrm{mm}$ $\mathrm{Hg}$ vs $(75.44 \pm 7.57) \mathrm{mm} \mathrm{Hg}$ ] were much lower in therapy group than the control group $(\mathrm{P}<0.01)$. Besides physical functioning of quality of life, the other dimensions were much higher ( role physical $70.84 \pm$ 9.90 vs $62.31 \pm 10.16$, bodily pain $74.71 \pm 8.51$ vs $63.33 \pm 8.48$, general health $62.19 \pm 8.39$ vs $50.64 \pm 7.07$, vitality $64.03 \pm 8.26$ vs $56.11 \pm 6.82$, social functioning $70.94 \pm 6.94$ vs $62.92 \pm 7.46$, role-emotional $70.32 \pm$ 6.93 vs $62.14 \pm 8.18$, mental health $69.94 \pm 6.44$ vs $61.69 \pm 8.83$, total 
score $68.70 \pm 3.29$ vs $60.40 \pm 3.40$, all $\mathrm{P}<0.01$ ).

Conclusion Besides antihypertensive therapy, antidepression therapy also should be put more attention in the elderly hypertensive people with depression.

\section{Prevalence and cardio-metabolic characteristics of primary aldosteronism \\ Nanfang Li, Xiaoguang Yao, Abulikem Suofeiya, Delian Zhang, Guijuan Chang, Jianqiong Kong, \\ Maimaiti Nuerguli, Keming Zhou, Qin Luo, Menghui Wang, Junli Hu, Lei Wang, Guoliang Wang, Wen Jiang, Yu Zhang Hypertension Center of The People's Hospital Of Xinjiang Uygur Autonomous Region, Hypertension Institute of Xinjiang}

Objective To investigated the prevalence of primary aldosteronism (PA) and cardio-metabolic pattern of PA in general hypertensive patients in Xinjiang of China.

Methods Consecutive hypertensive patients referred to Hypertension Center of Xinjiang from January 2009 to January 2011 underwent a diagnostic protocol composed of measurement of $\mathrm{K}^{+}$and $\mathrm{Na}^{+}$in serum and 24-h urine, sitting plasma renin activity, and aldosterone at baseline and after saline loading test. The polysomnography, glucose, lipid profile, smoking and alcoholism and the clinical profiles were compared between PA and non-PA group. The related factors of PA were discussed by multivariate logistic regression.

Results 474 (28.03\%) out of 1677 hypertensive patients were diagnosed to have PA. The mean hypertension duration of PA was longer than that in non-PA groups (5.54 yrs vs $4.24 \mathrm{yrs}, \mathrm{P}<0.001)$. The detection rate of PA was around $30 \%$ in OSAS, obese, age $<45$ yrs and hyperglycemia population, respectively. The patients of PA showed worse blood control, lower serum $\mathrm{K}^{+}(3.72 \pm 0.38) \mathrm{mmol} / \mathrm{L}$, higher urine $\mathrm{K}^{+}$excretion $(42.68 \pm 16.62) \mathrm{mmol} / \mathrm{L}$ compared to non-PA. More patients with PA were reported to have the family history of hypertension than non-PA patients (66.6\% vs 75.1\%, P=0.001). 197 (43.2\%) cases in PA group and 416 (35.6\%) cases in non-PA group were evaluated as obese, but the fasting glucose, TC and LDL-C showed higher levels in non-PA patients. $43.2 \%$ PA patients and $35.6 \%$ hypertensive patients were diagnosed of OSAS by an AHI $>5$ during sleep. Multivariate logistic regression analysis showed that abnormal presence of adrenal CT, hypokalemia history, family history of hypertension and severity of OSAS would increase the risk of PA.

Conclusion A markedly prevalence of PA (28.03\%) was detected in Chinese general hypertensive population, the greater detection rate of PA in males and severe OSAS leading to a concept that PA identification should extend to such individuals.

\section{Hypertension with obesity patients serum adiponectin level and left ventricular hypertrophy and the relationship between the atherosclerosis}

Huan Lin, Xiuying Li, Jiyan Leng The First Hospital of Jilin University

Objective Observation of hypertension with obesity patients serum adiponectin (APN) level changes. Discuss it with left ventricular hypertrophy (LVH), the relationship between atherosclerosis (AS).

Methods Selection of 50 patients with essential hypertension were divided into pure hypertension group (23 cases) and hypertension obesity group ( 27 cases), an alternate 45 cases of normal blood pressure is divided into control group (24 cases) and the simple obesity group (21 cases); Enzyme-linked immunosorbent (ELISA) determination of serum APN level; Using color doppler ultrasound instrument measuring film thickness in the common carotid arteries - (CCA IMT) and carotid artery diameter (CADIA); Calculate the left ventricular mass index (LVMI).

Result Control group, simple obesity group, the pure hypertension group and hypertension group of fasting plasma glucose (FPG), three acyl glycerin (TG), low density lipoprotein cholesterol (LDL-C) and high density lipoprotein cholesterol (HDL-C) differences statistically significant $(\mathrm{F}=81.97,8.56,25.53,8.56, \mathrm{P}<0.05)$; Comparing the two results show that the FPG hypertension obesity group and control group, the difference of simple obesity group is statistically significant; Simple obesity, pure hypertension group, TG, LDL-C hypertension obesity group were higher than the control group, hdl-c leel is reduced, and high blood pressure, obesity group of TG, TC, LDL-C, increased in the pure hypertension group (all $\mathrm{P}<0.05$ ); Control group, simple obesity group, pure in high blood pressure, high blood pressure, obesity group, in turn, reduce the serum APN level, CADIA has shrunk, LVMI increase, CCA IMT increased $(\mathrm{F}=28.34,10.26,36.52,1,4.73, \mathrm{P}<0.01)$; the two comparative differences are statistically significant (all $\mathrm{P}<0.05$ ). Correlation analysis showed that APN and LVMI, CCA IMT was significantly negative correlation $(\mathrm{r}=0.870,0.710)$, are positively correlated $(\mathrm{P}<0.01)$, and the CADIA $(\mathrm{r}=0.742, \mathrm{P}<0.742)$; Stepwise regression analysis showed that $\mathrm{APN}$, systolic blood pressure and body mass index (BMI) is the main factor affecting the left ventricular hypertrophy (to decide the value $0.909,0.126$ and 0.153 , respectively).

Conclusion High blood pressure and obesity exist at the same time, both may play a synergy, APN levels of reduction, increase in BMI could accelerate hypertension with LVH and AS the occurrence and development of obesity patients.

\section{Effects of peridopril on vascular inflammation and endothelium function \\ Zhangqiang Chen \\ Jiangxi Province People' s Hospital}

Objective To explore the effects of peridopril on vascular inflammation and vascular endothelium function in mild to moderate essential hypertensive $(\mathrm{EH})$ patients.

Methods $140 \mathrm{EH}$ patients were divided into peridopril group (70 cases) and conventional treatment group (70 cases). 70 cases of healthy volunteers were selected as a control group. We examined the blood express levels of high sensitivity C-reactive protein (hs-CRP), IL-6, ICAM-1, Fibrinogen C (FIB-C) and endothelium 1 (ET-1), nitric oxide (NO) levels and flow mediated dilatation (FMD) in brachial artery blood samples as well as angiotension before beginning treatment in each group, The peridopril group received a $4 \mathrm{mg}$ tablet daily for 4 weeks. in addition to similar drug therapies as those in the conventional treatment group.

Result All EH patients blood levels of hs-CRP, IL-6, ICAM-1, FIB-C and ET-1 increased significantly (all $\mathrm{P}<0.01$ ), while NO and FMD decreased significantly compared with control group (all $\mathrm{P}<$ 0.01 ). In the peridopril group after 4 weeks treatment, the serum levels of hs-CRP, IL-6, ICAM-1, FIB-C and ET-1 as well as angiotension II 
decreased significantly (all $\mathrm{P}<0.01$ ), the level of $\mathrm{NO}$ and FMD increased significantly $(\mathrm{P}<0.01, \mathrm{P}<0.05)$ compared with pre-treatment, The levels of angiotension II decreased significantly $(\mathrm{P}<0.05)$, compared with pre-treatment in the conventional treatment group, while those other index above appeared to have no significant change compared with pretreatment in the conventional treatment group.

Conclusion Peridopril tablet can inhibit vascular inflammation and protect the vascular endothelium function of $\mathrm{EH}$ patients. It suggests this plays an important role in the prevention of thrombotic diseases induced by hypertension.

\section{Comparison of ABPM evaluation and synergy on treatment of combining tianma gouteng decoction and I-amlodipine besylate versus I-amlodipine besylate in patients with essential hypertension of type of yang-hyperactivity due to yin-deficiency \\ Yong Liu, Lunfei Du \\ Department of Cardiavascular Medicine, Zigong City Third People's Hospital}

Objective To investigate the treatment effect and safety of combined tianma gouteng decoction and l-amlodipine besylate in patients with essential hypertension (EH) of type of Yang-hyperactivity due to Yin-deficiency; To evaluate the advantages and limitationso combining TCM and Western medicine in treatment of hypertension.

Methods 100 out-hospitalized patients with essential hypertension of type of yang-hyperactivity due to yin-deficiency were enrolled from May 2013 to April 2014. The patients were randomly divided into two groups: control group $(n=50)$ were treated with l-amlodipine besylate $2.5 \mathrm{mg}$ qd; treatment group $(\mathrm{n}=50)$ were treated with the same dose of 1-amlodipine besylate and plused Tianma gouteng decoction $150 \mathrm{ml}$ tid. The levels of symptoms, CBP, $24 \mathrm{hSBP}, 24 \mathrm{hDBP}, \mathrm{dSBP}$, dDBP, nSBP, nDBP, BPL, BPV, TPR and HR were assessed at baseline and after treatment for 4 weeks by follow-up, CBPM and ABPM. The side effects from therapies were recorded.

Result After 4 weeks treatment, the clinical symptoms of headache, dizziness, palpitation, insomnia, etc in the two groups were relieved, but the treatment group was earlier and more significant $(\mathrm{P}<0.05)$. The casual blood pressure levels of SBP and DBP were significantly reduced compared to baseline (all $\mathrm{P}<0.01$ ) in two groups respectively, and the reduction amplitude was more significant in trea-tment group than in control group $[(148.33 \pm 14.56) /(90.73 \pm 10.24) \mathrm{mm} \mathrm{Hg}$ vs $(139.63 \pm 11.82) /(84.16 \pm 8.79) \mathrm{mm} \mathrm{Hg}, \mathrm{P}<0.05]$. The values of 24 hSBP, 24 hDBP, dSBP, dDBP, nSBP, nSBP, dSBPL, dDBPL, nSBPL and $\mathrm{nDBPL}$ were declined obviously compared to baseline (all $\mathrm{P}<$ 0.01 ) in every group. $24 \mathrm{hSBP}, \mathrm{dSBP}, \mathrm{nSBP}, \mathrm{dSBPL}$ and nSBPL were declined significantly compared to those in treatment group than in control group after treatment $[(136.80 \pm 13.60) \mathrm{mm} \mathrm{Hg}$ vs $(130.02 \pm$ $10.69) \mathrm{mm} \mathrm{Hg}$, (138.96 \pm 14.40$) \mathrm{mm} \mathrm{Hg}$ vs $(131.84 \pm 10.65) \mathrm{mm} \mathrm{Hg}$, (130.84 \pm 13.24$) \mathrm{mm} \mathrm{Hg}$ vs (123.64 \pm 14.53$) \mathrm{mm} \mathrm{Hg},(47.68 \pm 33.34) \%$ vs $(28.91 \pm 24.48) \%,(62.43 \pm 32.99) \%$ vs $(44.38 \pm 31.46) \%$, all $\mathrm{P}<$ 0.05 ]. However, $24 \mathrm{hDBP}, \mathrm{dDBP}, \mathrm{nDBP}, \mathrm{dDBPL}$ and $\mathrm{nDBPL}$ were no significant difference between in treatment group and in control group after treat-ment $[(82.93 \pm 10.70) \mathrm{mm} \mathrm{Hg}$ vs $(83.47 \pm 9.05) \mathrm{mm} \mathrm{Hg},(84.44$ $\pm 11.26) \mathrm{mm} \mathrm{Hg}$ vs $(85.16 \pm 9.40) \mathrm{mm} \mathrm{Hg},(78.29 \pm 110.73) \mathrm{mm} \mathrm{Hg}$ vs $(77.44 \pm 11.60) \mathrm{mm} \mathrm{Hg},(39.41 \pm 32.87) \%$ vs $(36.49 \pm 29.03) \%$, $(45.69 \pm 33.45) \%$ vs $(47.47 \pm 29.41) \%$, all $\mathrm{P}>0.05]$. The dSSD and dDSD were significantly reduced compared to baseline (all $\mathrm{P}<0.01$ ), but nSSD and nDSD were not reduced compared to baseline $(\mathrm{P}>0.05)$ in every group. Furthermore, dSSD, dDSD, nSSD and nDSD were not significant difference between in treatment group and in control group after treatment $[(13.53 \pm 3.84) \mathrm{mm} \mathrm{Hg}$ vs $(12.81 \pm 3.27) \mathrm{mm} \mathrm{Hg}$, (9.50 \pm 2.47) $\mathrm{mm} \mathrm{Hg}$ vs $(9.03 \pm 2.21) \mathrm{mm} \mathrm{Hg},(12.17 \pm 3.59) \mathrm{mm} \mathrm{Hg}$ vs $(12.37$ $\pm 4.30) \mathrm{mm} \mathrm{Hg},(9.34 \pm 3.02)$ vs $(9.42 \pm 2.25) \mathrm{mm} \mathrm{Hg}$, all $\mathrm{P}>0.05)$. The $\mathrm{T} / \mathrm{p}$ ratio was no significant difference between two groups $(\mathrm{P}>$ $0.05)[(0.71 \pm 0.08) /(0.65 \pm 0.09)$ vs $(0.71 \pm 0.06) /(0.68 \pm 0.07), \mathrm{P}>$ $0.05]$. No serious side effects were observed during the treatment in two groups.

Conclusion The clinical symptoms of essential hypertension of type of Yang-hyperactivity due to Yin-deficiency can be relieved obviously by Tianma gouteng decoction or 1-amlodipine besylate. Tianma gouteng decoction or l-amlodipine besylate is useful on CBP. Tianma gouteng decoction can reduce $24 \mathrm{hSBP}, \mathrm{dSBP}$ and $\mathrm{nSBP}$ and can not reduce $24 \mathrm{hDBP}, \mathrm{dDBP}$ and nDBP. In addition, Tianma gouteng decoction do not to influence the BPV and T/P ratio. Contrast CBP and ABPM, this study showed that the anti-hypertensive effect of Tianma gouteng on DBP was over-evaluated by CBP. Exception for anti-hypertensive effect, Tianma gouteng decoction was safe and could not increase heart rate. There are mult-aspect advantages of symptomatic improvement, SBP droping lesser side effective, etc. that in the patients by the therapy of combining Tianma gouteng decoction with L-amlodipinebesylate, but the long term adherence is poor.

\section{Relationship between SOCS-3 gene polymorphism and abnormal glucose metabolism in the Xinjiang uygur population Keming Zhou, Nanfang Li, Juhong Zhang, Ling Zhou, Xiaoguang Yao, Na Lin, Jing Hong Hypertension Center of The Peoples Hospital Of Xinjiang Uygur \\ Autonomous Region, Hypertension Institute of Xinjiang}

Objective To investigate the relationship between SOCS-3 gene polymorphism and abnormal glucose metabolism in Xinjiang Uygur population.

Methods According to different plasma glucose levels, 1232 individual in Xinjiang hetian area were divided into 3 groups that is 451 patients with pre-diabetes (PD group), 252 patients with type 2 diabetes mellitus (T2DM group) and 529 healthy people as normal controls (NC group). Based on HapMap we selected the polymorphismrs rs12953258, rs4969168, rs9914220 as haplotypes, tagging SNP (htSNP) sufficiently covering the genetic variation of the whole gene. We therefore examined the association between rs12953258, rs4949168 and rs914220 within SOCS-3 and abnormal glucose metabolism in the Xinjiang Uygur population in three independent study populations; The genotype and allele frequencies and relative clinic data were compared among groups.

Result The T2DM study with 451 individuals showed the thomozygosity for the $\mathrm{C}$ allele of rs 12953258 polymorphism of SOCS3 was associated with increased diabetes risk $(\mathrm{OR}=1.756,95 \% \mathrm{CI}$ : 1.168-2.640). In addition, association between rs4949168, rs914220 and abnormal glucose metabolism in the Xinjiang Uygurpopulation was not found. Age, total cholesterol (CHOL) and BMI were risk factors of DM development, total cholesterol (CHOL) and low high-density lipoprotein (HDL-C) was risk factors of PD development in Uygur people.

Conclusion The C allele of rs12953258 polymorphism of SOCS-3 gene may be an independent risk factor for abnormal glucose metabolism in Xinjiang Uygur population. 


\section{Pulmonary Vascular Disease}

\author{
Oral targeted therapies in the treatment of \\ pulmonary arterial hypertension: a meta- \\ analysis of clinical trials \\ Yaguo Zheng, Changming Xiong \\ State Key Laboratory of Cardiovascular Disease, Fuwai Hospital, \\ National Center for Cardiovascular Diseases, Chinese Academy of \\ Medical Sciences and Peking Union Medical College
}

Background Oral targeted therapies have been widely used in the treatment of pulmonary arterial hypertension (PAH). Many new oral agents emerge for PAH in recent years. In this study, we performed a meta-analysis to evaluate the efficacy and safety of oral targeted therapies in $\mathrm{PAH}$, focusing on overall survival improvement.

Methods Trials were searched in the Cochrane Library, EMBASE, and PUBMED databases through September 2013. We calculated risk ratios for dichotomous data and weighted mean differences with $95 \%$ confidence intervals for continuous data.

Result 18 trials with a total of 4363 subjects were indentified in the meta-analysis. Analysis by drug class revealed that phosphodiesterase type 5 inhibitors (PDE-5Is) were associated with a statically significant reduction in mortality $(\mathrm{RR}=0.22,95 \% \mathrm{CI}$ : $0.07-0.71, \mathrm{P}=0.011)$, while other drugs only showed a trend toward reducing mortality. Compared with placebo, endothelin receptor antagonists (ERAs), PDE-5Is and riociguatsignificantly reduced clinical worsening, ameliorated WHO function class, and increased the 6-min walk distance. However, oral prostanoids only showed a mild effect on 6-min walk distance (19.88 $\mathrm{m}$; 95\% CI: 10.12-29.64, $\mathrm{P}=0.000)$, and did not have any effect on reducing mortality and clinical worsening. Moreover, oral prostanoids significantly increased the incidence of withdrawal due to adverse effects $(\mathrm{RR}=3.41 ; 95 \% \mathrm{CI}: 2.06-5.63, \mathrm{P}=0.000)$.

Conclusion This Meta-analysis suggests that all oral agents confer a therapeutic benefit. Of these, only PDE-5Is has a proven survival benefit. ERAs and riociguat are efficient in reducing clinical worsening, and ameliorating exercise capacity. Oral prostanoids have the significant adverse effects and weak therapeutic effects.

\section{ProBNP in patients with pulmonary vein electrical isolation predicts the recurrence of late atrial fibrillation after ablation in paroxysmal and persistent atrial fibrillation \\ Bo Zhang \\ Cardiovascular Department of Xinqiao Hospital, Third Military Medical University}

Objective To determine the protective value of serum ProBNP in the recurrence of late atrial fibrillation after pulmonary vein isolation in paroxysmal and persistent atrial fibrillation (AF).

Methods 94 patients in the present study had symptomatic paroxysmal AF and had failed to respond to at least one anti- arrhythmic drug. All patients underwent electrophysiological study and circumferential pulmonary vein isolation (CPVI) till to achieve $\mathrm{PV}$ isolation. Cardiac structure and function were measured with 2-D echocardiogram, and the cardiac biomarker N-terminal pro-brain natriuretic peptide (NT-proBNP) was measured before ablation and at long-term follow-up.

Result After follow-ups for 12 months, 72 patients did not recurred anylateatrial fibrillation. There was no significant difference of atrioventricular structure and function parameters and pro-BNP in patients between success group and recurrence group before ablation. Left atrial dimension (LAD) and proBNP levels fell only after successful $\mathrm{AF}$ ablation and NT-proBNP after 12 months follow-up. A decrease up of $>25 \%$ of the baseline value of NT-pro-BNP at follow-up had a specificity of $90.6 \%$, a sensitivity of 80.6 and a accuracy $84.04 \%$ for ablation success.

Conclusion A decrease in NT-proBNP after CPVI may be a marker of longterm ablation success.

\section{The effects of ACE2 on monocrotaline-induced pulmonary arterial hypertension in rats \\ Mingyu Pu, Zeqi Zheng \\ Department of Cardiology, First Affiliated Hospital of Nanchang \\ University}

Objective In our study, we focused on the effects of ACE2 on monocrotaline-induced PAH in rats, to investigate whether ACE2 activator resorcinol naphthalein (Rec) can prevent monocrotaline (MCT) induced pulmonary arterial hypertension in rats, and what related cell signal transduction pathway might play a role in it.

Methods Male Sprague-Dawley rats (12 wk old) were randomly divided into 4 groups: control group, MCT group, ACE2 group and Mas receptor antagonist (A779) group. First day, control rats received vehicle subcutaneous, whereas others received a single injection of MCT subcutaneous. Second day, osmotic minipumps containing vehicle were implanted subcutaneously in control rats, at the same time, osmotic minipumps containing vehicle, Rec or Rec + A779 were implanted respectively in MCT rats, ACE2 rats and A779 rats. Used rat tail non invasive blood pressure measurement to measure the rats blood pressure after14 days of pumps implant, once a week, 3 times every time, the average of three data as the final measure blood pressure values. Used the right cardiac catheterization measurement to measure the rats mean pulmonary artery pressure after 14 days of pumps implant, and to calculate the right heart index; HE staining analyze of film thickness of the pulmonary artery and pulmonary small artery obstruction as well. Detected ACE2, Mas, Akt, P-Akt, eNOS and P-eNOS expression level in lung tissue by Western-blot. Detected Ang- (1-7) level in lung tissue by ELISA. Measured NO concentration in lung tissue.

Result The effects of ACE2 on mean pulmonary artery pressure and blood pressure in rats. There was no difference between ACE2 group and the control group in mean pulmonary artery pressure; Elevated mean pulmonary artery pressure is observed in the MCT group and A779 group has resulted in; there was no differenceamong the four groups in rats' blood pressure. The effects of ACE2 on pulmonary artery in rats. We observed increased pulmonary vessel wall thickness inMCT group and A779 group, whereas no obviously thickened pulmonary vessel wall in ACE2 group and the control group. The effects of ACE2 on right ventricle hypertrophy index in rats.MCT group and A779 group has resulted in right ventricular hypertrophy, whereas ACE2 group had no difference with the control group. The effects of ACE2 
on each component in the lung tissue of rats.Compared with MCT group and A779 group, the level of ACE2, Mas, P-akt, P-eNOS and the concentration of $\mathrm{NO}$ have elevated in $\mathrm{ACE} 2$ group.

Conclusion Rec can activate the ACE2 in vivo. ACE2 can prevent monocrotaline induced pulmonary arterial hypertension, increased pulmonary vessel wall thickness and right ventricular hypertrophy in rats. ACE2 had no obvious effect on systemic blood pressure of rats. By Akt/eNOS downstream signaling pathway, ACE2 can activate eNOS to generate $\mathrm{NO}$ to lower mean pulmonary artery pressure in rats.

\section{Relation of elevated C-reactive protein and interleukin-6 levels to recurrence of atrial fibrillation after catheter ablation of paroxysmal atrial fibrillation \\ Bo Zhang, Yi Zhang, Guoqiang Wang, Xiaofeng Cheng, Xiaoyan Liu, Xuejun Yu, Jun Jin, Aimin Li, Yaomin Song, Lan Huang \\ Cardiovascular Department of Xinqiao Hospital, Third Military Medical University}

Objective It is prevalent of recurrence of atrial fibrillation after catheter ablation of paroxysmal atrial fibrillation. The objective of this study was to investigate the relation of elevated C-reactive protein and interleukin- 6 levels to recurrence of atrial fibrillation after catheter ablation of paroxysmal atrial fibrillation.

Methods To analyze retrospectively the clinical data of 94 patients with paroxysmal atrial fibrillation who underwent radiofrequency catheter ablation by circumferential pulmonary vein isolation (CPVI). Patients were allocated to success group $(\mathrm{n}=72)$ and recurrence group (n $=22$ ) according to the symptoms, 12-Leads ECG and Holter ECG after 6 months follow-up. The levels of cardiac troponin T (cTnT), lactate dehydrogenase (LDH), and creatine kinase myocardial bound (CKMB) were determined at different time point. Enzym-linked immunosorbent assay and immune turbidimetric method were used to determine the concentration of interleukin-6 (IL-6) and high specific C-reaetive Protein (hsCRP). Cardiac structure and function were measured with 2-D echocardiogram.

Result There was no significant difference of atrioventricular structure and function parameters, hsCRP, IL-6 and serum cardiac biomarkers in patients between success group and recurrence group before ablation. The levels of hsCRP and IL-6 were significantly lower in success group than recurrence group. However, There was no significant difference of atrioventricular structure and function parameters and serum cardiac biomarkers between success group and recurrence group before ablation.

Conclusion High levels of hsCRP and IL-6 after long periods ablation have some predictive value in evaluating early recurrence of atrial fibrillation after radiofrequency catheter ablation.

\section{A novel mechanism of suppression on monocrotaline-induced pulmonary hypertension by estrogen-regulated microRNAs}

Zhichao Sun, Ping Yuan, Dong Liu, Jinming Liu Departmentof Cardio-Pulmonary Circulation, Shanghai Pulmonary Hospital, Tongji University, School of Medicine

Background and Objective Pulmonary arterial hypertension
(PAH) is a hyperproliferative vascular disorder observed predominantly in women. Estrogen has been shown to attenuate development of PAH in rats. However, little is known about the mechanism. Since microRNAs (miRNAs) were recently reported to play an important role in the pathogenesis of PAH and a host of them could be regulated by estrogen in other diseases, we hypothesis miRNAs are involved in the process of estrogen regulation of $\mathrm{PAH}$.

Methods PAH was induced in male Sprague-Dawleyrats (180-200 g) with monocrotaline $(\mathrm{MCT}, 60 \mathrm{mg} / \mathrm{kg})$. At day-21, rats were treated with 17ß-estradiol [E2, $75 \mu \mathrm{g} /$ ( $\mathrm{kg} \cdot$ day] for 10 days. After measuring right ventricular systolic pressure (RVSP) and mean pulmonary artery pressure (mPAP) of the three groups $(\mathrm{C}, \mathrm{M}, \mathrm{ME}, \mathrm{n}=8)$, we isolated their pulmonary arteries (PAs) for Exqion's microRNA array $(\mathrm{n}=3)$. The aberrant expression of miRNAs were validated independently in PAs and lung tissues from the three groups by qRT-PCR $(n=8)$.

Result E2 significantly attenuated RVSP and mPAP in MCT induced PAH rats. MiRNA array showed five miRNAs (miR-187-5p, miR-328b, miR-483-5p, miR-7b, miR-300-5p) aberrantly expressed in PAs of MCT group compared with the control while E2 could reversed the expression. Three miRNAs (miR-483-5p, miR-7b, miR-300-5p) could be further validated by qRT-PCR. However, we didn't find any abnormal expression of the three miRNAs in the whole lung tissues (data not shown).

Conclusion We therefore propose for the first time that a novel mechanism of $\mathrm{E} 2$ attenuates $\mathrm{PAH}$ in rats by regulating expression of miRNAs (miR-483-5p, miR-7b, miR-300-5p) specifically in pulmonary arteries.

\section{Serum high-density lipoprotein cholesterol levels as a prognostic indicator in patients with idiopathic pulmonary arterial hypertension \\ Qinhua Zhao, Jing He, Rong Jiang, Jinming Liu Department of Cardio-Pulmonary Circulation, Shanghai Pulmonary Hospital, Tongji University School of Medicine}

Objective High-density lipoprotein (HDL) cholesterol levels are a strong, independent inverse predictor of cardiovascular disease. The present study aimed to determine whether serum HDL cholesterol levels correlated with disease severity and clinical outcomes in patients with idiopathic pulmonary arterial hypertension (IPAH).

Methods The serum HDL cholesterol levels were measured in 76 Chinese patients with IPAH and 45 healthy controls, together with other clinical variables. Univariate and multivariate Cox proportional hazards analysis was performed to assess the prognostic value of HDL cholesterol and event-free survival. Event-free survival was estimated using the Kaplan-Meier method.

Result Serum HDL cholesterol levels were significantly decreased in patients with IPAH compared with controls $[(1.0 \pm 0.3) \mathrm{mmol} / \mathrm{L}$ vs $(1.5 \pm 0.3) \mathrm{mmol} / \mathrm{L}, \mathrm{P}<0.001]$. The serum HDL cholesterol levels decreased in proportion to the severity of World Health Organization functional class. Compared to the high HDL cholesterol group, the low HDL cholesterol group demonstrated a significantly lower 6-minute walking distance, cardiac index, mixed venous saturation, and arterial carbon dioxide pressure but significantly greater pulmonary vascular resistance and serum uric acid levels. The serum HDL cholesterol levels correlated positively with the cardiac index $(\mathrm{r}=0.42, \mathrm{P}=0.002)$ and negatively with the pulmonary vascular resistance $(\mathrm{r}=-0.25, \mathrm{P}=0.04)$. Serum HDL cholesterol was independently related to event-free survival on multivariate Cox proportional hazards analysis. Kaplan-Meier 
survival curves according to the median HDL cholesterol value showed that lower HDL cholesterol levels were associated with lower event-free survival.

Conclusion Serum HDL cholesterol levels might serve as an indicator of disease severity and prognosis in patients with IPAH.

\section{3-Bromopyruvate prevent monocrotaline- induced pulmonary arterial hypertension in rats \\ Rui Zhang, Hongda Zhang, Xiaoqing Sun, Jinming Liu Department of Cardio-Pulmonary Circulation, ShanghaiPulmonary \\ Hospital, Tongji University School of Medicine}

Objective Basis of the "Warburg effect", the metabolism shifts from oxidative mitochondria to glycolysis and the elevated enzyme hexokinase-2 (HK2) in PAH. An inhibitor of HK2, 3-bromopyruvate (3-BrPA), promptly and substantially suppresses ATP and lactic acid, however, it could prevents the development of PAH is not yet known.

Methods and Results Sprague-Dawley rats with monocrotaline (MCT, $60 \mathrm{mg} / \mathrm{kg}$ ) -induced PAH were administered subcutaneous injection of 3-BrPA [7.5 mg/ $(\mathrm{kg} \cdot \mathrm{d}), 15 \mathrm{mg} /(\mathrm{kg} \cdot \mathrm{d})$ and $30 \mathrm{mg} /(\mathrm{kg} \cdot \mathrm{d})$, respectively] for 14 days. Hemodynamic parameters were acquired by right heart catheterization. Histopathology, immunohistochemisty, and assessments of relative enzyme expressions were performed in rat lung tissue. Compared with MCT rats, 3-BrPA significantly decreased mean pulmonary arterial pressure, pulmonary vascular resistance (PVR) and right heart hypertrophy (RVH), and increased cardiac output. In additional, 3-BrPA treatment markedly reduced MCT-induced increase in both the wall thickness and area. 3-BrPA significantly suppressed proliferation and enhanced apoptosis of pulmonary artery smooth muscle cells, attenuating small pulmonary artery remodeling. Furthermore, treatment with 3-BrPA significantly increased endothelial NO synthase expression, cytochrome $\mathrm{c}$ in cytoplasm and superoxide dismutase activity, and down-regulated HK2 expression and ATP production.

Conclusion These investigations demonstrated the importance of glycolytic inhibition in $\mathrm{PAH}$ pathogenesis and highlight 3-BrPA may beneficial to the therapy of PAH.

\section{Effects of Inhaled iloprost on gas exchange and hemodynamics in patients with pulmonary hypertension due to lung diseases \\ LanWang, Lei Bai, Qinhua Zhao, Jing He, Jinming Liu Departmentof Cardio-Pulmonary Circulation, Shanghai Pulmonary \\ Hospital, Tongji University, School of Medicine}

Objective To investigate the acute effects of inhaled iloprost on v/ q matching, and hemodynamics in patients with pulmonary hypertension (PH) due to chronic lung diseases.

Methods Sixty-nine patients with chronic lung diseases and $\mathrm{PH}$ were diagnosed by right heart catheterization. All patients received a single dose of $20 \mu \mathrm{g}$ iloprost during the process of right heart catheterization, cardiopulmonary data included hemodynamic parameters and $\mathrm{PaO}_{2}, \mathrm{Paco}_{2}, \mathrm{SaO}_{2}, \mathrm{P}$ (a-a) $\mathrm{O}_{2}$, venous admixture/shunt fraction (qs $/$ / qt) were measured or calculated at baseline, and $20 \mathrm{~min}$ after inhalation iloprost respectively.

Result Inhalated iloprost caused a decrease in mPAP from (44.5 \pm 10.5) $\mathrm{mm} \mathrm{Hg}$ to $(41.6 \pm 11.4) \mathrm{mm} \mathrm{Hg}$, and PVR from (7.6 \pm 3.6$)$ wood units to $(6.6 \pm 3.9)$ wood units $(\mathrm{P}<0.001)$, cardiac index increase from
$(3.2 \pm 0.8) \mathrm{L} /\left(\min \cdot \mathrm{m}^{2}\right)$ to $(3.5 \pm 1.0) \mathrm{L} /\left(\min \cdot \mathrm{m}^{2}\right)(\mathrm{P}<0.001)$. Arterial blood gas and calculated qs $/$ qt were maintained after iloprost. No adverse effects on systemic blood pressure were seen.

Conclusion Iloprost inhalation was safe in patients with $\mathrm{PH}$ due to lung diseases, and improved hemodynamics but didn't affect v/q matching and gas change.

\section{Pulmonary embolism, pulmonary hypertension, hypertension and renal artery stenosis in polycythemia vera: report of one case and review of literature \\ Dan Yang, Qing Gu, Zhihong Liu, Zhihui Zhao, Changming Xiong, Xinhai Ni, Jianguo He, Xiansheng Cheng Pulmonary Cardiovascular Disease Center, State Key Laboratory of Cardiovascular Disease, Fuwai Hospital, National Center for Cardiovascular Diseases, Chinese Academy of Medical Sciences and Peking Union Medical College}

Objective To investigate the mechanism, therapy, and prognosis of polycythemia vera, a case of polycythemia vera with pulmonary embolism, pulmonary hypertension, hypertension and renal polycythemia verartery stenosis was analyzed.

Methods Analytic study was performed on the clinical features, imaging, laboratory test and treatment of this patient according to the literature.

Result The patient showed pulmonary embolism, pulmonary hypertension, hypertension and renal artery stenosis, which were related to polycythemia vera. The mechanism may be due to prothrombotic state leading to repeated endothelial injury, thrombosis and resultant intimal hyperplasia. Right heart catheterization was performed revealing acute vascular reactive test was positive. The patient was accepted not only the treatment of primary disease but also anticoagulant therapy and calcium antagonist. Three months followed, lung perfusion of the patient was not change but he had improved his cardiac function, and a decrease in his pulmonary artery systolic pressure on echocardiogram from $100 \mathrm{~mm}$ $\mathrm{Hg}$ to $73 \mathrm{~mm} \mathrm{Hg}$. His exercise capacity was improved. These changes suggested his clinical improvements maybe related to the mechanism of pulmonary arterial hypertension.

Conclusion Polycythemia vera may account for the incidence of pulmonary embolism, pulmonary hypertension, hypertension and renal artery stenosis. We should perform a comprehensive analysis in order to get an early diagnosis and treatment.

\section{Elevated plasma YKL-40 as a prognostic indicator in patients with idiopathic pulmonary arterial hypertension \\ Guo Chen, Tao Yang, Qing Gu, Xinhai Ni, Zhihui Zhao, Jue Ye, Xianmin Meng, Zhihong Liu, Jianguo He, Changming Xiong State Key Laboratory of Cardiovascular Disease, Fuwai Hospital, National Center for Cardiovascular Diseases, Chinese Academy of Medical Sciences and Peking Union Medical College}

Objective Pulmonary vascular remodeling and inflammation have been implicated in pulmonary arterial hypertension (PAH). YKL40 , a marker of tissue remodeling and inflammation, has recently been recognized as a risk predictor of cardiovascular and inflammatory diseases. We aimed to investigate a potential role of YKL-40 in 
predicting prognosis in idiopathic PAH (IPAH).

Methods Plasma YKL-40 levels were measured in 82 IPAH patients without current or previous $\mathrm{PAH}$-specific treatment during right heart catheterization and in 54 healthy volunteers. Concurrent data included clinical, hemodynamic, and biochemical variables.

Result Plasma YKL-40 levels were increased in IPAH patients compared with control subjects (median, interquartile range: IPAH: 24.90, 17.68-39.78; controls: 16.58, 14.20-19.64 ng/ml; P < 0.001). YKL-40 levels correlated with cardiac index $(\mathrm{r}=-0.244, \mathrm{P}=0.027)$ and $\mathrm{N}$-terminal pro-brain natriuretic peptide (NT-proBNP, $\mathrm{r}=0.263, \mathrm{P}=$ 0.017). After a median follow-up of 578 days, YKL-40 outperformed NT-proBNP, uric acid, and 6-minute-walk distance in receiver operating characteristic (ROC) analyses in predicting both clinical worsening (AUC: 0.681 ) and death (AUC: 0.717). Compared to patients with YKL40 below the ROC-derived cut-off point $(24.5 \mathrm{ng} / \mathrm{ml})$, the high YKL40 group showed higher pulmonary vascular resistance and serum uric acid levels, and showed more clinical worsening events and deaths in Kaplan-Meier analyses. Plasma YKL-40 was independently associated with clinical worsening in univariate and multivariate Cox analyses (all $\mathrm{P}$ $<0.05)$.

Conclusion Plasma YKL-40 might serve as a promising indicator of disease severity and prognosis in patients with IPAH. 


\section{Extracorporeal Circulation and Anesthesia}

\author{
The clinical study of infusion residual pump \\ blood after CPB processed by leukocyte filter \\ Jinxiao Hu, Huiying Wang, Cun Long, Song Lou \\ Department of Cardiopulmonary Bypass, State Key Laboratory \\ of Cardiovascular Disease Fuwai Hospital National Center for \\ Cardiovascular Diseases Chinese Academy of Medical Sciences and \\ Peking Union Medical College
}

Objective To study the effects of leukocyte filter processed residual pump blood infusion on the systemic inflammatory response and patients' recovery.

Methods 40 patients were randomly divided into 2 groups. Trial group (filter group) and control group (non-filtered group). Patients requiring heterogenous blood transfusion were excluded. The concentration of interleukin-6 (IL-6), interleukin-10 (IL-10), tumor necrosis factor- $\alpha$ (TNF- $\alpha)$, colloid osmotic pressure COP, free hemoglobin $\mathrm{FHb}$ were measured at the following time points: before $\mathrm{CPB}$, at the end of $\mathrm{CPB}, 6 \mathrm{~h}$ after the residual blood infusion finished. We also recorded patient's temperature and leukocyte counts before $\mathrm{CPB}$, at the end of $\mathrm{CPB}, 6 \mathrm{~h}$ after the residual blood infusion finished and next morning after operation. Mechanical ventilation time was recorded. The count of platelet, the concentration of free hemoglobin and colloid osmotic pressure of the residual bloodwere measured

Result The counts of leucocyte increased significantly in trial group at the following time points: $6 \mathrm{~h}$ after the residual blood infusion and first morning after operation.

Conclusion We could not demonstrate that infusion residual pump blood after CPB processed with leucocyte filter could inhibit systemic inflammatory response.

\section{Evaluation of cerebral injury after different hypothermic circulatory arrests \\ Yanhua Sun ${ }^{1}$, Xian Zhu ${ }^{1}$, Yaoyao Xiong ${ }^{1}$, \\ Yingjie $\mathrm{Du}^{2}$, Bingyang $\mathrm{Ji}^{1}$ \\ State Key Laboratory of Cardiovascular Disease Fuwai Hospital \\ National Center for Cardiovascular Diseases Chinese Academy of \\ Medical Sciences and Peking Union Medical College \\ 1. Department of Cardiopulmonary Bypass \\ 2. Department of Anesthesiology}

Objective Deep hypothermic circulatory arrest (DHCA) is widely used in aortic and complex congenital heart operations, in order to provide bloodless surgery field by arresting circulation, and to protect organs by lowering body temperature. However, the mechanism of cerebral injury after DHCA is still far away from fully understood. We evaluated the injury of different arrest temperature to search for new aspect of cerebral protection during DHCA.

Methods 24 SD rats were randomly divided to 4 groups: deep hypothermic circulatory arrest $\left(15-20^{\circ} \mathrm{C}\right)$, moderate hypothermic circulatory arrest $\left(20-25^{\circ} \mathrm{C}\right)$, mild hypothermic circulatory arrest $(25-$ $30^{\circ} \mathrm{C}$ ) and sham group. Brains of rats were harvest after surgery. Injury of neurons was evaluated after HE staining. The expression pattern of HIF- $1 \alpha$ was observed by immunohistochemical staining. TUNEL technique was used for evaluate apoptosis. Transmission electronic microscope was used to observe the ultrastructure injury of neuro (corpus neuroni, dendrite and axon) and blood brain barrier.

Result Neuron injury appeared in 3 hypothermic circulatory arrest groups, but there was no apparent difference after HE staining. There was no apparent apoptosis neuron observed after TUNEL. HIF-1 $\alpha$ was overexpressed in deep hypothermic circulatory arrest, moderate hypothermic circulatory arrest and mild hypothermic circulatory arrest group, but there was no different. Microtubules and mitochondrial cristaes were dissolved in dendrite and axon. Podocyticprocess of astrocyte which constitutes blood brain barrier swelled.

Conclusion Different hypothermic circulatory arrests contributed to cerebral injury, but there was no linear correlation between the injury severity and temperature. This indicated that the mechanism of cerebral injury after DHCA was complicated.

\section{Effect of a new blood-sparing approach on bloodless transfusion in low body weight infants underwent cardiac surgery \\ Jinping Liu, Zhengyi Feng, Ju Zhao, Cun Long Department of Cardiopulmonary Bypass, State Key Laboratory of Cardiovascular Disease Fuwai Hospital National Center for Cardiovascular Diseases Chinese Academy of Medical Sciences and Peking Union Medical College}

Objective To evaluate the feasibility of modified blood-sparing approach in low-weight infants $(\leq 15 \mathrm{~kg})$ with congenital heart disease, we analyzed the effect of blood-free priming strategy on transfusion requirements, clinic data, and the risk factors which may predict blood transfusion, for the purpose of finding a relatively safe hemodilution, as well as indication and contraindication of transfusion-free pericardiopulmonary bypass.

Methods A total of 531 infants were included between February 2011 and October 2013, according to internalize standard strictly, infants were divided into tow groups: control group (group C, $\mathrm{n}=$ 248), used traditional priming strategy, and modified group (group M, $\mathrm{n}=283$ ), applied a new blood-sparing approach, known as without homologous blood priming. Infants in M group were allowed to enter into intraoperative transfusion group (group IT, $n=106$ ) once having transfusion requirement during operation; infants in group $\mathrm{M}$ without transfusion during operation divided into postoperative transfusion group (group PT, $\mathrm{n}=56$ ) or transfusion-free group (group TF, $\mathrm{n}=$ 121), according to post-operative transfusion. All infants experienced routine heart surgery with cardiopulmonary bypass. Blood samples were collected as following time points: pre-CPB, $10 \mathrm{~min}$ after CPB, before termination of CPB after modified ultrafiltration, clinic data and transfusion requirements were collected during operation, ICU and ward.

Result In the first section of the study, packed red blood cell and fresh frozen plasma transfusion during admission of group $\mathrm{M}$ were less than group $\mathrm{C}(\mathrm{P}<0.01)$, as well as endotracheal intubation time, ICU stay, and total 24-hour chest tube output $(\mathrm{P}=0.028, \mathrm{P}=0.034$, $\mathrm{P}=0.012$ ), however there was no difference in admission time, reintubation, peritonealdialysis and death in two groups $(\mathrm{P}>0.05)$. In the second section, group IT had lower body weight and size, younger age, Lower pre-operation HCT than groupTF $(\mathrm{P}<0.01)$. Pre-operation HCT was also lower in IT group than that in group PT $(\mathrm{P}=0.01)$, reversely, admission time in IT group was longer $(\mathrm{P}<0.01)$, there was 
significant difference in admission stay among the three groups $(\mathrm{P}=$ 0.047). Multivariate logistic regression showed that pre-operation HCT, body weight, size can reduce the risk of intra-operative transfusion (OR $=0.811, \mathrm{P}<0.001 ; \mathrm{OR}=0.717, \mathrm{P}<0.001 ; \mathrm{OR}=0.909, \mathrm{P}=0.008)$, priming volume was the risk factor $(\mathrm{OR}=1.006 ; \mathrm{P}=0.032)$. Similarly, body weight could reduce the risk of post-operative transfusion $(\mathrm{OR}=$ $0.833, \mathrm{P}=0.009)$; however preoperative cyanosis can increase the risk of post-operative transfusion $(\mathrm{OR}=2.597, \mathrm{P}=0.019)$. For the infants with body weight less than $10 \mathrm{~kg}$ pre-operation HCT $39.5 \%$ or lowest HCT $23.5 \%$ had very good discriminatory ability (AUC: 0.80 ; sensitivity 0.774 , specificity 0.727 ; AUC: 0.802 , SE 0.750 , SP 0.731). And for the infants with body weight more than $10 \mathrm{~kg}$, pre-operation HCT $35.5 \%$ or lowest HCT $21.8 \%$ had good discriminatory ability (AUC: 0.749 , SE 0.583 , SP 0.931; AUC: 0.712, SE 0.750, SP 0.766).

Conclusion Our research elucidated that modified blood-sparing approach can reduce blood transfusion during admission, and without poorer outcome. Better understanding of tansfusion risk factor, protective factor, pre-operation HCT and safe hemodilution will benefit for blood sparing in infant with body weight less than $15 \mathrm{~kg}$.

\section{The effects of balanced ultrafiltration on plasma concentration of antimicrobial agents in vitro \\ Yinghui Fang, Yulong Guan, Cun Long \\ Departmentof Extracorporeal Circulation, State Key Laboratory \\ of Cardiovascular Disease, Fuwai Hospital, National Center for \\ Cardiovascular Diseases, Chinese Academy of Medical Sciences and \\ Peking Union Medical College}

Objective Patients with cardiac surgery are at risk for postoperative infections, and prophylactic antibiotics may help to reduce this risk. But whether balanced ultrafiltration during cardiopulmonary bypass (CPB) can remove antibiotics remains unknown. In this pseudo-extracorporeal circulation model, we measured the concentrations of antibiotics in plasma and ultrafiltrate samples.

Methods Extracorporeal circulation model consisted of a cardiotomy reservoir, a membrane oxygenator and a pediatric arterial line filter. Hemoconcentrator was placed between arterial purge line and oxygenator venous reservoir. Fresh human whole blood from donor mixed with Ringer's solution was added into the circuit and maintained hematocrit of $24 \%-28 \%$. After 30 minutes of CPB, zero -balanced ultrafiltration was started and arterial line pressure was keeped at approximately $100 \mathrm{~mm} \mathrm{Hg}$ with Hoffman clamp. By controlling ultrafiltrate outlet pressure, the rate of ultrafiltration was keeped at $12 \mathrm{ml} /$ min. During 45 minutes of experiment, identical volume of plasmaslyte A was dripped into the circuit to maintain stable hematocrit. Plasma and ultrafiltrate samples were drawn every 5 minutes, and concentrations of antibiotics (including Cefmetazole and cefotiam) were measured with high performance liquid chromatography.

Result Cefmetazole and cefotiam were both detected in ultrafiltrate, demonstrating hemoconcentration may remove antibiotics. The concentration of plasma antibiotics decreased lineally with the increase of ultrafiltrate volume. At end of balanced ultrafiltration, the concentration of plasma cefotiam was $(104.96 \pm 44.36) \mathrm{mg} / \mathrm{ml}$, which is about $(44.38 \pm 7.42) \%$ of the initial concentration $(238.95 \pm 101.12)$ $\mathrm{mg} / \mathrm{ml}(\mathrm{P}<0.001)$; the concentration of plasma cefmetazole decreased linearly to $(25.76 \pm 14.78) \mathrm{mg} / \mathrm{ml}$, which is about $(49.69 \pm 10.49) \%$ of the initial concentration $(51.49 \pm 28.03) \mathrm{mg} / \mathrm{ml}(\mathrm{P}<0.001)$. The total amount of cefotiam in ultrafiltrate is $(27.16 \pm 12.17) \%$ of the total dose administered, whereas cefmetazole in ultrafiltrate is $(7.74 \% \pm 4.17) \%$.
Conclusion Balanced ultrafiltration may remove antibiotics from plasma, and has significant influence on plasma concentration of antibiotics. The strategy of surgical prophylaxis should consider this unique technique during $\mathrm{CPB}$.

\section{Real-time integrated monitoring is useful approaches during aortic arch aneurysm surgery \\ Yulong Guan, Yinghui Fang, Qian wang, Zhida Fu, Xiaolong Wei, Xiaolong Wang, Jiuguang Yang, Fuqing Jiang, Peng Sun, Cun Long \\ Department of ExtracorporealCirculation, State Key Laboratory of Cardiovascular Disease, Fuwai Hospital, National Center for Cardiovascular Diseases, Chinese Academy of Medical Sciences and Peking Union Medical College}

Objective To monitor the distribution of blood supply and oxygen metabolism during aortic arch aneurysm surgery under selective antegrade cerebral perfusion (SACP).

Methods 37 patients underwent aortic arch repair and reconstruction surgery were enrolled in this study. The distribution of blood perfusion through separated axillary artery and tetrafurcate graft cannulation was monitored using TS410 transit-time tubing flowmeters. The cerebral blood supply was measured with transcranial cerebral Doppler and the oxygen with near-infrared spectroscopy cerebral oxygen saturation.

Result The blood flow through axillary artery canuulation was $27 \mathrm{ml} /(\mathrm{kg} \cdot \mathrm{min})$ during cooling and rewarming phases and ranged from $34 \%-41 \%$ of whole body perfusion. Left regional cerebral oximetry [rSO (2) ] maintained stable during whole procedure and right $[\mathrm{rSO}$ (2) ] elevated significantly compared with base levels. The middle cerebral artery blood flow velocity may be detected in more than $90 \%$ patients (24/26). 2 cases have an uneventful recovery process following increment of blood flow of SACP. Abnormal rSO (2) was recorded in other 2 patients without interventions that 1 patient died and the other patient recovered with serious neuropathic pain.

Conclusion Double arterial lines with single pump head may be performed safely with self-regulation of separated upper body and lower body. Real-time integrated monitoring is useful approaches during aortic arch aneurysm surgery.

\section{The effects of balanced ultrafiltration on plasma concentration of inflammatory factors and antimicrobial agents in pediatric cardiac surgery \\ Yinghui Fang, Yulong Guan, Cun Long Department of Extracorporeal Circulation, State Key Laboratory of Cardiovascular Disease, Fuwai Hospital, National Center for Cardiovascular Diseases, Chinese Academy of Medical Sciences and Peking Union Medical College}

Objective With the initiation of $\mathrm{CPB}$ the children's blood is diluted with the prime fluid present in the CPB-circuit, and ultrafiltraton has been routinely used for removing the excessive fluid in the body. We had reported that balanced ultrafiltration can remove antibiotics form plasma in a pseudo-extracorporeal circulation model, but the effects of ultrafiltration on antibiotic in pediatric cardiac surgery are unclear. The purpose of this investigation was to determine whether or not the ultrafiltration will affect the plasma antibiotics in pediatric cardiac 
surgery.

Methods From July 2012 to May 2013, children who received atrial septal defect repair or ventricular septal defect repair with heart function I-II were included in this study. Patients were randomly assigned to receive balanced ultrafiltration (BUF) or non-balanced ultrafiltration (nBUF), cefmetazole was administered within 30 minutes of the skin incision. Blood sample were collected before ultrafiltration (T1), at the end of ultrafiltration (T2), end of CPB (T3) for testing the blood gas analysis, oncotic pressure, inflammatory factors, and the concentrations of antibiotics. Ultrafiltrate samples were also collected for measuring the concentration and total amount of inflammatory factors and antibiotics.

Result At time point T3, plasma concentration of IL-1, IL-6, IL$10, \mathrm{TNF}-\alpha$ and NE were significantly higher than T2. There were no difference of IL-1, IL-6, and NE level between groups at T3. In BUF group, plasma concentration of IL-10, TNF-awere higher than nBUF group at T3. All kinds of inflammatory factors can be tested in the ultrafiltrate, but there were no difference of concentration between groups. After calculated by the volume of ultrafiltrate, BUF can remove more amount of inflammatory factors. In BUF group, at the end of balanced ultrafiltration, the concentration of plasma cefmetazole was $(40.04 \pm 12.38) \mathrm{mg} / \mathrm{ml}$, which is about $(53.47 \pm 13.60) \%$ of the initial concentration $(72.03 \pm 23.77) \mathrm{mg} / \mathrm{ml}$. In the nBUF group, the concentration of plasma cefmetazole decreased to $(58.02 \pm 18.86) \mathrm{mg} /$ $\mathrm{ml}$, which is about $(69.11 \pm 9.0) \%$ of the initial concentration $(80.04 \pm$ $30.30) \mathrm{mg} / \mathrm{ml}$. The difference of reduction of plasma antibiotics between groups are statisticalsignificant $(\mathrm{P}=0.018)$. In $\mathrm{BUF}$ group, the total amount of cefmetazole in ultrafiltrate is $(7.25 \pm 3.83) \%$ of the total dose administered, whereas in nBUFgroup cefmetazole in ultrafiltrate is $(2.83$ $\pm 1.71) \%$, the difference is statistically significant $(\mathrm{P}=0.004)$.

Conclusion In pediatric cardiac surgery, conventional ultrafiltration may remove inflammatory factors and antibiotics from plasma, and balanced ultrafiltration may further influence plasma concentration of inflammatory factors and antibiotics.

\section{The effects of continuous ultrafiltration on plasma concentration of inflammatory factors and antibioticsin aortic arch replacementsurgery under deep hypothermic circulatory arrest \\ Ying hui Fang, Yulong Guan, Long Long \\ DepartmentofExtracorporeal Circulation, State Key Laboratory \\ of Cardiovascular Disease, Fuwai Hospital, National Center for \\ Cardiovascular Diseases, Chinese Academy of Medical Sciences and \\ Peking Union Medical College}

Objective The aortic arch replacement surgery under deep hypothermic circulatory arrest (DHCA) often accompanied with prolonged surgery duration, deep hypothermia, and ischemia reperfusion injury, which causing the acid-base imbalance and oxidative stress, and produce large amounts of inflammatory cytokines. Thus the routine use of ultrafiltration technology could filter out excessive fluid, regulate electrolyte and acid-base balance, filter inflammatory cytokines. However, our previously research found that ultrafiltration can filter out antibiotics, and positively correlated with the amount of ultrafiltration, so the blood concentration of antibiotics in surgery under DHCA might be affected by the amount of ultrafiltration. The purpose of our study was to investigate the effect of high-volume ultrafiltration on plasma concentration of antibiotics in aortic arch replacement surgery under DHCA.
Methods The patients who need aortic arch replacement surgery with DHCA were enrolled in the study, and were assigned to receive continuous ultrafiltration which started at the initiation of CPB or the control group with ultrafiltration started at rewarming. Cefuroxime was administered within $30 \mathrm{~min}$ of the skin incision. Blood samples were collected at just before start $\mathrm{CPB}$, start $\mathrm{CPB}$, before ultrafiltration, end of $\mathrm{CPB}$. And ultrafiltrate was also collected at the end of ultrafiltration. Cefuroxime concentrations were tested by high performance liquid chromatography. IL-1, IL-6, IL-10, NE, TNF- $\alpha$ were tested by ELISA.

Result After two hours of CPB plasma concentration of Cefuroxime significantly decreased $(\mathrm{P}<0.05)$. The plasma concentration of Cefuroxime decreased $(17.86 \pm 11.52) \%$ in experimental group, whereas decreased $(12.16 \pm 6.85) \%$ in control group, but the difference between the groups was not statistical significant $(P>0.05)$. The differences of plasma concentration of Cefuroxime between the groups were not statistical significant at the end of CPB or $4 \mathrm{~h}$ after CPB. The plasma concentration of IL-1 and TNF- $\alpha$ were not significantly changed in the operation $(\mathrm{P}>0.05)$. After two hours of $\mathrm{CPB}$ plasma concentration of IL-6, IL-10 and NE were not significantly changed, but significantly elevated at the end of $\mathrm{CPB}(\mathrm{P}<0.05)$. Compared with the end of CPB (319.03 \pm 67.51$) \mathrm{ng} / \mathrm{L}$, plasma concentration of IL-10 decreased to $(148.57 \pm 83.28) \mathrm{ng} / \mathrm{L}(\mathrm{P}<0.05)$, but the concentration of IL-6 and NE were not significantly decreased $(\mathrm{P}>0.05)$. The plasma concentration of inflammatory factors at every time point were not significantly different between the groups $(\mathrm{P}>0.05)$. At the end of $\mathrm{CPB}$, plasma hemoglobin and glucose were significantly superior in continuous ultrafiltration group.

Conclusion There are no differences of two types of ultrafiltration on plasma concentration of Cefuroxime. The inflammatory factor elimination effect of continuous ultrafiltration is not superior to ultrafiltration after rewarming. But the glucose control is better in continuous group.

\section{The impact of priming without plasma during cardiopulmonary bypass on postoperative coagulation and clinical recovery in infants underwent cardiac surgery \\ Jinping Liu, Zhengyi Feng, Ju Zhao, Xiaolei Miao, Cun Long Department of Cardiopulmonary Bypass, State Key Laboratory of Cardiovascular Disease Fuwai Hospital National Center for Cardiovascular Diseases Chinese Academy of Medical Sciences and Peking Union Medical College}

Objective Fresh frozen plasma (FFP) has been considered as a vital component of the priming solution of cardiopulmonary bypass (CPB) circuits. It was thought to be useful to protect the postoperative coagulation function by prophylactic use of FFP during CPB. However, the clinical efficiency of prophylactic use of FFP was controversy and the practices in infants were limit. Considering the shortage and side effects of blood products transfusion, it is high time to clarify the clinical efficacy of FFP transfusion and to evaluate whether artificial colloid Gelofusine could be used safely and effectively as substitute for FFP in pump priming. In this study, we aimed to investigate the impact of priming without plasma during $\mathrm{CPB}$ on postoperative coagulation and clinical recovery in infants receiving cardiac surgery, then supply new strategies for blood saving.

Methods From December 2012 to August 2013, 115 pediatric patients who scheduled for cardiac surgery were enrolled in this investigation. All of the participants were less than three years old 
and ranging in body weight between 5 to $15 \mathrm{~kg}$. They were randomly divided into two groups according to the kind of priming solution, $\mathrm{G}$ group (Gelofusine, $\mathrm{n}=58$ ) and $\mathrm{F}$ group (FFP, $\mathrm{n}=57$ ). Either 10-20 $\mathrm{ml} / \mathrm{kg}$ Gelofusine of 1-2 units FFP was added to the CPB circuits in $\mathrm{G}$ or $\mathrm{F}$ group, respectively. Patients in both groups also received 100-200 $\mathrm{ml}$ acetated Ringer's solution in the pump circuits, with/without red blood cell depending upon the preoperative levels of hematocrit. Rapid-thromboelastography (r-TEG) and functional fibrinogen level (FLEV) were measured before skin incision and 15 minutes after heparin reversal. We recorded the postoperative renal and hepatic function, 6-hour, 24-hour and the total volume of chest tube drainage, transfusion requirements and recovery time which including the ventilation time, ICU length of stay and hospitalization time after the surgery. The relationships between hematologic data and TEG parameters and postoperative bleeding volume were also analyzed.

Result The baseline values were all comparable between the two groups. All cases recovered smoothly, and without re-open thoracotomy. After heparin reversal, there were significant elevated levels of fibrinogen, which were manifested by r-TEG parameters MAf $[(5.6 \pm 2.1)$ vs $(9.2 \pm 2.6), \mathrm{P}=0.008)$ and FLEV [(154.3 \pm 37.6) vs $(195.2 \pm 39.0), P=0.006]$, in the F group compared to the $G$ group. However, no significant differences of R, MA and Map were observed between the $\mathrm{G}$ and $\mathrm{F}$ groups $(\mathrm{P}>0.05)$. Moreover, FFP priming could not significantly reduce the blood loss, transfusion requirements and recovery time $(\mathrm{P}>0.05)$. The post-CPB MA showed moderate correlation with 6-hour postoperative bleeding $(\mathrm{r}=$ $-0.615, \mathrm{P}<0.001)$. The univariate correlations analysis revealed that the bypass time $(\mathrm{r}=0.263, \mathrm{P}=0.047)$, cyanotic disease $(\mathrm{r}=0.307$, $\mathrm{P}=0.033)$ and preoperative FLEV value $(\mathrm{r}=-0.468, \mathrm{P}=0.013)$ correlated with 6-hour postoperative bleeding.In multivariate testing, only FLEV tested preoperative could independently predict an increased risk of postoperative bleeding $(\mathrm{r}=-0.331, \mathrm{P}=0.037)$.

Conclusion Although prophylactic use of FFP for CPB circuit priming in pediatric patients could cause a significant increase in fibrinogen level after heparin reversal, no consensus clinical benefits were observed. FFP is not recommended as the priming solution during pediatric CPB. It is safe and effective to us Gelofusine as substitute for plasma in pump priming for children with certain congenital heart disease.TEG, which is a "point-of-care" assessment tool of hemostasis, could be an effective tool in blood protection.

\section{Influence of reinfusion of the residual blood containing blood cardioplegia after cardiopulmonary bypass on the concentration of potassiumin adult patients \\ Huiying Wang, Song Lou, Cun Long, Jingwen Li Department of Extracorporeal Circulation, State Key Laboratory of Cardiovascular Disease, Fuwai Hospital, National Center for Cardiovascular Diseases, Chinese Academy of Medical Sciences and Peking Union Medical College}

Objective To study the influence of reinfusion of the residual blood containing blood cardioplegia after $\mathrm{CPB}$ on the concentration of potassium in adult patients.

Methods From March 2013 to July 2013, 40 patients undergoing elective valve replacement were randomly divided into 2 groups, recycling blood cardioplegia group $(n=20)$, none recycling group $(n=$ 20). The blood gas was performed at the following time points: before
CPB, 5 minutes after administration of cold blood cardioplegia, 5 minutes after last potassium supplement during $\mathrm{CPB}, 15$ minutes after $\mathrm{CPB}$, before the ICU administration, $1 \mathrm{~h}$ and $4 \mathrm{~h}$ after ICU administration. The Blood gas of residual blood was also tested.

Result The concentration of potassium in residual blood was significantly different between two groups ( recycling group $5.81 \pm$ 0.18 vs non-recycling group $5.24 \pm 0.11, \mathrm{P}<0.05$ ). The concentration of $\mathrm{K}^{+}, \mathrm{Ca}^{2+}, \mathrm{Mg}^{2+}$ and $\mathrm{PH}$ were comparable between two groups after reinfusion of the residual blood $(\mathrm{P}>0.05)$

Conclusion In adult patients, the reinfusion of the residual blood containing blood cardioplegia after CPB didnt increase the potassium concentration.

\section{The effect of different temperature circulatory arrest to micro-tubule associated protein-2 Yanhua Sun ${ }^{1}$, Xian Zhu ${ }^{1}$, Yaoyao Xiong ${ }^{1}$, Yingjie $\mathrm{Du}^{2}$, Bingyang Ji \\ 1. Department of Cardiopulmonary Bypass, State Key Laboratory of Cardiovascular Disease Fuwai Hospital National Center for Cardiovascular Diseases Chinese Academy of Medical Sciences and Peking Union Medical College \\ 2. Department of Anesthesiology, State Key Laboratory of Cardiovascular Disease Fuwai Hospital National Center for Cardiovascular Diseases Chinese Academy of Medical Sciences and Peking Union Medical College}

Objective Our previous research indicated that miR-194 dramaticlly decreased in hippocampus after DHCA. And the target predicted web inferred micro-tubule associated protein-2 (MAP-2) is the modulated target of miR-194. In addition, we also observed the microtubule of rat dissolved after hypothermic circulatory arrest. We researched for a profound understanding of molecular mechanism of cerebral injury and protection associated with MAP-2 after DHCA.

Methods 30 SD rats were randomly divided to 5 groups: deep hypothermic circulatory arrest $\left(15-20^{\circ} \mathrm{C}\right)$, moderate hypothermic circulatory arrest $\left(20-25^{\circ} \mathrm{C}\right)$, mild hypothermic circulatory arrest $\left(25-30^{\circ} \mathrm{C}\right)$, normal temperature cardiopulmonary bypass, and sham group. Brains and blood of rats were harvest after surgery, and the hippocampus was isolated. Elisa test was used to measure the plasma concentration of MAP-2 and S100B, which is a classical biomarker for cerebral injury. The mRNA and protein content of hippocampus was tested with PCR and western blot. Wild type and mutant type of MAP-2 3'UTR vector were established, Dual Luciferase Reporter system was used to verify the modulate relationship between miR194 and MAP-2.

Result There was no statistical difference between the groups on plasma concentration of S100B after DHCA. But the plasma MAP-2 was dramatically increased in mild hypothermic circulatory arrest group compared with normal cardiopulmonary bypass group and sham group. The MAP-2 mRNA content was also increased in mild hypothermic circulatory arrest group compared with sham group. But the MAP-2 protein content showed no difference. The activity of luciferase showed that miR-194 did not modulate the expression of MAP-2.

Conclusion Microtubules were dissolved after hypothermic circulatory arrest, the plasma concentration of MAP-2 was increased dramatically in mild hypothermic circulatory arrest when S100B didn't show difference. MiR-194 did not modulate the expression of MAP-2, despite that miR-194 and MAP-2 both changed after DHCA. 


\section{Application of blood saving strategy in low- weight adults with heart disease during extracorporeal circulation \\ Huiying Wang ${ }^{1}$, Xianghui Zhu ${ }^{2}$, Cun Long ${ }^{1}$, Jingwen $\mathrm{Li}^{1}$ 1. Department of cardiopulmonary, State Key Laboratory of Cardiovascular Disease, Fuwai Hospital, National Center for Cardiovascular Diseases, Chinese Academy of Medical Sciences and Peking Union Medical College \\ 2. Department of anesthesiology, HezePeople 'hospital}

Objective To evaluate the effect of decreasing the extracorporeal circulation (ECC) priming volume for low-weight adults on reducing allergenic blood transfusion in cardiac surgery.

Methods Sixty low-weight adults (40-60 kg) were randomly divided into the following three groups: Group A (Dideco905, children ECC circuit), group B (Xijian adult oxygenator, children ECC circuit), group C (JostraVKMO7000 oxygenator, adult ECC circuit). Intra and post-operative (up to $24 \mathrm{~h}$ after ICU administration) transfusion were collected. Re-warming time, priming volume and extubation time were also recorded. The blood gas analysis was performed at the following time points, before ECC $\left(\mathrm{T}_{0}\right)$, after X-clamp $\left(\mathrm{T}_{1}\right)$, re-warming $\left(\mathrm{T}_{2}\right)$, conclusion of ECC $\left(\mathrm{T}_{3}\right)$, arriving at ICU $\left(\mathrm{T}_{4}\right), 1 \mathrm{~h}$ and $3 \mathrm{~h}$ after ICU administration $\left(\mathrm{T}_{5}\right.$ and $\left.\mathrm{T}_{6}\right)$.

Result There was no significant difference of re-warming time, extubation time and peri-operative $\mathrm{RBC}$ transfusionamong the three groups $(\mathrm{P}>0.05)$. Compared with group $\mathrm{A}$ and $\mathrm{B}$, group Chad larger priming volume $(\mathrm{P}<0.05)$.Hemoglobin $(\mathrm{Hb})$ concentrations at $\mathrm{T}_{1}$ and $\mathrm{T}_{2}$ were lower in group $\mathrm{C}$.

Conclusion The application of decreasing priming volume for lowweight adults has mitigated hemodilution and reduced the probability of peri-operative $\mathrm{RBC}$ transfusion.

\section{Change and effects of sumolytion in Rat hippocampus after hypothermic circulatory arrest \\ Yanhua Sun ${ }^{1}$, Xian Zhu ${ }^{1}$, Yaoyao Xiong ${ }^{1}$, Yingjie $\mathrm{Du}^{2}$, Bingyang $\mathrm{Ji}^{1}$ \\ State Key Laboratory of Cardiovascular Disease Fuwai Hospital National Center for Cardiovascular Diseases Chinese Academy of Medical Sciences and Peking Union Medical College 1.Department of Cardiopulmonary Bypass 2.Department of Anesthesiology}

Objective Sumolytion is up-regulated after hypothermic condition, in order to increase the cerebral tolerance to ischemia and reperfusion injury. However, the pattern of sumolytion, the expression and modulation of SUMO2, and the relationship between sumolytion and other pathway are still unkown.

Methods 30 SD rats were randomly divided to 5 groups: deep hypothermic circulatory arrest $\left(15^{\circ} \mathrm{C}-20^{\circ} \mathrm{C}\right)$, moderate hypothermic circulatory arrest $\left(20^{\circ} \mathrm{C}-25^{\circ} \mathrm{C}\right)$, mild hypothermic circulatory arrest $\left(25^{\circ} \mathrm{C}-30^{\circ} \mathrm{C}\right)$, normal temperature cardiopulmonary bypass, and sham group. Brains of rats were harvest after surgery, and the hippocampus was isolated. PCR was used to test the content of SUMO2 mRNA. Western blot was used to quantify SUMO2/3, Caspase3, Bax, Bcl-2, CytC, Beclin1, HIF-1a, Akt, p44/42 MAPK (Erk1/2) and pMEK.

Result Free SUMO2/3 and total SUMO2/3 decreased in moderate hypothermic circulatory arrest group compared with normal temperature cardiopulmonary bypass group. Total SUMO2/3 of mild hypothermic circulatory arrest group was also decreased compared with normal temperature cardiopulmonary bypass group. There was no statistical difference on other results.

Conclusion The content of SUMO2/3 changed after DHCA, but the effects and mechanism were still unknown.

\section{The clinical study of the autologous blood salvage effects of xijing XJ-12-05 type cell saver device during cardiac surgery \\ Kai Liu, Jian Ma, Mingxia Zhao, Yuan Yuan, Jingwen Li, Cun Long \\ Department of Cardiopulmonary Bypass, State Key Laboratory of Cardiovascular Diseases, Fuwai Hospital, National Center for Cardiovascular Diseases, Chinese Academy of Medical Sciences and Peking Union Medical College}

Objective To evaluate the autologous blood salvage effects of Xijing XJ-12-05 type cell saver device during cardiac surgery.

Methods 68 patients were divided randomly 2 groups according to the different cell saver devices: Group XJ (XJ-12-05, Xijing, $n=34$ ), Group CS (Cell saver 5+, USA, $n=34$ ). In order to compare the effects autologous blood salvage between the two different cell saver devices. Processed blood were analyzed for the content of hemoglobin $(\mathrm{Hb})$, hemotacrit (Hct), and the change of hemorheology, as well as the salvage rate of red blood cell (RBC) and the clearance rate of free hemoglobin (FHb), Glu, BUN, lactate (Lac).

Result The contents of $\mathrm{Hb}$ and Hct in Group XJ were higher than that in Group CS $(\mathrm{P}<0.05)$. No significant differences were observed in the other indexes.

Conclusion The RBC concentration ability of Group XJ was better than that of Group CS. The concentration ability was same between the two Groups. There were no significant differences among the other indexes.

\section{Perioperative dexmedetomidine not improves long term outcomes in chinese cardiac surgery Fei Xu, Hushan Ao, Qian Wang, Sipeng Chen, Qingshui Zheng, Yang Wang Department of Anesthesiology, State Key Laboratory of Cardiovascular Disease Fuwai Hospital National Center for Cardiovascular Diseases Chinese Academy of Medical Sciences and Peking Union Medical College}

Background Dexmedetomidine (DEX) is a novel type of a2-adrenergic receptor agonist with a high selectivity has been predominantly used as a sedative or adjuvant anesthetic drug in clinical settings. It is widespread use in the perioperaive period, howeverits shortterm outcomes and long-term outcomes remains uncertainty in cardiac surgeries.

Methods This study was a single center, retrospective and cohort study involving 1953 consecutive patients who underwent cardiac surgery at Fuwai Hospital from June 2012 to September 2012. 1477 patients met the inclusion criteria and were divided into two groups: those who received dexmedetomidine (DEX group, $\mathrm{n}=1077$ ) or those who did not receive dexmedetomidine (Non-DEX group, $n=400$, ) during the perioperative period. All patients were followed up by 1 year. Short-and long-term outcomes were compared between Dex and nonDex. Data were adjusted with propensity scores, and multivariate logistic 
regression was used.

Result Compared with non-Dex, the observed reduction postoperative atrial fibrillation $(\mathrm{P}=0.0419)$ in patients receiving perioperative DEX. Other effects of Dex on perioperative mortality and cardiovascular complication were not statistically significant. After 1 year follow-up, no differences of all-cause deaths (adjusted $\mathrm{HR}=1.89$; 95\% CI: $0.71-5.05 ; \mathrm{P}=0.7000)$ and MACCE were noted between two groups (adjusted $\mathrm{HR}=1.11 ; 95 \% \mathrm{CI}: 0.66-1.85 ; \mathrm{P}=0.7193$ )

Conclusion Compared with non-Dex, Dex is associated with small short-term gain as reduced postoperative atrial fibrillation occurrence but not effective for long-term risks of mortality and cardiovascular complications.

\section{Impact of the pulmonary artery catheter in CABG patients}

Fei Xu, Hushan Ao, Sipeng Chen

Department of Anesthesiology, State Key Laboratory of Cardiovascular

Disease Fuwai Hospital National Center for Cardiovascular Diseases

Chinese Academy of Medical Sciences and Peking Union Medical

College

Background Over the past 30 years the pulmonary artery catheter (PAC) has become a widely used for monitoring of hemodynamic in patients undergoing coronary bypass graft (CABG) surgery though recent studies have raised concerns regarding both effectiveness and safety. Our aim was, therefore, to determine the impact of PAC use on short- and long-term outcomes in patients undergoing CABG.

Methods 861 Chinese who consecutively underwent isolated, primary CABG at the Cardiovascular Institute of Fuwai Hospital from June 1, 2012 to September 31, 2012 were included in this study. Propensity score matched-pair analysis was used to adjust for differences in likelihood of PAC insertion, from which propensity paring yielded 228 matched pairs, for a total of 456 patients with comparable risk factors. Short (in-hospital) and long-term (1-year) major post-operative complications and mortalities were compared between PAC and nonPAC groups after initialrevascularization

Result Patients managed with PAC received intraoperative vasoactive drugs more frequently: adrenaline $(7.89 \%$ vs $3.07 \%, \mathrm{P}=$ $0.027)$ and noradrenaline ( $8.77 \%$ vs $3.95 \% \mathrm{P}=0.043)$. In addition, $\mathrm{PAC}$ patients were also associated with prolonged postoperative stay $(11.57 \pm$ 5.325 vs $8.97 \pm 6.793, \mathrm{P}<0.01)$. We noted no difference for in-hospital plus 1 -year mortality and other morbidity.

Conclusion Our findings indicate no clear evidence of benefit or harm by managing CABG patients with PAC. A randomized controlled trial would be ideal to either confirm or refute our findings. 


\section{Cardiovascular Drug Therapy}

\author{
Impact of coronary angiography and stent \\ implantation on platelet aggregation in $\mathrm{CHD}$ \\ patients under chronic clopidogrel therapy \\ Xiaoxing Zhang, Hong Liu, Lirong Yan, Dongxue Wang, \\ Xiaoyuan Guan, Jue Chen, Jinqing Yuan, Yishi Li \\ State key Laboratory of Clinical Trial Research in Cardiovascular Drugs, \\ Ministry of Health, State Key Laboratory of Cardiovascular Diseases, \\ Fuwai Hospital, National Center for Cardiovascular Diseases, Chinese \\ Academy of Medical Sciences and Peking Union Medical College
}

Objective To evaluate the impact of coronary angiography and stent implantation procedure on platelet aggregation in coronary heart disease (CHD) patients under chronic Clopidogrel therapy.

Methods CHD patients administered in Fuwai hospital who had been treated with clopidogrel $(75 \mathrm{mg} / \mathrm{d})$ and aspirin $(100 \mathrm{mg} / \mathrm{d})$ for at least 7 consecutive days undergoing elective percutaneous coronary intervention (PCI) were prospectively enrolled during October 2012 to May 2013. Based on whether a coronary stent was implanted, all of the included patients were assigned into two groups: the coronary angiography (CAG) group and the coronary stent implantation (CSI) group. The differences in platelet aggregation (PA) as measured by the $10 \mu \mathrm{mol} / \mathrm{L}$ ADP induced light transmission aggregation between the preoperative baseline level and the level at $24 \mathrm{~h}$ after the procedures in both patient groups were determined.

Result A total of 343 patients receiving coronary intervention were enrolled in this study, including 173 patients in the CAG group and 170 patients in the CSI group. Compared with the preoperative baseline, there was no significant difference in PA after the operation in the CAG group $[(54.21 \pm 11.44) \%$ vs $(53.15 \pm 11.80) \%, \mathrm{P}=0.062]$, while a significant postoperative PA increase was observed in the CSI group [(55.59 \pm 10.47$) \%$ vs $(52.47 \pm 11.97) \%, \mathrm{P}<0.001)$. Compared with the CAG group, the PA increase in the CSI group was significantly greater (the mean PA increase was $2.07 \%, 95 \%$ CI: $0.40 \%-3.74 \% ; \mathrm{P}=0.010$ ). A gradually increasing trend in the postoperative PA related to the number of coronary stents implanted was observed without significant difference $[(54.76 \pm 10.52) \%$ at 1 stent, $(55.44 \pm 10.50) \%$ at 2 stents, $(57.57 \pm 10.34) \%$ at 3 or more stents].

Conclusion The operation of coronary stent implantation induced a higher platelet reactivity increase than coronary angiography in $\mathrm{CHD}$ patients treated with Clopidogrel.

\section{Frequency of warfarin associated gene polymorphisms in chinese han patients \\ Yan Li, Ying Lou, Lulu Han, Xiaowei Xiong, \\ Xiaoxing Zhang, Lirong Yan, Hui Zhao, Yuexin Jiang, Yishi $\mathrm{Li}$ \\ Key Laboratory of Clinical Trial Research in Cardiovascular Drugs, Ministry of Health, State Key Laboratory of Cardiovascular Diseases, Fuwai Hospital, National Center for Cardiovascular Diseases, Chinese Academy of Medical Sciences and Peking Union Medical College}

Objective The known two significant genes influencing warfarin dose are *2 and *3 allele of Cytochrome P450 (CYP) 2C9 [CYP2C9] and vitamin $\mathrm{K}$ epoxide reductase complex subunit 1 (VKORC1). Cytochrome P450 4F2 (CYP4F2) is a minor significant genetic factor of warfarin dose. The aim of this research was to determine the frequency of the above three genes polymorphisms in Chinese Han patients.

Methods Inpatients and outpatients from 27 provinces undergoing anticoagulation with warfarin were recruited. And the clinical and demographic information was collected. DNA was extracted from whole blood samples. All the genes were genotyped by using TaqMan MGB probe. We tested the single nucleotide polymorphisms (SNPs) including *2 (C430T) and *3 (A1075C) allele of CYP2C9, VKORC1 (C1173T) and CYP4F2 (rs2108622 C > T). For all statistical comparisons, a P value $<0.05$ was considered significant. We used a chi-squared analysis to compare the prevalence of SNPs in the male and female groups and test for Hardy-Weinberg Equilibrium. All statistic analysis was preformed in SPSS 19.0.

Result Nine hundred and nine patients receiving warfarin therapy were genotyped including 454 males (49.9\%) and 455 females (50.1\%) of whom 311 person $(68.4 \%)$ have hit menopause. The enrolled population aged $(54.1 \pm 12.9) \mathrm{yrs},(164.9 \pm 8.2) \mathrm{cm}$ high, and weight $(65.9 \pm 12.5) \mathrm{kg}$. The results are in Hardy-Weinberg equilibrium. The frequencies of detected gene polymorphiams were as follows: VKORC1 (177/909) 19.5\%; CYP2C9*2 (6/909) 0.7\%, CYP2C9*3 (78/909) 8.6\%; CYP4F2 (433/909) 47.6\%. No gender differences in polymorphism frequency had been found in VKORC1 (93 in males and 84 in female, $\mathrm{P}$ $=0.66), \mathrm{CYP} 2 \mathrm{C} 9 * 2$ ( 5 in males and 1 in female, $\mathrm{P}=0.10), \mathrm{CYP} 2 \mathrm{C} 9 * 3$ ( 36 in males and 42 in female, $\mathrm{P}=0.71$ ), CYP4F2 (218 in males and 215 in female, $\mathrm{P}=0.96)$. The genotype results had been compared with other researches and populations.

Conclusion The results are in accordance with other studies that the mutation of VKORC1 is less than Caucasian people but the mutation of CYP2C9*3 is more than Africa-America.

\footnotetext{
Hemodynamic effects of short-term infusion of recombinant human atrial natriuretic peptide (rhANP) for congestive heart failure: a randomized, double-blind, placebo-controlled study

Pengbo Wang ${ }^{1}$, Guogan Wang ${ }^{1}$, Jie Huang ${ }^{1}$, Huiqiong Tan ${ }^{1}$, WenXian Liu ${ }^{2}$, Jian Zhang ${ }^{1}$, Zhongkai Liao ${ }^{1}$, Litian $\mathrm{Yu}^{1}$, Yi Mao ${ }^{1}$, Shaodong Ye ${ }^{1}$, Lei Feng ${ }^{1}$, Yuhui Zhang ${ }^{1}$, Yang Wang ${ }^{1}$, Wei Li ${ }^{1}$, Xiaojun Luan ${ }^{1}$, Sijia Wang ${ }^{1}$, Shugong Bai ${ }^{2}$

1. State Key Laboratory of Cardiovascular Diseases, Fuwai Hospital, National Center for Cardiovascular Diseases, Chinese Academy of Medical Sciences and Peking Union Medical College

2. Beijing Anzhen Hospital
}

Objective This study was designed to confirm hemodynamic effects of short-term infusion of recombinant human atrial natriuretic peptide (rhANP) at the prespecified dose in patients with congestive heart failure compared to placebo when both were added to standard care.

Methods After the placement of a Swan-Ganz catheter, 121 patients with congestive heart failure were randomly assigned to doubleblind treatment with placebo or rhANP [initiated at a rate of $0.1 \mu \mathrm{g}$ / ( $\mathrm{kg} \cdot \mathrm{min}$ ), adjusted to $0.15 \mu \mathrm{g} /(\mathrm{kg} \cdot \mathrm{min})$ half an hour later if the systolic blood pressure was more than $100 \mathrm{~mm} \mathrm{Hg}$ and the PCWP was 15 $\mathrm{mm} \mathrm{Hg}$ or higher, and stopped one hour later from the initiation]. The 
hemodyamic parameters were measured at $0.5,1,3,6$ and $12 \mathrm{~h}$ after the start of study drug. The primary end-point was PCWP at $1 \mathrm{~h}$. Adverse events were monitored through study, and mortality was assessed through a month.

Result 93 patients were randomized to rhANP group and 28 to the placebo group at a ratio of 3: 1 using block of size 4 . Baseline characteristics were similar among patients in the study groups, and PCWP were $(23.71 \pm 7.0) \mathrm{mm} \mathrm{Hg}$ and $(25.66 \pm 8.78) \mathrm{mm} \mathrm{Hg}$ in rhANP and placebo group, respectively $(\mathrm{P}=0.226)$. The mean reduction in PCWP was greater with rhANP $(-5.45 \mathrm{~mm} \mathrm{Hg})$ than placebo $(-2.03$ $\mathrm{mm} \mathrm{Hg}$ ) at $30 \mathrm{~min}$, and there was significant difference for the mean PCWP between groups $(\mathrm{P}=0.002)$. The maximum decrease of PCWP in rhANP group was observed at $1 \mathrm{~h}(-7.74 \mathrm{~mm} \mathrm{Hg}$ vs $-1.82 \mathrm{~mm} \mathrm{Hg}$ with placebo), and the mean PCWP of the two groups were significantly different $(\mathrm{P}<0.001)$. At $3 \mathrm{~h}$, PWCP was sustainedly lower in rhANP group $(19.52 \pm 6.55) \mathrm{mm} \mathrm{Hg}$ than in placebo group $(24.79 \pm 8.42) \mathrm{mm}$ $\mathrm{Hg}(\mathrm{P}<0.001)$. However, no significant differences were found between the two groups for PCWP at 6 hours [ $(21.43 \pm 6.51) \mathrm{mm} \mathrm{Hg}$ and $(24.79$ $\pm 10.64) \mathrm{mm} \mathrm{Hg}, \mathrm{P}=0.125]$. The rate of hypotension and other adverse event were even in the two groups $(\mathrm{P}=0.111)$.

Conclusion The short-term infusion of rhANP has prompt hemodynamic improvement in patients with congestive heart failure compared to placebo added to standard care.

\section{Angiotensin II type 1 receptor blocker attenuates cardiac remodeling through DLL4/ notch1 activation in pressure overload mice Jieyun You, Jian Wu, Guoliang Jiang, Shijun Wang, Lei Li, Junbo Ge, Yunzeng Zou \\ Shanghai Institute of Cardiovascular Diseases, Zhongshan Hospital, \\ Fudan University}

Background Notch1 signaling controls the cardiac adaptation to stress. We aimed to validate whether angiotensin II type 1 receptor blocker ameliorates cardiac remodeling and dysfunction via DLL4/ Notchl pathway in mice with chronic pressure overload.

Methods Cardiac pressure overload was produced by transverse aortic constriction (TAC). A total of 35 wide-type C57BL/6 J mice were randomly divided into sham group, TAC group, TAC+olmesartan group (olmesartan: angiotensin II type 1 receptor blocker) and TAC+olmsartan+DAPT group (DAPT: Notch signaling inhibitor). Saline $(10 \mathrm{ml} /(\mathrm{kg} \cdot \mathrm{d})$ or the same volume of olmesartan liquor $3 \mathrm{ml} /$ $(\mathrm{kg} \cdot \mathrm{d})]$ was administered by gavage, and DAPT $(10 \mu \mathrm{mol} /(\mathrm{kg} \cdot \mathrm{d})$ by peritoneal injection. After 28 days of treatment, cardiac hemodynamics, echocardiography and histology were evaluated, followed by qPCR of fetal gene (ANP and SAA) expression. Notch1-related proteins and ERK1/2 were examined by western blot, and the serum level of AngII was determined by ELISA Kits.

Result Persistent pressure overload induced left ventricular hypertrophy, dysfunction, fibrosis and microcirculation dysfunction, together with upregulation of AngII, ERK1/2 and fetal gene expression. By the activation of DLL4/Notch1, olmesartan decreased left ventricular hypertrophy and fibrosis, preserved cardiac function, and improved capillary density and coronary perfusion. All these curative effects were suppressed by pharmacological blockade of Notch signaling with DAPT.

Conclusion Our findings identify a heretofore unknown pharmacological mechanism that olmesartan improves cardiac remodeling and function via DLL4/Notch1 pathway activation in mice with chronic pressure overload, which may present a new therapeutic target for hypertension.

\section{Protective effects of blueberry anthocyanin on ang II -induced apoptosis to human umbilical vein endothelial cells \\ Huan Lin, Jiyan Leng, Jian Du \\ The First Hospital of Jilin University}

Objective To establish HUVEC apoptosis model, using Western blot to detect changes of apoptosis-related protein Bcl-2, Bax and caspase-3 before and after Ang II caused HUVEC apoptosis. Measure the content of intracellular ROS after Ang II reaction, and observe the impact of blueberry anthocyanin in above circumstances, investigate the protective effect of blueberry anthocyanin in HUVEC.

Methods Chosen human umbilical vein endothelial cells, conventional recovery and passaged, setup HUVEC apoptosis model. Cell groups: in vitro cultured human umbilical vein endothelial cells (HUVEC) were divided into control group (Control), AngII group (AngII), blueberry anthocyanin Group (BBA), AngII+ blueberry anthocyanin group (AngII+BBA); Determine the drug concentration for the next experiment: Using MTT assay to check out cell activity, determine the optimal concentration of AngII in inducing HUVEC apoptosis and the effectively protect concentration of blueberry anthocyanin; Cell apoptosis were detected by Annexin V-FITC-labeled flow cytometry; Using Western Blot to detect the expression of Bcl-2, Bax and Caspase-3 protein; To detect the intracellular ROS level.

Results HUVEC apoptosis was significantly increased after AngII stimulated. Compared to AngII group, Bcl-2 protein level of the blueberry anthocyanin Group was significantly increased, the expression of Bax and Caspase-3 protein decreased, the intracellular ROS level also in a downward trend.

Conclusion AngII can induce cultured endothelial cells apoptosis. AngII induced HUVEC apoptosis, may be related to the imbalance expression of $\mathrm{Bcl}-2$ and Bax protein and Caspase- 3 activation. Blueberry anthocyanin has inhibition effect of HUVEC apoptosis, the blueberry anthocyanin can protect vascular endothelial cells, which play a role in anti-atherosclerosis. 


\section{Cardiovascular Imaging and Laboratory Medicine}

\author{
The markedly enlarged pulmonary artery: case \\ report and literature review \\ Jiang Rong, QinHua Zhao, Lan Wang, Suang gong, Jing \\ $\mathrm{He}$, Jinming Liu \\ Department of Cardio-Pulmonary Circulation, Shanghai Pulmonary \\ Hospital, Tongji University, School of Medicine, Shanghai, China.
}

Extrinsic compression of the left main coronary artery (LMCA) can occur in patients with severe pulmonary hypertension $(\mathrm{PH})$ and enlarged pulmonary artery trunk which have been treated with LMCA stent implantation for the management of this condition. Such cases have been reported. We also performed a literature review of the reported cases concerning patients treated with LMCA stent implantation for the management of this condition. However, we report a case of not extrinsic compression of the LMCA in a symptomatic patient with Idiopathic pulmonary artery hypertension (IPAH). The IPAH patient also complained of left heart dysfunction. However her PH was IPAH not due to her left heart disease. The case highlighted dualism. We report the case of severe IPAH with an unusually long survival of nearly 30 years. We analyze the possible explanation. Severe dilation of the main pulmonary arteries may be related to long-term survival with idiopathic pulmonary arterial hypertension. This remodelling of pulmonary vasculature may represent a compensatory adaptive response to high pulmonary artery pressures and an attempt to optimize hemodynamics and reduce right heart dysfunction. Pulmonary arterial capacitance (PAC) describes the degree to which the pulmonary arterial tree dilates during systole. It could be postulated that the dilation of the pulmonary arteries seen in our patients was also a reflection of increased total PAC that resulted in improved right ventricular function.

\section{The effect of cardiac resynchronization therapy on left ventricular diastolic function: assessed by two-dimensional speckle tracking echocardiography \\ Tingyu Lan, Hao Wang, Xin Sun \\ State Key Laboratory of Cardiovascular Disease, Fuwai Hospital, National Center for Cardiovascular Disease, Chinese Academy of Medical Sciences and Peking Union Medical College}

Background There are conflicting data about the effects of CRT on LV diastolic function, and definition of response to CRT is still a controversial topic. The present study evaluated the effect of CRT on LV diastolic function in different degrees of response, focusing on the changes in novel global load-independent indices of LV relaxation as measured by 2D speckle tracking echocardiography.

Methods 100 patients with New York Heart Association class II to IV heart failure, LV ejection fractions $\leq 35 \%$, underwent echocardiography before CRT and 12 months follow-up. The following indices of LV diastolic function were obtained from 2D speckle tracking: early diastolic strain rate and atrial contraction strain rate. We divided our population into 3 subgroups. A group: reduction of LV end-systolic volume (LVESV) of $\geq 15 \%$ at follow-up, and an increase of $\geq 20 \%$ in the distance walked at the 6-minute walk test or, if that test was not performed, an improvement of $\geq 1$ NYHA functional class compared with baseline; B group: an increase of $\geq 20 \%$ in the distance walked at the 6-minute walk test or, if that test was not performed, an improvement of $\geq 1$ NYHA functional class compared with baseline, but reduction of LV end-systolic volume (LVESV) of $<15 \%$ at follow-up; C group: reduction of LV end-systolic volume (LVESV) of $<15 \%$ at followup, and increase of $<20 \%$ in the distance walked at the 6-minute walk test or, if that test was not performed, an improvement of $<1$ NYHA functional class compared with baseline.

Result An improvement in estimates of LV filling pressure and a decrease in left atrial dimensions were observed only in responders to CRT. Patients with clinical but no echocardiographic response had significant improvement in E-wave and deceleration time and nonsignificant improvement in other parameters. Compared with early diastole strain and atrial contraction strain rate parameters, LSRE exhibited the highest correlation with E/E'. Compared to other parameters LSRE yielded a significant higher area under the ROC curve.

Conclusion Diastolic function was significantly improved only exists in the CRT echocardiography responders. The LSRE had greater value in prediction of left ventricular function improved, which could be used as the best parameter to predict response to CRT therapy in heart failure patients.

\section{Septal reverse mismatch of myocardial perfusion and metabolism in patients with coronary artery disease and left bundle branch block}

Jianguang Wang ${ }^{1}$, Wei Fang ${ }^{1}$, Minfu Yang ${ }^{2}$, Yueqin Tian ${ }^{1}$, Xiaoli Zhang ${ }^{1}$, Rui Shen ${ }^{1}$, Xiaoxin Sun ${ }^{1}$, Feng Guo ${ }^{1}$,

Daoyu Wang ${ }^{1}$, Zuoxiang $\mathrm{He}^{1}$

1. Fu Wai Hospital, National Center for Cardiovascular Diseases, Beijing 2. Beijing Chaoyang Hospital

Objective Left bundle branch block (LBBB) affects on the myocardial perfusion of left ventricle, however, its effect on myocardial metabolism of left ventricle has not been well investigated. This study aimed to evaluate the effect of LBBB on myocardial perfusion and metabolism in patients with coronary artery disease (CAD).

Methods 65 patients with complete LBBB (mean age of $58 \pm 19.8$ ) underwent ${ }^{99 \mathrm{~m}} \mathrm{Tc}-\mathrm{MIBI}$ single photon emission computed tomography, ${ }^{18} \mathrm{~F}-\mathrm{FDG}$ positron emission tomography were retrospectively. All of them underwent contrast coronary angiography. The relationship between myocardial perfusion and metabolism were assessed, and the relationship between reverse mismatch score and QRS length and between reverse mismatch score and wall motion scores were evaluated.

Result The reverse mismatch of myocardial perfusion and metabolism in the left ventricular septum was observed in $56.9 \%$ of the total cohort. The septal reverse mismatch of myocardial perfusion and metabolism was observed in $100 \%$ of patients with normal coronary angiography or absence of LAD stenosis, in $50 \%$ of patients with LAD stenosis but normal anterior/septal myocardial perfusion, and in 54\% of patients with LAD stenosis and abnormal anterior and/or septal myocardial perfusion respectively. The septal reverse mismatch was observed in $82.8 \%$ of those patients with normal myocardial perfusion, and $36.1 \%$ of those patients with abnormal myocardial perfusion 
respectively. The correlation between reverse mismatch score and QRS length was significant in the septum $\mathrm{r}=-0.371, \mathrm{P}=0.005$.

Conclusion In patients with CAD and LBBB, the septal reverse mismatch of myocardial perfusion and metabolism was frequently present. The reverse mismatch score of the septum negatively correlates with the QRS interval.

\section{Study in the anatomy and morphology in patients with paroxysmal atrial fibrillation \\ Yankun yang, Fan Yang, Hong Zheng, Bin Lv, Tian Lan, \\ Xinling Yang \\ Fuwai hospital department of radiology}

Objective To explore the anatomical and morphological characteristics in patients with PAF and provide a direction for LAA occlusion.

Methods 46 patients with AF prior to RFCA (radiofrequency catheter ablation) and 92 patients in snus rhythm were enrolled prospectively from 15th December 2012 and 15th April, all patients were performed with CTCA (CT coronary angiography), postprocedure images were analysed, the morphology of LAA were classified into different types and related anatomical data were measured.

Result LAA morphology: Chicken-wing (55, 9.9\%), Cauliflower (40, 29\%), Cactus (17, 12.3\%), Windsock (26, 18.8\%). Anatomical relationship of the orifice of left atrial appendage to left superior pulmonary vein (LSPV): high type (superior to LSPV, 2.2\%), middle type (parallel to LSPV, 70.3\%), low type (inferior to LSPV, 27.5\%). LAA orifice morphology: oval (122, 88.4\%), round-like (12, 8.7\%) and drop-like $(4,2.9 \%)$. Analysis of anatomical parameter values: Patients with atrial fibrillation have a larger LAA volume than who with sinus rhythm. Patients with PAF have a larger LA volume $\left(97.32 \pm 26.12 \mathrm{~cm}^{3}\right.$ vs $\left.85.67 \pm 17.87 \mathrm{~cm}^{3}\right)$, a larger LAA volume $\left(10.88 \pm 4.09 \mathrm{~cm}^{3}\right.$ vs $9.20 \pm$ $\left.3.33 \mathrm{~cm}^{3}\right)$ and a larger diameter of LAA $(26.42 \pm 4.70 \mathrm{~mm}$ vs $24.05 \pm 5.02$ $\mathrm{mm}$ ) orifice than patients with sinus rhythm. There is a slightly positive correlation between LAV and LAAV, and the correlation coefficient $(r)$ is $0.291(\mathrm{P}<0.01)$. There is a moderate positive relationship between LAA orifice area and LAAV, $\mathrm{r}=0.869(\mathrm{P}<0.001)$. There is no significant correlation between age and LAAV, and there was no significant gender differences in LAAV.

Conclusion LAA has a complicated structure with large heterogeneity. Patients with PAF have a larger LAAV than patients with sinus rhythm.

\section{Left ventricular diastolic function improves after septal myectomy in patients with hypertrophic obstructive cardiomyopathy \\ Jingjin Wang, Xin Sun, Hao Wang, Shuiyun Wang, \\ Minghu Xiao, Hongtao Sun, Haibo Chen \\ Fuwai Hospital, National Center for Cardiovascular Diseases, Chinese \\ Academy of Medical Sciences and Peking Union Medical College, \\ Beijing, China}

Objective Left ventricular outflow tract obstruction, mitral regurgitation, small coronary disease, and ischemia are factors of left ventricular diastolic dysfunction in hypertrophic obstructive cardiomyopathy (HOCM). Echocardiographic Tissue Doppler image provides direct information on myocardial function and improves the diagnostic of diastolic dysfunction in HOCM patients. The objective of this study was to evaluate the improvement of left ventricular diastolic function after Modified Morrow Surgery by echocardiography.

Methods A total of 70 consecutive patients (45 male and 25 female) underwent Modified Morrow Surgery were enrolled. The mean age of the 70 patients was $42 \pm 14$ years (10-67years), We collected echocardiographic images before and after surgery (in 15 days). Left ventricular diastolic function was evaluated by echocardiography based on American Society of Echocardiography Guidelines.

Result After Modified Morrow, Left ventricular outflow gradient decreased dramatically (from $77.5 \pm 30.4 \mathrm{~mm} \mathrm{Hg}$ to $13.3 \pm 5.7 \mathrm{~mm} \mathrm{Hg}$, $\mathrm{P}<0.05$ ), left atrium diameter decreased (from $44.5 \pm 7.1 \mathrm{~mm}$ to $38.7 \pm$ $6.7 \mathrm{~mm}, \mathrm{P}<0.05$ ), Peak early diastolic mitral annulus velocity (lateral e') increased (from $6.2 \pm 1.9 \mathrm{~cm} / \mathrm{s}$ to $7.3 \pm 2.2 \mathrm{~cm} / \mathrm{s}, \mathrm{P}<0.05$ ), Peak early diastolic mitral flow (E) / Peak early diastolic mitral annulus velocity ( $\mathrm{e}^{\prime}$ ) decreased (from $16.2 \pm 5.4$ to $13.6 \pm 5, \mathrm{P}<0.05$ ), Mitral regurgitation decreased (from moderate to none, $\mathrm{P}<0.05$ ). Patients had significant symptomatic alleviation.

Conclusion In patients with HOCM, Modified Morrow Surgery relieved obstruction, alleviated patients' symptoms, reduced mitral regurgitation. Left atrial diameter and E/e' decreased, demonstrating the improvement of left ventricular diastolic function.

\section{Double chambered left ventricle: diagnosis, classification and treatment \\ Hong Meng, Jianrong Li, Kunjing Pang, Hao Wang Department of Echocardiography, State Key Laboratory of \\ Cardiovascular Disease, Fuwai Hospital, National Center for Cardiovascular Diseases, Chinese Academy of Medical Sciences and Peking Union Medical College, Beijing}

Objective Left ventricular (LV) morphological anormalies co mprise a heterogeneous group of diseases that include LV diverticula, congenital or acquired LV aneurysms and double chambered left ventricle (DCLV). DCLV is characterized by the subdivision of the left ventricular (LV) cavity into two chambers. Here, we report a single institution's experiences to highlight the imaging morphologies of DCLV, describe the associated congenital heart defects and clinical presentations , and discuss management plans.

Methods 7 patients diagnosed with DCLV at Fuwai Hospital were involved. Clinical data, electrocardiography (ECG), echocardiography and other imaging modalities were reviewed.

Result There were anormal ridges or bands in the LV inflow chambers, at mid or apical level. Two anatomic varieties of DCLV were exhibited in our series of patients. In the first type, intraventricu lar divisions were due to abnormal muscle bands. In the second type, abnormal fibrous ridges partitioned LV chamber. In most cases, the normally contractile LV main chambers (LV ejection fraction, EF, 60\%$65 \%$ ) received mitral inflow and ejected to the aorta, while the apical one only had an orifice communicating with the main chamber during sys tole and diastole. There were 2 cases, the apical accessory chambers were akinetic, different from the other 5 patients who had contractile apices. We found the flow could freely cross the bundles in five cases: peak velocity range of $1.2-1.9 \mathrm{~m} / \mathrm{s}$ in diastole and $1.3-2.6 \mathrm{~m} / \mathrm{s}$ in systole, however, there were mild obstruction in 2 patients, peak systolic velocity were 5.2 and $3.0 \mathrm{~m} / \mathrm{s} .3$ patient in our study suffered from th e other congenital heart disorders: multiple ventricular septal defects (perimembranous and 'swiss-cheese' muscular VSD), double cha mbered right ventricle (DCRV), and pulmonary vavular stenosis. These two patients underwent cardiac surgeries: recetion of anormal 
right ventricular hypertrophied muscle and VSD repair. No further surgi cal procedures for $\mathrm{LV}$ abnormal muscles or accessory chamber resection were taken. During a 1.5-year follow-up, these patients had nonspecific complainings, normally contractile main chambers, and no significant en largements of chambers.

Conclusion DCLV has distinct appearances that can be easily identified on TTE. It can occur in isolation or in conjunction with other congenital lesions, as we have demonstrated in our study. Furthermore, the treatment and prognosis of DCLV might depend on the symptoms, the severity of the obstruction and the associated cardiac diseases.

\section{Low backscattering detected by optical coherence tomography may be valuable in predicting prognosis of patients after $\mathrm{PCl}$}

\author{
Dewei Wu, Mengyue Yu, Jie Qian, Shubin Qiao, \\ Yuejin yang, Yongjian wu \\ National Center for Cardiovascular Diseases and Fuwai Hospital, \\ Chinese Academy of Medical Sciences and Peking Union Medical \\ College
}

Backgrounds Morphologic characteristics of stent restenosis by optical coherence tomography (OCT) ranges among patients. Low backscattering is an infrequent but poorly understood morphology pattern in OCT images. We investigated whether factors influencing low backscattering can be identified and whether clinical outcome is different.

Methods Patients presenting stent restenosis documented by OCT reexamination after percutaneous coronary intervention (PCI) were included from February 2009 through September 2011. Backscattering of restenotic tissue was qualitatively analyzed and classified into two patterns, namely, low backscattering and high backscattering. White blood cells, platelet, glycated hemoglobin $\left(\mathrm{HbA}_{1 \mathrm{c}}\right)$, hsCRP, Serum total cholesterol (TC), triglyceride, low density lipoprotein (LDL) and creatinine were analyzed in order to assess their contribution to backscattering characteristics. Follow-up were performed in patients who didn't receive revascularization during OCT reexamination. We prospectively evaluated the relationship between morphologic patterns and prognosis of the patients.

Result 128 patients undergoing OCT reexamination $2.2 \pm 2.6$ years after stent implantation were included. Low backscattering was detected in $22(17.2 \%)$ patients. The incidence of low backscattering was positively correlated with stent implantation time $(\mathrm{P}<0.001)$. Higher serum TC $(\mathrm{OR}=2.592,95 \% \mathrm{CI}: 1.239-5.423, \mathrm{P}=0.011)$ and LDL $(\mathrm{OR}=2.686,95 \% \mathrm{CI}: 1.158-6.230, \mathrm{P}=0.021)$ were associated with more frequent low backscattering. 84 patients who didn't undergo revascularization during reexamination were followed-up for 3.7 \pm 0.5 years. Major adverse cardiac events (MACEs) occurred in 7 (7.3\%) patients with a predominance of target-vessel revascularization while showed no correlation with OCT morphology patterns. 16 (16.7\%) patients experienced unstable angina (UA) and showed great significance with low backscattering $(\mathrm{RR}=3.48,95 \% \mathrm{CI}: 1.033-11.722$, $\mathrm{P}=0.044)$.

Conclusion OCT detected low backscattering is positively correlated with stent implantation time. Higher serum TC and LDL are associated with higher incidence of low backscattering, which is associated with more frequency of UA. Low backscattering should be paid more attention in the future, it may be valuable in predicting prognosis of patients after PCI.

\section{Serum levels of NOX4 are associated with non-valvular atrial fibrillation \\ Tong Liu ${ }^{1}$, Qingmiao Shao ${ }^{1}$, Panagiotis Korantzopoulos ${ }^{2}$, Enzhao Liu ${ }^{1}$, Gang $\mathrm{Xu}^{1}{ }^{1}$, Guangping $\mathrm{Li}^{1}$ \\ 1. Tianjin Key Laboratory of lonic-Molecular Function of Cardiovascular disease, Department of Cardiology, Tianjin Institute of Cardiology, \\ Second Hospital of Tianjin Medical University, China \\ 2. Department of Cardiology, University of loannina Medical School, loannina, Greece}

Background Recent evidence indicates that NADPH oxidases (NOX) -derived reactive oxygen species (ROS) play a pivotal role in the development of atrial fibrillation (AF). We sought to investigate the potential association between serum levels of NOX4, as well as inflammatory biomarkers, and AF.

Methods We enrolled 108 patients with AF (71 with paroxysmal $\mathrm{AF}$ and 37 with persistent/permanent $\mathrm{AF}$ ) and 68 patients without $\mathrm{AF}$ as controls. Demographic, clinical, laboratory, electrocardiographic and echocardiographic characteristics were carefully recorded. Serum levels of myeloperoxidase (MPO), hs-CRP and NOX4 were assessed.

Result Left atrial diameter (LAD), left ventricular end diastolic diameter (LVEDD) and $\mathrm{P}$ wave dispersion (PWD) were significantly increased in patients with paroxysmal AF and persistent/permanent AF compared with controls while NOX4 levels were significantly higher in patients with paroxysmal $\mathrm{AF}$ and persistent/permanent $\mathrm{AF}$ compared to controls $(155.57 \pm 90,155.88 \pm 64.79$ vs $126.72 \pm 23.51$ $\mathrm{pg} / \mathrm{mL}$, respectively, $\mathrm{P}<0.05)$. A significant correlation between serum NOX4 levels and hs-CRP, and between NOX4 levels and MPO was also evident $(r=0.170 ; r=0.227, \mathrm{P}<0.05$, respectively). Multivariate analysis demonstrated that baseline serum NOX4 level was independently associated with paroxysmal $\mathrm{AF}(\mathrm{OR}=1.014,95 \% \mathrm{CI}$ : $1.001-1.027, \mathrm{P}<0.05)$ and with persistent/permanent $\mathrm{AF}(\mathrm{OR}=1.022$, $95 \%$ CI: $1.000-1.044, \mathrm{P}<0.05)$.

Conclusion There seems to be an association between increased NOX4 levels and AF, suggesting NOX4 involvement in the pathophysiology of human AF. Further studies are needed to elucidate its role in atrial remodeling and to examine its potential prognostic impact.

\section{Red blood cell distribution width predicts adverse outcomes in patients with heart failure: a systematic review meta-analysis \\ Tong Liu, Qingmiao Shao, Guangping Li Tianjin Key Laboratory of lonic-Molecular Function of Cardiovascular disease, Department of Cardiology, Tianjin Institute of Cardiology, Second Hospital of Tianjin Medical University, China}

Objective We aimed to perform a comprehensive meta-analysis to evaluate the potential association between red blood cell distribution width (RDW) and adverse outcomes in the patients with heart failure (HF).

Methods We conducted a systematic literature published August 2013 or earlier using electronic database (PubMed, Ovid, Embase and Web of Science) to identify the studies reporting on the association between RDW and adverse outcomes of the patients with HF. Prespecified adverse outcomes of interest included all-cause mortality, HF hospitalization, and combined adverse events included combined allcause mortality and HF hospitalization, all-cause mortality and heart transplantation, and cardiovascular death and HF hospitalization. 
Result We retrieved 17 studies (18 data sets), involving a total of 41311 participants. The results showed that high RDW level was associated with increased risk of all-cause mortality, HF hospitalization, and combined adverse events in the patients with HF. For every $1 \%$ increment in RDW, the risk of all-cause mortality, HF hospitalization, and combined adverse events increased by $12 \%(\mathrm{HR}=1.12,95 \% \mathrm{CI}$ : $1.08-1.16), 9.1 \%$ (HR $=1.091,95 \%$ CI: $1.025-1.162)$, and $18.9 \%$ (HR $=1.189,95 \%$ CI: $1.084-1.303)$ respectively. Subgroup analyses showed a significant association between RDW and all-cause mortality in the patients with acute $\mathrm{HF}(\mathrm{HR}=1.093,95 \% \mathrm{CI}: 1.040-1.150)$, but the association was even stronger in the patients with chronic HF (HR = 1.126, 95\% CI: $1.075-1.179)$

Conclusion Our meta-analysis suggests that the high level of RDW increases the risk of adverse outcomes in the HF patients, especially in chronic HF subjects.

\section{Evaluation of the left ventricular systolic function of stunned myocardium after ischemia and reperfusion in canines with $3 \mathrm{D}-\mathrm{STI}$ \\ Yuanyuan Xing, Yuhong Li Department of Ultrasound, First Affiliated Hospital of Liaoning Medical University}

Objective There were ischemia-reperfusion Dog Stunned myocardium model established, and Three-dimensional speckle tracking imaging (3D-STI) used to evaluate the local left ventricular function and overall, in this way, there was the structural and functional changes of stunned myocardial was clarified. This study will provide a new idea about the early and non-invasive diagnosis on angina.

Methods Left anterior descending arteries of 9 canines were ligated to induce myocardial ischemia for $15 \mathrm{~min}$, and followed by reperfusion. A series of two-dimensional and real-time three-dimensional ultrasound images strain results of the left ventricle before ischemia were collected, $5 \mathrm{~min}$ and $15 \mathrm{~min}$ after ischemia, $30 \mathrm{~min}, 60 \mathrm{~min}, 90 \mathrm{~min}$ and 120 min after reperfusion. Apply biplane Simpson's method to measure conventional indicators in the apical two-chamber view and fourchamber view: left ventricular end of the diastolic volume (LVEDV), left ventricular end of the systolic volume (LVESV), left ventricular diastolic diameter (LVDD), left ventricular systolic diameter (LVDS), left ventricular ejection fraction (LVEF). Using Three-dimensional speckle tracking imaging to detect peak longitudinal strain, peak radial strain, peak circumferential strain and peak area strain $\left(\mathrm{LS}_{\text {peak }}, \mathrm{RS}_{\text {peak }}\right.$, $\mathrm{CS}_{\text {peak }}, \mathrm{AS}_{\text {peak }}$ ) in ischemia [basal anterior (BA), mid anterior (MA), basal anteroseptal (BAS), mid anteroseptal (MAS) segment] and nonischemic [basal posterior (BP), mid posterior (MP) segment], the global peak strain $\left(\mathrm{GLS}_{\text {peak }}, \mathrm{GRS}_{\text {peak }}, \mathrm{GCS}_{\text {peak }}, \mathrm{GAS}_{\text {peak }}\right)$, and to analyze the correlationship of global peak strain with LVEF, so that we can evaluate the local left ventricular function and overall.

Result Comparison of left ventricular conventional indicators: LVESV, LVEDV, LVDS, LVDD increased during ischemia, the parameters have significant difference $(\mathrm{P}<0.05)$; LVEF decreased during ischemia, the parameters have significant difference $(\mathrm{P}<0.05)$, all parameters reached to normal value with $30 \mathrm{~min}$ reperfusion, there is no significant difference compared with before ischemia $(\mathrm{P}>0.05)$. Real time 3D-STI measure left ventricular total systolic function: The left ventricle global strain parameters are decreased during ischemia, there is significant difference compared with before $(\mathrm{P}<0.05)$. With the prolongation of reperfusion gradually restored. $\mathrm{GAS}_{\text {peak }}$ take the longest recovery time $(120 \mathrm{~min})$, there is no significant difference compared with before $(\mathrm{P}>0.05)$. The global strain parameters of 3D-STI before and after ischemia were positively correlated with LVEF, GAS peak $_{\text {has }}$ the highest correlation with LVEF. Real time 3D-STI measure left ventricular regional systolic function: $\mathrm{S}_{\text {peak }}$ Changes of the ischemia segment: The ischemia segments strain parameters of left ventricle are decreased during ischemia, there is significant difference compared with before $(\mathrm{P}<0.05)$, With the prolongation of reperfusion gradually restored, however, none of the $\mathrm{LS}_{\text {peak }}, \mathrm{RS}_{\text {peak }}, \mathrm{CS}_{\text {peak }}, \mathrm{AS}_{\text {peak }}$ recovered even in $120 \mathrm{~min}$, there is significance compared with before $(\mathrm{P}<0.05)$. $\mathrm{S}_{\text {peak }}$ Changes of the non-ischemia segment: The non-ischemia segments strain parameters of left ventricle are increased during ischemia, reached to normal value with $30 \mathrm{~min}$ reperfusion, there is no significant difference compared with before $(\mathrm{P}>0.05)$.

Conclusion Conventional ultrasound can only evaluate global left ventricular systolic function during ischemia, and 3D-STI is a better index to assess the local function of the stunned myocardium after ischemia and reperfusion. Total systolic function of left ventricular delayed recover after reperfusion, the myocardial stunning phenomenon. Regional systolic function of left ventricular stunned myocardial delayed recover after reperfusion, and local function recovery takes longer time than the total function. Area strain is the most sensitive 3D-STI parameter.

\section{Matrix metalloproteinase-2 and Adiponectin as biomarkers for coronary atherosclerotic plaques \\ Chun Gu, Lin Cai, Xuesong Fan, Fang Wang, \\ Xiangfeng Cong, Xi Chen \\ State Key Laboratory of Cardiovascular Disease, Fuwai Hospital, \\ National Center for Cardiovascular Disease, Chinese Academy of \\ Medical Sciences, Peking Union Medical College}

Objective To investigate the probability of serum MMP-2 and APN as the biomarkers for coronary atherosclerotic plaque.

Methods Patients of Fuwai Hospital, from September 2012 to November 2013, were divided into three groups through 64-slice spiral CT examination combined with clinical symptoms of ACS. Noncalcified plaque group: with symptoms of ACS, typical non-calcified plaque (CT value $<50 \mathrm{HU}$ ) on the main coronary arteries by coronary CT examination, without mixed or calcified plaque. Calcified plaque group: no symptoms of ACS, existence of typical calcified plaque (CT values $>50 \mathrm{HU}$ ), without non-calcified as well as mixed plaque. Normal control group: without clinical symptoms and history of other chronic diseases, normal function of liver and kidney, smoothcoronary and no plaques on CT. Fasting venous blood serum was separated and stored at $-80^{\circ}$ c. 20 cases were selected for each group. ELISA was used to detect serum levels of the plaque-related factors, including adiponectin (APN), matrix metalloproteinase-2 (MMP-2), myeloperoxidase (MPO) and tumor necrosis factor- $\alpha(\mathrm{TNF}-\alpha)$. The results were analyzed by SPSS17.0.

Result Univariate analysis showed that age, sex and biochemical parameters had no significant difference among the three groups. ELISA data indicated that although serum levels of MPO and TNF- $\alpha$ increased sequentially among the normal, calcified and non-calcified plaque groups and ANOVA showed no significant difference between the groups. While serum levels of MMP-2 and APN had a decreasing trend, which was statistically significant. Furthermore MMP-2 can distinguish the presence of plaques with $\mathrm{AUC}=0.728(\mathrm{P}=0.001)$, but not calcified plaques. APN in non-calcified plaque group was significantly lower than calcified plaque and normal groups by multiple comparison $(\mathrm{P}=0.017)$, 
and AUC $=0.721(\mathrm{P}<0.001)$. Further analysis of the data showed there was positive correlation between MMP-2 and APN, and pearson correlation was $0.543(\mathrm{P}<0.001)$.

Conclusion Serum MMP-2 can identify the presence of plaques. Serum APN levels associated with coronary plaque stability, which may become biomarker of unstable coronary plaque.

\section{Is tissue edema a potential cause of myocardial injury in HCM patients: A CMR study}

Shi Chen, Yucheng Chen, Qing Zhang, Yong Luo

Department of Cardiology, West China Hospital

Background Elevated cardiac troponin can be detected in patients with hypertrophic cardiomyopathy (HCM). However, the underlying mechanism of myocardial injury is unclear. Previous studies observed tissue edema with T2-weighted (T2W) imagine on cardiovascular magnet resonance (CMR) in HCM patients. A reasonable hypothesis is that edema is a potential cause for elevated cardiac troponin. We sought to investigate the association between cardiac troponin and the extent of myocardial edema based on T2W imagine in HCM patients.

Methods Forty six HCM patients underwent 1.5T CMR scanning including $\mathrm{T} 2 \mathrm{~W}$, fast spin echo triple inversion recovery sequences 5-7 days after hospital admission. On T2W images, edema was measured as percentage of high-signal area ( $>2 \mathrm{SD}$ above remote tissue) and the number of segments with high-signal intensity (myocardium to skeletal muscle signal intensity ratio $>2$ ) in 16 myocardial segments along the LV mid-myocardial circumference on 3 short-axis images. The level of high sensitivity cardiac troponin $\mathrm{T}$ (hs-cTnT) was also assessed.

Result 366 segments with high-signal intensity were observed in total 736 myocardial segments $(49.73 \%)$. The average percentage of high-signal area is $10.53 \%$. Compared with patients with normal hs$\mathrm{cTnT}$, the number of segments and average percentage of myocardium with high T2 signal intensity were significantly increased $(10.18 \pm 2.48$ vs $4.50 \pm 2.12, \mathrm{P}<0.001 ; 12.01 \pm 4.79 \%$ vs $8.24 \pm 3.95 \%, \mathrm{P}=0.008$; in 28 patients with elevate hs-cTnT ( $>14 \mathrm{ng} / \mathrm{l})$. Although the level of hs-cTnT was not associated with the percentage of high-signal area $(r=0.257, P=0.085)$, it significantly correlated with the number of myocardial segments with high-signal intensity $(r=0.454, \mathrm{P}=0.002)$.

Conclusion Our data indicated that tissue edema may contribute to myocardial injury in HCM patients. Further studies are needed to assess the clinical and prognostic impact of these findings.

\section{T1 mapping for discrimination between diffusely diseased myocardium and healthy myocardium in hypertrophic cardiomyopathy \\ Gang Yin, Shihua Zhao, Minjie Lu, Huaibing Cheng, \\ Yao Chen, Wei Xiangli, Chen Cui \\ Fu Wai Hospital, National Center for Cardiovascular Diseases, Beijing}

Objective The aim of this study was to determine the utility of cardiac magnetic resonance T1 mapping for discriminating between diffusely diseased and healthy myocardium in patients with hypertrophic cardiomyopathy.

Methods Consecutive 22 patients with known hypertrophic cardiomyopathy (HCM) and with hypertrophic and diffusely diseased myocardium in ventricular septum by cardiac MR imaging were included in this study. 15 healthy volunteers with normal cardiac MR findings were severed as control subjects. A modified Look-Locker with inversion recovery (MOLLI) sequence was used to determine myocardial T1 values pre-, and $15 \mathrm{~min}$ post-administration of $0.2 \mathrm{mmol} /$ $\mathrm{kg}$ of gadolinium-based contrast agent on a 1.5-T MR scanner. Late gadolinium enhancement imaging was used to indentify diffuse fibrosis in myocardium. Pre-contrast and post-contrast T1 values in diffusely diseased ventricular septal myocardium in patients with HCM and in ventricular septal myocardium in healthy volunteers were measured.

Result Pre-contrast T1 values were significantly longer in patients with HCM with control subjects $(1061 \pm 36$ and $1010 \pm 45$ $\mathrm{ms}$, respectively; $\mathrm{P}=0.001)$. Conversely, post-contrast $\mathrm{T} 1$ values were significantly shorter in patients with $\mathrm{HCM}(501 \pm 52$ and 544 $\pm 46 \mathrm{~ms}$, respectively; $\mathrm{P}=0.017$ ). Receiver operating characteristic (ROC) curve analysis showed pre-contrast T1 values could differentiate between healthy and diffusely diseased myocardium with $82 \%$ sensitivity and $80 \%$ specificity with cutoff value of 1028 . The area under the curve was 0.858 . Meanwhile, the ROC curve analysis showed post-contrast $\mathrm{T} 1$ values had poor ability to discriminate diffusely diseased myocardium from healthy myocardium.

Conclusion HCM patients have longer pre-contrast T1 values and shorter post-contrast $\mathrm{T} 1$ values in diffusely diseased myocardium as compared with healthy myocardium in controls. The pre-contrast $\mathrm{T} 1$ values can be used to differentiate between healthy and diffusely diseased myocardium.

\section{Usefulness of decrease in oxygen uptake efficiency to identify gas exchange abnormality in patients with idiopathic pulmonary arterial hypertension

\author{
Heart-Lung Function Testing Center, Fuwai Hospital, National Center
} \\ Xiaoyue Tan, Xingguo Sun for Cardiovascular Disease, Chinese Academy of Medical Sciences, Peking Union Medical College}

Objective Decline in oxygen uptake efficiency (OUE), especially during exercise is found in patients with chronic heart failure. In this study we aimed to test the validity and usefulness of OUE in evaluating gas exchange abnormality of patients with idiopathic pulmonary arterial hypertension (IPAH).

Methods We retrospectively investigated the cardiopulmonary exercise test (CPET) with gas exchange measurements in 32 patients with confirmed IPAH. All patients also had resting hemodynamic measurements and pulmonary function test (PFT). Sixteen healthy subjects, matched by age, sex, and body size were used as controls, also had CPET and PFT measurements.

Result In IPAH patients, the magnitude of absolute and percentage of predicted (\%pred) oxygen uptake efficiency slope (OUES) and oxygen uptake efficiency plateau (OUEP), as well as several other CPET parameters, were strikingly worse than healthy subjects $(\mathrm{P}<0.0001)$. Pattern of changes in OUE in patients is similar to that in controls. In IPAH patients, OUE values at rest, warming up, anaerobic threshold and peak exercise were all significantly lower than in normal $(\mathrm{P}<0.0001)$. IPAH patients had significantly greater $\mathrm{VE} / \mathrm{VCO}_{2}$ than normal subjects at all activity levels $(\mathrm{P}<0.001)$. From rest to the $\mathrm{AT}, \mathrm{VE} / \mathrm{VCO}_{2}$ values in control group reduced greatly, however in IPAH group decreased hardly. The similarities of both IPAH and Control groups for $\mathrm{VO}_{2}$ and $\mathrm{VCO}_{2}$ at rest and warm-up. There were significant differences at AT $(\mathrm{P}<0.05)$ and peak exercise $(\mathrm{P}<0.001)$. However, for VE, IPAH patients had higher values than those of Control subjects at rest $(\mathrm{P}<0.05)$ and warm- 
up $(\mathrm{P}<0.001)$, but no difference at AT and peak exercise $(\mathrm{P}>0.05)$. This indicates that at any required metabolic rate (as $\mathrm{VO}_{2}$ and $\mathrm{VCO}_{2}$ ), the ventilation is over driven by lung compensation for a limited heart function, i.e. mismatched Q/VA. OUEP\%pred, better than OUES\%pred, correlated significantly with New York Heart Association (NYHA) functional Class $(r=-0.724, \mathrm{P}<0.005)$. Total Pulmonary Vascular Resistance (TPVR) $(\mathrm{r}=-0.694, \mathrm{P}<0.005)$, diffusing capacity for carbon monoxide (DLCO) $(\mathrm{r}=0.577, \mathrm{P}<0.05)$, and the lowest ventilation versus $\mathrm{CO}_{2}$ output ratio during exercise (Lowest $\left.\mathrm{VE} / \mathrm{VCO}_{2}\right)(\mathrm{r}=-0.902$, $\mathrm{P}<0.0001$ ). In addition, the coefficient of variation (COV) of OUEP was lower $(20.9 \%)$ markedly than OUES $(34.3 \%)(\mathrm{P}<0.0001)$.

Conclusion In patients with IPAH, OUES and OUEP are both significantly lower than the healthy subjects. OUEP is a better physiological parameter than OUES in evaluating the gas exchange abnormality of patients with IPAH.

\section{Association between left ventricular twist mechanics and myocardial fibrosis in patients with hypertrophic cardiomyopathy by speckle tracking echocardiography \\ Hongju Zhang, Hao Wang, Minjie Lu, Nan Xu, Weichun Wu, Xin Sun, Wugang Wang, Qiongwen Lin Department of Ultrasound, State Key Laboratory of Cardiovascular Disease, Fuwai Hospital, National Center for Cardiovascular Diseases, Chinese Academy of Medical Sciences and Peking Union Medical College}

Objective Late gadolinium enhancement (LGE) on contrastenhanced magnetic resonance imaging (MRI) in hypertrophic cardiomyopathy (HCM) has been reported to be associated with myocardial fibrosis and cardiac events. In patients with HCM, speckle tracking echocardiography (STE) can identify subclinical global systolic dysfunction despite normal left ventricular (LV) chamber function. Our aim was to assess LV twist mechanics and its relation to myocardial fibrosis in patients with HCM.

Methods and result Echocardiography and MRI were performed in 76 consecutive patients with HCM and normal chamber function. As controls, 46 age and sex-matched normal subjects were studied. For speckle tracking analysis of LV twist and rotation, the dedicated software was used. The presence of myocardial fibrosis was detected by MRI. The patients were divided into fibrosis and non-fibrosis subgroups. Compared with the controls, the patients with HCM showed increased peak LV twist mainly because of increased apical rotation of those with myocardial fibrosis and left atrium end-systolic diameter (LADs) in patients with $\mathrm{HCM}$ were significantly higher than controls $(\mathrm{P}<0.001)$. The difference in left ventricular ejection fraction (LVEF) between the two groups was not statistically significant $(\mathrm{P}>0.05)$. Myocardial fibrosis was detected in $49(65.5 \%)$ patients with HCM. LV twist, LV apical rotation, inter-ventricular septal thickness (IVST), mitral valvular early diastolic in flow velocity/mitral annular early diastolic velocity (E/ $\mathrm{Em})$ and left atrial maximum volume index (LAVi) in fibrosis subgroups were significantly higher than those in non-fibrosis subgroups $(\mathrm{P}<0.001)$ while LV basal rotation, LADs, left ventricular end-diastolic diameter (LVDd), and LVEF did not show significant differences between the two subgroups $(\mathrm{P}>0.05)$. LV twist was significantly correlated with LGE segments $(r=0.785, \mathrm{P}<0.001)$. The area under the receiver operating characteristic (ROC) curve was 0.814 using LV twist to detect myocardial fibrosis $(\mathrm{P}<0.001,95 \%$ confidence interval $(0.625-0.890)$.

Conclusion LV twist and LV rotation in patients with $\mathrm{HCM}$ can be accurately measured using STE, These results suggest that it is increased LV twist that is associated with more myocardial fibrosis. Thus, LV twist might provide useful information on myocardial fibrosis and cardiac events in HCM patients with normal chamber function.

\section{Usefulness of red blood cell distribution width as a predictor of responsiveness in acute pulmonary vasodilator testing in patients with idiopathic pulmonary arterial hypertension \\ Qunying Xi, Zhihong Liu, Zhihui Zhao, Qin Luo Fuwai Hospital, National Center for Cardiovascular Diseases, Chinese Academy of Medical Sciences and Peking Union Medical College, Beijing, China}

Objective To examine the predictive ability of red blood cell distribution width (RDW) for responsiveness in acute pulmonary vasodilator testing in patients with idiopathic pulmonary arterial hypertension (IPAH).

Methods 113 consecutive IPAH patients were enrolled in this study. Right heart catheterization (RHC) and acute pulmonary vasodilator testing were performed in all patients to define diagnosis and positive acute responders. Laboratory tests including RDW and echocardiographic values were obtained before RHC procedures. Cardiopulmonary exercise testing variables were obtained after RHC procedures. Forward stepwise method multivariate logistic regression models were used to determine the independent predictors for vasodilator testing. The receiver-operating characteristic curve was obtained to assess the predictive value of RDW.

Result Of the 113 study subjects, $73 \%$ were women, the mean age of the population was $32.9 \pm 10.9$ years. $11.5 \%$ were positive responder in acute pulmonary vasodilator testing. Demographic characteristics of the 2 groups divided by median RDW value were similar except that the patients with higher RDW values ( $>13.7 \%$ ) had poorer World Health Organization (WHO) functional status $(63.6 \%$ vs $36.2 \%$ in WHO functional class III or IV, $\mathrm{P}=0.004)$. The rate of acute positive response was reduced dramatically along with increased RDW levels $(\mathrm{P}=0.001)$. In multivariate model, only $\mathrm{RDW} \leq 13.7 \%$ (Odds ratio 20.552, $\mathrm{P}=$ 0.009 ) and pulmonary vascular resistance (Odds ratio $0.711, \mathrm{P}=0.003$ ) independently associated with responsiveness. RDW levels predicted the acute positive response with optimal cutoff value of $13.45 \%$ (sensitivity $66 \%$ and specificity $84.6 \%$ ).

Conclusion RDW is an independent and additive predictor of responsiveness in acute pulmonary vasodilator testing in patients with IPAH.

The ultrasonic diagnosis value for the diagnosis of congenital coronary artery fistula, compared with findings of surgery and/or coronary angiography

Zhenxing Sun, Mingxing Xie, Ling Li, Jing Wang, Li Zhang Department of Ultrasonography, Union Hospital of Tongji Medical

College, Huazhong University of Science and Technology

Objective To evaluate the application of transthoracic echocardiography for the diagnosis of congenital coronary artery fistula.

Methods The echocardiographic appearances of 63 patients with 
coronary artery fistulas who had undergone coronary angiography and/or operation in our hospital, were analyzed retrospectively, and the results were compared with findings of surgery and/ or coronary angiography.

Result Right CAFs were detected in thirty-three patients (52.4\%): eleven of them had drainages to the right atrium, ten to the right ventricle, two to the left ventricle, nine to the main pulmonary artery, and one to the coronary sinus. Left CAFs were detected in twenty-nine patients $(46.0 \%)$ : six of them had drainages to the right atrium, twelve to the right ventricle, one to the left atrium, two to the left ventricle, seven to the main pulmonary artery, and one to the coronary sinus. In one case $(1.6 \%)$, there is origin in both coronary arteries. Most often the entry point was a single orifice $(96.8 \%)$, but, rarely, it was multiple (3.2\%). Fifty-seven patients were diagnosed simplex coronary fistulas (90.5\%). Six patients had other congenital cardiac malformations $(9.5 \%)$. Twelve patients had also undergo conventional coronary angiography (CAG) before surgery to confirm the diagnosis. The ultrasonographic diagnosis of sixty was in lin e with surgical findings and/or coronary angiography. The accurate rate for the diagnosis of coronary artery fistula was $95.2 \%$. Preoperative transthoracic echocardiography had three patients who were neglected CAF. The misdiagnosis rate was $4.8 \%$.

Conclusion Transthoracic echocardiography could be a primary method for diagnosis of congenital artery fistula.

\section{Characteristics of pulmonary arterial flow derived from phase contrast magnetic resonance imaging in patients with pulmonary arterial hypertension \\ Wen Li, Tao Yang, Yan Zhang, Qing Gu, Zhihui Zhao, Xinhai Ni, Zhihong Liu, Shihua Zhao, Changming Xiong, Jianguo $\mathrm{He}$ \\ State Key Laboratory of Cardiovascular Disease, Fuwai Hospital, National Center for Cardiovascular Diseases, Chinese Academy of Medical Sciences and Peking Union Medical College}

Background The phage contrast CMR can encode the flow character, which enables flow velocity and volume to be assessed. We designed this study to validate the value of phase contrast CMR in the assessment of pulmonary arterial hypertension (PAH).

Methods Patients with PAH were observed prospectively. Right heart catheterization, echocardiography and CMR were performed in all patients within 1 week interval. The correlations between parameters derived from phase contrast CMR and right ventricular function as well as hemodynamics were analyzed.

Result A total of 33 patients were enrolled in this study, including 21 patients with IPAH (59.1\%), 2 patients with heritable PAH (5.1\%), 5 patients with $\mathrm{PAH}$ associated with congenital heart disease after repair surgery (12.8\%), 5 patients with PAH associated with connective tissue disease $(12.8 \%)$. The average velocity pulmonary arterial flow correlated with PVR negatively $(\mathrm{r}=-0.578, \mathrm{P}=0.001)$ and correlated with TAPSE and S' positively $(\mathrm{r}=0.475, \mathrm{P}=0.006$ and $\mathrm{r}=0.450, \mathrm{P}=$ 0.014). Pulmonary arterial retrograde flow volume augmented with PVR increased $(r=0.426, P=0.015)$, and correlated positively with average pulmonary arterial area and pulmonary arterial distensibility (both $\mathrm{r}=$ $0.630, \mathrm{P}<0.001$ ). Right ventricular stroke volume measured by CMR and thermodilution method had significant correlation $(\mathrm{r}=0.704, \mathrm{P}<$ 0.001) and consistency.

Conclusion The average velocity of flow in main pulmonary artery can reflect PVR, and phase contrast CMR can be used as a noninvasive method to measure stroke volume.

\section{Myocarditis evaluated by three dimensional speckle tracking \\ Jingjin Wang ${ }^{1}$, Hao Wang ${ }^{1}$, Minghu Xiao ${ }^{1}$, Jingping Sun ${ }^{2}$, Jian Zhang ${ }^{1}$ \\ 1. Fuwai Hospital, National Center for Cardiovascular Diseases, \\ Chinese Academy of Medical Sciences and Peking Union Medical College, Beijing, China \\ 2. Prince of Wales Hospital, The Chinese University of Hong Kong, \\ Shatin, N.T., Hong Kong}

Objective The current gold standard for myocarditis is endomyocardial biopsy, but it is invasive to monitor the course of myocarditis and follow-up. Conventional two dimensional echocardiography plays a limited role in the course of myocarditis. Three dimensional speckle tracking is a new and noninvasive echocardiographic method to evaluate myocardial function. The objective was to evaluate myocardial function with three dimensional speckle tracking in myocarditis.

Methods One 47-year-old man who was diagnosed as acute myocarditis, echocardiographic image was collected (including three dimensional echocardiographic images) at acute stage, 1 week, 2 weeks, 5 weeks, and 12 weeks after conservative therapy respectively with Vivid E9 and analyzed with 4D Auto LVQ (FPS > 30).

Result Left ventricular end-diastolic diameter was $56 \mathrm{~mm}, 54$ $\mathrm{mm}, 49 \mathrm{~mm}, 46 \mathrm{~mm}$ and $46 \mathrm{~mm}$ at acute stage, 1 week, 2 weeks, 5 weeks, and 12 weeks after conservative therapy, respectively; Left atrium diameter was $42 \mathrm{~mm}, 42 \mathrm{~mm}, 42 \mathrm{~mm}, 42 \mathrm{~mm}$ and $36 \mathrm{~mm}$, respectively; Left ventricular ejection fraction was 30\%, 38\%, 43\%, 56\% and $68 \%$, respectively; Peak early diastolic mitral flow (E) /Peak early diastolic mitral annulus velocity ( $\mathrm{e}^{\prime}$ ) was $17,17,10.4,7.3,6.6$, respectively. Three dimensional speckle tracking parameters, global longitudinal strain $(-4 \%,-8 \%,-10 \%,-9 \%,-13 \%$, respectively), global circumferential strain $(-8 \%,-12 \%,-11 \%,-16 \%,-19 \%$, respectively), radial strain $(14 \%, 24 \%$, $30 \%, 37 \%, 56 \%$, respectively), area strain $(-11 \%,-17 \%,-19 \%,-22 \%,-27 \%$, respectively). Conventional echocardiographic parameters restored 5 weeks after therapy but three dimensional speckle tracking remained decreased.

Conclusion Three dimensional speckle tracking is convenient way to detect myocardial function and monitor the course of myocarditis, which is more sensitive than conventional echocardiography.

\section{The diagnostic value of three-dimensional vectorcardiograph on pulmonary hypertension and right ventricular hypertrophy in patients with COPD \\ Dianzhu Pan ${ }^{1}$, Renguang Liu ${ }^{2}$, Shuzhen Ren ${ }^{2}$ \\ 1. Department of respiratory Medicine, First Affiliated Hospital of Liaoning Medical University \\ 2. Institute of Cardiovascular, First Affiliated Hospital of Liaoning Medical University}

Objective This study were conducted to assess the potential diagnostic value of three-dimensional Vectorcardiography (3D-VCG) in detecting the changes of the Pulmonary Hypertension $(\mathrm{PH})$ and Right ventricular hypertrophy $(\mathrm{RVH})$ in COPD patients

Methods 74 COPD patients were divided into three groups: 26 in Non-PH group, 28 in PH group and 20 in PH with RV hypertrophy 
group. PH, based on the Color Doppler Echocardiography findings, was defined as pulmonary artery systolic pressure (PASP) beyond $37 \mathrm{~mm} \mathrm{Hg}$ and pulmonary hypertension was defined as right ventricular anterior wall thickness was $5.0 \mathrm{~mm}$ or more. The differences of 3D-VCG in three groups were compared statistically by one-way analysis of variance and Pearson correlation analysis were used between PASP and Ventricular Gradient magnitude $\left(\mathrm{VG}_{\text {magnitude }}\right)$ or Spatial Orientation, $\left(\mathrm{VG}_{\text {azimuth }}\right.$ and $\left.\mathrm{VG}_{\text {elevation }}\right)$.

Result The transition from Non-PH to $\mathrm{PH}$ negatively increased $\mathrm{QRS}_{\text {azimuth }}$ and $\mathrm{VG}_{\text {azimuth }}$ (from $-23.54 \pm 32.97^{\circ}$ to $-62.20 \pm 57.01^{\circ}$ and from $-9.22 \pm 27.92^{\circ}$ to $-56.72 \pm 28.18^{\circ}$, respectively; $\mathrm{P}<0.01$ for both indices). $\mathrm{QRS}_{\text {azimuth }}$ and $\mathrm{VG}_{\text {azimuth }}$ also increased negatively when shifting from $\mathrm{PH}$ to PAH with RV hypertrophy (from $-62.20 \pm 57.01^{\circ}$ to- 139.74 $\pm 26.03^{\circ}$ and from $-56.72 \pm 28.18^{\circ}$ to $-135.36 \pm 40.70^{\circ}$, respectively; $\mathrm{P}$ $<0.01$ for both indices). The spatial QRS-T angle was larger in patients with PH than those with Non-PH $\left(114.62 \pm 46.14^{\circ}\right.$ vs $68.71 \pm 34.60^{\circ}, \mathrm{P}$ $<0.01)$. There was a linear correlation between the spatial QRS-T angle and PASP in pulmonary hypertension $(\mathrm{r} 1=0.89, \mathrm{P} 1<0.05)$. Simple linear regression analysis rendered the following formula for prediction of the presence of increased PASP by the spatial QRS-T angle: PASP = $0.08 *$ the spatial QRS-T angle $+39.61(\mathrm{r} 1=0.80, \mathrm{P} 1<0.05)$. In addition, PASP also showed an inverse relation with VGazimuth ( $\mathrm{r} 2=-0.86, \mathrm{P} 2<$ 0.05 ). Simple linear regression analysis rendered the following formula for prediction of the presence of increased PASP by the $\mathrm{VG}_{\text {azimuth }}$ : PASP $=-0.13 \mathrm{VG}_{\text {azimuth }}+41.01(\mathrm{R} 22=0.74, \mathrm{P} 2<0.05)$.

Conclusion 3D-VCG is useful to detect the changes of PAH and RV hypertrophy in COPD patients and may be useful in detecting the development of early chronic pulmonary heart disease in COPD patients.

\section{Combined HDL associated apoA1 and SAA in diagnosis of coronary heart disease \\ Hui Zhao, Hong Liu, Lin Chai, Lirong Yan, Yuexin Jiang, Bing Duan, Xiaoyuan Guan, Lu Hua, Lei Tian, Yiling Huang, Yishi $\mathrm{Li}$ \\ Fuwai Hospital, National Center for Cardiovascular Diseases, Chinese Academy of Medical Sciences and Peking Union Medical College}

Objective Recent researches have demonstrated that the compositions of high-density lipoprotein (HDL) correlated with the risks of coronary heart disease (CHD). Our study aimed to investigate the relationship between HDL associated apolipoprotein A1 (apoA1), serum amyloid A (SAA) and CHD. The diagnostic value of each index was further tested.

Methods The study was a case-control design. 62 CHD patients diagnosed by coronary angiography was enrolled. And another 58 age and sex matched individuals were also included as non-CHD controls. Fasting venous plasma was collected and then plasma HDL was prepared and isolated by density gradient ultracentrifugation. ELISA method was used to measure the concentration of apoA1 and SAA in HDL sample. Lipid profiles and hsCRP were also detected. Differences between groups were compared and logistic regression model was applied to analyse the correlation between CHD risk and these indexes. Receiver operating characteristic curve was constructed to assess the diagnostic value.

Result Compared with non-CHD controls, the concentration of HDL associated SAA (HDL-SAA) was significantly higher in the patients with CHD $[(21.63 \pm 21.31) \mu \mathrm{g} / \mathrm{ml}$ vs. $(13.33 \pm 10.61) \mu \mathrm{g} / \mathrm{ml}$, $\mathrm{P}=0.014]$, while the concentration of HDL associated apoA1 (HDLapoA1) was markedly lower $[(10.67 \pm 5.64) \mathrm{g} / \mathrm{ml}$ vs $(8.04 \pm 3.33) \mathrm{g} / \mathrm{ml}$,
$\mathrm{P}=0.003]$. Multiple logistic regression analysis demonstrated that both HDL-SAA (OR $=1.084,95 \%$ CI: $1.023-1.149, \mathrm{P}=0.007)$ and HDLapoA1 $(\mathrm{OR}=0.837,95 \% \mathrm{CI}: 0.744-0.942, \mathrm{P}=0.003)$ correlated with CHD risk independently. ROC curve revealed that only HDL-SAA but not HDL-apoA1 had a diagnostic value to differentiate CHD patients (AUC $=0.648,95 \%$ CI: 0.549-0.748, $\mathrm{P}=0.005$ ). Combined HDL-SAA and HDL-apoA1 (HDL-SAA/HDL-apoA1) showed a higher diagnostic value $(\mathrm{AUC}=0.716,95 \% \mathrm{CI}: 0.624-0.808, \mathrm{P}<0.001)$, and the best cutoff value was 1.42 .

Conclusion Present study confirmed an independent correlationship between CHD risk and HDL-SAA and HDL-apoA1. Combined HDLSAA and HDL-apoA1 improved the CHD diagnostic efficacy.

\section{Genetic testing of FBN1 gene in marfan syndrome patients \\ Hang Yang, Mingyao Luo, Qinqin Xiang, Siyao Zhang, Kunlun Yin, Xiaogang Sun, Xiangyang Qian, Zhou Zhou, Qian Chang \\ State Key Laboratory of Cardiovascular Disease, Fuwai Hospital, National Center for Cardiovascular Diseases, Chinese Academy of Medical Sciences and Peking Union Medical College}

Objective Marfan syndrome is a common autosomal dominant connective tissue disorder. The major reason of mortality for Marfan patients is at high risk for aortic dissection/aortic aneurysm. Here, we screened FBN1 gene in 6 familial cases and 10 sporadic cases, who was clinically diagnosed as Marfan syndrome.

Methods Genomic DNA was extracted from peripheral blood samples. PCR and subsequent Sanger sequencing detected exons of FBN1 gene in the proband. Once a pathogenic mutation was identified, the family members would be screened for the same mutation. We also used Polyphen and SIFT program to predict possible impact of amino acid substitution on the secondary structure and function of FBN1.

Result We found 9 different mutations/deletions in FBN1 gene, 4 of them being novel.

A Marfan syndrome family had four generations with 21 family members, and 5 members passed away as sudden death at a younger age (18-40 years old). 3 family members carried out aortic replacement operation at our hospital. The testing results showed that $11(50 \%)$ had a heterozygous mutation in exon 56 (c.6992G > A, p.Cys2331Tyr), which was reported in the Universal Mutation Database as a pathogenic mutation.Patient MFS006 suffered a severe aortic disease. Her whole aorta, including ascending aorta, aortic arch and descending aorta, as well as aortic valves, was significantly affected. The testing results showed she had a novel complicated change in FBN1 gene with 57bp deletion in exon6/intron6 and subsequently a 57bp duplication, which led to a premature termination codon. The patients also had a probable pathogenic mutation in exon21 (c.2613A $>$ C, p.Leu871Phe), which may not be the pathogenic cause for her.

Two novel small deletions (c.4894_4897delCGCT, c.8038delC), which resulted in a premature termination codon, were identified in another two sporadic patients. We have also found five reported mutations/deletions, including three truncating mutations c.6241C $>$ T, (p.Gln2081Term), c.1285T > C (p.Arg429Term), c.296G > A (p.Ser988Term) and two splicing mutations (c.IVS14-1G > A, c.IVS54+1G $>$ C).

FBN1 mutation was not identified in four patients. One of the possible reasons could be the loose diagnostic criteria of Marfan syndrome clinically. Therefore, a 13-gene panel would be conducted to 
identify the genetic causes for Marfan syndrome and related diseases (such as Loeys-Dietz syndrome, thoracic aortic aneurysms and aortic dissection et. al.).

Conclusion Genetic testing of FBN1 gene can provide molecular diagnosis of Marfan syndrome. This allows prompt medical intervention for the patients and early surveillance for genotype-positive family members.

\section{Evaluation of patients with diastolic heart failure using NT-proBNP and tissue doppler imaging Yi Yang, Hua Xiao Department of Cardiology of the First Affiliated Hospital of Chongqing Medical University}

Objective To explore the significance of diagnosis of diastolic heart failure (DHF) and evaluated the degree of heart failure using mitral peak velocity of early filling/early diastolic mitral annular velocity (E/Ea) and N-terminal pro-brain natriuretic peptide (NT-proBNP).

Methods 80 cases of DHF patients were admitted to our department, and divided into II, III, IV grade according to the NYHA classification. Meanwhile, another 23 patients in hospitalization or with normal heart function after medical examinations were selected as the control group. They underwent conventional and tissue Doppler imaging and serum NT-proBNP level, high-sensitive $\mathrm{C}$ reactive protein and lipids were measured at the same time, and the results were analyzed statistically.

Result Compared with the control group, E/Ea and NT-proBNP of DHF patients were significantly higher and statistically significant $(\mathrm{P}<0.001)$. With the aggravation of heart failure, concentration of NT-proBNP and E/Ea ratio were gradually increased. The Multiple regression analysis showed lg NT-proBNP level were independently related to left ventricular ejection fraction and $\mathrm{E} / \mathrm{Ea}\left(\mathrm{R}^{2}=0.323, \mathrm{P}=\right.$ $0.000)$, and the relation to LVEF was the strongest $(\beta=-0.440, P=$ $0.000)$. Specificity, positive predictive value ratio and positive likelihood ratio of diagnose DHF with NT-proBNP joint E/Ea was better than the application of NT-proBNP or E/Ea alone.

Conclusion With the NT-proBNP and E/Ea ratio by tissue Doppler echocardiography, diagnosis of DHF and evaluate left ventricular function will be more easier and accurate.

\section{The comparison of the ankle brachial index and pulse wave velocity between the patients with aortic stenosis and patients with bilateral lower extremity artery stenosis \\ Meng Peng, Xiongjing Jiang, Hui Dong, Yubao Zou, Lei Song, Huimin Zhang, Xianliang Zhou, Haiying Wu Fuwai Hospital, Chinese academy of Medical Sciences and Peking Union Medical College}

Objective To compare the ankle brachial index (ABI) and pulse wave velocity (PWV) between the patients with aortic stenosis (AS) and patients with bilateral lower extremity artery stenosis (BLEAS) and investigate the diagnostic value of the difference.

Methods 56 patients with AS admitted to Fuwai Hospital during April 2010 to April 2013 were selected. 56 patients with BLEAS ageand sex-matched with the same exclusion criteria were selected. The general information was collected. ABI and ba-PWV were measured.

Result There were no significant difference in ABI $(0.69 \pm 0.15$ vs
$0.68 \pm 0.23, \mathrm{P}=0.729)$ or ba-PWV $(14.18 \pm 12.18 \mathrm{~m} / \mathrm{s}$ vs $12.19 \pm 5.4 \mathrm{~m} /$ s) between the two groups (AS vs BLEAS). However, the differences of the bilateral ABIs $(0.06 \pm 0.08$ vs $0.37 \pm 0.28, \mathrm{P}<0.001)$ and bilateral ab-PWVs $(1.82 \pm 2.96 \mathrm{~m} / \mathrm{s}$ vs $7.37 \pm 11.35 \mathrm{~m} / \mathrm{s}, \mathrm{P}=0.001)$ differed significantly between the two groups. To diagnose aortic stenosis in the two groups of patients, the area under the ROC curve of the bilateral difference in $\mathrm{ABI}$ was 0.90 , and the area under the ROC curve of the bilateral difference in ba-PWV was 0.82 .

Conclusion There were no significant difference in ABI or baPWV between patients with AS and patients with BLEAS. However, the differences of the bilateral ABIs and bilateral ab-PWVs differed significantly between the two groups. The differences in bilateral ABI and in bilateral ab-PWVs have a good diagnostic value.

\section{Identification and characterization of a novel HMBS gene mutation in an acute intermittent porphyria family \\ Honglian Wang ${ }^{1}$, Jing Yang ${ }^{2}$, Kunlun Yin ${ }^{1}$, Ricong Wang ${ }^{2}$, Wei Wu ${ }^{2}$, Zhou Zhou ${ }^{1}$ \\ 1. State Key Laboratory of Cardiovascular Disease, Center of Molecular \\ Diagnostics, Fuwai Hospital, National Center for Cardiovascular \\ Diseases; Chinese Academy of Medical Sciences and Peking Union Medical College, Beijing \\ 2. Department of Emergency, Department of Clinical Chemistry, Peking Union Medical College Hospital, Chinese Academy of Medical Sciences and Peking Union Medical College, Beijing}

Objective Acute intermittent porphyria (AIP) is characterized by acute neurovascular attacks that are precipitated by several factors including certain drugs, steroid hormones, alcohol and fasting. This disease is an autosomal dominant disorder with incomplete penetrance, which due to mutations in hydroxymethylbilane synthase (HMBS; or porphobilinogen deaminase, PBGD) gene. So far, more than 300 HMBS gene mutations had been in identified in AIP patients and nearly half were missense mutation. The purpose of this study was to make a genetic diagnosis of a patient with suspected AIP. We will also screen the relatives of the patient for early identification of asymptomatic affected individuals.

Methods A 21-year-old woman was admitted to our hospital for suffering from frequent severe abdominal pain frequent severe abdominal pain, convulsion and confusion. She also had hyponatremia, anemia and a high urinary $\delta$-aminolevulinic acid (ALA) concentration. Two of her cousin sisters have the similar symptoms. We investigated HMBS gene mutation in (18) members of a large, consanguineous three-generation family. All 18 family members were enrolled in the study, and peripheral blood was obtained from 17 members for the genomic DNA analysis. We also used Polyphen and SIFT programto predict possible impact of amino acid substitution on the secondary structure and function of HMBS.

Result We reported a novel HMBS missense mutation c.988G $>$ C (p.Ala330Pro) in an AIP family. The same mutation was also identified in another six family numbers, who were also diagnosed as AIP or asymptomatic affected individuals. The results of polyphen-2 and SIFT analysis showed that this variant is probably damaging. We also found the Alanine at codon 330 is absolutely conserved among all 23 known species, suggesting the residue may play a critical role in HMBS function.

Conclusion In this study, we identified a novel HMBS gene mutation in a Chinese pedigree and make the genetic diagnosis of the 
proband with AIP and the other asymptomatic family members. So we can give the patient proper treatment and advise those asymptomatic family members to avoid drugs and other compounds that may provoke the life-threatening acute neurovascular crises.

\section{Electrocardiographic difference between ventricular arrhythmias from the right ventricular outflow tract and idiopathic right ventricular arrhythmias}

Lan Ren, Zheng Liu, Yuhe Jia, Pihua Fang, Jielin Pu, Shu Zhang Cardiac Amhythmia Center, State Key Laboratory of cardiovascular disease, Fuwai hospital, National center for cardiovascular diseases, Chinese Academy of Medical Sciences and Peking Union Medical College, Beijing, China

Objective Ventricular arrhythmias (VA) arising from arrhythmogenic right ventricular dysplasia/cardiomyopathy (ARVD/ C) and idiopathic right ventricular outflow tract ventricular arrhythmias (RVOT-VA) share the pattern of left bundle branch block (LBBB) / inferior axis. The existence of QRS notching showed a discriminating effect of the two conditions in recent research, however, there are little data regarding the difference in the distribution of QRS notching. Aim of this study was to compare the VA morphology between the two conditions, especially evaluate the diagnostic role of QRS notching.

Methods Electrocardiographic (ECG) recordings of VA episode with $\mathrm{LBBB} /$ inferior axis of $16 \mathrm{ARVD} / \mathrm{C}$ and 45 idiopathic RVOT-VA patients ( 30 originated from the septum, 15 from the free-wall) were gathered and compared.

Result ARVD/C had longer mean QRS duration in all 12 leads, and significant differences existed in leads I, II, III, aVL, aVF, and $\mathrm{V}_{1}$ $(\mathrm{P}<0.05)$. Lead I had the largest mean difference of $25.1 \pm 5.8 \mathrm{~ms}$. In addition, $\mathrm{ARVD} / \mathrm{C}$ had more $\mathrm{R}$ wave transition in lead $\mathrm{V}_{5}$ or later $(37.5 \%$ vs $8.9 \%, \mathrm{P}<0.01)$. The presence of QRS notching [15/16 $(93.8 \%)$ vs $36 / 45(80.0 \%), \mathrm{P}=0.20$ ] and the total number of leads expressing notching $(2.88 \pm 2.0$ vs $2.80 \pm 2.0, \mathrm{P}=0.90)$ were not different between ARVD/C and idiopathic RVOT-VA. However, QRS notching existing simultaneously in lead I and aVL was more common in ARVD/C (43.8\% vs $13.3 \%, \mathrm{P}=0.011$ ).

Conclusion Longer QRS duration, later precordial R/S transition and QRS notching in lateral leads (lead I and aVL) are useful in discriminating ARVD/C from idiopathic RVOT-VA.

\section{A compound heterozygous lipoprotein lipase mutation in a patient with severe hypertriglyceridemia}

Kunlun Yin, Chenggang Zhu, Yuanyuan Fu, Siyao Zhang,

Yuanlin Guo, Meng Zhang, Zhou Zhou, Jianjun Li

State Key Laboratory of Cardiovascular Disease, Fuwai Hospital,

National Center for Cardiovascular Diseases, Chinese Academy of

Medical Sciences and Peking Union Medical College, Beijing

Objective Patients with severe hypertriglyceridemia (HTG, TG > $10 \mathrm{mmol} / \mathrm{L}$ ) usually suggest genetic reasons and show clinical features of recurrent attacks of pancreatitis, hepatosplenomegaly, lipemia retinalis and eruptive xanthomas. Familial HTG are caused by mutations in six genes, including lipoprotein lipase (LPL), apolipoprotein C-II (APOC2), ApoA-V (APOA5), lipase maturation factor 1 (LMF1), glycosylphosphatidyl-inositol-anchored HDL-binding protein (GPIHBP1), and glycerol-3-phosphate dehydrogenase 1 (GPD1). Here we investigated the molecular basis of severe HTG in one patient with his parents referred to our hospital.

Methods The patient was diagnosed as type 1 HTG with severe hypertriglyceride and recurrent pancreatitis. We designed primers flanking all exons and intron-exon boundaries of LPL, APOC2, APOA5, LMF1, GPIHBP1, GPD1 genes and performed PCR+Sanger sequencing in the patient diagnosed as type 1 HTG and phenotypic normal parents. We also used Polyphen and SIFT program to predict the possible impact of amino acid substitution on the function of genes.

Result We have identified that the patient has compound heterozygous mutations in LPL gene: c.997C > T (p.Arg333Cys) and c.1012T > C (p.Tyr338His). Further study has shown that c.1012T > C (p.Tyr338His) is from his father and c. $997 \mathrm{C}>\mathrm{T}$ (p.Arg333Cys) is from his mother. There have two SNPs in LMF1 (rs142481016) and GPIHBP1 (rs11538388). Interestingly, we also found a homozygous deletion of 19 bp (GGCGGGGCCTCCGAGAATG, g.1021033 -1021053) located at the 5' link area of LMF1 and both parents have the same deletion. No mutations were found in APOC2, APOA5 and GPD1 genes.

Conclusion HTG caused by LPL deficiency due to LPL gene mutations is the most common form, with an estimated prevalence of 1 in 106. The compound heterozygous mutations in LPL gene caused HTG in this patient. Later study will investigate how these mutations affect LPL enzymatic activity. The frequency and function of $19 \mathrm{bp}$ deletion at the 5' linker area of LMF1 gene needs further studies. Routine genetic testing of candidate genes in severe HTG has improved our understanding of the molecular basis of this phenotype, and may help to guide the individualized therapeutics.

\section{Population-based variations in thrombophilia genes \\ Siyao Zhang, Yuanyuan Fu, Wenke Li, Xiang Yang, \\ Zhou Zhou \\ State Key Laboratory of Cardiovascular Disease, Center of Molecular \\ Diagnostics, Fuwai Hospital, National Center for Cardiovascular \\ Diseases, Chinese Academy of Medical Sciences and Peking Union \\ Medical College}

Objective Thrombophilia is an abnormality of blood coagulation and an inherited or acquired predisposition to thrombosis, which leads to deep vein thrombosis (DVT) and pulmonary embolism (PE). The leading causes of the inherited thrombophilia are the factor V Leiden and prothrombin G20210A, and the deficiency of natural anticoagulants, such as antithrombin (AT), protein $\mathrm{C}$ (PC) and protein $\mathrm{S}$ (PS). Genetic diagnosis of thrombophilia relies on complete sequencing of the gene coding regions, and most pathogenic variants are rare. The "1000 Genomes Project" is an ongoing consortium designed to deliver whole genome information from an ethnically diverse population and, therefore, is a rich source to determine both common and rare genetic variants.

Methods We queried the "1000 Genomes Project" database of 1092 individuals for exonic variants from 3 natural anticoagulants genes, SERPINC1, PROC and PROS1. We focused on protein-altering variation, including nonsynonymous single nucleotide polymorphism (SNP), insertion/deletion polymorphisms, or splice site altering variants. False positive variants caused by pseudogenes were removed and rare variants with minor allele frequency (MAF) no more than 5\% were kept for further analysis. We predicted the functional consequences of 
variations using SIFT and Polyphen-2 tools. The numbers of proteinaltering variants (PAVs) carried by each individual among four ethnic groups were calculated, and a Kruskal-Wallis one-way analysis was performed in Prism software to test the differences between ethnic groups using variant load.

Result There were 157142 and 989 variants in SERPINC1, PROC, and PROS1, respectively. Among them, 1252 were SNPs, 35 were indels, and 1 was a structure variation (SV). All of indels and SV were in the intronic regions. Of $39 \mathrm{PAVs}, 97.4 \%$ were very rare (MAF $<0.5 \%$ ), and $2.6 \%$ were rare variants $(0.5 \%<\mathrm{MAF}<$ in 6 and 06 , were tools both by deleterious be to predicted Variants $>$ SERPINC1, PROC, and PROS1, respectively. On multiple comparison tests, we found African have significant more variations in PROC than American and European, and have more variation in PROS1 than all other groups.

Conclusion These results highlight the complexity of SERPINC1, PROC, and PROS1 variations in different ethnic groups and emphasize the importance of interrogating variations on multiple ethnic backgrounds for associations with thrombosis.

\section{Assessment of the left atrial appendage using real-time three dimensional transesophageal echocardiography \\ Wenjuan Bai, Zhongxiu Chen, Hui Wang, Xin Wei, Li Rao Laboratory of Cardiovascular Research, West China Hospital, Sichuan University, Chengdu, China}

Objective The left atrial appendage (LAA) is a common source of cardiac thrombi formation associated with systemic embolism, particularly in patients with nonvalvular atrial fibrillation (AF). Transesophageal echocardiography is performed routinely among AF patients in order to exclude LAA thrombi prior to radiofrequency cardioversion. In present study, the LAA structure was assessed using real-time three dimensional transesophageal echocardiographic (RT3DTEE) and cardiac compute tomography (CT) scanning. Our aims were to compare RT-3DTEE measurements of the LAA with cardiac $\mathrm{CT}$, analyze the relation of LAA size and $\mathrm{CHADS}_{2}$ score, and find the sensitive parameters to forecast thrombi risk.

Methods Patients with nonvalvular AF and prepared to take radiofrequency cardioversion were enrolled consecutively. Transesophageal echocardiography was performed using the Philips iE33 ultrasound system equipped with RT-3DTEE probe X7-2t (frequency range from 2 to $7 \mathrm{MHz}$ ). Cardiac $\mathrm{CT}$ was performed using Siemens Definition flash CT system. All data were imported to off-cart workstation for LAA quantification. The following parameters were measured: LAA orifice area, length (curvilinear distance from the middle of the orifice to the end of the tail), maximum volume (the phase defined by observer due to most images are acquired when AF rhythm). The patients were divided into 3 groups according to the $\mathrm{CHADS}_{2}$ score system, which used for reflect atrial fibrillation stroke risk. Whose score equal to 0 are low risk group, score equal to 1 are moderate risk group, and the others are high risk group.

Result A total of 114 patients were included, 75 patients are paroxysmal $\mathrm{AF}, 27$ patients are persistent $\mathrm{AF}$, and 12 patients are long persistent AF. Based on the $\mathrm{CHADS}_{2}$ score, low risk group has 53 patients, moderate risk group has 42 patients, and high risk group has 19 patients. There are only 2 patients be proved thrombi in LAA in high risk group. The average age is $60.3 \pm 11.7$ years. The LAA morphology was demonstrated clearly both in RT-3DTEE and CT. There is a positive correlation of LAA orifice area, length and volume between RT-3DTEE and CT measurements $(r=0.66,0.48,0.60$, respectively). In three groups, there is a significant difference of the LAA orifice area, LAA length, the maximum LAA volume by RT-3DTEE $(\mathrm{P}=0.010,0.014$, 0.032 , respectively), whereas there is no significant difference of the LAA emptying velocity $(\mathrm{P}>0.05)$.

Conclusion LAA size can be assessed reliably by RT-3DTEE. The LAA size enlarged with the $\mathrm{CHADS}_{2}$ score. The present study has proved cardioembolic incidence rate correlated with LAA dilation.

\section{Cardiopulmonary Exercise Testing, NT-proBNP and Ultrasound cardiogram assessment of cardiac function in patients with chronic heart failure \\ Zhinan $\mathrm{Lu}^{1}$, Xingguo Sun ${ }^{2}$, Shengshou $\mathrm{Hu}^{1}$, Jie Huang ${ }^{1}$ 1. Department of heart transplant center, State Key Laboratory of Cardiovascular Disease, Fuwai Hospital, National Center for Cardiovascular Diseases, Chinese Academy of Medical Sciences and Peking Union Medical College, Beijing, China \\ 2. Heart-Lung Function Testing Center, State Key Laboratory of Cardiovascular Disease, Fuwai Hospital, National Center for Cardiovascular Diseases, Chinese Academy of Medical Sciences and Peking Union Medical College, Beijing, China}

Objective To assess the relationship between cardiopulmonary exercise testing (CPET) parameters, the plasma concentrations of NTproBNP and ultrasound cardiogram indicators in patients with chronic heart failure (CHF).

Methods 70 CHF patients who underwent CPET were enrolled in this study. The patients were divided into three groups according to their peak oxygen uptake $\left(\right.$ Peak $\left.\mathrm{VO}_{2}\right)$ : severe group $\left(\right.$ Peak $\mathrm{VO}_{2}$ below 10 $\mathrm{ml} \cdot \mathrm{kg}^{-1} \cdot \mathrm{min}^{-1}$ ), moderate group ( Peak $\mathrm{VO}_{2}$ between $10-14 \mathrm{ml} \cdot \mathrm{kg}^{-}$ ${ }^{1} \cdot \mathrm{min}^{-1}$ ) and mild group (Peak $\mathrm{VO}_{2}$ above $14 \mathrm{ml} \cdot \mathrm{kg}^{-1} \cdot \mathrm{min}^{-1}$ ). The basic clinical information were collected, and the plasma concentrations of NTproBNP at rest, ultrasound cardiogram and cardiopulmonary exercise testing were all measured to assess the relationship between NT-proBNP, ultrasound cardiogram indicators and $\mathrm{Peak} \mathrm{VO}_{2}$ in patients with $\mathrm{CHF}$.

Results There are no significant differences in NT-proBNP, left atrium (LA), left ventricular end-diastolic diameter (LVEDD) and left ventricular ejection fraction (LVEF) in three group. NT-proBNP shows significant correlations with Peak $\mathrm{VO}_{2}$. NT-proBNP values $\geq 1322.4$ $\mathrm{pmol} / \mathrm{L}$ shows $88.9 \%$ sensitivity and $41.2 \%$ specificity for detecting Peak VO2 values $\leq 14 \mathrm{ml} \cdot \mathrm{kg}-1 \cdot \mathrm{min}-1$ ( $\mathrm{AUC}=0.643, \mathrm{P}<0.05)$.

Conclusion CPET indicator Peak $\mathrm{VO}_{2}$ is more sensitive to discover the aggravation of the chronic heart failure than NT-proBNP and ultrasound cardiogram. Peak $\mathrm{VO}_{2}$ and NT-proBNP good correlation can be combined to assess disease severity.

\section{The herb protosappanin A diminished recipient $T$ cell migration into allograft via inhibition of IP- 10 in rat heart transplant \\ Maomao Zhang, Jian Wu, Meng Sun, Jingbo Hou, Bo Yu Department of Cardiology, Second Affliated Hospital of Harbin Medical University, Harbin, Heilongjiang Province, China}

Protosappanin A ( $\operatorname{PrA})$, an immunosuppressive ingredient of the medicinal herb Caesalpinia sappan L., prolongs cardiac allograft survival, diminishes inflammatory cell infiltration, and inhibits interferon g-induced 
protein $10 \mathrm{kDa}$ (IP-10) mRNA expression in rats cardiac grafts. Binding of the chemokine IP-10 to its cognate receptor, CXCR3, plays crucial roles in allograft immunity, especially by mediating the recruitment of effector T cells to allografted tissues. In this study, we attempted to determine whether PrA-mediated inhibition of IP-10 contributes to the effect of reduced $\mathrm{T}$ cell infiltration into cardiac allograft within a rat model. Administration of $\operatorname{PrA}(25 \mathrm{mg} / \mathrm{kg}$ daily $)$ via oral gavage following heart transplantation significantly reduced the increase of IP-10 mRNA expression in the graft and prevented IP-10 secretion by peripheral blood mononuclear cells (PBMCs) isolated from recipient rats seven days posttransplantation. Furthermore, in vitro experiments demonstrated that PrA addition to control PBMCs prevented IP-10 secretion. Chemotactic migration assays were utilized to evaluate recipient $\mathrm{T}$ cell migration towards PBMC supernatant. PrA administration impaired PBMCs supernatant-induced $\mathrm{T}$ cell migration. Additional in vitro experiments revealed that PrA slightly reduced naïve $\mathrm{T}$ cell migration towards chemokines. Presence of IP-10 in the PBMC supernatant prevented PrA from reducing $\mathrm{T}$ cell migration in PrA-treated recipients. Neither CXCR3 chemokine ligand Mig nor non-CXCR3 chemokine ligand SDF-1 had any effect on T cell migration in PrA-treated recipients. Addition of antiCXCR3 antibody restored PrA-mediated inhibition of T cell migration. Immunofluorescence microscopy showed IP-10 expressed mainly in CD68 positive infiltrating monocytes. Furthermore, PrA consistently reduced $\mathrm{CXCR}^{+}{ }^{+} \mathrm{T}$ cell infiltration into cardiac allografts. The reduced intensity of CXCR3 staining in PrA-treated allografts contributed to the previously depressed naïve T cell migrating activity by PrA. Collectively, these data indicate that PrA inhibition of IP-10 activity reduced recipient $\mathrm{T}$ cell migration and infiltration of cardiac allografts, thus partially explaining the immunosuppressive effect of PrA. 


\section{Peripheral Vascular Disease}

\author{
Association of dyslipoproteinemia with the \\ disease activity of Takayasu arteritis \\ Xu Wang \\ State Key Laboratory of Cardiovascular Disease, Fuwai Hospital, \\ National Center for Cardiovascular Diseases, Chinese Academy of \\ Medical Sciences and Peking Union Medical College
}

Background and Objective The increased cardiovascular disease risk in Takayasu arteritis (TA) results from vasculitis, which affects the coronary artery, and accelerated atherosclerosis. Our study aimed to determine whether proatherogenic lipid profiles exist in patients with active TA and to assess the relationship between different lipid profiles and disease activity in TA.

Methods A total of 100 premenopausal female patients with TA and 92 sex-, age-, and body mass index-matched healthy controls were included in our study. The clinical data were collected in detail from all participants. Serumhs-Creactive protein, erythrocyte sedimentation rate, and lipid profiles were measured in all participants by using the blood samples collected after $10 \mathrm{~h}$ to $12 \mathrm{~h}$ fasting overnight.

Result Patients with active TA significantly have higher levels of apoB $[(1.05 \pm 0.34) \mathrm{mmol} / \mathrm{L}$ vs $(0.91 \pm 0.26) \mathrm{mmol} / \mathrm{L}, \mathrm{P}=0.008]$, higher ratios of apoB/apoA1 $(0.74 \pm 0.27$ vs $0.48 \pm 0.14, \mathrm{P}<0.001)$, and lower levels of apoA1 $[(1.47 \pm 0.30) \mathrm{mmol} / \mathrm{L}$ vs $(1.99 \pm 0.33) \mathrm{mmol} / \mathrm{L}$, $\mathrm{P}<0.001)$ and HDL-C $[(1.23 \pm 0.33) \mathrm{mmol} / \mathrm{L}$ vs $(1.68 \pm 0.38) \mathrm{mmol} /$ $\mathrm{L}, \mathrm{P}<0.001)$ compared with patients with inactive TA. No significant differences were found in the levels of LDL-C between patients at the active stage and those at the inactive stage. Multiple linear regression analysis found that the apoB/apoA1 ratio was independently associated with TA activity $(\beta=0.45, \mathrm{P}<0.001)$. Multivariate stepwise forward regression analysis found that the apoB/apoA1 ratio was the major determinant for hs-CRP.

Conclusion Our findings indicate that patients with active TA have proatherogenic lipid and lipoprotein profiles. In addition, the ratio of apoB to apoA1 could be a marker to be monitored and a target that needs to be treated in patients with active TA.

\section{The safety and influencing factors of carotid artery stenting for surgically high-risk carotid stenosis \\ Hui Dong, Xiongjing Jiang, Yubao Zou, Meng Peng, Lei Song, Bin Li, Wuqiang Che, Yuejin Yang, Runlin Gao Department of Cardiology, State Key Laboratory of Cardiovascular Disease, Fuwai Hospital, National Center for Cardiovascular Disease, Chinese Academy of Medical Sciences and Peking Union Medical College}

Background and Objective Large-scale randomized trials have demonstrated that carotid endarterectomy is an effective treatment strategy for the prevention of ischemic stroke in patients with significant carotid stenosis. However, some patients with carotid stenosis have anatomic or physiologic co-morbidities that place them at high risk for carotid endarterectomy. The purpose of this study was to evaluate the safety and influencing factors of carotid artery stenting (CAS) for the treatment of surgically high-riskcarotid stenosis.

Methods In this single-center retrospective study, 548 consecutive surgically high-risk patients undergoing carotid stenting from January 2005 to December 2012 were collected. All patients were required to have at least one anatomic or physiologic co-morbidities that placed them at high risk for surgery. Indication for CAS was defined as carotid artery diameter reduction of $60 \%$ (symptomatic) or $80 \%$ (asymptomatic). The primary end point was the composite of major stroke, myocardial infarction (MI), or death within 30 days.

Result The patients were 43-84 (65.7 \pm 7.6$)$ yrs, and there were $447(81.6 \%)$ male. $502(91.6 \%)$ patients suffered from coronary artery disease. $340(62.0 \%)$ patients had symptomatic carotid stenosis. 513 (93.6\%) patients had physiologic co-morbidity and 237 (43.2\%) patients had anatomic risk factors. The procedural success rate of CAS was $99.5 \%$. Cerebral protection devices were used in 538 patients (98.7\%). Among them, 120 (21.9\%) patients received simultaneous bilateral carotid stenting and $207(37.8 \%)$ patients underwent simultaneous percutaneous intervention of other arteries. The angiographic diameter stenosis reduced from $(86.2 \pm 8.1) \%$ to $(8.9 \pm 6.8) \%$ after stenting $(\mathrm{P}<$ $0.001)$. The rate of major stroke, minor stroke, death, $\mathrm{MI}$ and the primary end point from time of CAS to 30 days was $1.1 \%, 2.6 \%, 0.7 \%, 0.9 \%$ and $2.2 \%$ respectively. There was no significant statistical difference in primary end point among patients with pure physiologic risk factors, pure anatomic risk factors, and both anatomic and physiologic risk factors ( $1.9 \%$ vs $0 \%$ vs $3.0 \%, \mathrm{P}=0.632$ ). Moreover, congestive heart failure $(\mathrm{OR}=5.742,95 \% \mathrm{CI}: 1.620-20.354 ; \mathrm{P}=0.007)$ and renal insufficiency $(\mathrm{OR}=5.335,95 \%$ CI: $1.507-18.890 ; \mathrm{P}=0.009)$ were identified as independent risk factors of primary end point by multivariate analysis.

Conclusion This study indicated that CAS was safe and feasible with a low incidence of periprocedural complications in patients with surgically high-risk carotid stenosis. Congestive heart failure and renal insufficiency were independent predictors of periprocedural major adverse cardiovascular events.

\section{The safety of synchronous or staged carotid artery stenting and open heart surgery \\ Hui Dong, Xiongjing Jiang, Yubao Zou, Meng Peng, Lei Song, Huimin Zhang, Haiying Wu, Yuejin Yang, Runlin Gao \\ Department of Cardiology, State Key Laboratory of Cardiovascular Disease, Fuwai Hospital, National Center for Cardiovascular Disease, Chinese Academy of Medical Sciences and Peking Union Medical College}

Background and Objective Even if asymptomatic, patients with significant carotid stenosis undergoing open heart surgery (OHS) face a high risk of perioperative stroke. Thus, planning appropriate treatment for patients with severe carotid stenosis before OHS is an important clinical issue. The purpose of this studywas to evaluate the safety of synchronous or staged carotid artery stenting (CAS) and in patients with coexistent severe carotid and cardiac disease.

Methods The clinical data of 323 consecutive patients with coexistent severe carotid and cardiac disease undergoing CAS and OHS in our institution from January 2005 to December 2012 were collected and analyzed retrospectively. Indication for CAS was defined as carotid artery diameter reduction of $>60 \%$ (symptomatic) or $>80 \%$ 
(asymptomatic). The end points of the study included major stroke, minor stroke, death, myocardial infarction (MI), acute kidney injury (AKI) and their combination from time of CAS to 30 days after cardiac surgery.

Result There were 263 (81.4\%) men and 60 (18.6\%) women, with a mean age of $(65.2 \pm 7.3)$ yrs. $73(22.6 \%)$ had bilateral carotid stenosis, $193(59.8 \%)$ patients had symptomatic carotid stenosis, and 18 (5.6\%) had contralateral carotid artery occlusion. Three hundred twenty two $(99.7 \%)$ patients suffered from severe coronary artery disease, of whom $175(54.3 \%)$ had three-vessel disease and $166(51.6 \%)$ had left stem involvement by coronary angiography. The overall procedural success rate of CAS was $99.4 \%(321 / 323)$. Cerebral protection devices were used in 320 patients (99.7\%). Among them, 61 (18.9\%) patients received simultaneous bilateral CAS and 110 (34.1\%) patients underwent simultaneous percutaneous intervention of other arteries, including $32(9.9 \%)$ left subclavian arteries. The mean angiographic degree of stenosis was reduced from $(86.5 \pm 7.6) \%$ to $(7.8 \pm 6.8) \% \quad(\mathrm{P}<0.001)$. The mean interval time of CAS and OHS were $(22.4 \pm 15.7) \mathrm{d}$ in all 321 patients undergoing OHS. 320 patients underwent CABG after CAS. Simultaneous aneurysm resection, modified Maze procedure for atrial fibrillation and valve replacement were performed in $4(1.3 \%), 3(0.9 \%)$, and 17 (5.3\%) patients undergoing CABG respectively. The remaining patient only had valve replacement.The rate of major stroke, minor stroke, death, MI, AKI and the composite of major stroke, MI or death from time of CAS to 30 days after OHS was $1.9 \%, 2.2 \%, 1.5 \%, 2.2 \%$, $5.0 \%$ and $4.6 \%$ respectively.

Conclusion CAS before OHS was safe and feasible for patients with severe carotid artery stenosis and cardiac disease.

\footnotetext{
Aneurysm caused by takayasu's arteritis in the abdominal aorta: a case report

Lirui Yang ${ }^{1}$, Huimin Zhang ${ }^{1}$, Menggengtuya $\mathrm{Cai}^{2}$, Xiongjing Jiang ${ }^{1}$, Lei Song1, Ying Xu' ${ }^{3}$, Yubao Zou ${ }^{1}$, Yaxin Liu ${ }^{1}$, Lianjun $\mathrm{Xu}^{4}$, Xianliang Zhou ${ }^{1}$, Haiying $\mathrm{Wu}^{1}$, Rutai Hui ${ }^{1}$

1. Hypertension Division, State Key Laboratory of Cardiovascular Disease, Fuwai Hospital, National Center for Cardiovascular Diseases, Chinese Academy of Medical Sciences and Peking Union Medical College

2. Chronic Disease department, the Centers for Disease Control, Hebukesaier Country, the Xinjiang Uygur Autonomous Region

3. The Elderly and Cadre department, the Third People's hospital

4. Cardiology, State Key Laboratory of Cardiovascular Disease, Fuwai

Hospital, National Center for Cardiovascular Diseases, Chinese

Academy of Medical Sciences and Peking Union Medical College
}

A 26-year-old woman presented with five years of repeated headache and dizzy. She had suffered hypertension during pregnancy three years ago and had been taking amlodipine continuously over the last two years, but the blood pressure was uncontrolled. The patient was admitted to the hospital due to chest distress exacerbated by activity fourteen days ago. Upon physical examination, her blood pressure was about 196/134 mm Hg on the double arms. There was a significant level II systolic abdominal bruit. The laboratory biomarkers, including antinuclear antibodies, erythrocyte sedimentation rate, rheumatoid factor, serum C-reactive protein, anti-streptolysin "O", urine microalbumin, blood creatinine, and blood urea nitrogen were normal. Aortic computed tomography demonstrated a significant abdominal aortic aneurysm (maximal diameter, $5.0 \mathrm{~cm}$ ). The peripheral vascular angiography demonstrated a huge aneurysm on the upper and middle abdominal aorta. The double renal arteries were originated from the aneurysm, and the proximal segment of the double renal arteries were occluded with collateral blood flow through the abdominal aorta. The patient was diagnosed with Takayasu's arteritis (TA), with abdominal aortic aneurysm and hypertension due to renal artery involvement. The patient was administered prednisone $40 \mathrm{mg} / \mathrm{d}$, cyclophosphamide $100 \mathrm{mg} / \mathrm{d}$, and antihypertensive agents (metoprolol, nifidipine sustained release tablets, terazosin and hydrochlorothiazide). Her blood pressure remained elevated, so we suggested her perform abdominal aortic stent placement, renal autotransplantation and renal artery bypass surgery.

Three months after discharged from hospital, while still waiting for the operation, the glucocorticoid was reduced to $27.5 \mathrm{mg} / \mathrm{d}$, and the patient's blood pressure had decreased to $160 / 110 \mathrm{~mm} \mathrm{Hg}$. The aortic CT showed that the maximal diameter of the abdominal aortic aneurysm had decreased to $4.0 \mathrm{~cm}$.

The indication of the present case was renovascular hypertension, without fever or abdominal pain. The physical examination showed a level II systolic vascular murmur at the abdominal region. Due to the long course of the disease, the renal arteries were originated from the abdominal aneurysm, as well as a long occlusion of the proximal segment of the renal arteries, intervention therapy was forbidden. The diameter of the aortic aneurysm had been decreased after three months of regular drug treatment. The reason might be that with the aggressive anti-inflammatory and antihypertensive therapy, the pressure of the vessel had been declined. With the double renal arteries occluded, open revascularization should be performed when her condition was stable in order to prevent the renal function from deteriorating and to decrease blood pressure to normal. 


\section{Traditional Chinese medicine}

\author{
The laws of compatibility of proprietary chinese \\ medicines on dyslipidemia \\ Guifang Zhao ${ }^{1,2}$, Qingyong $\mathrm{He}^{1}$ \\ 1. China Academy of Chinese Medical Sciences, Guang An Men \\ hospital \\ 2. Beijing University of Chinese Medicine
}

Objective To analyze the laws of compatibility of proprietary Chinese medicine on dyslipidemia.

Methods Referenced books about Chinese medicine formulations published from 1949 to 2013, and finally selected 54 kinds of proprietary Chinese medicine formulations on dyslipidemia from the "national standard proprietary Chinese medicine heart sinks branch" and other 16 books. Using double dual independent entry method, first, the data is recorded in wps office 2013 version wps spreadsheet and analyzed in frequency. Then import SAS 6.12 software for variable clustering analysis.

Result The most common indications for Chinese medicine syndrome are blood stasis syndrome (33.33\%), phlegm stasis $(9.26 \%)$; the most common single herbs are hawthorn 23 (42.59\%), Polygonum 15 (27.78\%), Panax 12 times (22.22\%), etc. the most common compatibility of double drugs are hawthorn compatibility Salvia 8 (14.81\%), hawthorn compatibility Polygonum 7 (12.96\%), etc. the most common compatibility of there drugs are hawthorn - Polygonum Cassia 5 (9.26\%), hawthorn - Kudzu - Salvia 4 (7.41\%) and so on. The main compatible methods are promoting blood circulation + digestion + Water dampness (explained variance ratio of 0.3920), supplement Qi + Bushenyijing (explained variance ratio 0.3657), heat relief and laxative + dampness (explained variance ratio of 0.7243 ).

Conclusion There are 5 tips for the development of dyslipidemia Chinese medicine: in terms of treating the syndrome may prefer to consider blood stasis, phlegm stasis, etc. Single herb can select hawthorn, Polygonum, Panax etc. The compatibility of double drugs can select hawthorn compatibility Salvia, hawthorn compatibility Polygonum etc. The compatibility of three drugs can select hawthorn - Polygonum - cassia, hawthorn - Salvia - Pueraria; in terms of compatibility, giving priority to promoting blood circulation + digestion + Water dampness, supplement Qi + Bushenyijing, heat relief and laxative + dampness, etc.

\section{Panax quinquefolius saponin improves antithrombotic effect of dual anti-platelets in rats with acute myocardial infarction \\ Qinghua Shang ${ }^{1}$, Baojun Wang ${ }^{2}$, Qingxiang Zhang ${ }^{1}$, \\ Lei Zhang ${ }^{1}$, Jiangang Liu ${ }^{1}$, Dazhuo Shi ${ }^{1}$ \\ 1. China Academy of Chinese Medical Sciences, Xiyuan Hospital \\ 2.Oriental Hospital Affliliated to Beijing University of Chinese Medicine}

Objective To examine the cooperative antithrombotic effect and antiplatelet activity of panax quinquefolius saponin (PQS) combinated with dual anti-platelets (Aspirin and Clopidogrel) in rats with acute myocardial infarction (AMI).

Methods Four groups were designed in this study, 12 male SD rats in each group. Except for the sham group, the other rats with acute myocardial infarction (AMI) in model group, dual anti-platelets group and PQS \& dual anti-platelets group were induced by ligation of left anterior descending coronary artery (LAD). Rats in each group were intragastrically administrated with distilled water, $9 \mathrm{mg} / \mathrm{kg} / \mathrm{d}$ of aspirin and $6.75 \mathrm{mg} /(\mathrm{kg} \cdot \mathrm{d})$ of Clopidogrel or PQS $162 \mathrm{mg} / \mathrm{kg} / \mathrm{d}$ in addition to dual anti-platelets respectively for 28 days. The ratio of ventricular cavity area and cardiac transverse area, plasma endothelin (ET) concentration, serum nitric oxide (NO) concentration, platelet aggregation rate, thrombosis time, the length, wet and dry weights of thrombus and concentration of plasma thromboxane B2 (TXB2), 6 ketone prostaglandin F1 $\alpha$ (6-keto-PGF1 $\alpha$ ) and coagulation indicators (activated partial thromboplastin time, prothrombin time, thrombin time, fibrinogen, international normalized ratio) were tested in this study.

Result The ratio of ventricular cavity area and cardiac transverse area, plasma ET concentration, and the length, wet and dry weights of thrombus in vitro and FIB increased significantly $(\mathrm{P}<0.01, \mathrm{P}<0.01$, $\mathrm{P}<0.05$ and $\mathrm{P}<0.01$ ) in model group compared with the sham group, whereas, the serum NO, plasma 6-keto-PGF1 $\alpha$ concentrations, the thrombosis time and ATPP decreased significantly $(\mathrm{P}<0.01, \mathrm{P}<0.01$, $\mathrm{P}<0.05$ and $\mathrm{P}<0.01$, respectively) in model group. Rats in dual antiplatelets and $\mathrm{PQS} \&$ anti-platelets groups showed a remarkable decrease in $\mathrm{PAgT} \%$, plasma TXB2 concentration, FIB, thrombus length, wet and dry weights of thrombus $(\mathrm{P}<0.05, \mathrm{P}<0.01, \mathrm{P}<0.01$ and $\mathrm{P}<0.01$ respectively), and prolongated thrombosis time, APTT, $\mathrm{PT}(\mathrm{P}<0.01)$ compared with the model group. The ratio of ventricular cavity area and cardiac transverse area decreased significantly $(\mathrm{P}<0.01)$ and plasma 6-keto-PGF1 $\alpha$ concentration increased significantly $(\mathrm{P}<0.05)$ in $\mathrm{PQS}$ $\&$ anti-platelets group compared with the model group. Rats in PQS $\&$ anti-platelets group demonstrated a significant decrease in plasma ET-1 concentration $(\mathrm{P}<0.05)$ and an obvious increase in serum NO concentration $(\mathrm{P}<0.01)$ compared with dual anti-platelets group.

Conclusion As compared with dual anti-platelets treatment alone, the combination therapy of PQS \& dual anti-platelets improved vascular endothelial function and prevent the ventricular remodeling in rats with AMI. However, the antithrombotic effect and antiplatelet activity of PQS should be studied further.

\section{Chinese medicines for activating blood and removing blood stasis on the clinical outcome after percutaneous coronary intervention: a meta-analysis of randomized controlled trials \\ Ruixi Xi, Qiaoning Yang, Keji Chen, Ruina Bai, \\ Dazhuo Shi, Lizhi Li \\ China Acadamy of Chinese Medical Sciences, Xiyuan Hospita}

Objective This Meta-analysis was designed to evaluate the efficacy of Chinese medicines (CM) for activating blood and removing blood stasis (ABRBS) on the cardiovascular events in coronary heart disease (CHD) patients after percutaneous coronary intervention (PCI).

Methods The randomized controlled trials on the efficacy of $\mathrm{CM}$ for ABRBS, approved by State Food and Drug Administration, on the treatment of CHD patients after PCI were searched. The literature from 1979 to September 2012 was collected from Cochrane Library, clinical trial registration database, US National Library of Medicine of PubMed, ChinaInfo, China National Knowledge Infrastructure (CNKI), Chinese Biological Medical literature database (CBM), China Science and 
Technology Journal Database (VIP), China Proceedings of Conference Full-text Database, and Chinese Doctoral Dissertations \& Master's Theses Full-Text Databases. The primary end point was a composite of recurrent angina pectoris, myocardial infarction, cardiac death, heart failure; and the secondary end point was restenosis without limitation of blinding method and language. Quality evaluation of the literature was performed according to Jadad scoring. Meta-analysis was carried out using Review Manager (RevMan) 5.2 software.

Result Twenty-six randomized controlled trials using additional $\mathrm{CM}$ to Western medicine 000000000 (WM) with Jadad scores $\geq 2$ were included. In comparison to the WM treatment alone, the combination treatments significantly reduced events of the primary composite end points $(\mathrm{RR}=0.27,95 \% \mathrm{CI}: 0.22-0.34 ; \mathrm{P}<0.01)$, of them, the recurrence rate of angina pectoris ( $\mathrm{RR}=0.32,95 \% \mathrm{CI}$ : $0.26-0.40 ; \mathrm{P}<0.01$ ), acute myocardial infarction ( $\mathrm{RR}=0.28,95 \% \mathrm{CI}: 0.15-0.51 ; \mathrm{P}<0.01$ ), cardiac death $(\mathrm{RR}=0.29,95 \% \mathrm{CI}: 0.09-0.88 ; \mathrm{P}=0.03)$ and heart failure $(\mathrm{RR}=$ $0.22,95 \%$ CI: $0.09-0.56 ; \mathrm{P}=0.001)$; and also reduced restenosis rate $(\mathrm{RR}$ $=0.41,95 \%$ CI: $0.32-0.52 ; \mathrm{P}<0.01$ ).

Conclusion Addtional use of CM to WM treatment reduced cardiovascular events in CHD patients after PCI.

\section{Wenxin granule versus Anti-arrhythmic drugs for premature ventricular complexes}

Ruina Bai, Bo Li, Ruixi Xi, Lizhi Li

China Academy of Chinese Medical Sciences, Xiyuan Hospital

Objective This systematic review aims to evaluate current evidence of Wenxin granule and to compare it with anti-arrhythmic drugs for the benefits and side effects for premature ventricular complexes (PVCs).

Methods Relevant studies of Wenxin granule for PVCs were collected from databases including Pub med, Cochrane library, Embase, CNKI, Wanfang Database and CBM disk. Two reviewers screened literature according to the inclusion and exclusion criteria, extracted data and assessed the quality of the included studies independently.

Result We included 2 papers about Wenxin granule for PVCs (2 papers and 308 participants). In the end no study was included in this system review because of the different observed outcome, thus unable to combine those data.

Conclusion Although those trials that included in this system review showed that Wenxin granule can reduce the occurrence of PVCs in Holter and attenuate the symptoms, the evidence is inadequate because of the potential biases existed in those trials influencing the reliability of this conclusion. We think that the evidence is inadequate and it needs more rigorous trials with high quality to give high level evidence to support the effectiveness of Wenxin granule for premature ventricular complexes. 


\section{Cardiovascular Nursing}

\author{
Superiority of $5 \% \mathrm{NaHCO}_{3}$ for preoperative \\ hair removal in patients undergoing coronary \\ artery bypass graft surgery with a limb vein: a \\ randomized controlled trial \\ Honglei Wu \\ Affiliated Hospital of Nantong University
}

Objective A wet, alkaline environment with the $\mathrm{NaHCO}_{3}$ solution is better than a dry one with talcum powder, which can soften and expand the hair, decrease the friction and shear forces on the skin, then making hair removal easier and more efficient. The purpose of this study was to compare the effects of a preoperative skin preparation of $5 \% \mathrm{NaHCO}_{3}$ to talcum powder for softening hair in patients undergoing coronary artery bypass graft $(\mathrm{CABG})$ surgery with the saphenous vein.

Methods A prospective randomized, single-blinded, controlled study was established. Between March 2012 and June 2013, 120 patients who met the inclusion and exclusion criteria underwent elective $\mathrm{CABG}$ surgery with the saphenous vein in our hospital. The same doctor obtained the saphenous vein of each patient by a uniform method. CABG surgery was performed through an open or endoscopic technique, according to each patient's condition. Patients were divided into two groups of 60 patients each, according to whether the preoperative skin preparation procedure was performed with a $5 \%$ solution of $\mathrm{NaHCO}_{3}$ at 45 to $50{ }^{\circ} \mathrm{C}$ (Experimental group) or with talcum powder (Control group). Main outcome measures were the incision inflammation rate, skin preparation time, presence of shaving-induced macroscopic hemorrhagic spots, verbal rating scale (VRS) pain score, and satisfaction.

Result Neither group had any evidence of incision inflammation. The average (mean \pm standard deviation) skin preparation time of the experimental group was shorter than that of the control group [ 5.58 $\pm 1.52) \mathrm{min}$ vs $(9.74 \pm 1.23) \mathrm{min}, \mathrm{P}<0.001]$. Three patients in the experimental group and 17 patients in the control group had shavinginduced macroscopic hemorrhagic spots after skin preparation ( $\mathrm{P}=$ 0.001). The VRS pain scores were different between the two groups ( $\mathrm{P}$ $<0.001$ ). In the control group, $40 \%$ of patients and $37 \%$ of nurses were satisfied with the skin preparation procedure, compared to $95 \%$ and $90 \%$, respectively, in the experimental group (both $\mathrm{P}<0.001$ ).

Conclusion Hair softening with a $5 \% \mathrm{NaHCO}_{3}$ solution protected the skin integrity, alleviated pain, and improved patient satisfaction to a greater extent than talcum powder. Nurses found the $5 \% \mathrm{NaHCO}_{3}$ solution to be more convenient and less time-consuming to use.

\section{The functions of tidal volume and other parameters in the SIMV/AC mode of AVEA ventilator \\ Honglei Wu \\ Affliiated Hospital of Nantong University}

Objective The purpose of this study was to determine the functional relationship between tidal volume (VT) and 4 other ventilator parameters.

Methods The following parameters were collected from an AVEA ventilator operating in the SIMV/AC volume-controlled ventilation mode: VT (in L), peak velocity $\left(\mathrm{V}_{\max }\right.$, in $\left.\mathrm{L} / \mathrm{min}\right)$, breathrate (f, in bpm), inspiratory-to-expiratory time ratio (I: E), and plateau time (TP, in S). The relationship between VT and each of the other variables was determined.

Result When the other parameters were held constant, VT was positively correlated with Vmax and I: E, but negatively correlated with $\mathrm{f}$ and $\mathrm{TP}$. When the velocity versus time curve was a 50\% linearly decreasing wave, the functional relationship among the 5 parameters was governed by the equation: $\mathrm{VT}=180 \mathrm{~V}_{\max }[60 \mathrm{f}-60 \mathrm{f}(1+\mathrm{I}: \mathrm{E})-\mathrm{TP}]$.

Conclusion The functional relationship among the 5 parameters for the AVEA ventilator in the SIMV/AC mode, these parameters should be controlled in accordance with the patient's pathophysiological needs.

\section{The effectiveness of multimedia nursing education on reducing anxiety in myocardial infarction patients after percutaneous coronary intervention \\ Xinlei Ma, Chengfei Wen \\ The First Hospital of Jilin University}

Objective It is a so difficult work for all the nurses in the extremely busy cardiac care unit (CCU) to do the proper education to the myocardial infarction (MI) patients. This study aimed to confirm the effectiveness of multimedia nursing education on reducing anxiety in MI patients undergoing percutaneous coronary intervention (PCI).

Methods A total of $80 \mathrm{MI}$ patients undergoing percutaneous coronary intervention (PCI) were recruited and were randomly divided into the experimental group and the control group, 40 patients each group. The control group received written nursing education material and the experimental group received multimedia nursing education. All the 80 patients were measured at these periods: at admission to the $\mathrm{CCU}$, before undergoing the PCI, go back to the general ward and at discharge. We used the state-trait anxiety inventory (STAI), and Mishel's uncertainty in illness scale (MUIS) to assess the condition of the anxiety of all patients.

Result Before undergoing the PCI, all the patients would feel much more anxiety than other periods. The both two education methods could reduce the anxiety after the patients undergoing PCI. The experimental group achieved significantly greater reductions than the control group in terms of anxiety in each of the four periods.

Conclusion The both two education methods could reduce the anxiety of MI patients undergoing PCI in CCU. But we should to find out some new education to reduce the anxiety to the Mi patients before PCI.

\section{Digital monitoring of the dynamic pressure change in the pressure infusion bag using a CVP catheter \\ Honglei Wu \\ Affliated Hospital of Nantong University}

Objective This study investigated the feasibility and effectiveness of using a central venous pressure (CVP) catheter for digitally and continuously monitoring the pressure change in a pressure infusion bag after surgery, to increase the effectiveness of arterial (ART) and CVP 
catheters.

Methods This prospective study included 309 patients who underwent open-heart surgery between January 2013 and February 2014. Patients were divided into two groups. In the experimental group ( $\mathrm{n}=$ 144), the pressure change in the infusion bag was digitally monitored by a pressure transducer. In the control group $(n=165)$, the pressure change was checked manually every 4 hours. Blockage rates of the ART and CVP catheters were recorded 1,2 , and 3 days postoperation (DPO).

Result When the pressure transducer was placed parallel with the pressure gauge (or parallel with the heart), the line for $300 \mathrm{~mm} \mathrm{Hg}$ on the monitor for the infusion bag overlapped with (or was below) the pressure change curve. There was no significant difference in the CVP catheter blockage rate between the two groups. After 2 DPO, the effectiveness of the ART catheter was less reduced in the experimental group than in the control group $(\mathrm{P}<0.05)$.

Conclusion Using a CVP catheter for digitally and continuously monitoring the pressure change in the infusion bag is a feasible approach that can enhance the effectiveness of the ART catheter and can help nurses respond quickly to pressure changes.

\section{Nursing of patients received minimally invasive clinical pathway after cardiac interventional therapy \\ Hong Wang, Yamei Liu, Yaying Zhang \\ The First Hospital of Jilin University}

Objective Observe the effect of minimally invasive clinical pathway in patient after cardiac interventional therapy.

Methods According to the needs of the patients according to the cardiovascular interventional therapy and nursing characteristics, combined with the actual situation of the hospital, in chronological order as horizontal axis, with clinical assessment, examination and assay, drug therapy, diet, activity, hospitalization and hospital health education for the vertical axis, formulated the cardiovascular interventional therapy path table. The control group carried out according to the traditional model of medical care. The observation group begun first by responsibility nurse according to the clinical pathway table that prompts admission introduction, health assessment, contact a doctor in charge since the admission, the content and effect to explain the path. To gain understanding cooperation of patients, when necessary, and family participation, patients completed the inspection and evaluation of time to the fastest speed, the most short, and understand the treatment process, is expected to hospitalization, treatment programs, medical care and family should cooperate with the treatment nursing. Observe and and record each the average hospitalization time, cost of hospitalization, complications, patient care and job satisfaction, evaluation of patients knowledge on health. Health knowledge evaluation table for the selfmade questionnaire, health knowledge assessment scale consists of 10 questions, each question according "to know, know, do not know" the 3 grade, is $3-1$, out of 30 points. The survey of patient satisfaction unified formulation of the nursing department of our hospital, a total of $13 \mathrm{Q}, \mathrm{A}$, B, C, D 4 grades, scores of 3, 2, 1, 0, out of 39 points. These 2 form the higher the score, the better the effect. Table respectively before operation and on the day of discharge by responsibility nurse patients were instructed to fill in. The nursing effect in the two groups was compared.

Result The mean hospitalized duration, satisfaction degree and health knowledge level in the observation group were superior to those of control group $(\mathrm{P}<0.05)$.

Conclusion The adoption of minimally invasive clicical pathway in patients after cardiac interventional therapy could increase working efficiency and ensure the nursing quality. The multidiscipinary cooperation, deepen the holistic nursing. And help the patients to master knowledge of health, improve nursing satisfaction.

\section{Research on the effects of motivational interviewing in smoking cessation for patients with coronary heart diseases \\ Yingying Zhou, XiaoxingFeng, Liyan Yu, Yan Gu Cardiology Department, The First Hospital of Jilin University}

Objective To investigate the efficacy of motivational interviewingbased intervention in smoking cessation for patients with coronary heart diseases.

Methods Motivational Interviewing (MI) is a patient-centered way to improve intrinsic motivation of behavior change, contents of MI can be summarized as risks, benefits, obstacles, repeat. 100 coronary heart diseases patients were assigned to the usual care $(\mathrm{UC} n=50)$ or motivational interviewing intervention ( $\mathrm{MII} n=50$ ) groups. UC participants received the usual clinic care and health education.MII participants received a six time MI sessions over 3 months. Interviews include: (1) To help patients recognize that smoking and coronary heart disease are closely related. (2) To help patients realize the dangers of smoking on cardiovascular. (3) To help patients recognize the potential benefits of quitting smoking. (4) Encourage the patients to face obstacles and setbacks in the process of smoking cessation bravely, and provide a solution available for the patients, enhancing patient confidence and motivation. (5) Encountering with the patients do not want to try to change, repeat the above explanation optionally. Compare the cigarette consumptions, frequency and duration of smoking between the study groups to test the efficacy of the intervention.

Result The results showed cigarette consumptions of MII groups declined significantly in comparison with UC groups.

Conclusion Motivational Interviewing can enhance patient willingness to adhere to smoking cessation. MI is an effective interpersonal communication mode to help the patients identify their internal problems and promote behavior change. Findings support MI can improve health outcomes and adherence to healthy behavior in the study population.

\section{Application of the transtheoretical model in rehabilitative exercise of coronary stent implantation patients \\ Yingying Zhou, Dan Zhang, Yongchun Chen, Jinan Li The First Hospital of Jilin University, Cardiology department}

Objective To explore the effects and outcome of rehabilitative exercise based on the transtheoretical model (TTM) in patients with coronary stent implantation.

Methods The transtheoretical model consist of five major physical exercise behavior change stages: precontemplate stage, contemplate stage, preparation stage, action stage and maintenance stage. 120 cases of patients after coronary stent implantation were divided into the experimental $(n=60)$ and control $(n=60)$ groups randomly. Experimental group were received Trans-theoretical Modelbased education and planned intervention, they were divided into different physical exercise change stages according to the assessment of willingness to behavioral change and the movement baseline, then 
received corresponding intervention strategies. Control group were given routine health education and post-operative instructions.

Result There were significant differences in the patients physical exercise change stages between the two group, $76.67 \%$ of the control group have not take action, including 21 cases in precontemplate stage, 17 cases in contemplate stage, 8 cases in preparation stage. 49 cases (81.67\%) of experimental group began regular rehabilitative exercise, including 27 cases in the action stage, 22 cases in maintenance stage. The frequency, duration of physical activities from the experimental group was significantly improved

Conclusion Considering physical condition and specific situation of the each individual, targeted intervention were applied to the process of cardiac rehabilitation exercise on the basis of TTM, which can effectively improve the patients exercise compliance, promote the willingness of physical exercise and help the patients establish healthy behaviors. The TTM can provide clinical health education and behavior change with theoretical support and behavioral guidance.

\section{QCC in reducing the incidence of low molecular weight heparin injection petechiae \\ Yue Liu, Chengfei Wen, Xue Jin \\ The First Hospital of Jilin University}

Objective Our department carry out quality control circles from August 2013, aimed at reducing the subcutaneous low molecular weight heparin incidence of complications. This paper evaluated QCC subcutaneous low molecular weight heparin to reduce the incidence of bruising effect.

Methods Established QCC team, a clear division of labor, time to carry out quality control circle meeting to analyze the reasons for the occurrence of petechiae, standardize operations and injections assessment, strengthening communication and patient education, patient wards and paste missionary board for memory, petechiae standards bruising area $\geq 4 \mathrm{~cm}$, a search by the night nurse on a night shift nurse petechiae number occurs, the leader cycle length and calculated daily by the incidence. QCC launch time was from August 2013 to April 2014, the calculated results were compared with between February 2013 to August 2013.

Result QCC activities carried out before the subcutaneous low molecular weight heparin ecchymosis lower incidence rate intervention to $4.77 \%$ from $16.35 \%$ after the intervention significantly reduced, reaching the predetermined criteria, the difference was statistically significant $(\mathrm{P}<0.01)$, and in the activities of the QCC, mobilize the enthusiasm of everyone played a department nurses' creativity and initiative, departments atmosphere well swap.

Conclusion QCC activities carried out by subcutaneous injection to reduce the incidence of low molecular weight heparin petechiae have a good effect, worthy of promotion and practical in clinical.

\section{The effectiveness of rehabilitation guidance to patients undergoing pacemaker after leaving hospital \\ He Guan, Xinlei Ma, Yue Liu \\ The First Hospital of Jilin University}

Objective In order to improve life quality of patients undergoing pacemaker, reduce complications.

Methods A total of 56 patients were randomly recruited. All of them were primary school degree or above and no communication barriers. (1) Function exercise: position: lying left or horizontal in the first one month. the operation upper limb: Arm outreach is prohibited in the first one week. physical exercise: Do aerobic exercise in 2 to 4 weeks, such as Tai Chi, walking, jogging and so on. After half a year they can work and living as usual according to their own condition. (2) Mental nursing: Communicate with patients to make them in sunny mood and to ensure $6-8 \mathrm{~h}$ sleeping. (3) Wound care: Dressing: Avoiding to rub and impact the wound. Wearing cotton but not polyester fiber clothes. Two weeks after operation can take a bath but do not touch the wound. (4) Diet: Keep nutrition balanced, having more meals a day but less food at each, feeding more high dietary fiber, vitamin, protein to keep defecating expedite. (5) Self check: Teaching patients to take pulse by themselves. If the measurements are not normal should be timely medical treatment. If there are dizziness, palpitations, syncope, hiccup, timely medical treatment. (6) Keeping away from radiation: Keeping away the radiation, such as power station, transformers, CT, nuclear magnetism and so on. Daily radiation: keeping away from oven, electric blanket and other radiation electrical appliances. During using time, if uncomfortable, must stop using and keep away from it above 1.8 meters. (7) Carrying pacemaker card all times: Patients detail data, pacemaker model, date of surgery, pacing rate must be included. (8) Regular reexamination: In the first one year must be regular reexamination. before to replace the batteries, reexamination once a year, when the power is closely finished, do reexamination frequently.

Result None of these 56 patients went to hospital again because of the complications by using these outside hospital methods.

Conclusion A specific rehabilitation guidance to the patients undergoing pacemaker, not only improve patients life quality, but also reduce complications after pacemaker. It is deserved to spread.

\section{Analysis of the common psychological problems in the elderly \\ Zengjuan Guo, Xiaoyuan Huang, Bo Gao The First Hospital of Jilin University}

Objective The Cardiovascular disease incidence in the elderly is often in the firstplace. Because the symptoms of cardiovascular disease are untypical, longer course, higher probability of recurrent hospitalization, andvery easy to produce negative psychologicalproblems. Therefore, this study focuses on the analysis of the common mental problems of the elderly.

Methods All subjects were comefrom diagnosis and treatment center of cardiovascular diseasein our hospital. Among them, 94 were male, mean age 51 years; female, 81 cases, with an average age of 54 yrs. Randomly divided into 2 groups: the observation group and the control group. The control group was given conventional nursing, the implementation of personalized psychological care on the basis of the observation group. Psychological evaluation for the elderly; Psychological adjustment; Family and society`s participation; Psychological problems and common impact factors in the elderlywere analyzed, so as to put forward countermeasures.

Result The common mental problem of the elderly is loneliness, frustration, fear, depression and forgetfulness. Influence factors include physiological factors and social factors. Physiological factors: ageing of sense organ. The infuence of the diseases. threaten of death. Social factors: social role change, widowed "empty nest family". Loneliness, sense of loss, fear, depression and forgetfulness.

Conclusion Only by grasping the psychological characteristics of the elderly, understanding the common psychological problems of 
the elderly and their causes, to take targeted measures, can effectively reduce the psychological problems of the elderly.

\section{Influence of carrying out clinical nursing pathway on the psychological status of elderly patients with cardiovascular disease \\ Ying Liu, Ling Qi, Juan Xie \\ The First Hospital of Jilin University}

Objective Due to various reasons, physiological, psychological and social, environmental, elderly cardiovascular disease patients prone to loneliness and sense of loss, need to get sympathy and help. If the psychological needs are not met, patients often appear anxiety, depression, fear, guilt, role cognition disorder, psychological dependence and other adverse psychological reactions. These adverse psychological reaction causing the body regulating function and disease resistant ability, increase the difficulty of nursing work. Therefore, this study examines the impact of the implementation of clinical nursing pathway on the psychological state of elderlypatients with cardiovascular disease.

Methods The inclusion criteria: All the subjects suffered from cardiovascular disease. Do not have concurrent diseases. In accordance with the principle of informed consent. Exclusion criteria: Do not meet the above criteria. Clinical data collection is not complete or not subject tomanagement. Evaluation criteria of mental state: Self-rating Anxiety Scale (SAS): Less than 50 points were normal; 50-60 formild anxiety; 61-70 for moderate anxiety, 70 points or more for severe anxiety. Self rating Depression Scale (SDS): 53-62 points for mild depression, 63-71 for moderate depression; 70 points or more for severe depression. The control group received routine nursing, the observation group based on the application of nursing pathway model.

Result Self-rating Anxiety Scale (SAS) and self rating Depression Scale (SDS) score of two groups after treatment were decreased $(\mathrm{P}<$ 0.05 ), and the observation group decreased more obviously than in the control group $(\mathrm{P}<0.05)$. Total nursing satisfaction rate was $94.83 \%$ in the observation group, $82.76 \%$ in the control group.

Conclusion For each specific patient personalized clinical nursing path, the provisions of clear project examination, treatment, nursing, health education programs and counseling expectations are detailed, standard, make the nursing service with planning and foresight, will more patient in daily care of patients, to pay attention to patients' psychological feelings, to enhance the treatment effect, significantlyimprove the physiological and psychological status of patients.

\section{Nursing of patients with mental disorder associated with cardiovascular disease \\ Lei Wang, Shuqing Wang, Li Feng \\ The first hospital of Jilin University}

Objective The prevalence of mental disorder in patients suffering from cardiovascular disease was high.Cohherr reported, patients with cardiovascular disease accompanied depression or anxiety disorder was $8 \%-44 \%$. After tracking acute myocardial infarction patient found that, $15 \%$ patients may have anxiety or depression. 101 cases of hypertension and 97 cases of atrial fibrillation survey found that, the prevalence rate of depression and anxiety disorders in patients with atrial fibrillation were $38 \%$ and $28 \%$; in patients with essential hypertension prevalence rate of depression and anxiety disorder were $30 \%$ and $23 \%$. Cardiovascular disease and depression, anxiety disorders exist mutual influence, and are related to a negative. Depression, anxiety disorders have unhealth influence to the development of cardiovascular disease and its prognosis. Therefore, this study discuss the nursing of patients with mental disorder associated with cardiovascular disease.

Methods All samples were from diagnosis and treatment central of cardiovascular disease in our hospital, 42 male cases, 26 female cases, age 42 to 80 years, 29 cases of coronary heart disease, 20 cases of hypertensive heart disease, rheumatic heart disease in 15 cases, 4 cases of myocardial infarction, hospitalization duration for the longest time 48 days, the shortest 17 days, with an average of 26 days. In addition to monitoring the symptoms and vital signs ofthe patients, at admission, during hospitalization, one month after discharge psychological questionnaire was investigated respectively. Personalized psychological analysis and adjustment was done to the patients with mental disorder.

Result 31 cases were cured, 36 cases improved and 1 case died. The days of hospitalization shorten. The psychological characteristics of the patients are significantly improved; the effective rate was $98.5 \%$.

Conclusion Pay attention to psychological influence factors on cardiovascular disease. The control of the possible stress factors. Promptly adjust with the psychological balance in patients. The good feedback, so as to grasp the mentality of the patients. To correct unhealth behavior.

\section{A retrospective analysis on cardiovascular disease incidence associations with individual risk factor \\ Shui Yu, Xiaosu Zhao, Ling Song, Wenshuai Wang First Hospital of Jilin University}

Objective Cardiovascular disease, as its high incidence, morbidity, mortality, recurrence rate, and complications, has becoming a serious disease. it threat human especially elderly healthy. The main risk factors for cardiovascular disease, including high blood pressure, high cholesterol, high blood sugar, smoking and overweight, However, the prevalence of these risk factors differ among individuals in the role. This paper discusses the relationship between the major risk factors for cardiovascular disease and cardiovascular disease in individuals.

Methlrds A retrospective analysis a total of 100 patients from September 2012 to September 2013 at the First Hospital of Jilin University suffering from cardiovascular disease (50-60 yrs, mean age 57 yrs), including 56 males and 44 females. Detailed history combined with physical examination and related laboratory tests, then statistic the main five correlation risk factors and analysis cardiovascular disease incidence associations with individual risk factor.

Result $90 \%$ of individuals with more than one risk factor (of which $53.3 \%$ have either one, $17.8 \%$ had either $2,15.6 \%$ have either three, $7.8 \%$ had either 4, 5.6\% had either 5), Male with a high incidence than female. With the increase of the risk number the incidence of cardiovascular disease increase, there are significant synergy between risk factors which induced cardiovascular disease. Different combinations of risk factors has different role for cardiovascular disease, but hard to clear doseresponse relationship between each other.

Conclusion Risk factors of cardiovascular disease in individuals and their aggregates in the elderly population is prevalent in our country, but the proportion for cardiovascular disease induced by multiple of factors is not high. A retrospective analysis to explore the different risk factors aggregates effect which could cause cardiovascular disease and the pathophysiology and dose-effect relationship is important for the prevention and treatment of cardiovascular disease. 


\section{Influence of evidence-based nursing on prognosis of patients with cardiovascular disease}

Yaying Zhang, Ying Liu, Zengjuan Guo

The First Hospital of Jilin University

Objective Cardiovascular diseases, including hypertension, myocardial infarction, angina and heart failure, long-term repeated attacks, which may lead to emergency, danger, severe cases, even result in the death of thecrisis. Because of the disease itself, and physiological factors, patients in the hospital often exposed many psychological problems, new diseases and complications, not good for the treatment of patients, and they influence each other, forming avicious spiral. Therefore, this study aims to investigate the effect of evidence-based nursing on prognosis of patients with cardiovascular disease.

Methods Knowledge of economic status, family background, marital status, educational level, social support, pathogenetic condition, living environment and other factors that can influence the prognosis of cardiovascular disease. Personal skills and clinical experience of each primary nurse. Patient value, desire and reality. The above three elements combined organically, formulate the complete nursing program. Analyze the effect of the implementation.

Result In both groups the adverse reactions, incidence of complications decreased, hospital duration shorten, the satisfaction of patients to medical staff improved. All th items in the observation group was significantly different than that of control group $(\mathrm{P}<0.05)$.

Conclusion The evidence-based nursing in patients with cardiovascular disease can help nursing staff to consult massive literature, find empirical scientific and credible evidence, so as to make clinical decision making in nursing care plan appropriate, reasonable and effective.

\section{Analysis the cardiovascular states in peritoneal dialysis patients with end stage diabetic nephropathy \\ Guiying Wang, Runduo Pan, Juan Xie \\ The First Hospital of Jilin University}

Objective To analysis the cardiovascular states of peritoneal dialysis patients with end-stage diabetic nephropathy, so as to provide a basis for nursing countermeasures after peritoneal dialysis.

Methods All the peritoneal dialysis patients were divided into the diabetic nephropathy group and chronic glomerinephritis group, and the cardiovascular ultrasound, blood pressure, body weight index, cardiochest radio, hemoglobin, serum creatinine, serum urea nitrogen, serum albumin, urine volume were compared a week before the peritoneal dialysis treatment.

Result All the preperitoneal dialysis patients had a significant cardio-vascular abnormality. There were older age, wore serious cardiac injury, higher cardi-chest radio, lager left atrium, higher hypertension incidence rate, lower hemoglobin and serum albumin level in the diabetic nephropathy patients compared to those in the chronic glomerinephritis group $(\mathrm{P}<0.05)$.

Conclusion The diabetic nephropathy patients had higher cardiovascular risk before peritoneal dialysis treatment and required effective nursing countermeasures on initial stage of peritoneal dialysis. It is necessary to improve the anemia, the chronic infection states, the cardiovascular volume states and the control of blood pressure to reduce the cardiovascular complications and nursing risk. To improve compliance and ensure the safety in the process of patients with anemia treatment. For the diabetic nephropathy patients, we should make strict capacity management.

\section{The application of health education path in patients with acute myocardial infarction in $\mathrm{PCl}$ Haiting Zhou, Xiaoxing Feng, Yue Liu, Liyan Yu, Jinan Li The First Hospital of Jilin University}

Objective To explore the implement of health education path in patients with acute myocardial infarction in PCI.

Methods Choose 176 patients with acute myocardial infarction of implement of PCI during January to February in 2014, divided into two groups at random: the matched group and experimental group. The matched group carrys out regular Health-Education, consist of offering health guidance to being admitted to hospital, preoperation, postoperation, prior to diacharge, and proceeding health education randomly at the rest of the time, experimental group carrys out health education path. Using the homemade score to questionnaire the patients before leaving the hospital on health education effect, patient compliance and nursing satisfaction, assign points corresponding to each problem, take those patients that who can repeat the related knowledge and points for attention and score 80 points.

Result All the indexes of the experimental group on health education effect were higher than the matched group; the experimental group was superior to the matched group on compliance and satisfaction; The differences were statistically significant $(\mathrm{P}<0.05)$.

Conclusion The application of health education path, it makes the health education work on acute myocardial infarction programmatically, standardization, specifically, and the effects on health education are more significant, which can contribute to obtainning better curative effect to improve the satisfaction of patients. 


\title{
Hospital management
}

\author{
Data quality in the China cardiovascular surgery \\ registry (CCSR): results from a national data \\ validation audit \\ Kun Hua, Zhe Zheng, Wei Li \\ State Key Laboratory of Cardiovascular Diseases, Fuwai Hospital, \\ National Center for Cardiovascular Diseases, Chinese Academy of \\ Medical Sciences and Peking Union Medical College
}

Background Chinese Adult Cardiovascular Surgery Registry (CACSR) is a national cardiovascular surgery program initiated in 2004 by Ministry of Health to promote cardiovascular surgery quality improvement efforts, reduce disparities in cardiovascular surgical care, and improve clinical outcomes through increased adherence to guideline recommendations. We examined the accuracy and reliability of data entered in CACSR.

Methods Data entered by website in the CACSR database were compared with that abstracted from de-identified medical records by trained auditors. Accuracy for each individual data element and a composite accuracy measure were calculated. Reliability was assessed using kappa $(\kappa)$ statistics for categorical variables and intra class correlation (ICC) for continuous variables.

Result A random selection of 793 medical records from 86 CACSR hospitals was obtained. Overall accuracy was above $90 \%$ for all variables abstracted except for height $(84.9 \%)$, serum creatinine (88.1\%), peri-operative blood production use $(79.0 \%)$, and mechanical ventilation time $(85.3 \%)$ and ICU stay time $(85.3 \%)$. Intermediate to

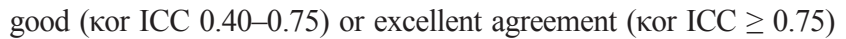
was observed for nearly all audited variables, including time-related performance measures. The overall composite accuracy rate was $96.1 \%$. The composite measure varied slightly by region and hospital academic status, but there were no significant differences in composite accuracy by bed size, cardiac surgery volume.

Conclusion This audit establishes the reliability of CACSR registry data. Individual data elements with suboptimal accuracy should be targeted for further data quality improvement. 


\section{Author index}

B

Bai, Ruina $\quad 133$

Bai, Wenjuan 128

Bai, Yuan 68

$\mathrm{Bu}$, Xiaoqing 3

\section{C}

Cao, Fangfang 78

Chen, Guo $\quad 108$

Chen, Guocai $\quad 62$

Chen, Guozhu $\quad 67$

Chen, Huiqiang 26

Chen, Jinliang $\quad 64$

Chen, Junxiong $\quad 38$

Chen, Ken 29

Chen, Mengmeng $\quad$ 47; 47

Chen, Panpan 25

Chen, Shi 122

Chen, Shufeng 4

Chen, Tao 96

Chen, Wei $\quad 66$

Chen, Yang 93

Chen, Zhangqiang $\quad 76 ; 104$

Chen, Zhen $\quad 74$

Chen, Zhenglian $\quad 45$

Cheng, Zhaoyun 85

Cui, Bin $\quad 45$

D

Dai, Hailong 53

Dong, Hui $130 ; 130$

Dou, Kefei $\quad 70$

Du, Yingjie 79

\section{F}

Fan, Fan

Fan, Guangpu

Fan, Wei

Fang, Yinghui

Fang, Zhoufei

Feng, Tianjie

Fu, Biao

Fu, Yuanyuan

G

Gao, Jie $\quad 37$

Gao, Xiao $\quad 41$

Gao, Xiaojin 62

Ge, Zeng 8

Gong, Xuhe 33

$\mathrm{Gu}$, Chun $\quad 121$

Gu, Hongqiu 13

Gu, Jian $\quad 102$

Gu, Shujun 3

\begin{tabular}{ll} 
Guan, He & 136 \\
Guan, Wenchi & 6 \\
Guan, Yulong & 111 \\
Guo, Daoxia & $20 ; 22$ \\
Guo, Liwei & 56 \\
Guo, Shujie & 47 \\
Guo, Xiying & 61 \\
Guo, Ying & 63 \\
Guo, Zengjuan & 136 \\
& \\
H & \\
Hai, Bo & 17 \\
Hao, Guang & 11 \\
He, Fengping & 28 \\
He, Jia & 95 \\
He, Peiyuan & $69 ; 69$ \\
He, Yan & 99 \\
Hu, Jinxiao & 110 \\
Hu, Junlong & 84 \\
Hu, Shengshou & 83 \\
Hu, Tao & $73 ; 74$ \\
Hu, Xinyang & $25 ; 60$ \\
Hu, Zunsong & 21 \\
Hua, Kun & $86 ; 139$ \\
Huang, Bi & $93 ; 93 ; 94$ \\
Huang, Chen & 21 \\
Huang, Yuqing & $101 ; 101 ; 102$ \\
Huang, Zhiwei & 60 \\
\hline &
\end{tabular}

$\mathrm{J}$

Jia, Yanjun 28

Jiang, Long $\quad 52$

Jiang, Tiannan 45

Jiang, Yuexin 59;59

Jiang, Zhiyan $\quad 82$

L

Lan, Tingyu 118

$\mathrm{Li}$, Chen $\quad 28$

Li, Dianjiang $\quad 13 ; 13$

$\mathrm{Li}$, Fang $\quad 90$

Li, Haixia $\quad 19$

$\mathrm{Li}, \mathrm{He} \quad 18$

Li, Hongjian $\quad 102$

Li, Jiehui $\quad 70 ; 78 ; 78 ; 80$

Li, Liangpeng $\quad 34$

Li, Meixiang $\quad 29$

Li, Nanfang $\quad 104$

Li, Sha $\quad 14 ; 14$

$\mathrm{Li}$, Shoujun $\quad 90$

Li, Wen $15 ; 124$

Li, Wenlei $\quad 28$

Li, Xiangjun 14

$\mathrm{Li}$, Yan $\quad 116$ 


\begin{tabular}{|c|c|c|c|}
\hline Li, Yanyang & 43 & $\mathrm{R}$ & \\
\hline $\mathrm{Li}$, Yongjun & $52 ; 55 ; 55$ & Rao, Chenfei & $81 ; 81 ; 81$ \\
\hline Li, Yuehua & $6 ; 7 ; 66$ & Rao, Mingyue & 17 \\
\hline Liang, Xiaohua & 1 & Ren, Lan & 127 \\
\hline Lie, Ning & 30 & Ren, Xiaoqing & 98 \\
\hline Lin, Huan & $30 ; 104 ; 117$ & Rong, Jiang & 118 \\
\hline Lin, Yaowang & 11 & & \\
\hline Liu, Gang & $10 ; 59 ; 101$ & $\mathrm{~S}$ & \\
\hline Liu, Haibo & 71 & Shang, Qinghua & 132 \\
\hline Liu, Hanning & $8 ; 83$ & Shang, Qingqing & 31 \\
\hline Liu, Jinping & $110 ; 112$ & Shao, XingHui & $92 ; 92$ \\
\hline Liu, Jun & 97 & Shen, Xiaoxia & 11 \\
\hline Liu, Junwei & 57 & Sheng, Tao & 37 \\
\hline Liu, Kai & 114 & Shi, Liying & 63 \\
\hline Liu, Liang & 5 & Shi, Xiaojing & 51 \\
\hline Liu, Miao & 8 & Song, Lei & $68 ; 70$ \\
\hline Liu, Nana & 31 & Song, Mingbao & $46 ; 46$ \\
\hline Liu, Pei & 15 & Song, Pan & 16 \\
\hline Liu, Peiliang & 68 & Song, Yan & 39 \\
\hline Liu, Pinxiao & 23 & Song, Zhiming & 33 \\
\hline Liu, Shen & $85 ; 85$ & Sun, Baojing & 51 \\
\hline Liu, Tong & $36 ; 120 ; 120$ & Sun, Yanhua & $110 ; 113 ; 114$ \\
\hline Liu, Xi & 16 & Sun, Zhenxing & 123 \\
\hline Liu, Yaowu & 40 & Sun, Zhichao & 107 \\
\hline Liu, Ying & 137 & & \\
\hline Liu, Yong & 105 & $\mathrm{~T}$ & \\
\hline Liu, Yue & $32 ; 136$ & Tan, Xiaoyue & 122 \\
\hline Liu, Zheng & $96 ; 96 ; 97$ & Tang, Xugang & 17 \\
\hline Liu, Zhouying & 42 & Tao, Quanwei & 27 \\
\hline Lu, Jia & 6 & Tian, Maozhou & 80 \\
\hline Lu, Wei & 30 & & \\
\hline Lu, Zhinan & 128 & $\mathrm{~W}$ & \\
\hline \multirow[t]{2}{*}{ Luo, Qin } & 3 & Wang, Chen & 25 \\
\hline & & Wang, Chunmao & $49 ; 49 ; 51$ \\
\hline M & & Wang, Dezheng & 2 \\
\hline Ma, Lina & 103 & Wang, Guiying & 138 \\
\hline Ma, Xinlei & 134 & Wang, Guoliang & $42 ; 43$ \\
\hline Ma, Zhifei & 26 & Wang, Hong & 135 \\
\hline Meng, Hong & 119 & Wang, Honglian & 126 \\
\hline Meng, Zili & $75 ; 76$ & Wang, Huiying & 113 \\
\hline Mi, Xuenan & 54 & Wang, Jian & 79 \\
\hline \multirow[t]{2}{*}{ Mo, Xingbo } & 54 & Wang, Jiang & 46 \\
\hline & & Wang, Jianguang & 118 \\
\hline $\mathrm{P}$ & & Wang, Jingjin & $119 ; 124$ \\
\hline Pan, Dianzhu & 124 & Wang, Jizheng & 40 \\
\hline Peng, Meng & 126 & Wang, Juan & 5 \\
\hline Peng, Kuang & 39 & Wang, Lan & 108 \\
\hline Peng, Yaguang & 1 & Wang, Lei & 137 \\
\hline \multirow[t]{2}{*}{$\mathrm{Pu}$, Mingyu } & 106 & Wang, Li & 43 \\
\hline & & Wang, Linhong & 22 \\
\hline Q & & Wang, Pengbo & 116 \\
\hline Qi, Kang & $35 ; 36$ & Wang, Shixiong & $87 ; 89$ \\
\hline \multirow[t]{3}{*}{ Qu, Yanji } & 20 & Wang, Wenyao & 64 \\
\hline & & Wang, Xiaozhan & 35 \\
\hline & & Wang, Xifu & 40 \\
\hline
\end{tabular}




\begin{tabular}{|c|c|c|c|}
\hline Wang, Xinquan & 57 & Yuan, Jiamin & 39 \\
\hline Wang, Xinyu & 69 & & \\
\hline Wang, Xu & 130 & Z & \\
\hline Wang, Xule & 94 & Zhan, Lijuan & 24 \\
\hline Wang, Yao & 48 & Zhang, Siyao & 127 \\
\hline Wang, Yishi & 44 & Zhang, Bo & $106 ; 107$ \\
\hline Wang, Zengwu & $12 ; 12$ & Zhang, Dong & 99 \\
\hline Wu, Dewei & 120 & Zhang, Haibo & 9 \\
\hline Wu, Honglei & $134 ; 134 ; 134$ & Zhang, Haifeng & 48 \\
\hline \multirow[t]{2}{*}{ Wu, Xiaojing } & 18 & Zhang, Han & $5 ; 95$ \\
\hline & & Zhang, Hao & 35 \\
\hline $\mathrm{X}$ & & Zhang, Heng & $87 ; 87$ \\
\hline Xi, Qunying & 123 & Zhang, Hongju & 123 \\
\hline Xi, Ruixi & 132 & Zhang, Jiahui & 75 \\
\hline Xia, Xiangyang & 61 & Zhang, Jing & 88 \\
\hline Xiang, Quanyong & 19 & Zhang, Kuo & 23 \\
\hline Xiao, Fucheng & $86 ; 88$ & Zhang, Lihua & 7 \\
\hline Xiao, Yan & 34 & Zhang, Linfeng & 10 \\
\hline Xing, Yuanyuan & 121 & Zhang, Maomao & 128 \\
\hline Xiong, Qinmei & 58 & Zhang, Meng & 64 \\
\hline $\mathrm{Xu}, \mathrm{Bo}$ & $71 ; 74$ & Zhang, $\mathrm{Na}$ & 26 \\
\hline $\mathrm{Xu}, \mathrm{Fei}$ & $114 ; 115$ & Zhang, Qianrong & 31 \\
\hline $\mathrm{Xu}$, Jianchao & 20 & Zhang, Rongyuan & 84 \\
\hline $\mathrm{Xu}$, Juan & $2 ; 2$ & Zhang, Rui & 108 \\
\hline $\mathrm{Xu}$, Yang & 7 & Zhang, Siyao & 127 \\
\hline \multirow[t]{2}{*}{ Xue, Sheliang } & 94 & Zhang, Xiaoxing & 116 \\
\hline & & Zhang, Yan & 29 \\
\hline $\mathrm{Y}$ & & Zhang, Yaying & 138 \\
\hline Yan, Jianyun & 37 & Zhang, Yi & $9 ; 9$ \\
\hline Yan, Jun & $82 ; 83$ & Zhang, Yingjie & 50 \\
\hline Yan, Lirong & $21 ; 49$ & Zhang, Yonghui & 89 \\
\hline Yang, Dan & 108 & Zhao, Gang & 46 \\
\hline Yang, Fan & 44 & Zhao, Guifang & 132 \\
\hline Yang, Hang & 125 & Zhao, Hailong & 23 \\
\hline Yang, Jingang & 65 & Zhao, Hui & $44 ; 125$ \\
\hline Yang, $\mathrm{Ke}$ & 52 & Zhao, Kai & 10 \\
\hline Yang, Kunqi & 50 & Zhao, Qinhua & 107 \\
\hline Yang, Lirui & $62 ; 131$ & Zhao, Wen & $53 ; 56$ \\
\hline Yang, Xinwei & $98 ; 100$ & Zhao, Xiaohui & $75 ; 76$ \\
\hline Yang, Yankun & 119 & Zhao, Xin & 63 \\
\hline Yang, Yi & 125 & Zhao, Xue & 99 \\
\hline Yang, Yinjian & 65 & Zhao, Yelin & $72 ; 72 ; 73$ \\
\hline Yang, Yuejin & 61 & Zhen, Yisong & 54 \\
\hline Yao, Xiaoguang & 103 & Zheng, Yaguo & 106 \\
\hline Yao, Yi & 53 & Zhou, Chi & 56 \\
\hline Yao, Yingshui & 4 & Zhou, Gongbu & 100 \\
\hline Ye, Wenxue & 34 & Zhou, Haiting & 138 \\
\hline $\mathrm{Yi}, \mathrm{Fu}$ & 55 & Zhou, Keming & 105 \\
\hline Yin, Dong & 72 & Zhou, Yan & 89 \\
\hline Yin, Gang & 122 & Zhou, Yingying & $135 ; 135$ \\
\hline Yin, Kunlun & 127 & Zhou, Yu & 27 \\
\hline Yin, Ting & 103 & Zhou, Yuzi & 66 \\
\hline You, Jieyun & 117 & Zhou, Zhengyuan & 12 \\
\hline Yu, Shui & 138 & Zou, Changhong & 65 \\
\hline Yu, Shujie & 57 & Zuo, Junli & 19 \\
\hline Yu, Xia & 90 & & \\
\hline
\end{tabular}

